\title{
Molecular Imprinting Science and Technology: A Survey of the Literature for the Years from 2004 to 2011
}

Michael J. Whitcombe, ${ }^{\text {a }}$ Nicole Kirsch ${ }^{\mathrm{b}}$ and lan A. Nicholls ${ }^{\star b, c}$

${ }^{a}$ Department of Chemistry, University of Leicester, LE1 7RH, UK

${ }^{\mathrm{b} B i o o r g a n i c} \&$ Biophysical Chemistry Laboratory, Linnæus University Centre for Biomaterials Chemistry, Linnæus University, SE-39182 Kalmar, Sweden

'Department of Chemistry - BMC, Uppsala University, Box 576, SE-75123 Uppsala, Sweden

*Authors for correspondence: M.J. Whitcombe: ${ }^{a}$ Department of Chemistry, University of Leicester, LE1 7RH, UK. E-mail: mike@mipdatabase.com, Tel: +44-116-2522100, or I.A. Nicholls: ${ }^{\mathrm{b}}$ Bioorganic \& Biophysical Chemistry Laboratory, Linnæus University Centre for Biomaterials Chemistry, Linnæus University, SE-39182 Kalmar, Sweden, E-mail: ian.nicholls@Inu.se Tel: +46-480-446258.

\section{Summary}

Herein we present a survey of the literature covering the development of molecular imprinting science and technology over the years 2004 to 2011. In total, 3779 references to the original papers, reviews, edited volumes and monographs from this period are included, along with recently identified uncited materials from prior to 2004 which were omitted in the first instalment of this series covering the years 1930 to $2003 .{ }^{1}$ In the presentation of the assembled references, a section presenting reviews and monographs covering the area is followed by sections describing fundamental aspects of molecular imprinting including the development of novel polymer formats. Thereafter, literature describing efforts to apply these polymeric materials to a range of application areas is presented. Current trends and areas of rapid development are discussed. 
Keywords: Molecular imprinting, molecularly imprinted polymer, template polymerization

\section{Abbreviations used:}

CE: capillary electrophoresis

CEC: capillary electrochromatography

LC: liquid chromatography

MIP: molecularly imprinted polymer

MISPE: molecular-imprint based solid-phase extraction

NMR: nuclear magnetic resonance spectroscopy

SPE: solid phase extraction

\section{INTRODUCTION}

The continued increase in the volume of literature describing the design, development and application of molecularly imprinted polymers (MIPs) reflects the maturation of this field of study and the broad interest it has attracted from the scientific community in general, Fig. 1.

Insert Figure 1 about here

Figure 1. The number of publications within the field of molecular imprinting science and technology per year for the period 1931-2011. ${ }^{2}$

We previously ${ }^{1}$ presented a comprehensive survey of the field spanning the literature from the seminal work of Polyakov in the 1930s, ${ }^{3}$ until 2003. In this, the second instalment in this series, we cover first reviews, monographs and contributions to edited volumes; followed by work dealing with fundamental aspects of molecular imprinting; the development of novel polymer formats and attempts to apply these polymeric materials to a range of application areas. We conclude with a brief commentary regarding prospects for future developments within this exciting and 
dynamic field of research endeavour. Papers are only cited once, and our classification of papers is based upon our joint assessment of the perceived primary area of impact of each paper.

We have included all material known to us to have been published within the timeframe covered by this survey. With the exception of a limited number of papers of historical significance, we have limited the survey primarily to works published in English. It should be stated that the past few years have witnessed a dramatic increase in the volume of molecular imprinting related literature published in Chinese, and the appearance of some English translations of some key Chinese journals. Furthermore, a number of reports from the period prior to 2004 that were not included in the previous survey have been identified and are herein presented. Of particular historical interest is a series of eight publications in German from the late 1960s by Rackow and colleagues describing efforts to develop non-covalent molecularly imprinted materials using condensation polymers. ${ }^{4-11}$

A small number of errata, corrigenda, comments and replies to comments have been published in the period covered by this review. These are cited immediately following the original paper, with a single exception, ${ }^{12}$ which relates to a paper cited in our previous survey ${ }^{1}$ as reference number 34 .

For the purpose of this survey we have elected to define molecular imprinting as:

"The construction of ligand selective recognition sites in synthetic polymers where a template (atom, ion, molecule, complex or a molecular, ionic or macromolecular assembly, including micro-organisms) is employed in order to facilitate recognition site formation during the covalent assembly of the bulk phase by a polymerization or polycondensation process, with subsequent removal of some or all of the template being necessary for recognition to occur in the spaces vacated by the templating species".

Schematically this can be represented by Figure 2 .

Insert Figure 2 about here

Figure 2. Highly schematic representation of the molecular imprinting process: ${ }^{1}$ The formation of reversible interactions between the template and polymerizable functionality may involve one or more of the following interactions: [(A) reversible 
covalent bond(s), (B) covalently attached polymerizable binding groups that are activated for non-covalent interaction by template cleavage, (C) electrostatic interactions, (D) hydrophobic or van der Waals interactions or (E) co-ordination with a metal centre; each formed with complementary functional groups or structural elements of the template, (a-e) respectively]. A subsequent polymerization in the presence of crosslinker(s), a cross-linking reaction or other process, results in the formation of an insoluble matrix (which itself can contribute to recognition through steric, van der Waals and even electrostatic interactions) in which the template sites reside. Template is then removed from the polymer through disruption of polymertemplate interactions, and extraction from the matrix. The template, or analogues thereof, may then be selectively rebound by the polymer in the sites vacated by template, the 'imprints'. While the representation here is specific to vinyl polymerization, the same basic scheme can equally be applied to sol-gel, polycondensation etc.

\section{REVIEWS AND MONOGRAPHS}

The number of reviews on the topic of molecular imprinting has continued to increase ${ }^{1,13-214}$ which is a direct reflection of the rapidly expanding primary literature covering the area. The increasing incidence of molecular imprinting related material in reviews from other fields indicates that molecular imprinting science and technology has established itself within the broader scientific community. ${ }^{215-501}$ Similar trends are observed in the cases of multi-authored monographs and general scientific reference works. ${ }^{502-627}$ Since the initial survey, a number of books dedicated to the topic have been forthcoming, these provide the scientific public with consolidated presentations of various aspects of the design, preparation, characterization and application of molecularly imprinted polymers. ${ }^{628-681}$

\section{FUNDAMENTAL ASPECTS}

While the evolution of the field runs apace, there is undoubtedly a significant and continuing need for improved fundamental understanding of the mechanisms underlying the molecular imprinting effect and their consequences for the nano-, micro- and macro-level structure and function of imprinted materials. The establishment of a capacity to predict material structure and function is possibly the single factor most likely to hasten the development of molecular imprinting. Over recent years, a range of theoretical and experimental strategies has been deployed to address fundamental aspects of MIPs at nano-, micro- and macro-levels. These efforts, summarized below, have yielded new insights, novel tools, and new formats, 
the latter, in particular, having had considerable impact on the development of MIP application areas.

\section{Theoretical and Computational Studies}

Recent years have seen significant progress in the development and application of theoretical and computational strategies for the elucidation of the mechanisms underlying MIP synthesis and ligand-polymer recognition events, and as a tool for MIP design. ${ }^{682-768}$ The impact of these techniques is seen in the increasing frequency with which they are being used as a predictive tool in the development of new MIP systems. We suggest this trend shall continue and that the further development of computational methods and increases in computing power shall help drive this process.

\section{Spectroscopic Studies}

Traditionally, spectroscopic methods have been invaluable for the study of fundamental aspects of the molecular imprinting process, in particular events taking place in prepolymerization mixtures. The work of recent years ${ }^{769-805}$ has seen a continued use of NMR, IR and UV-Vis spectroscopies for characterizing monomertemplate interactions, to the point where such studies are becoming essential aspects of MIP design, either for the screening of monomers for interaction with template, or for the validation of computational design data. A recent trend is the significant increase in the number of spectroscopic studies of ligand-MIP interactions. To these ends, Raman spectroscopy, light scattering, circular dichroism and various fluorescence and chemiluminescence based techniques have also been deployed. The use of XAFS, diffraction studies, XPS and high resolution AFM has become more prominent. AFM and various fluorescence techniques have played important roles in the characterization of thin-film MIPs. We perceive that these trends shall continue, especially for probing polymer structure and for the direct study of polymer-ligand binding events.

\section{Recognition}

The demonstration of template (or template analog)-selective recognition by a MIP remains a cornerstone in the development of all new MIP systems. Various strategies have been employed to characterize ligand-polymer binding events, ${ }^{806-1196}$ which have in turn provided fundamental insights, and in some cases inspiration, for the development of new applications; such as protein-imprinted hydrogels capable of nucleating protein crystallization. ${ }^{1197,1198}$ Radioligand binding studies and chromatographic, electrochemical, gravimetric and spectroscopic based analyses, or combinations thereof, have been important for demonstrating molecular imprinting effects. 


\section{Formats}

The development of alternatives to the synthesis of traditional monolithic polymers has been driven by both practical considerations, for example: ease of scale-up, requirements for integration with other technologies, such as sensors, and for performance issues, e.g. mass transfer or flow properties. Methods for preparation of discrete MIP particles, composites and molecularly imprinted polymer surfaces and fibers are areas that have been gaining in prominence over recent years and now constitute a significant portion of the original literature published in the molecular imprinting field. ${ }^{1199-1704}$ The importance of new methods for MIP synthesis and regulation of polymer morphology and structure in general, is a critical factor in the development of materials for new applications.

\section{APPLICATIONS}

Historically, the development of molecularly imprinted polymer science and technology has been driven by application development, as reflected in the abundant patent literature covering the area. The dramatic increase in the volume of scientific literature in the field has been closely paralleled by developments in the patent literature. Interestingly, while companies and governmental agencies are well represented in the MIP intellectual property sector, it is academic institutions that have so-far been the greatest contributors. Here we categorized application oriented papers under seven application headings.

\section{Solid Phase Extraction}

The development of MIPs as selective adsorbents in solid phase extraction (often referred to as MISPE) was a natural progression from early work aimed at developing MIP chromatographic stationary phases. This is the application area that has found most commercial viability, as witnessed by the presence of several products on the market. The bulk of the MISPE studies reported to date $e^{1705-2331}$ has been directed to (small) organic structures, with substances of clinical and environmental impact of particular interest. SPE systems for analysis of ionic and inorganic materials have also received considerable attention. ${ }^{2332-2535}$ As the demand for more sensitive analytical techniques continues to grow, so too should the need for robust technologies such as MISPE. Access to MIPs in new formats should further improve the use of these selective materials. 
Assays

Efforts to employ MIPs in assays has extended well beyond the large volume of literature focused upon MIPSE, most notably capillary electrophoresis (CE) and capillary electrochromatography (CEC), ${ }^{2536-2588}$ and liquid chromatography (LC). ${ }^{2589-}$ 2722 In contrast to the 90's and early 00's, the proportion of studies devoted to, or heavily dependent upon, liquid chromatography (LC) has reduced significantly. In the cases of CE and CEC, however, interest in these techniques has been sustained. We suggest that this development is in part fuelled by the sensitivities of these techniques and in part by developments in the range of chemistries used for preparing MIP-containing capillaries.

A number of papers report assays based upon competitive ligand binding studies. ${ }^{2723-2763}$ The high sensitivities of assays using either radiolabeled or nonisotopically labeled studies (fluorescence and chemiluminescence) afford access to events taking place at very low concentration regimes that are not generally accessible with chromatographic methods. Interestingly these methods appear to be being superseded by the development of chemical sensors and biosensors based on MIPs where the binding event gives rise to a measurable response by various transduction mechanisms.

\section{Sensors}

The application of MIPs in the development of sensors has continued to flourish. It could be argued that the increased use of MIPs in sensor development has been in part driven by developments in MIP formats, not least by the establishment of reproducible strategies for thin film, fiber and (nano)particle preparation. Optical, ${ }^{2764-}$ 2995 electrochemical $^{2996-3310}$ and acoustic $^{3311-3430}$ sensor platforms are those most frequently reported in conjunction with MIP recognition elements are based upon either platforms. The potential of molecular imprinting in sensor development is reflected in the current rapid growth in the number of reports of devices with MIPbased electrochemical transduction mechanisms. The appearance of a limited number of studies using calorimetric ${ }^{3431-3434}$ and mass spectrometric detection ${ }^{3435,3436}$ over recent years may provide an indication of areas were MIP-based sensors may find increasing use in the future. 


\section{Membranes}

The majority of reported molecularly imprinted membrane studies have as an ultimate goal either process development (in particular for separation and purification) or incorporation with sensors. ${ }^{3437-3554}$ While efforts to develop and apply molecularly imprinted membranes continue, it remains clear that more work is required to address the mechanisms underlying membrane function.

\section{Catalysis and Synthesis}

While much has been achieved in terms of the development of MIPs capable of recognizing a given molecular species, relatively few examples exist of polymers with enzyme-like activity. In principle, a MIP with enzyme-like activity should recognize substrate(s), orchestrate the making and/or breaking of bonds, and expel the product to prepare for a new cycle. This may rightly be deemed a daunting task. Nonetheless, MIP-based catalytic systems and the use of MIPs in synthesis continues to evolve. ${ }^{3555-3672}$ Recent reports of several systems with significant catalytic power bodes well for the continued development in this sector.

\section{MIPs meet biology}

The integration of MIPs in, or interaction of MIPs with, biological systems is perhaps the area that has drawn most attention from the scientific community at large over recent years. Two themes dominate this sector, namely the use of MIPs for drug delivery ${ }^{3673-3737}$ and the development of MIPs with biological function. ${ }^{3738-3777}$ The use of molecular imprinted materials in conjunction with ocular drug delivery is arguably the sector that has come closest to clinical use. Perhaps even more challenging is the concept of developing MIPs that interact directly with biological systems; efforts to this end appear to be gaining in pace and number. The first use of MIPs in vivo, ${ }^{3778}$ for sequestration of a toxin, constitutes a highly significant breakthrough for the field as a whole, Fig. 3. Finally, the idea of molecular imprinting-related processes constituting a possible mechanism for the creation of Life has been proposed. ${ }^{3779}$

Insert Figure 3 about here 
Figure 3. The sequestration of the toxic peptide melittin (a component of bee venom) from the blood stream of living mice by MIP nanoparticles (NPs) presented by Hoshino and Shea $(178,3778)$. a) Survival rates over a period of $24 \mathrm{~h}$ following the intravenous administration of melittin $(4.5 \mathrm{mg} / \mathrm{Kg})$. NPs $(30 \mathrm{mg} / \mathrm{Kg})$ were administered 20s after the toxin: MIPs (red), NIPs (grey), control group (green). Significant survival rates were seen in the case of MIP-adminstered mice (Wilcoxon $P$ values). b) Fluorescent imaging of Cy5-labelled melittin shows that binding to MIP NPs results in clearance of the toxin from the peripheral circulation and transportation to the liver in the treated mice (right) while in the untreated control group (left) the toxin remains widely distributed throughout the body. c) Fluorescence intensity imaging of the livers of mice treated with different concentrations of Cy5melittin and MIP NPs. Reproduced from Ref. 189 with permission from The Royal Society of Chemistry.

\section{SUMMARY AND FUTURE OUTLOOK}

The continued growth of research activity within the field of molecular imprinting is reflected in the dramatic increase in the scientific literature covering the area over recent years. While significant improvements in our understanding of the molecular imprinting process have been achieved, and many new types of polymer, polymer format and application area have been investigated, a number of significant fundamental challenges regarding the mechanisms underlying the technique remain to be explored and many opportunities for applications are still to be exploited. Accordingly, we envisage a continued growth in research activity in the area over the coming years, and a concomitant increase in the volume of literature dealing with the science and technology of molecular imprinting.

\section{ACKNOWLEDGEMENTS}

We gratefully acknowledge the financial support of: Cranfield University to MJW; and the Swedish Research Council (Vetenskapsrådet), Swedish Knowledge Foundation, Linnæus University and Uppsala University to IAN.

\section{REFERENCES}

1. Alexander C, Andersson HS, Andersson LI, Ansell RJ, Kirsch N, Nicholls IA, O'Mahony J, Whitcombe MJ. 2006. Molecular imprinting science and technology: a survey of the literature for the years up to and including 2003. J. Mol. Recognit. 19: 106-180. DOI: 10.1002/jmr.760

2. Data taken from mipdabase.com: (http://mipdatabase.com/all_items.php) date accessed March 262013. 
3. Polyakov MV. 1931. Adsorption properties and structure of silica gel. Zhur. Fiz. Khim. 2: 799-805.

4. Rackow B, Curdt H. 1969. Stereospecific imprint of molecular information on resin surfaces and testing with stereoisomers by crossing experiments. Ber. Bunsenges. 73: 452-455.

5. Rackow B, Curdt H. 1968. Information chemical cross experiments on stereospecific imprinting of functional group distances on resin surfaces. XXVII. Z. Chem. 8: 438.

6. Rackow B. 1968. Imprinting of molecular information on resin surfaces and mathematical and graphical determination of their information content. Ber. Bunsenges. 72: 110-116.

7. Rackow B, Süssenbach D. 1967. Imprinting of molecular information on resin surfaces and testing of a multidentate imprint with monodentate "guest." XXII. Z. Chem. 7: 444-445.

8. Rackow B. 1967. Imprinting of molecular information on surfaces of organic high polymers. Z. Chem. 7: 398-399.

9. Rackow B. 1967. Deviation of "fundamental equation of information chemistry" for molecularly imprinted polymers. Z. Chem. 7: 472-473.

10. Rackow B. 1968. Application of the "fundamental equation of information chemistry" for a resin imprinted with copper-phthalocyaninetetrasulfonic acid and graphic determination of its information via sulfonic acid and methyl orange. XXIV. Z. Chem. 8: 33.

11. Rackow B, Süssenbach D. 1968. In vitro experiments to impinge molecular folding information on organic polymers. Vol. 1st Biokybernetik, Mater. Int. Symp., Drischel H (ed). Karl Marx University: Leipzig; 246-249. 
12. Rimmer S. 2008. Synthesis of Molecular Imprinted Polymer Networks. Chromatographia 67: 343. DOI: 10.1365/s10337-007-0496-z

13. Amato I. 1991. Making choosy molecules. Science 253: 1358. DOI: 10.1126/science.253.5026.1358

14. Pickup J. 1995. Where sensors lead. The Lancet 346: 1572-1573. DOI: 10.1016/S0140-6736(95)91924-4

15. Wallich P. 1996. Molecular molds - Plastic replicas mimic complex molecules. Scientific American 275: 45-46.

16. Derrington A. 27-Sep-1997. A dainty print and a glass slipper: The Nature of Things: If the shoe fits, chemists will detect drugs and poisons more readily, finds Andrew Derrington. Financial Times, London 2.

17. Dickert FL, Forth $\mathrm{P}$, Lieberzeit $\mathrm{P}$, Tortschanoff M, Bulst WE, Knauer U, Fischerauer G. 1997. Molecular imprinting of chemically sensitive coatings New strategies for sensor design and fabrication. In Proceedings of Sensor '97, Vol. 1, S137-S142.

18. Dickert FL, Greibl W, Hayden O, Lieberzeit P, Tortschanoff M, Bulst WE, Knauer U, Fischerauer G. 1998. Chemosensoren mit molekulargeprägten Erkennungsschichten - Technologie der analytspezifischen Sensoradaption. In ITG-Fachbericht, VDE-Verlag: Berlin; 133-138.

19. Dickert FL, Hayden O, Halikias K, Lieberzeit P, Rohrer A, Bulst WE, Wolff U, Voigt G. 2000. Molekular geprägte Chemosensoren für die Qualitätskontrolle. VDI Berichte 1530: 1051-1056.

20. Severin K. 2002. Molecularly Imprinted Polymers. Man-Made Mimics of Antibodies and their Applications in Analytical Chemistry. (Book Review). Angew. Chem. Int. Edit. 41: 1071. DOI: 10.1002/15213773(20020315)41:6<1071::AID-ANIE1071>3.0.CO;2-C 
21. Kindschy LM, Alocilja EC. 2003. A review of molecularly imprinted polymers for biosensor development for food and agricultural applications. In Proceedings, 2003 ASAE Annual Meeting, American Society of Agricultural and Biological Engineers: Paper No. 037004.

22. Shea JJ. 2003. Molecular Imprinting-From Fundamentals to Applications [Book Review]. IEEE Electrical Insulation Magazine 19: 74. DOI: 10.1109/MEI.2003.1238726

23. Dickert FL, Bindeus R, Fassnauer M, Hayden O, Lieberzeit $\mathrm{P}$, Mann KJ, Horner G. 2003. Parallel-Processing of Chemically Sensitive Layers by SelfAssembling - Structuring of Bulk Materials and Surfaces. In Proceedings of Sensors 2003, Vol. 1, 135-141.

24. Lai EPC. 2003. New Developments Towards the Use of Molecularly Imprinted Polymers in Drug Discovery. Business Briefings. http://www.touchbriefings.com/pdf/17/pt031_p_lai.pdf

25. Wilson ID. 2000. Breaking the mould: molecular imprints in separation and analysis. CAST, Chromatography and Separation Technology 6-10.

26. Nicholls IA, Andersson LI. 2001. Foreword. Bioseparation 10: 255. DOI: 10.1023/A:1021502000885

27. Andersson LI, Nicholls IA. 2004. Molecularly imprinted polymers in separation science - Foreword. J. Chromatogr. B 804: 1. DOI: 10.1016/j.jchromb.2004.02.041

28. Ansell RJ. 2004. Molecularly imprinted polymers in pseudoimmunoassay. J. Chromatogr. B 804: 151-165. DOI: 10.1016/j.jchromb.2004.02.022

29. Becker JJ, Gagné MR. 2004. Exploiting the synergy between coordination chemistry and molecular imprinting in the quest for new catalysts. Acc. Chem. Res. 37: 798-804. DOI: 10.1021/ar000102u 
30. Boyd JW, Cobb GP, Southard GE, Murray GM. 2004. Development of molecularly imprinted polymer sensors for chemical warfare agents. Johns Hopkins APL Techn. Dig. 25: 44-49.

31. Chapuis F, Pichon V, Hennion MC. 2004. Molecularly imprinted polymers: Developments and applications of new selective solid-phase extraction materials. LC-GC Europe 17: 408-417.

32. Cormack PAG, Elorza AZ. 2004. Molecularly imprinted polymers: synthesis and characterisation. J. Chromatogr. $B$ 804: 173-182. DOI: 10.1016/j.jchromb.2004.02.013

33. Davies MP, de Biasi V, Perrett D. 2004. Approaches to the rational design of molecularly imprinted polymers. Anal. Chim. Acta 504: 7-14. DOI: 10.1016/S0003-2670(03)00812-2

34. Dickert FL, Lieberzeit PA, Gazda-Miarecka S, Halikias K, Bindeus R. 2004. Imprinting with chemical sensors - Challenges in molecular recognition and universal application. In Molecularly Imprinted Materials-2003, MRS Symposium Proceedings, Vol. 787, Kofinas P, Sellergren B, Roberts MJ (eds). Materials Research Society: Warrendale; 71-77. DOI: 10.1557/PROC787-G5.4

35. Dmitrienko SG, Irkha VV, Kuznetsova AY, Zolotov YA. 2004. Use of molecular imprinted polymers for the separation and preconcentration of organic compounds. J. Anal. Chem. 59: 808-817. DOI: 10.1023/B:JANC.0000040694.23348.45

36. Haginaka J. 2004. Molecularly imprinted polymers for solid-phase extraction. Anal. Bioanal. Chem. 379: 332-334. DOI: 10.1007/s00216-004-2621-2

37. Hilt JZ, Byrne ME. 2004. Configurational biomimesis in drug delivery: molecular imprinting of biologically significant molecules. Adv. Drug Deliv. Rev. 56: 1599-1620. DOI: 10.1016/j.addr.2004.04.002 
38. Kandimalla VB, Ju HX. 2004. Molecular imprinting: a dynamic technique for diverse applications in analytical chemistry. Anal. Bioanal. Chem. 380: 587605. DOI: 10.1007/s00216-004-2793-9

39. Kindschy LM, Alocilja EC. 2004. A review of molecularly imprinted polymers for biosensor development for food and agricultural applications. Trans. ASAE 47: 1375-1382.

40. Lanza F, Sellergren B. 2004. Molecularly imprinted polymers via highthroughput and combinatorial techniques. Macromol. Rapid Commun. 25: 59-68. DOI: 10.1002/marc.200300211

41. Lavignac N, Allender CJ, Brain KR. 2004. Current status of molecularly imprinted polymers as alternatives to antibodies in sorbent assays. Anal. Chim. Acta 510: 139-145. DOI: 10.1016/j.aca.2003.12.066

42. Liu CY, Lin CC. 2004. An insight into molecularly imprinted polymers for capillary electrochromatography. Electrophoresis 25: 3997-4007. DOI: 10.1002/elps.200406160

43. Martin-Esteban A. 2004. Molecular imprinting technology: a simple way of synthesizing biomimetic polymeric receptors. Anal. Bioanal. Chem. 378: 1875. DOI: 10.1007/s00216-004-2502-8

44. Nilsson J, Spégel P, Nilsson S. 2004. Molecularly imprinted polymer formats for capillary electrochromatography. J. Chromatogr. B 804: 3-12. DOI: 10.1016/j.jchromb.2003.12.036

45. Striegler S. 2004. Designing selective sites in templated polymers utilizing coordinative bonds. J. Chromatogr. B 804: 183-195. DOI: 10.1016/j.jchromb.2004.01.065

46. Turiel E, Martin-Esteban A. 2004. Molecularly imprinted polymers: towards highly selective stationary phases in liquid chromatography and capillary 
electrophoresis. Anal. Bioanal. Chem. 378: 1876-1886. DOI: 10.1007/s00216-003-2331-1

47. Ulbricht M. 2004. Membrane separations using molecularly imprinted polymers. J. Chromatogr. B 804: 113-125. DOI: 10.1016/j.jchromb.2004.02.007

48. Umpleby RJ, Baxter SC, Rampey AM, Rushton GT, Chen YZ, Shimizu KD. 2004. Characterization of the heterogeneous binding site affinity distributions in molecularly imprinted polymers. J. Chromatogr. B 804: 141149. DOI: $10.1016 /$ j.jchromb.2004.01.064

49. Xu XJ, Zhu LL, Chen LR. 2004. Separation and screening of compounds of biological origin using molecularly imprinted polymers. J. Chromatogr. $B$ 804: 61-69. DOI: 10.1016/j.jchromb.2004.02.012

50. Ye L, Haupt K. 2004. Molecularly imprinted polymers as antibody and receptor mimics for assays, sensors and drug discovery. Anal. Bioanal. Chem. 378: 1887-1897. DOI: 10.1007/s00216-003-2450-8

51. Allender CJ. 2005. Molecularly imprinted polymers: technology and applications. Adv. Drug Deliv. Rev. 57: 1731-1732. DOI: 10.1016/j.addr.2005.08.001

52. Anon. 2005. Imprints are not footprints. Chem. Eng. News 83: 52.

53. Ansell RJ. 2005. Molecularly imprinted polymers for the enantioseparation of chiral drugs. Adv. Drug Deliv. Rev. 57: 1809-1835. DOI: 10.1016/j.addr.2005.07.014

54. Cunliffe D, Kirby A, Alexander C. 2005. Molecularly imprinted drug delivery systems. Adv. Drug Deliv. Rev. 57: 1836-1853. DOI: 10.1016/j.addr.2005.07.015 
55. Díaz-García ME, Laíño RB. 2005. Molecular imprinting in sol-gel materials: Recent developments and applications. Microchim. Acta 149: 19-36. DOI: 10.1007/s00604-004-0274-7

56. Haginaka J. 2005. Selectivity of affinity media in solid-phase extraction of analytes. TrAC, Trends Anal. Chem. 24: 407-415. DOI: 10.1016/j.trac.2004.12.005

57. Henry OYF, Cullen DC, Piletsky SA. 2005. Optical interrogation of molecularly imprinted polymers and development of MIP sensors: a review. Anal. Bioanal. Chem. 382: 947-956. DOI: 10.1007/s00216-005-3255-8

58. Hillberg AL, Brain KR, Allender CJ. 2005. Molecular imprinted polymer sensors: Implications for therapeutics. Adv. Drug Deliv. Rev. 57: 1875-1889. DOI: 10.1016/j.addr.2005.07.016

59. Karim K, Breton F, Rouillon R, Piletska EV, Guerreiro A, Chianella I, Piletsky SA. 2005. How to find effective functional monomers for effective molecularly imprinted polymers? Adv. Drug Deliv. Rev. 57: 1795-1808. DOI: 10.1016/j.addr.2005.07.013

60. O'Mahony J, Nolan K, Smyth MR, Mizaikoff B. 2005. Molecularly imprinted polymers - potential and challenges in analytical chemistry. Anal. Chim. Acta 534: 31-39. DOI: 10.1016/j.aca.2004.07.043

61. Mayes AG, Whitcombe MJ. 2005. Synthetic strategies for the generation of molecularly imprinted organic polymers. Adv. Drug Deliv. Rev. 57: 17421778. DOI: 10.1016/j.addr.2005.07.011

62. Piacham T, Ayudhya CIN, Boonpangrak S, Nantasenamat C, Suksrichavalit T, Bülow L, Ye L, Prachayasittikul V. 2005. Molecularly imprinted polymer as a tool for nanobiosensors and enzyme mimics. Vol. Proceedings of the $31 \mathrm{st}$ Congress on Science and Technology of Thailand at Suranaree University of Technology, Art. No. E0119. 
63. Rathbone DL. 2005. Molecularly imprinted polymers in the drug discovery process. Adv. Drug Deliv. Rev. 57: 1854-1874. DOI: 10.1016/j.addr.2005.07.017

64. Sadecka J, Polonsky J. 2005. Molecularly imprinted polymers in analytical chemistry. Chem. Listy 99: 222-230.

65. Sellergren B, Allender CJ. 2005. Molecularly imprinted polymers: A bridge to advanced drug delivery. Adv. Drug Deliv. Rev. 57: 1733-1741. DOI: 10.1016/j.addr.2005.07.010

66. Spivak DA. 2005. Optimization, evaluation, and characterization of molecularly imprinted polymers. Adv. Drug Deliv. Rev. 57: 1779-1794. DOI: 10.1016/j.addr.2005.07.012

67. Spivak DA. 2006. Corrigendum to "Optimization, evaluation, and characterization of molecularly imprinted polymers" [Advanced Drug Delivery Reviews 57 (2005) 1779-1794]. Adv. Drug Deliv. Rev. 58: 116. DOI: 10.1016/j.addr.2005.07.020

68. Takeuchi T, Mukawa T, Shinmori H. 2005. Signaling molecularly imprinted polymers: Molecular recognition-based sensing materials. Chem. Rec. 5: 263-275. DOI: 10.1002/tcr.20052

69. Takeuchi T, Mukawa T, Shinmori H. 2005. Corrigendum to 'Signaling molecularly imprinted polymers: Molecular recognition-based sensing materials'. Chem. Rec. 5: 410-411. DOI: 10.1002/tcr.20068

70. Tarley CRT, Taboada-Sotomayor MDP, Kubota LT. 2005. Biomimetic polymers in analytical chemistry. Part 1: Preparation and applications of MIP (molecularly imprinted polymers) in extraction and separation techniques. Quimica Nova 28: 1076-1086. DOI: 10.1590/S0100-40422005000600023

71. Tarley CRT, Taboada-Sotomayor MDP, Kubota LT. 2005. Biomimetic polymers in analytical chemistry. Part 2: Applications of MIP (Molecularly 
Imprinted Polymers) in the development of chemical sensors. Quimica Nova 28: 1087-1101. DOI: 10.1590/S0100-40422005000600025

72. Thiesen PH, Niemeyer B. 2005. Customised adsorbent materials in the application spectra of Bio-, medicine- and environmental technology. Chem. -Ing. -Tech. 77: 373-383. DOI: 10.1002/cite.200500003

73. Turiel E, Martin-Esteban A. 2005. Molecular imprinting technology in capillary electrochromatography. J. Sep. Sci. 28: 719-728. DOI: $10.1002 /$ jssc.200400076

74. van Nostrum CF. 2005. Molecular imprinting: A new tool for drug innovation. Drug Discov. Today Technol. 2: 119-124. DOI: 10.1016/j.ddtec.2005.05.004

75. Zhang ZY, Zhang SC, Zhang XR. 2005. Recent developments and applications of chemiluminescence sensors. Anal. Chim. Acta 541: 37-46. DOI: 10.1016/j.aca.2004.11.069

76. Tóth B, Pap T, Horváth V, Horvai G. 2005. Molecularly imprinted polymers: optimization of their preparation by combinatorial methods and applications in non-linear chromatography and binding assays. Molekuláris lenyomatú polimerek: kombinatorikus elõállítás, nemlineáris kromatográfi a és pszeudo-immunanalitika. Magyar Kémiai Folyóirat 111: 110-113.

77. Anon. 2006. Nanocytes ${ }^{\circledR}$ - Nanostrukturierte Biomimetische Partikel für die Molekulare Erkennung. Bunsen Mag. 122-123.

78. Baggiani C, Anfossi L, Giovannoli C. 2006. Molecular imprinted polymers: Useful tools for pharmaceutical analysis. Curr. Pharm. Anal. 2: 219-247. DOI: $10.2174 / 157341206777934662$

79. Caro E, Marce RM, Borrull F, Cormack PAG, Sherrington DC. 2006. Application of molecularly imprinted polymers to solid-phase extraction of compounds from environmental and biological samples. TrAC, Trends Anal. Chem. 25: 143-154. DOI: 10.1016/j.trac.2005.05.008 
80. Díaz-García ME, Pina-Luis G, Rivero IA. 2006. Combinatorial solid-phase organic synthesis for developing materials with molecular recognition properties. TrAC, Trends Anal. Chem. 25: 112-121. DOI: 10.1016/j.trac.2005.12.001

81. Fernández-González A, Guardia L, Badía-Laíno R, Díaz-García ME. 2006. Mimicking molecular receptors for antibiotics - analytical implications. TrAC, Trends Anal. Chem. 25: 949-957. DOI: 10.1016/j.trac.2006.08.001

82. Lisichkin GV, Krutyakov YA. 2006. Molecularly imprinted materials: Synthesis, properties, applications. Russ. Chem. Rev. 75: 901-918. DOI: 10.1070/RC2006v075n10ABEH003618

83. Mosbach K. 2006. The promise of molecular imprinting. Scientific American 295: 86-91. DOI: 10.1038/scientificamerican1006-86

84. Niece KL. 2006. Molecularly imprinted polyurethane offers economically viable route to blood typing. MRS Bull. 31: 437-438. DOI: $10.1557 / \mathrm{mrs} 2006.110$

85. Peppas NA. 2006. Intelligent biomaterials as pharmaceutical carriers in microfabricated and nanoscale devices. MRS Bull. 31: 888-893.

86. Piletsky SA, Turner NW, Laitenberger P. 2006. Molecularly imprinted polymers in clinical diagnostics - Future potential and existing problems. Med. Eng. Phys. 28: 971-977. DOI: 10.1016/j.medengphy.2006.05.004

87. Qiao FX, Sun HW, Yan HY, Row KH. 2006. Molecularly Imprinted Polymers for Solid Phase Extraction. Chromatographia V64: 625-634. DOI: 10.1365/s10337-006-0097-2

88. Rao TP, Kala R, Daniel S. 2006. Metal ion-imprinted polymers--Novel materials for selective recognition of inorganics. Anal. Chim. Acta 578: 105116. DOI: 10.1016/j.aca.2006.06.065 
89. Row KH. 2006. Molecularly Imprinted Polymers for Solid Phase Extraction. Chromatographia 64: 625-634. DOI: 10.1365/s10337-006-0097-2

90. Schillemans JP, van Nostrum CF. 2006. Molecularly imprinted polymer particles: synthetic receptors for future medicine. Nanomedicine 1: 437-447. DOI: $10.2217 / 17435889.1 .4 .437$

91. Turner NW, Jeans CW, Brain KR, Allender CJ, Hlady V, Britt DW. 2006. From 3D to 2D: A Review of the Molecular Imprinting of Proteins. Biotechnol. Prog. 22: 1474-1489. DOI: 10.1021/bp060122g

92. Wei ST, Jakusch M, Mizaikoff B. 2006. Capturing molecules with templated materials--Analysis and rational design of molecularly imprinted polymers. Anal. Chim. Acta 578: 50-58. DOI: 10.1016/j.aca.2006.06.077

93. Widstrand C, Yilmaz E, Boyd B, Rees A. 2006. Selective extractions by molecularly imprinted polymers (MIPs). The Column 2: 20-24.

94. Widstrand C, Bjork H, Yilmaz E. 2006. Analysis of analytes - The use of MIPs in solid-phase extraction increases efficiency and improves detection limits. Laboratory News 14-15.

95. Widstrand C, Yilmaz E, Boyd B, Billing J, Rees A. 2006. Molecularly imprinted polymers: A new generation of affinity matrices. Am. Lab. 38: 12.

96. Xu XJ. 2006. New concepts and approaches for drug discovery based on traditional Chinese medicine. Drug Discov. Today Technol. 3: 247-253. DOI: 10.1016/j.ddtec.2006.09.008

97. Yan H, Row KH. 2006. Characteristic and synthetic approach of molecularly imprinted polymer. Int. J. Mol. Sci. 7: 155-178. DOI: 10.3390/i7050155

98. Zhang HQ, Ye L, Mosbach K. 2006. Non-covalent molecular imprinting with emphasis on its application in separation and drug development. J. Mol. Recognit. 19: 248-259. DOI: 10.1002/jmr.793 
99. Baggiani C, Anfossi L, Giovannoli C. 2007. Solid phase extraction of food contaminants using molecular imprinted polymers. Anal. Chim. Acta 591: 29-39. DOI: 10.1016/j.aca.2007.01.056

100. Binet C, Ferrere S, Lattes A, Laurent E, Marty JD, Mauzac M, Mingotaud AF, Palaprat G, Weyland M. 2007. Benefit of liquid crystal moieties in the MIP technique. Anal. Chim. Acta 591: 1-6. DOI: 10.1016/j.aca.2006.12.042

101. Bossi A, Bonini F, Turner APF, Piletsky SA. 2007. Molecularly imprinted polymers for the recognition of proteins: The state of the art. Biosens. Bioelectron. 22: 1131-1137. DOI: 10.1016/j.bios.2006.06.023

102. Dickert F. 2007. Molecular imprinting (editorial). Anal. Bioanal. Chem. 389: 353-354. DOI: 10.1007/s00216-007-1479-5

103. García-Calzón JA, Díaz-García ME. 2007. Characterization of binding sites in molecularly imprinted polymers. Sens. Actuators B 123: 1180-1194. DOI: 10.1016/j.snb.2006.10.068

104. Hansen DE. 2007. Recent developments in the molecular imprinting of proteins. Biomaterials 28: 4178-4191. DOI: 10.1016/j.biomaterials.2007.06.017

105. He CY, Long YY, Pan JL, Li K, Liu F. 2007. Application of molecularly imprinted polymers to solid-phase extraction of analytes from real samples. J. Biochem. Biophys. Meth. 70: 133-150. DOI: 10.1016/j.jbbm.2006.07.005

106. Holthoff EL, Bright FV. 2007. Molecularly templated materials in chemical sensing. Anal. Chim. Acta 594: 147-161. DOI: 10.1016/j.aca.2007.05.044

107. Holthoff EL, Bright FV. 2007. Molecularly Imprinted Xerogels as Platforms for Sensing. Acc. Chem. Res. 40: 756-767. DOI: 10.1021/ar700087t 
108. Janiak DS, Kofinas P. 2007. Molecular imprinting of peptides and proteins in aqueous media. Anal. Bioanal. Chem. 389: 399-404. DOI: 10.1007/s00216007-1327-7

109. Jiang XM, Jiang N, Zhang HX, Liu MC. 2007. Small organic molecular imprinted materials: their preparation and application. Anal. Bioanal. Chem. 389: 355-368. DOI: 10.1007/s00216-007-1336-6

110. Lachová M, Lehotay J, Cizmárik A. 2007. Applications of molecularly imprinted polymers in analytical and pharmaceutical chemistry. Ceska Slov. Farm. 56: 159-164.

111. Liu ZS, Zheng C, Yan C, Gao RY. 2007. Molecularly imprinted polymers as a tool for separation in CEC. Electrophoresis 28: 127-136. DOI: 10.1002/elps.200600544

112. Maier NM, Lindner W. 2007. Chiral recognition applications of molecularly imprinted polymers: a critical review. Anal. Bioanal. Chem. 389: 377-397. DOI: $10.1007 /$ s00216-007-1427-4

113. McCluskey A, Holdsworth Cl, Bowyer MC. 2007. Molecularly imprinted polymers (MIPs): sensing, an explosive new opportunity? Org. Biomol. Chem. 5: 3233-3244. DOI: 10.1039/b708660a

114. Mehta R. 2007. Making a mark. Materials World 15: 11.

115. Pichon V. 2007. Selective sample treatment using molecularly imprinted polymers. J. Chromatogr. A 1152: 41-53. DOI: 10.1016/j.chroma.2007.02.109

116. Stephenson CJ, Shimizu KD. 2007. In focus: Colorimetric and fluorometric molecularly imprinted polymer sensors and binding assays. Polym. Int. 56: 482-488. DOI: 10.1002/pi.2206 
117. Tamayo FG, Turiel E, Martín-Esteban A. 2007. Molecularly imprinted polymers for solid-phase extraction and solid-phase microextraction: Recent developments and future trends. J. Chromatogr. A 1152: 32-40. DOI: 10.1016/j.chroma.2006.08.095

118. Tan CJ, Tong YW. 2007. Molecularly imprinted beads by surface imprinting. Anal. Bioanal. Chem. 389: 369-376. DOI: 10.1007/s00216-007-1362-4

119. Uludag Y, Piletsky SA, Turner APF, Cooper MA. 2007. Piezoelectric sensors based on molecular imprinted polymers for detection of low molecular mass analytes. FEBS J. 274: 5471-5480. DOI: 10.1111/j.1742-4658.2007.06079.x

120. Wei ST, Mizaikoff B. 2007. Recent advances on noncovalent molecular imprints for affinity separations. J. Sep. Sci. 30: 1794-1805. DOI: $10.1002 /$ jssc.200700166

121. Zhou X, Li WY, He XW, Chen LX, Zhang YK. 2007. Recent Advances in the Study of Protein Imprinting. Sep. Purif. Rev. 36: 257-283. DOI: $10.1080 / 15422110701771425$

122. Anon. 2008. Honey trap. Nature 456: 284.

123. Ávila M, Zougagh M, Ríos A, Escarpa A. 2008. Molecularly imprinted polymers for selective piezoelectric sensing of small molecules. TrAC, Trends Anal. Chem. 27: 54-65. DOI: 10.1016/j.trac.2007.10.009

124. Baggiani C, Anfossi L, Giovannoli C. 2008. Molecular imprinted polymers as synthetic receptors for the analysis of myco- and phyco-toxins. Analyst 133: 719-730. DOI: 10.1039/b711352h

125. BelBruno JJ, Richter A, Gibson UJ. 2008. Amazing pores: Processing, morphology and functional states of molecularly imprinted polymers as sensor materials. Mol. Cryst. Liq. Cryst. 483: 179-190. DOI: 10.1080/15421400801905135 
126. Bergmann NM, Peppas NA. 2008. Molecularly imprinted polymers with specific recognition for macromolecules and proteins. Prog. Polym. Sci. 33: 271-288. DOI: 10.1016/j.progpolymsci.2007.09.004

127. Byrne ME, Salian V. 2008. Molecular imprinting within hydrogels II: Progress and analysis of the field. Int. J. Pharm. 364: 188-212. DOI: 10.1016/j.jpharm.2008.09.002

128. De Smet D, De Saeger S, Van Peteghem C. 2008. Molecularly imprinted polymers for selective extraction of mycotoxins in food. New Food. http://www.newfoodmagazine.com/1213/new-food-magazine/pastissues/molecularly-imprinted-polymers-for-selective-extraction-ofmycotoxins-in-food/

129. Ge Y, Turner APF. 2008. Too large to fit? Recent developments in macromolecular imprinting. Trends Biotechnol. 26: 218-224. DOI: 10.1016/j.tibtech.2008.01.001

130. Giovannoli C, Baggiani C, Anfossi L, Giraudi G. 2008. Aptamers and molecularly imprinted polymers as artificial biomimetic receptors in affinity capillary electrophoresis and electrochromatography. Electrophoresis 29: 3349-3365. DOI: 10.1002/elps.200800004

131. Guan GJ, Liu BH, Wang ZY, Zhang ZP. 2008. Imprinting of Molecular Recognition Sites on Nanostructures and Its Applications in Chemosensors. Sensors 8: 8291-8320. DOI: 10.3390/s8128291

132. Haginaka J. 2008. Monodispersed, molecularly imprinted polymers as affinity-based chromatography media. J. Chromatogr B 866: 3-13. DOI: 10.1016/j.jchromb.2007.07.019

133. Hillberg AL, Tabrizian M. 2008. Biomolecule imprinting: Developments in mimicking dynamic natural recognition systems. IRBM 29: 89-104. DOI: 10.1016/j.rbmret.2007.11.023 
134. Kubo T. 2008. Development and Applications of Fragment Imprinting Technique. Chromatography 29: 9-17.

135. Lieberzeit PA, Dickert FL. 2008. Rapid bioanalysis with chemical sensors: novel strategies for devices and artificial recognition membranes. Anal. Bioanal. Chem. 391: 1629-1639. DOI: 10.1007/s00216-008-1909-z

136. Longo L, Vasapollo G. 2008. Molecularly imprinted polymers as nucleotide receptors. Mini-Rev. Org. Chem 5: 163-170. DOI: $10.2174 / 157019308785161620$

137. Moreno-Bondi MC, Navarro-Villoslada F, Benito-Peña E, Urraca JL. 2008. Molecularly imprinted polymers as selective recognition elements in optical sensing. Curr. Anal. Chem. 4: 316-340. DOI: 10.2174/157341108785914925

138. Pichon V, Chapuis-Hugon F. 2008. Role of molecularly imprinted polymers for selective determination of environmental pollutants--A review. Anal. Chim. Acta 622: 48-61. DOI: 10.1016/j.aca.2008.05.057

139. Puoci F, lemma F, Picci N. 2008. Stimuli-responsive molecularly imprinted polymers for drug delivery: A review. Curr. Drug Delivery 5: 85-96. DOI: $10.2174 / 156720108783954888$

140. Rao TP, Kala R. 2008. Potentiometric transducer based biomimetic sensors for priority envirotoxic markers--An overview. Talanta 76: 485-496. DOI: 10.1016/j.talanta.2008.03.044

141. Takeuchi T, Hishiya T. 2008. Molecular imprinting of proteins emerging as a tool for protein recognition. Org. Biomol. Chem. 6: 2459-2467. DOI: $10.1039 / \mathrm{b} 715737 \mathrm{c}$

142. Weetall HH. 2008. Electrochemical Polymerization for Preparation of Electrochemical Sensors. In Handbook of Biosensors and Biochips, Marks RS, Cullen DC, Karube I, Lowe CR, Weetall HH (eds). Wiley: DOI: 10.1002/9780470061565.hbb021 
143. Ye L, Mosbach K. 2008. Molecular Imprinting: Synthetic Materials As Substitutes for Biological Antibodies and Receptors. Chem. Mater. 20: 859868. DOI: $10.1021 / \mathrm{cm} 703190 \mathrm{w}$

144. Agbenyega J. 2009. Molecular imprinted polymers: Polymers and soft materials. Materials Today 12: 7. DOI: 10.1016/S1369-7021(09)70023-9

145. Allender C, Mosbach K. 2009. MIP2008 Kobe, Japan, September 2008. Biosens. Bioelectron. 25: 539-542. DOI: 10.1016/j.bios.2009.08.001

146. Bompart M, Haupt K. 2009. Molecularly Imprinted Polymers and Controlled/Living Radical Polymerization. Aust. J. Chem. 62: 751-761. DOI: $10.1071 /$ ch09124

147. Bunte G, Heil M, Röseling D, Hürttlen J, Pontius H, Krause H. 2009. Trace Detection of Explosives Vapours by Molecularly Imprinted Polymers for Security Measures. Propel. Explos. Pyrot. 34: 245-251. DOI: 10.1002/prep.200800111

148. Dickert FL, Lieberzeit PA, Seifner A, Schirhagl R, Jungbauer C. 2009. Sensors for Healthcare Monitoring - Proteins, Viruses and Blood-GroupTyping. In Micro- and Nanosystems in Medicine, Active Implants, Biosensors, IFMBE Proceedings, Vol. 25/VIII, Dössel O, Schlegel WC (eds). 325-328. DOI: 10.1007/978-3-642-03887-7_94

149. Flavin K, Resmini M. 2009. Imprinted nanomaterials: a new class of synthetic receptors. Anal. Bioanal. Chem. 393: 437-444. DOI: $10.1007 / \mathrm{s} 00216-008-2496-8$

150. Ge Y, Turner APF. 2009. Molecularly Imprinted Sorbent Assays: Recent Developments and Applications. Chem. Eur. J. 15: 8100-8107. DOI: 10.1002/chem.200802401

151. Greibrokk T, Sellergren B. 2009. Molecular imprinting in Separation Science (Editorial). J. Sep. Sci. 32: 3263-3264. DOI: 10.1002/jssc.200990072 
152. Huang YP, Liu ZS, Zheng C, Gao RY. 2009. Recent developments of molecularly imprinted polymer in CEC. Electrophoresis 30: 155-162. DOI: 10.1002/elps.200800410

153. Izenberg NR, Murray GM, Pilato RS, Baird LM, Levin SM, Van Houten KA. 2009. Astrobiological molecularly imprinted polymer sensors. Planet. Space Sci. 57: 846-853. DOI: 10.1016/j.pss.2009.02.015

154. Kloskowski A, Pilarczyk M, Przyjazny A, Namiesnik J. 2009. Progress in Development of Molecularly Imprinted Polymers as Sorbents for Sample Preparation. Crit. Rev. Anal. Chem. 39: 43-58. DOI: 10.1080/10408340802570223

155. Lok CM, Son R. 2009. Application of molecularly imprinted polymers in food sample analysis - A perspective. Int. Food Res. J. 16: 127-140.

156. Martin-Esteban A. 2009. Molecularly Imprinted Polymers: Providing Selectivity to Sample Preparation. J. Chromatogr. Sci. 47: 254-256.

157. Nicholls IA, Andersson HS, Charlton C, Henschel H, Karlsson BCG, Karlsson JG, O'Mahony J, Rosengren AM, Rosengren KJ, Wikman S. 2009. Theoretical and computational strategies for rational molecularly imprinted polymer design. Biosens. Bioelectron. 25: 543-552. DOI: 10.1016/j.bios.2009.03.038

158. Öpik A, Menaker A, Reut J, Syritski V. 2009. Molecularly imprinted polymers: a new approach to the preparation of functional materials. Proc. Est. Acad. Sci. 58: 3-11. DOI: 10.3176/proc.2009.1.01

159. Rehman A, lqbal N, Lieberzeit PA, Dickert FL. 2009. Multisensor biomimetic systems with fully artificial recognition strategies in food analysis. Monatsh. Chem. 140: 931-939. DOI: 10.1007/s00706-009-0151-5 
160. Sergeyeva TA. 2009. Molecularly imprinted polymers as synthetic mimics of bioreceptors. 1. General principles of molecular imprinting. Biopolym. Cell 25: 253-265.

161. Sergeyeva TA. 2009. Molecularly-imprinted polymers as synythetic mimics of bioreceptors. 2. Applications in modern biotechnology. Biopolym. Cell 25: 431-444.

162. Tokonami S, Shiigi H, Nagaoka T. 2009. Review: Micro- and nanosized molecularly imprinted polymers for high-throughput analytical applications. Anal. Chim. Acta 641: 7-13. DOI: 10.1016/j.aca.2009.03.035

163. Turiel E, Martín-Esteban A. 2009. Molecularly imprinted polymers for solidphase microextraction. J. Sep. Sci. 32: 3278-3284. DOI: $10.1002 /$ jssc. 200900218

164. Yilmaz E, Karlsson O, Rees A. 2009. MIP-based processing. Innov. Pharmaceut. Technol. 88-91.

165. Beltran A, Borrull F, Marcé RM, Cormack PAG. 2010. Molecularly-imprinted polymers: useful sorbents for selective extractions. TrAC, Trends Anal. Chem. 29: 1363-1375. DOI: 10.1016/j.trac.2010.07.020

166. Coghlan A. 2010. World's first plastic antibody works in mice. The New Scientist 206: 12. DOI: 10.1016/S0262-4079(10)61466-2

167. Du JX. 2010. Molecular Imprinting-Based Chemiluminescence Techniques in Pharmaceutical Analysis. Curr. Pharm. Anal. 6: 30-38.

168. Haupt K. 2010. Biomaterials: Plastic antibodies. Nat. Mater. 9: 612-614. DOI: $10.1038 / \mathrm{nmat} 2818$

169. Hu J, Mao XM, Cao SS, Yuan XH. 2010. Recognition of proteins and peptides: Rational development of molecular imprinting technology. Polym. Sci. Ser. A 52: 328-339. DOI: 10.1134/S0965545X10030156 
170. Li SJ, Tiwari A. 2010. Molecularly imprinted sensors (editorial). Adv. Mat. Lett. 1: 178. DOI: 10.5185/amlett.2010.1201

171. Mao XA, Liang WA, Peng XH, Tang SQ. 2010. Recent Progress in Protein Imprinting Technology. Recent Patents on Nanotechnology 4: 85-99. DOI: $10.2174 / 187221010791208830$

172. Mujahid A, Lieberzeit PA, Dickert FL. 2010. Chemical Sensors Based on Molecularly Imprinted Sol-Gel Materials. Materials 3: 2196-2217. DOI: 10.3390/ma3042196

173. Poma A, Turner APF, Piletsky SA. 2010. Advances in the manufacture of MIP nanoparticles. Trends Biotechnol. 28: 629-637. DOI: 10.1016/j.tibtech.2010.08.006

174. Schreiber T, Niedergall K, Wojciukiewicz D, Gose T, Gruber-Traub C, Weber A, Hirth T, Tovar GEM. 2010. NANOCYTES-technology - Biomimetic nanoparticles for molecular recognition by molecular imprinting. In Technical Proceedings of the 2010 NSTI Nanotechnology Conference and Expo, NSTI-Nanotech 2010, Vol. 3, Nanoscience and Technology Institute: 242245.

175. Sellergren B. 2010. Molecularly imprinted polymers: Shaping enzyme inhibitors. Nat. Chem. 2: 7-8. DOI: 10.1038/nchem.496

176. Sharma A, Sharma R. 2010. Molecular imprinted nanoPolymer nanomaterials: Application in biomolecule recognition. In Technical Proceedings of the 2010 NSTI Nanotechnology Conference and Expo, NSTI-Nanotech 2010, Vol. 3, Nanoscience and Technology Institute: 270273.

177. Shimizu KD, Stephenson CJ. 2010. Molecularly imprinted polymer sensor arrays. Curr. Opin. Chem. Biol. 14: 743-750. DOI: 10.1016/j.cbpa.2010.07.007 
178. Suryanarayanan V, Wu CT, Ho KC. 2010. Molecularly Imprinted Electrochemical Sensors. Electroanalysis 22: 1795-1811. DOI: 10.1002/elan.200900616

179. Tiwari A, Li SJ. 2010. Deoxyribonucleic acid imprinted biosensor technology (Editorial). Adv. Mat. Lett. 1: 85. DOI: 10.5185/amlett.2010.8111

180. Tse Sum Bui B, Haupt K. 2010. Molecularly imprinted polymers: synthetic receptors in bioanalysis. Anal. Bioanal. Chem. 398: 2481-2492. DOI: $10.1007 / \mathrm{s} 00216-010-4158-x$

181. Turiel E, Martín-Esteban A. 2010. Molecularly imprinted polymers for sample preparation: A review. Anal. Chim. Acta 668: 87-99. DOI: 10.1016/j.aca.2010.04.019

182. Turley A. 2010. Polymers. Molecularly imprinted nanoparticles net bee toxin. Chemistry \& Industry 9.

183. White CJ, Byrne ME. 2010. Molecularly imprinted therapeutic contact lenses. Expert Opin. Drug Delivery 7: 765-780. DOI: $10.1517 / 17425241003770098$

184. Yu JCC, Lai EPC. 2010. Molecularly Imprinted Polymers for Ochratoxin A Extraction and Analysis. Toxins 2: 1536-1553. DOI: 10.3390/toxins2061536

185. Advincula RC. 2011. Engineering molecularly imprinted polymer (MIP) materials: Developments and challenges for sensing and separation technologies. Kor. J. Chem. Eng. 28: 1313-1321. DOI: 10.1007/s11814-0110133-2

186. Castell OK, Barrow DA, Kamarudin AR, Allender CJ. 2011. Current practices for describing the performance of molecularly imprinted polymers can be misleading and may be hampering the development of the field. $J$. Mol. Recognit. 24: 1115-1122. DOI: 10.1002/jmr.1161 
187. Chen LX, Xu SF, Li JH. 2011. Recent advances in molecular imprinting technology: current status, challenges and highlighted applications. Chem. Soc. Rev. 40: 2922-2942. DOI: 10.1039/C0CS00084A

188. Garcia R, Cabrita MJ, Freitas AMC. 2011. Application of Molecularly Imprinted Polymers for the Analysis of Pesticide Residues in Food-A Highly Selective and Innovative Approach. Am. J. Anal. Chem. 2: 16-25. DOI: 10.4236/ajac.2011.228119

189. Hoshino Y, Shea KJ. 2011. The evolution of plastic antibodies. J. Mater. Chem. 21: 3517-3521.

190. Huang YP, Zheng C, Liu ZS. 2011. Molecularly Imprinted Polymers for the Separation of Organic Compounds in Capillary Electrochromatography. Curr. Org. Chem. 15: 1863-1870. DOI: 10.2174/138527211795656651

191. Levi L, Raim V, Srebnik S. 2011. A brief review of coarse-grained and other computational studies of molecularly imprinted polymers. J. Mol. Recognit. 24: 883-891. DOI: 10.1002/jmr.1135

192. Nicholls IA, Andersson HS, Golker K, Henschel H, Karlsson BCG, Olsson GD, Rosengren AM, Shoravi S, Suriyanarayanan S, Wiklander JG, Wikman S. 2011. Rational design of biomimetic molecularly imprinted materials: theoretical and computational strategies for guiding nanoscale structured polymer development. Anal. Bioanal. Chem. 400: 1771-1786. DOI: 10.1007/s00216-011-4935-1

193. Pardeshi S, Kumar A, Dhodapkar R. 2011. Molecular Imprinting: Mimicking Molecular Receptors for Antioxidants. Materials Science Forum 675-677: 515-520. DOI: 10.4028/www.scientific.net/MSF.675-677.515

194. Puoci F, Cirillo G, Curcio M, Parisi OI, lemma F, Picci N. 2011. Molecularly imprinted polymers in drug delivery: state of art and future perspectives. 
Expert Opin. Drug Delivery 8: 1379-1393. DOI: $10.1517 / 17425247.2011 .609166$

195. Vasapollo G, del Sole R, Mergola L, Lazzoi MR, Scardino A, Scorrano S, Mele G. 2011. Molecularly Imprinted Polymers: Present and Future Prospective. Int. J. Mol. Sci. 12: 5908-5945. DOI: 10.3390/ijms12095908

196. Whitcombe MJ. 2011. Molecularly imprinted polymers: Smart hydrogel crystal gardens. Nat. Chem. 3: 657-658. DOI: 10.1038/nchem.1125

197. Whitcombe MJ, Chianella I, Larcombe L, Piletsky SA, Noble J, Porter R, Horgan A. 2011. The rational development of molecularly imprinted polymerbased sensors for protein detection. Chem. Soc. Rev. 40: 1547-1571. DOI: $10.1039 /$ COCSO0049C

198. Wulff G, Liu JQ. 2011. Design of Biomimetic Catalysts by Molecular Imprinting in Synthetic Polymers: The Role of Transition State Stabilization. Acc. Chem. Res. 45: 239-247. DOI: 10.1021/ar200146m

199. Xu ZX, Gao HJ, Zhang LM, Chen XQ, Qiao XG. 2011. The Biomimetic Immunoassay Based on Molecularly Imprinted Polymer: A Comprehensive Review of Recent Progress and Future Prospects. J. Food Sci. 76: R69R75. DOI: 10.1111/j.1750-3841.2010.02020.x

200. Yoshikawa M, Tanioka A, Matsumoto H. 2011. Molecularly imprinted nanofiber membranes. Current Opinion in Chemical Engineering 1: 18-26. DOI: $10.1016 /$ j.coche.2011.07.003

201. Zhang JJ, Shen XG, Chen QD. 2011. Separation Processes in the Presence of Cyclodextrins Using Molecular Imprinting Technology and Ionic Liquid Cooperating Approach. Curr. Org. Chem. 15: 74-85. DOI: $10.2174 / 138527211793797765$ 
202. Zheng C, Huang YP, Liu ZS. 2011. Recent developments and applications of molecularly imprinted monolithic column for HPLC and CEC. J. Sep. Sci. 34: 1988-2002. DOI: 10.1002/jssc.201100164

203. Verheyen E, Schillemans JP, van Wijk M, Demeniex MA, Hennink WE, van Nostrum CF. 2011. Challenges for the effective molecular imprinting of proteins. Biomaterials 32: 3008-3020. DOI: 10.1016/j.biomaterials.2011.01.007

204. DiCesare JC, Parker J, Horne SN, Kita J, Earni R, Peeples C. 2004. Progress in developing nerve agent sensors using combinatorial techniques. In Molecularly Imprinted Materials-2003, MRS Symposium Proceedings, Vol. 787, Kofinas P, Sellergren B, Roberts MJ (eds). Materials Research Society: Warrendale; 17-22. DOI: 10.1557/PROC-787-G2.3

205. Dickert FL, Lieberzeit P, Hayden O, Schirk C, Halikias K. 2004. Molecular imprinting - a universal strategy for the development of chemosensors. In Sensors And Measuring Systems 2004, VDI Berichte, Vol. 1829, 611-615.

206. Lieberzeit PA, Schirk C, Glanznig G, Gazda-Miarecka S, Bindeus R, Nannen H, Kauling J, Dickert FL. 2004. From nanopatterning to functionality - surface and bulk imprinting for analytical purposes. Superlattices Microstruct. 36: 133-142. DOI: 10.1016/j.spmi.2004.08.032

207. Lisichkin GV, Novotortsev RY, Bernadyuk SZ. 2004. Chemically modified oxide surfaces capable of molecular recognition. Colloid J. 66: 387-399. DOI: 10.1023/B:COLL.0000037442.92374.79

208. Lasáková M, Jandera P. 2009. Molecularly imprinted polymers and their application in solid phase extraction. J. Sep. Sci. 32: 799-812. DOI: 10.1002/jssc.200800506 
209. Alvarez-Lorenzo C, Yañez F, Concheiro A. 2010. Ocular drug delivery from molecularly-imprinted contact lenses. J. Drug. Deliv. Sci. Technol. 20: 237248.

210. Kempe M, Mosbach K. 1994. Preparation of synthetic polymers selective for amino acid derivatives and peptides by molecular imprinting. In Peptides: Chemistry, Structure and Biology, Proceedings of the Thirteenth American Peptide Symposium, Hodges RS, Smith JA (eds). Escom Science Publishers: Leiden, The Netherlands; 221-223.

211. Rao TP, Daniel S, Gladis JM. 2004. Tailored materials for preconcentration or separation of metals by ion-imprinted polymers for solid-phase extraction (IIP-SPE). TrAC, Trends Anal. Chem. 23: 28-35. DOI: 10.1016/S01659936(04)00106-2

212. Piletska EV, Guerreiro AR, Whitcombe MJ, Piletsky SA. 2009. Influence of the Polymerization Conditions on the Performance of Molecularly Imprinted Polymers. Macromolecules 42: 4921-4928. DOI: 10.1021/ma900432z

213. Haginaka J. 2009. Molecularly imprinted polymers as affinity-based separation media for sample preparation. J. Sep. Sci. 32: 1548-1565. DOI: 10.1002/jssc.200900085

214. Zimmerman SC, Lemcoff NG. 2004. Synthetic hosts via molecular imprinting - are universal synthetic antibodies realistically possible? Chem. Commun. 5-14. DOI: 10.1039/b304720b

215. Hodgson J. 1992. Elective affinities - The art of chromatography. Bio/Technology 10: 997-1000.

216. Malafaya PB, Silva GA, Baran ET, Reis RL. 2002. Drug delivery therapies II.: Strategies for delivering bone regenerating factors. Curr. Opin. Solid State Mater. Sci. 6: 297-312. DOI: 10.1016/S1359-0286(02)00077-3 
217. Strauss E. 1998. After the genome - IV - New ways to probe the molecules of life. Science 282: 1406-1407.

218. Fleischer CT, Boos KS. 2000. Bioanalytical solid-phase extraction. A classic in a new made-to-measure form. GIT Lab-Fachz. 89-92.

219. Reinbold J, Cammann K. 1998. Sensors for gases, odours and organic solvent vapours. GIT Lab-Fachz. 42: 396-400.

220. Adhikari B, Majumdar S. 2004. Polymers in sensor applications. Prog. Polym. Sci. 29: 699-766. DOI: 10.1016/j.progpolymsci.2004.03.002

221. Anderson J, Pestov D, Fischer R, Webb S, Tepper G. 2004. Fluorescence measurements of activity associated with a molecularly imprinted polymer imprinted to dipicolinic acid. In Chemical And Biological Point Sensors For Homeland Defense, Proceeding of SPIE, Vol. 5269, The International Society for Optical Engineering: 212-216. DOI: 10.1117/12.519480

222. Archibald $B$, Brümmer $O$, Devenney $M$, Giaquinta $G M$, Jandeleit $B$, Weinberg WH, Weskamp T. 2004. Combinatorial aspects of materials science. In Handbook of Combinatorial Chemistry, Drugs, Catalysts, Materials, Nicolaou KC, Hanko R, Hartwig W (eds). Wiley-VCH Verlag GmbH: Weinheim; 1017-1062. DOI: 10.1002/3527603034.ch34

223. Ariga K. 2004. Template-assisted nano-patterning: From the submicron scale to the submolecular level. J. Nanosci. Nanotechnol. 4: 23-34. DOI: 10.1166/jnn.2004.048

224. Balaji R, Boileau S, Guérin P, Grande D. 2004. Design of porous polymeric materials from miscellaneous macromolecular architectures: An overview. Polymer News 29: 205-212. DOI: 10.1080/748639192

225. Chen $X, Y i C Q$, Yang $X Q$, Wang XR. 2004. Liquid chromatography of active principles in Sophora flavescens root. J. Chromatogr B 812: 149-163. DOI: 10.1016/j.jchromb.2004.08.032 
226. Corma A. 2004. Attempts to fill the gap between enzymatic, homogeneous, and heterogeneous catalysis. Catal. Rev. Sci. Eng. 46: 369-417. DOI: 10.1081/CR-200036732

227. Cserhati T, Forgacs E, Deyl Z, Miksik I, Eckhardt A. 2004. Chromatographic determination of herbicide residues in various matrices. Biomed. Chromatogr. 18: 350-359. DOI: 10.1002/bmc.378

228. Dalko PI, Moisan L. 2004. In the golden age of organocatalysis. Angew. Chem. Int. Edit. 43: 5138-5175. DOI: 10.1002/anie.200400650

229. Gübitz G, Schmid MG. 2004. Recent advances in chiral separation principles in capillary electrophoresis and capillary electrochromatography. Electrophoresis 25: 3981-3996. DOI: 10.1002/elps.200406173

230. Guiochon GA, Beaver LA. 2004. Progress and future of instrumental analytical chemistry applied to the environment. Anal. Chim. Acta 524: 1-14. DOI: 10.1016/j.aca.2004.03.102

231. Hilder EF, Svec F, Fréchet JMJ. 2004. Development and application of polymeric monolithic stationary phases for capillary electrochromatography. J. Chromatogr. A 1044: 3-22. DOI: 10.1016/j.chroma.2004.04.057

232. Kim Y, Yi J. 2004. Advances in environmental technologies via the application of mesoporous materials. J. Ind. Eng. Chem. 10: 41-51.

233. Kist TBL, Mandaji M. 2004. Separation of biomolecules using electrophoresis and nanostructures. Electrophoresis 25: 3492-3497. DOI: 10.1002/elps.200406114

234. Kopecni MM, Comor JJ. 2004. New solid phases for chromatography. In Encyclopedia of Surface and Colloid Science, Somasunderan P (ed). Marcel Dekker: 3668-3676. 
235. Maragos CM. 2004. Emerging technologies for mycotoxin detection. J. Toxicol. -Toxin Rev. 23: 317-344. DOI: 10.1081/TXR-200027859

236. Mastrorilli P, Nobile CF. 2004. Supported catalysts from polymerizable transition metal complexes. Coord. Chem. Rev. 248: 377-395. DOI: 10.1016/j.ccr.2004.02.003

237. Monaci L, Palmisano F. 2004. Determination of ochratoxin A in foods: stateof-the-art and analytical challenges. Anal. Bioanal. Chem. 378: 96-103. DOI: 10.1007/s00216-003-2364-5

238. Monk DJ, Walt DR. 2004. Optical fiber-based biosensors. Anal. Bioanal. Chem. 379: 931-945. DOI: 10.1007/s00216-004-2650-x

239. Palmer CF, McCarney JP. 2004. Recent progress in the use of soluble ionic polymers as pseudostationary phases for electrokinetic chromatography. Electrophoresis 25: 4086-4094. DOI: 10.1002/elps.200406110

240. Park JK, Kan HY, Lee JW, Kim SJ, Kim IH. 2004. Preparation of molecularly imprinted polymer membrane using wet phase method (II) [separation of phenylalanine using D-PHE imprinted membrane]. Tong ZF, Kim SH (eds). World Scientific: 687-691.

241. Rao TP, Praveen RS, Daniel S. 2004. Styrene-divinyl benzene copolymers: Synthesis, characterization, and their role in inorganic trace analysis. Crit. Rev. Anal. Chem. 34: 177-193. DOI: 10.1080/10408340490888689

242. Reynolds JG, Hart BR. 2004. Nanomaterials and their application to defense and homeland security. JOM 56: 36-39.

243. Shinkai S, Takeuchi M. 2004. Molecular design of synthetic receptors with dynamic, imprinting, and allosteric functions. Biosens. Bioelectron. 20: 12501259. DOI: 10.1016/j.bios.2004.03.036 
244. Smith RK, Lewis PA, Weiss PS. 2004. Patterning self-assembled monolayers. Prog. Surf. Sci. 75: 1-68. DOI: 10.1016/j.progsurf.2003.12.001

245. Yang RT, Benton DF. 2003. Silica gel, MCM, and activated alumina. In Adsorbents: Fundamentals and Applications, Yang RT (ed). John Wiley \& Sons, Inc.: New York; 131-156. DOI: 10.1002/047144409X.ch6

246. Ulubayram K. 2004. Molecularly imprinted polymers. In Biomaterials: From Molecules To Engineered Tissues, Advances in Experimental Medicine and Biology, Vol. 553, Hasirci N, Hasirici V (eds). Springer: 123-138.

247. Zwiener C, Frimmel FH. 2004. LC-MS analysis in the aquatic environment and in water treatment - a critical review - Part I: Instrumentation and general aspects of analysis and detection. Anal. Bioanal. Chem. 378: 851861. DOI: 10.1007/s00216-003-2404-1

248. Anon. 2005. Research trends. Membr. Technol. 2005: 15-16. DOI: 10.1016/S0958-2118(05)70418-3

249. Anon. 2005. Shaanxi Normal University, Xian; Molecule imprintingchemiluminescence method used to determine tamoxifen in breast cancer sufferers' urine. Managed Care Business Week, Atlanta

250. Bergwerff AA. 2005. Rapid assays for detection of residues of veterinary drugs. In Rapid Methods: For Biological And Chemical Contaminants In Food And Feed, van Amerongen A, Barug D, Lauwaars M (eds). Waginingen Academic Publishers: Waginingen, Netherlands; 259-292.

251. Bojarski J, Aboul-Enein HY, Ghanem A. 2005. What's new in chromatographic enantioseparations? Curr. Anal. Chem. 1: 59-77.

252. Chapuis F, Pichon V, Hennion MC. 2005. Preconcentration by solid phase extraction: Principles and applications in the environmental and petroleum industries. Oil Gas Sci. Technol. Rev. IFP 60: 899-912. DOI: 10.2516/ogst:2005063 
253. Ciardelli G, Silvestri D, Cristallini C, Barbani N, Giusti P. 2005. The relevance of the transfer of molecular information between natural and synthetic materials in the realisation of biomedical devices with enhanced properties. J. Biomater. Sci. Polym. Ed. 16: 219-236. DOI: $10.1163 / 1568562053115417$

254. Gauglitz G. 2005. Direct optical sensors: principles and selected applications. Anal. Bioanal. Chem. 381: 141-155. DOI: 10.1007/s00216-0042895-4

255. Gavioli E, Maier NM, Haupt K, Mosbach K, Lindner W. 2005. Analyte templating: Enhancing the enantioselectivity of chiral selectors upon incorporation into organic polymer environments. Anal. Chem. 77: 50095018. DOI: 10.1021/ac050407s

256. Kashyap N, Kumar N, Kumar MNVR. 2005. Hydrogels for pharmaceutical and biomedical applications. Crit. Rev. Ther. Drug Carrier Syst. 22: 107-149. DOI: 10.1615/CritRevTherDrugCarrierSyst.v22.i2.10

257. Krska R, Welzig E, Berthiller F, Molinelli A, Mizaikoff B. 2005. Advances in the analysis of mycotoxins and its quality assurance. Food Addit. Contam. 22: 345-353. DOI: 10.1080/02652030500070192

258. Li XZ, Sun YP. 2005. Progress in solid-liquid extraction resin for separation of rare earth elements. J. Rare Earths 23: 581-592.

259. Lieberzeit PA, Glanznig G, Jenik M, Gazda-Miarecka S, Dickert FL, Leidl A. 2005. Softlithography in chemical sensing - Analytes from molecules to cells. Sensors 5: 509-518. DOI: 10.3390/s5120509

260. Nakano Y. 2005. Science and technology of polymer gels. J. Chem. Eng. Jpn. 38: 605-614. DOI: 10.1252/jcej.38.605

261. Pap T, Horvath V, Horvai G. 2005. Molecularly imprinted polymers for analytical chemistry. Chem. Anal. (Warsaw) 50: 129-137. 
262. Shinkai S, Takeuchi M. 2005. Molecular design of synthetic receptors with dynamic, imprinting, and allosteric functions. Bull. Chem. Soc. Jpn. 78: 4051. DOI: $10.1246 / \mathrm{bcsj} .78 .40$

263. Sims MR, Cullen DC, Bannister NP, Grant WD, Henry O, Jones R, McKnight D, Thompson DP, Wilson PK. 2005. The specific molecular identification of life experiment (SMILE). Planet. Space Sci. 53: 781-791. DOI: 10.1016/j.pss.2005.03.006

264. Soderberg D. 2005. Committee on residues and related topics - Pesticides and other chemical contaminants. J. AOAC Int. 88: 331-341.

265. Telford M. 2005. Imprinted nanowires for biorecognition: Nanotechnology. Materials Today 8: 10.

266. Trojanowicz M, Wcislo M. 2005. Electrochemical and piezoelectric enantioselective sensors and biosensors. Anal. Lett. 38: 523-547. DOI: 10.1081/AL-200050157

267. Venkatesh S, Byrne ME, Peppas NA, Hilt JZ. 2005. Applications of biomimetic systems in drug delivery. Expert Opin. Drug Delivery 2: 10851096. DOI: 10.1517/17425247.2.6.1085

268. Visconti A, De Girolamo A. 2005. Fitness for purpose - Ochratoxin A analytical developments. Food Addit. Contam. 22: 37-44. DOI: $10.1080 / 02652030500410307$

269. Vriezema DM, Aragones MC, Elemans JAAW, Cornelissen JJLM, Rowan AE, Nolte RJM. 2005. Self-assembled nanoreactors. Chem. Rev. 105: 14451489. DOI: $10.1021 / \mathrm{cr} 0300688$

270. Walcarius A, Mandler D, Cox JA, Collinson M, Lev O. 2005. Exciting new directions in the intersection of functionalized sol-gel materials with electrochemistry. J. Mater. Chem. 15: 3663-3689. DOI: 10.1039/b504839g 
271. Welbes LL, Borovik AS. 2005. Confinement of metal complexes within porous hosts: development of functional materials for gas binding and catalysis. Acc. Chem. Res. 38: 765-774. DOI: 10.1021/ar0402513

272. Zhang J, Albelda MT, Liu Y, Canary JW. 2005. Chiral nanotechnology. Chirality 17: 404-418. DOI: 10.1002/chir.20178

273. Anon. 2006. Quest for selectivity dominates column technology at Pittcon«'06. Am. Lab. 38: 12-21.

274. Aponte VM, Finch DS, Klaus DM. 2006. Considerations for non-invasive inflight monitoring of astronaut immune status with potential use of MEMS and NEMS devices. Life Sci. 79: 1317-1333. DOI: 10.1016/j.Ifs.2006.04.007

275. de Oliviera EC, Pires CTGVMT, Pastore HO. 2006. Why are carbon molecular sieves interesting? J. Braz. Chem. Soc. 17: 16-29. DOI: $10.1590 /$ S0103-50532006000100003

276. Dickert FL, Lieberzeit P, Glanzing G, Leidl A. 2006. "On-board" Qualitätskontrolle von Motorenölen basierend auf nanostrukturierten Chemosensoren. In Sensoren und Messsysteme 2006, VDE Verlag GmbH: Berlin; 161-164.

277. Dioos BML, Vankelecom IFJ, Jacobs PA. 2006. Aspects of Immobilisation of Catalysts on Polymeric Supports. Adv. Synth. Catal. 348: 1413-1446. DOI: 10.1002/adsc.200606202

278. Espinosa-Bosch M, Ruiz-Sánchez AJR, Sánchez-Rojas FS, Bosch-Ojeda C. 2006. Determination of paracetamol: Historical evolution. J. Pharm. Biomed. Anal. 42: 291-321. DOI: 10.1016/j.jpba.2006.04.007

279. Gimeno N, Vilar R. 2006. Anions as templates in coordination and supramolecular chemistry. Coord. Chem. Rev. 250: 3161-3189. DOI: 10.1016/j.ccr.2006.05.016 
280. Gübitz G, Schmid MG. 2006. Chiral separation principles in chromatographic and electromigration techniques. Mol. Biotechnol. 32: 159179. DOI: 10.1385/MB:32:2:159

281. He L, Toh CS. 2006. Recent advances in analytical chemistry--A material approach. Anal. Chim. Acta 556: 1-15. DOI: 10.1016/j.aca.2005.08.042

282. Jiang GB, Huang MJ, Cai YQ, Lv JX, Zhao ZS. 2006. Progress of solidphase microextraction coatings and coating techniques. J. Chromatogr. Sci. 44: 324-332.

283. Karakhanov EA, Karapetyan LM, Kardasheva YS, Maksimov AL, Runova EA, Skorkin VA, Terenina MV. 2006. Molecular recognition and catalysis: from macrocyclic receptors to molecularly imprinted metal complexes. Macromol. Symp. 235: 39-51. DOI: 10.1002/masy.200650306

284. Kruppa M, Konig B. 2006. Reversible Coordinative Bonds in Molecular Recognition. Chem. Rev. 106: 3520-3560. DOI: 10.1021/cr010206y

285. Lieberzeit PA, Glanzing G, Leidl A, Dickert FL. 2006. Ceramic Materials for Mass-Sensitive Sensors - Detection of VOCs and Monitoring Oil Degradation. In Proceedings of the 11th International Ceramics Congress, Advances in Science and Technology, Vol. 45, Vincenzini $P$ (ed). Scientific.Net: 1799-1802. DOI: 10.4028/www.scientific.net/AST.45.1799

286. Lucklum R, Hauptmann P. 2006. Acoustic microsensors - the challenge behind microgravimetry. Anal. Bioanal. Chem. 384: 667-682. DOI: 10.1007/s00216-005-0236-x

287. Magan N. 2006. Mycotoxin contamination of food in Europe: Early detection and prevention strategies. Mycopathologia 162: 245-253. DOI: 10.1007/s11046-006-0057-2 
288. Malhotra BD, Chaubey A, Singh SP. 2006. Prospects of conducting polymers in biosensors. Anal. Chim. Acta 578: 59-74. DOI: 10.1016/j.aca.2006.04.055

289. Ngundi MM, Kulagina NV, Anderson GP, Taitt CR. 2006. Nonantibodybased recognition: Alternative molecules for detection of pathogens. Expert Rev. Proteomics 3: 511-524. DOI: 10.1586/14789450.3.5.511

290. Nguyen BT, Anslyn EV. 2006. Indicator-displacement assays. Coord. Chem. Rev. 250: 3118-3127. DOI: 10.1016/j.ccr.2006.04.009

291. Oliferova LA, Statkus MA, Tsisin GI, Wang J, Zolotov YA. 2006. On-line coupling of sorption preconcentration to liquid-chromatographic methods of analysis. J. Anal. Chem. 61: 416-441. DOI: 10.1134/S1061934806050029

292. Potterat O, Hamburger M. 2006. Natural products in drug discovery Concepts and approaches for tracking bioactivity. Curr. Org. Chem. 10: 899920. DOI: $10.2174 / 138527206776894401$

293. Potyrailo RA. 2006. Polymeric sensor materials: Toward an alliance of combinatorial and rational design tools? Angew. Chem. Int. Edit. 45: 702723. DOI: $10.1002 /$ anie.200500828

294. Ramanaviciene A, Finkelsteinas A, Ramanavicius A. 2006. Basic electrochemistry meets nanotechnology: Electrochemical preparation of artificial receptors based on a nanostructured conducting polymer, polypyrrole. J. Chem. Educ. 83: 1212-1214.

295. Ramanavicius A, Ramanaviciene A, Malinauskas A. 2006. Electrochemical sensors based on conducting polymer--polypyrrole. Electrochim. Acta 51: 6025-6037. DOI: 10.1016/j.electacta.2005.11.052

296. Ravelet C, Peyrin E. 2006. Recent developments in the HPLC enantiomeric separation using chiral selectors identified by a combinatorial strategy. $\mathrm{J}$. Sep. Sci. 29: 1322-1331. DOI: 10.1002/jssc.200600010 
297. Sánchez-Barragán I, Costa-Fernández JM, Sanz-Medel A, Valledor M, Campo JC. 2006. Room-temperature phosphorescence (RTP) for optical sensing. TrAC, Trends Anal. Chem. 25: 958-967. DOI: 10.1016/j.trac.2006.07.009

298. Shelley T. 2006. Technical report: Medical design: Pushing the frontiers. Eureka 26: 38.

299. Svec F. 2006. Less common applications of monoliths: Preconcentration and solid-phase extraction. J. Chromatogr B 841: 52-64. DOI: 10.1016/j.jchromb.2006.03.055

300. Theodoridis GA, Papadoyannis LN. 2006. Novel advanced approaches in sample preparation and analyte detection for bioanalysis. Curr. Pharm. Anal. 2: 385-404. DOI: 10.2174/157341206778699591

301. Ulbricht M. 2006. Advanced functional polymer membranes. Polymer 47: 2217-2262. DOI: 10.1016/j.polymer.2006.01.084

302. Warsinke A, Nagel B. 2006. Towards Separation-Free Electrochemical Affinity Sensors by Using Antibodies, Aptamers, and Molecularly Imprinted Polymers-A Review. Anal. Lett. 39: 2507-2556. DOI: 10.1080/00032710600853903

303. Klabunovskii E, Smith GV, Zsigmond A. 2006. Asymmetric adsorption on minerals. In Heterogeneous Enantioselective Hydrogenation, Catalysis by Metal Complexes, Vol. 31, Klabunovskii E, Smith GV, Zsigmond A (eds). Springer Netherlands: 1-29. DOI: 10.1007/978-1-4020-4296-6_1

304. Bakker E, Qin Y. 2006. Electrochemical Sensors. Anal. Chem. 78: 39653984. DOI: $10.1021 / \mathrm{ac} 060637 \mathrm{~m}$

305. Arnaud CH. 2007. Testing our food. Chem. Eng. News 85: 92-94. 
306. Burnworth M, Rowan SJ, Weder C. 2007. Fluorescent Sensors for the Detection of Chemical Warfare Agents. Chem. Eur. J. 13: 7828-7836. DOI: 10.1002/chem.200700720

307. Campàs $M$, Prieto-Simón $B$, Marty $J-L$. 2007. Biosensors to detect marine toxins: Assessing seafood safety. Talanta 72: 884-895. DOI: 10.1016/j.talanta.2006.12.036

308. Chaterji S, Kwon IK, Park K. 2007. Smart polymeric gels: Redefining the limits of biomedical devices. Prog. Polym. Sci. 32: 1083-1122. DOI: 10.1016/j.progpolymsci.2007.05.018

309. Cooper MA, Singleton VT. 2007. A survey of the 2001 to 2005 quartz crystal microbalance biosensor literature: applications of acoustic physics to the analysis of biomolecular interactions. J. Mol. Recognit. 20: 154-184. DOI: $10.1002 / \mathrm{jmr} .826$

310. Díaz-Cruz MS, Barceló D. 2007. Recent advances in LC-MS residue analysis of veterinary medicines in the terrestrial environment. TrAC, Trends Anal. Chem. 26: 637-646. DOI: 10.1016/j.trac.2007.04.004

311. Dietz C, Sanz J, Sanz E, Muñoz-Olivas R, Cámara C. 2007. Current perspectives in analyte extraction strategies for tin and arsenic speciation. $J$. Chromatogr. A 1153: 114-129. DOI: 10.1016/j.chroma.2006.11.064

312. Giordano BC, Collins GE. 2007. Synthetic methods applied to the detection of chemical warfare nerve agents. Curr. Org. Chem. 11: 255-265. DOI: $10.2174 / 138527207779940883$

313. Guiochon G. 2007. Monolithic columns in high-performance liquid chromatography. J. Chromatogr. A 1168: 101-168. DOI: 10.1016/j.chroma.2007.05.090

314. Habib MK. 2007. Controlled biological and biomimetic systems for landmine detection. Biosens. Bioelectron. 23: 1-18. DOI: 10.1016/j.bios.2007.05.005 
315. Izake EL. 2007. Chiral discrimination and enantioselective analysis of drugs: An overview. J. Pharm. Sci. 96: 1659-1676. DOI: 10.1002/jps.20820

316. Lieberzeit PA, Dickert FL. 2007. Sensor technology and its application in environmental analysis. Anal. Bioanal. Chem. 387: 237-247. DOI: 10.1007/s00216-006-0926-z

317. Liu P. 2007. Modification of polymeric materials via surface-initiated controlled/"living" radical polymerization. E-Polymers Art. No. 062.

318. Marx S, Avnir D. 2007. The Induction of Chirality in Sol-Gel Materials. Acc. Chem. Res. 40: 768-776. DOI: 10.1021/ar6000236

319. Mullett WM. 2007. Determination of drugs in biological fluids by direct injection of samples for liquid-chromatographic analysis. J. Biochem. Biophys. Meth. 70: 263-273. DOI: 10.1016/j.jbbm.2006.10.001

320. Murkovic M. 2007. Analysis of heterocyclic aromatic amines. Anal. Bioanal. Chem. 389: 139-146. DOI: 10.1007/s00216-007-1306-z

321. Nilsson C, Birnbaum S, Nilsson S. 2007. Use of nanoparticles in capillary and microchip electrochromatography. J. Chromatogr. A 1168: 212-224. DOI: 10.1016/j.chroma.2007.07.018

322. O'Connor S, Aga DS. 2007. Analysis of tetracycline antibiotics in soil: Advances in extraction, clean-up, and quantification. TrAC, Trends Anal. Chem. 26: 456-465. DOI: 10.1016/j.trac.2007.02.007

323. Pasparakis G, Alexander C. 2007. Synthetic polymers for capture and detection of microorganisms. Analyst 132: 1075-1082. DOI: $10.1039 / \mathrm{b} 705097 f$

324. Pedersen-Bjergaard S, Rasmussen KE, Mills GA. 2007. Recent progress in sample extraction: A report on the ExTech 2007 Symposium at Alesund, 
Norway, 3-6 June 2007. TrAC, Trends Anal. Chem. 26: 843-846. DOI: 10.1016/j.trac.2007.07.002

325. Picó Y, Fernández M, Ruiz MJ, Font G. 2007. Current trends in solid-phasebased extraction techniques for the determination of pesticides in food and environment. J. Biochem. Biophys. Meth. 70: 117-131. DOI: 10.1016/j.jbbm.2006.10.010

326. Preinerstorfer B, Lämmerhofer M. 2007. Recent accomplishments in the field of enantiomer separation by CEC. Electrophoresis 28: 2527-2565. DOI: 10.1002/elps.200700070

327. Prieto-Simón B, Noguer T, Campàs M. 2007. Emerging biotools for assessment of mycotoxins in the past decade. TrAC, Trends Anal. Chem. 26: 689-702. DOI: 10.1016/j.trac.2007.05.012

328. Rao TP, Prasad K, Kala R, Gladis JM. 2007. Biomimetic Sensors for Toxic Pesticides and Inorganics based on Optoelectronic/Electrochemical Transducers-An Overview. Crit. Rev. Anal. Chem. 37: 191-210. DOI: $10.1080 / 10408340701244664$

329. Ridgway K, Lalljie SPD, Smith RM. 2007. Sample preparation techniques for the determination of trace residues and contaminants in foods. $J$. Chromatogr. A 1153: 36-53. DOI: 10.1016/j.chroma.2007.01.134

330. Rodriguez-Mozaz S, Lopez de Alda MJ, Barceló D. 2007. Advantages and limitations of on-line solid phase extraction coupled to liquid chromatography-mass spectrometry technologies versus biosensors for monitoring of emerging contaminants in water. J. Chromatogr. A 1152: $97-$ 115. DOI: 10.1016/j.chroma.2007.01.046

331. Sanz ML, Martinez-Castro I. 2007. Recent developments in sample preparation for chromatographic analysis of carbohydrates. J. Chromatogr. A 1153: 74-89. DOI: 10.1016/j.chroma.2007.01.028 
332. Schrader T, Koch S. 2007. Artificial protein sensors. Mol. BioSyst. 3: 241248. DOI: $10.1039 / \mathrm{b} 614103 \mathrm{j}$

333. Szumski M, Klodzinska E, Jarmalaviciene R, Maruska A, Buszewski B. 2007. Considerations on influence of charge distribution on determination of biomolecules and microorganisms and tailoring the monolithic (continuous bed) materials for bioseparations. J. Biochem. Biophys. Meth. 70: 107-115. DOI: 10.1016/j.jbbm.2006.09.013

334. Tada M, Iwasawa Y. 2007. Advanced design of catalytically active reaction space at surfaces for selective catalysis. Coord. Chem. Rev. 251: 27022716. DOI: 10.1016/j.ccr.2007.06.008

335. Wille SMR, Lambert WEE. 2007. Recent developments in extraction procedures relevant to analytical toxicology. Anal. Bioanal. Chem. 388: 1381-1391. DOI: 10.1007/s00216-007-1294-z

336. Alvarez-Lorenzo C, Concheiro A. 2008. Intelligent Drug Delivery Systems: Polymeric Micelles and Hydrogels. Mini-Rev. Med. Chem 8: 1065-1074. DOI: $10.2174 / 138955708785909952$

337. Bayer CL, Peppas NA. 2008. Advances in recognitive, conductive and responsive delivery systems. J. Controlled Release 132: 216-221. DOI: 10.1016/j.jconrel.2008.06.021

338. Budnikov G, Evtyugin G, Budnikova Y, Al'fonsov V. 2008. Chemically modified electrodes with amperometric response in enantioselective analysis. J. Anal. Chem. 63: 2-12. DOI: 10.1007/s10809-008-1002-y

339. Chen Y, Guo ZP, Wang XY, Qu CG. 2008. Sample preparation. J. Chromatogr. A 1184: 191-219. DOI: 10.1016/j.chroma.2007.10.026

340. Clapper JD, Sievens-Figueroa L, Guymon CA. 2008. Photopolymerization in Polymer Templating. Chem. Mater. 20: 768-781. DOI: 10.1021/cm702130r 
341. Gübitz G, Schmid MG. 2008. Chiral separation by capillary electromigration techniques. J. Chromatogr. A 1204: 140-156. DOI: 10.1016/j.chroma.2008.07.071

342. Holmes PF, Bohrer M, Kohn J. 2008. Exploration of polymethacrylate structure-property correlations: Advances towards combinatorial and highthroughput methods for biomaterials discovery. Prog. Polym. Sci. 33: 787796. DOI: 10.1016/j.progpolymsci.2008.05.002

343. John H, Worek F, Thiermann H. 2008. LC-MS-based procedures for monitoring of toxic organophosphorus compounds and verification of pesticide and nerve agent poisoning. Anal. Bioanal. Chem. 391: 97-116. DOI: $10.1007 /$ s00216-008-1925-z

344. Karakhanov EA, Karapetyan LM, Kardasheva YS, Maksimov AL, Runova EA, Terenina MV, Filippova TY. 2008. Molecules-Receptors: Different Approaches to Design Effective Catalysts. Macromol. Symp. 270: 106-116. DOI: 10.1002/masy.200851013

345. Karakhanov EA, Maksimov AL, Runova EA, Kardasheva YuS, Terenina MV, Kardashev SV, Skorkin VA, Karapetyan LM, Talanova MY. 2008. Design of supramolecular metal complex catalytic systems for petrochemical and organic synthesis. Russ. Chem. Bull. 57: 780-792. DOI: 10.1007/s11172008-0117-5

346. Lange U, Roznyatovskaya NV, Mirsky VM. 2008. Conducting polymers in chemical sensors and arrays. Anal. Chim. Acta 614: 1-26. DOI: 10.1016/j.aca.2008.02.068

347. Lemos VA, Teixeira LSG, Bezerra MdA, Costa ACS, Castro JT, Castro LAM, de Jesus DS, Santos ES, Baliza PX, Santos LN. 2008. New Materials for Solid-Phase Extraction of Trace Elements. Appl. Spec. Rev. 43: 303-334. DOI: 10.1080/05704920802031341 
348. Li XM, Cui YD, Lloyd AW, Mikhalovsky SV, Sandeman SR, Howel CA, Liao LW. 2008. Polymeric hydrogels for novel contact lens-based ophthalmic drug delivery systems: A review. Cont. Lens Anterior Eye 31: 57-64. DOI: 10.1016/j.clae.2007.09.002

349. Liu S, Yi LZ, Liang YZ. 2008. Traditional Chinese medicine and separation science. J. Sep. Sci. 31: 2113-2137. DOI: 10.1002/jssc.200800134

350. Martin MA, Olives Al, del Castillo B, Menéndez JC. 2008. Trends in the design and application of optical chemosensors in pharmaceutical and biomedical analysis. Curr. Pharm. Anal. 4: 106-117. DOI: $10.2174 / 157341208785161236$

351. Patek M, Drew M. 2008. Chemical synthesis in nanosized cavities. Curr. Opin. Chem. Biol. 12: 332-339. DOI: 10.1016/j.cbpa.2008.04.602

352. Potter OG, Hilder EF. 2008. Porous polymer monoliths for extraction: Diverse applications and platforms. J. Sep. Sci. 31: 1881-1906. DOI: $10.1002 /$ jssc.200800116

353. Potyrailo RA, Mirsky VM. 2008. Combinatorial and High-Throughput Development of Sensing Materials: The First 10 Years. Chem. Rev. 108: 770-813. DOI: 10.1021/cr068127f

354. Quintana MC, Ramos L. 2008. Sample preparation for the determination of chlorophenols. TrAC, Trends Anal. Chem. 27: 418-436. DOI: 10.1016/j.trac.2008.03.009

355. Szabelski P, Kaczmarski K. 2008. Phenomenological Modeling of Separation of Enantiomers by Nonlinear Chromatography. Acta Chromatogr. 20: 513-547. DOI: 10.1556/AChrom.20.2008.4.1

356. Thomas A, Goettmann F, Antonietti M. 2008. Hard Templates for Soft Materials: Creating Nanostructured Organic Materials. Chem. Mater. 20: 738-755. DOI: $10.1021 / \mathrm{cm} 702126 \mathrm{j}$ 
357. Wang ZZ, Ouyang J, Baeyens WRG. 2008. Recent developments of enantioseparation techniques for adrenergic drugs using liquid chromatography and capillary electrophoresis: A review. J. Chromatogr $B$ 862: 1-14. DOI: 10.1016/j.jchromb.2007.11.034

358. Wu RA, Hu LH, Wang FJ, Ye ML, Zou HF. 2008. Recent development of monolithic stationary phases with emphasis on microscale chromatographic separation. J. Chromatogr. A 1184: 369-392. DOI: 10.1016/j.chroma.2007.09.022

359. Zhang HT, Wang SL, Zhou ZQ, Pan CP, Zhang JP, Niu W. 2008. Food safety: Monitoring of organophosphate pesticide residues in crops and food. Phosphorus, Sulfur Silicon Relat. Elem. 183: 280-290. DOI: 10.1080/10426500701734273

360. Aranaz I, Mengíbar M, Harris R, Paños I, Miralles B, Acosta N, Galed G, Heras Á. 2009. Functional characterization of chitin and chitosan. Curr. Chem. Biol. 3: 203-230. DOI: 10.2174/187231309788166415

361. Arrua RD, Strumia MC, Igarzabal CIA. 2009. Macroporous Monolithic Polymers: Preparation and Applications. Materials 2: 2429-2466. DOI: 10.3390/ma2042429

362. Ballesteros-Gómez A, Rubio S, Pérez-Bendito D. 2009. Analytical methods for the determination of bisphenol $A$ in food. J. Chromatogr. A 1216: 449469. DOI: 10.1016/j.chroma.2008.06.037

363. Bogialli S, Di Corcia A. 2009. Recent applications of liquid chromatographymass spectrometry to residue analysis of antimicrobials in food of animal origin. Anal. Bioanal. Chem. 395: 947-966. DOI: 10.1007/s00216-009-29306

364. Brannon-Peppas L. 2009. Mimetic drug delivery systems for release with specific molecular triggers. Drug Delivery Technol. 9: 30-37. 
365. Caldorera-Moore M, Peppas NA. 2009. Micro- and nanotechnologies for intelligent and responsive biomaterial-based medical systems. Adv. Drug Deliv. Rev. 61: 1391-1401. DOI: 10.1016/j.addr.2009.09.002

366. Chen LG, Wang H, Zeng QL, Xu Y, Sun L, Xu HY, Ding L. 2009. On-line Coupling of Solid-Phase Extraction to Liquid Chromatography-A Review. J. Chromatogr. Sci. 47: 614-623.

367. Cigic IK, Prosen H. 2009. An Overview of Conventional and Emerging Analytical Methods for the Determination of Mycotoxins. Int. J. Mol. Sci. 10: 62-115. DOI: 10.3390/ijms10010062

368. Deng JP, Wang LF, Liu LY, Yang WT. 2009. Developments and new applications of UV-induced surface graft polymerizations. Prog. Polym. Sci. 34: 156-193. DOI: 10.1016/j.progpolymsci.2008.06.002

369. Fernández-Barbero A, Suárez IJ, Sierra-Martín B, Fernández-Nieves A, de las Nieves FJ, Marquez M, Rubio-Retama J, López-Cabarcos E. 2009. Gels and microgels for nanotechnological applications. Adv. Colloid Interface Sci. 147-148: 88-108. DOI: 10.1016/j.cis.2008.12.004

370. Haber J. 2009. The Role of Catalysis in Determining Men's Quality of Life. Top. Catal. 52: 970-981. DOI: 10.1007/s11244-009-9247-8

371. Ikai T, Okamoto Y. 2009. Structure Control of Polysaccharide Derivatives for Efficient Separation of Enantiomers by Chromatography. Chem. Rev. 109: 6077-6101. DOI: $10.1021 / \mathrm{cr} 8005558$

372. Jansen JC, Drioli E. 2009. Poly(ether ether ketone) derivative membranes a review of their preparation, properties and potential. Polym. Sci. Ser. A 51: 1355-1366. DOI: 10.1134/S0965545X09110200

373. Kinsella B, O'Mahony J, Malone E, Moloney M, Cantwell H, Furey A, Danaher M. 2009. Current trends in sample preparation for growth promoter 
and veterinary drug residue analysis. J. Chromatogr. A 1216: 7977-8015. DOI: 10.1016/j.chroma.2009.09.005

374. Kovensky J. 2009. Sulfated Oligosaccharides: New Targets for Drug Development? Curr. Med. Chem. 16: 2338-2344. DOI: $10.2174 / 092986709788453096$

375. Lattanzio VMT, Pascale M, Visconti A. 2009. Current analytical methods for trichothecene mycotoxins in cereals. TrAC, Trends Anal. Chem. 28: 758768. DOI: 10.1016/j.trac.2009.04.012

376. Lieberzeit PA, Dickert FL. 2009. Chemosensors in environmental monitoring: challenges in ruggedness and selectivity. Anal. Bioanal. Chem. 393: 467-472. DOI: 10.1007/s00216-008-2464-3

377. Maragos CM. 2009. Recent advances in the development of novel materials for mycotoxin analysis. Anal. Bioanal. Chem. 395: 1205-1213. DOl: $10.1007 / \mathrm{s} 00216-009-2728-6$

378. Marazuela MD, Bogialli S. 2009. A review of novel strategies of sample preparation for the determination of antibacterial residues in foodstuffs using liquid chromatography-based analytical methods. Anal. Chim. Acta 645: 517. DOI: 10.1016/j.aca.2009.04.031

379. Mmualefe LC, Torto N. 2009. Environmental and food sample handling challenges for developing countries. Toxicol. Environ. Chem. 91: 819-835. DOI: $10.1080 / 02772240802674592$

380. Nantasenamat C, Isarankura-Na-Ayudhya C, Naenna T, Prachayasittikul V. 2009. A practical overview of quantitative structure-activity relationship. EXCLI J. 8: 74-88.

381. Nesterenko IS, Nokel MA, Eremin SA. 2009. Immunochemical methods for the detection of sulfanylamide drugs. J. Anal. Chem. 64: 435-444. DOI: $10.1134 /$ S1061934809050025 
382. Nováková L, Vlcková H. 2009. A review of current trends and advances in modern bio-analytical methods: Chromatography and sample preparation. Anal. Chim. Acta 656: 8-35. DOI: 10.1016/j.aca.2009.10.004

383. Patel RP, Purohit NS, Suthar AM. 2009. An overview of silica aerogels. Int. J. ChemTech Res. 1: 1052-1057.

384. Ricanyova J, Gadzala-Kopciuch R, Reiffova K, Buszewski B. 2009. Estrogens and Their Analytics by Hyphenated Separation Techniques. Crit. Rev. Anal. Chem. 39: 13-31. DOI: 10.1080/10408340802569506

385. Rubio S, Pérez-Bendito D. 2009. Recent Advances in Environmental Analysis. Anal. Chem. 81: 4601-4622. DOI: 10.1021/ac900738s

386. Schneider HJ, Strongin RM. 2009. Supramolecular Interactions in Chemomechanical Polymers. Acc. Chem. Res. 42: 1489-1500. DOI: 10.1021/ar800274u

387. Shiju NR, Guliants VV. 2009. Recent developments in catalysis using nanostructured materials. Appl. Catal., $A$ 356: 1-17. DOI: 10.1016/j.apcata.2008.11.034

388. Striegler S. 2009. Emulsion and Miniemulsion Polymers in Catalysis. MiniRev. Org. Chem 6: 234-240.

389. Tang F, Zhang QL, Nie Z, Yao SZ, Chen B. 2009. Sample preparation for analyzing traditional Chinese medicines. TrAC, Trends Anal. Chem. 28: 1253-1262. DOI: 10.1016/j.trac.2009.09.004

390. Tothill IE. 2009. Biosensors for cancer markers diagnosis. Semin. Cell Dev. Biol. 20: 55-62. DOI: 10.1016/j.semcdb.2009.01.015

391. Trojanowicz M, Kaniewska M. 2009. Electrochemical Chiral Sensors and Biosensors. Electroanalysis 21: 229-238. DOI: 10.1002/elan.200804382 
392. Vial L, Dumy P. 2009. Artificial enzyme-based biosensors. New J. Chem. 33: 939-946. DOI: 10.1039/b822966j

393. Walcarius A, Collinson MM. 2009. Analytical Chemistry with Silica Sol-Gels: Traditional Routes to New Materials for Chemical Analysis. Annu. Rev. Anal. Chem. 2: 121-143. DOI: 10.1146/annurev-anchem-060908-155139

394. Yang YJ, Alexandratos SD. 2009. Affinity of Polymer-Supported Reagents for Lanthanides as a Function of Donor Atom Polarizability. Ind. Eng. Chem. Res. 48: 6173-6187. DOI: 10.1021/ie900074t

395. Yashima E, Maeda K, lida H, Furusho Y, Nagai K. 2009. Helical Polymers: Synthesis, Structures, and Functions. Chem. Rev. 109: 6102-6211. DOI: $10.1021 / \mathrm{cr} 900162 q$

396. Aguilar-Arteaga K, Rodriguez JA, Barrado E. 2010. Magnetic solids in analytical chemistry: A review. Anal. Chim. Acta 674: 157-165. DOI: 10.1016/j.aca.2010.06.043

397. Ariga K, Richards GJ, Ishihara S, Izawa H, Hill JP. 2010. Intelligent Chiral Sensing Based on Supramolecular and Interfacial Concepts. Sensors 10: 6796-6820. DOI: 10.3390/s100706796

398. Augusto F, Carasek E, Silva RGC, Rivellino SR, Batista AD, Martendal E. 2010. New sorbents for extraction and microextraction techniques. J. Chromatogr. A 1217: 2533-2542. DOI: 10.1016/j.chroma.2009.12.033

399. Calvo-Rolle JL, Meizoso-López MDC, González-Rodríguez MV, LópezVilariño JM, Cela-Pérez MC. 2010. Oil degradation monitoring with chemical sensors and molecular imprinted polymer. Dyna 85: 738-745.

400. Capriotti AL, Cavaliere C, Giansanti $P$, Gubbiotti R, Samperi R, Laganà A. 2010. Recent developments in matrix solid-phase dispersion extraction. $J$. Chromatogr. A 1217: 2521-2532. DOI: 10.1016/j.chroma.2010.01.030 
401. Chapuis-Hugon F, Pichon V. 2010. Selective tools for trace analysis in complex samples (Utilisation d'outils sélectifs pour l'analyse de traces dans des échantillons complexes). Ann. Toxicol. Anal. 22: 97-101. DOI: 10.1051/ata/2010016

402. Chen J, Duan CF, Guan YF. 2010. Sorptive extraction techniques in sample preparation for organophosphorus pesticides in complex matrices. $J$. Chromatogr B 878: 1216-1225. DOI: 10.1016/j.jchromb.2010.02.031

403. Cruz-Vera M, Lucena R, Cárdenas S, Valcárcel M. 2010. Highly selective and non-conventional sorbents for the determination of biomarkers in urine by liquid chromatography. Anal. Bioanal. Chem. 397: 1029-1038. DOI: 10.1007/s00216-010-3476-3

404. de Dios AS, Díaz-García ME. 2010. Multifunctional nanoparticles: Analytical prospects. Anal. Chim. Acta 666: 1-22. DOI: 10.1016/j.aca.2010.03.038

405. Deloncle R, Caminade AM. 2010. Stimuli-responsive dendritic structures: The case of light-driven azobenzene-containing dendrimers and dendrons. J. Photochem. Photobiol. C:Photochem. Rev. 11: 25-45. DOI: 10.1016/j.jphotochemrev.2010.02.003

406. Faraji M, Yamini Y, Rezaee M. 2010. Magnetic nanoparticles: Synthesis, stabilization, functionalization, characterization, and applications. J. Iranian Chem. Soc. 7: 1-37. DOI: 10.1007/BF03245856

407. Gawel K, Barriet D, Sletmoen M, Stokke BT. 2010. Responsive Hydrogels for Label-Free Signal Transduction within Biosensors. Sensors 10: 43814409. DOI: $10.3390 / \mathrm{s} 100504381$

408. Hennion MC, Gareil P, Hagege A, Kuhn A, Pichon V. 2010. Analytical chemistry and society. Actualité Chim. 55-63.

409. Higuchi A, Tamai M, Ko YA, Tagawa YI, Wu YH, Freeman BD, Bing JT, Chang Y, Ling QD. 2010. Polymeric Membranes for Chiral Separation of 
Pharmaceuticals and Chemicals. Polym. Rev. 50: 113-143. DOI: $10.1080 / 15583721003698853$

410. Huang X, Yin YZ, Liu JQ. 2010. Design of Artificial Selenoenzymes Based on Macromolecular Scaffolds. Macromol. Biosci. 10: 1385-1396. DOI: 10.1002/mabi.201000134

411. Jagur-Grodzinski J. 2010. Polymeric gels and hydrogels for biomedical and pharmaceutical applications. Polym. Adv. Technol. 21: 27-47. DOI: 10.1002/pat.1504

412. Khulbe KC, Feng C, Matsuura T. 2010. The art of surface modification of synthetic polymeric membranes. J. Appl. Polym. Sci. 115: 855-895. DOI: 10.1002/app.31108

413. Kloskowski A, Pilarczyk M, Chrzanowski W, Namiesnik J. 2010. Sol-Gel Technique A Versatile Tool for Adsorbent Preparation. Crit. Rev. Anal. Chem. 40: 172-186. DOI: 10.1080/10408347.2010.490486

414. Kumazawa T, Hasegawa C, Lee XP, Sato K. 2010. New and unique methods of solid-phase extraction for use before instrumental analysis of xenobiotics in human specimens. Forensic Toxicol. 28: 61-68. DOI: 10.1007/s11419-010-0097-7

415. Lämmerhofer M, Gargano A. 2010. Monoliths with chiral surface functionalization for enantioselective capillary electrochromatography. $\mathrm{J}$. Pharm. Biomed. Anal. 53: 1091-1123. DOI: 10.1016/j.jpba.2010.05.026

416. Lourenco TD, Cassiano NM, Cass QB. 2010. Chiral Stationary Phases for High-Performance Liquid Chromatography. Quimica Nova 33: 2155-2164. DOI: $10.1590 / S 0100-40422010001000028$

417. Matsuoka S, Yoshimura K. 2010. Recent trends in solid phase spectrometry: 2003-2009. A Review. Anal. Chim. Acta 664: 1-18. DOI: 10.1016/j.aca.2010.01.041 
418. Melde BJ, Johnson BJ. 2010. Mesoporous materials in sensing: morphology and functionality at the meso-interface. Anal. Bioanal. Chem. 398: 15651573. DOI: $10.1007 / \mathrm{s} 00216-010-3688-6$

419. Miyata T. 2010. Preparation of smart soft materials using molecular complexes. Polym. J. 42: 277-289. DOI: 10.1038/pj.2010.12

420. Pedrón S, Anseth K, Benton JA, Bosch P, Peinado C. 2010. Bioapplications of Networks Based on Photo-Cross-Linked Hyperbranched Polymers. Macromol. Symp. 291-292: 307-313. DOI: 10.1002/masy.201050536

421. Plata MR, Contento AM, Rios A. 2010. State-of-the-Art of (Bio)Chemical Sensor Developments in Analytical Spanish Groups. Sensors 10: 25112576. DOI: $10.3390 / \mathrm{s} 100402511$

422. Prodromidis MI. 2010. Impedimetric immunosensors--A review. Electrochim. Acta 55: 4227-4233. DOI: 10.1016/j.electacta.2009.01.081

423. Pustam AN, Alexandratos SD. 2010. Engineering selectivity into polymersupported reagents for transition metal ion complex formation. Reac. Func. Polym. 70: 545-554. DOI: 10.1016/j.reactfunctpolym.2010.05.002

424. Ramautar R, Somsen GW, de Jong GJ. 2010. Recent developments in coupled SPE-CE. Electrophoresis 31: 44-54. DOI: 10.1002/elps.200900510

425. Raynie DE. 2010. Modern Extraction Techniques. Anal. Chem. 82: 49114916. DOI: $10.1021 / \mathrm{ac} 101223 \mathrm{c}$

426. Spietelun A, Pilarczyk M, Kloskowski A, Namiesnik J. 2010. Current trends in solid-phase microextraction (SPME) fibre coatings. Chem. Soc. Rev. 39: 4524-4537. DOI: 10.1039/C003335A

427. Sun FX, Ma W, Xu LG, Zhu YY, Liu LQ, Peng CF, Wang LB, Kuang $H, X u$ CL. 2010. Analytical methods and recent developments in the detection of 
melamine. TrAC, Trends Anal. Chem. 29: 1239-1249. DOI: 10.1016/j.trac.2010.06.011

428. Tada M. 2010. Surface-mediated design and catalytic properties of active metal complexes for advanced catalysis creation. Bull. Chem. Soc. Jpn. 83: 855-876. DOI: 10.1246/bcsj.20090336

429. Uccello-Barretta G, Vanni L, Balzano F. 2010. Nuclear magnetic resonance approaches to the rationalization of chromatographic enantiorecognition processes. J. Chromatogr. A 1217: 928-940. DOI: 10.1016/j.chroma.2009.10.070

430. Urusov AE, Zherdev AV, Dzantiev BB. 2010. Immunochemical methods of mycotoxin analysis (review). Appl. Biochem. Microbiol. 46: 253-266. DOI: $10.1134 /$ S0003683810030038

431. Van Dorst B, Mehta J, Bekaert K, Rouah-Martin E, De Coen W, Dubruel P, Blust R, Robbens J. 2010. Recent advances in recognition elements of food and environmental biosensors: A review. Biosens. Bioelectron. 26: 11781194. DOI: 10.1016/j.bios.2010.07.033

432. Ward TJ, Ward KD. 2010. Chiral Separations: Fundamental Review 2010. Anal. Chem. 82: 4712-4722. DOI: 10.1021/ac1010926

433. Wistuba D. 2010. Chiral silica-based monoliths in chromatography and capillary electrochromatography. J. Chromatogr. A 1217: 941-952. DOI: 10.1016/j.chroma.2009.11.079

434. Yang GL, Liu HY. 2010. Application of Monolithic Stationary Phases in Solid-Phase Extraction and Pharmaceutical Analysis. Curr. Pharm. Anal. 6: 213-224. DOI: 10.2174/157341210791936777

435. Borsdorf H, Mayer T, Zarejousheghani M, Eiceman GA. 2011. Recent Developments in Ion Mobility Spectrometry. Appl. Spec. Rev. 46: 472-521. DOI: 10.1080/05704928.2011.582658 
436. Buchberger WW. 2011. Current approaches to trace analysis of pharmaceuticals and personal care products in the environment. $J$. Chromatogr. A 1218: 603-618. DOI: 10.1016/j.chroma.2010.10.040

437. Campbell K, Rawn DFK, Niedzwiadek B, Elliott CT. 2011. Paralytic shellfish poisoning (PSP) toxin binders for optical biosensor technology: problems and possibilities for the future: a review. Food Addit. Contam., Part A 28: 711-725. DOI: 10.1080/19440049.2010.531198

438. Chigome S, Torto N. 2011. A review of opportunities for electrospun nanofibers in analytical chemistry. Anal. Chim. Acta 706: 25-36. DOI: 10.1016/j.aca.2011.08.021

439. Chimuka L, Cukrowska E, Michel M, Buszewski B. 2011. Advances in sample preparation using membrane-based liquid-phase microextraction techniques. TrAC, Trends Anal. Chem. 30: 1781-1792. DOI: 10.1016/j.trac.2011.05.008

440. Cichna-Markl M. 2011. New strategies in sample clean-up for mycotoxin analysis. World Mycotoxin J. 4: 203-215. DOI: 10.3920/WMJ2010.1280

441. Cobzac SC, Gocan S. 2011. Sample preparation for high performance liquid chromatography: Recent progress. J. Liq. Chrom. Rel. Technol. 34: 11571267. DOI: 10.1080/10826076.2011.588064

442. Denizli A. 2011. Plasma fractionation: conventional and chromatographic methods for albumin purification. Hacettepe J. Biol. \& Chem. 39: 315-341.

443. Dong ZY, Wang YG, Yin YZ, Liu JQ. 2011. Supramolecular enzyme mimics by self-assembly. Curr. Opin. Colloid Interf. Sci. 16: 451-458. DOI: 10.1016/j.cocis. 2011.08 .006

444. Ekdahl KN, Lambris JD, Elwing $H$, Ricklin D, Nilsson PH, Teramura $\mathrm{Y}$, Nicholls IA, Nilsson B. 2011. Innate immunity activation on biomaterial 
surfaces: A mechanistic model and coping strategies. Adv. Drug Deliv. Rev. 63: 1042-1050. DOI: 10.1016/j.addr.2011.06.012

445. Fodey T, Leonard P, O'Mahony J, O'Kennedy R, Danaher M. 2011. Developments in the production of biological and synthetic binders for immunoassay and sensor-based detection of small molecules. TrAC, Trends Anal. Chem. 30: 254-269. DOI: 10.1016/j.trac.2010.10.011

446. García JM, García FC, Serna F, de la Peña JL. 2011. Fluorogenic and Chromogenic Polymer Chemosensors. Polym. Rev. 51: 341-390. DOI: 10.1080/15583724.2011.616084

447. Graham LM, Nguyen TM, Lee SB. 2011. Nanodetoxification: emerging role of nanomaterials in drug intoxication treatment. Nanomedicine 6: 921-928.

448. Hapiot F, Ponchel A, Tilloy S, Monflier E. 2011. Cyclodextrins and their applications in aqueous-phase metal-catalyzed reactions. Comptes Rendus Chimie 14: 149-166. DOI: 10.1016/j.crci.2010.04.003

449. Heimink J, Sieger $P$, Koller H. 2011. Two-dimensional pH mapping of release kinetics of silica-encapsulated drugs. J. Pharm. Sci. 100: 44014412. DOI: $10.1002 / j p s .22593$

450. Hu XH, Hao LY, Wang HQ, Yang XL, Zhang GJ, Wang GY, Zhang X. 2011. Hydrogel Contact Lens for Extended Delivery of Ophthalmic Drugs. Int. J. Polym. Sci. 2011: Article ID 814163. DOI: 10.1155/2011/814163

451. Huang X, Liu XM, Luo QA, Liu JQ, Shen JC. 2011. Artificial selenoenzymes: Designed and redesigned. Chem. Soc. Rev. 40: 1171-1184. DOI: $10.1039 /$ COCS00046A

452. Kaye PT. 2011. Designer ligands: The search for metal ion selectivity. South African Journal of Science 107: Art. No. 439. DOI: 10.4102/sajs.v107i3/4.439 
453. Kim HN, Guo ZQ, Zhu WH, Yoon J, Tian H. 2011. Recent progress on polymer-based fluorescent and colorimetric chemosensors. Chem. Soc. Rev. 40: 79-93. DOI: 10.1039/C0CS00058B

454. Kole PL, Venkatesh G, Kotecha J, Sheshala R. 2011. Recent advances in sample preparation techniques for effective bioanalytical methods. Biomed. Chromatogr. 25: 199-217. DOI: 10.1002/bmc.1560

455. Lu H, Chen GN. 2011. Recent advances of enantioseparations in capillary electrophoresis and capillary electrochromatography. Anal. Methods 3: 488508. DOI: 10.1039/COAY00489H

456. Lucena R, Simonet BM, Cárdenas S, Valcárcel M. 2011. Potential of nanoparticles in sample preparation. J. Chromatogr. A 1218: 620-637. DOI: 10.1016/j.chroma.2010.10.069

457. Mahouche-Chergui S, Gam-Derouich S, Mangeney C, Chehimi MM. 2011. Aryl diazonium salts: a new class of coupling agents for bonding polymers, biomacromolecules and nanoparticles to surfaces. Chem. Soc. Rev. 40: 4143-4166.

458. Mangelings D, Vander Heyden Y. 2011. Enantioselective capillary electrochromatography: Recent developments and new trends. Electrophoresis 32: 2583-2601. DOI: 10.1002/elps.201100009

459. Marchetti L, Levine M. 2011. Biomimetic Catalysis. ACS Catalysis 10901118. DOI: $10.1021 / \mathrm{cs} 200171 \mathrm{u}$

460. Martínez-Máñez R, Sancenón F, Biyikal M, Hecht M, Rurack K. 2011. Mimicking tricks from nature with sensory organic-inorganic hybrid materials. J. Mater. Chem. 21: 12588-12604.

461. Meggers E. 2011. From Conventional to Unusual Enyzme Inhibitor Scaffolds: The Quest for Target Specificity. Angew. Chem. Int. Edit. 50: 2442-2448. DOI: 10.1002/anie.201005673 
462. Miró M, Oliveira HM, Segundo MA. 2011. Analytical potential of mesofluidic lab-on-a-valve as a front end to column-separation systems. TrAC, Trends Anal. Chem. 30: 153-164. DOI: 10.1016/j.trac.2010.08.007

463. Paz Y. 2011. Nanoscale structures in photocatalysis: Dense films, molecular imprinting and composites. In Nanoelectronics Conference (INEC), 2011 IEEE 4th International, IEEE: 1-2. DOI: 10.1109/INEC.2011.5991622

464. Qiu JX, Zhang SQ, Zhao HJ. 2011. Recent applications of TiO2 nanomaterials in chemical sensing in aqueous media. Sens. Actuators $B$ 160: 875-890. DOI: 10.1016/j.snb.2011.08.077

465. Rickerby DG, Skouloudis AN. 2011. Biosensor networks for monitoring water pollution. In Proceedings of the Global Humanitarian Technology Conference (GHTC), 2011 IEEE, IEEE: 276-282. DOI: 10.1109/GHTC.2011.65

466. Rocha FRP, Raimundo IM, Teixeira LSG. 2011. Direct Solid-Phase Optical Measurements in Flow Systems: A Review. Anal. Lett. 44: 528-559. DOI: $10.1080 / 00032719.2010 .500790$

467. Ruigrok VJB, Levisson M, Eppink MHM, Smidt H, van der Oost J. 2011. Alternative affinity tools: more attractive than antibodies? Biochem. J. 436: 1-13. DOI: 10.1042/BJ20101860

468. Samanidou V, Kovatsi L, Fragou D, Rentifis K. 2011. Novel strategies for sample preparation in forensic toxicology. Bioanalysis 3: 2019-2046. DOI: 10.4155/bio.11.168

469. Singh K, Nair AB, Kumar A, Kumria A. 2011. Novel Approaches in Formulation and Drug Delivery using Contact Lenses. Journal of Basic and Clinical Pharmacy 2: 87-101.

470. Sproß J, Sinz A. 2011. Monolithic media for applications in affinity chromatography. J. Sep. Sci. 34: 1958-1973. DOI: 10.1002/jssc.201100400 
471. Stocka J, Tankiewicz M, Biziuk M, Namiesnik J. 2011. Green Aspects of Techniques for the Determination of Currently Used Pesticides in Environmental Samples. Int. J. Mol. Sci. 12: 7785-7805. DOI: doi:10.3390/ijms12117785

472. Sun SD, Hunag JY, Zhao CS. 2011. Polymeric Particles for the Removal of Endocrine Disruptors. Sep. Purif. Rev. 40: 312-337. DOI: 10.1080/15422119.2011.561395

473. Teles FSRR. 2011. Biosensors and rapid diagnostic tests on the frontier between analytical and clinical chemistry for biomolecular diagnosis of dengue disease: A review. Anal. Chim. Acta 687: 28-42. DOI: 10.1016/j.aca.2010.12.011

474. Thorne JB, Vine GJ, Snowden MJ. 2011. Microgel applications and commercial considerations. Colloid Polym. Sci. 289: 625-646. DOI: $10.1007 / \mathrm{s} 00396-010-2369-5$

475. Tomatsu I, Peng K, Kros A. 2011. Photoresponsive hydrogels for biomedical applications. Adv. Drug Deliv. Rev. 63: 1257-1266. DOI: 10.1016/j.addr.2011.06.009

476. Tuantranont A, Wisitsora-at A, Sritongkham P, Jaruwongrungsee K. 2011. A review of monolithic multichannel quartz crystal microbalance: A review. Anal. Chim. Acta 687: 114-128. DOI: 10.1016/j.aca.2010.12.022

477. Vashisy SK, Vashist P. 2011. Recent Advances in Quartz Crystal Microbalance-Based Sensors. J. Sens. Article ID 571405. DOI: $10.1155 / 2011 / 571405$

478. Wang NX, von Recum HA. 2011. Affinity-Based Drug Delivery. Macromol. Biosci. 11: 321-332. DOI: 10.1002/mabi.201000206

479. Welch M, Rastogi A, Ober C. 2011. Polymer brushes for electrochemical biosensors. Soft Matter 7: 297-302. DOI: 10.1039/C0SM00035C 
480. White CJ, Tieppo A, Byrne ME. 2011. Controlled drug release from contact lenses: a comprehensive review from 1965-present. J. Drug. Deliv. Sci. Technol. 21: 369-384.

481. Yamamoto T, Yoshikiyo K. 2011. The Effects of Cyclodextrins on the Conformation of Proteins. Curr. Org. Chem. 15: 831-838. DOI: $10.2174 / 138527211794518943$

482. Llorent-Martínez EJ, Ortega-Barrales P, Fernández-De Córdova ML, RuizMedina A. 2011. Contribution to Automation for Determination of Drugs Based on Flow-Through Optosensors. Appl. Spec. Rev. 46: 339-367. DOI: 10.1080/05704928.2011.555897

483. Rao TP, Metilda P, Gladis JM. 2006. Preconcentration techniques for uranium(VI) and thorium(IV) prior to analytical determination--an overview. Talanta 68: 1047-1064. DOI: 10.1016/j.talanta.2005.07.021

484. Kataoka H, Saito K. 2011. Recent advances in SPME techniques in biomedical analysis. J. Pharm. Biomed. Anal. 54: 926-950. DOI: 10.1016/j.jpba.2010.12.010

485. Dickert FL, Hayden O, Haderspöck C, Wirl B, Scholl G, Wolff U. 2002. Biomimetische Chemosensoren - Selektive Detektion von Mikroorganismen, Viren und Enzymen. In Sensoren- und Mess-Systeme 2002, 331-334.

486. Cheng WH, Lo MY, Hwang CL, Wu KC, Ting C, Lin Cl, Lee CH. 2004. Recent advances in catalyst and polymer related nanotechnology at UCL/ITRI. Asian Pacific Confederation of Chemical Engineering congress program and abstracts, Vol. 2004, The Society of Chemical Engineers, Japan: 56-67.

487. Song CE, Park YS. 2005. Improved Catalytic Performances of Supported Catalysts. Adv. Org. Synth. 1: 233-260. DOI: 10.2174/1574087054582941 
488. Lieberzeit PA, Rehman A, Najafi B, Mujahid A, Dickert FL. 2008. Acoustic chemosensors for real-life environments. In Frequency Control Symposium, 2008 IEEE International, IEEE: 431-433. DOI: 10.1109/FREQ.2008.4623033

489. Lieberzeit PA, Rehman A, Najafi B, Dickert FL. 2008. Generating bioanalogous recognition of artificial materials - Sensors and electronic noses for odours. In Mining Smartness from Nature, Advances in Science and Technology, Vol. 58, Vincenzini P, Graziani S (eds). Scientific.net: 103-107. DOI: 10.4028/www.scientific.net/AST.58.103

490. Miyata T. 2008. Smart hydrogels that respond to target biomolecules. In Biomedical Applications of Smart Materials, Advances in Science and Technology, Vol. 57, Vincenzini P, de Rossi D (eds). Scientific.net: 15-21. DOI: 10.4028/www.scientific.net/AST.57.15

491. Nehra D. 2010. Bile in the Esophagus-Model for a Bile Acid Biosensor. Journal of Gastrointestinal Surgery 14: 6-8. DOI: 10.1007/s11605-009-10260

492. Cavazzini A, Pasti L, Massi A, Marchetti N, Dondi F. 2011. Recent applications in chiral high performance liquid chromatography: A review. Anal. Chim. Acta 706: 205-222. DOI: 10.1016/j.aca.2011.08.038

493. Lakshmi D, Whitcombe MJ, Davis F, Sharma PS, Prasad BB. 2011. Electrochemical Detection of Uric Acid in Mixed and Clinical Samples: A Review. Electroanalysis 23: 305-320. DOI: 10.1002/elan.201000525

494. Varma AJ, Deshpande SV, Kennedy JF. 2004. Metal complexation by chitosan and its derivatives: a review. Carbohydr. Polym. 55: 77-93. DOI: 10.1016/j.carbpol.2003.08.005

495. Meier MAR, Hoogenboom R, Schubert US. 2004. Combinatorial methods, automated synthesis and high-throughput screening in polymer research: 
The evolution continues. Macromol. Rapid Commun. 25: 21-33. DOI: 10.1002/marc.200300147

496. Cruz-Vera M, Lucena R, Cárdenas S, Valcárcel M. 2009. Sorptive microextraction for liquid-chromatographic determination of drugs in urine. TrAC, Trends Anal. Chem. 28: 1164-1173. DOI: 10.1016/j.trac.2009.07.010

497. Kataoka H. 2011. Current Developments and Future Trends in Solid-phase Microextraction Techniques for Pharmaceutical and Biomedical Analyses. Anal. Sci. 27: 893-905. DOI: 10.2116/analsci.27.893

498. Tran-Thi TH, Dagnelie R, Crunaire S, Nicole L. 2011. Optical chemical sensors based on hybrid organic-inorganic sol-gel nanoreactors. Chem. Soc. Rev. 40: 621-639. DOI: 10.1039/CoCS00021C

499. Bakker E. 2004. Electrochemical sensors. Anal. Chem. 76: 3285-3298. DOI: $10.1021 / \mathrm{ac} 049580 z$

500. Alvarez-Lorenzo C, Hiratani H, Concheiro A. 2006. Contact lenses for drug delivery: Achieving sustained release with novel systems. Am. J. Drug Deliv. 4: 131-151.

501. Barbu E, Verestiuc L, Nevell TG, Tsibouklis J. 2006. Polymeric materials for ophthalmic drug delivery: trends and perspectives. J. Mater. Chem. 16: 3439-3443. DOI: 10.1039/b605640g

502. Burleigh MC, Dai S, Hagaman EW, Barnes CE, Xue ZL. 2000. Stepwise Assembly of Surface Imprint Sites on MCM-41 for Selective Metal Ion Separations. In Nuclear Site Remediation, ACS Symposium Series, Vol. 778, Eller PG, Heineman WR (eds). American Chemical Society: Washington, DC; 146-158. DOI: 10.1021/bk-2001-0778.ch010

503. Byrne ME, Henthorn DB, Huang YB, Peppas NA. 2002. Micropatterning Biomimetic Materials for Bioadhesion and Drug Delivery. In Biomimetic Materials and Design: Interactive Biointerfacial Strategies, Tissue 
Engineering, and Targeted Drug Delivery, Dillow AK, Lowman AM (eds). Marcel Dekker: New York;

504. Collinson MM. 2002. Imprinted Functionalized Silica. In The Supramolecular Chemistry of Organic-Inorganic Hybrid Materials, Rurack K, Martínez-Máñez R (eds). John Wiley \& Sons Inc.: Hoboken; 581-598. DOI: 10.1002/9780470552704.ch20

505. Cormack PAG, Haupt K. 2000. Molecular imprints for solid-phase extraction. In Encyclopedia of Separation Science, Vol. 7, Wilson ID, Adlard TR, Poole CF, Cook M (eds). Elsevier Ltd: Oxford; 3395-3400. DOI: 10.1016/B0-12226770-2/06741-7

506. Cormack PAG, Haupt K, Mosbach K. 2000. Affinity Separation I Imprint Polymers. In Encyclopedia of Separation Science, Vol. 1, Wilson ID, Adlard TR, Poole CF, Cook M (eds). Elsevier Ltd: Oxford; 288-296. DOI: 10.1016/B0-12-226770-2/00571-8

507. Crudden CM, Allen DP, Motorina I, Fairgrieve M. 2003. Late Transition Metal Complexes Immobilized on Structured Surfaces as Catalysts for Hydrogenation and Oxidation Reactions. In Nanostructured Catalysts, Nanostructure Science and Technology, Scott SL, Crudden CM, Jones CW (eds). Springer US: 113-155. DOI: 10.1007/978-0-387-30641-4_5

508. Dickert FL, Greibl W, Hayden O, Lieberzeit P, Sikorski R, Tortschanoff M, Weber K. 1999. Development of materials for chemical sensors - From molecular cavities to imprinting techniques. In Smart Material Systems, Advances in Science and Technology, Vol. 25, Vincenzini P (ed). 175-182.

509. Dickert FL, Lieberzeit PA. 2000. Solid-State Sensors for Field Measurements of Gases and Vapors. In Encyclopedia of Analytical Chemistry, Meyers RA (ed). John Wiley \& Sons Ltd.: Chichester; 38313855. DOI: 10.1002/9780470027318.a0921 
510. Flavin K, Resmini M. 2002. Molecular Imprinting with Nanomaterials. In Advanced Nanomaterials, Vol. 2, Geckeler KE, Nishide H (eds). Wiley-VCH: Weinheim; 651-675. DOI: 10.1002/9783527628940.ch20

511. Jones CW. 2003. Strategies for the Control of Porosity around Organic Active Sites in Inorganic Matrices. In Nanostructured Catalysts, Nanostructure Science and Technology, Scott SL, Crudden CM, Jones CW (eds). Springer US: 277-295. DOI: 10.1007/978-0-387-30641-4_12

512. Kempe M. 2000. Chiral Separations | Molecular Imprints as Stationary Phases. In Encyclopedia of Separation Science, Wilson ID, Adlard TR, Poole CF, Cook M (eds). Elsevier Ltd: Oxford; 2387-2397. DOI: 10.1016/B012-226770-2/03161-6

513. Kozulic B, Heimgartner U. 1996. Hydrophilic and Amphiphatic Monomers and use of their Gels as Separation Media. In Biochemical Technology, Advances in Molecular and Cell Biology, Vol. 15B, Bittar EE, Danielsson B, Bulow L (eds). Elsevier: Amsterdam; 593-604. DOI: 10.1016/S15692558(08)60139-3

514. Meredith JC, Smith AP, Karim A, Amis EJ. 2002. Combinatorial Polymer Science: Synthesis and Characterization. In Combinatorial Materials Development, ACS Symposium Series, Vol. 814, Malhotra R (ed). American Chemical Society: Washington, DC; 23-47. DOI: 10.1021/bk-20020814.ch002

515. Nicholls IA. 1996. Molecular Recognition: An Introduction. In Biochemical Technology, Advances in Molecular and Cell Biology, Vol. 15B, Bittar EE (ed). Elsevier: 621-622. DOI: 10.1016/S1569-2558(08)60141-1

516. Piletsky SA, Turner APF. 2002. New materials based on imprinted polymers and their application in optical biosensors. In Optical bioensors: Present and future, Ligler FS, Rowe Taitt CA (eds). Elsevier: Amsterdam; 397-426. 
517. Ramström O. 2000. Selectivity of imprinted polymers: Affinity separation. In Encyclopedia of Separation Science, Wilson ID, Adlard TR, Poole CF, Cook M (eds). Elsevier Ltd: Oxford; 4104-4111. DOI: 10.1016/B0-12-2267702/06141-X

518. Sellergren B. 2003. Imprinted Monoliths. In Monolithic Materials Preparation, Properties and Applications, Journal of Chromatography Library, Vol. 67, Svec F, Tennikova TB, Deyl Z (eds). Elsevier: 277-300. DOI: 10.1016/S0301-4770(03)80029-5

519. Spivak D. 2000. Molecular Imprinting. In Kirk-Othmer Encyclopedia of Chemical Technology, John Wiley \& Sons, Inc.: DOI: 10.1002/0471238961.molespiv.a01

520. Ichinose I, Lee SW, Kunitake T. 2001. Nanofabrication by the surface sol-gel process and molecular imprinting. In Supramolecular Organization and Materials Design, Jones W, Rao CNR (eds). Cambridge University Press: Cambridge; 172-213. DOI: 10.1017/CBO9780511564987.007

521. Alexander C, Kirsch N, Whitcombe MJ. 2004. New methodologies in the preparation of imprinted polymers. In Polymer Chemistry A Practical Approach, Practical Approach to Chemistry, Davis FJ (ed). Oxford University Press: Oxford; 201-214.

522. Vilar R. 2004. Hydrogen-bonding templated assemblies. In Supramolecular Assembly via Hydrogen Bonds II, Structure and Bonding, Vol. 111, Mingos DMP (ed). Springer Verlag: Berlin; 85-137. DOI: 10.1007/b14141

523. Baggiani C, Giovannoli C. 2005. Molecular Imprinted Solid-Phase Extraction for Cleanup of Chlorinated Phenoxyacids From Aqueous Samples. In Pesticide Protocols, Methods in Biotechnology, Vol. 19, Vidal JLM, Frenich AG (eds). Springer: 421-433. DOI: 10.1385/1-59259-929-X:421 
524. Dickert FL, Lieberzeit PA. 2005. Chemical Recognition and Sensing by SelfOrganization. In Encyclopedia of Supramolecular Chemistry, Atwood JL, Steed JW (eds). Taylor \& Francis: 1-13. DOI: 10.1081/E-ESMC-120012776

525. Hall AJ, Emgenbroich M, Sellergren B. 2005. Imprinted polymers. In Templates in Chemistry II, Topics in Current Chemistry, Vol. 249, Schalley CA, Vögtle F, Dötz KH (eds). Springer Verlag: Berlin; 317-349. DOI: $10.1007 /$ b104333

526. Komiyama M. 2005. Molecular Imprinting. In Supramolecular Polymers, Ciferri A (ed). CRC Press: 711-724.

527. Marty JD, Mauzac M. 2005. Molecular imprinting: State of the art and perspectives. In Microlithography/Molecular Imprinting, Advances In Polymer Science, Ito H, Marty JD (eds). Springer-Verlag GmbH: 1-35. DOI: $10.1007 /$ b97573

528. Molinelli A, Janotta M, Mizaikoff B. 2005. Molecularly imprinted polymers for biomolecular recognition. In Protein Nanotechnology: Protocols, Instrumentation, and Applications, Methods in Molecular Biology, Vol. 300, Vo-Dinh T (ed). Humana Press: 243-254. DOI: 10.1385/1-59259-858-7:243

529. Turkewitsch $P$, Massé R, Powell WS. 2005. Molecular Imprinting. In Advanced Concepts in Fluorescence Sensing, Pt. B: Macromolecular Sensing, Topics in Fluoresence Spectroscopy, Vol. 10, Geddes CD, Lakowicz JR (eds). Springer: New York; 157-209.

530. Turner APF, Piletsky S. 2005. Biosensors and biomimetic sensors for the detection of drugs, toxins and biological agents. In Defense Against Bioterror: Detection Technologies, Implementation Strategies And Commercial Opportunities 1, NATO Security Through Science Series B: Physics And Biophysics, Morrison D, Milanovich F, Ivnitski D, Austin TR (eds). Springer: Dordrecht, The Netherlands; 261-272. 
531. Végvári A. 2005. Peptide and protein separations by capillary electrophoresis and electrochromatography. In Proteomics and Peptiodomics: New Technology Platforms Elucidating Biology, Comprehensive Analytical Chemistry, Vol. 46, Marko-Varga G (ed). Elsevier: Amsterdam; 149-252. DOI: 10.1016/S0166-526X(05)46003-1

532. Haupt K. 2005. Molecularly Imprinted Polymers: Artificial Receptors for Affinity Separations. In Handbook of Affinity Chromatography, Second Edition, Chromatographic Science Series, Hage DS (ed). CRC Press: 837856. DOI: $10.1201 / 9780824751982 . c h 30$

533. Alvarez-Lorenzo C, Concheiro A. 2006. Molecularly imprinted materials as advanced excipients for drug delivery systems. In Biotechnology Annual Review, Vol. 12, El-Gewely MR (ed). Elsevier: 225-268. DOI: 10.1016/S1387-2656(06)12007-4

534. Bunte G, Hurttlen J, Krause H, Pontius H, Schreiter M, Weber J. 2006. MIPbased low-cost sensor for short-range detection of explosives. In Stand-off Detection of Suicide Bombers and Mobile Subjects, NATO Security through Science Series B: Physics and Biophysics, Schubert H, Rimski-Korsakov A (eds). Springer: 17-27. DOI: 10.1007/1-4020-5159-X_4

535. Cormack PAG. 2006. Imprinted polymers. In Encyclopedia of Supramolecular Chemistry, Atwood JL, Steed JW (eds). Taylor and Francis: DOI: 10.1081/E-ESMC-120012798

536. Dickert FL, Lieberzeit PA, Hayden O. 2006. Patterning Materials - QCM and SAW Applications. In Encyclopedia of Sensors, Grimes CA, Dickey EC, Pishko MV (eds). American Science Publishers: 19 pages.

537. Krska R, Welzig E. 2006. Mycotoxin analysis: An overview of classical, rapid and emerging techniques. In Mycotoxin Factbook: Food \& Feed Topics, Barug D, Bhatnagar D, van Egmond HP, van der Kamp JW, van 
Osenbruggen WA, Visconti A (eds). Wageningen Academic Publishers: Wageningen; 225-247.

538. Lee SW, Kunitake T. 2006. Molecular Imprinting by the Surface Sol-Gel Process: Templated Nanoporous Metal Oxide Thin Films for Molecular Recognition. In Self-Organized Nanoscale Materials, Nanostructure Science and Technology, Adachi M, Lockwood CJ (eds). Springer: New York; 186220. DOI: 10.1007/0-387-27976-8_5

539. Maier NA, Lindner W. 2006. Stereoselective Chromatographic Methods for Drug Analysis. In Chirality in Drug Research, Methods and Principles in Medicinal Chemistry, Francotte E, Lindner W (eds). Wiley-VCH Verlag GmbH \& Co. KGaA: 189-260. DOI: 10.1002/9783527609437.ch7

540. Maschmeyer T, van de Water L. 2006. An Overview of Zeolite, Zeotype and Mesoporous Solids Chemistry: Design, Synthesis and Catalytic Properties. In Microporous and Mesoporous Solid Catalysts, Catalysts for Fine Chemical Synthesis, Vol. 4, Derouane EG (ed). John Wiley \& Sons, Ltd.: Chichester, UK; 1-38. DOI: 10.1002/0470094214.ch1

541. Bogliotti N, Dalko PI. 2007. Shape- and Site-Selective Asymmetric Reactions. In Enantioselective Organocatalysis: Reactions and Experimental Procedures, Dalko PI (ed). Wiley-VCH Verlag GmbH \& Co.: Weinheim; 425-449. DOI: 10.1002/9783527610945.ch13

542. Davis F, Higson SJ. 2007. Polymers in biosensors. In Biomedical Polymers, Jenkins M (ed). Woodhead Publishing Ltd.: Cambridge, UK;

543. Dhruv H, Turner N, Britt D. 2007. Nanoscale Prion Detection Strategies. In Encyclopedia of Agricultural, Food, and Biological Engineering, Taylor \& Francis: 1-3. DOI: 10.1081/E-EAFE-120042375 
544. Farré M, Barceló D. 2007. Sensor, biosensors and MIP based sensors. In Food Toxicants Analysis, Picó Y (ed). Elsevier: Amsterdam; 599-636. DOI: 10.1016/B978-044452843-8/50017-1

545. Koesdjojo MT, Tennico YH, Remcho VT. 2007. Molecularly imprinted polymers as sorbents for separations and extractions. In Separation Science and Technology: HPLC Method Development for Pharmaceuticals, Vol. 8, Ahuja S, Rasmussen H (eds). Academic Press: 479-503. DOI: 10.1016/S0149-6395(07)80024-9

546. Li WK, Li SJ. 2007. Molecular Imprinting: A Versatile Tool for Separation, Sensors and Catalysis. In Oligomers - Polymer Composites - Molecular Imprinting, Advances in Polymer Science, Vol. 206, Springer-Verlag: Berlin; 191-210. DOI: 10.1007/978-3-540-46830-1

547. Sellergren B. 2007. Separation of Enantiomers Using Molecularly Imprinted Polymers. In Chiral Separation Techniques, Subramanian G (ed). WILEY$\mathrm{VCH}$ Verlag $\mathrm{GmbH} \&$ Co.: Weinheim; 399-431. DOI: 10.1002/9783527611737.ch12

548. Southard GE, Van Houten KA, Ott EWJr, Murray GM. 2007. Synthesis and spectroscopic characterization of molecularly imprinted polymer phosphonate sensors. In Antiterrorism and Homeland Defense: Polymers and Materials, ACS Symposium Series, Vol. 980, Reynolds JG, Lawson GE (eds). Oxford University Press, USA: 19-38. DOI: 10.1021/bk-20070980.ch002

549. Wang XD, Li WY, Rasmussen H. 2007. Chiral separations. In Separation Science and Technology: HPLC Method Development for Pharmaceuticals, Vol. 8, Ahuja S, Rasmussen H (eds). Academic Press: 111-144. DOI: 10.1016/S0149-6395(07)80011-0

550. Wulff G. 2007. Templated Synthesis of Polymers - Molecularly Imprinted Materials for Recognition and Catalysis. In Templated Organic Synthesis, 
Diederich F, Stang PJ (eds). Wiley-VCH: Weinheim; 39-73. DOI: 10.1002/9783527613526.ch02

551. Zheng J, Shamsi SA. 2007. Chiral Analysis in Capillary Electrochromatography (CEC) and CEC Coupled to Mass Spectrometry. In Chiral Separation Techniques, Subramanian G (ed). WILEY-VCH Verlag GmbH \& Co.: Weinheim; 441-504. DOI: 10.1002/9783527611737.ch14

552. Shimizu KD, Rushton GT. 2007. Molecularly Imprinted Polymers. In Encyclopedia of Chemical Processing, Lee SG (ed). Taylor \& Francis: 17371746. DOI: 10.1081/E-ECHP-120041040

553. Afzal A, Chen X, Jenik M, Krassnig S, Dickert FL. 2008. Detection of cells and viruses with mass sensitive devices - application of synthetic antibodies. In Commercial and Pre-Commercial Cell Detection Technologies for Defence against Bioterror: Technology, Market and Society, NATO Science for Peace and Security Series E: Human and Societal Dynamics, Vol. 39, Lechuga LM, Milanovich FP, Skládal P, Ignatov O, Austin TR (eds). IOS Press: Amsterdam; 60-76.

554. Anwander R. 2008. Immobilization of Molecular Catalysts. In Handbook of Heterogeneous Catalysis, Ertl G, Knözinger H, Schüth F, Weitkamp J (eds). Wiley: 583-614. DOI: 10.1002/9783527610044.hetcat0029

555. Appell M, Maragos CM, Kendra DF. 2008. Molecularly Imprinted Polymers for Mycotoxins. In Food Contaminants, ACS Symposium Series, Vol. 1001, Siantar DS, Trucksess MW, Scott PM, Herman EM (eds). American Chemical Society: Washington, DC; 152-169. DOI: 10.1021/bk-20081001.ch008

556. Bing NC, Tian Z, Chen SW, Li QH, Xu ZL. 2008. Synthesis and characteristic of molecularly imprinted polymer immobilized mesoporous silica sphere for selective binding of S-naproxen. In Multi-Funtional Materials and Structures, Parts 1 \& 2, Advanced Materials Research, Vol. 47-50, Lau 
AKT, Lu J, Varadan VK, Chang FK, Tu JP, Lam PM (eds). Trans Tech Publications Ltd.: Zurich; 890-893. DOI: 10.4028/www.scientific.net/AMR.4750.890

557. Danielsson B. 2008. Artificial Receptors. In Biosensing for the 21st Century, Advances in Biochemical Engineering/Biotechnology, Renneberg R, Lisdat F (eds). Springer: Berlin; 97-122. DOI: 10.1007/10_2007_088

558. Dufaud V, Bonneviot L. 2008. Molecular Imprinting. In Nanomaterials and Nanochemistry, Bréchignac C, Houdy P, Lahmani M (eds). Springer: Berlin; 597-614. DOI: 10.1007/978-3-540-72993-8_27

559. Ewen SL, Steinke JHG. 2008. Molecularly Imprinted Polymers Using Anions as Templates. In Recognition of Anions, Structure and Bonding, Vol. 129, Vilar R (ed). Springer: Berlin / Heidelberg; 207-248. DOI: 10.1007/430_2008_084

560. Farré M, Martínez E, Barceló D. 2008. Immunochemical and Receptor Technologies: The Role of Immunoassay, Immunoaffinity Chromatography, Immunosensors and Molecularly Imprinted Polymeric Sensors. In Food Contaminants and Residue Analysis, Comprehensive Analytical Chemistry, Vol. 51, Picó Y (ed). Elsevier: Amsterdam; 91-130. DOI: 10.1016/S0166$526 \times(08) 00004-4$

561. Góra L. 2008. Monocrystal-thin b-oriented silicalite-1 assemblies by molecular imprinting: from membrane to micro-reactor applications. In Zeolites and related materials: Trends, targets and challenges, Proceedings of the 4th International FEZA Conference, Studies in Surface Science and Catalysis, Vol. 174, Part 1, Gédéon A, Massiani P, Babonneau F (eds). Elsevier: 629-634. DOI: 10.1016/S0167-2991(08)80278-9

562. Gros M, Petrovic M, Barceló D. 2008. Analysis of Emerging Contaminants of Municipal and Industrial Origin. In Emerging Contaminants from Industrial 
and Municipal Waste, The Handbook of Environmental Chemistry, Barceló D, Petrovic M (eds). Springer: 37-104. DOI: 10.1007/698_5_102

563. Haupt K, Belmont AS. 2008. Molecularly Imprinted Polymers as Recognition Elements in Sensors. In Handbook of Biosensors and Biochips, Marks RS, Cullen DC, Karube I, Lowe CR, Weetall HH (eds). Wiley: DOI: 10.1002/9780470061565.hbb020

564. Khajeh M. 2008. Molecular Imprinted Polymer (MIP) for nickel(II) extraction from environmental water and biological samples. In Metal lons in Biology and Medicine, Metal lons in Biology and Medicine, Vol. 10, Collery P, Maynard I, Theophanides T, Khassanova L, Collery T (eds). John Libby Eurotext: Montrouge, France; 252-256.

565. la Farré M, Martínez E, Barceló D. 2008. Immunochemical and Receptor Technologies: The Role of Immunoassay, Immunoaffinity Chromatography, Immunosensors and Molecularly Imprinted Polymeric Sensors. In Food Contaminants and Residue Analysis, Comprehensive Analytical Chemistry, Vol. 51, Picó Y (ed). Elsevier: Amsterdam; 91-130. DOI: 10.1016/S0166526X(08)00004-4

566. Molina-Díaz A, García-Reyes JF. 2008. Luminescence Detection in Flow Analysis. In Advances in Flow Analysis, Trojanowicz M (ed). Wiley-VCH: Weinheim; 343-393. DOI: 10.1002/9783527623259.ch13

567. Silvestri D, Cristallini C, Barbani N. 2008. Bioartificial Polymer Matrices as Biomaterials with Enzymatically Controlled Functional Properties. In Polymer Biocatalysis and Biomaterials II, ACS Symposium Series, Vol. 999, Cheng HN, Gross RA (eds). American Chemical Society: Washington, DC; 52-75. DOI: 10.1021/bk-2008-0999.ch004

568. Warriner K, Lai E, Namvar A, Hawkins DM, Reddy SM. 2008. Molecular Imprinted Polymers for Biorecognition of Bioagents. In Principles of Bacterial Detection: Biosensors, Recognition Receptors and Microsystems, Zourob M, 
Elwary S, Turner A (eds). Springer: New York; 785-814. DOI: 10.1007/9780-387-75113-9_29

569. Wu XY, Shimizu KD. 2008. Molecular Imprinting for Sensor Applications. In Molecular Recognition and Polymers, Rotello VM, Thayumanavan S (eds). Wiley: Hoboken, NJ; 395-429. DOI: 10.1002/9780470384053.ch15

570. Ashraf SA, Mercado C, Mueller A. 2009. Imprinted Polymers for the Removal of Hydrophilic and Hydrophobic Metal Complexes. In New Membranes and Advanced Materials for Wastewater Treatment, ACS Symposium Series, Vol. 1022, Mueller A, Guieysse B, Sarkar A (eds). American Chemical Society: Washington DC; 53-70. DOI: 10.1021/bk-20091022.ch004

571. Berrocal MJ, Johnson RD, Bachas LG. 2009. Design of Molecular Recognition Elements for Environmental Potentiometric Sensors. In Chemical and Biological Sensors for Environmental Monitoring, ACS Symposium Series, Vol. 762, Mulchandani A, Sadik OA (eds). American Chemical Society: Washington, DC; 8-22. DOI: 10.1021/bk-20000762.ch002

572. Borchers K, Genov S, Gruber-Traub C, Niedergall K, Plankalayil J, PufkyHeinrich D, Riegler J, Schreiber T, Tovar GEM, Weber A, Wojciukiewicz D. 2009. Biomimetic Nanoparticles Providing Molecularly Defined Binding Sites - Protein-Featuring Structures versus Molecularly Imprinted Polymers. In Cellular and Biomolecular Recognition: Synthetic and Non-Biological Molecules, Jelinek R (ed). Wiley-VCH: Weinheim; 31-67. DOI: 10.1002/9783527627011.ch2

573. Dean JR. 2009. Solid Phase Extraction. In Extraction Techniques in Analytical Sciences, Analytical Techniques in the Sciences (AnTS), John Wiley \& Sons Ltd.: Chichester; 49-84. DOI: 10.1002/9780470682494.ch3 
574. Díaz-García ME, Luis GP, Rivero-Espejel IA. 2009. Combinatorial Chemistry for Optical Sensing Applications. In Combinatorial Methods for Chemical and Biological Sensors, Integrated Analytical Systems, Potyrailo RA, Mirsky VM (eds). Springer: New York; 373-391. DOI: 10.1007/978-0-387-737133_15

575. Dunn IS. 2009. Evolving and Creating Recognition Molecules. In Searching for Molecular Solutions - Empirical Discovery and its Future, John Wiley \& Sons Inc.: Hoboken; 234-274. DOI: 10.1002/9780470551455.ch7

576. Guieysse B, Le NM, Mattiasson B. 2009. Removal of Endocrine Disrupting Compounds Using Molecularly Imprinted Polymers: A Review. In New Membranes and Advanced Materials for Wastewater Treatment, ACS Symposium Series, Vol. 1022, Mueller A, Guieysse B, Sarkar A (eds). American Chemical Society: Washington DC; 7-23. DOI: 10.1021/bk-20091022.ch002

577. Kempe H, Kempe M. 2009. Molecularly Imprinted Polymers. In The Power of Functional Resins in Organic Synthesis, Tulla-Puche J, Albericio F (eds). Wiley-VCH: Weinheim; 15-44. DOI: 10.1002/9783527626175.ch2

578. Le NM, Plieva F, Mattiasson B. 2009. Removal of Endocrine Disrupting Contaminants from Water Using Macroporous Molecularly Imprinted Selective Media. In New Membranes and Advanced Materials for Wastewater Treatment, ACS Symposium Series, Vol. 1022, Mueller A, Guieysse B, Sarkar A (eds). American Chemical Society: Washington DC; 25-51. DOI: 10.1021/bk-2009-1022.ch003

579. Marx S. 2009. Chiral Molecular Imprinting as a Tool for Drug Sensing. In Cellular and Biomolecular Recognition: Synthetic and Non-Biological Molecules, Jelinek R (ed). Wiley-VCH: Weinheim; 97-109. DOI: 10.1002/9783527627011.ch4 
580. Sellergren B, Schillinger E, Lanza F. 2009. Experimental Combinatorial Methods in Molecular Imprinting. In Combinatorial Methods for Chemical and Biological Sensors, Integrated Analytical Systems, Potyrailo RA, Mirsky VM (eds). Springer: New York; 173-198. DOI: 10.1007/978-0-387-737133_7

581. Spörl R, Sarkisov L. 2009. Computer Simulation of Enantioselective Adsorption in A Simple Model of Molecularly Imprinted Polymers. In Characterisation of Porous Solids VIII, Royal Society of Chemistry Special Publications, Vol. 318, Kaskel S, Llewellyn P, Rodriguez-Reinoso F, Seaton NA (eds). Royal Society of Chemistry: Cambridge, UK; 204-210. DOI: $10.1039 / 9781847559418$

582. Subrahmanyam S, Piletsky SA. 2009. Computational Design of Molecularly Imprinted Polymers. In Combinatorial Methods for Chemical and Biological Sensors, Integrated Analytical Systems, Potyrailo RA, Mirsky VM (eds). Springer: New York; 135-172. DOI: 10.1007/978-0-387-73713-3_6

583. Tada M, Iwasawa Y. 2009. Advanced Design of Catalyst Surfaces with Metal Complexes for Selective Catalysis. In Modern Surface Organometallic Chemistry, Basset JM, Psaro R, Roberto D, Ugo R (eds). Wiley: Weinheim; 375-415. DOI: 10.1002/9783527627097.ch10

584. Xu ZK, Wan LS, Huang XJ. 2009. Molecularly Imprinted Membranes. In Surface Engineering of Polymer Membranes, Advanced Topics in Science and Technology in China, Xu ZK, Huang XJ, Wan LS (eds). Springer: Berlin; 225-262. DOI: 10.1007/978-3-540-88413-2_8

585. Theodoridis GA, Manesiotis P. 2009. Affinity Chromatography: Molecularly Imprinted Polymers. In Encyclopedia of Chromatography, Third Edition, Cazes J (ed). Taylor \& Francis: 24-30. DOI: 10.1081/E-ECHR3-120045288

586. Baggiani C, Anfossi L, Giovannoli C. 2010. Artificial Systems for Molecular Recognition of Mycotoxins. In Mycotoxins in Food, Feed and Bioweapons, 
Rai M, Varma A (eds). Springer: Berlin, Heidelberg; 3-20. DOI: 10.1007/9783-642-00725-5_1

587. Bezbaruah AN, Kalita H. 2010. Sensors and biosensors for endocrine disrupting chemicals: State-of-the-art and future trends. In Treatment of Micropollutants in Water and Wastewater, Integrated Environmental Technology Series, Virkutyte J, Jegatheesan V, Varma RS (eds). IWA Publishing: London; 93-128.

588. Cragg PJ. 2010. Supramolecular Enzyme Mimics. In Supramolecular Chemistry, Springer Netherlands: Dordrecht; 113-151. DOI: 10.1007/978-90481-2582-1_4

589. Fuchiwaki Y, Kubo I. 2010. Electrochemical Sensor Based on Biomimetic Recognition Utilizing Molecularly Imprinted Polymer Receptor. In Biomimetics Learning from Nature, Mukherjee A (ed). InTech: 385-398.

590. Hatti-Kaul R. 2010. Downstream Processing in Industrial Biotechnology. In Industrial Biotechnology, Soetaert W, Vandamme EJ (eds). Wiley-VCH: Weinheim; 279-321. DOI: 10.1002/9783527630233.ch8

591. Lai E, Dzhun A, De Maleki Z. 2010. Molecularly Imprinted Polymer Submicron Particles Tailored for Extraction of Trace Estrogens in Water. In Trace Analysis with Nanomaterials, Pierce DT, Zhao JXJ (eds). Wiley-VCH Verlag GmbH \& Co. KGaA: Weinheim; 133-159.

592. Lange U, Roznyatovskaya NV, Hao QL, Mirsky VM. 2010. Conducting Polymers as Artificial Receptors in Chemical Sensors. In Artificial Receptors for Chemical Sensors, Mirsky VM, Yatsimirsky AK (eds). Wiley-VCH Verlag $\mathrm{GmbH} \quad \& \quad$ Co. KGaA: Weinheim; 361-390. DOI: 10.1002/9783527632480.ch12

593. Margelefsky EL, Zeidan RK, Davis ME. 2010. Heterogeneous Catalyst Design by Multiple Functional Group Positioning in Organic-Inorganic 
Materials: On the Route to Analogs of Multifunctional Enzymes. In Model Systems in Catalysis, Rioux RM (ed). Springer: New York; 495-516. DOI: 10.1007/978-0-387-98049-2_23

594. Meier F, Mizaikoff B. 2010. Molecularly Imprinted Polymers as Artificial Receptors. In Artificial Receptors for Chemical Sensors, Mirsky VM, Yatsimirsky AK (eds). Wiley-VCH Verlag GmbH \& Co. KGaA: Weinheim; 391-437. DOI: 10.1002/9783527632480.ch13

595. Miyata T. 2010. Biomolecule-Responsive Hydrogels. In Biomedical Applications of Hydrogels Handbook, Park K, Okano T (eds). Springer: New York; 65-86. DOI: 10.1007/978-1-4419-5919-5_4

596. Puoci F, Cirillo G, Curcio M, lemma F, Parisi OI, Spizzirri UG, Picci N. 2010. Molecularly Imprinted Polymers (MIPs) in Biomedical Applications. In Biopolymers, Elnashar M (ed). Sciyo: 547-574.

597. Sellergren B, Martín-Esteban A. 2010. The Use of Molecularly Imprinted Polymers for Sampling and Sample Preparation. In Handbook of Sample Preparation, Pawliszyn J, Lord HL (eds). John Wiley \& Sons: Hoboken, New Jersey; 445-474.

598. Southard GE, Murray GM. 2010. Molecularly Imprinted Polymer Receptors for Sensors and Arrays. In Recognition Receptors in Biosensors, Zourob M (ed). Springer: 751-775. DOI: 10.1007/978-1-4419-0919-0_19

599. Tada M, Iwasawa Y. 2010. Molecular-Imprinted Metal Complexes for the Design of Catalytic Structures. In Model Systems in Catalysis: Single Crystal to Supported Enzyme Mimics, Rioux RM (ed). Springer: New York; 475-493. DOI: 10.1007/978-0-387-98049-2_22

600. Tothill IE. 2010. Peptides as Molecular Receptors. In Recognition Receptors in Biosensors, Zourob M (ed). Springer: 249-274. DOI: 10.1007/978-1-44190919-0_6 
601. Whitcombe MJ, Lakshmi D. 2010. Imprinted Polymers. In Electropolymerisation, Cosnier S, Karyakin A (eds). Wiley-VCH: Weinheim; 133-151. DOI: 10.1002/9783527630592.ch7

602. Yoshida R, Okano T. 2010. Stimuli-Responsive Hydrogels and Their Application to Functional Materials. In Biomedical Applications of Hydrogels Handbook, Park K, Okano T (eds). Springer: New York; 19-43. DOI: 10.1007/978-1-4419-5919-5_2

603. Zhang SL, Cao XJ, Chu J, Qian JC, Zhuang YP. 2010. Bioreactors and Bioseparation. In Biotechnology in China II, Advances in Biochemical Engineering/Biotechnology, Vol. 122, Tsao GT, Ouyang PK, Chen JA (eds). Springer: Heidelberg; 105-150. DOI: 10.1007/10_2010_70

604. Aránzazu Goicolea M, Gómez-Caballero A, Barrio RJ. 2011. New materials in electrochemical sensors for pesticides monitoring. In Pesticides Strategies for Pesticides Analysis, Stoytcheva M (ed). InTech: 333-358.

605. Berti F, Marrazza G, Mascini M, Todros S, Baratto C, Ferroni M, Faglia G, Lakshmi D, Chianella I, Whitcombe MJ, Piletsky S, Turner APF. 2011. OneDimensional Polyaniline Nanotubes for Enhanced Chemical and Biochemical Sensing. In Sensors and Microsystems, Lecture Notes in Electrical Engineering, Vol. 91, Neri G, Donato N, D'Amico A, Di Natale C (eds). Springer Netherlands: 311-315. DOI: 10.1007/978-94-007-1324-6_48

606. Booker K, Holdsworth Cl, Bowyer MC, McCluskey A. 2011. Ionic Liquids as Porogens in the Synthesis of Molecularly Imprinted Polymers. In Applications of Ionic Liquids in Science and Technology, Handy S (ed). InTech: 197-212.

607. Chiono V, Descrovi E, Sartori S, Gentile P, Ballarini M, Giorgis F, Ciardelli G. 2011. Biomimetic Tailoring of the Surface Properties of Polymers at the Nanoscale: Medical Applications. In Scanning Probe Microscopy in Nanoscience and Nanotechnology, Volume 2, Nanoscience and 
Technology, Bhushan B (ed). Springer: Heidelberg; 645-689. DOI: 10.1007/978-3-642-10497-8_22

608. El Kaoutit M. 2011. Application of Conducting Polymers in Electroanalysis. In Aspects on Fundaments and Applications of Conducting Polymers, de Jesus Motheo A (ed). InTech Open: 43-66.

609. Fan MT, He J. 2011. Pesticide immunoassay. In Pesticides - Strategies for Pesticides Analysis, Stoytcheva M (ed). InTech: Rikeka, Croatia; 293-314.

610. Ju HX, Zhang XJ, Wang J. 2011. Biosensing Applications of Molecularly Imprinted Nanomaterials. In NanoBiosensing: Principles, Development and Applications, Biological and Medical Physics, Biomedical Engineering, Ju HX, Zhang XJ, Wang J (eds). Springer: New York; 265-303. DOI: 10.1007/978-1-4419-9622-0_9

611. Ju HX, Zhang XJ, Wang J. 2011. Nanostructured Mimic Enzymes for Biocatalysis and Biosensing. In NanoBiosensing: Principles, Development and Applications, Biological and Medical Physics, Biomedical Engineering, Ju HX, Zhang XJ, Wang J (eds). Springer: New York; 85-109. DOI: 10.1007/978-1-4419-9622-0_3

612. Kim YS, Moh SH, Cho SH, Kim YJ, Cho JC. 2011. Preparation and Characteristics of Poly(St-co-MAA) Microgels. In Grid and Distributed Computing, Communications in Computer and Information Science, Kim T, Adeli H, Cho H, Gervasi O, Yau SS, Kang BH, Villalba JG (eds). Springer Berlin Heidelberg: 263-270. DOI: 10.1007/978-3-642-27180-9_32

613. Liu ZL, Gao AG, Harrison LA, Yau KY, Lawry J, Shan GM. 2011. Future Perspectives and Challenges. In Immunoassays in Agricultural Biotechnology, Shan GM (ed). John Wiley \& Sons, Inc.: Hoboken, New Jersey; 325-339. DOI: 10.1002/9780470909935.ch16 
614. Mascini M, Del Carlo M, Compagnone D, Perez G, Montero-Cabrera LA, Gonzalez S, Yamanaka H. 2011. Multiple Minima Hypersurfaces Procedures for Biomimetic Ligands Screening. In Sensors and Microsystems, Lecture Notes in Electrical Engineering, Vol. 91, Neri G, Donato N, D'Amico A, Di Natale C (eds). Springer Netherlands: 403-407. DOI: 10.1007/978-94-007-1324-6_65

615. Mazzotta E, Malitesta C. 2011. Electrochemically Synthesized Molecularly Imprinted Polymers for Sensing Applications. In Sensors and Microsystems, Lecture Notes in Electrical Engineering, Vol. 91, Neri G, Donato N, D'Amico A, Di Natale C (eds). Springer Netherlands: 409-413. DOI: 10.1007/978-94007-1324-6_66

616. Medina DD, Mastai Y. 2011. Biomimetic Polymers for Chiral Resolution and Antifreeze Applications. In On Biomimetics, Pramatarova LD (ed). InTech: 321-354.

617. Morais S, Correia M, Domingues V, Delerue-Matos C. 2011. Urea pesticides. In Pesticides - Strategies for Pesticides Analysis, Stoytcheva M (ed). InTech: Rikeka, Croatia; 241-262.

618. Palchetti I, Mascini M. 2011. Biosensor Techniques for Environmental Monitoring. In Nucleic Acid Biosensors for Environmental Pollution Monitoring, Mascini M, Palchetti I (eds). The Royal Society of Chemistry: Cambridge; 1-16.

619. Paolesse R, Monti D, Dini F, Di Natale C. 2011. Fluorescence Based Sensor Arrays. In Luminescence Applied in Sensor Science, Topics in Current Chemistry, Vol. 300, Prodi L, Montalti M, Zaccheroni N (eds). Springer: Berlin / Heidelberg; 139-174. DOI: 10.1007/128_2010_97

620. Pyrzynska K. 2011. Solid-Phase Extraction for Enrichment and Separation of Herbicides. In Herbicides, Theory and Applications, Soloneski S, Larramendy ML (eds). InTech: 325-344. 
621. Pyrzynska K. 2011. The Use of Novel Materials as Solid-Phase Extractors for Chromatographic Analysis. In Advances in Chromatography, Volume 49, Advances in Chromatography, Vol. 49, Grushka E, Grinberg N (eds). CRC Press: Boca Raton, FI.; 365-399. DOI: 10.1201/b10721-10

622. Rosellini E, Cristallini C, Barbani N, Giusti P. 2011. Engineering of Multifunctional Scaffolds for Myocardial Repair Through Nanofunctionalization and Microfabrication of Novel Polymeric Biomaterials. In Myocardial Tissue Engineering, Studies in Mechanobiology, Tissue Engineering and Biomaterials, Vol. 6, Boccaccini AR, Harding SE (eds). Springer Berlin Heidelberg: 187-214. DOI: 10.1007/8415_2010_42

623. Wilson AD. 2011. Future Applications of Electronic-Nose Technologies in Healthcare and Biomedicine. In Wide Spectra of Quality Control, Akyar I (ed). InTech:

624. Dai S, Zhang ZT, Liang CD. 2004. Hierarchically imprinted nanostructures for separation of metal ions. In Dekker Encyclopedia of Nanoscience and Nanotechnology, Schwarz JA, Contescu Cl, Putyera K (eds). Marcel Dekker: New York; 1369-1379. DOI: 10.1081/E-ENN-120023656

625. Haupt K. 2004. Molecularly imprinted polymers as recognition elements in sensors. In Ultrathin Electrochemical Chemo- And Biosensors: Technology And Performance, Springer Series on Chemical Sensors and Biosensors, Vol. 2, Mirsky VM (ed). Springer Verlag: Berlin; 23-39. DOI: 10.1007/978-3662-05204-4_2

626. Breton F, Piletska EV, Karim K, Rouillon R, Piletsky SA. 2006. Mimicking the plastoquinone-binding pocket of Photosystem II using molecularly imprinted polymers. In Biotechnological Applications of Photosynthetic Proteins: Biochips, Biosensors and Biodevices, Biotechnology Intelligence Unit, Giardi MT, Piletska EV (eds). Landes Bioscience: Georgetown, Texas; 155-165. DOI: 10.1007/978-0-387-36672-2_14 
627. Vukovic R, Kuzmic AE, Bogdanic G, Fles D. 2005. Preparation of molecularly imprinted cross-linked copolymers by thermal degradation of poly(methacryl-N,N '-diisopropylurea-co-ethylene glycol dimethacrylate). In New Polymeric Materials, ACS Symposium Series, Vol. 916, Korugic-Karasz LS, MacKnight WJ, Martuscelli E (eds). The American Chemical Society: Washington DC; 229-237. DOI: 10.1021/bk-2005-0916.ch017

628. Komiyama M, Takeuchi T, Mukawa T, Asanuma H.(eds). 2003 Molecular Imprinting: From Fundamentals to Applications, Wiley-VCH: Weinheim, Germany.

629. Komiyama M, Takeuchi T, Mukawa T, Asanuma H. 2004. Introduction. In Molecular Imprinting, Komiyama M, Takeuchi T, Mukawa T, Asanuma $\mathrm{H}$ (eds). Wiley-VCH Verlag GmbH \& Co. KGaA: Weinheim; 1-8. DOI: 10.1002/352760202X.ch1

630. Komiyama M, Takeuchi T, Mukawa T, Asanuma H. 2004. Fundamentals of Molecular Imprinting. In Molecular Imprinting, Komiyama M, Takeuchi T, Mukawa $\mathrm{T}$, Asanuma $\mathrm{H}$ (eds). Wiley- $\mathrm{VCH}$ Verlag $\mathrm{GmbH}$ \& Co. KGaA: Weinheim; 9-19. DOI: 10.1002/352760202X.ch2

631. Komiyama M, Takeuchi T, Mukawa T, Asanuma H. 2004. Experimental Methods (1) - Procedures of Molecular Imprinting. In Molecular Imprinting, Komiyama M, Takeuchi T, Mukawa T, Asanuma H (eds). Wiley- $\mathrm{VCH}$ Verlag GmbH \& Co. KGaA: Weinheim; 21-45. DOI: 10.1002/352760202X.ch3

632. Komiyama M, Takeuchi T, Mukawa T, Asanuma H. 2004. Experimental Methods (2) - Evaluation of Imprinting Efficiency. In Molecular Imprinting, Komiyama M, Takeuchi T, Mukawa T, Asanuma H (eds). Wiley-VCH Verlag GmbH \& Co. KGaA: Weinheim; 47-52. DOI: 10.1002/352760202X.ch4

633. Komiyama M, Takeuchi T, Mukawa T, Asanuma H. 2004. Spectroscopic Anatomy of Molecular Imprinting Reactions. In Molecular Imprinting, 
Komiyama M, Takeuchi T, Mukawa T, Asanuma H (eds). Wiley-VCH Verlag GmbH \& Co. KGaA: Weinheim; 53-64. DOI: 10.1002/352760202X.ch5

634. Komiyama M, Takeuchi T, Mukawa T, Asanuma H. 2004. Flow Chart of a Typical Molecular Imprinting. In Molecular Imprinting, Komiyama M, Takeuchi T, Mukawa T, Asanuma H (eds). Wiley-VCH Verlag GmbH \& Co. KGaA: Weinheim; 65-73. DOI: 10.1002/352760202X.ch6

635. Komiyama M, Takeuchi T, Mukawa T, Asanuma H. 2004. Applications of Molecularly Imprinted Polymers. In Molecular Imprinting, Komiyama M, Takeuchi T, Mukawa T, Asanuma H (eds). Wiley-VCH Verlag GmbH \& Co. KGaA: Weinheim; 75-118. DOI: 10.1002/352760202X.ch7

636. Komiyama M, Takeuchi T, Mukawa T, Asanuma H. 2004. Recent Challenges and Progress. In Molecular Imprinting, Komiyama M, Takeuchi $\mathrm{T}$, Mukawa T, Asanuma H (eds). Wiley-VCH Verlag GmbH \& Co. KGaA: Weinheim; 119-139. DOI: 10.1002/352760202X.ch8

637. Komiyama M, Takeuchi T, Mukawa T, Asanuma H. 2004. Conclusions and Prospects. In Molecular Imprinting, Komiyama M, Takeuchi T, Mukawa T, Asanuma H (eds). Wiley-VCH Verlag GmbH \& Co. KGaA: Weinheim; 141. DOI: $10.1002 / 352760202 X . c h 9$

638. Yan M, Ramström O.(eds). 2005 Molecularly Imprinted Materials: Science and Technology, Marcel Dekker Ltd: New York.

639. Ramström O, Yan M. 2005. Molecular imprinting - an introduction. In Molecularly Imprinted Materials: Science and Technology, Yan M, Ramström O (eds). Marcel Dekker: New York; 1-12. DOI: 10.1201/9781420030303.pt1

640. Mayes AG. 2005. A brief history of the "new era" of molecular imprinting. In Molecularly Imprinted Materials: Science and Technology, Yan M, 
Ramström O (eds). Marcel Dekker: New York; 13-23. DOI: 10.1201/9781420030303.ch2

641. Yilmaz E, Schmidt RH, Mosbach K. 2005. The noncovalent approach. In Molecularly Imprinted Materials: Science and Technology, Yan M, Ramström O (eds). Marcel Dekker: New York; 25-57. DOI: 10.1201/9781420030303.pt2

642. Wulff G. 2005. The covalent and other stoichiometric approaches. In Molecularly Imprinted Materials: Science and Technology, Yan M, Ramström O (eds). Marcel Dekker: New York; 59-92. DOI: 10.1201/9781420030303.ch4

643. Kirsch N, Whitcombe MJ. 2005. The semi-covalent approach. In Molecularly Imprinted Materials: Science and Technology, Yan M, Ramström O (eds). Marcel Dekker: New York; 93-122. DOI: 10.1201/9781420030303.ch5

644. Conrad PGI, Shea KJ. 2005. The use of metal coordination for controlling the microenvironment of imprinted polymers. In Molecularly Imprinted Materials: Science and Technology, Yan M, Ramström O (eds). Marcel Dekker: New York; 123-180. DOI: 10.1201/9781420030303.ch6

645. Ramström O. 2005. Synthesis and selection of functional and structural monomers. In Molecularly Imprinted Materials: Science and Technology, Yan M, Ramström O (eds). Marcel Dekker: New York; 181-224. DOI: 10.1201/9781420030303.pt3

646. Lanza F, Dirion B, Sellergren B. 2005. Combinatorial approaches to molecular imprinting. In Molecularly Imprinted Materials: Science and Technology, Yan M, Ramström O (eds). Marcel Dekker: New York; 225-248. DOI: $10.1201 / 9781420030303 . c h 8$ 
647. Cunliffe D, Alexander C. 2005. Surface imprinting. In Molecularly Imprinted Materials: Science and Technology, Yan M, Ramström O (eds). Marcel Dekker: New York; 249-283. DOI: 10.1201/9781420030303.ch9

648. Kobayashi T. 2005. Scaffold imprinting. In Molecularly Imprinted Materials: Science and Technology, Yan M, Ramström O (eds). Marcel Dekker: New York; 285-306. DOI: 10.1201/9781420030303.ch10

649. Defreese JL, Katz A. 2005. Imprinting in inorganic matrices. In Molecularly Imprinted Materials: Science and Technology, Yan M, Ramström O (eds). Marcel Dekker: New York; 307-327. DOI: 10.1201/9781420030303.ch11

650. Shimizu KD. 2005. Post modification of imprinted polymers. In Molecularly Imprinted Materials: Science and Technology, Yan M, Ramström O (eds). Marcel Dekker: New York; 329-345. DOI: 10.1201/9781420030303.ch12

651. Dai S. 2005. Molecular imprinting using hybrid materials as host matrices. In Molecularly Imprinted Materials: Science and Technology, Yan M, Ramström $O$ (eds). Marcel Dekker: New York; 347-361. DOI: 10.1201/9781420030303.ch13

652. Nicholls IA, Piletsky SA, Chen B, Chianella I, Turner APF. 2005. Thermodynamic considerations and the use of molecular modeling as a tool for predicting MIP performance. In Molecularly Imprinted Materials: Science and Technology, Yan M, Ramström O (eds). Marcel Dekker: New York; 363393. DOI: 10.1201/9781420030303.pt4

653. Spivak DA. 2005. Selectivity in molecularly imprinted matrices. In Molecularly Imprinted Materials: Science and Technology, Yan M, Ramström $O$ (eds). Marcel Dekker: New York; 395-417. DOI: 10.1201/9781420030303.ch15 
654. Shimizu KD. 2005. Binding isotherms. In Molecularly Imprinted Materials: Science and Technology, Yan M, Ramström O (eds). Marcel Dekker: New York; 419-434. DOI: 10.1201/9781420030303.ch16

655. Ye L, Yilmaz E. 2005. Molecularly imprinted polymer beads. In Molecularly Imprinted Materials: Science and Technology, Yan M, Ramström O (eds). Marcel Dekker: New York; 435-454. DOI: 10.1201/9781420030303.pt5

656. Ulbricht M. 2005. Molecularly imprinted polymer films and membranes. In Molecularly Imprinted Materials: Science and Technology, Yan M, Ramström $O$ (eds). Marcel Dekker: New York; 455-490. DOI: 10.1201/9781420030303.ch18

657. Brazier JJ, Yan M. 2005. Micromonoliths and microfabricated molecularly imprinted polymers. In Molecularly Imprinted Materials: Science and Technology, Yan M, Ramström O (eds). Marcel Dekker: New York; 491-516. DOI: $10.1201 / 9781420030303 . c h 19$

658. Baggiani C. 2005. Chromatographic techniques. In Molecularly Imprinted Materials: Science and Technology, Yan M, Ramström O (eds). Marcel Dekker: New York; 517-552. DOI: 10.1201/9781420030303.pt6

659. Brüggemann O. 2005. Capillary electrophoresis. In Molecularly Imprinted Materials: Science and Technology, Yan M, Ramström O (eds). Marcel Dekker: New York; 553-578. DOI: 10.1201/9781420030303.ch21

660. Murray GM, Southard GE. 2005. Metal ion selective molecularly imprinted polymers. In Molecularly Imprinted Materials: Science and Technology, Yan M, Ramström O (eds). Marcel Dekker: New York; 579-602. DOI: 10.1201/9781420030303.ch22

661. Ye L. 2005. Solid phase extraction and by-product removal. In Molecularly Imprinted Materials: Science and Technology, Yan M, Ramström O (eds). Marcel Dekker: New York; 603-618. DOI: 10.1201/9781420030303.ch23 
662. Severin K. 2005. Applications of molecularly imprinted materials as enzyme mimics. In Molecularly Imprinted Materials: Science and Technology, Yan M, Ramström O (eds). Marcel Dekker: New York; 619-640. DOI: 10.1201/9781420030303.ch24

663. Ansell RJ. 2005. Application of MIPs as antibody mimics in immunoassays. In Molecularly Imprinted Materials: Science and Technology, Yan M, Ramström $O$ (eds). Marcel Dekker: New York; 641-683. DOI: 10.1201/9781420030303.ch25

664. Haupt K. 2005. Molecularly imprinted polymers as recognition elements in sensors: mass and electrochemical sensors. In Molecularly Imprinted Materials: Science and Technology, Yan M, Ramström O (eds). Marcel Dekker: New York; 685-700. DOI: 10.1201/9781420030303.ch26

665. Gao SH, Wang W, Wang BH. 2005. Molecularly imprinted polymers as recognition elements in optical sensors. In Molecularly Imprinted Materials: Science and Technology, Yan M, Ramström O (eds). Marcel Dekker: New York; 701-726. DOI: 10.1201/9781420030303.ch27

666. Piletsky S, Turner A.(eds). 2006 Molecular Imprinting of Polymers, Biotechnology Intelligence Unit, Landes Bioscience: Georgetown, Texas.

667. Pérez-Moral N, Mayes AG. 2006. MIP formats for analytical applications. In Molecular imprinting of polymers, Intelligence Unit Series, Piletsky S, Turner A (eds). Landes Bioscience: Georgetown, Texas; 1-11.

668. Baggiani C, Giovannoli C. 2006. Bioimprinting. In Molecular imprinting of polymers, Intelligence Unit Series, Piletsky S, Turner A (eds). Landes Bioscience: Georgetown, Texas; 12-25.

669. Katada N, Niwa M. 2006. Re-birth of molecular imprinting on silica. In Molecular imprinting of polymers, Intelligence Unit Series, Piletsky S, Turner A (eds). Landes Bioscience: Georgetown, Texas; 26-40. 
670. Katada N, Niwa M. 2006. Chemical vapour deposition of silica overlayer using an organic molecule as template surface: Application to molecular seiving sensor and adsorbent. In Molecular imprinting of polymers, Intelligence Unit Series, Piletsky S, Turner A (eds). Landes Bioscience: Georgetown, Texas; 41-49.

671. Dickert F, Lieberzeit PA, Hayden O. 2006. Molecularly imprinted polymers for mass sensitive sensors - from cells to viruses and enzymes. In Molecular imprinting of polymers, Intelligence Unit Series, Piletsky S, Turner A (eds). Landes Bioscience: Georgetown, Texas; 50-63.

672. Piletsky S, Turner A. 2006. A new generation of chemical sensors based on MIPs. In Molecular imprinting of polymers, Intelligence Unit Series, Piletsky S, Turner A (eds). Landes Bioscience: Georgetown, Texas; 64-79.

673. Ulbricht M. 2006. Molecularly imprinted membranes. In Molecular imprinting of polymers, Intelligence Unit Series, Piletsky S, Turner A (eds). Landes Bioscience: Georgetown, Texas; 80-94.

674. Sellergren B. 2006. Recognition of enantiomers using molecularly imprinted polymers. In Molecular imprinting of polymers, Intelligence Unit Series, Piletsky S, Turner A (eds). Landes Bioscience: Georgetown, Texas; 95-121.

675. Whitcombe M. 2006. MIP catalysts - from theory to practice. In Molecular imprinting of polymers, Intelligence Unit Series, Piletsky S, Turner A (eds). Landes Bioscience: Georgetown, Texas; 122-139.

676. Andersson LI. 2006. Solid-phase extraction on molecularly imprinted polymers - requirements, achievements and future work. In Molecular imprinting of polymers, Intelligence Unit Series, Piletsky S, Turner A (eds). Landes Bioscience: Georgetown, Texas; 140-148.

677. Bossi A, Righetti PG, Nilsson S. 2006. Imprinted polymers in capillary electrophoresis and capillary electrochromatography. In Molecular imprinting 
of polymers, Intelligence Unit Series, Piletsky S, Turner A (eds). Landes Bioscience: Georgetown, Texas; 149-163.

678. Allender C. 2006. Molecularly imprinted polymers in drug screening. In Molecular imprinting of polymers, Intelligence Unit Series, Piletsky S, Turner A (eds). Landes Bioscience: Georgetown, Texas; 164-181.

679. Spivak DA. 2006. MIPs in biotechnology, perspective and reality. In Molecular imprinting of polymers, Intelligence Unit Series, Piletsky S, Turner A (eds). Landes Bioscience: Georgetown, Texas; 182-190.

680. Leverkus P. 2006. Business models for the commercialisation of MIPs. In Molecular imprinting of polymers, Intelligence Unit Series, Piletsky S, Turner A (eds). Landes Bioscience: Georgetown, Texas; 191-198.

681. McIntyre JB. 2006. A general survey of patents in the field of molecularly imprinted polymers. In Molecular imprinting of polymers, Intelligence Unit Series, Piletsky S, Turner A (eds). Landes Bioscience: Georgetown, Texas; 199-204.

682. Rzysko W, Sokolowski S, Pizio O. 2002. Theory of adsorption in a polydisperse templated porous material: Hard sphere systems. J. Chem. Phys. 116: 4286-4292. DOI: 10.1063/1.1450556

683. Baggiani C, Anfossi L, Giovannoli C, Tozzi C. 2004. Multivariate analysis of the selectivity for a pentachlorophenol-imprinted polymer. J. Chromatogr. $B$ 804: 31-41. DOI: 10.1016/j.jchromb.2004.01.037

684. Berezkin AV, Solov'ev MA, Khalatur PG, Khokhlov AR. 2004. Template copolymerization near a patterned surface: Computer simulation. J. Chem. Phys. 121: 6011-6020. DOI: 10.1063/1.1783874

685. Pap T, Horvai G. 2004. Binding assays with molecularly imprinted polymers - why do they work? J. Chromatogr. B 804: 167-172. DOI: 10.1016/j.jchromb.2003.12.007 
686. Wu LQ, Li YZ. 2004. Study on the recognition of templates and their analogues on molecularly imprinted polymer using computational and conformational analysis approaches. J. Mol. Recognit. 17: 567-574. DOI: $10.1002 / \mathrm{jmr} .688$

687. Burridge DJ, Mao Y, Warner M. 2005. Chiral-mechanical transitions in topologically imprinted elastomers. Phys. Rev. $E$ 72: DOI: 10.1103/PhysRevE.72.051718

688. Dong WG, Yan M, Zhang ML, Liu Z, Li YM. 2005. A computational and experimental investigation of the interaction between the template molecule and the functional monomer used in the molecularly imprinted polymer. Anal. Chim. Acta 542: 186-192. DOI: 10.1016/j.aca.2005.03.032

689. Holdsworth $\mathrm{Cl}$, Bowyer MC, Lennard C, McCluskey A. 2005. Formulation of cocaine-imprinted polymers utilizing molecular modelling and NMR analysis. Aust. J. Chem. 58: 315-320. DOI: 10.1071/CH04138

690. Nantasenamat C, Tansila N, Naenna T, Ayudhya CIN, Prachayasittikul V. 2005. The use of artificial neural network for the prediction of imprinting factor of molecularly imprinted polymer. Vol. Proceedings of the $31 \mathrm{st}$ Congress on Science and Technology of Thailand at Suranaree University of Technology, Art. No. E0118.

691. Pavel D, Lagowski J. 2005. Computationally designed monomers and polymers for molecular imprinting of theophylline and its derivatives. Part I. Polymer 46: 7528-7542. DOI: 10.1016/j.polymer.2005.04.099

692. Pavel D, Lagowski J. 2005. Computationally designed monomers and polymers for molecular imprinting of theophylline - part II. Polymer 46: 75437556. DOI: 10.1016/j.polymer.2005.05.146 
693. Rosengren AM, Karlsson JG, Andersson PA, Nicholls IA. 2005. Chemometric models of template-molecularly imprinted polymer binding. Anal. Chem. 77: 5700-5705. DOI: 10.1021/ac050765v

694. Rossinsky E, Srebnik S. 2005. Monte Carlo simulation of protein folding in the presence of residue-specific binding sites. Biopolymers 79: 259-268. DOI: 10.1002/bip.20365

695. Chianella I, Karim K, Piletska EV, Preston C, Piletsky SA. 2006. Computational design and synthesis of molecularly imprinted polymers with high binding capacity for pharmaceutical applications-model case: Adsorbent for abacavir. Anal. Chim. Acta 559: 73-78. DOI: 10.1016/j.aca.2005.11.068

696. Courty S, Tajbakhsh AR, Terentjev EM. 2006. Chirality transfer and stereoselectivity of imprinted cholesteric networks. Phys. Rev. E 73: Art. No. 011803. DOI: 10.1103/PhysRevE.73.011803

697. Diñeiro Y, Menéndez Ml, Blanco-López MC, Lobo-Castañón MJ, MirandaOrdieres AJ, Tuñón-Blanco P. 2006. Computational predictions and experimental affinity distributions for a homovanillic acid molecularly imprinted polymer. Biosens. Bioelectron. 22: 364-371. DOI: 10.1016/j.bios.2006.03.027

698. Garcés JL, Mas F, Puy J. 2006. Conditional equilibrium constants in multicomponent heterogeneous adsorption: The conditional affinity spectrum. J. Chem. Phys. 124: Art. No. 044710. DOI: 10.1063/1.2162876

699. Gomy C, Schmitzer AR. 2006. Rational design of new polymerizable oxyanion receptors. J. Org. Chem. 71: 3121-3125. DOI: 10.1021/jo060088k

700. Kim H, Kaczmarski K, Guiochon G. 2006. Isotherm parameters and intraparticle mass transfer kinetics on molecularly imprinted polymers in 
acetonitrile/buffer mobile phases. Chem. Eng. Sci. 61: 5249-5267. DOI: 10.1016/j.ces.2006.03.043

701. Monti S, Cappelli C, Bronco S, Giusti P, Ciardelli G. 2006. Towards the design of highly selective recognition sites into molecular imprinting polymers: A computational approach. Biosens. Bioelectron. 22: 153-163. DOI: 10.1016/j.bios.2006.05.017

702. Nantasenamat C, Naenna T, Ayudhya C, Prachayasittikul V. 2006. Quantitative prediction of imprinting factor of molecularly imprinted polymers by artificial neural network. J. Comput. -Aided Mol. Des. 19: 509-524. DOI: $10.1007 / \mathrm{s} 10822-005-9004-4$

703. O'Mahony J, Molinelli A, Nolan K, Smyth MR, Mizaikoff B. 2006. Anatomy of a successful imprint: Analysing the recognition mechanisms of a molecularly imprinted polymer for quercetin. Biosens. Bioelectron. 21: 1383-1392. DOI: 10.1016/j.bios.2005.05.015

704. O'Mahony J, Wei ST, Molinelli A, Mizaikoff B. 2006. Imprinted Polymeric Materials. Insight into the Nature of Prepolymerization Complexes of Quercetin Imprinted Polymers. Anal. Chem. 78: 6187-6190. DOI: 10.1021/ac060446j

705. Pavel D, Lagowski J, Lepage CJ. 2006. Computationally designed monomers for molecular imprinting of chemical warfare agents - Part V. Polymer 47: 8389-8399. DOI: 10.1016/j.polymer.2006.09.067

706. Tóth B, Pap T, Horvath V, Horvai G. 2006. Nonlinear adsorption isotherm as a tool for understanding and characterizing molecularly imprinted polymers. J. Chromatogr. A 1119: 29-33. DOI: 10.1016/j.chroma.2005.10.048

707. Wu XY, Shimizu KD. 2006. Affinity distributions and selectivities of molecularly imprinted polymers simulated by a probabilty-based computer model. Polym. Prepr. 47: 61. 
708. Breton F, Rouillon R, Piletska EV, Karim K, Guerreiro A, Chianella I, Piletsky SA. 2007. Virtual imprinting as a tool to design efficient MIPs for photosynthesis-inhibiting herbicides. Biosens. Bioelectron. 22: 1948-1954. DOI: 10.1016/j.bios.2006.08.017

709. Castro-Garay P, Reyes JA, Ramos-Garcia R. 2007. Band structure controlled by chiral imprinting. Appl. Phys. Lett. 91: Art. No. 113519. DOI: $10.1063 / 1.2784174$

710. Dong WG, Yan M, Liu Z, Wu GS, Li YM. 2007. Effects of solvents on the adsorption selectivity of molecularly imprinted polymers: Molecular simulation and experimental validation. Sep. Purif. Technol. 53: 183-188. DOI: 10.1016/j.seppur.2006.06.023

711. Fang CL, Li SJ. 2007. Thermodynamic and Kinetic Considerations on the Specific Adsorption and Molecular Recognition by Molecularly Imprinted Polymer. J. Inorg. Organomet. Polym. Mater. 17: 623-629. DOI: 10.1007/s10904-007-9156-x

712. Henthorn DB, Peppas NA. 2007. Molecular Simulations of Recognitive Behavior of Molecularly Imprinted Intelligent Polymeric Networks. Ind. Eng. Chem. Res. 46: 6084-6091. DOI: 10.1021/ie061369|

713. Kriz K, Debeljak N, Warnmark I, Kriz D. 2007. An experimental and theoretical study of the morphine binding capacity and kinetics of an engineered opioid receptor. Biosens. Bioelectron. 22: 1168-1171. DOI: 10.1016/j.bios.2006.09.024

714. Liu Y, Wang F, Tan TW, Lei M. 2007. Study of the properties of molecularly imprinted polymers by computational and conformational analysis. Anal. Chim. Acta 581: 137-146. DOI: 10.1016/j.aca.2006.08.015

715. O'Mahony J, Karlsson BCG, Mizaikoff B, Nicholls IA. 2007. Correlated theoretical, spectroscopic and X-ray crystallographic studies of a non- 
covalent molecularly imprinted polymerisation system. Analyst 132: 11611168. DOI: $10.1039 / \mathrm{b} 706258 \mathrm{c}$

716. Sarkisov L, VanTassel PR. 2007. Integral Equation Theory of Adsorption in Templated Materials: Influence of Molecular Attraction. J. Phys. Chem. C 111: 15726-15735. DOI: 10.1021/jp073536g

717. Si HZ, Zhang KJ, Hu ZD, Fan BT. 2007. QSAR Model for Prediction Capacity Factor of Molecular Imprinting Polymer Based on Gene Expression Programming. QSAR Comb. Sci. 26: 41-50. DOI: 10.1002/qsar.200530187

718. Azenha M, Kathirvel P, Nogueira P, Fernando-Silva A. 2008. The requisite level of theory for the computational design of molecularly imprinted silica xerogels. Biosens. Bioelectron. 23: 1843-1849. DOI: 10.1016/j.bios.2008.02.023

719. Isarankura-Na-Ayudhya $C$, Nantasenamat $C$, Buraparuangsang $P$, Piacham T, Ye L, Bulow L, Prachayasittikul V. 2008. Computational Insights on Sulfonamide Imprinted Polymers. Molecules 13: 3077-3091. DOI: 10.3390/molecules13123077

720. Jacob R, Tate M, Banti Y, Rix C, Mainwaring DE. 2008. Synthesis, Characterization, and ab Initio Theoretical Study of a Molecularly Imprinted Polymer Selective for Biosensor Materials. J. Phys. Chem. A 112: 322-331. DOI: $10.1021 / j p 074405 i$

721. Koohpaei AR, Shahtaheri SJ, Ganjali MR, Forushani AR, Golbabaei F. 2008. Application of multivariate analysis to the screening of molecularly imprinted polymers (MIPs) for ametryn. Talanta 75: 978-986. DOI: 10.1016/j.talanta.2007.12.046

722. Lv YQ, Lin ZX, Tan TW, Feng W, Qin PY, Li C. 2008. Application of molecular dynamics modeling for the prediction of selective adsorption 
properties of dimethoate imprinting polymer. Sens. Actuators B 133: 15-23. DOI: 10.1016/j.snb.2008.01.067

723. Sarkisov L. 2008. Theory of pair connectedness in templated quenchedannealed systems. J. Chem. Phys. 128: Art. No. 044707. DOI: $10.1063 / 1.2823734$

724. Wu XY, Carroll WR, Shimizu KD. 2008. Stochastic Lattice Model Simulations of Molecularly Imprinted Polymers. Chem. Mater. 20: 43354346. DOI: $10.1021 / \mathrm{cm} 8002645$

725. Yao JH, Li X, Qin W. 2008. Computational design and synthesis of molecular imprinted polymers with high selectivity for removal of aniline from contaminated water. Anal. Chim. Acta 610: 282-288. DOI: 10.1016/j.aca.2008.01.042

726. Zhao ZJ, Wang Q, Zhang L, Wu T. 2008. Structured Water and WaterPolymer Interactions in Hydrogels of Molecularly Imprinted Polymers. J. Phys. Chem. B 112: 7515-7521. DOI: 10.1021/jp800836d

727. Bhaskarapillai A, Chandra S, Sevilimedu NV, Sellergren B. 2009. Theoretical investigations of the experimentally observed selectivity of a cobalt imprinted polymer. Biosens. Bioelectron. 25: 558-562. DOI: 10.1016/j.bios.2009.03.043

728. Dong CK, Li X, Guo ZC, Qi JY. 2009. Development of a model for the rational design of molecular imprinted polymer: Computational approach for combined molecular dynamics/quantum mechanics calculations. Anal. Chim. Acta 647: 117-124. DOI: 10.1016/j.aca.2009.05.040

729. Dourado EMA, Sarkisov L. 2009. Computer simulation of adsorption in simple models of molecularly imprinted polymers. In MODELING AND SIMULATION OF NEW MATERIALS: Proceedings of Modeling and Simulation of New Materials: Tenth Granada Lectures, AIP Conference 
Proceedings, Vol. 1091, American Institute of Physics: 256-261. DOI: $10.1063 / 1.3082297$

730. Dourado EMA, Sarkisov L. 2009. Emergence of molecular recognition phenomena in a simple model of imprinted porous materials. J. Chem. Phys. 130: 214701-214709. DOI: 10.1063/1.3140204

731. Herdes C, Sarkisov L. 2009. Computer Simulation of Volatile Organic Compound Adsorption in Atomistic Models of Molecularly Imprinted Polymers. Langmuir 25: 5352-5359. DOI: 10.1021/la804168b

732. Karlsson BCG, O'Mahony J, Karlsson JG, Bengtsson H, Eriksson LA, Nicholls IA. 2009. Structure and Dynamics of Monomer-Template Complexation: An Explanation for Molecularly Imprinted Polymer Recognition Site Heterogeneity. J. Am. Chem. Soc. 131: 13297-13304. DOI: 10.1021/ja902087t

733. Kowalska A, Stobiecka A, Wysocki S. 2009. A computational investigation of the interactions between harmane and the functional monomers commonly used in molecular imprinting. J. Mol. Struct.: THEOCHEM 901: 88-95. DOI: 10.1016/j.theochem.2009.01.008

734. Li YQ, Li X, Li Y, Dong CK, Jin PF, Qi JY. 2009. Selective recognition of veterinary drugs residues by artificial antibodies designed using a computational approach. Biomaterials 30: 3205-3211. DOI: 10.1016/j.biomaterials.2009.03.007

735. Medina-Castillo AL, Fernandez-Sanchez JF, Segura-Carretero A, Fernandez-Gutierrez A. 2009. A semi-empirical model to simplify the synthesis of homogeneous and transparent cross-linked polymers and their application in the preparation of optical sensing films. Biosens. Bioelectron. 25: 442-449. DOI: 10.1016/j.bios.2009.07.034 
736. Piacham T, Nantasenamat C, Suksrichavalit T, Puttipanyalears C, Pissawong T, Maneewas S, Isarankura-Na-Ayudhya C, Prachayasittikul V. 2009. Synthesis and Theoretical Study of Molecularly Imprinted Nanospheres for Recognition of Tocopherols. Molecules 14: 2985-3002. DOI: 10.3390/molecules 14082985

737. Prathish KP, Vishnuvardhan V, Rao TP. 2009. Rational Design of In Situ Monolithic Imprinted Polymer Membranes for the Potentiometric Sensing of Diethyl Chlorophosphate - a Chemical Warfare Agent Simulant. Electroanalysis 21: 1048-1056. DOI: 10.1002/elan.200804515

738. Riahi S, Edris-Tabrizi F, Javanbakht M, Ganjali MR, Norouzi P. 2009. A computational approach to studying monomer selectivity towards the template in an imprinted polymer. J. Mol. Model. 15: 829-836. DOI: $10.1007 / \mathrm{s} 00894-008-0437-2$

739. Rosengren AM, Golker K, Karlsson JG, Nicholls IA. 2009. Dielectric constants are not enough: Principal component analysis of the influence of solvent properties on molecularly imprinted polymer-ligand rebinding. Biosens. Bioelectron. 25: 553-557. DOI: 10.1016/j.bios.2009.06.042

740. Stille W. 2009. Deformation of cholesteric elastomers by uniaxial stress along the helix axis. Eur. Phys. J. E: Soft Matter Biol. Phys. 28: 57-71. DOI: 10.1140/epje/i2008-10415-3

741. Atta NF, Hamed MM, Abdel-Mageed AM. 2010. Computational investigation and synthesis of a sol-gel imprinted material for sensing application of some biologically active molecules. Anal. Chim. Acta 667: 63-70. DOI: 10.1016/j.aca.2010.04.005

742. Gómez-Pineda LE, Pina-Luis GE, Cortés-Romero CM, Palomar-Pardavé ME, Rosquete-Pina GA, Díaz-García ME, Hernández MC. 2010. Quantum Chemical Calculations on the Interaction between Flavonol and Functional 
Monomers (Methacrylic Acid and 4-Vinylpyridine) in Molecularly Imprinted Polymers. Molecules 15: 4017-4032. DOI: 10.3390/molecules 15064017

743. Levi L, Srebnik S. 2010. Simulation of Protein-Imprinted Polymers. 1. Imprinted Pore Properties. J. Phys. Chem. B 114: 107-114. DOI: 10.1021/jp9087767

744. Levi L, Srebnik S. 2010. Simulation of Protein-Imprinted Polymers. 2. Imprinting Efficiency. J. Phys. Chem. B 114: 16744-16751. DOI: 10.1021/jp108762t

745. Levi L, Srebnik S. 2010. Structural Characterization of Protein-Imprinted Gels Using Lattice Monte Carlo Simulation. Macromol. Symp. 291-292: 258270. DOI: 10.1002/masy.201050530

746. Liu Y, Wang F, Tan TW, Lei M. 2010. Rational Design and Study on Recognition Property of Paracetamol-Imprinted Polymer. Appl. Biochem. Biotechnol. 160: 328-342. DOI: 10.1007/s12010-008-8299-8

747. Losev VV, Roshchin AV, Epinat'ev ID, Ivanova NA, Kumpanenko IV. 2010. The effect of selective absorption sites in the diffusion processes in polymer films (Original Russian Text @ V.V. Losev, A.V. Roshchin, I.D. Epinat'ev, N.A. Ivanova, and I.V. Kumpanenko, 2010, published in Vysokomolekulyarnye Soedineniya, Ser. A, 2010, Vol. 52, No. 3, pp. 456465.). Polym. Sci. Ser. A 52: 308-316. DOI: 10.1134/S0965545X10030132

748. Riahi S, Eynollahi S, Ganjali MR, Norouzi P. 2010. Computational Approach to Investigation of Template/Monomer Complex in Imprinted Polymers; Dinitrobenzene Sensor. Int. J. Electrochem. Sci. 5: 509-516.

749. Saloni J, Dasary SSR, Anjaneyulu Y, Yu HT, Hill G. 2010. Molecularly imprinted polymers for detection of explosives: computational study on molecular interactions of 2,6-dinitrotoluene and methacrylic acid complex. Struct. Chem. 21: 1171-1184. DOI: 10.1007/s11224-010-9657-z 
750. Yañez F, Chianella I, Piletsky SA, Concheiro A, Alvarez-Lorenzo C. 2010. Computational modeling and molecular imprinting for the development of acrylic polymers with high affinity for bile salts. Anal. Chim. Acta 659: 178185. DOI: 10.1016/j.aca.2009.11.054

751. Ahmadi F, Ahmadi J, Rahimi-Nasrabadi M. 2011. Computational approaches to design a molecular imprinted polymer for high selective extraction of 3,4-methylenedioxymethamphetamine from plasma. J. Chromatogr. A 1218: 7739-7747. DOI: 10.1016/j.chroma.2011.08.020

752. Azenha M, Szefczyk B, Loureiro D, Kathirvel P, Cordeiro MN, FernandoSilva A. 2011. Molecular Dynamics Simulations of Pregelification Mixtures for the Production of Imprinted Xerogels. Langmuir 27: 5062-5070. DOI: $10.1021 /$ la200032e

753. Fernández-González A, Badía-Laíño R, Díaz-García ME. 2011. Improving the synthesis of a molecularly imprinted sol-gel for serine using a PlackettBurman design. Microchim. Acta 172: 351-356. DOI: 10.1007/s00604-0100497-8

754. Khajeh M, Sanchooli E. 2011. A pre-concentration procedure employing a new imprinted polymer for the determination of copper in water. Int. J. Environ. Anal. Chem. 91: 1310-1319. DOI: 10.1080/03067310903020334

755. Kryscio DR, Shi Y, Ren PY, Peppas NA. 2011. Molecular Docking Simulations for Macromolecularly Imprinted Polymers. Ind. Eng. Chem. Res. 50: 13877-13884. DOI: 10.1021/ie201858n

756. Levi L, Srebnik S. 2011. Simulation of Protein-Imprinted Polymers. 3. Imprinting Selectivity. J. Phys. Chem. B 115: 14469-14474. DOI: 10.1021/jp206940j

757. Liu Y, Wu LF, Sun LQ, Luo AQ. 2011. Computational and experimental investigation of 4-nitrotoluene molecularly imprinted polymers. In Remote 
Sensing, Environment and Transportation Engineering (RSETE), 2011 International Conference on, IEEE: 8140-8143. DOI: 10.1109/RSETE.2011.5964045

758. Mojica ER, Autschbach J, Bright FV, Aga DS. 2011. Synthesis and evaluation of tetracycline imprinted xerogels: Comparison of experiment and computational modeling. Anal. Chim. Acta 684: 72-80. DOI: 10.1016/j.aca.2010.11.005

759. Saloni J, Lipkowski P, Dasary SSR, Anjaneyulu Y, Yu HT, Hill GJr. 2011. Theoretical study of molecular interactions of TNT, acrylic acid, and ethylene glycol dimethacrylate - Elements of molecularly imprinted polymer modeling process. Polymer 52: 1206-1216. DOI: 10.1016/j.polymer.2010.11.057

760. Shariatinia Z, Arabzadeh N, Abdous M. 2011. Ab initio calculations on the hydrogen bonding interactions among pseudoephedrinium cation isomers and methacrylic acid. Main Group Chem. 10: 1-16. DOI: http://iospress.metapress.com/content/j00250g112qlm371/

761. Zolek T, Lulinski P, Maciejewska D. 2011. A computational model for selectivity evaluation of 2-(3,4-dimethoxyphenyl)ethylamine (homoveratrylamine) imprinted polymers towards biogenic compounds. Anal. Chim. Acta 693: 121-129. DOI: 10.1016/j.aca.2011.03.009

762. Shariatinia Z, Erben MF, Della Védova CO, Abdous M, Azodi S. 2011. Hydrogen bonding interactions between $\alpha$-, $\alpha$-glucose, and methacrylic acid. Struct. Chem. 22: 1347-1352. DOI: 10.1007/s11224-011-9831-y

763. Diñeiro Y, Menéndez Ml, Blanco-López MC, Lobo-Castañón MJ, MirandaOrdieres AJ, Tuñón-Blanco P. 2005. Computational approach to the rational design of molecularly imprinted polymers for voltammetric sensing of homovanillic acid. Anal. Chem. 77: 6741-6746. DOI: 10.1021/ac0513461 
764. Navarro-Villoslada F, Takeuchi T. 2005. Multivariate analysis and experimental design in the screening of combinatorial libraries of molecular imprinted polymers. Bull. Chem. Soc. Jpn. 78: 1354-1361. DOI: $10.1246 /$ bcsj. 78.1354

765. Sarkisov L, Van Tassel PR. 2005. Replica Ornstein-Zernike theory of adsorption in a templated porous material: Interaction site systems. J. Chem. Phys. 123: DOI: 10.1063/1.2049247

766. Burridge DJ, Mao Y, Warner M. 2006. Mechanical strains and electric fields applied to topologically imprinted elastomers. Phys. Rev. E 74: Article Number 021708. DOI: 10.1103/PhysRevE.74.021708

767. Yungerman I, Srebnik S. 2006. Factors contributing to binding-site imperfections in imprinted polymers. Chem. Mater. 18: 657-663. DOI: $10.1021 / \mathrm{cm} 050598 f$

768. Navarro-Villoslada F, San Vicente B, Moreno-Bondi MC. 2004. Application of multivariate analysis to the screening of molecularly imprinted polymers for bisphenol A. Anal. Chim. Acta 504: 149-162. DOI: 10.1016/S00032670(03)00766-9

769. Svenson J, Karlsson JG, Nicholls IA. 2004. H-1 nuclear magnetic resonance study of the molecular imprinting of (-)-nicotine: template self-association, a molecular basis for cooperative ligand binding. J. Chromatogr. A 1024: $39-$ 44. DOI: 10.1016/j.chroma.2003.09.064

770. Wandelt B, Mielniczak A, Cywinski P. 2004. Monitoring of cAMP-imprinted polymer by fluorescence spectroscopy. Biosens. Bioelectron. 20: 10311039. DOI: 10.1016/j.bios.2004.07.035

771. McStay D, Al-Obaidi AH, Hoskins R, Quinn PJ. 2005. Raman spectroscopy of molecular imprinted polymers. J. Opt. A: Pure Appl. Opt. 7: S340-S345. DOI: 10.1088/1464-4258/7/6/013 
772. Molinelli A, O'Mahony J, Nolan K, Smyth MR, Jakusch M, Mizaikoff B. 2005. Analyzing the mechanisms of selectivity in biomimetic self-assemblies via IR and NMR spectroscopy of prepolymerization solutions and molecular dynamics simulations. Anal. Chem. 77: 5196-5204. DOI: 10.1021/ac050525f

773. O'Mahony J, Molinelli A, Nolan K, Smyth MR, Mizaikoff B. 2005. Towards the rational development of molecularly imprinted polymers: ${ }^{1} \mathrm{H}$ NMR studies on hydrophobicity and ion-pair interactions as driving forces for selectivity. Biosens. Bioelectron. 20: 1884-1893. DOI: 10.1016/j.bios.2004.07.036

774. Courtois J, Fischer G, Schauff S, Albert K, Irgum K. 2006. Interactions of bupivacaine with a molecularly imprinted polymer in a monolithic format studied by NMR. Anal. Chem. 78: 580-584. DOI: 10.1021/ac0515733

775. Chen YC, Wang ZM, Yan MD, Prahl SA. 2006. Fluorescence anisotropy studies of molecularly imprinted polymers. Luminescence 21: 7-14. DOI: 10.1002/bio.874

776. Heo K, Jin KS, Oh W, Yoon J, Jin S, Ree M. 2006. Synchrotron X-ray Scattering Study of the Mechanism of Nanopore Generation in Nanoporous Organosilicate Thin Films Imprinted with a Reactive Six-Armed Porogen. J. Phys. Chem. B 110: 15887-15895. DOI: 10.1021/jp061372i

777. Hawkins DM, Trache A, Ellis EA, Stevenson D, Holzenburg A, Meininger GA, Reddy SM. 2006. Quantification and Confocal Imaging of Protein Specific Molecularly Imprinted Polymers. Biomacromolecules 7: 2560-2564. DOI: $10.1021 / \mathrm{bm} 060494 \mathrm{~d}$

778. Palaprat G, Marty JD, Routaboul C, Lattes A, Mingotaud AF, Mauzac M. 2006. Study of Hydrogen Bonding in Liquid Crystalline Solvent by Fourier Transform Infrared Spectroscopy. J. Phys. Chem. A 110: 12887-12890. DOI: 10.1021/jp063987I 
779. Salvador JP, Estevez MC, Marco MP, Sánchez-Baeza F. 2007. A New Methodology for the Rational Design of Molecularly Imprinted Polymers. Anal. Lett. 40: 1294-1306. DOI: 10.1080/00032710701326668

780. Skogsberg U, Meyer C, Rehbein J, Fischer G, Schauff S, Welsch N, Albert K, Hall AJ, Sellergren B. 2007. A solid-state and suspended-state magic angle spinning nuclear magnetic resonance spectroscopic investigation of a 9-ethyladenine molecularly imprinted polymer. Polymer 48: 229-238. DOI: 10.1016/j.polymer.2006.10.036

781. Wei ST, Jakusch M, Mizaikoff B. 2007. Investigating the mechanisms of $17 \alpha$-estradiol imprinting by computational prediction and spectroscopic analysis. Anal. Bioanal. Chem. 389: 423-431. DOI: 10.1007/s00216-0071358-0

782. Ansell RJ, Wang DY, Kuah JKL. 2008. Imprinted polymers for chiral resolution of $( \pm$ )-ephedrine. Part 2: probing pre-polymerisation equilibria in different solvents by NMR. Analyst 133: 1673-1683. DOI: 10.1039/b806376a

783. Cai ZF, Ren FL. 2008. The imprinted mechanism of metallothioneinimprinted polymers. Iran. Polym. J. (Eng. Ed. ) 17: 289-296.

784. Gué AM, Lattes A, Laurent E, Mauzac M, Mingotaud AF. 2008. Characterization of recognition sites for diethyl 4-nitrobenzylphosphonate, an organophosphate pesticide analogue. Anal. Chim. Acta 614: 63-70. DOI: 10.1016/j.aca.2008.03.016

785. Malosse L, Palmas P, Buvat P, Siove A. 2008. Novel Stoichiometric, Noncovalent Pinacolyl Methylphosphonate Imprinted Polymers: A Rational Design by NMR Spectroscopy. Macromolecules 41: 7834-7842. DOI: $10.1021 / \mathrm{ma} 801171 \mathrm{~g}$

786. Osmani Q, Hughes H, Flavin K, Hedin-Dahlstrom J, Allender C, Frisby J, McLoughlin P. 2008. The use of FTIR and NMR spectroscopies to study 
prepolymerisation interactions in nitrogen heterocycles. Anal. Bioanal. Chem. 391: 1229-1236. DOI: 10.1007/s00216-008-1867-5

787. Allender CJ, Castell OK, Davies PR, Fiddy S, Hedin-Dahlström J, Stockenhuber M. 2009. A glimpse of the inner workings of the templated site. Chem. Commun. 165-167. DOI: 10.1039/b811578h

788. Ansell RJ, Wang DY. 2009. Imprinted polymers for chiral resolution of ( \pm )ephedrine. Part 3: NMR predictions and HPLC results with alternative functional monomers. Analyst 134: 564-576. DOI: 10.1039/b815145h

789. Bompart M, Gheber LA, De Wilde Y, Haupt K. 2009. Direct detection of analyte binding to single molecularly imprinted polymer particles by confocal Raman spectroscopy. Biosens. Bioelectron. 25: 568-571. DOI: 10.1016/j.bios.2009.01.020

790. Che AF, Wan LS, Ling J, Liu ZM, Xu ZK. 2009. Recognition Mechanism of Theophylline-Imprinted Polymers: Two-Dimensional Infrared Analysis and Density Functional Theory Study. J. Phys. Chem. B 113: 7053-7058. DOI: $10.1021 /$ jp902163s

791. del Sole R, Lazzoi MR, Arnone M, Della Sala F, Cannoletta D, Vasapollo G. 2009. Experimental and Computational Studies on Non-Covalent Imprinted Microspheres as Recognition System for Nicotinamide Molecules. Molecules 14: 2632-2649. DOI: 10.3390/molecules 14072632

792. El Kirat K, Bartkowski M, Haupt K. 2009. Probing the recognition specificity of a protein molecularly imprinted polymer using force spectroscopy. Biosens. Bioelectron. 24: 2618-2624. DOI: 10.1016/j.bios.2009.01.018

793. Kantarovich K, Belmont AS, Haupt K, Bar I, Gheber LA. 2009. Detection of template binding to molecularly imprinted polymers by Raman microspectroscopy. Appl. Phys. Lett. 94: 820-825. DOI: 10.1063/1.3132061 
794. Odukale AA. 2009. EXAFS Offers Evaluation of Intrinsic Template Binding Site of Molecularly Imprinted Polymers. MRS Bull. 34: 76-77.

795. Pasetto P, Flavin K, Resmini M. 2009. Simple spectroscopic method for titration of binding sites in molecularly imprinted nanogels with hydrolase activity. Biosens. Bioelectron. 25: 572-578. DOI: 10.1016/j.bios.2009.03.042

796. Malitesta C, Guascito MR, Mazzotta E, Picca RA. 2010. X-Ray Photoelectron Spectroscopy characterization of electrosynthesized poly(3thiophene acetic acid) and its application in Molecularly Imprinted Polymers for atrazine. Thin Solid Films 518: 3705-3709. DOI: 10.1016/j.tsf.2009.10.016

797. Men'shikova AYu, Moskalenko YuE, Gribanov AV, Shevchenko NN, Faraonova VB, Yakimanskii AV, Goikhman MYa, Loretsyan NL, Koshkin AV, Alfimov MV. 2010. Sorption of vapors of aromatic compounds by crosslinked polymer particles containing luminophores: A spectroscopic study. Russ. J. Appl. Chem. 83: 1997-2005. DOI: 10.1134/S1070427210110200

798. Nicholls IA, Karlsson BCG, Rosengren AM, Henschel H. 2010. Warfarin: an environment-dependent switchable molecular probe. J. Mol. Recognit. 23: 604-608. DOI: 10.1002/jmr.1058

799. Paik P, Gedanken A, Mastai Y. 2010. Chiral-mesoporous-polypyrrole nanoparticles: Its chiral recognition abilities and use in enantioselective separation. J. Mater. Chem. 20: 4085-4093. DOI: 10.1039/c000232a

800. Paik P, Gedanken A, Mastai Y. 2010. Chiral separation abilities: Aspartic acid block copolymer-imprinted mesoporous silica. Microporous Mesoporous Mater. 129: 82-89. DOI: 10.1016/j.micromeso.2009.09.001

801. Tao YG, Gu H, Xu YQ, Ye LB, Wang QJ. 2010. Pb (II)-Imprinted Chitosan/ $/ \mathrm{TiO}_{2}$ Hybrid Film for High Selectivity of Adsorption Lead Ion in Aqueous Solution. In New Materials and Advanced Materials, Advanced 
Materials Research, Vol. 152-153, Jiang ZY, Han JT, Liu XH (eds). Scientific.net: 484-488. DOI: 10.4028/www.scientific.net/AMR.152-153.484

802. Long YY, Philip JYN, Schillén K, Liu F, Ye L. 2011. Insight into molecular imprinting in precipitation polymerization systems using solution NMR and dynamic light scattering. J. Mol. Recognit. 24: 619-630. DOI: 10.1002/jmr.1097

803. Qin PY, Yang J, Karim MN. 2011. Characterization and selectivity studies of molecular imprinted membranes of Puerarin using scanning electron microscopy. Scanning 33: 7-12. DOI: 10.1002/sca.20212

804. Uibel RH, Harris JM. 2005. Templating of multiple ligand metal ion complexation sites in 8-hydroxyquinoline-modified silica sol-gel materials investigated by in situ Raman spectroscopy. Anal. Chem. 77: 991-1000. DOI: $10.1021 / \operatorname{ac} 048807 n$

805. Fireman-Shoresh S, Marx S, Avnir D. 2007. Induction and detection of chirality in doped sol-gel materials: NMR and circular dichroism studies. J. Mater. Chem. 17: 536-544. DOI: 10.1039/b612822j

806. Klabunovskii El, Volkova LM, Agronomov AE. 1961. A new method of preparation of stereospecific silica gels. Izv. Akad. Nauk SSSR, Ser. Khim. 2101.

807. Shin YS, Liu J, Wang LQ, Nie ZM, Samuels WD, Fryxell GE, Exarhos GJ. 2000. Ordered Hierarchical Porous Materials: Towards Tunable Size- and Shape-Selective Microcavities in Nanoporous Channels. Angew. Chem. Int. Edit. 39: 2702-2707. DOI: 10.1002/1521-3773(20000804)39:15<2702::AIDANIE2702>3.0.CO;2-F

808. Ki CD, Oh C, Oh SG, Chang JY. 2002. Synthesis and characterization of estrone imprinted silica powders. Polym. Prepr. 43: 787-788. 
809. Oral E, Peppas NA. 2002. Molecular imprinting with small molecules: effects on polymerization, material and imprinting properties. Polym. Prepr. 43: 393394.

810. Shimizu KD. 2003. Understanding the fundamental recognition behavior of molecularly imprinted polymers. Polym. Prepr. 44: 481-482.

811. Baggiani C, Anfossi L, Giovannoli C, Tozzi C. 2004. Binding properties of 2,4,5-trichlorophenoxyacetic acid-imprinted polymers prepared with different molar ratios between template and functional monomer. Talanta 62: 10291034. DOI: 10.1016/j.talanta.2003.10.027

812. Cacho C, Turiel E, Martin-Esteban A, Perez-Conde C, Cámara C. 2004. Characterisation and quality assessment of binding sites on a propazineimprinted polymer prepared by precipitation polymerisation. J. Chromatogr. B 802: 347-353. DOI: 10.1016/j.jchromb.2003.12.018

813. Cacho C, Turiel E, Martin-Esteban A, Pérez-Conde C, Cámara C. 2004. Characterisation and quality assessment of binding sites on a propazineimprinted polymer prepared by precipitation polymerisation. J. Chromatogr. B 804: 83. DOI: $10.1016 /$ j.jchromb.2004.03.035

814. Dirion B, Schillinger E, Sellergren B. 2004. Development of a high throughput synthesis technique for the optimization of MIPs for $17 \alpha-$ estradiol. In Molecularly Imprinted Materials-2003, MRS Symposium Proceedings, Vol. 787, Kofinas P, Sellergren B, Roberts MJ (eds). Materials Research Society: Warrendale; 53-60. DOI: 10.1557/PROC-787-G4.3

815. Garcés JL, Mas F, Puy J. 2004. Affinity distribution functions in multicomponent heterogeneous adsorption. Analytical inversion of isotherms to obtain affinity spectra. J. Chem. Phys. 120: 9266-9276. DOI: $10.1063 / 1.1710857$ 
816. Gong SL, Yu ZJ, Meng LZ, Hu L, He YB. 2004. Dye-molecular-imprinted polysiloxanes. II. Preparation, characterization, and recognition behavior. J. Appl. Polym. Sci. 93: 637-643. DOI: 10.1002/app.20486

817. Gorbatchuk VV, Mironov NA, Solomonov BN, Habicher WD. 2004. Biomimetic cooperative interactions of dried cross-linked poly $(\mathrm{N}-6$ aminohexylacrylamide) with binary mixtures of solvent vapors. Biomacromolecules 5: 1615-1623. DOI: 10.1021/bm049743t

818. Gore MA, Karmalkar RN, Kulkarni MG. 2004. Enhanced capacities and selectivities for cholesterol in aqueous media by molecular imprinting: role of novel cross-linkers. J. Chromatogr. $B$ 804: 211-221. DOI: 10.1016/j.jchromb.2003.12.028

819. Guo TY, Xia YQ, Hao GJ, Zhang BH. 2004. Chemically modified chitosan beads as molecularly imprinted polymer matrix for adsorptive separation of proteins. Chin. Chem. Lett. 15: 1339-1341.

820. Habaue S, Satonaka T, Nakano T, Okamoto Y. 2004. Synthesis of polymer gel with chiral helical cavity by molecular imprinting using bifunctional vinyl monomers. Polymer 45: 5095-5100. DOI: 10.1016/j.polymer.2004.04.045

821. Haginaka J, Kagawa C. 2004. Chiral resolution of derivatized amino acids using uniformly sized molecularly imprinted polymers in hydro-organic mobile phases. Anal. Bioanal. Chem. 378: 1907-1912. DOI: 10.1007/s00216-003-2208-3

822. Hu Y, Orwoll RA. 2004. Molecular imprinting of 3-hydroxybenzoic acid: Special and general binding sites. In Molecularly Imprinted Materials-2003, MRS Symposium Proceedings, Vol. 787, Kofinas P, Sellergren B, Roberts MJ (eds). Materials Research Society: Warrendale; 41-46. DOI: 10.1557/PROC-787-G3.4 
823. Ikegami T, Lee WS, Nariai H, Takeuchi T. 2004. Synthetic polymers adsorbing bisphenol $\mathrm{A}$ and its analogues prepared by covalent molecular imprinting using bisphenol A dimethacrylate as a template molecule. Anal. Bioanal. Chem. 378: 1898-1902. DOI: 10.1007/s00216-004-2490-8

824. Karlsson JG, Karlsson B, Andersson LI, Nicholls IA. 2004. The roles of template complexation and ligand binding conditions on recognition in bupivacaine molecularly imprinted polymers. Analyst 129: 456-462. DOI: 10.1039/b316716j

825. Kempe M. 2004. Studies on the cross-reactivity of synthetic oxytocin receptors prepared by molecular imprinting. In Peptides. Peptide Revolution: Genomics, Proteomics \& Therapeutics, Chorev M, Sawyer TK (eds). American Peptide Society: San Diego, CA; 756-757.

826. Lee K, Chang DK, Kim H, Chang JY. 2004. Selectivity control by chemical modification of the recognition sites in two-point binding molecularly imprinted polymer. Macromolecules 37: 5544-5549. DOI: $10.1021 / \mathrm{ma} 0496089$

827. Lehmann M, Dettling M, Brunner H, Tovar GEM. 2004. Affinity parameters of amino acid derivative binding to molecularly imprinted nanospheres consisting of poly[(ethylene glycol dimethacrylate)-co-(methacrylic acid)]. J. Chromatogr. B 808: 43-50. DOI: 10.1016/j.jchromb.2004.03.068

828. Li H, Nie LH, Yao SZ. 2004. Adsorption isotherms and sites distribution of caffeic acid - imprinted polymer monolith from frontal analysis. Chromatographia 60: 425-431. DOI: 10.1365/s10337-004-0403-9

829. Li P, Rong F, Xie YB, Hu V, Yuan CW. 2004. Study on the binding characteristic of S-naproxen imprinted polymer and the interactions between templates and monomers. J. Anal. Chem. 59: 939-944. DOI: 10.1023/B:JANC.0000043909.32186.a0 
830. Lu CY, He HC, He XW, Zeng XS. 2004. Molecularly imprinted polymer with calix[4]arene derivative for the recognition of acetanilide. Chin. J. Chem. 22: 275-278. DOI: $10.1002 /$ cjoc.20040220313

831. Lu Y, Li CX, Wang XD, Sun PC, Xing XH. 2004. Influence of polymerization temperature on the molecular recognition of imprinted polymers. $J$. Chromatogr. B 804: 53-59. DOI: 10.1016/j.jchromb.2003.10.013

832. Pap T, Horvai G. 2004. Characterization of the selectivity of a phenytoin imprinted polymer. J. Chromatogr. A 1034: 99-107. DOI: 10.1016/j.chroma.2004.01.064

833. Parmpi P, Bolisay LDV, Kofinas P. 2004. Isomeric glucose recognition using molecularly imprinted polymer hydrogels. In Molecularly Imprinted Materials2003, MRS Symposium Proceedings, Vol. 787, Kofinas P, Sellergren B, Roberts MJ (eds). Materials Research Society: Warrendale; 79-84. DOI: 10.1557/PROC-787-G6.2

834. Patel A, Fouace S, Steinke JHG. 2004. Novel stereoselective molecularly imprinted polymers via ring-opening metathesis polymerisation. Anal. Chim. Acta 504: 53-62. DOI: 10.1016/j.aca.2003.08.069

835. Pérez-Moral N, Mayes AG. 2004. Comparative study of imprinted polymer particles prepared by different polymerisation methods. Anal. Chim. Acta 504: 15-21. DOI: 10.1016/S0003-2670(03)00533-6

836. Pestov D, Levit N, Maniscalco V, Deveney B, Tepper G. 2004. Molecular imprinting using monomers with solid-state polymerization. Anal. Chim. Acta 504: 31-35. DOI: 10.1016/S0003-2670(03)00672-X

837. Piletsky SA, Guerreiro A, Piletska EV, Chianella I, Karim K, Turner APF. 2004. Polymer cookery. 2. Influence of polymerization pressure and polymer swelling on the performance of molecularly imprinted polymers. Macromolecules 37: 5018-5022. DOI: 10.1021/ma0498161 
838. Rosengren JP, Karlsson JG, Nicholls IA. 2004. Enantioselective synthetic thalidomide receptors based upon DNA binding motifs. Org. Biomol. Chem. 2: 3374-3378. DOI: 10.1039/b407996e

839. Schwarz L, Holdsworth Cl, McCluskey A, Bowyer MC. 2004. Synthesis and evaluation of a molecularly imprinted polymer selective to 2,4,6trichlorophenol. Aust. J. Chem. 57: 759-764. DOI: 10.1071/CH04004

840. Sibrian-Vazquez M, Spivak DA. 2004. Characterization of molecularly imprinted polymers employing crosslinkers with nonsymmetric polymerizable groups. J. Polym. Sci. A,Polym. Chem. 42: 3668-3675. DOI: 10.1002/pola.20186

841. Simon RL, Spivak DA. 2004. Performance analysis of molecularly imprinted polymers for carboxylate and aminophosphate templates using commercially available basic functional monomers. J. Chromatogr. B 804: 203-209. DOI: 10.1016/j.jchromb.2003.12.040

842. Spivak DA. 2004. A new mechanistic diagram for molecularly imprinted polymers. In Molecularly Imprinted Materials-2003, MRS Symposium Proceedings, Vol. 787, Kofinas P, Sellergren B, Roberts MJ (eds). Materials Research Society: Warrendale; 23-28. DOI: 10.1557/PROC-787-G2.6

843. Srebnik S. 2004. Theoretical investigation of the imprinting efficiency of molecularly imprinted polymers. Chem. Mater. 16: 883-888. DOI: $10.1021 / \mathrm{cm} 034705 \mathrm{~m}$

844. Subat M, Borovik AS, König B. 2004. Synthetic creatinine receptor: Imprinting of a Lewis acidic zinc(II)cyclen binding site to shape its molecular recognition selectivity. J. Am. Chem. Soc. 126: 3185-3190. DOI: $10.1021 / \mathrm{ja} 038980 \mathrm{I}$

845. Sun RF, Yu HM, Luo H, Shen ZY. 2004. Construction and application of a stoichiometric displacement model for retention in chiral recognition of 
molecular imprinting. J. Chromatogr. A 1055: 1-9. DOI: 10.1016/j.chroma.2004.08.161

846. Turner NW, Piletska EV, Karim K, Whitcombe M, Malecha M, Magan N, Baggiani C, Piletsky SA. 2004. Effect of the solvent on recognition properties of molecularly imprinted polymer specific for ochratoxin $A$. Biosens. Bioelectron. 20: 1060-1067. DOI: 10.1016/j.bios.2004.06.052

847. Ansell RJ, Kuah KL. 2005. Imprinted polymers for chiral resolution of (+/-)ephedrine: understanding the pre-polymerisation equilibrium and the action of different mobile phase modifiers. Analyst 130: 179-187. DOI: 10.1039/b408751h

848. Baggiani C, Anfossi L, Baravalle P, Giovannoli C, Tozzi C. 2005. Selectivity features of molecularly imprinted polymers recognising the carbamate group. Anal. Chim. Acta 531: 199-207. DOI: 10.1016/j.aca.2004.10.025

849. Bolisay LD, Culver JN, Kofinas P. 2005. Virus recognition using molecularly imprinted polymer hydrogels. Polym. Prepr. 46: 1184.

850. Carabias-Martínez R, Rodríguez-Gonzalo E, Herrero-Hernández E, DíazGarcía ME. 2005. Development and characterisation of a molecularly imprinted polymer prepared by precipitation polymerisation for the determination of phenylurea herbicides. J. Sep. Sci. 28: 453-461. DOI: $10.1002 /$ jssc.200400051

851. Green BS, Strikovsky AG, Pergament-Tzomik I, Arad-Yellin R, Ashani Y. 2005. Selective molecularly imprinted polymers for fluorophosphonate nerve gases - Proof of principle. Polym. Prepr. 46: 1209-1210.

852. Hall AJ, Manesiotis P, Emgenbroich M, Quaglia M, De Lorenzi E, Sellergren B. 2005. Urea host monomers for stoichiometric molecular imprinting of oxyanions. J. Org. Chem. 70: 1732-1736. DOI: 10.1021/jo048470p 
853. Hall AJ, Lanza-Sellergren F, Manesiotis P, Sellergren B. 2005. Non-covalent imprinting of phosphorous esters. Anal. Chim. Acta 538: 9-14. DOI: 10.1016/j.aca.2005.02.030

854. Hall AJ, Lanza-Sellergren F, Manesiotis P, Sellergren B. 2005. Erratum to "Non-covalent imprinting of phosphorous esters": [Analytica Chimica Acta 538 (2005) 9-14]. Anal. Chim. Acta 540: 417. DOI: 10.1016/j.aca.2005.05.004

855. Jin $\mathrm{YZ}$, Row $\mathrm{KH}$. 2005. Adsorption isotherm of ibuprofen on molecular imprinted polymer. Kor. J. Chem. Eng. 22: 264-267. DOI: 10.1007/BF02701495

856. Kim H, Guiochon G. 2005. Thermodynamic studies on the solvent effects in chromatography on molecularly imprinted polymers. 1. Nature of the organic modifier. Anal. Chem. 77: 1708-1717. DOI: 10.1021/ac040155f

857. Kim H, Guiochon G. 2005. Thermodynamic studies on solvent effects in molecularly imprinted polymers. 2. Concentration of the organic modifier. Anal. Chem. 77: 1718-1726. DOI: 10.1021/ac0401640

858. Kim H, Kaczmarski K, Guiochon G. 2005. Mass transfer kinetics on the heterogeneous binding sites of molecularly imprinted polymers. Chem. Eng. Sci. 60: 5425-5444. DOI: 10.1016/j.ces.2005.04.057

859. Kim H, Guiochon G. 2005. Adsorption on molecularly imprinted polymers of structural analogues of a template. single-component adsorption isotherm data. Anal. Chem. 77: 6415-6425. DOI: 10.1021/ac050914+

860. Kim H, Guiochon G. 2005. Thermodynamics and mass transfer kinetics in molecularly imprinted polymers. Polym. Prepr. 46: 1178-1179.

861. Lee JD, Greene NT, Rushton GT, Shimizu KD, Hong JI. 2005. Carbohydrate recognition by porphyrin-based molecularly imprinted polymers. Org. Lett. 7: 963-966. DOI: 10.1021/ol0476180 
862. Liu HY, Yang GL, Liu SB, Wang MM, Chen Y. 2005. Molecular recognition properties and adsorption isotherms of diniconaziole-imprinted polymers. $\mathrm{J}$. Liq. Chrom. Rel. Technol. 28: 2315-2323. DOI: 10.1080/10826070500187509

863. Manesiotis P, Hall AJ, Sellergren B. 2005. Improved imide receptors by imprinting using pyrimidine-based fluorescent reporter monomers. J. Org. Chem. 70: 2729-2738. DOI: 10.1021/jo0477906

864. Manesiotis P, Hall AJ, Courtois J, Irgum K, Sellergren B. 2005. An artificial riboflavin receptor prepared by a template analogue imprinting strategy. Angew. Chem. Int. Edit. 44: 3902-3906. DOI: 10.1002/anie.200500342

865. Nelsen DL, White PS, Gagné MR. 2005. Synthesis and characterization of bifunctional compounds: Templates for metal crown ether assemblies. Organometallics 24: 5479-5483. DOI: 10.1021/om050639h

866. Park HR, Yoon SD, Bang EY, Rogers KR, Chough SH. 2005. Molecular imprinting polymers for the separation of toluic acid isomers. J. Appl. Polym. Sci. 96: 650-654. DOI: 10.1002/app.21491

867. Park HR, Chough SH, Yun YH, Yoon SD. 2005. Synthesis of molecular imprinting polymers separating toluic acid isomers (II). J. Polym. Environ. 13: 81-86. DOI: 10.1007/s10924-004-1232-5

868. Park JK, Lee JW. 2005. Characteristics of selective adsorption using DPhenylalanine imprinted terpolymer beads. Kor. J. Chem. Eng. 22: 927-931. DOI: 10.1007/BF02705677

869. Piletska EV, Romero-Guerra M, Guerreiro AR, Karim K, Turner APF, Piletsky SA. 2005. Adaptation of the molecular imprinted polymers towards polar environment. Anal. Chim. Acta 542: 47-51. DOI: 10.1016/j.aca.2005.01.034 
870. Piletsky SA, Andersson KS, Nicholls IA. 2005. On the role of electrostatic interactions in the enantioselective recognition of phenylalanine in molecularly imprinted polymers incorporating $\alpha$-cyclodextrin. Polym. J. 37: 793-796. DOI: 10.1295/polymj.37.793

871. Rushton GT, Karns CL, Shimizu KD. 2005. A critical examination of the use of the Freundlich isotherm in characterizing molecularly imprinted polymers (MIPs). Anal. Chim. Acta 528: 107-113. DOI: 10.1016/j.aca.2004.07.048

872. Striegler S. 2005. Discrimination of epimeric disaccharides by templated polymers. Anal. Chim. Acta 539: 91-95. DOI: 10.1016/j.aca.2005.03.014

873. Svenson J, Ning Z, Föhrman U, Nicholls IA. 2005. The role of functional monomer-template complexation on the performance of atrazine molecularly imprinted polymers. Anal. Lett. 38: 57-69. DOI: 10.1081/AL-200043443

874. Syu MJ, Deng JH, Nian YM, Chiu TC, Wu AH. 2005. Binding specificity of $\alpha-$ bilirubin-imprinted poly(methacrylic acid-co-ethylene glycol dimethylacrylate) toward $\alpha$-bilirubin. Biomaterials 26: 4684-4692. DOI: 10.1016/j.biomaterials.2004.11.024

875. Syu MJ, Nian YM. 2005. An allosteric model for the binding of bilirubin to the bilirubin imprinted poly(methacrylic acid-co-ethylene glycol dimethylacrylate). Anal. Chim. Acta 539: 97-106. DOI: 10.1016/j.aca.2005.03.031

876. Tai DF, Lin CY, Wu TZ, Chen LK. 2005. Recognition of dengue virus protein using epitope-mediated molecularly imprinted film. Anal. Chem. 77: 51405143. DOI: $10.1021 / \mathrm{ac} 0504060$

877. Takeda K, Kobayashi T. 2005. Bisphenol A imprinted polymer adsorbents with selective recognition and binding characteristics. Sci. Technol. Adv. Mater. 6: 165-171. DOI: 10.1016/..stam.2004.11.008 
878. Takeuchi T, Minato Y, Takase M, Shinmori H. 2005. Molecularly imprinted polymers with halogen bonding-based molecular recognition sites. Tetrahedron Lett. 46: 9025-9027. DOI: 10.1016/j.tetlet.2005.10.098

879. Tokuyama H, Kanazawa R, Sakohara S. 2005. Equilibrium and kinetics for temperature swing adsorption of a target metal on molecular imprinted thermosensitive gel adsorbents. Sep. Purif. Technol. 44: 152-159. DOI: 10.1016/j.seppur.2005.01.004

880. Tsai HA, Syu MJ. 2005. Synthesis and characterization of creatinine imprinted poly(4-vinylpyridine-co-divinylbenzene) as a specific recognition receptor. Anal. Chim. Acta 539: 107-116. DOI: 10.1016/j.aca.2005.03.016

881. Wang JC, Guo RB, Chen JP, Zhang Q, Liang XM. 2005. Phenylurea herbicides-selective polymer prepared by molecular imprinting using $\mathrm{N}$-(4isopropylphenyl)- $N$-butyleneurea as dummy template. Anal. Chim. Acta 540: 307-315. DOI: 10.1016/j.aca.2005.03.057

882. Wu LQ, Zhu KC, Zhao MP, Li YZ. 2005. Theoretical and experimental study of nicotinamide molecularly imprinted polymers with different porogens. Anal. Chim. Acta 549: 39-44. DOI: 10.1016/j.aca.2005.06.009

883. Yang ML, Yuan ZL. 2005. Molecularly imprinted polymer with salicylaldehyde-Cu(OAc) $)_{2}$ as template. J. Mol. Recognit. 18: 103-108. DOI: 10.1002/jmr.692

884. Yoshikawa M, Hotta N, Kyoumura J, Osagawa Y, Aoki T. 2005. Chiral recognition sites from carbonyldioxyglyceryl moiety by an alternative molecular imprinting. Sens. Actuators $B$ 104: 282-288. DOI: 10.1016/j.snb.2004.05.025

885. Yoshikawa M, Nagai Y, Moriguchi K, Hiraoka S. 2005. Chiral recognition ability of oligopeptide derivatives consisting of glutamyl residues. J. Appl. Polym. Sci. 95: 1302-1309. DOI: 10.1002/app.21307 
886. Baggiani C, Baravalle P, Giovannoli C, Tozzi C. 2006. Binding behaviour of pyrimethanil-imprinted polymers prepared in the presence of polar comonomers. J. Chromatogr. A 1117: 74-80. DOI: 10.1016/j.chroma.2006.03.067

887. Boopathi M, Suryanarayana MVS, Nigam AK, Pandey P, Ganesan K, Singh B, Sekhar K. 2006. Plastic antibody for the recognition of chemical warfare agent sulphur mustard. Biosens. Bioelectron. 21: 2339-2344. DOI: 10.1016/j.bios.2006.01.036

888. Carabias-Martínez R, Rodríguez-Gonzalo E, Herrero-Hernández E. 2006. Behaviour of triazine herbicides and their hydroxylated and dealkylated metabolites on a propazine-imprinted polymer: Comparative study in organic and aqueous media. Anal. Chim. Acta 559: 186-194. DOI: 10.1016/j.aca.2005.11.081

889. Chronakis IS, Jakob A, Hagstrom B, Ye L. 2006. Encapsulation and Selective Recognition of Molecularly Imprinted Theophylline and 17 $\beta$ Estradiol Nanoparticles within Electrospun Polymer Nanofibers. Langmuir 22: 8960-8965. DOI: 10.1021/la0613880

890. Defreese JL, Katz A. 2006. Shape-selective covalent binding in bulk, microporous imprinted silica. Microporous Mesoporous Mater. 89: 25-32. DOI: 10.1016/j.micromeso.2005.09.023

891. Dmitrienko SG, Irkha VV, Duisebaeva TB, Mikhailik YV, Zolotov YA. 2006. Synthesis and study of the sorption properties of 4-hydroxybenzoic acidimprinted polymers. J. Anal. Chem. 61: 14-19. DOI: $10.1134 /$ S1061934806010047

892. Dmitrienko SG, Irkha VV, Mikhailik Y, Klokova EV. 2006. Influence of the functional monomer/template ratio in the prepolymerization mixture on the sorption properties of molecularly imprinted polymers of organic compounds. Moscow Uni. Chem. Bull. 61: 54-61. 
893. Gallego-Gallegos M, Muñoz-Olivas R, Cámara C, Mancheño MJ, Sierra MA. 2006. Synthesis of a pH dependent covalent imprinted polymer able to recognize organotin species. Analyst 131: 98-105. DOI: 10.1039/b511946d

894. Hall AJ, Quaglia M, Manesiotis P, DeLorenzi E, Sellergren B. 2006. Polymeric Receptors for the Recognition of Folic Acid and Related Compounds via Substructure Imprinting. Anal. Chem. 78: 8362-8367. DOI: 10.1021/ac0615652

895. He JF, Liu L, Yang GL, Deng QY. 2006. Preparation, characterization and properties studies of quinine-imprinted polymer in the aqueous phase. Frontiers Chem. Chin. 1: 211-216. DOI: 10.1007/s11458-006-0011-3

896. Hsieh RY, Tsai HA, Syu MJ. 2006. Designing a molecularly imprinted polymer as an artificial receptor for the specific recognition of creatinine in serums. Biomaterials 27: 2083-2089. DOI: 10.1016/j.biomaterials.2005.09.024

897. Hsu CY, Lin HY, Chou TC, Thomas JL. 2006. Synthesis of and recognition by ribonuclease A imprinted polymers. Nanotechnology 17: S77-S83. DOI: $10.1088 / 0957-4484 / 17 / 4 / 012$

898. Hsu CY, Lin HY, Thomas JL, Wu BT, Chou TC. 2006. Incorporation of styrene enhances recognition of ribonuclease $A$ by molecularly imprinted polymers. Biosens. Bioelectron. 22: 355-363. DOI: 10.1016/j.bios.2006.05.008

899. Hung $\mathrm{CY}$, Huang $\mathrm{YT}$, Huang HH, Hwang CC. 2006. Preparation of (S)ibuprofen-imprinted polymer and its molecular recognition study. J. Appl. Polym. Sci. 102: 2972-2979. DOI: 10.1002/app.24575

900. Jayasundera S, Zeinali M, Miller JB, Velea LM, Gaber BP, Markowitz MA. 2006. Investigation of Surface Interactions in Molecular Recognition of 
Phosphonate Imprinted Organosilicates and the Role of Water. J. Phys. Chem. B 110: 18121-18125. DOI: 10.1021/jp063880y

901. Kim H, Kaczmarski K, Guiochon G. 2006. Thermodynamic analysis of the heterogenous binding sites of molecularly imprinted polymers. J. Chromatogr. A 1101: 136-152. DOI: 10.1016/j.chroma.2005.09.092

902. Kugimiya A, Takei H. 2006. Preparation of molecularly imprinted polymers with thiourea group for phosphate. Anal. Chim. Acta 564: 179-183. DOI: 10.1016/j.aca.2006.01.099

903. Kuzmic AE, Podolski S, Bogdanic G, Vukovic R. 2006. Synthesis of N-acryl$\mathrm{N}, \mathrm{N}$-di-tert-butylurea and copolymerization with ethylene glycol dimethacrylate. J. Macromol. Sci. A 43: 879-887. DOI: $10.1080 / 10601320600653673$

904. Lavignac N, Brain KR, Allender CJ. 2006. Concentration dependent atrazine-atrazine complex formation promotes selectivity in atrazine imprinted polymers. Biosens. Bioelectron. 22: 138-144. DOI: 10.1016/j.bios.2006.03.017

905. Lin LQ, Li YC, Fu Q, He LC, Zhang J, Zhang QQ. 2006. Preparation of molecularly imprinted polymer for sinomenine and study on its molecular recognition mechanism. Polymer 47: 3792-3798. DOI: 10.1016/j.polymer.2006.03.083

906. Liu XJ, Ouyang CB, Zhao R, Shangguan DH, Chen Y, Liu GQ. 2006. Monolithic molecularly imprinted polymer for sulfamethoxazole and molecular recognition properties in aqueous mobile phase. Anal. Chim. Acta 571: 235-241. DOI: 10.1016/j.aca.2006.05.005

907. Luo Y, Liu L, Li LH, Deng QY. 2006. Preparation of high selective molecularly imprinted polymers for (S)-4-phenyl-2-oxazolidinone. Chin. Chem. Lett. 17: 1551-1554. 
908. Nomachi M, Kubo T, Hosoya K, Kaya K. 2006. Solvent effects in the preparation of molecularly imprinted polymers for melatonin using $\mathrm{N}$ propionyl-5-methoxytryptamine as the pseudo template. Anal. Bioanal. Chem. 384: 1291-1296. DOI: 10.1007/s00216-006-0310-z

909. Osawa T, Shirasaka K, Matsui T, Yoshihara S, Akiyama T, Hishiya T, Asanuma H, Komiyama M. 2006. Importance of the position of vinyl group on $\alpha$-cyclodextrin for the effective imprinting of amino acid derivatives and oligopeptides in water. Macromolecules 39: 2460-2466. DOI: $10.1021 / \mathrm{ma} 060064 \mathrm{f}$

910. Özcan AA, Say R, Denizli A, Ersöz A. 2006. L-Histidine Imprinted Synthetic Receptor for Biochromatography Applications. Anal. Chem. 78: 7253-7258. DOI: 10.1021/ac060536o

911. Rezeli M, Kilar F, Hjertén S. 2006. Monolithic beds of artificial gel antibodies. J. Chromatogr. A 1109: 100-102. DOI: 10.1016/j.chroma.2005.11.076

912. Schwarz L, Bowyer MC, Holdsworth Cl, McCluskey A. 2006. Synthesis and evaluation of a molecularly imprinted polymer selective to 2,4,6trichloroanisole. Aust. J. Chem. 59: 129-134. DOI: 10.1071/CH05318

913. Sellergren B. 2006. Ionization State Selective Modification of Carboxyl Groups in Molecularly Imprinted Polymers: Supporting Evidence for a Binding Site Model. Macromolecules 39: 6306-6309. DOI: $10.1021 / \mathrm{ma} 060941 \mathrm{~h}$

914. Sezgin S, Weber A, Herold M, Gruber-Traub C, Brunner H, Tovar GEM. 2006. Kinetic and thermodynamic behaviour of recognition processes employing nano-spherical L-boc-phenylalanine anilide molecularly imprinted poly(MAA-co-EGDMA) monoliths. Polym. Prepr. 47: 860-861. 
915. Shahgaldian P, Pieles U. 2006. Cyclodextrin derivatives as chiral supramolecular receptors for enantioselective sensing. Sensors 6: 593-615. DOI: $10.3390 / \mathrm{s} 6060593$

916. Shlyapnikov YA, Kolesnikova NN. 2006. Physical chemistry of topological disorder in polymers: Some remarks on polymer memory. J. Appl. Polym. Sci. 100: 4208-4211. DOI: 10.1002/app.23621

917. Syu MJ, Nian YM, Chang YS, Lin XZ, Shiesh SC, Chou TC. 2006. Ionic effect on the binding of bilirubin to the imprinted poly(methacrylic acid-coethylene glycol dimethylacrylate). J. Chromatogr. A 1122: 54-62. DOI: 10.1016/j.chroma.2006.04.024

918. Takeuchi T, Murase N, Maki H, Mukawa T, Shinmori H. 2006. Dopamine selective molecularly imprinted polymers via post-imprinting modification. Org. Biomol. Chem. 4: 565-568. DOI: 10.1039/b514432a

919. Taranekar P, Huang CY, Advincula RC. 2006. Pinacolyl methyl phosphonate (PMP) detection by molecularly imprinted polymers (MIP): A labile covalent bonding approach. Polymer 47: 6485-6490. DOI: 10.1016/j.polymer.2006.07.025

920. Tominey A, Andrew D, Oliphant L, Rosair GM, Dupre J, Kraft A. 2006. Supramolecular binding of protonated amines to a receptor microgel in aqueous medium. Chem. Commun. 2492-2494. DOI: 10.1039/b604393c

921. Urraca JL, Hall AJ, Moreno-Bondi MC, Sellergren B. 2006. A Stoichiometric Molecularly Imprinted Polymer for the Class-Selective Recognition of Antibiotics in Aqueous Media. Angew. Chem. Int. Edit. 45: 5158-5161. DOI: 10.1002/anie.200601636

922. Wang GS, Cao QE, Xiong J, Zhu XF, Hou NB, Ding ZT. 2006. Preparation and Recognition Properties of Vanillin-Imprinted Polymers. Helv. Chim. Acta 89: 3032-3040. DOI: 10.1002/hlca.200690273 
923. Wang HY, Jiang JG, Ma LY, Pang YL. 2006. Syntheses of molecularly imprinted polymers and their molecular recognition study for benzotriazole. Reac. Func. Polym. 66: 1081-1086. DOI: 10.1016/j.reactfunctpolym.2006.01.022

924. Xu ZF, Liu L, Deng QY. 2006. Study on the mechanism of binding specificity of metoclopramide-imprinted polymers. J. Pharm. Biomed. Anal. 41: 701706. DOI: $10.1016 /$ j.jpba.2006.01.043

925. Xu ZF, Liu L, Deng QY. 2006. Molecularly imprinted polymers with bifunctional monomers of polymerizable cyclodextrin derivatives and 2(diethylamino)-ethyl methacrylate for recognition of norfloxacin in aqueous media. Chin. Chem. Lett. 17: 641-644.

926. Yan H, Row KH. 2006. Characteristic and molecular recognition mechanism of theophylline monolithic molecularly imprinted polymer. J. Liq. Chrom. Rel. Technol. 29: 1393-1404. DOI: 10.1080/10826070600674778

927. Yang DH, Ju MJ, Maeda A, Hayashi K, Toko K, Lee SW, Kunitake T. 2006. Design of highly efficient receptor sites by combination of cyclodextrin units and molecular cavity in TiO2 ultrathin layer. Biosens. Bioelectron. 22: 388392. DOI: 10.1016/j.bios.2006.06.013

928. Zhang DN, Li SJ, Huang J, Luo G. 2006. Selective adsorption and steric recognition by molecularly imprinted polymers: A study on molecular selfassembly and its effect on selectivity. High Perform. Polym. 18: 949-960. DOI: 10.1177/0954008306069132

929. Zhao Z, Wang CH, Guo MJ, Shi LQ, Fan YG, Long Y, Mi HF. 2006. Molecular imprinted polymer with cloned bacterial protein template enriches authentic target in cell extract. FEBS Lett. 580: 2750-2754. DOI: 10.1016/j.febslet.2006.04.037 
930. BelBruno JJ, Richter A, Campbell SE, Gibson UJ. 2007. Detection of functional states of molecularly imprinted thin films with multi-cycling nanoindentation. Polymer 48: 1679-1687. DOI: 10.1016/j.polymer.2006.11.054

931. Belyakova LA, Besarab LN. 2007. The influence of the structure of the surface of hydrophilic-hydrophobic silicas on the adsorption of cholic acid. Russ. J. Phys. Chem. 81: 1537-1541.

932. Chou TC, Rick J, Weng YC. 2007. Nanocavity protein biosensor Fabricated by molecular imprinting. In Proceedings of the 7th IEEE International Conference on Nanotechnology, IEEE: 16-20. DOI: 10.1109/NANO.2007.4601131

933. Corton E, García-Calzón JA, Díaz-García ME. 2007. Kinetics and binding properties of cloramphenicol imprinted polymers. J. Non-Cryst. Solids 353: 974-980. DOI: 10.1016/j.jnoncrysol.2006.12.066

934. del Sole R, de Luca A, Catalano M, Mele G, Vasapollo G. 2007. Noncovalent imprinted microspheres: Preparation, evaluation and selectivity of DBU template. J. Appl. Polym. Sci. 105: 2190-2197. DOI: 10.1002/app.26208

935. Farrington K, Regan F. 2007. Investigation of the nature of MIP recognition: The development and characterisation of a MIP for Ibuprofen. Biosens. Bioelectron. 22: 1138-1146. DOI: 10.1016/j.bios.2006.06.025

936. Fazal FM, Hansen DE. 2007. Glucose-specific poly(allylamine) hydrogels--A reassessment. Bioorg. Med. Chem. Lett. 17: 235-238. DOI: 10.1016/j.bmcl.2006.09.054

937. Gao JG, Liu ZL, Liu PY, Jiang N. 2007. Preparation of MAA/TRIM molecularly imprinted polymers and binding selectivity for ciprofloxacin. Chem. J. Internet 9: 6. 
938. He JF, Zhu QH, Deng QY. 2007. Investigation of imprinting parameters and their recognition nature for quinine-molecularly imprinted polymers. Spectrochim. Acta, A 67: 1297-1305. DOI: 10.1016/j.saa.2006.09.040

939. Kimhi O, Bianco-Peled H. 2007. Study of the Interactions between ProteinImprinted Hydrogels and Their Templates. Langmuir 23: 6329-6335. DOI: $10.1021 /$ la700248s

940. Krupadam RJ, Ahuja R, Wate SR. 2007. Benzo([a])pyrene imprinted polyacrylate nanosurfaces: Adsorption and binding characteristics. Sens. Actuators B 124: 444-451. DOI: 10.1016/j.snb.2007.01.020

941. Le Jeune J, Spivak DA. 2007. Chiral effects of alkyl-substituted derivatives of N,O-bismethacryloyl ethanolamine on the performance of one monomer molecularly imprinted polymers (OMNiMIPs). Anal. Bioanal. Chem. 389: 433-440. DOI: 10.1007/s00216-007-1364-2

942. Li SJ, Liao C, Li WK, Chen YF, Hao X. 2007. Rationally Designing Molecularly Imprinted Polymer towards Predetermined High Selectivity by Using Metal as Assembled Pivot. Macromol. Biosci. 7: 1112-1120. DOI: 10.1002/mabi.200700047

943. Li WK, Li SJ, Luo G, Ding K. 2007. A kinetic consideration on the selective adsorption and molecular recognition by molecularly imprinted polymer. Bull. Kor. Chem. Soc. 28: 1346-1352.

944. Liao TB, Tu X, Li SJ. 2007. Selective adsorption and recognition by molecularly imprinted polymer: A study on molecular self-assembly and its effect on selectivity. Polym. -Plast. Technol. Eng. 46: 613-619. DOI: 10.1080/03602550701304784

945. Longo L, Vasapollo G. 2008. Phthalocyanine-Based Molecularly Imprinted Polymers as Nucleoside Receptors. Met. -Based Drugs 2008: Article ID 281843. DOI: $10.1155 / 2008 / 281843$ 
946. Ma SJ, Zhuang XL, Wang HB, Liu HM, Li J, Dong XC. 2007. Preparation and Characterization of Trans-Resveratrol Imprinted Polymers. Anal. Lett. 40: 321-333. DOI: 10.1080/00032710600867689

947. Matsunaga T, Hishiya T, Takeuchi T. 2007. Optimization of Functional Monomer Content in Protein-Imprinted Polymers. Anal. Lett. 40: 2633-2640. DOI: 10.1080/00032710701588275

948. Nantasenamat C, Isarankura-Na-Ayudhya C, Naenna T, Prachayasittikul V. 2007. Quantitative structure-imprinting factor relationship of molecularly imprinted polymers. Biosens. Bioelectron. 22: 3309-3317. DOI: 10.1016/j.bios.2007.01.017

949. O'Connor NA, Paisner DA, Huryn D, Shea KJ. 2007. Screening of 5-HT1A Receptor Antagonists using Molecularly Imprinted Polymers. J. Am. Chem. Soc. 129: 1680-1689. DOI: 10.1021/ja067276c

950. Palaprat G, Marty JD, Langevin D, Finkelmann H, Mauzac M. 2007. Enantioselective Absorption of Chirally Doped Liquid Crystalline Networks Studied by the Use of an Electronic Microbalance. J. Phys. Chem. B 111: 9239-9243.

951. Pan HH, Lee WC, Hung CY, Hwang CC. 2007. Synthesis of molecularly imprinted polymer and its molecular recognition properties of $\mathrm{N}$ acetylneuraminic acid. E-J. Chem. 4: 611-619.

952. Papaioannou EH, Liakopoulou-Kyriakides M, Papi RM, Kyriakidis DA. 2007. Molecularly Imprinted Polymers for Cholecystokinin C-Terminal Pentapeptide Recognition. Macromol. Chem. Phys. 208: 2621-2627. DOI: 10.1002/macp.200700371

953. Park HR, Yoon SD, Lee JC, Chough SH. 2007. Separation of hydroxybenzoic acid isomers using the molecular imprinting technique. $\mathrm{J}$. Appl. Polym. Sci. 105: 2824-2829. DOI: 10.1002/app.26265 
954. Puoci F, lemma F, Cirillo G, Trombino S, Cassano R, Picci N. 2007. Molecularly imprinted polymers for selective adsorption of cholesterol from aqueous environment. E-Polymers Art. No. 013.

955. Rossi C, Haupt K. 2007. Application of the Doehlert experimental design to molecularly imprinted polymers: surface response optimization of specific template recognition as a function of the type and degree of cross-linking. Anal. Bioanal. Chem. 389: 455-460. DOI: 10.1007/s00216-007-1484-8

956. Saenkasa Z, Chaiyasut C, Srichana R, Piyamongkol S. 2007. Comparison of molecular adsorption ability of the molecularly imprinted polymers prepared by ethylene glycol dimethacrylate and trimethylolpropane trimethacrylate as cross linkers. J. Appl. Polym. Sci. 103: 2325-2330. DOI: 10.1002/app.24741

957. Simon R, Collins ME, Spivak DA. 2007. Shape selectivity versus functional group pre-organization in molecularly imprinted polymers. Anal. Chim. Acta 591: 7-16. DOI: 10.1016/j.aca.2006.12.045

958. Siñeriz F, Ikeda Y, Petit E, Bultel L, Haupt K, Kovensky J, Papy-Garcia D. 2007. Toward an alternative for specific recognition of sulfated sugars. Preparation of highly specific molecular imprinted polymers. Tetrahedron 63: 1857-1862. DOI: 10.1016/j.tet.2006.12.026

959. Spivak DA, Sibrian-Vazquez M, Houck S. 2007. Discovery and Development of OMNiMIPs: One MoNomer Molecularly Imprinted Polymers. In Advances in Photo-Initiated Polymer Processes and Materials, Materials Research Society Symposium Proceedings, Vol. 1005E, Guymon A, Hoyle C, Shirai M, Nelson E (eds). Materials Research Society: Paper No. 1005-Q05-05.

960. Sreenivasan K. 2007. Synthesis and evaluation of multiply templated molecularly imprinted polyaniline. J. Mater. Sci. 42: 7575-7578. DOI: 10.1007/s10853-007-1625-7 
961. Su HJ, Chen S, Tan TW. 2007. Surface active site model for $\mathrm{Ni2+}$ adsorption of the surface imprinted adsorbent. Process Biochem. 42: 612619. DOI: 10.1016/j.procbio.2006.11.013

962. Takeuchi T, Goto D, Shinmori H. 2007. Protein profiling by protein imprinted polymer array. Analyst 132: 101-103. DOI: 10.1039/b614532a

963. Toth B, Pap T, Horvath V, Horvai G. 2007. Which molecularly imprinted polymer is better? Anal. Chim. Acta 591: 17-21. DOI: 10.1016/j.aca.2007.01.016

964. Turner NW, Liu X, Piletsky SA, Hlady V, Britt DW. 2007. Recognition of Conformational Changes in $\beta$-Lactoglobulin by Molecularly Imprinted Thin Films. Biomacromolecules 8: 2781-2787. DOI: 10.1021/bm7004774

965. Villar P, Whitcombe MJ, Vulfson EN. 2007. Matrix effects on the selectivity of a cholesterol-imprinted polymer. Polymer 48: 1483-1489. DOI: 10.1016/j.polymer.2007.01.043

966. Wang XJ, Xu ZL, Bing NC, Yang ZG. 2007. Preparation and Aqueous Recognition of Metal Complex Imprinted Polymer Using N-vinyl-2pyrrolidone as Functional Monomer. Chin. J. Chem. Eng. 15: 595-599. DOI: 10.1016/S1004-9541(07)60130-X

967. Wang YJ, Zhang J, Zhu XX, Yu A. 2007. Specific binding of cholic acid by cross-linked polymers prepared by the hybrid imprinting method. Polymer 48: 5565-5571. DOI: 10.1016/j.polymer.2007.07.003

968. Wei ST, Mizaikoff B. 2007. Binding site characteristics of $17 \alpha$-estradiol imprinted polymers. Biosens. Bioelectron. 23: 201-209. DOI: 10.1016/j.bios.2007.03.031

969. Xu ZF, Kuang DZ, Liu L, Deng QY. 2007. Selective adsorption of norfloxacin in aqueous media by an imprinted polymer based on hydrophobic and 
electrostatic interactions. J. Pharm. Biomed. Anal. 45: 54-61. DOI: 10.1016/j.jpba.2007.05.024

970. Yan SL, Gao ZX, Fang YJ, Cheng YY, Zhou HY, Wang HY. 2007. Characterization and quality assessment of binding properties of malachite green molecularly imprinted polymers prepared by precipitation polymerization in acetonitrile. Dyes Pigm. 74: 572-577. DOI: 10.1016/j.dyepig.2006.03.021

971. An FQ, Gao BJ, Feng XQ. 2008. Adsorption and recognizing ability of molecular imprinted polymer MIP-PEI/SiO2 towards phenol. J. Haz. Mater. 157: 286-292. DOI: 10.1016/j.jhazmat.2007.12.095

972. Chen ZY, Hua ZD, Xu L, Huang Y, Zhao MP, Li YZ. 2008. Proteinresponsive imprinted polymers with specific shrinking and rebinding. J. Mol. Recognit. 21: 71-77. DOI: 10.1002/jmr.870

973. Dmitrienko SG, Irkha VV, Apyari VV, Klokova EV, Zolotov YA. 2008. Recognition of hydroxybenzoic acids and their esters by molecularly imprinted polymers. Mendeleev Commun. 18: 315-317. DOI: 10.1016/j.mencom.2008.11.009

974. Feng QZ, Zhao LX, Chu BL, Yan W, Lin JM. 2008. Synthesis and binding site characteristics of 2,4,6-trichlorophenol-imprinted polymers. Anal. Bioanal. Chem. 392: 1419-1429. DOI: 10.1007/s00216-008-2390-4

975. Fu GQ, Yu H, Zhu J. 2008. Imprinting effect of protein-imprinted polymers composed of chitosan and polyacrylamide: A re-examination. Biomaterials 29: 2138-2142. DOI: 10.1016/j.biomaterials.2008.01.019

976. Haginaka J, Tabo H, Kagawa C. 2008. Uniformly sized molecularly imprinted polymers for d-chlorpheniramine: Influence of a porogen on their morphology and enantioselectivity. J. Pharm. Biomed. Anal. 46: 877-881. DOI: 10.1016/j.jpba.2007.05.030 
977. Hsu CW, Yang MC. 2008. Enhancement of the imprinting effect in cholesterol-imprinted microporous silica. J. Non-Cryst. Solids 354: 40374042. DOI: 10.1016/j.jnoncrysol.2008.05.037

978. Huang X, Li SJ. 2008. Rationally Designing Molecularly Imprinted Polymers Toward a Highly Specific Recognition by Using a Stoichiometric Molecular Self-assembly. J. Inorg. Organomet. Polym. Mater. 18: 277-283. DOI: 10.1007/s10904-008-9200-5

979. Ikeda Y, Siñeriz F, Bultel L, Grand E, Kovensky J, Papy-Garcia D. 2008. Synthesis of a trisulfated heparan sulfate disaccharide analog and its use as a template for preliminary molecular imprinting studies. Carbohydr. Res. 343: 587-595. DOI: 10.1016/j.carres.2007.12.020

980. Jin Y, Choi DK, Row KH. 2008. Adsorption isotherms of caffeine on molecular imprinted polymer. Kor. J. Chem. Eng. 25: 816-818. DOI: $10.1007 / \mathrm{s} 11814-008-0135-\mathrm{x}$

981. Kugimiya A, Takei H. 2008. Selectivity and recovery performance of phosphate-selective molecularly imprinted polymer. Anal. Chim. Acta 606: 252-256. DOI: 10.1016/j.aca.2007.11.025

982. Lee WC, Cheng CH, Pan HH, Chung TH, Hwang CC. 2008. Chromatographic characterization of molecularly imprinted polymers. Anal. Bioanal. Chem. 390: 1101-1109. DOI: 10.1007/s00216-007-1765-2

983. Li SJ, Huang X, Zheng MX, Li WK, Tong KJ. 2008. Molecularly imprinted polymers: Thermodynamic and kinetic considerations on the specific sorption and molecular recognition. Sensors 8: 2854-2864. DOI: $10.3390 / \mathrm{s} 8042854$

984. Li SJ, Zheng MX, Zhang JM. 2008. Selective Adsorption and Molecular Recognition by a Molecularly Imprinted Polymer: Kinetic Study and Analysis. J. Chin. Chem. Soc. 55: 121-128. 
985. Li SJ, Li WK, Zheng MX, Zhang JM. 2008. Rationally Designing Molecularly Imprinted Polymer Toward a High Specific Recognition by Using a Stoichiometric Self-Assembly. Polym. -Plast. Technol. Eng. 47: 936-942. DOI: 10.1080/03602550802274522

986. Liu PY, Liu L, Zhang L, Jiang N, Liu ZL, Wang Y. 2008. Synthesis and characterization of molecularly imprinted polymers for recognition of ciprofloxacin. Frontiers Chem. Chin. 3: 378-383. DOI: 10.1007/s11458-0080085-1

987. Mookda P, Singha K, Weeranuch K, Chatchai T. 2008. Synthesis of nicotinamide-imprinted polymers and their binding performances in organic and aqueous media. E-Polymers Art. No. 091.

988. Murakami S, Yamamoto KI, Shinmori H, Takeuchi T. 2008. A molecularly imprinted polymer for the reconstruction of a molecular recognition region. Chem. Lett. 37: 1028-1029. DOI: 10.1246/cl.2008.1028

989. Noss KR, Vaughan AD, Byrne ME. 2008. Tailored binding and transport parameters of molecularly imprinted films via macromolecular structure: The rational design of recognitive polymers. J. Appl. Polym. Sci. 107: 3435-3441. DOI: 10.1002/app.27308

990. Peeples CJ, Earni RR, DiCesare JC. 2008. The synthesis of 1-(4triethoxysilyl)phenyl-4,4,4-trifluoro-1,3- butanedione, a novel trialkoxysilane monomer for the preparation of functionalized sol-gel matrix materials. Molecules 13: 2601-2607. DOI: 10.3390/molecules13102601

991. Philip CM, Mathew B. 2008. Design of EGDMA-Crosslinked Theophylline Imprinted Polymer with High Specificity and Selectivity. J. Macromol. Sci. A 45: 335-343. DOI: 10.1080/10601320701865978

992. Shen ZL, Zhu XL, Yang J, Cai JB, Su QD. 2008. Study on the Binding Characteristic of Methamidophos-specific Molecularly Imprinted Polymer 
and the Interactions between Template and Monomers. J. Chin. Chem. Soc. 55: 587-593.

993. Suedee R, Seechamnanturakit V, Suksuwan A, Canyuk B. 2008. Recognition Properties and Competitive Assays of a Dual Dopamine/Serotonin Selective Molecularly Imprinted Polymer. Int. J. Mol. Sci. 9: 2333-2356. DOI: 10.3390/ijms9122333

994. Uysal A, Demirel G, Turan E, Çaykara T. 2008. Hemoglobin recognition of molecularly imprinted hydrogels prepared at different pHs. Anal. Chim. Acta 625: 110-115. DOI: 10.1016/j.aca.2008.07.010

995. Wang HF, Li WY, He XW, Chen LX, Zhang YK. 2008. mAminophenylboronic acid as a functional monomer for fabricating molecularly imprinted polymer for the recognition of bovine serum albumin. Reac. Func. Polym. 1291-1296. DOI: 10.1016/j.reactfunctpolym.2008.06.002

996. Wu XY, Goswami K, Shimizu KD. 2008. Comparison of monofunctional and multifunctional monomers in phosphate binding molecularly imprinted polymers. J. Mol. Recognit. 21: 410-418. DOI: 10.1002/jmr.912

997. Xia YQ, Guo TY, Song MD, Zhang BH, Zhang BL. 2008. Adsorption dynamics and thermodynamics of $\mathrm{Hb}$ on the $\mathrm{Hb}$-imprinted polymer beads. Reac. Func. Polym. 68: 63-69. DOI: 10.1016/j.reactfunctpolym.2007.10.018

998. Xu ZF, Xu L, Kuang DZ, Zhang FX, Wang JQ. 2008. Exploiting $\alpha-$ cyclodextrin as functional monomer in molecular imprinting for achieving recognition in aqueous media. Mater. Sci. Eng.: C 28: 1516-1521. DOI: 10.1016/j.msec.2008.04.007

999. Yu BY, Zhang XL, He J, Yang KG, Zhao CS. 2008. Effect of the template molecules and nonsolvent additives on the recognition property of molecular 
imprinted polyethersulfone particles. J. Appl. Polym. Sci. 108: 3859-3866. DOI: 10.1002/app.28041

1000. Zhang HT, Song T, Zong FL, Chen TC, Pan CP. 2008. Synthesis and characterization of molecularly imprinted polymers for phenoxyacetic acids. Int. J. Mol. Sci. 9: 98-106. DOI: 10.3390/ijms9010098

1001. Zhao KY, Huang JJ, Ying XG, Cheng GX. 2008. Macromolecularly imprinted calcium phosphate/alginate hybrid polymer microspheres with the surface imprinting of bovine serum albumin in inverse-phase suspension. J. Appl. Polym. Sci. 109: 2687-2693. DOI: 10.1002/app.28354

1002. Zheng MX, Li SJ, Zhang JM. 2008. Molecularly imprinted polymer: Using the template-monomer strategy towards a highly selective sorbent. Polym. Polym. Compos. 16: 47-54.

1003. Zsebi Z, Horvath V, Safrany A, Horvai G. 2008. Analytical followup of the gamma initiated synthesis of a molecularly imprinted polymer. Anal. Chim. Acta 608: 197-203. DOI: 10.1016/j.aca.2007.12.014

1004. Akdamar HA, Sariözlü NY, Özcan AA, Ersöz A, Denizli A, Say RI. 2009. Separation and purification of hyaluronic acid by glucuronic acid imprinted microbeads. Mater. Sci. Eng.: C 29: 1404-1408. DOI: 10.1016/j.msec.2008.10.038

1005. Anfossi L, Baggiani C, Baravalle P, Giovannoli C, Guzzella L, Pozzoni F. 2009. Molecular Recognition of the Fungicide Carbendazim by a Molecular Imprinted Polymer Obtained through a Mimic Template Approach. Anal. Lett. 42: 807-820. DOI: 10.1080/00032710802677183

1006. Athikomrattanakul U, Promptmas C, Katterle M. 2009. Synthetic receptors for neutral nitro derivatives. Tetrahedron Lett. 50: 359-362. DOI: 10.1016/j.tetlet.2008.11.015 
1007. Athikomrattanakul U, Katterle M, Gajovic-Eichelmann N, Scheller FW. 2009. Development of molecularly imprinted polymers for the binding of nitrofurantoin. Biosens. Bioelectron. 25: 82-87. DOI: 10.1016/j.bios.2009.06.003

1008. Benito-Peña E, Martins S, Orellana G, Moreno-Bondi MC. 2009. Watercompatible molecularly imprinted polymer for the selective recognition of fluoroquinolone antibiotics in biological samples. Anal. Bioanal. Chem. 393: 235-245. DOI: 10.1007/s00216-008-2405-1

1009. Breton F, Delépée R, Agrofoglio LA. 2009. Molecular imprinting of AMP by an ionic-noncovalent dual approach. J. Sep. Sci. 32: 3285-3291. DOI: $10.1002 /$ jssc.200900226

1010. Chaitidou S, Kotrotsiou O, Kiparissides C. 2009. On the synthesis and rebinding properties of $[\mathrm{Co}(\mathrm{C} 2 \mathrm{H} 3 \mathrm{O} 2) 2(\mathrm{z}$-Histidine $)]$ imprinted polymers prepared by precipitation polymerization. Mater. Sci. Eng.: C 29: 1415-1421. DOI: 10.1016/j.msec.2008.11.011

1011. Chen ZH, Luo AQ, Sun LQ. 2009. Studies on molecular recognition of thymidines with molecularly imprinted polymers. In Proceedings of SPIE The International Society for Optical Engineering, Vol. 7493, SPIE: Art. No. 74931Y. DOI: 10.1117/12.847732

1012. Chou SK, Syu MJ. 2009. Via zinc(II) protoporphyrin to the synthesis of poly(ZnPP-MAA-EGDMA) for the imprinting and selective binding of bilirubin. Biomaterials 30: 1255-1262. DOI: 10.1016/j.biomaterials.2008.11.029

1013. Çimen D, Akbulut E, Demirel G, Caykara T. 2009. Molecularly imprinted hydrogels for fibrinogen recognition. Reac. Func. Polym. 69: 655-659. DOI: 10.1016/j.reactfunctpolym.2009.04.009 
1014. Curcio M, Parisi Ol, Cirillo G, Spizzirri UG, Puoci F, lemma F, Picci N. 2009. Selective recognition of methotrexate by molecularly imprinted polymers. $E$ Polymers Art. No. 078.

1015. Farber S, Green BS, Domb AJ. 2009. Selective 17- $\alpha$-estradiol molecular imprinting. J. Polym. Sci. A,Polym. Chem. 47: 5534-5542. DOI: 10.1002/pola.23604

1016. Farrington K, Regan F. 2009. Molecularly imprinted sol gel for ibuprofen: An analytical study of the factors influencing selectivity. Talanta 78: 653-659. DOI: 10.1016/j.talanta.2008.12.013

1017. Feás X, Seijas JA, Vázquez-Tato MP, Regal P, Cepeda A, Fente C. 2009. Syntheses of molecularly imprinted polymers: Molecular recognition of cyproheptadine using original print molecules and azatadine as dummy templates. Anal. Chim. Acta 631: 237-244. DOI: 10.1016/j.aca.2008.10.054

1018. Gao BJ, Lu JH, Chen ZP, Guo JF. 2009. Preparation and recognition performance of cholic acid-imprinted material prepared with novel surfaceimprinting technique. Polymer 50: 3275-3284. DOI: 10.1016/j.polymer.2009.05.008

1019. Guo JJ, Cai JB, Su QD. 2009. Ion imprinted polymer particles of neodymium: synthesis, characterization and selective recognition. J. Rare Earths 27: 22-27. DOI: 10.1016/S1002-0721(08)60183-0

1020. Hu CH, Chou TC. 2009. Albumin molecularly imprinted polymer with high template affinity -- Prepared by systematic optimization in mixed organic/aqueous media. Microchem. J. 91: 53-58. DOI: 10.1016/j.microc.2008.07.005

1021. Kirk C, Jensen M, Kjaer CN, Smedskjaer MM, Larsen KL, Wimmer R, Yu $\mathrm{DH}$. 2009. Aqueous batch rebinding and selectivity studies on sucrose 
imprinted polymers. Biosens. Bioelectron. 25: 623-628. DOI: 10.1016/j.bios.2009.01.021

1022. Kotrotsiou O, Chaitidou S, Kiparissides C. 2009. Boc-I-tryptophan imprinted polymeric microparticles for bioanalytical applications. Mater. Sci. Eng.: C 29: 2141-2146. DOI: 10.1016/j.msec.2009.04.014

1023. Krupadam RJ, Bhagat B, Khan MS, Wate PS. 2009. Molecularly Imprinted Nanoporous Polyacrylate Surface for Benzo( $\alpha)$ Pyrene Recognition. J. Nanosci. Nanotechnol. 9: 5441-5447. DOI: 10.1166/jnn.2009.1142

1024. Kudrinskaya VA, Dmitrienko SG, Zolotov YuA. 2009. Synthesis and study of sorption properties of molecularly imprinted polymers for quercetin. Moscow Uni. Chem. Bull. 64: 124-129. DOI: 10.3103/S0027131409030031

1025. Kyzas GZ, Bikiaris DN, Lazaridis NK. 2009. Selective separation of basic and reactive dyes by molecularly imprinted polymers (MIPs). Chem. Eng. J. 149: 263-272. DOI: 10.1016/j.cej.2008.11.002

1026. Lee SC, Lin HM, Chen H. 2009. Studies on the preparation and properties of inorganic molecularly imprinted polymer (MIP) based on tetraethoxysilane and silane coupling agents. J. Appl. Polym. Sci. 114: 3994-3999. DOI: 10.1002/app.30974

1027. LeJeune J, Spivak DA. 2009. Analyte separation by OMNiMIPs imprinted with multiple templates. Biosens. Bioelectron. 25: 604-608. DOI: 10.1016/j.bios.2009.02.014

1028. Li SH, Wang J, Zhao MP. 2009. Cupric ion enhanced molecular imprinting of bovine serum albumin in hydrogel. J. Sep. Sci. 32: 3359-3363. DOI: $10.1002 /$ jssc. 200900250

1029. Liao PC, Tyan YC, Wang CY, Hsu JF, Chou TC, Lin HY. 2009. Assessing the binding selectivity of molecularly imprinted polymer artificial antibodies 
by mass spectrometry-based profiling system. J. Biomed. Mater. Res. Part A 91A: 597-604. DOI: 10.1002/jbm.a.32257

1030. Lu YK, Liu Y, Bian C, Lu GD, Qin XY. 2009. Preparation and evaluation of molecularly imprinted monolithic column for liquid chromatographic determination of enrofloxacin in eggs. Chem. J. Internet 11: Article No. 115026pe.

1031. Lulinski P, Maciejewska D. 2009. Examination of Imprinting Process with Molsidomine as a Template. Molecules 14: 2212-2225. DOI: doi:10.3390/molecules 14062212

1032. Matsui J, Nagano J, Miyoshi D, Tamaki K, Sugimoto N. 2009. An approach to peptide-based ATP receptors by a combination of random selection, rational design, and molecular imprinting. Biosens. Bioelectron. 25: 563-567. DOI: 10.1016/j.bios.2009.01.031

1033. Okutucu B, Ünal S, Telefoncu A. 2009. Noncovalently galactose imprinted polymer for the recognition of different saccharides. Talanta 78: 1190-1193. DOI: 10.1016/j.talanta.2009.01.047

1034. Paik P, Gedanken A, Mastai Y. 2009. Enantioselective Separation Using Chiral Mesoporous Spherical Silica Prepared by Templating of Chiral Block Copolymers. ACS Appl. Mater. Interf. 1: 1834-1842. DOI: 10.1021/am9003842

1035. Pan J, Xue XH, Wang JH, Xie HM, Wu ZY. 2009. Recognition property and preparation of Staphylococcus aureus protein A-imprinted polyacrylamide polymers by inverse-phase suspension and bulk polymerization. Polymer 50: 2365-2372. DOI: 10.1016/j.polymer.2009.04.004

1036. Papaioannou E, Koutsas C, Liakopoulou-Kyriakides M. 2009. Molecularly imprinted polymers for RGD selective recognition and separation. Amino Acids 36: 563-569. DOI: 10.1007/s00726-008-0118-6 
1037. Petcu M, Karlsson JG, Whitcombe MJ, Nicholls IA. 2009. Probing the limits of molecular imprinting: strategies with a template of limited size and functionality. J. Mol. Recognit. 22: 18-25. DOI: 10.1002/jmr.918

1038. Rosengren-Holmberg JP, Karlsson JG, Svenson J, Andersson HS, Nicholls IA. 2009. Synthesis and ligand recognition of paracetamol selective polymers: semi-covalent versus non-covalent molecular imprinting. Org. Biomol. Chem. 7: 3148-3155. DOI: 10.1039/b900014c

1039. Su WX, Rick J, Chou TC. 2009. Selective recognition of ovalbumin using a molecularly imprinted polymer. Microchem. J. 92: 123-128. DOI: 10.1016/j.microc.2008.12.010

1040. Turner NW, Holmes N, Brisbane C, McGeachie AB, Bowyer MC, McCluskey A, Holdsworth Cl. 2009. Effect of template on the formation of phaseinversed molecularly imprinted polymer thin films: an assessment. Soft Matter 5: 3663-3671. DOI: 10.1039/b902468a

1041. Wang HF, He YH, He XW, Li WY, Chen LX, Zhang YK. 2009. BSAimprinted synthetic receptor for reversible template recognition. J. Sep. Sci. 32: 1981-1986. DOI: 10.1002/jssc.200800562

1042. Wang J, Hua ZD, Chen ZY, Li YZ, Zhao MP. 2009. Molecular imprinting of protein by coordinate interaction. Chin. Chem. Lett. 20: 747-750. DOI: 10.1016/j.cclet.2008.12.035

1043. Wang JY, Xu ZL, Wu P, Yin SJ. 2009. Binding constant and transport property of S-Naproxen molecularly imprinted composite membrane. J. Membr. Sci. 331: 84-90. DOI: 10.1016/j.memsci.2009.01.016

1044. Xia YQ, Guo TY, Zhao HL, Song MD, Zhang BH, Zhang BL. 2009. Protein recognition onto silica particles using chitosan as intermedium substrate. $J$. Biomed. Mater. Res. Part A 90A: 326-332. DOI: 10.1002/jbm.a.32084 
1045. Xu PP, Xu WZ, Zhang XJ, Pan JM, Yan YS. 2009. Molecularly-imprinted Material for Dibenzothiophene Recognition Prepared by Surface Imprinting Methods. Adsorpt. Sci. Technol. 27: 975-987. DOI: 10.1260/02636174.27.10.975

1046. Yun YH, Shon HK, Yoon SD. 2009. Preparation and characterization of molecularly imprinted polymers for the selective separation of 2,4dichlorophenoxyacetic acid. J. Mater. Sci. 44: 6206-6211. DOI: 10.1007/s10853-009-3863-3

1047. Zakaria ND, Yusof NA, Haron J, Abdullah AH. 2009. Synthesis and Evaluation of a Molecularly Imprinted Polymer for 2,4-Dinitrophenol. Int. J. Mol. Sci. 10: 354-365. DOI: 10.3390/ijms10010354

1048. Zhai CX, Lu Q, Chen XM, Peng Y, Chen LN, Du SH. 2009. Molecularly imprinted layer-coated silica nanoparticles toward highly selective separation of active diosgenin from Dioscorea nipponica Makino. J. Chromatogr. A 1216: 2254-2262. DOI: 10.1016/j.chroma.2009.01.030

1049. Zhang YQ, Xiang L, Du TD, Zhang Y, Lu M. 2009. Preparation and Characterization of Imprinted Porous Materials with High Selectivity for Luteolin. Chem. Eng. Technol. 32: 1512-1519. DOI: 10.1002/ceat.200900134

1050. Zhang ZL, Wang B. 2009. Synthesis of highly efficient D-naproxen imprinted polymer and investigation of their specific performance. J. Appl. Polym. Sci. 113: 1050-1062. DOI: 10.1002/app.30048

1051. Zhu XF, Cao QE, Yang XQ, Li F, Wang GS, Ding ZT. 2009. Preparation and Recognition Mechanism of Gallic Acid Imprinted Polymers. Helv. Chim. Acta 92: 78-87. DOI: 10.1002/hlca.200800209

1052. Baggiani C, Baravalle P, Giovannoli C, Anfossi L, Giraudi G. 2010. Molecularly imprinted polymers for corticosteroids: Analysis of binding 
selectivity. Biosens. Bioelectron. 26: 590-595. DOI: 10.1016/j.bios.2010.07.023

1053. Cohen T, Starosvetsky J, Cheruti U, Armon R. 2010. Whole Cell Imprinting in Sol-Gel Thin Films for Bacterial Recognition in Liquids: Macromolecular Fingerprinting. Int. J. Mol. Sci. 11: 1236-1252. DOI: 10.3390/ijms11041236

1054. Fan PM, Wang B. 2010. Regulatory effects of $\mathrm{Zn}(\mathrm{II})$ on the recognition properties of metal coordination imprinted polymers. J. Appl. Polym. Sci. 116: 258-266. DOI: 10.1002/app.31454

1055. Fang G, Xie W, Wang H. 2010. Study on the interaction between glutathione and functional monomers. In Proceedings of the 2010 International Conference on Biomedical Engineering and Computer Science (ICBECS), IEEE: DOI: 10.1109/ICBECS.2010.5462328

1056. Gai QQ, Qu F, Zhang YK. 2010. The Preparation of BHb-Molecularly Imprinted Gel Polymers and Its Selectivity Comparison to $\mathrm{BHb}$ and BSA. Sep. Sci. Technol. 45: 2394-2399. DOI: 10.1080/01496395.2010.484409

1057. Gao BJ, Li YB, Zhang ZG. 2010. Preparation and recognition performance of creatinine-imprinted material prepared with novel surface-imprinting technique. J. Chromatogr B 878: 2077-2086. DOI: 10.1016/j.jchromb.2010.06.007

1058. Gao BJ, Niu QY, Du RK. 2010. Preparation and recognition performance of cytisine alkaloid-imprinted material prepared using novel surface molecular imprinting technique. J. Sep. Sci. 33: 1338-1348. DOI: 10.1002/jssc.200900762

1059. Gao BJ, Liu SY, Li YB. 2010. Preparation and recognition performance of uric acid-imprinted material prepared with novel surface imprinting technique. J. Chromatogr. A 1217: 2226-2236. DOI: 10.1016/j.chroma.2010.02.027 
1060. Gao BJ, Fu HY, Li YB, Du RK. 2010. Preparation of surface molecularly imprinted polymeric microspheres and their recognition property for basic protein lysozyme. J. Chromatogr $B$ 878: 1731-1738. DOI: 10.1016/j.jchromb.2010.04.033

1061. Chai ZH, Chen HC, Kong J, Wang Y, Fu GQ. 2011. Comment on "Preparation of surface molecularly imprinted polymeric microspheres and their recognition property for basic protein lysozyme": Molecularly imprinted polymer or cation exchanger? J. Chromatogr B 879: 669-670. DOI: 10.1016/j.jchromb.2011.01.024

1062. Hu LY, Orwoll RA. 2010. Molecular Imprinting by 4-Hydroxybenzoic Acid: A Two-Site Model. Sep. Sci. Technol. 45: 2337-2344. DOI: $10.1080 / 01496395.2010 .491811$

1063. Janiak DS, Ayyub OB, Kofinas P. 2010. Effects of charge density on the recognition properties of molecularly imprinted polyampholyte hydrogels. Polymer 51: 665-670. DOI: 10.1016/j.polymer.2009.12.022

1064. Karlsson BCG, Rosengren AM, Näslund I, Andersson PO, Nicholls IA. 2010. Synthetic Human Serum Albumin Sudlow I Binding Site Mimics. J. Med. Chem. 53: 7932-7937. DOI: 10.1021/jm100491v

1065. Kempe H, Kempe M. 2010. Influence of salt ions on binding to molecularly imprinted polymers. Anal. Bioanal. Chem. 396: 1599-1606. DOI: 10.1007/s00216-009-3329-0

1066. Kempe $H$, Kempe M. 2010. QSRR analysis of $\alpha$-lactam antibiotics on a penicillin G targeted MIP stationary phase. Anal. Bioanal. Chem. 398: 30873096. DOI: 10.1007/s00216-010-4254-y

1067. Kusunoki T, Kobayashi T. 2010. Molecular imprinting micropolymerbeads having cooperative effect of both surfactant and inosine template. J. Appl. Polym. Sci. 117: 565-571. DOI: 10.1002/app.31128 
1068. Lachová M, Lehotay J, Skacáni I, Cižmárik J. 2010. Study of Selectivity of Molecularly Imprinted Polymers Prepared Under Different Conditions. J. Chromatogr. Sci. 48: 395-398.

1069. Lee SC, Chuang FL, Tsai YL, Chen H. 2010. Studies on the preparation and properties of sol-gel molecularly imprinted polymer based on tetraethoxysilane for recognizing sulfonamides. J. Polym. Res. 17: 737-744. DOI: 10.1007/s10965-009-9364-x

1070. Li Y, Li X, Dong CK, Qi JY, Han XJ. 2010. A graphene oxide-based molecularly imprinted polymer platform for detecting endocrine disrupting chemicals. Carbon 48: 3427-3433. DOI: 10.1016/j.carbon.2010.05.038

1071. Liang SA, Wan JF, Zhu JL, Cao XJ. 2010. Effects of porogens on the morphology and enantioselectivity of core-shell molecularly imprinted polymers with ursodeoxycholic acid. Sep. Purif. Technol. 72: 208-216. DOI: 10.1016/j.seppur.2010.02.011

1072. Liu HJ, Han RF, Feng M, Gao JF, Long Y, Zhao Z, Wang Y, Mi HF. 2010. Preparation of molecular imprinted polymer with quaternary ammonium groups as recognition sites for separation of pig cyclophilin 18 and bovine serum albumin. J. Sep. Sci. 33: 1856-1862. DOI: 10.1002/jssc.201000038

1073. Liu XY, Zhou T, Wang XC, Zhang JH. 2010. Investigation of selective molecular interactions using two-dimensional Fourier transform IR spectroscopy. Anal. Bioanal. Chem. 397: 339-343. DOI: 10.1007/s00216009-3403-7

1074. Longo L, Scorrano S, Vasapollo G. 2010. RNA nucleoside recognition by phthalocyanine-based molecularly imprinted polymers. J. Polym. Res. 17: 683-687. DOI: 10.1007/s10965-009-9356-x 
1075. Luk Y, Allender CJ, Wirth T. 2010. Molecular imprinted polymers binding low functionality templates. Tetrahedron Lett. 51: 5883-5885. DOI: 10.1016/j.tetlet.2010.08.108

1076. Sasaki S, Ooya T, Takeuchi T. 2010. Highly selective bisphenol A-imprinted polymers prepared by atom transfer radical polymerization. Polym. Chem. 1: 1684-1688. DOI: 10.1039/COPY00140F

1077. Singh DK, Mishra S. 2010. Synthesis and characterization of $\mathrm{Hg}(\mathrm{II})$-ionimprinted polymer: Kinetic and isotherm studies. Desalination 257: 177-183. DOI: 10.1016/j.desal.2010.02.026

1078. Song SH, Shirasaka K, Hirokawa Y, Asanuma H, Wada T, Sumaoka J, Komiyama M. 2010. Molecular imprinting of cyclodextrin to physiologically active oligopeptides in water. Supramol. Chem. 22: 149-155. DOI: $10.1080 / 10610270902980622$

1079. Tov OY, Luvitch S, Bianco-Peled H. 2010. Molecularly imprinted hydrogel displaying reduced non-specific binding and improved protein recognition. $J$. Sep. Sci. 33: 1673-1681. DOI: 10.1002/jssc.200900874

1080. Wang Y, Chai ZH, He HY, Jiang YZ, Fu GQ. 2010. Polyacrylamide hydrogels with phenylboronic acid moieties for the imprinting of proteins. Chin. Chem. Lett. 21: 1487-1489. DOI: 10.1016/j.cclet.2010.06.005

1081. Xu ZF, Kuang DZ, Feng YL, Zhang FX. 2010. Combination of hydrophobic effect and electrostatic interaction in imprinting for achieving efficient recognition in aqueous media. Carbohydr. Polym. 79: 642-647. DOI: 10.1016/j.carbpol.2009.09.010

1082. Yu JY, Hu XL, Song RY, Xi S. 2010. Molecularly imprinted polymer microspheres prepared by precipitation polymerization for atenolol recognition. In Manufacturing Processes and Systems, Advanced Materials 
Research, Vol. 148-149, Liu XH, Jiang ZY, Han JT (eds). Scientific.net: 1192-1198. DOI: 10.4028/www.scientific.net/AMR.148-149.1192

1083. Yu JY, Hu XL, Li DP, Wang HL. 2010. Molecularly imprinted polymer microspheres prepared by precipitation polymerization for the binding and recognition of roxithromycin. In 4th International Conference on Bioinformatics and Biomedical Engineering (iCBBE), 2010, IEEE: Art. No. 5515319. DOI: 10.1109/ICBBE.2010.5515319

1084. Yu S, Puleo DA, Luo AQ. 2010. Preliminary study on repeatability of molecular recognition capability of a silica-based molecularly imprinted polymer. In 4th International Conference on Bioinformatics and Biomedical Engineering (iCBBE), 2010, IEEE: Art. No. 5516486. DOI: 10.1109/ICBBE.2010.5516486

1085. Zhang W, Qin L, Chen RR, He XW, Li WY, Zhang YK. 2010. Protein imprinted polymer using acryloyl- $\alpha$-cyclodextrin and acrylamide as monomers. Appl. Surf. Sci. 256: 3000-3005. DOI: 10.1016/j.apsusc.2009.11.064

1086. Zhang YG, Song D, Lanni LM, Shimizu KD. 2010. Importance of Functional Monomer Dimerization in the Molecular Imprinting Process. Macromolecules 43: 6284-6294. DOI: 10.1021/ma101013c

1087. Zhang ZB, Hu JY. 2010. Effect of Environmental Factors on Estrogenic Compounds Adsorption by MIP. Water, Air, \& Soil Pollution 210: 255-264. DOI: $10.1007 /$ s11270-009-0247-x

1088. Zhao KY, Cheng GX, Wei JF, Zhou JY, Zhang JL, Chen L. 2010. The Rebinding Properties of Bovine Serum Albumin Imprinted Calcium Alginate/Phosphate Hybrid Microspheres Via the Adjustment of $\mathrm{pH}$ Values and Salt Concentration. Macromol. Symp. 297: 126-137. DOI: 10.1002/masy.200900105 
1089. Zhao KY, Wei JF, Zhou JY, Zhao YP, Cheng GX. 2010. The rebinding properties of bovine serum albumin imprinted calcium phosphate/polyacrylate/alginate hybrid polymer microspheres. In New Materials and Advanced Materials, Advanced Materials Research, Vol. 152153, Jiang ZY, Han JT, Liu XH (eds). Scientific.net: 1636-1640. DOI: 10.4028/www.scientific.net/AMR.152-153.1636

1090. Bhagat B, Krupadam RJ. 2010. Effect of Solvents on the Adsorption Properties of Benzoopyrene-imprinted Polymers. Adsorpt. Sci. Technol. 28: 79-88. DOI: 10.1260/0263-6174.28.1.79

1091. Cela-Pérez MC, Castro-López MM, Lasagabáster-Latorre A, López-Vilariño JM, González-Rodríguez MV, Barral-Losada LF. 2011. Synthesis and characterization of bisphenol-A imprinted polymer as a selective recognition receptor. Anal. Chim. Acta 706: 275-284. DOI: 10.1016/j.aca.2011.09.002

1092. Chen $\mathrm{CY}$, Wang $\mathrm{CH}$, Chen $\mathrm{AH} .2011$. Recognition of molecularly imprinted polymers for a quaternary alkaloid of berberine. Talanta 84: 1038-1046. DOI: 10.1016/j.talanta.2011.03.009

1093. Ho YS. 2011. Comments on "Recognition of molecularly imprinted polymers for a quaternary alkaloid of berberine $\forall$. Talanta 87: 311. DOI: 10.1016/j.talanta.2011.10.007

1094. Dana M, Lulinski P, Maciejewska D. 2011. Synthesis of Homoveratric AcidImprinted Polymers and Their Evaluation as Selective Separation Materials. Molecules 16: 3826-3844. DOI: 10.3390/molecules 16053826

1095. Ding J, Chen SS, Li X, Ying XG, Jiang SF, Weng S, Zhang WY. 2011. Preparation of L-Tryptophan Imprinted Polymer Microspheres in Aqueous Media by In Situ Grafting on Surface. In Emerging Focus on Advanced Materials, Advanced Materials Research, Vol. 306-307, Liu SQ, Zuo M (eds). Scientific.net: 1658-1662. DOI: 10.4028/www.scientific.net/AMR.306307.1658 
1096. Dmitrienko SG, Popov I, Chumichkina YuA, Zolotov YuA. 2011. The sorption properties of polymers with molecular imprints of 2,4-dichlorophenoxyacetic acid synthesized by various methods (Original Russian Text (c) S.G. Dmitrienko, S.A. Popov, Yu.A. Chumichkina, Yu.A. Zolotov, 2011, published in Zhurnal Fizicheskoi Khimii, 2011, Vol. 85, No. 3, pp. 542-547.). Russ. J. Phys. Chem. A 85: 472-477. DOI: 10.1134/S0036024411020087

1097. Esfandyari-Manesh M, Javanbakht M, Atyabi F, Badiei A, Dinarvand R. 2011. Effect of porogenic solvent on the morphology, recognition and release properties of carbamazepine-molecularly imprinted polymer nanospheres. J. Appl. Polym. Sci. 121: 1118-1126. DOI: 10.1002/app.33812

1098. Fang LJ, Chen SJ, Zhang Y, Zhang HQ. 2011. Azobenzene-containing molecularly imprinted polymer microspheres with photoresponsive template binding properties. J. Mater. Chem. 21: 2320-2329. DOI: 10.1039/COJM02898C

1099. Fu GQ, He HY, Chai ZH, Chen HC, Kong J, Wang Y, Jiang YZ. 2011. Enhanced Lysozyme Imprinting Over Nanoparticles Functionalized with Carboxyl Groups for Noncovalent Template Sorption. Anal. Chem. 83: 14311436. DOI: $10.1021 /$ ac1029924

1100. Gao BJ, Chen YX, Men JY. 2011. Constructing chiral caves and efficiently separating enantiomers of glutamic acid with novel surface-imprinting technique. J. Chromatogr. A 1218: 5441-5448. DOI: 10.1016/j.chroma.2011.06.003

1101. Gao RX, Kong X, Wang X, He XW, Chen LX, Zhang YK. 2011. Preparation and characterization of uniformly sized molecularly imprinted polymers functionalized with core-shell magnetic nanoparticles for the recognition and enrichment of protein. J. Mater. Chem. 21: 17863-17871. DOI: 10.1039/C1JM12414E 
1102. Gavrilovic I, Mitchell K, Brailsford AD, Cowan DA, Kicman AT, Ansell RJ. 2011. A molecularly imprinted receptor for separation of testosterone and epitestosterone, based on a steroidal cross-linker. Steroids 76: 478-483. DOI: 10.1016/j.steroids.2011.01.004

1103. Ghasemzadeh N, Rossbach UL, Johansson BM, Nyberg F. 2011. Application of artificial gel antibodies for investigating molecular polymorphisms of human pituitary growth hormone. Amino Acids 40: 12491255. DOI: $10.1007 / \mathrm{s} 00726-011-0840-3$

1104. Gong XY, Cao XJ. 2011. Preparation of molecularly imprinted polymers for artemisinin based on the surfaces of silica gel. Journal of Biotechnology 153: 8-14. DOI: 10.1016/j.jbiotec.2011.02.005

1105. Gómez-Pineda LE, Pina-Luis GE, Cuán Á, García-Calzón JA, Díaz-García ME. 2011. Physico-chemical characterization of flavonol molecularly imprinted polymers. Reac. Func. Polym. 71: 402-408. DOI: 10.1016/j.reactfunctpolym.2010.12.013

1106. Gupta R, Kumar A. 2011. Synthesis and characterization of sol-gel-derived molecular imprinted polymeric materials for cholesterol recognition. J. SolGel Sci. Technol. 58: 182-194. DOI: 10.1007/s10971-010-2376-5

1107. He JX, Fang GZ, Deng QL, Wang S. 2011. Preparation, characterization and application of organic-inorganic hybrid ractopamine multi-template molecularly imprinted capillary monolithic column. Anal. Chim. Acta 692: 5762. DOI: 10.1016/j.aca.2011.02.056

1108. Hou JY, Han XY, Zhang Y, Ding S, Ye L. 2011. Molecularly Imprinted Microspheres for Highly Selective Separation of Diosgenin. In Advanced Research on Advanced Structure, Materials and Engineering, Vol. 382, Zhang $H$, Jin $D$ (eds). Scientific.net: 303-306. DOI: 10.4028/www.scientific.net/AMR.382.303 
1109. luga C, Ortíz E, Noreña L. 2011. Interaction between volatile organic compounds and functional monomers in molecularly imprinted materials. In Technical Proceedings of the 2011 NSTI Nanotechnology Conference and Expo, NSTI-Nanotech 2011, Vol. 3, 777-780.

1110. Jiang HM, Zhang SF, Sui Q. 2011. Removal of Cholesterol by $\alpha$ Cyclodextrin. Asian J. Chem. 23: 3783-3786.

1111. Jiang JB, Song KS, Chen Z, Zhou Q, Tang YW, Gu FL, Zuo XJ, Xu ZG. 2011. Novel molecularly imprinted microsphere using a single chiral monomer and chirality-matching (S)-ketoprofen template. J. Chromatogr. A 1218: 3763-3770. DOI: 10.1016/j.chroma.2011.04.043

1112. Jiang JG. 2011. Molecular recognition characteristics of cobalt(II)-complex molecularly imprinted polymer. Asian J. Chem. 23: 4470-4472.

1113. Jo SH, Park C, Yi SC, Kim D, Mun S. 2011. Development of a four-zone carousel process packed with metal ion-imprinted polymer for continuous separation of copper ions from manganese ions, cobalt ions, and the constituent metal ions of the buffer solution used as eluent. J. Chromatogr. A 1218: 5664-5674. DOI: 10.1016/j.chroma.2011.06.094

1114. Khomutov S, Donova MV. 2011. Nanodimer cyclodextrin ligands with high affinity to steroids. J. Inclusion Phenom. Macrocyl. Chem. 70: 353-357. DOI: 10.1007/s10847-010-9896-z

1115. Kong Y, Yao C, Ni JH, Zhou YS, Chen ZD. 2011. Identification of Aspartic Acid Enantiomers Based on Molecularly Imprinted Polyaniline. Chin. J. Chem. 29: 2659-2663. DOI: 10.1002/cjoc.201180436

1116. Kong Y, Wei JX, Wang WC, Chen ZD. 2011. Separation of tryptophan enantiomers with polypyrrole electrode column by potential-induced technique. Electrochim. Acta 56: 4770-4774. DOI: 10.1016/j.electacta.2011.02.082 
1117. Lacasta S, Sebastiàn V, Casado C, Mayoral Ál, Romero P, Larrea Á, Vispe E, López-Ram-de-Viu P, Uriel S, Coronas J. 2011. Chiral Imprinting with Amino Acids of Ordered Mesoporous Silica Exhibiting Enantioselectivity after Calcination. Chem. Mater. 23: 1280-1287. DOI: 10.1021/cm1032546

1118. Li JH, Zheng ZJ, Fu SP, Zhu JB. 2011. Preparation and Characterization of Paclitaxel Imprinted Silica Nanoparticles. In New Materials, Applications and Processes, Vol. 399-401, Zeng JM, Kim YH, Chen YF (eds). Scientific.net: 1894-1897. DOI: 10.4028/www.scientific.net/AMR.399-401.1894

1119. Li SJ, Ge Y, Piletsky SA, Turner APF. 2011. A Zipper-Like On/OffSwitchable Molecularly Imprinted Polymer. Adv. Funct. Mater. 21: 33443349. DOI: 10.1002/adfm.201100593

1120. Liu XY, Zhou T, Du ZW, Wei Z, Zhang JH. 2011. Recognition ability of temperature responsive molecularly imprinted polymer hydrogels. Soft Matter 7: 1986-1993. DOI: 10.1039/COSM00192A

1121. Lu FG, Xi YL, Lv Z, Fan LL, Qiu HM, Luo CN. 2011. Molecularly Imprinted Polymer for Preparation and Adsorption of Phenylalanine. In Emerging Focus on Advanced Materials, Advanced Materials Research, Vol. 306-307, Liu SQ, Zuo M (eds). Scientific.net: 638-641. DOI: 10.4028/www.scientific.net/AMR.306-307.638

1122. Lulinski P, Maciejewska D. 2011. Impact of functional monomers, crosslinkers and porogens on morphology and recognition properties of 2-(3,4dimethoxyphenyl)ethylamine imprinted polymers. Mater. Sci. Eng.: C 31: 281-289. DOI: 10.1016/j.msec.2010.09.010

1123. Luo XB, Luo SL, Zhan YC, Shu HY, Huang YN, Tu XM. 2011. Novel Cu (II) magnetic ion imprinted materials prepared by surface imprinted technique combined with a sol-gel process. J. Haz. Mater. 192: 949-955. DOI: 10.1016/j.jhazmat.2011.05.042 
1124. Ma XL, Chen Z, Chen RY, Zheng X, Chen X, Lan RF. 2011. Imprinted $\alpha-$ cyclodextrin polymers using naringin as template. Polym. Int. 60: 1455-1460. DOI: 10.1002/pi.3101

1125. Mahon CS, Jackson AW, Murray BS, Fulton DA. 2011. Templating a polymer-scaffolded dynamic combinatorial library. Chem. Commun. 47: 7209-7211. DOI: 10.1039/C1CC11998B

1126. Mizutani N, Yang DH, Selyanchyn R, Korposh S, Lee SW, Kunitake T. 2011. Remarkable enantioselectivity of molecularly imprinted TiO2 nano-thin films. Anal. Chim. Acta 694: 142-150. DOI: 10.1016/j.aca.2011.02.042

1127. Mojica ER, Autschbach J, Bright FV, Aga DS. 2011. Tetracycline speciation during molecular imprinting in xerogels results in class-selective binding. Analyst 136: 749-755. DOI: 10.1039/COAN00707B

1128. Nematollahzadeh A, Sun W, Aureliano CSA, Lütkemeyer D, Stute J, Abdekhodaie MJ, Shojaei A, Sellergren B. 2011. High-Capacity Hierarchically Imprinted Polymer Beads for Protein Recognition and Capture. Angew. Chem. Int. Edit. 50: 495-498. DOI: 10.1002/anie.201004774

1129. Nguyen TH, Ansell RJ. 2011. N-isopropylacrylamide as a functional monomer for noncovalent molecular imprinting. J. Mol. Recognit. 25: 1-10. DOI: $10.1002 / j m r .1163$

1130. Okutucu B, Önal S. 2011. Molecularly imprinted polymers for separation of various sugars from human urine. Talanta 87: 74-79. DOI: 10.1016/j.talanta.2011.09.043

1131. Pan JM, Xu LC, Dai JD, Li XX, Hang H, Huo PW, Li CX, Yan YS. 2011. Magnetic molecularly imprinted polymers based on attapulgite/Fe3O4 particles for the selective recognition of 2,4-dichlorophenol. Chem. Eng. J. 174: 68-75. DOI: 10.1016/j.cej.2011.08.046 
1132. Patachia S, Croitoru C. 2011. Imprinted poly (vinyl alcohol) as a promising tool for xanthine derivatives separation. J. Appl. Polym. Sci. 122: 2081-2089. DOI: 10.1002/app.34305

1133. Patachia S, Croitoru C, Scarneciu I. 2011. Selectivity studies of caffeine molecularly imprinted poly (vinyl alcohol) hydrogels. Environ. Eng. Manag. J. 10: 175-179.

1134. Qiu HM, Luo CN, Fan LL, Lv Z, Lu FG. 2011. Molecularly Imprinted Polymer Prepared by Precipitation Polymerization for Quercetin. In Emerging Focus on Advanced Materials, Advanced Materials Research, Vol. 306-307, Liu SQ, Zuo M (eds). Scientific.net: 646-648. DOI: 10.4028/www.scientific.net/AMR.306-307.646

1135. Schumacher S, Grüneberger F, Katterle M, Hettrich C, Hall DG, Scheller FW, Gajovic-Eichelmann N. 2011. Molecular imprinting of fructose using a polymerizable benzoboroxole: Effective complexation at pH 7.4. Polymer 52: 2485-2491. DOI: 10.1016/j.polymer.2011.04.002

1136. Shah N, Ha J, Ul-Islam M, Park JK. 2011. Highly improved adsorption selectivity of L-phenylalanine imprinted polymeric submicron/nanoscale beads prepared by modified suspension polymerization. Kor. J. Chem. Eng. 28: 1936-1944. DOI: 10.1007/s11814-011-0043-3

1137. Shamsipur M, Besharati-Seidani A. 2011. Synthesis of a novel nanostructured ion-imprinted polymer for very fast and highly selective recognition of copper(II) ions in aqueous media. Reac. Func. Polym. 71: 131-139. DOI: 10.1016/j.reactfunctpolym.2010.11.002

1138. Shan JJ, Wang B. 2011. Preparation and Characterization of a MetalComplexing Imprinted Polymer for Improved Quercetin Recognition. Sep. Sci. Technol. 46: 164-171. DOI: 10.1080/01496391003789189 
1139. Shi XZ, Meng YA, Liu JH, Sun AL, Li DX, Yao CX, Lu Y, Chen J. 2011. Group-selective molecularly imprinted polymer solid-phase extraction for the simultaneous determination of six sulfonamides in aquaculture products. $\mathrm{J}$. Chromatogr B 879: 1071-1076. DOI: 10.1016/j.jchromb.2011.03.019

1140. Sun $Y$, Jin L, Wang H, Yang YJ. 2011. Polymerized organogel particles formed and imprinted by chiral gelators and their selective adsorption for phenylalanine racemates. Soft Matter 7: 348-350. DOI: 10.1039/COSM00460J

1141. Tamahkar E, Bereli N, Say RI, Denizli A. 2011. Molecularly imprinted supermacroporous cryogels for cytochrome c recognition. J. Sep. Sci. 34: 3433-3440. DOI: 10.1002/jssc.201100623

1142. Taranum N, Singh M. 2011. Selective Recognition and Detection of LAspartic Acid by Molecularly Imprinted Polymer in Aqueous Solution. Am. J. Anal. Chem. 2: 909-918. DOI: 10.4236/ajac.2011.28105

1143. Tian M, Bi W, Row KH. 2011. Molecular imprinting in ionic liquid-modified porous polymer for recognitive separation of three tanshinones from Salvia miltiorrhiza Bunge. Anal. Bioanal. Chem. 399: 2495-2502. DOI: $10.1007 / \mathrm{s} 00216-010-4641-4$

1144. Tiwari MP, Madhuri R, Kumar D, Jauhari D, Prasad BB. 2011. Double imprinting in a single molecularly imprinted polymer format for the determination of ascorbic acid and dopamine. Adv. Mat. Lett. 2: 276-280. DOI: 10.5185/amlett.indias.202

1145. Tong YJ, Guan HM, Wang SF, Xu JW, He CB. 2011. Syntheses of chitinbased imprinting polymers and their binding properties for cholesterol. Carbohydr. Res. 346: 495-500. DOI: 10.1016/j.carres.2010.12.013

1146. Walsh R, Osmani Q, Hughes H, Duggan P, McLoughlin P. 2011. Synthesis of imprinted beads by aqueous suspension polymerisation for chiral 
recognition of antihistamines. J. Chromatogr B 879: 3523-3530. DOI: 10.1016/j.jchromb.2011.09.036

1147. Wang CY, Howell M, Raulji P, Davis Y, Mohapatra S. 2011. Preparation and Characterization of Molecularly Imprinted Polymeric Nanoparticles for Atrial Natriuretic Peptide (ANP). Adv. Funct. Mater. 21: 4423-4429. DOI: 10.1002/adfm.201100946

1148. Wang JS, Peng RT, Yang JH, He QH, Liu YJ. 2011. Selective Adsorption of Uranium(VI) on $\mathrm{U}(\mathrm{VI})$ Ion-Imprinted Chitosan Composite Magnetic Microsphers. In Proceedings of the 2011 International Conference on Computer Distributed Control and Intelligent Environmental Monitoring, IEEE computer society: 1714-1717. DOI: 10.1109/CDCIEM.2011.296

1149. Wang P, Fu XF, Li J, Luo J, Zhao XY, Sun MJ, Shang YZ, Ye C. 2011. Preparation of hydrophilic molecularly imprinted polymers for tetracycline antibiotics recognition. Chin. Chem. Lett. 22: 611-614. DOI: 10.1016/j.cclet.2010.12.004

1150. Wang X, Pan JM, Guan W, Zou XH, Huo PW, Yan YS, Hu W. 2011. Selective recognition of sesamol using molecularly imprinted polymers containing magnetic wollastonite. J. Sep. Sci. 34: 3287-3294. DOI: $10.1002 /$ jssc.201100358

1151. Wiklander J, Karlsson BCG, Aastrup T, Nicholls IA. 2011. Towards a synthetic avidin mimic. Anal. Bioanal. Chem. 400: 1397-1404. DOI: $10.1007 / \mathrm{s} 00216-011-4907-5$

1152. Wu HG, Ju XJ, Xie R, Liu YM, Deng JG, Niu CH, Chu LY. 2011. A novel ionimprinted hydrogel for recognition of potassium ions with rapid response. Polym. Adv. Technol. 22: 1389-1394. DOI: 10.1002/pat.1843

1153. Xu WZ, Zhou W, Xu PP, Pan JM, Wu XY, Yan YS. 2011. A molecularly imprinted polymer based on $\mathrm{TiO} 2$ as a sacrificial support for selective 
recognition of dibenzothiophene. Chem. Eng. J. 172: 191-198. DOI: 10.1016/j.cej.2011.05.089

1154. Xu ZX, Song JM, Zhao DY, Zhou J, Qiao XG. 2011. Preparation and Characterization of Hydrophilic Olaquindox Molecularly Imprinted Polymer in Aqueous Environment. Int. J. Polym. Anal. Charact. 16: 67-77. DOI: 10.1080/1023666X.2011.537477

1155. Yang HA, Guo TY, Zhou DZ. 2011. Surface hydrophilic modification with well-defined glycopolymer for protein imprinting matrix. Int. J. Biol. Macromol. 48: 432-438. DOI: 10.1016/j.ijbiomac.2011.01.002

1156. Yang M, Sun Q, Li CY. 2011. Preparation and Characterization of Molecularly Imprinted Polymer Microspheres of $\mathrm{p}$-Nitroaniline. In Frontiers of Green Building, Materials and Civil Engineering, Vol. 71-78, Sun DY, Sung WP, Chen R (eds). Trans Tech Publications: Switzerland; 3411-3414. DOI: 10.4028/www.scientific.net/AMM.71-78.3411

1157. Yang YJ, Li JY, Liu YR, Zhang JY, Li B, Cai XP. 2011. Optimization of polymerization parameters for the sorption of oseltamivir onto molecularly imprinted polymers. Anal. Bioanal. Chem. 400: 3665-3674. DOI: 10.1007/s00216-011-5063-7

1158. Yao CY, Li QQ, Lu YH. 2011. Preparation of MIP Microspheres by Precipitation Polymerization with 1-Phenyl-1-Propanol as Template. In Advanced Materials, Vol. 415-417, Bu JL, Jiang ZY, Jiao SH (eds). Trans Tech Publications: Switzerland; 1225-1230. DOI: 10.4028/www.scientific.net/AMR.415-417.1225

1159. Yin XY, Xu XH, Jiang YF, Luo YM, Luo LY. 2011. The Effect on Adsorption Performance of Molecularly Imprinted Polymer Using the Different Functional Monomers. In Applications of Engineering Materials, Advanced Materials Research, Vol. 287-290, Bu JL, Wang PC, Ai LQ, Sang XM, Li YG 
(eds). Trans Tech Publications: 334-337. DOI: 10.4028/www.scientific.net/AMR.287-290.334

1160. Yu LZ, Yun YB, Zhang WJ, Wang LH. 2011. Preparation, recognition characteristics and properties for quercetin molecularly imprinted polymers. Desal. Water Treat. 34: 309-314.

1161. Yu Z, Zhang X, Huo P, Yan Y. 2011. Selective adsorption copper (II) behavior by surface molecular imprinted polymers. Fresenius Environ. Bull. 20: 3186-3193.

1162. Yuan ZG, Liu YZ, An FQ. 2011. Preparation and phenol-recognizing ability of a poly(methacrylic acid) molecular imprint on the surface of a silica gel. Microchim. Acta 172: 89-94. DOI: 10.1007/s00604-010-0461-7

1163. Zayats M, Kanwar M, Ostermeier M, Searson PC. 2011. Molecular Imprinting of Maltose Binding Protein: Tuning Protein Recognition at the Molecular Level. Macromolecules 44: 3966-3972. DOI: 10.1021/ma200355j

1164. Zhan YC, Luo XB, Nie SS, Huang YN, Tu XM, Luo SL. 2011. Selective Separation of $\mathrm{Cu}(\mathrm{II})$ from Aqueous Solution with a Novel $\mathrm{Cu}(\mathrm{II})$ Surface Magnetic Ion-Imprinted Polymer. Ind. Eng. Chem. Res. 50: 6355-6361. DOI: 10.1021/ie102177e

1165. Zhang SJ, Huang YP, Liu ZS, Duan HQ. 2011. Via protoporphyrin to the synthesis of levofloxacin-imprinted polymer. Polym. Adv. Technol. 22: 286292. DOI: $10.1002 /$ pat.1766

1166. Zhao DY, Jia JF, Yu XL, Sun XJ. 2011. Preparation and characterization of a molecularly imprinted polymer by grafting on silica supports: a selective sorbent for patulin toxin. Anal. Bioanal. Chem. 401: 2259-2273. DOI: 10.1007/s00216-011-5282-y

1167. Zheng X, Ma XL, Xie HF, Li SB, Ren YX, Chen Z. 2011. Imprinted $\alpha$ Cyclodextrin Polymer for Recognition of Naringin. In Materials and Design, 
Advanced Materials Research, Vol. 284-286, Sang XM, Wang PC, Ai LQ, Li YG, Bu JL (eds). Trans Tech Publications: 1850-1853. DOI: 10.4028/www.scientific.net/AMR.284-286.1850

1168. Zheng Y, Ji YB. 2011. Monoliths with Proteins as Chiral Selectors for Enatiomer Separation. In Proceedings of the World Congress on Engineering and Computer Science 2011 Vol II, Ao SI, Douglas C, Grundfest WS, Burgstone J (eds). Newswood Limited: 679-684.

1169. Zhu LM, Fu SP, Li LY, Zhu JB. 2011. Characterization and Evaluation of Binding Properties of Salvianolic Acid a Imprinted Polymers Prepared by Precipitation Polymerization. In Advanced Materials, Advanced Materials Research, Vol. 239 - 242, Cao Z, Cao XQ, Sun LX, He YH (eds). Scientific.net: 2423-2426. DOI: 10.4028/www.scientific.net/AMR.239242.2423

1170. Zhu LY, Zhu ZL, Zhang RH, Hong J, Qiu YL. 2011. Synthesis and adsorption performance of lead ion-imprinted micro-beads with combination of two functional monomers. J. Environ. Sci. 22: 1955-1961. DOI: 10.1016/S1001-0742(10)60611-0

1171. Lorenzo RA, Carro AM, Alvarez-Lorenzo C, Concheiro A. 2011. To Remove or Not to Remove? The Challenge of Extracting the Template to Make the Cavities Available in Molecularly Imprinted Polymers (MIPs). Int. J. Mol. Sci. 12: 4327-4347. DOI: 10.3390/ijms 12074327

1172. Rong F, Li P. 2011. Optimization of S-naproxen imprinted polymers: the combination of theoretical and experimental study. High Perform. Polym. 23: 585-591. DOI: 10.1177/0954008311425973

1173. Rong F, Fu DG, Wu W. 2011. Study on the Effect of Functional Monomer on the Binding Characteristics of Molecularly Imprinted Polymer. In Advanced Materials, Advanced Materials Research, Vol. 239 - 242, Cao Z, Cao XQ, 
Sun LX, He YH (eds). Scientific.net: 821-824. DOI: 10.4028/www.scientific.net/AMR.239-242.821

1174. Rong F, Li P. 2011. Study on the Weakest Interaction Model for Chiral Resolution Using Molecularly Imprinted Polymer. In Chemical Engineering and Material Properties, Advanced Materials Research, Vol. 391-392, Zhang HM, Wu B (eds). Scientific.net: 111-115. DOI: 10.4028/www.scientific.net/AMR.391-392.111

1175. Dourado EMA, Herdes C, Van Tassel PR, Sarkisov L. 2011. Molecular Recognition Effects in Atomistic Models of Imprinted Polymers. Int. J. Mol. Sci. 12: 4781-4804. DOI: 10.3390/ijms12084781

1176. Gong GL, Jia L, Li H, Qi XJ. 2011. Preparation and Characterization of Molecular Imprinted Polymers of Tetracycline Antibiotics by MixedTemplates. In Measuring Technology and Mechatronics Automation IV, Vol. 128-129, Hou ZX (ed). Trans Tech Publications: Switzerland; 407-410. DOI: 10.4028/www.scientific.net/AMM.128-129.407

1177. Bolisay LD, Kofinas P. 2010. Imprinted Polymer Hydrogels for the Separation of Viruses. Macromol. Symp. 291-292: 302-306. DOI: 10.1002/masy.201050535

1178. Hachulka K, Lekka M, Okrajni J, Ambroziak W, Wandelt B. 2010. Polymeric sensing system molecularly imprinted towards enhanced adhesion of Saccharomyces cerevisiae. Biosens. Bioelectron. 26: 50-54. DOI: 10.1016/j.bios.2010.05.009

1179. Lago MA, Grinberg VY, Burova TV, Concheiro A, Alvarez-Lorenzo C. 2011. Ionic and Polyampholyte N-Isopropylacrylamide-Based Hydrogels Prepared in the Presence of Imprinting Ligands: Stimuli-Responsiveness and Adsorption/Release Properties. Journal of Functional Biomaterials 2: 373390. DOI: 10.3390/jfb2040373 
1180. Oral E, Peppas NA. 2006. Hydrophilic molecularly imprinted poly(hydroxyethyl-methacrylate) polymers. J. Biomed. Mater. Res. Part $A$ 78A: 205-210. DOI: 10.1002/jbm.a.30725

1181. Erlenmeyer H, Bartels H. 1964. Über das Problem der Ähnlichkeit in der Chemie. Über spezifisch adsorbierende Silikagele II. Helv. Chim. Acta 47: 1285-1288. DOI: 10.1002/hlca.19640470523

1182. Ou SH, Wu MC, Chou TC, Liu CC. 2004. Polyacrylamide gels with electrostatic functional groups for the molecular imprinting of lysozyme. Anal. Chim. Acta 504: 163-166. DOI: 10.1016/S0003-2670(03)00531-2

1183. Su LQ, Qiao S, Zhang WB. 2007. Studies on the synthesis and properties of malachite green imprinted polymer. Chin. Chem. Lett. 18: 229-232. DOI: 10.1016/j.cclet.2006.12.013

1184. Rampey AM, Umpleby RJ, Rushton GT, Iseman JC, Shah RN, Shimizu KD. 2004. Characterization of the imprint effect and the influence of imprinting conditions on affinity, capacity, and heterogeneity in molecularly imprinted polymers using the Freundlich isotherm-affinity distribution analysis. Anal. Chem. 76: 1123-1133. DOI: 10.1021/ac0345345

1185. Sibrian-Vazquez M, Spivak DA. 2004. Molecular imprinting made easy. J. Am. Chem. Soc. 126: 7827-7833. DOI: 10.1021/ja038961b

1186. Spivak DA, Simon R, Campbell J. 2004. Evidence for shape selectivity in non-covalently imprinted polymers. Anal. Chim. Acta 504: 23-30. DOI: 10.1016/S0003-2670(03)00946-2

1187. Sreenivasan K. 2004. Imparting affinity sites for adenosine triphosphate on the surface of polyurethane through molecular imprinting. J. Appl. Polym. Sci. 94: 2088-2090. DOI: 10.1002/app.21142

1188. Syu MJ, Deng JH, Nian YM. 2004. Towards bilirubin imprinted poly(methacrylic acid-co-ethylene glycol dimethylacrylate) for the specific 
binding of $\alpha$-bilirubin. Anal. Chim. Acta 504: 167-177. DOI: 10.1016/S00032670(03)00879-1

1189. Piletsky SA, Mijangos I, Guerreiro A, Piletska EV, Chianella I, Karim K, Turner APF. 2005. Polymer cookery: Influence of polymerization time and different initiation conditions on performance of molecularly imprinted polymers. Macromolecules 38: 1410-1414. DOI: 10.1021/ma048021r

1190. Shimizu KD. 2005. Molecularly imprinted polymer sensors and sensor arrays. Polym. Prepr. 46: 1132.

1191. Nemoto K, Kubo T, Nomachi M, Sano T, Matsumoto T, Hosoya K, Hattori T, Kaya K. 2007. Simple and Effective 3D Recognition of Domoic Acid Using a Molecularly Imprinted Polymer. J. Am. Chem. Soc. 129: 13626-13632. DOI: $10.1021 / \mathrm{ja} 0741426$

1192. Nemoto K, Kubo T, Nomachi M, Sano T, Matsumoto T, Hosoya K, Hattori T, Kaya K. 2008. Simple and Effective 3D Recognition of Domoic Acid Using a Molecularly Imprinted Polymer [J. Am. Chem. Soc. 2007, 129, 1362613632]. J. Am. Chem. Soc. 130: 774. DOI: 10.1021/ja7106788

1193. Wei HS, Tsai YL, Wu JY, Chen H. 2006. Preparation of inorganic molecularly imprinted polymers with higher adsorption and selectivity by solgel method. J. Chromatogr B 836: 57-62. DOI: 10.1016/j.jchromb.2006.03.047

1194. Luo Y, Liu L, Li LH, Deng QY. 2006. Chromatographic Separation of the Enantiomers of a Series of C2-Asymmetric Bi-Naphthyl Compounds by Molecularly Imprinted Polymers. Chromatographia 64: 393-397. DOI: 10.1365/s10337-006-0017-5

1195. Tsai HA, Syu MJ. 2005. Synthesis of creatinine-imprinted poly $(\beta-$ cyclodextrin) for the specific binding of creatinine. Biomaterials 26: 27592766. DOI: 10.1016/j.biomaterials.2004.07.037 
1196. Bereli N, Andaç M, Baydemir G, Say R, Galaev IY, Denizli A. 2008. Protein recognition via ion-coordinated molecularly imprinted supermacroporous cryogels. J. Chromatogr. A 1190: 18-26. DOI: 10.1016/j.chroma.2008.02.110

1197. Saridakis E, Khurshid S, Govada L, Phan Q, Hawkins D, Crichlow GV, Lolis E, Reddy SM, Chayen NE. 2011. Protein crystallization facilitated by molecularly imprinted polymers. Proc. Natl. Acad. Sci. USA 108: 1108111086. DOI: 10.1073/pnas. 1016539108

1198. Saridakis E, Khurshid S, Govada L, Phan Q, Hawkins D, Crichlow GV, Lolis E, Reddy SM, Chayen NE. 2011. Protein crystallization facilitated by molecularly imprinted polymers (vol 108, pg 11081, 2011). Proc. Natl. Acad. Sci. USA 108: 18566. DOI: 10.1073/pnas.1116413108

1199. Zimmerman SC, Schultz LG, Lemcoff NG. 2003. Monomolecular imprinting: synthetic hosts via molecular imprinting inside of dendrimers. Polym. Prepr. 44: 466-467.

1200. Aburto J, Le Borgne S. 2004. Selective adsorption of dibenzothiophene sulfone by an imprinted and stimuli-responsive chitosan hydrogel. Macromolecules 37: 2938-2943. DOI: 10.1021/ma049961e

1201. Beil JB, Zimmerman SC. 2004. A monomolecularly imprinted dendrimer (MID) capable of selective binding with a tris(2-aminoethyl)amine guest through multiple functional group interactions. Chem. Commun. 488-489. DOI: 10.1039/b316248f

1202. Beil JB, Zimmerman SC. 2004. Synthesis of nanosized "cored" star polymers. Macromolecules 37: 778-787. DOI: 10.1021/ma034556t

1203. Beil JB, Lemcoff NG, Zimmerman SC. 2004. On the nature of dendrimer cross-linking by ring-closing metathesis. J. Am. Chem. Soc. 126: 1357613577. DOI: $10.1021 / \mathrm{ja} 045885 \mathrm{j}$ 
1204. Carter SR, Rimmer S. 2004. Surface molecularly imprinted polymer coreshell particles. Adv. Funct. Mater. 14: 553-561. DOI: 10.1002/adfm.200305069

1205. Cheng GX, Pei GL, Zeng LG, Zhang LY, Liu C. 2004. The surface imprinted polystyrene beads prepared via emulsion templates. Chin. Chem. Lett. 15: 242-245.

1206. Elmer SL, Zimmerman SC. 2004. Cross-linking dendrimers with allyl ether end-groups using the ring-closing metathesis reaction. J. Org. Chem. 69: 7363-7366. DOI: 10.1021/j0049368v

1207. Fujiwara M, Nishiyama M, Yamamura I, Ohtsuki S, Nomura R. 2004. A solgel method using acetic anhydride in the presence of cholesterol in organic solution media: Preparation of silicas that recognize steroid hormones. Anal. Chem. 76: 2374-2381. DOI: 10.1021/ac035173w

1208. Guo TY, Xia YQ, Hao GJ, Song MD, Zhang BH. 2004. Adsorptive separation of hemoglobin by molecularly imprinted chitosan beads. Biomaterials 25: 5905-5912. DOI: 10.1016/j.biomaterials.2004.01.032

1209. Hsu HC, Chen LC, Ho KC. 2004. Colorimetric detection of morphine in a molecularly imprinted polymer using an aqueous mixture of $\mathrm{Fe}^{3+}$ and $[\mathrm{Fe}(\mathrm{CN})(6)]^{3-}$. Anal. Chim. Acta 504: 141-147. DOI: 10.1016/j.aca.2003.11.021

1210. Jiang $Y$, Tong AJ. 2004. Synthesis of molecularly imprinted microspheres for recognition of trans-aconitic acid. J. Appl. Polym. Sci. 94: 542-547. DOI: 10.1002/app.20954

1211. Kanazawa R, Mori K, Tokuyama H, Sakohara S. 2004. Preparation of thermosensitive microgel adsorbent for quick adsorption of heavy metal ions by a temperature change. J. Chem. Eng. Jpn. 37: 804-807. DOI: 10.1252/jcej.37.804 
1212. Katada N, Akazawa S, Niwa M. 2004. Improvement of selectivity in specific adsorption by the addition of acetic acid during the CVD of silicon alkoxide to form a silica overlayer with a molecular sieving property. Chem. Vap. Deposition 10: 103-107. DOI: 10.1002/cvde.200306278

1213. Kirsch N, Alexander C, Davies S, Whitcombe MJ. 2004. Sacrificial spacer and non-covalent routes toward the molecular imprinting of "poorlyfunctionalized" N-heterocycles. Anal. Chim. Acta 504: 63-71. DOI: $10.1016 /$ S0003-2670(03)00510-5

1214. Kornysova O, Jarmalaviciene R, Marsuka A. 2004. A simplified synthesis of polymeric nonparticulate stationary phases with macrocyclic antibiotic as chiral selector for capillary electrochromatography. Electrophoresis 25: 2825-2829. DOI: 10.1002/elps.200405933

1215. Kunitake T, Lee SW. 2004. Molecular imprinting in ultrathin titania gel films via surface sol-gel process. Anal. Chim. Acta 504: 1-6. DOI: 10.1016/S00032670(03)00811-0

1216. Lemcoff NG, Spurlin TA, Gewirth AA, Zimmerman SC, Beil JB, Elmer SL, Vandeveer HG. 2004. Organic nanoparticles whose size and rigidity are finely tuned by cross-linking the end groups of dendrimers. J. Am. Chem. Soc. 126: 11420-11421. DOI: 10.1021/ja047055b

1217. Li ZJ, Jaroniec M. 2004. Mesoporous carbons synthesized by imprinting ordered and disordered porous structures of silica particles in mesophase pitch. J. Phys. Chem. B 108: 824-826. DOI: 10.1021/jp0368233

1218. Liu XY, Ding XB, Guan $Y$, Peng $Y X$, Long XP, Wang XC, Chang K, Zhang Y. 2004. Fabrication of temperature-sensitive imprinted polymer hydrogel. Macromol. Biosci. 4: 412-415. DOI: 10.1002/mabi.200300057

1219. Liu XY, Guan Y, Ding XB, Peng YX, Long XP, Wang XC, Chang K. 2004. Design of temperature sensitive imprinted polymer hydrogels based on 
multiple-point hydrogen bonding. Macromol. Biosci. 4: 680-684. DOI: 10.1002/mabi.200400031

1220. Maier P, Werner-Allen J, Gibson UJ, Richter A, BelBruno JJ. 2004. Scanning force microscopy study of the morphology of spin-cast molecularimprinted nylon thin films. Surf. Interf. Sci. 36: 1340-1343. DOI: 10.1002/sia.1928

1221. Marty JD, Labadie L, Mauzac M, Fournier C, Rico-Lattes I, Lattes A. 2004. Liquid crystalline networks: Potential uses in molecular imprinting technique. Mol. Cryst. Liq. Cryst. 411: 561-568. DOI: 10.1080/15421400490436520

1222. Marx S, Zaltsman A, Turyan I, Mandler D. 2004. Parathion sensor based on molecularly imprinted sol-gel films. Anal. Chem. 76: 120-126. DOI: $10.1021 /$ ac034531s

1223. Matsui J, Minamimura N, Nishimoto K, Tamaki K, Sugimoto N. 2004. Synthetic cinchonidine receptors obtained by cross-linking linear poly(methacrylic acid) derivatives as an alternative molecular imprinting technique. J. Chromatogr. B 804: 223-229. DOI: 10.1016/j.jchromb.2004.01.063

1224. Mertz E, Elmer SL, Balija AM, Zimmerman SC. 2004. Integrating chemosensors for amine-containing compounds into cross-linked dendritic hosts. Tetrahedron 60: 11191-11204. DOI: 10.1016/j.tet.2004.08.100

1225. Oral E, Peppas NA. 2004. Responsive and recognitive hydrogels using star polymers. J. Biomed. Mater. Res. Part A 68A: 439-447. DOI: 10.1002/jbm.a.20076

1226. Oya T, Nakano Y. 2004. Creation of hetero polymer gels with function of memorizing conformation. Asian Pacific Confederation of Chemical Engineering congress program and abstracts, Vol. 2004, The Society of Chemical Engineers, Japan: 671-678. 
1227. Park JK, Khan H, Lee JW. 2004. Preparation of phenylalanine imprinted polymer by the sol-gel transition method. Enzyme Microb. Technol. 35: 688693. DOI: 10.1016/j.enzmictec.2004.08.023

1228. Pérez-Moral N, Mayes AG. 2004. Noncovalent imprinting in the shell of core-shell nanoparticles. Langmuir 20: 3775-3779. DOI: 10.1021/la0356755

1229. Petcu M, Schaare PN, Cook CJ. 2004. Propofol-imprinted membranes with potential applications in biosensors. Anal. Chim. Acta 504: 73-79. DOI: 10.1016/S0003-2670(03)00673-1

1230. Puoci F, lemma F, Muzzalupo R, Spizzirri UG, Trombino S, Cassano R, Picci N. 2004. Spherical molecularly imprinted polymers (SMIPs) via a novel precipitation polymerization in the controlled delivery of sulfasalazine. Macromol. Biosci. 4: 22-26. DOI: 10.1002/mabi.200300035

1231. Roberts MJ, Johnson SK. 2004. Nanorods produced on the surface of thin films of TiOx by surface-imprinting. In Molecularly Imprinted Materials-2003, MRS Symposium Proceedings, Vol. 787, Kofinas P, Sellergren B, Roberts MJ (eds). Materials Research Society: Warrendale; 47-51. DOI: 10.1557/PROC-787-G3.8

1232. Say R, Ersöz A, Sener I, Atilir A, Diltemiz S, Denizli A. 2004. Comparison of adsorption and selectivity characteristics for 4-nitrophenol imprinted polymers prepared via bulk and suspension polymerization. Sep. Sci. Technol. 39: 3471-3484. DOI: 10.1081/SS-200028939

1233. Schmidt RH, Mosbach K, Haupt K. 2004. A simple method for spin-coating molecularly imprinted polymer films of controlled thickness and porosity. Adv. Mater. 16: 719-722. DOI: 10.1002/adma.200306374

1234. Titirici MM, Sellergren B. 2004. Peptide recognition via hierarchical imprinting. Anal. Bioanal. Chem. 378: 1913-1921. DOI: 10.1007/s00216003-2445-5 
1235. Tokuyama H, Kanazawa R, Sakohara S. 2004. Adsorption/desorption of target metal on molecular imprinted thermosensitive gel adsorbent: equilibrium isotherms and kinetics behavior. Asian Pacific Confederation of Chemical Engineering congress program and abstracts, Vol. 2004, The Society of Chemical Engineers, Japan: 670-677.

1236. Watabe $\mathrm{Y}$, Kondo $\mathrm{T}$, Imai H, Morita M, Tanaka N, Haginaka J, Hosoya K. 2004. Improved detectability with a polymer-based trapping device in rapid HPLC analysis for ultra-low levels of bisphenol A (BPA) in environmental samples. Anal. Sci. 20: 133-137. DOI: 10.2116/analsci.20.133

1237. Xi FN, Wu JM. 2004. Macroporous chitosan layer coated on non-porous silica gel as a support for metal chelate affinity chromatographic adsorbent. J. Chromatogr. A 1057: 41-47. DOI: 10.1016/j.chroma.2004.09.059

1238. Ye L. 2004. Molecularly imprinted micro- and nano-particles by precipitation polymerization. In Molecularly Imprinted Materials-2003, MRS Symposium Proceedings, Vol. 787, Kofinas P, Sellergren B, Roberts MJ (eds). Materials Research Society: Warrendale; 97-102. DOI: 10.1557/PROC-787-G7.3

1239. Zhang YH, Tong AJ, Li LD. 2004. Synthesis of molecularly imprinted polymer with 7-chloroethyl-theophylline-immobilized silica gel as template and its molecular recognition function. Spectrochim. Acta, A 60: 241-244. DOI: 10.1016/S1386-1425(03)00225-7

1240. Boonpangrak S, Prachayasittikul V, Bülow L, Ye L. 2005. Molecularly imprinted polymer microspheres prepared by precipitation polymerization using a sacrificial covalent bond. Vol. Proceedings of the 31 st Congress on Science and Technology of Thailand at Suranaree University of Technology, Art. No. E0117.

1241. Carter SR, Rimmer S. 2005. Aqueous compatible polymers in bionanotechnology. IEE Proc. -Nanobiotechnol. 152: 169-176. DOI: 10.1049/ip-nbt:20050007 
1242. Chen WC, Lin HY, Chou TC. 2005. Design of creatinine-imprinted polymers. In Proceedings of the IEEE 31St Annual Northeast Bioengineering Conference, 2005, IEEE: New York; 38-39. DOI: 10.1109/NEBC.2005.1431915

1243. Chen ZY, Zhao R, Shangguan DH, Liu GQ. 2005. Preparation and evaluation of uniform-sized molecularly imprinted polymer beads used for the separation of sulfamethazine. Biomed. Chromatogr. 19: 533-538. DOI: $10.1002 / \mathrm{bmc} .476$

1244. Chou PC, Rick J, Chou TC. 2005. C-reactive protein thin-film molecularly imprinted polymers formed using a micro-contact approach. Anal. Chim. Acta 542: 20-25. DOI: 10.1016/j.aca.2004.12.074

1245. Clavier CW, Rodman DL, Sinski JF, Allain LR, Im HJ, Yang Y, Clark JC, Xue ZL. 2005. A method for the preparation of transparent mesoporous silica solgel monoliths containing grafted organic functional groups. J. Mater. Chem. 15: 2356-2361. DOI: 10.1039/b417014h

1246. Cummins W, Duggan P, McLoughlin P. 2005. A comparative study of the potential of acrylic and sol-gel polymers for molecular imprinting. Anal. Chim. Acta 542: 52-60. DOI: 10.1016/j.aca.2005.01.042

1247. Daniel S, Rao PP, Rao TP. 2005. Investigation of different polymerization methods on the analytical performance of palladium(II) ion imprinted polymer materials. Anal. Chim. Acta 536: 197-206. DOI: 10.1016/j.aca.2004.12.052

1248. Demirel G, Özçetin G, Turan E, Çaykara T. 2005. pH/Temperature Sensitive Imprinted lonic Poly(N-tert-butylacrylamide-co-acrylamide/maleic acid) Hydrogels for Bovine Serum Albumin. Macromol. Biosci. 5: 1032-1037. DOI: 10.1002/mabi.200500085 
1249. Donato L, Figoli A, Drioli E. 2005. Novel composite poly(4vinylpyridine)/polypropylene membranes with recognition properties for (S)naproxen. J. Pharm. Biomed. Anal. 37: 1003-1008. DOI: 10.1016/j.jpba.2004.09.020

1250. Egawa Y, Shimura Y, Nowatari Y, Aiba D, Juni K. 2005. Preparation of molecularly imprinted cyclodextrin microspheres. Int. J. Pharm. 293: 165170. DOI: 10.1016/j.jpharm.2004.12.020

1251. Farnik D, Binder WH, Hüsing N. 2005. Novel carbohydrate-based surfactants for the preparation of imprinted silica. Polym. Prepr. 46: 11401141.

1252. Fireman-Shoresh S, Popov I, Avnir D, Marx S. 2005. Enantioselective, chirally templated sol-gel thin films. J. Am. Chem. Soc. 127: 2650-2655. DOI: $10.1021 / \mathrm{ja} 0454384$

1253. Fish WP, Ferreira J, Sheardy RD, Snow NH, O'Brien TP. 2005. Rational design of an imprinted polymer: Maximizing selectivity by optimizing the monomer-template ratio for a cinchonidine MIP, prior to polymerization, using microcalorimetry. J. Liq. Chrom. Rel. Technol. 28: 1-15. DOI: 10.1081/JLC-200038551

1254. Ghosh S, Ramakrishnan S. 2005. Small-molecule-induced folding of a synthetic polymer. Angew. Chem. Int. Edit. 44: 5441-5447. DOI: 10.1002/anie.200501448

1255. Gill RS, Marquez M, Larsen G. 2005. Molecular imprinting of a cellulose/silica composite with caffeine and its characterization. Microporous Mesoporous Mater. 85: 129-135. DOI: 10.1016/j.micromeso.2005.06.003

1256. Goto H, Nomura N, Akagi K. 2005. Electrochemical polymerization of 3,4ethylenedioxythiophene in a DNA liquid-crystal electrolyte. J. Polym. Sci. A,Polym. Chem. 43: 4298-4302. DOI: 10.1002/pola.20909 
1257. Guo TY, Zhang LY, Hao GJ, Song MD, Zhang BH. 2005. Preparation and properties of uniform-sized polymer beads imprinted with N-CBZ-Lphenylalanine. Int. J. Polym. Mater. 54: 743-755. DOI: $10.1080 / 00914030490463124$

1258. Guo TY, Xia YQ, Hao GJ, Zhang BH, Fu GQ, Yuan Z, He BL, Kennedy JF. 2005. Chemically modified chitosan beads as matrices for adsorptive separation of proteins by molecularly imprinted polymer. Carbohydr. Polym. 62: 214-221. DOI: 10.1016/j.carbpol.2005.03.012

1259. Hawkins DM, Stevenson D, Reddy SM. 2005. Investigation of protein imprinting in hydrogel-based molecularly imprinted polymers (HydroMIPs). Anal. Chim. Acta 542: 61-65. DOI: 10.1016/j.aca.2005.01.052

1260. Herold M, Tovar GEM, Gruber C, Dettling M, Sezgin S, Brunner H. 2005. Molecular recognition by imprinted polymer nanospheres - fundamental research and applications. Polym. Prepr. 46: 1125-1126.

1261. Hirsch T, Zharnikov M, Shaporenko A, Stahl J, Weiss D, Wolfbeis OS, Mirsky VM. 2005. Size-controlled electrochemical synthesis of metal nanoparticles on monomolecular templates. Angew. Chem. Int. Edit. 44: 6775-6778. DOI: 10.1002/anie.200500912

1262. Katada N, Akazawa S, Nishiaki N, Yano Y, Yamakita S, Hayashi K, Niwa M. 2005. Formation of selective adsorption cavity by chemical vapor deposition of molecular sieving silica overlayer on alumina using molecular template in the presence of acetic acid. Bull. Chem. Soc. Jpn. 78: 1001-1007. DOI: $10.1246 / \mathrm{bcsj} .78 .1001$

1263. Kim H, Guiochon G. 2005. Thermodynamic functions and intraparticle mass transfer kinetics of structural analogues of a template on molecularly imprinted polymers in liquid chromatography. J. Chromatogr. A 1097: 84-97. DOI: 10.1016/j.chroma.2005.08.020 
1264. Kim JS, Kim HC, Lee B, Ree M. 2005. Imprinting of nanopores in organosilicate dielectric thin films with hyperbranched ketalized polyglycidol. Polymer 46: 7394-7402. DOI: 10.1016/j.polymer.2005.06.024

1265. Kim KS, Lee JH, Kim MH, Cho SH. 2005. Preparation and characterization of molecularly imprinted uniform-sized Poly(4VP-co-EGDMA) microgels. Polym. J. 37: 669-676. DOI: 10.1295/polymj.37.669

1266. Kim TH, Do Ki C, Cho H, Chang TY, Chang JY. 2005. Facile preparation of core-shell type molecularly imprinted particles: Molecular imprinting into aromatic polyimide coated on silica spheres. Macromolecules 38: 64236428. DOI: $10.1021 / \mathrm{ma} 0502708$

1267. Koenig S, Chechik V. 2005. Au nanoparticle-imprinted polymers. Chem. Commun. 4110-4112. DOI: 10.1039/b503243a

1268. Kubo T, Hosoya K, Nomachi M, Tanaka N, Kaya K. 2005. Preparation of a novel molecularly imprinted polymer using a water-soluble crosslinking agent. Anal. Bioanal. Chem. 382: 1698-1701. DOI: 10.1007/s00216-005$3341-y$

1269. Lauceri R, Purrello R. 2005. Transfer, memory and amplification of chirality in porphyrin aggregates. Supramol. Chem. 17: 61-66. DOI: 10.1080/10610270412331328934

1270. Lee B, Oh W, Yoon J, Hwang Y, Kim J, Landes BG, Quintana JP, Ree M. 2005. Scattering studies of nanoporous organosilicate thin films imprinted with reactive star porogens. Macromolecules 38: 8991-8995. DOI: $10.1021 / \mathrm{ma} 0501951$

1271. Lee WS, Takeuchi T. 2005. Bisphenol A analog-imprinted polymers prepared by an immobilized template on a modified silica microsphere matrix. Anal. Sci. 21: 1125-1128. DOI: 10.2116/analsci.21.1125 
1272. Lei JD, Tong AJ. 2005. Preparation of Z-I-Phe-OH-NBD imprinted microchannel and its molecular recognition study. Spectrochim. Acta, A 61: 1029-1033. DOI: 10.1016/j.saa.2004.06.001

1273. Li Z, Day M, Ding JF, Faid K. 2005. Synthesis and characterization of functional methacrylate copolymers and their application in molecular imprinting. Macromolecules 38: 2620-2625. DOI: 10.1021/ma0478308

1274. Liang C, Fréchet JMJ. 2005. Applying key concepts from nature: transition state stabilization, pre-concentration and cooperativity effects in dendritic biomimetics. Prog. Polym. Sci. 30: 385-402. DOI: 10.1016/j.progpolymsci.2005.01.004

1275. Lieberzeit PA, Gazda-Miarecka S, Halikias K, Schirk C, Kauling J, Dickert FL. 2005. Imprinting as a versatile platform for sensitive materials nanopatterning of the polymer bulk and surfaces. Sens. Actuators $B$ 111112: 259-263. DOI: 10.1016/j.snb.2004.12.064

1276. Lin HY, Lin CP, Lee GB, Chou TC. 2005. The recognition of lysozyme by patterned molecularly imprinted polymers. In Proceedings of the IEEE 31St Annual Northeast Bioengineering Conference, 2005, IEEE: New York; 4041. DOI: $10.1109 /$ NEBC.2005.1431916

1277. Ling TR, Syu YZ, Tasi YC, Chou TC, Liu CC. 2005. Size-selective recognition of catecholamines by molecular imprinting on silica-alumina gel. Biosens. Bioelectron. 21: 901-907. DOI: 10.1016/j.bios.2005.02.009

1278. Marty JD, Mauzac M, Lavabre D. 2005. High capacity molecular imprinted mesomorphous networks usable as antibody mimics. Mol. Cryst. Liq. Cryst. 437: 1307-1314. DOI: 10.1080/15421400590956090

1279. Marty JD, Mauzac M, Gornitzka H. 2005. Chiral molecular imprinting in liquid-crystalline network. Eur. Phys. J. E: Soft Matter Biol. Phys. 17: 515520. DOI: 10.1140/epje/i2004-10156-3 
1280. Maury P, Escalante M, Reinhoudt DN, Huskens J. 2005. Directed assembly of nanoparticles onto polymer-imprinted or chemically patterned templates fabricated by nanoimprint lithography. Adv. Mater. 17: 2718-2723. DOI: 10.1002/adma.200501072

1281. Pang XS, Cheng GX, Li RS, Lu SL, Zhang YH. 2005. Bovine serum albumin-imprinted polyacrylamide gel beads prepared via inverse-phase seed suspension polymerization. Anal. Chim. Acta 550: 13-17. DOI: 10.1016/j.aca.2005.06.067

1282. Poovarodom S, Bass JD, Hwang SJ, Katz A. 2005. Investigation of the coreshell interface in gold@silica nanoparticles: A silica imprinting approach. Langmuir 21: 12348-12356. DOI: 10.1021/la052006d

1283. Rathbone DL, Bains A. 2005. Tools for fluorescent molecularly imprinted polymers. Biosens. Bioelectron. 20: 1438-1442. DOI: 10.1016/j.bios.2004.05.021

1284. Rick J, Chou TC. 2005. Imprinting unique motifs formed from protein-protein associations. Anal. Chim. Acta 542: 26-31. DOI: 10.1016/j.aca.2004.12.051

1285. Rick J, Chou TC. 2005. Enthalpy changes associated with protein binding to thin films. Biosens. Bioelectron. 20: 1878-1883. DOI: 10.1016/j.bios.2004.11.015

1286. Roy S, Claverie J, Barasc M, Ogier J, Durant Y. 2005. Molecularly imprinted nanoparticles. Polym. Prepr. 46: 1130-1131.

1287. Rückert B, Kolb U. 2005. Distribution of molecularly imprinted polymer layers on macroporous silica gel particles by STEM and EDX. Micron 36: 247-260. DOI: 10.1016/j.micron.2004.11.003

1288. Rushton GT, Furmanski B, Shimizu KD. 2005. Plastic antibodies: Molecular recognition with imprinted polymers - An introductory polymer chemistry laboratory investigation. J. Chem. Educ. 82: 1374-1377. 
1289. Schmidt RH, Haupt K. 2005. Molecularly imprinted polymer films with binding properties enhanced by the reaction-induced phase separation of a sacrificial polymeric porogen. Chem. Mater. 17: 1007-1016. DOI: $10.1021 / \mathrm{cm} 048392 \mathrm{~m}$

1290. Schmidt RH, Belmont AS, Haupt K. 2005. Porogen formulations for obtaining molecularly imprinted polymers with optimized binding properties. Anal. Chim. Acta 542: 118-124. DOI: 10.1016/j.aca.2005.03.064

1291. Schneider F, Piletsky S, Piletska E, Guerreiro A, Ulbricht M. 2005. Comparison of thin-layer and bulk MIPs synthesized by photoinitiated in situ crosslinking polymerization from the same reaction mixtures. J. Appl. Polym. Sci. 98: 362-372. DOI: 10.1002/app.22112

1292. Shiomi T, Matsui M, Mizukami F, Sakaguchi K. 2005. A method for the molecular imprinting of hemoglobin on silica surfaces using silanes. Biomaterials 26: 5564-5571. DOI: 10.1016/j.biomaterials.2005.02.007

1293. Spivak DA, Sibrian-Vazquez M, Houck S. 2005. Development of one monomer molecularly imprinted polymers (OmniMIPs). Polym. Prepr. 46: 1103-1104.

1294. Spizzirri UG, Peppas NA. 2005. Structural analysis and diffusional behavior of molecularly imprinted polymer networks for cholesterol recognition. Chem. Mater. 17: 6719-6727. DOI: 10.1021/cm0478531

1295. Stancil KA, Feld MS, Kardar M. 2005. Correlation and cross-linking effects in imprinting sites for divalent adsorption in gels. J. Phys. Chem. B 109: 66366639. DOI: $10.1021 / \mathrm{jp} 045858 f$

1296. Tan G, Singh M, He J, John VT, McPherson GL. 2005. Use of a selfassembling organogel as a reverse template in the preparation of imprinted porous polymer films. Langmuir 21: 9322-9326. DOI: 10.1021/la051080t 
1297. Tokuyama H, Fujioka M, Sakohara S. 2005. Development and performance of a novel molecular imprinted thermosensitive gel with a cross-linked chelating group for the temperature swing adsorption of a target metal. $J$. Chem. Eng. Jpn. 38: 633-640. DOI: 10.1252/jcej.38.633

1298. Ulbricht M, Malaisamy R. 2005. Insights into the mechanism of molecular imprinting by immersion precipitation phase inversion of polymer blends via a detailed morphology analysis of porous membranes. J. Mater. Chem. 15: 1487-1497. DOI: 10.1039/b416186f

1299. Von Werne TA, Beinhoff M, Hagberg EC, Jhaveri SB, Sogah DY, Hawker CJ, Carter KR. 2005. Patterned polymer brushes from molded polymer surfaces. Polym. Prepr. 46: 46-47.

1300. Voshell SM, Gagné MR. 2005. Rigidified dendritic structures for imprinting chiral information. Organometallics 24: 6338-6350. DOI: 10.1021/om050647y

1301. Wang HY, Jiang JG, Ma LY, Pang YL. 2005. Syntheses of molecularly imprinted polymers and their molecular recognition study for doxazosin mesylate. Reac. Func. Polym. 64: 119-126. DOI: 10.1016/j.reactfunctpolym.2005.05.010

1302. Wang SF, Xu JW, Tong YJ, Wang L, He CB. 2005. Cholesterol-imprinted polymer receptor prepared by a hybrid imprinting method. Polym. Int. 54: 1268-1274. DOI: 10.1002/pi.1841

1303. Watabe Y, Hosoya K, Tanaka N, Kubo T, Kondo T, Morita M. 2005. Shielded molecularly imprinted polymers prepared with a selective surface modification. J. Polym. Sci. A,Polym. Chem. 43: 2048-2060. DOI: 10.1002/pola.20674 
1304. Wei XL, Li X, Husson SM. 2005. Surface molecular imprinting by atom transfer radical polymerization. Biomacromolecules 6: 1113-1121. DOI: 10.1021/bm049311i

1305. Wei XL, Samadi A, Husson SM. 2005. Synthesis and characterization of molecularly imprinted polymers for chromatographic separations. Sep. Sci. Technol. 40: 109-129. DOI: 10.1081/SS-200041880

1306. Xi FN, Wu JM, Luan MM. 2005. Silica-supported macroporous chitosan bead for affinity purification of trypsin inhibitor. Chin. Chem. Lett. 16: 10891092.

1307. Xia YQ, Guo TY, Song MD, Zhang BH, Zhang BL. 2005. Hemoglobin recognition by imprinting in semi-interpenetrating polymer network hydrogel based on polyacrylamide and chitosan. Biomacromolecules 6: 2601-2606. DOI: $10.1021 / \mathrm{bm} 050324 \mathrm{I}$

1308. Yamakita S, Katada N, Niwa M. 2005. Shape-selective adsorption of substituted benzaldehyde isomers by a molecular sieving silica overlayer prepared by the chemical vapor deposition method using organic template on tin oxide. Bull. Chem. Soc. Jpn. 78: 1425-1430. DOI: $10.1246 /$ bcsj.78.1425

1309. Yan H, Jin L, Row KH. 2005. Special selectivity of molecularly imprinted monolithic stationary phase. J. Liq. Chrom. Rel. Technol. 28: 3147-3155. DOI: $10.1080 / 10826070500330661$

1310. Yang $\mathrm{DH}$, Lee SW, Kunitake T. 2005. Facile fabrication of molecularly imprinted cavities in spin-coated $\mathrm{TiO}_{2}$ nanofilms. Chem. Lett. 34: 1686-1687. DOI: 10.1246/cl.2005.1686

1311. Yang $H$, Lazos D, Ulbricht M. 2005. Thin, highly crosslinked polymer layer synthesized via photoinitiated graft copolymerization on a self-assembled- 
monolayer-coated gold surface. J. Appl. Polym. Sci. 97: 158-164. DOI: 10.1002/app.21621

1312. Yang $H H$, Zhang SQ, Tan F, Zhuang ZX, Wang XR. 2005. Surface molecularly imprinted nanowires for biorecognition. J. Am. Chem. Soc. 127: 1378-1379. DOI: 10.1021/ja0467622

1313. Yang KG, Liu ZB, Mao M, Zhang XH, Zhao CS, Nishi N. 2005. Molecularly imprinted polyethersulfone microspheres for the binding and recognition of bisphenol A. Anal. Chim. Acta 546: 30-36. DOI: 10.1016/j.aca.2005.05.008

1314. Yoshikawa M, Guiver MD, Robertson GP. 2005. Molecularly imprinted films derived from Torlon ${ }^{\mathrm{TM}}$ polyamide-imide. J. Mol. Struc. 739: 41-46. DOI: 10.1016/j.molstruc.2004.04.031

1315. Zdyrko B, Hoy O, Luzinov I. 2005. Protein imprinting via solvent assisted grafting of polymer brush. Polym. Prepr. 46: 76-77.

1316. Advincula R, Lemons J, Bellis S, Advincula M. 2006. Bioactive surfaces derived from nanostructured hybrid polymer-sol-gel titanium oxide coatings. Polym. Prepr. 47: 11-12.

1317. Bass JD, Katz A. 2006. Bifunctional surface imprinting of silica: thermolytic synthesis and characterization of discrete thiol-amine functional group pairs. Chem. Mater. 18: 1611-1620. DOI: 10.1021/cm052382j

1318. Booker K, Bowyer MC, Holdsworth Cl, McCluskey A. 2006. Efficient preparation and improved sensitivity of molecularly imprinted polymers using room temperature ionic liquids. Chem. Commun. 1730-1732. DOI: 10.1039/b517886j

1319. Boonpangrak S, Prachayasittikul V, Bülow L, Ye L. 2006. Molecularly imprinted polymer microspheres prepared by precipitation polymerization using a sacrificial covalent bond. J. Appl. Polym. Sci. 99: 1390-1398. DOI: 10.1002/app.22519 
1320. Boonpangrak S, Whitcombe MJ, Prachayasittikul V, Mosbach K, Ye L. 2006. Preparation of molecularly imprinted polymers using nitroxide-mediated living radical polymerization. Biosens. Bioelectron. 22: 349-354. DOI: 10.1016/j.bios.2006.04.014

1321. Che AF, Yang YF, Wan LS, Wu J, Xu ZK. 2006. Molecular imprinting fibrous membranes of poly(acrylonitrile-co-acrylic acid) prepared by electrospinning. Chem. Res. Chin. Univ. 22: 390-393. DOI: 10.1016/S1005-9040(06)60124-4

1322. Chronakis IS, Milosevic B, Frenot A, Ye L. 2006. Generation of molecular recognition sites in electrospun polymer nanofibers via molecular imprinting. Macromolecules 39: 357-361. DOI: 10.1021/ma052091w

1323. Ciardelli G, Borrelli C, Silvestri D, Cristallini C, Barbani N, Giusti P. 2006. Supported imprinted nanospheres for the selective recognition of cholesterol. Biosens. Bioelectron. 21: 2329-2338. DOI: 10.1016/j.bios.2005.12.027

1324. Courtois J, Fischer G, Sellergren B, Irgum K. 2006. Molecularly imprinted polymers grafted to flow through poly(trimethylolpropane trimethacrylate) monoliths for capillary-based solid-phase extraction. J. Chromatogr. A 1109: 92-99. DOI: 10.1016/j.chroma.2005.12.014

1325. Cummins W, Duggan P, McLoughlin P. 2006. Systematic cross-selectivity study of the factors influencing template receptor interactions in molecularly imprinted nitrogen heterocycles. Biosens. Bioelectron. 22: 372-380. DOI: 10.1016/j.bios.2006.05.003

1326. Defreese JL, Hwang SJ, Parra-Vasquez ANG, Katz A. 2006. Molecular motion of tethered molecules in bulk and surface-functionalized materials: A comparative study of confinement. J. Am. Chem. Soc. 128: 5687-5694. DOI: 10.1021/ja0556474 
1327. Dhruv H, Pepalla R, Taveras M, Britt DW. 2006. Protein insertion and patterning of PEG-bearing Langmuir monolayers. Biotechnol. Prog. 22: 150155. DOI: 10.1021/bp050173y

1328. Du ZX, Liu H, Fu ZF, Yang WT. 2006. Molecularly imprinted polymers on chloromethyl polystyrene resin prepared via RAFT polymerization. Chin. Chem. Lett. 17: 549-552.

1329. Enholm EJ, Allais F, Martin RT, Mohamed R. 2006. A Comparison of a Radical Polymerization vs ROMP Matrix for Molecular Imprinting. Macromolecules 39: 7859-7862. DOI: 10.1021/ma061429z

1330. Gruber-Traub C, Weber A, Dettling M, Herz M, Herold M, Brunner H, Tovar GEM. 2006. NANOCYTES ${ }^{\mathrm{TM}}$ - inverse miniemulsion polymerization technology for specific protein recognition. Polym. Prepr. 47: 901-902.

1331. Guo MJ, Zhao Z, Fan YG, Wang CH, Shi LQ, Xia JJ, Long Y, Mi HF. 2006. Protein-imprinted polymer with immobilized assistant recognition polymer chains. Biomaterials 27: 4381-4387. DOI: 10.1016/j.biomaterials.2006.04.002

1332. He CY, Liu F, Li K, Liu HW. 2006. Molecularly imprinted polymer film grafted from porous silica for selective recognition of testosterone. Anal. Lett. 39: 275-286. DOI: 10.1080/00032710500476946

1333. Herold M, Müller E, Dettling M, Weber A, Brunner H, Tovar GEM. 2006. A detailed investigation of the co-polymerization kinetics and particle formation of nanoscopical poly(EGDMA-co-MAA) by miniemulsion polymerization. Polym. Prepr. 47: 835-836.

1334. Hicks JC, Dabestani R, Buchanan AC, Jones CW. 2006. Spacing and Site Isolation of Amine Groups in 3-Aminopropyl-Grafted Silica Materials: The Role of Protecting Groups. Chem. Mater. 18: 5022-5032. DOI: $10.1021 / \mathrm{cm} 061382 \mathrm{w}$ 
1335. Hilt JZ, Byrne ME, Peppas NA. 2006. Microfabrication of Intelligent Biomimetic Networks for Recognition of D-Glucose. Chem. Mater. 18: 58695875. DOI: $10.1021 / \mathrm{cm} 061343 \mathrm{k}$

1336. Ikawa T, Hoshino F, Matsuyama T, Takahashi H, Watanabe O. 2006. Molecular-shape imprinting and immobilization of biomolecules on a polymer containing azo dye. Langmuir 22: 2747-2753. DOI: 10.1021/la0528394

1337. Ishida Y, Amano S, Iwahashi N, Saigo K. 2006. Switching of Structural Order in a Cross-Linked Polymer Triggered by the Desorption/Adsorption of Guest Molecules. J. Am. Chem. Soc. 128: 13068-13069. DOI: $10.1021 / \mathrm{ja} 064969 \mathrm{k}$

1338. Kempe H, Kempe M. 2006. Development and evaluation of spherical molecularly imprinted polymer beads. Anal. Chem. 78: 3659-3666. DOI: 10.1021/ac060068i

1339. Khan H, Park JK. 2006. The preparation of D-phenylalanine imprinted microbeads by a novel method of modified suspension polymerization. Biotechnol. Bioprocess Eng. 11: 503-509. DOI: 10.1007/BF02932074

1340. Kim H, Kaczmarski K, Guiochon G. 2006. Intraparticle mass transfer kinetics on molecularly imprinted polymers of structural analogues of a template. Chem. Eng. Sci. 61: 1122-1137. DOI: 10.1016/j.ces.2005.08.012

1341. Ko DY, Lee HJ, Jeong B. 2006. Surface-Imprinted, Thermosensitive, CoreShell Nanosphere for Molecular Recognition. Macromol. Rapid Commun. 27: 1367-1372. DOI: 10.1002/marc.200600259

1342. Kubo A, Shinmori H, Takeuchi T. 2006. Atrazine-imprinted microspheres prepared using a microfluidic device. Chem. Lett. 35: 588-589. DOI: 10.1246/cl.2006.588 
1343. Lee I, Zaera F. 2006. Chiral templating of surfaces: Adsorption of (S)-2methylbutanoic acid on Pt(111) single-crystal surfaces. J. Am. Chem. Soc. 128: 8890-8898. DOI: $10.1021 / \mathrm{ja} 061654 \mathrm{w}$

1344. Li Y, Yang HH, You QH, Zhuang ZX, Wang XR. 2006. Protein recognition via surface molecularly imprinted polymer nanowires. Anal. Chem. 78: 317320. DOI: $10.1021 / \mathrm{ac} 050802 \mathrm{i}$

1345. Li Y, Yin XF, Chen FR, Yang HH, Zhuang ZX, Wang XR. 2006. Synthesis of magnetic molecularly imprinted polymer nanowires using a nanoporous alumina template. Macromolecules 39: 4497-4499. DOI: $10.1021 / \mathrm{ma0526185}$

1346. Li YC, Fu QQ, Zhang QQ, He LC. 2006. Preparation and evaluation of uniform-size (-)-ephedrine-imprinted polymeric microspheres by multi-step swelling and suspension polymerization. Anal. Sci. 22: 1355-1360. DOI: 10.2116/analsci.22.1355

1347. Li Z, Ding J, Day M, Tao Y. 2006. Molecularly imprinted polymeric nanospheres by diblock copolymer self-assembly. Macromolecules 39: 2629-2636. DOI: 10.1021/ma0526793

1348. Lin HY, Hsu CY, Thomas JL, Wang SE, Chen HC, Chou TC. 2006. The microcontact imprinting of proteins: The effect of cross-linking monomers for lysozyme, ribonuclease A and myoglobin. Biosens. Bioelectron. 22: 534543. DOI: 10.1016/j.bios.2006.07.038

1349. Liu ZH, Huan SY, Jiang JH, Shen GL, Yu RQ. 2006. Molecularly imprinted $\mathrm{TiO}_{2}$ thin film using stable ground-state complex as template as applied to selective electrochemical determination of mercury. Talanta 68: 1120-1125. DOI: 10.1016/j.talanta.2005.07.014

1350. Longo L, Vasapollo G, Scardino A, Picca RA, Malitesta C. 2006. Synthesis of a new substituted zinc phthalocyanine as functional monomer in the 
preparation of MIPs. J. Porphyrins Phthalocyanines 10: 1061-1065. DOI: $10.1142 / S 1088424606000417$

1351. Lu SL, Cheng GX, Pang XS. 2006. Protein-imprinted soft-wet gel composite microspheres with magnetic susceptibility. II. Characteristics. J. Appl. Polym. Sci. 99: 2401-2407. DOI: 10.1002/app.22812

1352. Lu SL, Cheng GX, Zhang HG, Pang XS. 2006. Preparation and characteristics of Tryptophan-imprinted $\mathrm{Fe}_{3} \mathrm{O}_{4} / \mathrm{P}$ (TRIM) composite microspheres with magnetic susceptibility by inverse emulsion-suspension polymerization. J. Appl. Polym. Sci. 99: 3241-3250. DOI: 10.1002/app.22997

1353. Lu SL, Cheng GX, Pang XS. 2006. Study on preparation of protein-imprinted soft-wet gel composite microspheres with magnetic susceptibility and their characteristics. I. Preparation and particle morphology. J. Appl. Polym. Sci. 100: 684-694. DOI: 10.1002/app.23411

1354. Martin-Esteban A, Tadeo JL. 2006. Selective molecularly imprinted polymer obtained from a combinatorial library for the extraction of bisphenol $A$. Comb. Chem. High Throughput Screening 9: 747-751. DOI: $10.2174 / 138620706779026024$

1355. Matsui J, Sodeyama T, Tamaki K, Sugimoto N. 2006. Molecularly-imprinted polymeric logic gates selective for predetermined chemical input species. Chem. Commun. 3217-3219. DOI: 10.1039/b604354b

1356. Matsui T, Osawa T, Shirasaka K, Katayama M, Hishiya T, Asanuma H, Komiyama M. 2006. Improved Method of Molecular Imprinting of Cyclodextrin on Silica-gel Surface for the Preparation of Stable Stationary HPLC Phase. J. Inclusion Phenom. Macrocyl. Chem. 56: 39-44. DOI: $10.1007 / \mathrm{s} 10847-006-9058-5$

1357. Matsunaga T, Takeuchi T. 2006. Crystallized Protein-imprinted Polymer Chips. Chem. Lett. 35: 1030-1031. DOI: 10.1246/cl.2006.1030 
1358. Mijangos I, Navarro-Villoslada F, Guerreiro A, Piletska E, Chianella I, Karim K, Turner A, Piletsky S. 2006. Influence of initiator and different polymerisation conditions on performance of molecularly imprinted polymers. Biosens. Bioelectron. 22: 381-387. DOI: 10.1016/j.bios.2006.05.012

1359. Miyata T, Jige $M$, Nakaminami T, Uragami T. 2006. Tumor markerresponsive behavior of gels prepared by biomolecular imprinting. Proc. Natl. Acad. Sci. USA 103: 1190-1193. DOI: 10.1073/pnas.0506786103

1360. Miyata T, Okawa K, Jige M, Ohba C, Uragami T. 2006. Preparation of bioconjugated hydrogels that respond to target biomolecules. Polym. Prepr. 47: 128-129.

1361. Mizutani N, Yang DH, Mitsushita N, Lee SW, Kunitake T. 2006. Fabrication and Application of Enantioselective TiO2 Nanoflims by Molecular Imprinting. Vol. Proceeding of the 5th IEEE Conference on Sensors, 2006, IEEE: New York; 1249-1252. DOI: 10.1109/ICSENS.2007.355856

1362. Nishino H, Huang CS, Shea KJ. 2006. Selective protein capture by epitope imprinting. Angew. Chem. Int. Edit. 45: 2392-2396. DOI: 10.1002/anie.200503760

1363. Palaprat G, Weyland M, Phou T, Binet C, Marty JD, Mingotaud AF, Mauzac M. 2006. Introduction of unusual properties into polymers by the use of liquid-crystalline moieties. Polym. Int. 55: 1191-1198. DOI: 10.1002/pi.1983

1364. Pang XS, Cheng GX, Lu SL, Tang EJ. 2006. Synthesis of polyacrylamide gel beads with electrostatic functional groups for the molecular imprinting of bovine serum albumin. Anal. Bioanal. Chem. 384: 225-230. DOI: $10.1007 / \mathrm{s} 00216-005-0147-\mathrm{x}$ 
1365. Pang XS, Cheng GX, Zhang YH, Lu SL. 2006. Soft-wet polyacrylamide gel beads with the imprinting of bovine serum albumin. Reac. Func. Polym. 66: 1182-1188. DOI: 10.1016/j.reactfunctpolym.2006.03.001

1366. Paniagua-González G, Fernández-Hernando P, Durand-Alegría JS. 2006. A morphological study of molecularly imprinted polymers using the scanning electron microscope. Anal. Chim. Acta 557: 179-183. DOI: 10.1016/j.aca.2005.10.034

1367. Richter A, Gibson UJ, Nowicki M, BelBruno JJ. 2006. Processing and morphology of molecularly imprinted nylon thin films. J. Appl. Polym. Sci. 101: 2919-2926. DOI: 10.1002/app.23369

1368. Richter A, Gruner M, BelBruno JJ, Gibson UJ, Nowicki M. 2006. Nanomechanical measurements on glutamine molecularly imprinted nylon films. Colloids Surf., A 284-285: 401-408. DOI: 10.1016/j.colsurfa.2005.10.087

1369. Rick J, Chou TC. 2006. Using protein templates to direct the formation of thin-film polymer surfaces. Biosens. Bioelectron. 22: 544-549. DOI: 10.1016/j.bios.2006.06.035

1370. Sagawa T, Kudo M, Steinke JHG, Morii T. 2006. Surface molecularly imprinted $\mathrm{TiO} 2$ nanoparticle for photoreduction of viologen. MRS Proceedings, Vol. 945, Ellingson R (ed). Materials Research Society: 25-30.

1371. Sambe H, Hoshina K, Moaddel R, Wainer IW, Haginaka J. 2006. Uniformlysized, molecularly imprinted polymers for nicotine by precipitation polymerization. J. Chromatogr. A 1134: 88-94. DOI: 10.1016/j.chroma.2006.08.073

1372. Say R. 2006. Creation of recognition sites for organophosphate esters based on charge transfer and ligand exchange imprinting methods. Anal. Chim. Acta 579: 74-80. DOI: 10.1016/j.aca.2006.07.010 
1373. Skrdla PJ, Shnayderman M, Wright L, O'Brien TP. 2006. GC-MS Study of the formation of alkoxysilanes from a sol-gel precursor in a hydrophobic solution: A potential new route to hybrid molecular imprinted polymers. $J$. Non-Cryst. Solids 352: 3302-3309. DOI: 10.1016/j.jnoncrysol.2006.04.010

1374. Sreenivasan K. 2006. Surface imprinted polyurethane film as a chiral discriminator. Talanta 68: 1037-1039. DOI: 10.1016/j.talanta.2005.05.005

1375. Su HJ, Li Q, Tan TW. 2006. Double-functional characteristics of a surface molecular imprinted adsorbent with immobilization of nano-TiO ${ }_{2}$. J. Chem. Technol. Biotechnol. 81: 1797-1802. DOI: 10.1002/jctb.1606

1376. Svenson J. 2006. Ultrasound-assisted preparation of molecularly imprinted polymers: Effects on polymer morphology, binding, and chromatographic behavior. Anal. Lett. 39: 2749-2760. DOI: 10.1080/00032710600867358

1377. Takátsy A, Sedzik J, Kilár F, Hjertén S. 2006. Universal method for synthesis of artificial gel antibodies by the imprinting approach combined with a unique electrophoresis technique for detection of minute structural differences of proteins, viruses, and cells (bacteria): II. Gel antibodies against virus (Semliki Forest Virus). J. Sep. Sci. 29: 2810-2815. DOI: $10.1002 /$ jssc.200600212

1378. Titirici MM, Sellergren B. 2006. Thin molecularly imprinted polymer films via reversible addition-fragmentation chain transfer polymerization. Chem. Mater. 18: 1773-1779. DOI: 10.1021/cm052153x

1379. Tunc Y, Hasirci N, Yesilada A, Ulubayram K. 2006. Comonomer effects on binding performances and morphology of acrylate-based imprinted polymers. Polymer 47: 6931-6940. DOI: 10.1016/j.polymer.2006.07.043

1380. Wan D, Satoh K, Kamigaito M. 2006. Triple Hydrogen Bonding for Stereospecific Radical Polymerization of a DAD Monomer and Simultaneous 
Control of Tacticity and Molecular Weight. Macromolecules 39: 6882-6886. DOI: $10.1021 / \mathrm{ma} 0615654$

1381. Wan LS, Wu J, Xu ZK. 2006. Porphyrinated Nanofibers via Copolymerization and Electrospinning. Macromol. Rapid Commun. 27: 1533-1538. DOI: 10.1002/marc.200600381

1382. Wang HF, Zhu YZ, Yan XP, Gao RY, Zheng JY. 2006. A Room Temperature Ionic Liquid (RTIL)-Mediated, Non-Hydrolytic Sol-Gel Methodology to Prepare Molecularly Imprinted, Silica-Based Hybrid Monoliths for Chiral Separation. Adv. Mater. 18: 3266-3270. DOI: 10.1002/adma.200601024

1383. Wang HJ, Zhou WH, Yin XF, Zhuang ZX, Yang HH, Wang XR. 2006. Template Synthesized Molecularly Imprinted Polymer Nanotube Membranes for Chemical Separations. J. Am. Chem. Soc. 128: 15954-15955. DOI: 10.1021/ja065116v

1384. Wang XB, Ding XB, Zheng ZH, Hu XH, Cheng X, Peng YX. 2006. Magnetic Molecularly Imprinted Polymer Particles Synthesized by Suspension Polymerization in Silicone Oil. Macromol. Rapid Commun. 27: 1180-1184. DOI: $10.1002 /$ marc.200600211

1385. Wang XB, Zheng ZH, Bin Ding X, Cheng X, Hu XH, Peng YX. 2006. Synthesis of molecularly imprinted polymer particles by suspension polymerization in silicon oil. Chin. Chem. Lett. 17: 1243-1246.

1386. Wei ST, Molinelli A, Mizaikoff B. 2006. Molecularly imprinted micro and nanospheres for the selective recognition of $17 \alpha$-estradiol. Biosens. Bioelectron. 21: 1943-1951. DOI: 10.1016/j.bios.2005.09.017

1387. Wolman FJ, Smolko EE, Cascone O, Grasselli M. 2006. Peptide imprinted polymer synthesized by radiation-induced graft polymerization. Reac. Func. Polym. 66: 1199-1205. DOI: 10.1016/j.reactfunctpolym.2006.03.002 
1388. Wu JM, Luan MM, Zhao JY. 2006. Trypsin immobilization by direct adsorption on metal ion chelated macroporous chitosan-silica gel beads. Int. J. Biol. Macromol. 39: 185-191. DOI: 10.1016/j.ijbiomac.2006.03.017

1389. Wulff G, Chong BO, Kolb U. 2006. Soluble single-molecule nanogels of controlled structure as a matrix for efficient artificial enzymes. Angew. Chem. Int. Edit. 45: 2955-2958. DOI: 10.1002/anie.200503926

1390. Xi FN, Wu JM. 2006. Preparation of macroporous chitosan layer coated on silica gel and its application to affinity chromatography for trypsin inhibitor purification. Reac. Func. Polym. 66: 682-688. DOI: 10.1016/j.reactfunctpolym.2005.10.028

1391. Xi FN, Wu JM, Lin XF. 2006. Novel nylon-supported organic-inorganic hybrid membrane with hierarchical pores as a potential immobilized metal affinity adsorbent. J. Chromatogr. $A$ 1125: 38-51. DOI: 10.1016/j.chroma.2006.05.016

1392. Xie CG, Zhang ZP, Wang DP, Guan GJ, Gao DM, Liu JH. 2006. Surface Molecular Self-Assembly Strategy for TNT Imprinting of Polymer Nanowire/Nanotube Arrays. Anal. Chem. 78: 8339-8346. DOI: $10.1021 / \mathrm{ac} 0615044$

1393. Yan H, Row KH. 2006. Preparation and characterization of theophyllineimprinted monolithic column. Sep. Sci. Technol. 41: 1841-1855. DOI: $10.1080 / 01496390600725745$

1394. Ye L, Yoshimatsu K, Kolodziej D, Da Cruz Francisco J, Dey ES. 2006. Preparation of molecularly imprinted polymers in supercritical carbon dioxide. J. Appl. Polym. Sci. 102: 2863-2867. DOI: 10.1002/app.24648

1395. Zhang FJ, Cheng GX, Ying XG. 2006. Emulsion and macromolecules templated alginate based polymer microspheres. Reac. Func. Polym. 66: 712-719. DOI: 10.1016/j.reactfunctpolym.2005.10.022 
1396. Zourob M, Mohr S, Mayes AG, Macaskill A, Pérez-Moral N, Fielden PR, Goddard NJ. 2006. A micro-reactor for preparing uniform molecularly imprinted polymer beads. Lab Chip 6: 296-301. DOI: 10.1039/b513195b

1397. Al Kobaisi M, Tate M, Rix C, Jakubov TS, Mainwaring DE. 2007. The effect of molecular imprinting on the pore size distribution of polymers. Adsorption 13: 315-321. DOI: $10.1007 / \mathrm{s} 10450-007-9062-0$

1398. Amano S, Ishida Y, Saigo K. 2007. Solid-State Hosts by the Template Polymerization of Columnar Liquid Crystals: Locked Supramolecular Architectures around Chiral 2-Amino Alcohols. Chem. Eur. J. 13: 5186-5196. DOI: 10.1002/chem.200601656

1399. Basavaraja C, Do YP, Young MC, Hyun TP, Yan SZ, Yamaguchi T, Do SH. 2007. Dependence of molecular recognition for a specific cation on the change of the oxidation state of the metal catalyst component in the hydrogel network. Bull. Kor. Chem. Soc. 28: 805-810.

1400. Belmont AS, Sokuler M, Haupt K, Gheber LA. 2007. Direct writing of molecularly imprinted microstructures using a nanofountain pen. Appl. Phys. Lett. 90: Art. No. 193101. DOI: 10.1063/1.2730753

1401. Bi X, Yang KL. 2007. Complexation of Copper lons with Histidine-Containing Tripeptides Immobilized on Solid Surfaces. Langmuir 23: 11067-11073. DOI: 10.1021/la7016243

1402. Bi XY, Lau RJ, Yang KL. 2007. Preparation of Ion-Imprinted Silica Gels Functionalized with Glycine, Diglycine, and Triglycine and Their Adsorption Properties for Copper lons. Langmuir 23: 8079-8086. DOI: 10.1021/la7008072

1403. Bodugoz H, Güven O, Peppas NA. 2007. Glucose recognition capabilities of hydroxyethyl methacrylate-based hydrogels containing poly(ethylene glycol) chains. J. Appl. Polym. Sci. 103: 432-441. DOI: 10.1002/app.24906 
1404. Bonini F, Piletsky S, Turner APF, Speghini A, Bossi A. 2007. Surface imprinted beads for the recognition of human serum albumin. Biosens. Bioelectron. 22: 2322-2328. DOI: 10.1016/j.bios.2006.12.034

1405. Booker K, Bowyer MC, Lennard CJ, Holdsworth Cl, McCluskey A. 2007. Molecularly Imprinted Polymers and Room Temperature Ionic Liquids: Impact of Template on Polymer Morphology. Aust. J. Chem. 60: 51-56. DOI: $10.1071 / \mathrm{CH} 06284$

1406. Bossi A, Rivetti C, Mangiarotti L, Whitcombe MJ, Turner APF, Piletsky SA. 2007. Patterned gallium surfaces as molecular mirrors. Biosens. Bioelectron. 23: 290-294. DOI: 10.1016/j.bios.2007.06.001

1407. Choi K. 2007. Materials Strategies for Advanced NanoTechnology. In Materials and Strategies for Lab-on-a-Chip - Biological Analysis, Microfactories, and Fluidic Assembly of Nanostructures, Materials Research Society Symposium Proceedings, Vol. 1004E, Grego S, Ramsey JM, Velev O, Verpoorte S (eds). Materials Research Society: Warrendale, PA; Paper No. 1004-P06-08.

1408. Djourelov N, Ates Z, Güven O, Misheva M, Suzuki T. 2007. Positron annihilation lifetime spectroscopy of molecularly imprinted hydroxyethyl methacrylate based polymers. Polymer 48: 2692-2699. DOI: 10.1016/j.polymer.2007.03.006

1409. Elmer SL, Lemcoff NG, Zimmerman SC. 2007. Exploring the Reversibility of the Ring-Closing Metathesis Mediated Cross-linking of Dendrimers. Macromolecules 40: 8114-8118. DOI: 10.1021/ma071233e

1410. Espinosa-Garcia BM, Arguelles-Monal WM, Hernandez J, Felix-Valenzuela L, Acosta N, Goycoolea FM. 2007. Molecularly Imprinted Chitosan-Genipin Hydrogels with Recognition Capacity toward o-Xylene. Biomacromolecules 8: 3355-3364. DOI: 10.1021/bm700458a 
1411. Fireman-Shoresh S, Marx S, Avnir D. 2007. Enantioselective Sol-Gel Materials Obtained by Either Doping or Imprinting with a Chiral Surfactant. Adv. Mater. 19: 2145-2150. DOI: 10.1002/adma.200601793

1412. Fu GQ, Zhao JC, Yu H, Liu L, He BL. 2007. Bovine serum albumin-imprinted polymer gels prepared by graft copolymerization of acrylamide on chitosan. $\begin{array}{llll}\text { Reac. Func. } & \text { 67: }\end{array}$ 10.1016/j.reactfunctpolym.2007.02.006

1413. Fu XJ, Yang $Y$, Wang NX, Wang $H$, Yang $Y J$. 2007. A novel chiral separation material: polymerized organogel formed by chiral gelators for the separation of D- and L-phenylalanine. J. Mol. Recognit. 20: 238-244. DOI: 10.1002/jmr.831

1414. Fujiwara M, Yamamura I, Nishiyama M, Shiokawa K, Ohtsuki S, Nomura R. 2007. Bisphenol A imprinted SiO2-ZrO2 mixed oxide materials prepared by acetic anhydride sol-gel method. Synth. React. Inorg. Met. -Org. Nano-Met. Chem. 37: 771-777. DOI: 10.1080/15533170701740883

1415. Gao BJ, An FQ, Zhu Y. 2007. Novel surface ionic imprinting materials prepared via couple grafting of polymer and ionic imprinting on surfaces of silica gel particles. Polymer 48: 2288-2297. DOI: 10.1016/j.polymer.2006.12.041

1416. Gao DM, Zhang ZP, Wu MH, Xie CG, Guan GJ, Wang DP. 2007. A Surface Functional Monomer-Directing Strategy for Highly Dense Imprinting of TNT at Surface of Silica Nanoparticles. J. Am. Chem. Soc. 129: 7859-7866. DOI: $10.1021 / \mathrm{ja} 070975 \mathrm{k}$

1417. Gomy C, Schmitzer AR. 2007. Synthesis and Photoresponsive Properties of a Molecularly Imprinted Polymer. Org. Lett. 9: 3865-3868. DOI: 10.1021/ol701618n 
1418. Guan GJ, Zhang ZP, Wang ZY, Liu BH, Gao DM, Xie CG. 2007. Single-Hole Hollow Polymer Microspheres toward Specific High-Capacity Uptake of Target Species. Adv. Mater. 19: 2370-2374. DOI: 10.1002/adma.200700984

1419. Hawkins DM, Ellis EA, Stevenson D, Holzenburg A, Reddy SM. 2007. Novel critical point drying (CPD) based preparation and transmission electron microscopy (TEM) imaging of protein specific molecularly imprinted polymers (HydroMIPs). J. Mater. Sci. 42: 9465-9468. DOI: 10.1007/s10853007-1806-4

1420. Inoue K, Yamauchi T, Itoh T, Ihara E. 2007. Ionic Conductivity of Crosslinked Polymethacrylate Derivatives/Cyclophosphazenes/Li+ Salt Complexes. J. Inorg. Organomet. Polym. Mater. 17: 367-375. DOI: 10.1007/s10904-007-9126-3

1421. Jing $T$, Gao XD, Wang $P$, Wang $Y$, Lin $Y F$, Zong XC, Zhou YK, Mei SR. 2007. Preparation of high selective molecularly imprinted polymers for tetracycline by precipitation polymerization. Chin. Chem. Lett. 18: 15351538. DOI: 10.1016/j.cclet.2007.10.029

1422. Koesdjojo MT, Rasmussen HT, Fermier AM, Patel P, Remcho VT. 2007. The Development of a Semiautomated Procedure for the Synthesis and Screening of a Large Group of Molecularly Imprinted Polymers. J. Comb. Chem. 9: 929-934. DOI: 10.1021/cc0700243

1423. Lee K, Itharaju RR, Puleo DA. 2007. Protein-imprinted polysiloxane scaffolds. Acta Biomater. 3: 515-522. DOI: 10.1016/j.actbio.2007.01.003

1424. Lee KS, Kim DS, Kim BS. 2007. Biodegradable molecularly imprinted polymers based on poly(epsilon-caprolactone). Biotechnol. Bioprocess Eng. 12: $152-156$.

1425. Lin HY, Rick J, Chou TC. 2007. Optimizing the formulation of a myoglobin molecularly imprinted thin-film polymer--formed using a micro-contact 
imprinting method. Biosens. Bioelectron. 22: 3293-3301. DOI: 10.1016/j.bios.2006.11.015

1426. Liu XJ, Chen ZY, Zhao R, Shangguan DH, Liu GQ, Chen Y. 2007. Uniformsized molecularly imprinted polymer for metsulfuron-methyl by one-step swelling and polymerization method. Talanta 71: 1205-1210. DOI: 10.1016/j.talanta.2006.06.021

1427. Lu Y, Zhao BH, Ren Y, Xiao GS, Wang XD, Li CX. 2007. Water-assisted formation of novel molecularly imprinted polymer membranes with ordered porous structure. Polymer 48: 6205-6209. DOI: 10.1016/j.polymer.2007.08.045

1428. Matassa R, Carbone M, Lauceri R, Purrello R, Caminiti R. 2007. Supramolecular Structure of Extrinsically Chiral Porphyrin HeteroAssemblies and Achiral Analogues. Adv. Mater. 19: 3961-3967. DOI: 10.1002/adma.200602042

1429. Narita M, Hoshino F, Mouri M, Tsuchimori M, Ikawa T, Watanabe O. 2007. Photoinduced Immobilization of Biomolecules on the Surface of Azopolymer Films and Its Dependence on the Concentration and Type of the Azobenzene Moiety. Macromolecules 40: 623-629. DOI: $10.1021 / \mathrm{ma061601z}$

1430. Oh WG, Kim BS. 2007. Novel Biodegradable Molecularly Imprinted Polymers Based on Poly(3-hydroxybutyrate). Macromol. Symp. 249-250: 7680. DOI: 10.1002/masy.200750313

1431. Pérez-Moral N, Mayes AG. 2007. Molecularly Imprinted Multi-Layer CoreShell Nanoparticles - A Surface Grafting Approach. Macromol. Rapid Commun. 28: 2170-2175. DOI: 10.1002/marc.200700532 
1432. Rajkumar R, Warsinke A, Mohwald H, Scheller FW, Katterle M. 2007. Development of fructosyl valine binding polymers by covalent imprinting. Biosens. Bioelectron. 22: 3318-3325. DOI: 10.1016/j.bios.2007.03.001

1433. Shi F, Liu Z, Wu GL, Zhang M, Chen H, Wang ZQ, Zhang X, Willner I. 2007. Surface Imprinting in Layer-by-Layer Nanostructured Films. Adv. Funct. Mater. 17: 1821-1827. DOI: 10.1002/adfm.200700267

1434. Soares CMF, Zanin GM, de Moraes FF, dos Santos OAA, de Castro HF. 2007. Molecular imprinting of $\alpha$-cyclodextrin/cholesterol template into a silica polymer for cholesterol separation. J. Inclusion Phenom. Macrocyl. Chem. 57: 79-82. DOI: 10.1007/s10847-006-9218-7

1435. Sreenivasan K. 2007. Synthesis and evaluation of molecularly imprinted polymers for nucleic acid bases using aniline as a monomer. Reac. Func. Polym. 67: 859-864. DOI: 10.1016/j.reactfunctpolym.2007.05.017

1436. Takátsy A, Végvári Á, Hjertén S, Kilár F. 2007. Universal method for synthesis of artificial gel antibodies by the imprinting approach combined with a unique electrophoresis technique for detection of minute structural differences of proteins, viruses and cells (bacteria). Ib. Gel antibodies against proteins (hemoglobins). Electrophoresis 28: 2345-2350. DOI: 10.1002/elps.200600191

1437. Takeuchi T, Akeda K, Murakami S, Shinmori H, Inoue S, Lee WS, Hishiya T. 2007. Photoresponsive porphyrin-imprinted polymers prepared using a novel functional monomer having diaminopyridine and azobenzene moieties. Org. Biomol. Chem. 5: 2368-2374. DOI: 10.1039/b704830k

1438. Tan CJ, Tong YW. 2007. Preparation of Superparamagnetic Ribonuclease A Surface-Imprinted Submicrometer Particles for Protein Recognition in Aqueous Media. Anal. Chem. 79: 299-306. DOI: 10.1021/ac061364y 
1439. Fu GQ, Zhu J, Jiang YZ. 2008. Comment on "Preparation of Superparamagnetic Ribonuclease A Surface-Imprinted Submicrometer Particles for Protein Recognition in Aqueous Media". Anal. Chem. 80: 26342635. DOI: $10.1021 / \mathrm{ac} 8000868$

1440. Tan CJ, Wangrangsimakul S, Sankarakumar N, Tong YW. 2008. Response to Comment on "Preparation of Superparamagnetic Ribonuclease A Surface-Imprinted Submicrometer Particles for Protein Recognition in Aqueous Media". Anal. Chem. 80: 9375-9376. DOI: 10.1021/ac8010318

1441. Tan CJ, Tong YW. 2007. The Effect of Protein Structural Conformation on Nanoparticle Molecular Imprinting of Ribonuclease A Using Miniemulsion Polymerization. Langmuir 23: 2722-2730. DOI: 10.1021/la062178q

1442. Vandevelde F, Leichle T, Ayela C, Bergaud C, Nicu L, Haupt K. 2007. Direct Patterning of Molecularly Imprinted Microdot Arrays for Sensors and Biochips. Langmuir 23: 6490-6493. DOI: 10.1021/la700320n

1443. Vandevelde F, Belmont AS, Pantigny J, Haupt K. 2007. Hierarchically Nanostructured Polymer Films Based on Molecularly Imprinted SurfaceBound Nanofilaments. Adv. Mater. 19: 3717-3720. DOI: 10.1002/adma.200700600

1444. Vaughan AD, Sizemore SP, Byrne ME. 2007. Enhancing molecularly imprinted polymer binding properties via controlled/living radical polymerization and reaction analysis. Polymer 48: 74-81. DOI: 10.1016/j.polymer.2006.11.013

1445. Voicu R, Faid K, Farah AA, Bensebaa F, Barjovanu R, Py C, Tao Y. 2007. Nanotemplating for Two-Dimensional Molecular Imprinting. Langmuir 23: 5452-5458. DOI: 10.1021/la063562q

1446. Wang JF, Cormack PAG, Sherrington DC, Khoshdel E. 2007. Synthesis and characterization of micrometer-sized molecularly imprinted spherical 
polymer particulates prepared via precipitation polymerization. Pure Appl. Chem. 79: 1505-1519. DOI: 10.1351/pac200779091505

1447. Yan CL, Lu Y, Gao SY. 2007. Coating lysozyme molecularly imprinted thin films on the surface of microspheres in aqueous solutions. J. Polym. Sci. A,Polym. Chem. 45: 1911-1919. DOI: 10.1002/pola.21955

1448. Yang KG, Li BQ, Zhou H, Ma JJ, Bai PL, Zhao CS. 2007. Preparation and selective binding characterization of Bisphenol A imprinted polyethersulfone particles. J. Appl. Polym. Sci. 106: 2791-2799. DOI: 10.1002/app.26924

1449. Yavuz H, Karakoç V, Türkmen D, Say R, Denizli A. 2007. Synthesis of cholesterol imprinted polymeric particles. Int. J. Biol. Macromol. 41: 8-15. DOI: 10.1016/j.ijbiomac.2006.11.011

1450. Zeng G, Gao J, Chen S, Chen H, Wang Z, Zhang X. 2007. Combining Hydrogen-Bonding Complexation in Solution and Hydrogen-BondingDirected Layer-by-Layer Assembly for the Controlled Loading of a Small Organic Molecule into Multilayer Films. Langmuir 23: 11631-11636. DOI: 10.1021/la702054d

1451. Zhang YQ, Qin Z, Tu ZY. 2007. Study of the Preparation of Flavone Imprinted Silica Microspheres and Their Molecular Recognition Function. Chem. Eng. Technol. 30: 1014-1019. DOI: 10.1002/ceat.200700116

1452. Zheng MX, Li SJ, Luo X. 2007. Rationally Designing Molecularly Imprinted Polymer Toward a High Specific Adsorbent by Using Metal as Assembled Pivot. J. Macromol. Sci. A 44: 1187-1194. DOI: $10.1080 / 10601320701561122$

1453. Zhuang Y, Luo HP, Duan DL, Chen LR, Xu XJ. 2007. In situ synthesis of molecularly imprinted polymers on glass microspheres in a column. Anal. Bioanal. Chem. 389: 1177-1183. DOI: 10.1007/s00216-007-1526-2 
1454. Demirel M, Sevin SB, Say R, Yazan Y. 2007. Propranolol HCl Imprinted Polymeric Microspheres: Development, Characterization and Dissolution. FABAD J. Pharmaceut. Sci. 32: 147-157.

1455. Kotrotsiou O, Chaitidou S, Liakopoulou-Kyriakides M, Kiparissides C. 2007. Molecularly imprinted polymers for selective recognition of biomolecules. $\mathrm{J}$. Nanostruct. Polym. Nanocompos. 3: 35-45.

1456. Abu Bakar N, Ahmad M, Daik R. 2008. Molecularly imprinted polymer for recognition of p-xylene in organic medium. Sains Malaysiana 37: 373-377.

1457. Bergmann NM, Peppas NA. 2008. Configurational Biomimetic Imprinting for Protein Recognition: Structural Characteristics of Recognitive Hydrogels. Ind. Eng. Chem. Res. 47: 9099-9107. DOI: 10.1021/ie071270u

1458. Brown ME, Puleo DA. 2008. Protein binding to peptide-imprinted porous silica scaffolds. Chem. Eng. J. 137: 97-101. DOI: 10.1016/j.cej.2007.09.002

1459. Casey BJ, Kofinas P. 2008. Selective binding of carcinoembryonic antigen using imprinted polymeric hydrogels. J. Biomed. Mater. Res. Part A 87A: 359-363. DOI: 10.1002/jbm.a.31757

1460. Chaitidou S, Kotrotsiou O, Kotti K, Kammona O, Bukhari M, Kiparissides C. 2008. Precipitation polymerization for the synthesis of nanostructured particles. Mater. Sci. Eng. B 152: 55-59. DOI: 10.1016/j.mseb.2008.06.024

1461. Choi KM. 2008. Microfluidic Approach for the Synthesis of Micro- or Nanosized Molecularly Imprinted Polymer Particles. Res. Lett. Mater. Sci. 2008: Article ID 458158. DOI: 10.1155/2008/458158

1462. Dam AH, Kim D. 2008. Metal ion-imprinted polymer microspheres derived from copper methacrylate for selective separation of heavy metal ions. $J$. Appl. Polym. Sci. 108: 14-24. DOI: 10.1002/app.26923 
1463. Dergunov SA, Pinkhassik E. 2008. Functionalization of Imprinted Nanopores in Nanometer-Thin Organic Materials. Angew. Chem. Int. Edit. 47: 82648267. DOI: 10.1002/anie.200803261

1464. Dhakal RP, Oshima T, Baba Y. 2008. Planarity-recognition enhancement of $\mathrm{N}$-(2-pyridylmethyl)chitosan by imprinting planar metal ions. Reac. Func. Polym. 68: 1549-1556. DOI: 10.1016/j.reactfunctpolym.2008.08.008

1465. Gai QQ, Liu QY, Li WY, He XW, Chen LX, Zhang YK. 2008. Preparation of bovine hemoglobin-imprinted polymer beads via the photografting surfacemodified method. Frontiers Chem. Chin. 3: 370-377. DOI: 10.1007/s11458008-0089-x

1466. Gao BJ, Wang J, An FQ, Liu Q. 2008. Molecular imprinted material prepared by novel surface imprinting technique for selective adsorption of pirimicarb. Polymer 49: 1230-1238. DOI: 10.1016/j.polymer.2007.12.041

1467. Guardia L, Badía-Laíño R, Díaz-García ME, Ania CO, Parra JB. 2008. Role of surface adsorption and porosity features in the molecular recognition ability of imprinted sol-gels. Biosens. Bioelectron. 23: 1101-1108. DOI: 10.1016/j.bios.2007.10.028

1468. Guerreiro AR, Korkhov V, Mijangos I, Piletska EV, Rodins J, Turner APF, Piletsky SA. 2008. Influence of continuous magnetic field on the separation of ephedrine enantiomers by molecularly imprinted polymers. Biosens. Bioelectron. 23: 1189-1194. DOI: 10.1016/j.bios.2007.09.009

1469. He CY, Long YY, Pan JL, Li K, Liu F. 2008. Molecularly imprinted silica prepared with immiscible ionic liquid as solvent and porogen for selective recognition of testosterone. Talanta 74: 1126-1131. DOI: 10.1016/j.talanta.2007.08.009 
1470. He CY, Long YY, Pan JL, Li K, Liu F. 2008. A method for coating colloidal particles with molecularly imprinted silica films. J. Mater. Chem. 18: 28492854. DOI: 10.1039/b801848k

1471. Henry OYF, Piletsky SA, Cullen DC. 2008. Fabrication of molecularly imprinted polymer microarray on a chip by mid-infrared laser pulse initiated polymerisation. Biosens. Bioelectron. 23: 1769-1775. DOI: 10.1016/j.bios.2008.02.010

1472. Hua ZD, Chen ZY, Li YZ, Zhao MP. 2008. Thermosensitive and SaltSensitive Molecularly Imprinted Hydrogel for Bovine Serum Albumin. Langmuir 24: 5773-5780. DOI: 10.1021/la703963f

1473. Huo HY, Su HJ, Tan TW. 2008. The influence of trace TiO2 on adsorption of Ag+-imprinted adsorbents made from chitosan and mycelium. Biotechnol. Bioprocess Eng. 13: 77-83. DOI: 10.1007/s12257-007-0174-x

1474. Jin GY, Li W, Yu SN, Peng YY, Kong JL. 2008. Novel superparamagnetic core-shell molecular imprinting microspheres towards high selective sensing. Analyst 133: 1367-1372. DOI: 10.1039/b802120a

1475. Jin Y, Jiang M, Shi Y, Lin Y, Peng Y, Dai K, Lu B. 2008. Narrowly dispersed molecularly imprinted microspheres prepared by a modified precipitation polymerization method. Anal. Chim. Acta 612: 105-113. DOI: 10.1016/j.aca.2008.02.011

1476. Johnson BJ, Melde BJ, Charles PT, Cardona DC, Dinderman MA, Malanoski AP, Qadri SB. 2008. Imprinted Nanoporous Organosilicas for Selective Adsorption of Nitroenergetic Targets. Langmuir 24: 9024-9029. DOI: $10.1021 / \mathrm{la} 800615 y$

1477. Karabörk M, Ersöz A, Denizli A, Say R. 2008. Polymer-Clay Nanocomposite Iron Traps Based on Intersurface Ion-Imprinting. Ind. Eng. Chem. Res. 47: 2258-2264. DOI: 10.1021/ie070885o 
1478. Lee E, Park DW, Lee JO, Kim DS, Lee BH, Kim BS. 2008. Molecularly imprinted polymers immobilized on carbon nanotube. Colloids Surf., A 313314: 202-206. DOI: 10.1016/j.colsurfa.2007.04.093

1479. Li F, Li J, Zhang SS. 2008. Molecularly imprinted polymer grafted on polysaccharide microsphere surface by the sol-gel process for protein recognition. Talanta 74: 1247-1255. DOI: 10.1016/j.talanta.2007.08.032

1480. Li Q, Su HJ, Tan TW. 2008. Synthesis of ion-imprinted chitosan-TiO2 adsorbent and its multi-functional performances. Biochem. Eng. J. 38: 212218. DOI: 10.1016/j.bej.2007.07.007

1481. Li Q, Zhang WY, Li X. 2008. Preparation and Properties of Salicylic AcidImprinted Polymers from Emulsions. Macromol. Symp. 261: 91-96. DOI: 10.1002/masy.200850112

1482. Li SJ, Huang X, Zheng MX, Li WK. 2008. Molecularly imprinted polymers: modulating molecular recognition by a thermal phase transition in the binding framework. Anal. Bioanal. Chem. 392: 177-185. DOl: $10.1007 / s 00216-008-2254-y$

1483. Liu HM, Liu CH, Yang XJ, Zeng SJ, Xiong YQ, Xu WJ. 2008. Uniformly sized $\alpha$-cyclodextrin molecularly imprinted microspheres prepared by a novel surface imprinting technique for ursolic acid. Anal. Chim. Acta 628: 8794. DOI: 10.1016/j.aca.2008.08.042

1484. Long Y, Xing XC, Han RF, Sun Y, Wang Y, Zhao Z, Mi HF. 2008. Two-step purification of low-content cellular protein using protein-imprinted polymers. Anal. Biochem. 380: 268-275. DOI: 10.1016/j.ab.2008.05.045

1485. Niu J, Liu ZH, Fu L, Shi F, Ma HW, Ozaki Y, Zhang X. 2008. SurfaceImprinted Nanostructured Layer-by-Layer Film for Molecular Recognition of Theophylline Derivatives. Langmuir 24: 11988-11994. DOI: $10.1021 /$ la802165f 
1486. Okutucu B, Zihnioglu F, Telefoncu A. 2008. Shell-core imprinted polyacrylamide crosslinked chitosan for albumin removal from plasma. $J$. Biomed. Mater. Res. Part A 84A: 842-845. DOI: 10.1002/jbm.a.31714

1487. Ouyang RZ, Lei JP, Ju HX. 2008. Surface molecularly imprinted nanowire for protein specific recognition. Chem. Commun. 5761-5763. DOI: 10.1039/b810248a

1488. Ouyang RZ, Lei JP, Ju HX. 2008. Surface molecularly imprinted nanowire for protein specific recognition (pg 5761, 2008). Chem. Commun. 6614. DOI: 10.1039/b819970c

1489. Papaioannou EH, Liakopoulou-Kyriakides M, Papi RM, Kyriakidis DA. 2008. Artificial receptor for peptide recognition in protic media: The role of metal ion coordination. Mater. Sci. Eng. $B$ 152: 28-32. DOI: 10.1016/j.mseb.2008.06.017

1490. Piletska EV, Guerreiro AR, Romero-Guerra M, Chianella I, Turner APF, Piletsky SA. 2008. Design of molecular imprinted polymers compatible with aqueous environment. Anal. Chim. Acta 607: 54-60. DOI: 10.1016/j.aca.2007.11.019

1491. Plieva FM, Mattiasson B. 2008. Macroporous Gel Particles As Novel Sorbent Materials: Rational Design. Ind. Eng. Chem. Res. 47: 4131-4141. DOI: $10.1021 / \mathrm{ie} 0714060$

1492. Sreenivasan K. 2008. Improving the efficiency of imprinting in poly(HEMA) for polyaromatic hydrocarbon using silver ions. J. Appl. Polym. Sci. 109: 3275-3278. DOI: 10.1002/app.28509

1493. Su HJ, Li J, Tan TW. 2008. Adsorption mechanism for imprinted ion (Ni2+) of the surface molecular imprinting adsorbent (SMIA). Biochem. Eng. J. 39: 503-509. DOI: 10.1016/j.bej.2007.11.011 
1494. Tan CJ, Wangrangsimakul S, Bai R, Tong YW. 2008. Defining the Interactions between Proteins and Surfactants for Nanoparticle Surface Imprinting through Miniemulsion Polymerization. Chem. Mater. 20: 118-127. DOI: $10.1021 / \mathrm{cm} 702174 \mathrm{y}$

1495. Tan CJ, Chua HG, Ker KH, Tong YW. 2008. Preparation of Bovine Serum Albumin Surface-Imprinted Submicrometer Particles with Magnetic Susceptibility through Core-Shell Miniemulsion Polymerization. Anal. Chem. 80: 683-692. DOI: 10.1021/ac701824u

1496. Tokuyama H, Naohara S, Sakohara S. 2008. Preparation of molecular imprinted thermosensitive gels grafted onto polypropylene by plasmainitiated graft polymerization. Reac. Func. Polym. 68: 182-188. DOI: 10.1016/j.reactfunctpolym.2007.09.010

1497. Urraca JL, Carbajo MC, Torralvo MJ, González-Vázquez J, Orellana G, Moreno-Bondi MC. 2008. Effect of the template and functional monomer on the textural properties of molecularly imprinted polymers. Biosens. Bioelectron. 24: 155-161. DOI: 10.1016/j.bios.2008.04.004

1498. Vandevelde F, Pantigny J, Pezron I, Gheber LA, Haupt K. 2008. Nanostructured molecularly imprinted polymer films as synthetic recognition layers. Int. J. Nanotechnol. 5: 757-768. DOI: 10.1504/IJNT.2008.018695

1499. Wang B, Liu MZ, Liang R, Ding SL, Chen ZB, Chen SL, Jin SP. 2008. MMTCA Recognition by Molecular Imprinting in Interpenetrating Polymer Network Hydrogels Based on Poly(acrylic acid) and Poly(vinyl alcohol). Macromol. Biosci. 8: 417-425. DOI: 10.1002/mabi.200700176

1500. Wybranska K, Szczubialka K, Nowakowska M. 2008. Photochemical molecular imprinting of cholesterol. J. Inclusion Phenom. Macrocyl. Chem. 61: 147-151. DOI: 10.1007/s10847-007-9407-z 
1501. Xie CG, Liu BH, Wang ZY, Gao DM, Guan GJ, Zhang ZP. 2008. Molecular Imprinting at Walls of Silica Nanotubes for TNT Recognition. Anal. Chem. 80: 437-443. DOI: 10.1021/ac701767h

1502. Yao QZ, Zhou YM, Sun YQ, Ye XY. 2008. Synthesis of TiO2 Hybrid Molecular Imprinted Polymer for Ethofumesate Linked by Silane Coupling Agent. J. Inorg. Organomet. Polym. Mater. 18: 477-484. DOI: 10.1007/s10904-008-9227-7

1503. Yao W, Ning B, Zhou HY, Fang YJ, Gao ZX. 2008. Recognition of Staphylococcus enterotoxin via molecularly imprinted beads. J. Sep. Sci. 31: 413-418. DOI: 10.1002/jssc.200700429

1504. Yoshimatsu K, Ye L, Lindberg J, Chronakis IS. 2008. Selective molecular adsorption using electrospun nanofiber affinity membranes. Biosens. Bioelectron. 23: 1208-1215. DOI: 10.1016/j.bios.2007.12.002

1505. Zdyrko B, Hoy O, Kinnan MK, Chumanov G, Luzinov I. 2008. Nanopatterning with polymer brushes via solvent-assisted polymer grafting. Soft Matter 4: 2213-2219. DOI: 10.1039/b810038a

1506. Zhang SZ, Fu XJ, Wang H, Yang YJ. 2008. Selective adsorption of D- and L-phenylalanine on molecularly-imprinted polymerized organogels formed using polymerizable gelator $\mathrm{N}$-octadecyl maleamic acid. J. Sep. Sci. 31: 3782-3787. DOI: $10.1002 / j s s c .200800349$

1507. Zhang YQ, Zhang YH, Qin Z, Ma ZR. 2008. Synthesis of silica adsorbent and its selective separation for flavone (Translated from: Journal of Tianjin University, 2007, 40(4): 411-415). Frontiers Chem. Chin. 3: 320-324. DOI: $10.1007 / s 11458-008-0052-x$

1508. Zhao HL, Guo TY, Xia YQ, Song MD. 2008. Hemoglobin-imprinted polymer gel prepared using modified glucosamine as functional monomer. Chin. Chem. Lett. 19: 233-236. DOI: 10.1016/j.cclet.2007.10.045 
1509. Zhao KY, Cheng GX, Huang JJ, Ying XG. 2008. Rebinding and recognition properties of protein-macromolecularly imprinted calcium phosphate/alginate hybrid polymer microspheres. Reac. Func. Polym. 68: 732-741. DOI: 10.1016/j.reactfunctpolym.2007.11.011

1510. Zhao KY, Kan BH, Wei JF, Cheng GX, Chen L. 2008. Bovine serum albumin imprinted calcium phosphate/polyacrylate/alginate multi-hybrid polymer microspheres in inverse-phase suspension. E-Polymers Art. No. 100.

1511. Arrachart G, Carcel C, Trens P, Moreau JJE, Man MWC. 2009. Silylated Melamine and Cyanuric Acid as Precursors for Imprinted and Hybrid Silica Materials with Molecular Recognition Properties. Chem. Eur. J. 15: 62796288. DOI: 10.1002/chem.200900278

1512. Baydemir G, Bereli N, Andaç M, Say R, Galaev IY, Denizli A. 2009. Supermacroporous poly(hydroxyethyl methacrylate) based cryogel with embedded bilirubin imprinted particles. Reac. Func. Polym. 69: 36-42. DOI: 10.1016/j.reactfunctpolym.2008.10.007

1513. Baydemir G, Bereli N, Andaç M, Say R, Galaev IY, Denizli A. 2009. Bilirubin recognition via molecularly imprinted supermacroporous cryogels. Colloids Surf., B 68: 33-38. DOI: 10.1016/j.colsurfb.2008.09.008

1514. Berglin M, Pinori E, Sellborn A, Andersson M, Hulander M, Elwing H. 2009. Fibrinogen Adsorption and Conformational Change on Model Polymers: Novel Aspects of Mutual Molecular Rearrangement. Langmuir 25: 56025608. DOI: 10.1021/la803686m

1515. Bing NC, Zhen T, Chen SW, Xie LL, Wang LJ, Hao Y, Li QH, Xu ZL. 2009. Analysis on microstructure of polymers for selective separation of naproxen enantiomers. In Proceedings, 2009 Symposium on Photonics and Optoelectronics, SOPO 2009, Art. No. 5230138. DOI: 10.1109/SOPO.2009.5230138 
1516. Campbell SE, Collins M, Xie L, BelBruno JJ. 2009. Surface morphology of spin-coated molecularly imprinted polymer films. Surf. Interf. Sci. 41: 347356. DOI: 10.1002/sia.3030

1517. Chang YS, Ko TH, Hsu TJ, Syu MJ. 2009. Synthesis of an Imprinted Hybrid Organic-Inorganic Polymeric Sol-Gel Matrix Toward the Specific Binding and Isotherm Kinetics Investigation of Creatinine. Anal. Chem. 81: 2098-2105. DOI: 10.1021/ac802168w

1518. Chen PY, Nien PC, Wu CT, Wu TH, Lin CW, Ho KC. 2009. Fabrication of a molecularly imprinted polymer sensor by self-assembling monolayer/mediator system. Anal. Chim. Acta 643: 38-44. DOI: 10.1016/j.aca.2009.04.004

1519. Chen PY, Lin CY, Ho KC. 2009. Detection Of Uric Acid Based On MultiWalled Carbon Nanotubes Polymerized With A Layer Of Molecularly Imprinted PMAA. AIP Conference Proceedings, Vol. 1137, Pardo M, Sberveglieri G (eds). 284-287. DOI: 10.1063/1.3156526

1520. Chen YW, Rick J, Chou TC. 2009. A systematic approach to forming microcontact imprints of creatine kinase. Org. Biomol. Chem. 7: 488-494. DOI: 10.1039/b813361a

1521. Curcio P, Zandanel C, Wagner A, Mioskowski C, Baati R. 2009. SemiCovalent Surface Molecular Imprinting of Polymers by One-Stage Miniemulsion Polymerization: Glucopyranoside as a Model Analyte. Macromol. Biosci. 9: 596-604. DOI: 10.1002/mabi.200900056

1522. Fujikawa S, Muto E, Kunitake T. 2009. Nanochannel Design by Molecular Imprinting on a Free-Standing Ultrathin Titania Membrane. Langmuir 25: 11563-11568. DOI: 10.1021/la9014916

1523. Gallego-Gallegos M, Muñoz-Olivas R, Cámara C. 2009. Different formats of imprinted polymers for determining organotin compounds in environmental 
samples. J. Environ. Manag. 90: S69-S76. DOI: 10.1016/j.jenvman.2008.07.020

1524. Gao BJ, Wang J, Yang Y. 2009. Studies of Imprinting Conditions and Application Performance of Pirimicarb Molecule-Imprinted Material Prepared Using a Novel Surface-Imprinting Technique. Chromatographia 69: 13531361. DOI: $10.1365 / \mathrm{s} 10337-009-1062-7$

1525. Garcinuño RM, Chianella I, Guerreiro A, Mijangos I, Piletska EV, Piletsky SA. 2009. The stabilisation of receptor structure in low cross-linked MIPs by an immobilised template. Soft Matter 5: 313-317. DOI: 10.1039/b804476g

1526. Guan GJ, Liu RY, Wu MH, Li Z, Liu BH, Wang ZY, Gao DM, Zhang ZP. 2009. Protein-building molecular recognition sites by layer-by-layer molecular imprinting on colloidal particles. Analyst 134: 1880-1886. DOI: 10.1039/b820962f

1527. Guerreiro AR, Chianella I, Piletska E, Whitcombe MJ, Piletsky SA. 2009. Selection of imprinted nanoparticles by affinity chromatography. Biosens. Bioelectron. 24: 2740-2743. DOI: 10.1016/j.bios.2009.01.013

1528. Guillon S, Lemaire R, Linares AV, Haupt K, Ayela C. 2009. Single step patterning of molecularly imprinted polymers for large scale fabrication of microbiochips. Lab Chip 9: 2987-2991. DOI: 10.1039/b905608d

1529. Gültekin A, Diltemiz SE, Ersöz A, Sariözlü NY, Denizli A, Say RI. 2009. Gold-silver nanoclusters having dipicolinic acid imprinted nanoshell for Bacillus cereus spores recognition. Talanta 78: 1332-1338. DOI: 10.1016/j.talanta.2009.02.007

1530. Hashidzume A, Zimmerman SC. 2009. Switching the selectivity of a polyglycerol dendrimer monomolecularly imprinted with $\mathrm{d}$-(-)-fructose. Tetrahedron Lett. 50: 2204-2207. DOI: 10.1016/j.tetlet.2009.02.168 
1531. Horváth V, Lorántfy B, Tóth B, Bognár J, László K, Horvai G. 2009. Preparation of terbutylazine imprinted polymer microspheres using viscous polymerization solvents. J. Sep. Sci. 32: 3347-3358. DOI: 10.1002/jssc.200900230

1532. Hu CH, Chou TC. 2009. Albumin molecularly imprinted polymer prepared with a semi-rigid crosslinker in mixed organic/aqueous media. Microchim. Acta 165: 399-405. DOI: 10.1007/s00604-009-0151-5

1533. Hua ZD, Zhou S, Zhao MP. 2009. Fabrication of a surface imprinted hydrogel shell over silica microspheres using bovine serum albumin as a model protein template. Biosens. Bioelectron. 25: 615-622. DOI: 10.1016/j.bios.2009.01.027

1534. Huang YP, Zhang SJ, Wu X, Zhang QW, Liu ZS. 2009. Preparation and Characterization of a Low-Density Imprinted Monolithic Column. Chromatographia 70: 691-698. DOI: 10.1365/s10337-009-1226-5

1535. Janiak DS, Ayyub OB, Kofinas P. 2009. Effects of Charge Density on the Recognition Properties of Molecularly Imprinted Polymeric Hydrogels. Macromolecules 42: 1703-1709. DOI: 10.1021/ma8027722

1536. Kotrotsiou O, Chaitidou S, Kiparissides C. 2009. On the synthesis of peptide imprinted polymers by a combined suspension--Epitope polymerization method. Mater. Sci. Eng. B 165: 256-260. DOI: 10.1016/j.mseb.2009.05.006

1537. Lee HY, Kim S. 2009. Grafting of molecularly imprinted polymers on iniferter-modified carbon nanotube. Biosens. Bioelectron. 25: 587-591. DOI: 10.1016/j.bios.2009.03.040

1538. Lee SC, Chang JY. 2009. Preparation of Molecularly Imprinted Polymers Using Photocross-linkable Polyphosphazene and Selective Rebinding of Amino Acids. Macromol. Res. 17: 522-527. DOI: 10.1007/BF03218901 
1539. Li L, He XW, Chen LX, Zhang YK. 2009. Preparation of Core-shell Magnetic Molecularly Imprinted Polymer Nanoparticles for Recognition of Bovine Hemoglobin. Chem. Asian J. 4: 286-293. DOI: 10.1002/asia.200800300

1540. Li L, He XW, Chen LX, Zhang YK. 2009. Preparation of novel bovine hemoglobin surface-imprinted polystyrene nanoparticles with magnetic susceptibility. Sci. China Ser. B Chem. 52: 1402-1411. DOI: 10.1007/s11426-009-0182-0

1541. Li SJ, Pilla S, Gong SQ. 2009. Modulated molecular recognition by a temperature-sensitive molecularly-imprinted polymer. J. Polym. Sci. A,Polym. Chem. 47: 2352-2360. DOI: 10.1002/pola.23325

1542. Li Y, Zhou WH, Yang HH, Wang XR. 2009. Grafting of molecularly imprinted polymers from the surface of silica gel particles via reversible additionfragmentation chain transfer polymerization: A selective sorbent for theophylline. Talanta 79: 141-145. DOI: 10.1016/j.talanta.2009.03.014

1543. Lin Z, Yang F, He XW, Zhang YK. 2009. Organic-inorganic hybrid silica as supporting matrices for selective recognition of bovine hemoglobin via covalent immobilization. J. Sep. Sci. 32: 3980-3987. DOI: $10.1002 /$ jssc.200900295

1544. Linares AV, Vandevelde F, Pantigny J, Falcimaigne-Cordin A, Haupt K. 2009. Polymer Films Composed of Surface-Bound Nanofilaments with a High Aspect Ratio, Molecularly Imprinted with Small Molecules and Proteins. Adv. Funct. Mater. 19: 1299-1303. DOI: 10.1002/adfm.200801222

1545. Liu Z, Bucknall DG, Allen MG. 2009. Recognition and absorption of the water-soluble X-ray contrast medium iodixanol using molecularly imprinted polymers for biomedical applications. In Materials Research Society Symposium Proceedings, Vol. 1138, 39-44. 
1546. Lu CH, Wang Y, Li Y, Yang HH, Chen X, Wang XR. 2009. Bifunctional superparamagnetic surface molecularly imprinted polymer core-shell nanoparticles. J. Mater. Chem. 19: 1077-1079. DOI: 10.1039/b821299f

1547. Lu Y, Yan CL, Gao SY. 2009. Preparation and recognition of surface molecularly imprinted core-shell microbeads for protein in aqueous solutions. Appl. Surf. Sci. 255: 6061-6066. DOI: 10.1016/j.apsusc.2008.12.080

1548. Lu Y, Yan CL, Wang XJ, Wang GK. 2009. Protein imprinting and recognition via forming nanofilms on microbeads surfaces in aqueous media. Appl. Surf. Sci. 256: 1341-1346. DOI: 10.1016/j.apsusc.2009.08.003

1549. Meng AC, LeJeune J, Spivak DA. 2009. Multi-analyte imprinting capability of OMNiMIPs versus traditional molecularly imprinted polymers. J. Mol. Recognit. 22: 121-128. DOI: 10.1002/jmr.901

1550. Pan GQ, Zu BY, Guo XZ, Zhang Y, Li CX, Zhang HQ. 2009. Preparation of molecularly imprinted polymer microspheres via reversible additionfragmentation chain transfer precipitation polymerization. Polymer 50: 28192825. DOI: 10.1016/j.polymer.2009.04.053

1551. Puoci F, lemma F, Cirillo G, Curcio M, Parisi OI, Spizzirri UG, Picci N. 2009. New restricted access materials combined to molecularly imprinted polymers for selective recognition/release in water media. Eur. Polym. J. 45: 1634-1640. DOI: 10.1016/j.eurpolymj.2009.01.021

1552. Qin L, He XW, Zhang W, Li WY, Zhang YK. 2009. Macroporous Thermosensitive Imprinted Hydrogel for Recognition of Protein by Metal Coordinate Interaction. Anal. Chem. 81: 7206-7216. DOI: $10.1021 / a c 900676 t$

1553. Qiu HB, Inoue Y, Che SN. 2009. Supramolecular Chiral Transcription and Recognition by Mesoporous Silica Prepared by Chiral Imprinting of a Helical 
Micelle. Angew. Chem. Int. Edit. 48: 3069-3072. DOI: 10.1002/anie.200900303

1554. Roeseling D, Tuercke T, Krause H, Loebbecke S. 2009. Microreactor-Based Synthesis of Molecularly Imprinted Polymer Beads Used for Explosive Detection. Org. Process Res. Dev. 13: 1007-1013. DOI: 10.1021/op9001774

1555. Takeda K, Kuwahara A, Ohmori K, Takeuchi T. 2009. Molecularly Imprinted Tunable Binding Sites Based on Conjugated Prosthetic Groups and IonPaired Cofactors. J. Am. Chem. Soc. 131: 8833-8838. DOI: $10.1021 / \mathrm{ja9} 004317$

1556. Tominaga Y, Kubo T, Kaya K, Hosoya K. 2009. Effective Recognition on the Surface of a Polymer Prepared by Molecular Imprinting Using Ionic Complex. Macromolecules 42: 2911-2915. DOI: 10.1021/ma802880z

1557. Turan E, Özçetin G, Caykara T. 2009. Dependence of Protein Recognition of Temperature-Sensitive Imprinted Hydrogels on Preparation Temperature. Macromol. Biosci. 9: 421-428. DOI: 10.1002/mabi.200800273

1558. Wang X, Wang LY, He XW, Zhang YK, Chen LX. 2009. A molecularly imprinted polymer-coated nanocomposite of magnetic nanoparticles for estrone recognition. Talanta 78: 327-332. DOI: 10.1016/j.talanta.2008.11.024

1559. Yang DH, Ham YR, Oh MH, Yoon YS, Shin JS, Kim YD, Kim H. 2009. Simple method for the fabrication of 1-hydroxypyrene-imprinted $\mathrm{TiO} 2$ gel nanofilms. Curr. Appl. Phys. 9: e136-e139. DOI: 10.1016/j.cap.2008.12.043

1560. Yao QZ, Zhou YM. 2009. Surface Functional Imprinting of Bensulfuronmethyl at Surface of Silica Nanoparticles Linked by Silane Coupling Agent. J. Inorg. Organomet. Polym. Mater. 19: 215-222. DOI: 10.1007/s10904-0099253-0 
1561. Yao QZ, Zhou YM. 2009. Synthesis of TiO2 Hybrid Molecular Imprinted Nanospheres Linked by Silane Coupling Agent. J. Inorg. Organomet. Polym. Mater. 19: 466-472. DOI: 10.1007/s10904-009-9289-1

1562. Yoshimatsu K, LeJeune K, Spivak DA, Ye L. 2009. Peptide-imprinted polymer microspheres prepared by precipitation polymerization using a single bi-functional monomer. Analyst 134: 719-724. DOI: 10.1039/b814967d

1563. Zaidi SA, Cheong WJ. 2009. Preparation of an open-tubular capillary column with a monolithic layer of S-ketoprofen imprinted and 4styrenesulfonic acid incorporated polymer and its enhanced chiral separation performance in capillary electrochromatography. J. Chromatogr. A 1216: 2947-2952. DOI: 10.1016/j.chroma.2008.08.015

1564. Zdyrko B, Hoy O, Luzinov I. 2009. Toward protein imprinting with polymer brushes. Biointerphases 4: FA17-FA21. DOI: 10.1116/1.3101907

1565. Zeng JB, Chen JM, Chen WF, Huang XL, Chen LB, Chen X. 2009. Recent Development of Laboratory-made Solid-phase Microextraction Fibers on the Application of Food Safety Analysis. Food Sci. Biotechnol. 18: 579-585.

1566. Zeng ZY, Hoshino Y, Rodriguez A, Yoo HS, Shea KJ. 2009. Synthetic Polymer Nanoparticles with Antibody-like Affinity for a Hydrophilic Peptide. ACS Nano 4: 199-204. DOI: 10.1021/nn901256s

1567. Zhang QQ, Kobayashi T. 2009. Imprinting Effect Inducing Crystallization of Uracil on Polymer Membrane Prepared in Supercritical CO2 Phase Inversion. J. Chem. Eng. Jpn. 42: 21-28. DOI: 10.1252/jcej.08we085

1568. Zhang W, Qin L, He XW, Li WY, Zhang YK. 2009. Novel surface modified molecularly imprinted polymer using acryloyl- $\alpha$-cyclodextrin and acrylamide as monomers for selective recognition of lysozyme in aqueous solution. $J$. Chromatogr. A 1216: 4560-4567. DOI: 10.1016/j.chroma.2009.03.056 
1569. Zhang Y, Liu RJ, Hu YL, Li GK. 2009. Microwave Heating in Preparation of Magnetic Molecularly Imprinted Polymer Beads for Trace Triazines Analysis in Complicated Samples. Anal. Chem. 81: 967-976. DOI: 10.1021/ac8018262

1570. Zhao KY, Wei JF, Cheng GX, Yang CX, Chen L. 2009. Preparation of bovine serum albumin-imprinted calcium polyacrylate/alginate hybrid microspheres via Ca2+ crosslinking. J. Appl. Polym. Sci. 113: 1133-1140. DOI: 10.1002/app.29936

1571. Zheng XM, Tu WP, Fan RY. 2009. Preparation of molecularly imprinted polymer films on monodisperse macromolecular beads. J. Appl. Polym. Sci. 113: 2620-2627. DOI: 10.1002/app.30326

1572. Zhou X, He XW, Chen LX, Li WY, Zhang YK. 2009. Optimum Conditions of Separation Selectivity Based on Molecularly Imprinted Polymers of Bovine Serum Albumin Formed on Surface of Aminosilica. Chin. J. Anal. Chem. 37: 174-180. DOI: 10.1016/S1872-2040(08)60084-5

1573. Zill AT, Zimmerman SC. 2009. A Route to Water-Soluble Molecularly Templated Nanoparticles Using Click Chemistry and Alkyne-Functionalized Hyperbranched Polyglycerol. Isr. J. Chem. 49: 71-78. DOI: 10.1560/IJC.49.1.71

1574. Zu BY, Pan GQ, Guo XZ, Zhang Y, Zhang HQ. 2009. Preparation of molecularly imprinted polymer microspheres via atom transfer radical precipitation polymerization. J. Polym. Sci. A,Polym. Chem. 47: 3257-3270. DOI: 10.1002/pola.23389

1575. Schreiber T, Weber A, Niedergall K, Riegler J, Bryniok D, Hirth T, Tovar GEM. 2009. Water treatment by molecularly imprinted polymer nanoparticles. Vol. 1169, Article No. 1169-Q04-07. DOI: 10.1557/PROC1169-Q04-07 
1576. Abbate V, Frascione N, Bansal SS. 2010. Preparation, characterization, and binding profile of molecularly imprinted hydrogels for the peptide hepcidin. $J$. Polym. Sci. A,Polym. Chem. 48: 1721-1731. DOI: 10.1002/pola.23937

1577. Ates Z, Güven O. 2010. Radiation-induced molecular imprinting of d-glucose onto poly(2-hydroxyethyl methacrylate) matrices using various crosslinking agents. Radiation Physics and Chemistry 79: 219-222. DOI: 10.1016/j.radphyschem.2009.10.010

1578. Barahona F, Turiel E, Cormack PAG, Martín-Esteban A. 2010. Chromatographic performance of molecularly imprinted polymers: Core-shell microspheres by precipitation polymerization and grafted MIP films via iniferter-modified silica beads. J. Polym. Sci. A,Polym. Chem. 48: 10581066. DOI: 10.1002/pola.23860

1579. Berti F, Todros S, Lakshmi D, Whitcombe MJ, Chianella I, Ferroni M, Piletsky SA, Turner APF, Marrazza G. 2010. Quasi-monodimensional polyaniline nanostructures for enhanced molecularly imprinted polymerbased sensing. Biosens. Bioelectron. 26: 497-503. DOI: 10.1016/j.bios.2010.07.063

1580. Cao FM, Liao J, Yang KG, Bai PL, Wei Q, Zhao CS. 2010. Self-Assembly Molecularly Imprinted Nanofiber for 4-HA Recognition. Anal. Lett. 43: 27902797. DOI: $10.1080 / 00032711003731480$

1581. Chang LM, Li Y, Chu J, Qi JY, Li X. 2010. Preparation of core-shell molecularly imprinted polymer via the combination of reversible additionfragmentation chain transfer polymerization and click reaction. Anal. Chim. Acta 680: 65-71. DOI: 10.1016/j.aca.2010.09.017

1582. Choi KM. 2010. Microfluidic synthesis and functional patterning for advanced nanotechnology. In Materials Research Society Symposium Proceedings, Vol. 1260, 11-17. 
1583. Darder Ma, Aranda P, Burgos-Asperilla L, Llobera A, Cadarso VJ, Fernández-Sánchez C, Ruiz-Hitzky E. 2010. Algae-silica systems as functional hybrid materials. J. Mater. Chem. 20: 9362-9369.

1584. Duan YQ, Qin Y, Xu FF, Zhang HH, Yan YS, Zhang C, Ma HL. 2010. Optimization of the process parameters of synthesis of oligomeric procyanidins imprinted polymer. Sci. Res. Essays 5: 2953-2964.

1585. Dvorakova G, Haschick R, Chiad K, Klapper M, Müllen K, Biffis A. 2010. Molecularly Imprinted Nanospheres by Nonaqueous Emulsion Polymerization. Macromol. Rapid Commun. 31: 2035-2040. DOI: 10.1002/marc.201000406

1586. Dvorakova G, Biffis A. 2010. Room temperature preparation of highly crosslinked microgels. Polym. Bull. 64: 107-114. DOI: 10.1007/s00289-0090134-z

1587. Feás X, Ye L, Hosseini SV, Fente CA, Cepeda A. 2010. Molecularly imprinted polyallylamine hydrogels: another reassessment. Polym. Int. 59: 11-15. DOI: 10.1002/pi.2690

1588. Feng L, Pamidighantam B, Lauterbur PC. 2010. Microwave-assisted sol-gel synthesis for molecular imprinting. Anal. Bioanal. Chem. 396: 1607-1612. DOI: $10.1007 / \mathrm{s} 00216-009-3311-\mathrm{x}$

1589. Forschheimer D, Luo G, Montelius L, Ye L. 2010. Molecularly imprinted nanostructures by nanoimprint lithography. Analyst 135: 1219-1223. DOI: 10.1039/c0an00132e

1590. Gauczinski J, Liu Z, Zhang X, Schönhoff M. 2010. Mechanism of Surface Molecular Imprinting in Polyelectrolyte Multilayers. Langmuir 26: $10122-$ 10128. DOI: $10.1021 / \mathrm{la} 1002447$ 
1591. Ginzburg-Turgeman R, Mandler D. 2010. Nanometric thin polymeric films based on molecularly imprinted technology: Towards electrochemical sensing applications. Phys. Chem. Chem. Phys. 12: 11041-11050.

1592. Guo MJ, Gao T, Fan Z, Yao JX, Xia JJ, Mi HF. 2010. Molecularly imprinted polymers with assistant recognition polymer chains for bovine serum albumin. Sci. China Chem. 53: 905-911. DOI: 10.1007/s11426-010-0113-0

1593. He HY, Fu GQ, Wang Y, Chai ZH, Jiang YZ, Chen ZL. 2010. Imprinting of protein over silica nanoparticles via surface graft copolymerization using low monomer concentration. Biosens. Bioelectron. 26: 760-765. DOI: 10.1016/j.bios.2010.06.043

1594. Herrero EP, Martín Del Valle EM, Peppas NA. 2010. Protein Imprinting by Means of Alginate-Based Polymer Microcapsules. Ind. Eng. Chem. Res. 49: 9811-9814. DOI: 10.1021/ie101068z

1595. Holland N, Frisby J, Owens E, Hughes H, Duggan P, McLoughlin P. 2010. The influence of polymer morphology on the performance of molecularly imprinted polymers. Polymer 51: 1578-1584. DOI: 10.1016/j.polymer.2009.10.035

1596. Huang YP, Zhang SJ, Zhao LA, Zhang QW, Liu ZS. 2010. Characterization of Convection for Molecularly Imprinted Monolith. Chromatographia 71: 559569. DOI: 10.1365/s10337-010-1513-1

1597. Jung BM, Kim MS, Kim WJ, Chang JY. 2010. Molecularly imprinted mesoporous silica particles showing a rapid kinetic binding. Chem. Commun. 46: 3699-3701. DOI: 10.1039/c003173a

1598. Kan XW, Zhao Q, Shao DL, Geng ZR, Wang ZL, Zhu JJ. 2010. Preparation and Recognition Properties of Bovine Hemoglobin Magnetic Molecularly Imprinted Polymers. J. Phys. Chem. B 114: 3999-4004. DOI: 10.1021/jp910060c 
1599. Lalo H, Ayela C, Dague E, Vieu C, Haupt K. 2010. Nanopatterning molecularly imprinted polymers by soft lithography: a hierarchical approach. Lab Chip 10: 1316-1318. DOI: 10.1039/b924315a

1600. Li JY, Zu BY, Zhang Y, Guo XZ, Zhang HQ. 2010. One-pot synthesis of surface-functionalized molecularly imprinted polymer microspheres by iniferter-induced "living" radical precipitation polymerization. J. Polym. Sci. A,Polym. Chem. 48: 3217-3228. DOI: 10.1002/pola.24057

1601. Liu JX, Deng QL, Yang KG, Zhang LH, Liang Z, Zhang YK. 2010. Macroporous molecularly imprinted monolithic polymer columns for protein recognition by liquid chromatography. J. Sep. Sci. 33: 2757-2761. DOI: $10.1002 /$ jssc. 201000350

1602. Liu YQ, Hoshina K, Haginaka J. 2010. Monodispersed, molecularly imprinted polymers for cinchonidine by precipitation polymerization. Talanta 80: 1713-1718. DOI: 10.1016/j.talanta.2009.10.011

1603. Liu ZH, Yi Y, Xu HP, Zhang X, Ngo TH, Smet M. 2010. Cation-Selective Microcontact Printing Based on Surface-Molecular-Imprinted Layer-by-Layer Films. Adv. Mater. 22: 2689-2693. DOI: 10.1002/adma.201000469

1604. Pan GQ, Zhang Y, Guo XZ, Li CX, Zhang HQ. 2010. An efficient approach to obtaining water-compatible and stimuli-responsive molecularly imprinted polymers by the facile surface-grafting of functional polymer brushes via RAFT polymerization. Biosens. Bioelectron. 26: 976-982. DOI: 10.1016/j.bios.2010.08.040

1605. Turner NW, Holdsworth CI, Donne SW, McCluskey A, Bowyer MC. 2010. Microwave induced MIP synthesis: comparative analysis of thermal and microwave induced polymerisation of caffeine imprinted polymers. New J. Chem. 34: 686-692. DOI: 10.1039/b9nj00538b 
1606. Ying XG, Zhang FJ, Zhang LG, Cheng GX. 2010. Effect on rebinding behavior with different composition and structure of the dually imprinted alginate polymer microspheres using proteins and o/w emulsion drops as dual templates. J. Appl. Polym. Sci. 115: 3516-3526. DOI: 10.1002/app.30727

1607. Ying XG, Cheng GX, Zhao KY. 2010. Preparation and characterization of protein imprinted agarose microspheres. Polym. Bull. 65: 245-263. DOI: 10.1007/s00289-009-0239-4

1608. Ying XG, Cheng GX, Liu GP, Qu RJ, Wang YQ, Zhang LG. 2010. Specific rebinding property of protein macromolecularly imprinted polymer microspheres based on calcium alginate hydrogel via gas jetting-dropping method. J. Appl. Polym. Sci. 117: 2331-2339. DOI: 10.1002/app.32061

1609. Zhang ZL, Xu XH, Yan YS. 2010. Kinetic and thermodynamic analysis of selective adsorption of $\mathrm{Cs}(\mathrm{l})$ by a novel surface whisker-supported ionimprinted polymer. Desalination 263: 97-106. DOI: 10.1016/j.desal.2010.06.044

1610. Zu BY, Zhang Y, Guo XZ, Zhang HQ. 2010. Preparation of molecularly imprinted polymers via atom transfer radical "bulk" polymerization. J. Polym. Sci. A,Polym. Chem. 48: 532-541. DOI: 10.1002/pola.23750

1611. Yu SA, Luo AQ, Biswal D, Hilt JZ, Puleo DA. 2010. Lysozyme-imprinted polymer synthesized using UV free-radical polymerization. Talanta 83: 156161. DOI: $10.1016 / j$.talanta.2010.08.055

1612. Cirillo G, Puoci F, Curcio M, Parisi Ol, lemma F, Spizzirri UG, Picci N. 2010. Molecular imprinting polymerization by Fenton reaction. Colloid Polym. Sci. 288: 689-693. DOI: 10.1007/s00396-010-2198-6 
1613. Annamma KM, Beena M. 2011. Design of 2,4-Dichlorophenoxyacetic Acid Imprinted Polymer with High Specificity and Selectivity. Materials Sciences and Applications 2: 131-140. DOI: 10.4236/msa.2011.23017

1614. Athikomrattanakul U, Katterle M, Gajovic-Eichelmann N, Scheller FW. 2011. Preparation and characterization of novel molecularly imprinted polymers based on thiourea receptors for nitrocompounds recognition. Talanta 84: 274-279. DOI: 10.1016/j.talanta.2010.12.049

1615. Azizi ES, Ahmad MN, Islam AKMS, Arbain D, Tahir I. 2011. Porogen effect towards the quality of curcumin imprinted polymer. Indonesian Journal of Chemistry 11: 207-211.

1616. Baggiani C, Baravalle P, Giovannoli C, Anfossi L, Passini C, Giraudi G. 2011. Binding behaviour of molecularly imprinted polymers prepared by a hierarchical approach in mesoporous silica beads of varying porosity. $\mathrm{J}$. Chromatogr. A 1218: 1828-1834. DOI: 10.1016/j.chroma.2011.02.003

1617. Balamurugan S, Spivak DA. 2011. Molecular imprinting in monolayer surfaces. J. Mol. Recognit. 24: 915-929. DOI: 10.1002/jmr.1150

1618. Barahona F, Turiel E, Martín-Esteban A. 2011. Molecularly imprinted polymer grafted to porous polyethylene frits: A new selective solid-phase extraction format. J. Chromatogr. A 1218: 7065-7070. DOI: 10.1016/j.chroma.2011.07.092

1619. Batlokwa BS, Mokgadi J, Nyokong T, Torto N. 2011. Optimal Template Removal from Molecularly Imprinted Polymers by Pressurized Hot Water Extraction. Chromatographia 73: 589-593. DOI: 10.1007/s10337-010-1884-3

1620. Bayer CL, Herrero EP. 2011. Alginate Films as Macromolecular Imprinted Matrices. J. Biomater. Sci. Polym. Ed. 22: 1523-1534. DOI: $10.1163 / 092050610 \times 514115$ 
1621. Bereli N, Saylan Y, Uzun L, Say RI, Denizli A. 2011. I-Histidine imprinted supermacroporous cryogels for protein recognition. Sep. Purif. Technol. 82: 28-35. DOI: 10.1016/j.seppur.2011.08.011

1622. Bloshenko AV, Roshchin AV, Kumpanenko IV, Ivanova NA. 2011. An analysis of absorption-desorption of volatile organic compounds by molecularly imprinted polymer films. Russ. J. Phys. Chem. B 5: 332-344. DOI: 10.1134/S1990793111020187

1623. Burova TV, Grinberg NV, Kalinina EV, Ivanov RV, Lozinsky VI, AlvarezLorenzo C, Grinberg VY. 2011. Thermoresponsive Copolymer Cryogel Possessing Molecular Memory: Synthesis, Energetics of Collapse and Interaction with Ligands. Macromol. Chem. Phys. 212: 72-80. DOI: 10.1002/macp.201000382

1624. Chang LM, Wu S, Chen SN, Li X. 2011. Preparation of graphene oxidemolecularly imprinted polymer composites via atom transfer radical polymerization. J. Mater. Sci. 46: 2024-2029. DOI: 10.1007/s10853-0105033-z

1625. Chen EZ, Su HJ, Zhang WY, Tan TW. 2011. A novel shape-controlled synthesis of dispersed silver nanoparticles by combined bioaffinity adsorption and TiO2 photocatalysis. Powder Technol. 212: 166-172. DOI: 10.1016/j.powtec.2011.05.008

1626. Chen EZ, Su HJ, Tan TW. 2011. Antimicrobial properties of silver nanoparticles synthesized by bioaffinity adsorption coupled with $\mathrm{TiO} 2$ photocatalysis. J. Chem. Technol. Biotechnol. 86: 421-427. DOI: $10.1002 /$ jctb. 2533

1627. Deng QL, Li YL, Zhang LH, Zhang YK. 2011. Molecularly imprinted macroporous monolithic materials for protein recognition. Chin. Chem. Lett. 22: 1351-1354. DOI: 10.1016/j.cclet.2011.05.044 
1628. Ezhova NM, Garkushina IS, Pisarev OA. 2011. Molecularly imprinted hydrophilic polymer sorbents for selective sorption of erythromycin. Appl. Biochem. Microbiol. 47: 635-639. DOI: 10.1134/S0003683811060056

1629. Fertier L, Théron C, Carcel C, Trens P, Wong Chi Man M. 2011. pHResponsive Bridged Silsesquioxane. Chem. Mater. 23: 2100-2106. DOI: $10.1021 / \mathrm{cm} 103327 \mathrm{y}$

1630. Forchheimer D, Luo G, Ye L, Montelius L. 2011. Molecularly selective nanopatterns using nanoimprint lithography: A label-free sensor architecture. J. Vac. Sci. Technol. B 29: Article No. 011021. DOI: $10.1116 / 1.3527080$

1631. Fuchs Y, Linares AV, Mayes AG, Haupt K, Soppera O. 2011. Ultrathin Selective Molecularly Imprinted Polymer Microdots Obtained by Evanescent Wave Photopolymerization. Chem. Mater. 23: 3645-3651. DOI: $10.1021 / \mathrm{cm} 2009829$

1632. Gai QQ, Qu F, Zhang T, Zhang YK. 2011. The preparation of bovine serum albumin surface-imprinted superparamagnetic polymer with the assistance of basic functional monomer and its application for protein separation. $\mathrm{J}$. Chromatogr. A 1218: 3489-3495. DOI: 10.1016/j.chroma.2011.03.069

1633. Gam-Derouich S, Mahouche-Chergui S, Truong S, Ben Hassen-Chehimi D, Chehimi MM. 2011. Design of molecularly imprinted polymer grafts with embedded gold nanoparticles through the interfacial chemistry of aryl diazonium salts. Polymer 52: 4463-4470. DOI: 10.1016/j.polymer.2011.08.007

1634. Gao RX, Su XQ, He XW, Chen LX, Zhang YK. 2011. Preparation and characterisation of core-shell CNTs@MIPs nanocomposites and selective removal of estrone from water samples. Talanta 83: 757-764. DOI: 10.1016/j.talanta.2010.10.034 
1635. Gonzato C, Courty M, Pasetto P, Haupt K. 2011. Magnetic Molecularly Imprinted Polymer Nanocomposites via Surface-Initiated RAFT Polymerization. Adv. Funct. Mater. 21: 3947-3953. DOI: 10.1002/adfm.201100466

1636. Guo Y, Yang Y, Zhang L, Guo TY. 2011. Core/shell molecular imprinting microparticles prepared using RAFT technology for degradation of paraoxon. Macromol. Res. 19: 1202-1209. DOI: 10.1007/s13233-011-11072

1637. He MQ, Song CC, Yan YS, Chen YQ, Wan JC. 2011. Synthesis and recognition of molecularly imprinted polymers for gastrodin based on surface-modified silica nanoparticles. J. Appl. Polym. Sci. 121: 2354-2360. DOI: 10.1002/app.33887

1638. Jiang GS, Zhong SA, Chen L, Blakey I, Whitaker A. 2011. Synthesis of molecularly imprinted organic-inorganic hybrid azobenzene materials by solgel for radiation induced selective recognition of 2,4-dichlorophenoxyacetic acid. Radiation Physics and Chemistry 80: 130-135. DOI: 10.1016/j.radphyschem.2010.07.020

1639. Jiang XH, Liu ZM. 2011. Uniformly-Sized, Molecularly Imprinted Polymers for Naproxen by Precipitation Polymerization. In New Materials, Applications and Processes, Vol. 399-401, Zeng JM, Kim YH, Chen YF (eds). Scientific.net: 713-717. DOI: 10.4028/www.scientific.net/AMR.399-401.713

1640. Jiang Y, Kim D. 2011. Effect of solvent/monomer feed ratio on the structure and adsorption properties of Cu2+-imprinted microporous polymer particles. Chem. Eng. J. 166: 435-444. DOI: 10.1016/j.cej.2010.11.006

1641. Kim WJ, Jung BM, Kang SH, Chang JY. 2011. Molecular imprinting into organogel nanofibers. Soft Matter 7: 4160-4162. DOI: 10.1039/C1SM05166K 
1642. Kim WJ, Chang JY. 2011. Molecularly imprinted polyimide nanofibers prepared by electrospinning. Materials Letters 65: 1388-1391. DOI: 10.1016/j.matlet.2011.02.010

1643. Li TY, Wu L, Chen SX, Li HC, Xu XZ. 2011. A Simple Scheme for Grafting an Ion-Imprinted Layer onto the Surface of Poly(propylene) Fibers. Macromol. Chem. Phys. 212: 2166-2172. DOI: 10.1002/macp.201100195

1644. Li XX, Liu X, Bai LH, Duan HQ, Huang YP, Liu ZS. 2011. Preparation of imprinted monolithic column under molecular crowding conditions. Chin. Chem. Lett. 22: 989-992. DOI: 10.1016/j.cclet.2011.04.010

1645. Li Y, Ding MJ, Wang S, Wang RY, Wu XL, Wen TT, Yuan LH, Dai P, Lin YH, Zhou XM. 2011. Preparation of Imprinted Polymers at Surface of Magnetic Nanoparticles for the Selective Extraction of Tadalafil from Medicines. ACS Appl. Mater. Interf. 3: 3308-3315. DOI: 10.1021/am2007855

1646. Li Y, Dong CK, Chu J, Qi JY, Li X. 2011. Surface molecular imprinting onto fluorescein-coated magnetic nanoparticles via reversible addition fragmentation chain transfer polymerization: A facile three-in-one system for recognition and separation of endocrine disrupting chemicals. Nanoscale 3: 280-287. DOI: 10.1039/CONR00614A

1647. Li YB, Gao BJ, Du RK. 2011. Studies on Preparation and Recognition Characteristic of Surface-lon Imprinting Material IIP-PEI/SiO ${ }_{2}$ of Chromate Anion. Sep. Sci. Technol. 46: 1472-1481. DOI: $10.1080 / 01496395.2011 .561821$

1648. Linares AV, Falcimaigne-Cordin A, Gheber LA, Haupt K. 2011. Patterning Nanostructured, Synthetic, Polymeric Receptors by Simultaneous Projection Photolithography, Nanomolding, and Molecular Imprinting. Small 7: 23182325. DOI: 10.1002/smll.201100248 
1649. Liu JH, Fang H, Chien CC. 2011. Solvent-tunable colors in imprinted helical structures on polymer template via multiple UV-induced polymerization. $\mathrm{J}$. Polym. Sci. A,Polym. Chem. 49: 1256-1262. DOI: 10.1002/pola.24545

1650. Liu JX, Yang KG, Deng QL, Li QR, Zhang LH, Liang Z, Zhang YK. 2011. Preparation of a new type of affinity materials combining metal coordination with molecular imprinting. Chem. Commun. 47: 3969-3971. DOI: 10.1039/C0CC05317A

1651. Liu JZ, Wang WZ, Xie YF, Huang YY, Liu YL, Liu XJ, Zhao R, Liu GQ, Chen $Y$. 2011. A novel polychloromethylstyrene coated superparamagnetic surface molecularly imprinted core-shell nanoparticle for bisphenol A. J. Mater. Chem. 21: 9232-9238. DOI: 10.1039/C1JM10227C

1652. Liu Y, Liu ZC, Dai JD, Gao J, Xie JM, Yan YS. 2011. Selective Adsorption of Co(II) by Mesoporous Silica SBA-15-Supported Surface Ion Imprinted Polymer: Kinetics, Isotherms, and Thermodynamics Studies. Chin. J. Chem. 29: 387-398. DOI: 10.1002/cjoc.201190093

1653. Liu ZH, Yi Y, Gauczinski J, Xu HP, Schönhoff M, Zhang X. 2011. Surface Molecular Imprinted Layer-by-Layer Film Attached to a Porous Membrane for Selective Filtration. Langmuir 27: 11806-11812. DOI: 10.1021/la202093s

1654. Lofgreen JE, Moudrakovski IL, Ozin GA. 2011. Molecularly Imprinted Mesoporous Organosilica. ACS Nano 5: 2277-2287. DOI: $10.1021 / \mathrm{nn} 1035697$

1655. Luo XB, Deng F, Luo SL, Tu XM, Yang LX. 2011. Grafting of molecularly imprinted polymers from the surface of $\mathrm{Fe} 3 \mathrm{O} 4$ nanoparticles containing double bond via suspension polymerization in aqueous environment: $A$ selective sorbent for theophylline. J. Appl. Polym. Sci. 121: 1930-1937. DOI: 10.1002/app.33710 
1656. Lv YK, Zhang Q, Song YL, Yan SL. 2011. Preparation and Characterization of Imprinted Polymers Based on Cobalt(II)-Enrofloxacin Coordination. Asian J. Chem. 23: 4037-4041.

1657. Ma J, Yuan LH, Ding MJ, Wang S, Ren F, Zhang J, Du SH, Li F, Zhou XM. 2011. The study of core-shell molecularly imprinted polymers of $17 \alpha-$ estradiol on the surface of silica nanoparticles. Biosens. Bioelectron. 26: 2791-2795. DOI: 10.1016/j.bios.2010.10.045

1658. Ma XL, Chen Z, Chen X, Chen RY, Zheng X. 2011. Preparation of Imprinted $P V B / \alpha-C D$ Nanofiber by Electrospinning Technique and Its Selective Binding Abilities for Naringin. Chin. J. Chem. 29: 1753-1758. DOI: 10.1002/cjoc.201180312

1659. Men'shikova AYu, Pankova GA, Evseeva TG, Shabsel's BM, Shevchenko NN. 2011. Monodisperse polymer particles with molecular imprints of naphthalene in the shell: Synthesis and adsorption properties. Russ. J. Appl. Chem. 84: 1567-1574. DOI: 10.1134/S1070427211090187

1660. Mohajeri SA, Karimi G, Aghamohammadian J, Khansari MR. 2011. Clozapine recognition via molecularly imprinted polymers; bulk polymerization versus precipitation method. J. Appl. Polym. Sci. 121: 35903595. DOI: 10.1002/app.34147

1661. Mu LN, Wang XH, Zhao L, Huang YP, Liu ZS. 2011. Low cross-linked molecularly imprinted monolithic column prepared in molecular crowding conditions. J. Chromatogr. A 1218: 9236-9243. DOI: 10.1016/j.chroma.2011.10.079

1662. Nicolescu TV, Sârbu A, Ghiurea M, Donescu D. 2011. Influence of crosslinker/porogen ratio upon imprinted polymer parameters. U. P. B. Science Bulletin, Series B 73: 163-172. 
1663. Niroomandi Z, Otadi M, Panahi HA, Goharrokhi M. 2011. Isolation and Purification of $\alpha$-Amylase Enzyme Using MIP Technique. In Proceedings of the 2011 International Conference on Nanotechnology and Biosensors, International Proceedings of Chemical, Biological and Environmental Engineering, Vol. 25, IACSIT Press: Singapore; 100-105.

1664. Njikang G, Liu GJ, Hong LZ. 2011. Chiral Imprinting of Diblock Copolymer Single-Chain Particles. Langmuir 27: 7176-7184. DOI: 10.1021/la2006887

1665. Pan GQ, Ma Y, Zhang Y, Guo XZ, Li CX, Zhang HQ. 2011. Controlled synthesis of water-compatible molecularly imprinted polymer microspheres with ultrathin hydrophilic polymer shells via surface-initiated reversible addition-fragmentation chain transfer polymerization. Soft Matter 7: 84288439. DOI: 10.1039/C1SM05497J

1666. Pan JM, Hu W, Dai XH, Guan W, Zou XH, Wang X, Huo PW, Yan YS. 2011. Molecularly imprinted polymers based on magnetic fly-ash-cenosphere composites for bisphenol A recognition. J. Mater. Chem. 21: 15741-15751. DOI: 10.1039/C1JM12099A

1667. Pan JM, Yao H, Guan W, Ou HX, Huo PW, Wang X, Zou XH, Li CX. 2011. Selective adsorption of 2,6-dichlorophenol by surface imprinted polymers using polyaniline/silica gel composites as functional support: Equilibrium, kinetics, thermodynamics modeling. Chem. Eng. J. 172: 847-855. DOI: 10.1016/j.cej.2011.06.072

1668. Pan JM, Yao H, Xu LC, Ou HX, Huo PW, Li XX, Yan YS. 2011. Selective Recognition of 2,4,6-Trichlorophenol by Molecularly Imprinted Polymers Based on Magnetic Halloysite Nanotubes Composites. J. Phys. Chem. C 115: 5440-5449. DOI: $10.1021 / \mathrm{jp} 111120 x$

1669. Qian K, Fang GZ, Wang S. 2011. A novel core-shell molecularly imprinted polymer based on metal-organic frameworks as a matrix. Chem. Commun. 47: 10118-10120. DOI: 10.1039/C1CC12935J 
1670. Qiu HB, Che SN. 2011. Chiral mesoporous silica: Chiral construction and imprinting via cooperative self-assembly of amphiphiles and silica precursors. Chem. Soc. Rev. 40: 1259-1268. DOI: 10.1039/C0CS00002G

1671. Saifuddin N, Nur YAA, Abdullah SF. 2011. Microwave enhanced synthesis of chitosan-graft-polyacrylamide molecular imprinting polymer for selective removal of 17 $\alpha$-estradiol at trace concentration. Asian J. Biochem. 6: 38-54. DOI: 10.3923/ajb.2011.38.54

1672. Scorrano S, Mergola L, del Sole R, Vasapollo G. 2011. Synthesis of Molecularly Imprinted Polymers for Amino Acid Derivates by Using Different Functional Monomers. Int. J. Mol. Sci. 12: 1735-1743. DOI: 10.3390/ijms 12031735

1673. Shen XT, Ye L. 2011. Interfacial Molecular Imprinting in NanoparticleStabilized Emulsions. Macromolecules 44: 5631-5637. DOI: $10.1021 / \mathrm{ma} 200837 \mathrm{n}$

1674. Shen XT, Ye L. 2011. Molecular imprinting in Pickering emulsions: a new insight into molecular recognition in water. Chem. Commun. 47: 1035910361. DOI: 10.1039/C1CC13899E

1675. Tang QA, Gong CB, Lam MHW, Fu XK. 2011. Preparation of a photoresponsive molecularly imprinted polymer containing fluorinesubstituted azobenzene chromophores. Sens. Actuators B 156: 100-107. DOI: 10.1016/j.snb.2011.03.078

1676. Tang XS, Zhang D, Zhou TS, Nie DX, Yang QY, Jin LT, Shi GY. 2011. Fe3O4@Au sphere molecular imprinting with self-assembled monolayer for the recognition of parathion-methyl. Anal. Methods 3: 2313-2321. DOI: 10.1039/C1AY05279A

1677. Wang YX, Liu QM, Rong F, Fu DG. 2011. A facile method for grafting of bisphenol A imprinted polymer shells onto poly(divinylbenzene) 
microspheres through precipitation polymerization. Appl. Surf. Sci. 257: 6704-6710. DOI: 10.1016/j.apsusc.2011.02.105

1678. Wei Q, Cao FM, Zhang YJ, Li J, Zhao CS. 2011. Preparation of Surface Molecularly Imprinted Poly(dopamine) Film for 4-Hydroxybenzoic Acid (4BA) Recognition by One-Step Method. Anal. Lett. 44: 1796-1806. DOI: $10.1080 / 00032719.2010 .526270$

1679. Xu CG, Ye L. 2011. Clickable molecularly imprinted nanoparticles. Chem. Commun. 47: 6096-6098. DOI: 10.1039/C1CC11439E

1680. Xu JX, Gao Y, Li HM. 2011. Controlled Fabrication of Theophylline Imprinted Polymers on Multiwalled Carbon Nanotubes via Atom Transfer Radical Polymerization. J. Nanosci. Nanotechnol. 11: 1217-1224. DOI: 10.1166/jnn.2011.3095

1681. Yang BJ, Cao XJ. 2011. Synthesis of the artemisinin-imprinting polymers on silica surface and its adsorption behavior in supercritical $\mathrm{CO} 2$ fluid. AIChE $J$ 57: 3514-3521. DOI: 10.1002/aic.12547

1682. Yang LX, Fan ZJ, Wang T, Cai WS, Yang MX, Jiang P, Zhang M, Dong XC. 2011. Preparation of a Pyrazosulfuron-Ethyl Imprinted Polymer with Hydrophilic External Layers by Reversible Addition-Fragmentation Chain Transfer Precipitation and Grafting Polymerization. Anal. Lett. 44: 26172632. DOI: $10.1080 / 00032719.2011 .553007$

1683. Ye T, Lu SY, Hu QQ, Jiang X, Wei GF, Wang JJ, Lu JQ. 2011. One-bath synthesis of hydrophilic molecularly imprinted quantum dots for selective recognition of chlorophenol. Chin. Chem. Lett. 22: 1253-1256. DOI: 10.1016/j.cclet.2011.03.022

1684. Ying XG, Cheng GX, Li X. 2011. The imprinting induce-fit model of specific rebinding of macromolecularly imprinted polymer microspheres. J. Appl. Polym. Sci. 122: 1847-1856. DOI: 10.1002/app.34263 
1685. Zhang JJ, Riskin M, Freeman R, Tel-Vered R, Balogh D, Tian H, Willner I. 2011. Electrochemically Triggered Au Nanoparticles 'Sponges' for the Controlled Uptake and Release of a Photoisomerizable Dithienylethene Guest Substrate. ACS Nano 5: 5936-5944. DOI: 10.1021/nn201724g

1686. Zhang JW, Liu YL, Wu GL, Schönhoff M, Zhang X. 2011. Bolaform Supramolecular Amphiphiles as a Novel Concept for the Buildup of SurfaceImprinted Films. Langmuir 27: 10370-10375. DOI: 10.1021/la202490q

1687. Zhang Q, Zhang XY, Zhang WC, Pan J, Liu L, Zhang HT, Zhao D, Li Z. 2011. Preparation and Adsorption Properties of PA6/PSMA-OA Molecularly Imprinted Composite Membranes in Supercritical $\mathrm{CO}_{2}$. Bull. Kor. Chem. Soc. 32: 3348-3354. DOI: 10.5012/bkcs.2011.32.9.3348

1688. Zhang WC, Zhang HT, Zhang Q, Cui YF, Wu ZY, Zheng RJ, Liu L. 2011. Molecularly imprinted polymers prepared by precipitation polymerization and used for inducing crystallization of oleanolic acid in supercritical CO2. Sep. Purif. Technol. 81: 411-417. DOI: 10.1016/j.seppur.2011.08.012

1689. Zhang WL, Zhao YP, Liu XH, Chen L, Zhang QS, Zhao KY, Chen JJ. 2011. Preparation and Properties of Thermo-Sensitive Molecularly Imprinted Hydrogels. In Materials Processing Technology, Vol. 418-420, Liu XH, Jiang ZY, Han JT (eds). Trans Tech Publications: Switzerland; 490-493. DOI: 10.4028/www.scientific.net/AMR.418-420.490

1690. Zhang YG, Song D, Brown JC, Shimizu KD. 2011. Suppression of background sites in molecularly imprinted polymers via urea-urea monomer aggregation. Org. Biomol. Chem. 9: 120-126. DOI: 10.1039/C0OB00637H

1691. Zhang YJ, Wei QA, Zhang QC, Li J, Yang J, Zhao CS. 2011. Molecularly Imprinted Electrospinning Polyethersulfone Nano-Scale Fibers for the Binding and Recognition of Bisphenol A. Sep. Sci. Technol. 46: 1615-1620. DOI: 10.1080/01496395.2011.561822 
1692. Zhang ZL, Wang B, Li JA. 2011. Effect of the synthesis initiation mode on the structure and properties of sulfadiazine molecularly imprinted polymers. J. Appl. Polym. Sci. 119: 3189-3198. DOI: 10.1002/app.32834

1693. Zhao L, Ban L, Zhang QW, Huang YP, Liu ZS. 2011. Preparation and characterization of imprinted monolith with metal ion as pivot. $J$. Chromatogr. A 1218: 9071-9079. DOI: 10.1016/j.chroma.2011.10.027

1694. Qin L, He XW, Jia M, Li WY, Zhang YK. 2011. A Thermosensitive Monolithic Column as an Artificial Antibody for the On-line Selective Separation of the Protein. Chem. Eur. J. 17: 1696-1704. DOI: 10.1002/chem.201000875

1695. Qin L, He XW, Yuan X, Li WY, Zhang YK. 2011. Molecularly imprinted beads with double thermosensitive gates for selective recognition of proteins. Anal. Bioanal. Chem. 399: 3375-3385. DOI: 10.1007/s00216-0114736-6

1696. Piperno S, Tse Sum Bui B, Haupt K, Gheber LA. 2011. Immobilization of Molecularly Imprinted Polymer Nanoparticles in Electrospun Poly(vinyl alcohol) Nanofibers. Langmuir 27: 1547-1550. DOI: 10.1021/la1041234

1697. Kuzmic AE, Vukovic R, Bogdanic G, Smit I, Fles D. 2005. A new approach to the preparation of molecularly imprinted polymers [Novi pristup priredivanju molekulski tiskanih polimera]. Polimeri (Zagreb) 26: 63-68.

1698. Katada N, Akazawa S, Yamakita S, Niwa M. 2004. Molecular sieving overlayer prepared by chemical vapor deposition of silica using molecule as template on metal oxide surface. In Recent Advances In The Science And Technology Of Zeolites And Related Materials, Parts A -C, Studies in Surface Science and Catalysis, Vol. 154, van Steen E, Callanan H, Claeys M (eds). Elsevier: Amsterdam; 710-716. 
1699. Kempe H, Kempe M. 2004. Novel method for the synthesis of molecularly imprinted polymer bead libraries. Macromol. Rapid Commun. 25: 315-320. DOI: 10.1002/marc.200300189

1700. Choi KM, Rogers JA, Shea KJ. 2005. A fabrication of a novel microfluidic reactor microsynthesis of MIP's particles. In Micro- and Nanosystems Materials and Devices, Materials Research Society Symposium Proceedings, Vol. 872, Ozkan CS, La Van.D.A., McNie M, Prasad S (eds). Materials Research Society: Warrendale; 137-141.

1701. Dong HX, Jiang QL, Tong F, Wang ZX, Tang JY. 2009. Preparation of imprinted polymer with d-phenylalanin on silica surface. In Advanced Design and Manufacturing II, Key Engineering Materials, Su DZ, Zhang QB, Zhu SF (eds). Scientific.Net: 541-544. DOI: 10.4028/www.scientific.net/KEM.419420.541

1702. Ikegami T, Mukawa T, Nariai H, Takeuchi T. 2004. Bisphenol A-recognition polymers prepared by covalent molecular imprinting. Anal. Chim. Acta 504: 131-135. DOI: 10.1016/j.aca.2003.08.032

1703. Yang KG, Berg MM, Zhao CS, Ye L. 2009. One-Pot Synthesis of Hydrophilic Molecularly Imprinted Nanoparticles. Macromolecules 42: 8739-8746. DOI: $10.1021 / \mathrm{ma901761z}$

1704. Turson M, Zhuang XL, Liu HN, Jiang P, Dong XC. 2009. Evaluation of the clenbuterol imprinted monolithic column prepared by reversible additionfragmentation chain transfer polymerization. Chin. Chem. Lett. 20: 11361140. DOI: 10.1016/j.cclet.2009.04.012

1705. Kempe M, Fiaccabrino GC, Moreno-Bondi MC, French M, van Rhijn JA. 2000. CREAM: Cartridges with molecularly imprinted recognition elements for antibiotic residues monitoring in milk. In Proceedings of the Euroresidue IV Conference on Residues of Veterinary Drugs in Food, van Ginkel LA, 
Ruiter A (eds). Institute of Public Health and the Environment (RIVM): Bilthoven, The Netherlands; 655-657.

1706. Weiss R, Mizaikoff B. 2002. Towards analysis of mykotoxins in beverages with molecularly imprinted polymers for deoxynivalenol and zearalenone. Mycotoxin Research 18: 89-93. DOI: 10.1007/BF02946071

1707. Fleischer CT, Boos KS. 2000. Analyte-specific online solid-phase extraction. GIT Lab-Fachz. 15-17.

1708. Andersson LI, Hardenborg E, Sandberg-Ställ M, Möller K, Henriksson J, Bramsby-Sjöström I, Olsson LI, Abdel-Rehim M. 2004. Development of a molecularly imprinted polymer based solid-phase extraction of local anaesthetics from human plasma. Anal. Chim. Acta 526: 147-154. DOI: 10.1016/j.aca.2004.09.051

1709. Blahova E, Lehotay J, Skacani I. 2004. The use of molecularly imprinted polymer for selective extraction of (+)-catechin. J. Liq. Chrom. Rel. Technol. 27: 2715-2731. DOI: 10.1081/JLC-200029276

1710. Caro E, Marcé RM, Cormack PAG, Sherrington DC, Borrull F. 2004. Molecularly imprinted solid-phase extraction of naphthalene sulfonates from water. J. Chromatogr. A 1047: 175-180. DOI: 10.1016/j.chroma.2004.07.015

1711. Caro E, Marcé RM, Cormack PAG, Sherrington DC, Borrull F. 2004. A new molecularly imprinted polymer for the selective extraction of naproxen from urine samples by solid-phase extraction. J. Chromatogr B 813: 137-143. DOI: 10.1016/j.jchromb.2004.09.019

1712. Chapuis F, Pichon V, Lanza F, Sellergren B, Hennion MC. 2004. Retention mechanism of analytes in the solid-phase extraction process using molecularly imprinted polymers - Application to the extraction of triazines from complex matrices. J. Chromatogr. $B$ 804: 93-101. DOI: 10.1016/j.jchromb.2003.12.033 
1713. Chassaing C, Stokes J, Venn RF, Lanza F, Sellergren B, Holmberg A, Berggren C. 2004. Molecularly imprinted polymers for the determination of a pharmaceutical development compound in plasma using 96-well MISPE technology. J. Chromatogr. $B$ 804: 71-81. DOI: 10.1016/j.jchromb.2003.12.011

1714. Dong XC, Wang N, Wang SL, Zhang XW, Fan ZJ. 2004. Synthesis and application of molecularly imprinted polymer on selective solid-phase extraction for the determination of monosulfuron residue in soil. $J$. Chromatogr. A 1057: 13-19. DOI: 10.1016/j.chroma.2004.09.036

1715. Ersöz A, Denizli A, Sener I, Atilir A, Diltemiz S, Say R. 2004. Removal of phenolic compounds with nitrophenol-imprinted polymer based on $\pi-\pi$ and hydrogen-bonding interactions. Sep. Purif. Technol. 38: 173-179. DOI: 10.1016/j.seppur.2003.11.004

1716. Feng SY, Lai EPC, Dabek-Zlotorzynska E, Sadeghi S. 2004. Molecularly imprinted solid-phase extraction for the screening of antihyperglycemic biguanides. J. Chromatogr. A 1027: 155-160. DOI: 10.1016/j.chroma.2003.11.042

1717. Kubo T, Hosoya K, Watabe Y, Ikegami T, Tanaka N, Sano T, Kaya K. 2004. Polymer-based adsorption medium prepared using a fragment imprinting technique for homologues of chlorinated bisphenol A produced in the environment. J. Chromatogr. $A$ 1029: 37-41. DOI: 10.1016/j.chroma.2003.12.050

1718. Lai JP, Jiang Y, He XW, Huang JC, Chen F. 2004. Separation and determination of astaxanthin from microalgal and yeast samples by molecularly imprinted microspheres. J. Chromatogr. B 804: 25-30. DOI: 10.1016/j.jchromb.2003.12.010 
1719. Lai JP, Niessner R, Knopp D. 2004. Benzo[a]pyrene imprinted polymers: synthesis, characterization and SPE application in water and coffee samples. Anal. Chim. Acta 522: 137-144. DOI: 10.1016/j.aca.2004.07.003

1720. Martin PD, Jones GR, Stringer F, Wilson ID. 2004. Comparison of extraction of a $\beta$-blocker from plasma onto a molecularly imprinted polymer with liquidliquid extraction and solid phase extraction methods. J. Pharm. Biomed. Anal. 35: 1231-1239. DOI: 10.1016/j.jpba.2004.03.024

1721. Möller K, Crescenzi C, Nilsson U. 2004. Determination of a flame retardant hydrolysis product in human urine by SPE and LC-MS. Comparison of molecularly imprinted solid-phase extraction with a mixed-mode anion exchanger. Anal. Bioanal. Chem. 378: 197-204. DOI: 10.1007/s00216-0032267-5

1722. Möller K, Nilsson U, Crescenzi C. 2004. Investigation of matrix effects of urine on a molecularly imprinted solid-phase extraction. J. Chromatogr $B$ 811: 171-176. DOI: 10.1016/j.jchromb.2004.08.036

1723. Naczk M, Shahidi F. 2004. Extraction and analysis of phenolics in food. $J$. Chromatogr. A 1054: 95-111. DOI: 10.1016/j.chroma.2004.08.059

1724. Olwill A, Hughes $H$, O'Riordain M, McLoughlin P. 2004. The use of molecularly imprinted sol-gels in pharmaceutical separations. Biosens. Bioelectron. 20: 1045-1050. DOI: 10.1016/j.bios.2004.04.029

1725. Pichon V, Chapuis F, Hennion MC. 2004. A dual analytical approach for the selective extraction of organic pollutants in environmental matrices: immunosorbents and molecularly imprinted polymers. Actualité Chim. 277278: $15-26$.

1726. Puri BK, Muñoz-Olivas R, Cámara C. 2004. A new polymeric adsorbent for screening and pre-concentration of organotin compounds in sediments and 
seawater samples. Spectrochim. Acta, $B$ 59: 209-214. DOI: 10.1016/j.sab.2003.12.010

1727. San Vicente B, Navarro-Villoslada F, Moreno-Bondi MC. 2004. Continuous solid-phase extraction and preconcentration of bisphenol $A$ in aqueous samples using molecularly imprinted columns. Anal. Bioanal. Chem. 380: 115-122. DOI: $10.1007 / \mathrm{s} 00216-004-2744-5$

1728. Snowden EM, Bowyer PK, Grbin PR, Bowyer MC. 2004. The removal of mousy off-flavour from wine using molecular imprint technology. In Proceedings of the Twelfth Australian Wine Industry Technical Conference, Blair RJ, Williams PJ, Pretorius IS (eds). Winetitles: Adelaide, Australia; 301-302.

1729. Theodoridis G, Zacharis CK, Tzanavaras PD, Themelis DG, Economou A. 2004. Automated sample preparation based on the sequential injection principle - Solid-phase extraction on a molecularly imprinted polymer coupled on-line to high-performance liquid chromatography. J. Chromatogr. A 1030: 69-76. DOI: 10.1016/j.chroma.2003.12.036

1730. Wang D, Hong SP, Row KH. 2004. Solid extraction of caffeine and theophylline from green tea by molecular imprinted polymers. Kor. J. Chem. Eng. 21: 853-857. DOI: 10.1007/BF02705530

1731. Watabe Y, Hosoya K, Tanaka N, Kubo T, Kondo T, Morita M. 2004. Novel surface-modified molecularly imprinted polymer focused on the removal of interference in environmental water samples. Chem. Lett. 33: 806-807. DOI: $10.1246 / \mathrm{cl} .2004 .806$

1732. Widstrand C, Larsson F, Fiori M, Civitareale C, Mirante S, Brambilla G. 2004. Evaluation of MISPE for the multi-residue extraction of $\beta$-agonists from calves urine. J. Chromatogr. $B$ 804: 85-91. DOI: 10.1016/j.jchromb.2003.12.034 
1733. Wu SG, Lai EPC, Mayer PM. 2004. Molecularly imprinted solid phase extraction-pulsed elution-mass spectrometry for determination of cephalexin and $\alpha$-aminocephalosporin antibiotics in human serum. J. Pharm. Biomed. Anal. 36: 483-490. DOI: 10.1016/j.jpba.2003.05.001

1734. Zhou SN, Lai EPC, Miller JD. 2004. Analysis of wheat extracts for ochratoxin A by molecularly imprinted solid-phase extraction and pulsed elution. Anal. Bioanal. Chem. 378: 1903-1906. DOI: 10.1007/s00216-003-2409-9

1735. Bastide J, Cambon JP, Breton F, Piletsky SA, Rouillon R. 2005. The use of molecularly imprinted polymers for extraction of sulfonylurea herbicides. Anal. Chim. Acta 542: 97-103. DOI: 10.1016/j.aca.2005.02.054

1736. Carabias-Martínez R, Rodríguez-Gonzalo E, Herrero-Hernández E. 2005. Determination of triazines and dealkylated and hydroxylated metabolites in river water using a propazine-imprinted polymer. J. Chromatogr. A 1085: 199-206. DOI: 10.1016/j.chroma.2005.05.084

1737. Caro E, Marce RM, Cormack PAG, Sherrington DC, Borrull F. 2005. Synthesis and application of an oxytetracycline imprinted polymer for the solid-phase extraction of tetracycline antibiotics. Anal. Chim. Acta 552: 8186. DOI: 10.1016/j.aca.2005.07.047

1738. Caro E, Marcé RM, Cormack PAG, Sherrington DC, Borrull F. 2005. Selective enrichment of anti-inflammatory drugs from river water samples by solid-phase extraction with a molecularly imprinted polymer. J. Sep. Sci. 28: 2080-2085. DOI: 10.1002/jssc.200500027

1739. Cobb Z, Andersson LI. 2005. Determination of ropivacaine in human plasma using highly selective molecular imprint-based solid phase extraction and fast LC-MS analysis. Anal. Bioanal. Chem. 383: 645-650. DOI: $10.1007 / \mathrm{s} 00216-005-0042-5$ 
1740. Dong XC, Wang W, Ma SJ, Sun H, Li Y, Guo JQ. 2005. Molecularly imprinted solid-phase extraction of (-)-ephedrine from Chinese Ephedra. $\mathrm{J}$. Chromatogr. A 1070: 125-130. DOI: 10.1016/j.chroma.2005.03.017

1741. Fiori M, Civitareale C, Mirante S, Magaro E, Brambilla G. 2005. Evaluation of two different clean-up steps, to minimise ion suppression phenomena in ion trap liquid chromatography-tandem mass spectrometry for the multiresidue analysis of $\beta$-agonists in calves urine. Anal. Chim. Acta 529: 207210. DOI: 10.1016/j.aca.2004.08.068

1742. Gallego-Gallegos M, Muñoz-Olivas R, Martin-Esteban A, Cámara C. 2005. Synthesis and evaluation of molecularly imprinted polymers for organotin compounds: a screening method for tributyltin detection in seawater. Anal. Chim. Acta 531: 33-39. DOI: 10.1016/j.aca.2004.09.052

1743. Guzmán-Vázquez de Prada A, Martínez-Ruiz P, Reviejo AJ, Pingarrón JM. 2005. Solid-phase molecularly imprinted on-line preconcentration and voltammetric determination of sulfamethazine in milk. Anal. Chim. Acta 539: 125-132. DOI: 10.1016/j.aca.2005.02.068

1744. Han DM, Fang GZ, Yan XP. 2005. Preparation and evaluation of a molecularly imprinted sol-gel material for on-line solid-phase extraction coupled with high performance liquid chromatography for the determination of trace pentachlorophenol in water samples. J. Chromatogr. A 1100: 131136. DOI: 10.1016/j.chroma.2005.09.035

1745. Harvey SD. 2005. Molecularly imprinted polymers for selective analysis of chemical warfare surrogate and nuclear signature compounds in complex matrices. J. Sep. Sci. 28: 1221-1230. DOI: 10.1002/jssc.200301702

1746. Hu SG, Li L, He XW. 2005. Solid-phase extraction of esculetin from the ash bark of Chinese traditional medicine by using molecularly imprinted polymers. J. Chromatogr. A 1062: 31-37. DOI: 10.1016/j.chroma.2004.11.036 
1747. Hu SG, Li L, He XW. 2005. Comparison of trimethoprim molecularly imprinted polymers in bulk and in sphere as the sorbent for solid-phase extraction and extraction of trimethoprim from human urine and pharmaceutical tablet and their determination by high-performance liquid chromatography. Anal. Chim. Acta 537: 215-222. DOI: 10.1016/j.aca.2005.01.018

1748. Karasová G, Lehotay J, Sádecká J, Skacáni I, Lachová M. 2005. Selective extraction of derivates of $p$-hydroxy-benzoic acid from plant material by using a molecularly imprinted polymer. J. Sep. Sci. 28: 2468-2476. DOI: $10.1002 /$ jssc. 200500190

1749. Kawaguchi M, Hayatsu $Y$, Nakata H, Ishii $Y$, Ito R, Saito K, Nakazawa H. 2005. Molecularly imprinted solid phase extraction using stable isotope labeled compounds as template and liquid chromatography-mass spectrometry for trace analysis of bisphenol A in water sample. Anal. Chim. Acta 539: 83-89. DOI: 10.1016/j.aca.2005.03.005

1750. Kootstra PR, Kuijpers CJPF, Wubs KL, van Doorn D, Sterk SS, van Ginkel LA, Stephany RW. 2005. The analysis of $\beta$-agonists in bovine muscle using molecular imprinted polymers with ion trap LCMS screening. Anal. Chim. Acta 529: 75-81. DOI: 10.1016/j.aca.2004.09.053

1751. Kubo T, Hosoya K, Watabe Y, Tanaka N, Sano T, Kaya K. 2005. Dependence of the pretreatment efficiency of polymer-based adsorbents for environmental water on their uniformity and size. J. Polym. Sci. A,Polym. Chem. 43: 2112-2118. DOI: 10.1002/pola.20685

1752. Liu SX, Dong XC, Li FX. 2005. Evaluation of the (-)-ephedrine imprinted polymers with high affinity for template molecule synthesized using redox initiation system. Anal. Lett. 38: 227-236. DOI: 10.1081/AL-200045128 
1753. McElhiney J, Lawton LA. 2005. Detection of the cyanobacterial hepatotoxins microcystins. Toxicol. Appl. Pharm. 203: 219-230. DOI: 10.1016/j.taap.2004.06.002

1754. Meng ZH, Chen W, Mulchandani A. 2005. Removal of estrogenic pollutants from contaminated water using molecularly imprinted polymers. Environ. Sci. Technol. 39: 8958-8962. DOI: 10.1021/es0505292

1755. Musteata FM, Pawliszyn J. 2005. Study of ligand-receptor binding using SPME: Investigation of receptor, free, and total ligand concentrations. J. Proteome Res. 4: 789-800. DOI: 10.1021/pr049768z

1756. Nakamura M, Ono M, Nakajima T, Ito Y, Aketo T, Haginaka J. 2005. Uniformly sized molecularly imprinted polymer for atropine and its application to the determination of atropine and scopolamine in pharmaceutical preparations containing Scopolia extract. J. Pharm. Biomed. Anal. 37: 231-237. DOI: 10.1016/j.jpba.2004.10.017

1757. Puoci F, Garreffa C, lemma F, Muzzalupo R, Spizzirri UG, Picci N. 2005. Molecularly imprinted solid phase extraction for detection of sudan I in food matrices. Food Chem. 93: 349-353. DOI: 10.1016/j.foodchem.2004.11.014

1758. Tamayo FG, Casillas JL, Martin-Esteban A. 2005. Evaluation of new selective molecularly imprinted polymers prepared by precipitation polymerisation for the extraction of phenylurea herbicides. J. Chromatogr. $A$ 1069: 173-181. DOI: 10.1016/j.chroma.2005.02.029

1759. Tamayo FG, Casillas JL, Martin-Esteban A. 2005. Clean up of phenylurea herbicides in plant sample extracts using molecularly imprinted polymers. Anal. Bioanal. Chem. 381: 1234-1240. DOI: 10.1007/s00216-005-3071-1

1760. Tang YW, Huang ZF, Yang $T$, Hu XG, Jiang XO. 2005. The characteristic and application of molecularly imprinted polymer: Efficient sample preconcentration of antibiotic cefathiamidine from human plasma and serum 
by solid phase extraction. Anal. Lett. 38: 219-226. DOI: 10.1081/AL200045122

1761. Tarley CRT, Kubota LT. 2005. Molecularly-imprinted solid phase extraction of catechol from aqueous effluents for its selective determination by differential pulse voltammetry. Anal. Chim. Acta 548: 11-19. DOI: 10.1016/j.aca.2005.05.055

1762. van Hoof N, Courtheyn D, Antignac J-P, van de Weile M, Poelmans S, Noppe $\mathrm{H}$, de Brabander $\mathrm{H}$. 2005. Multi-residue liquid chromatography/tandem mass spectrometric analysis of $\beta$-agonists in urine using molecular imprinted polymers. Rapid Commun. Mass Spectrosc. 19: 2801-2808. DOI: 10.1002/rcm.2126

1763. Visser N, Lingeman H, Irth H. 2005. Sample preparation for peptides and proteins in biological matrices prior to liquid chromatography and capillary zone electrophoresis. Anal. Bioanal. Chem. 382: 535-558. DOI: 10.1007/s00216-005-3120-9

1764. Watabe Y, Hosoya K, Tanaka N, Kondo T, Morita M, Kubo T. 2005. LC/MS determination of bisphenol $A$ in river water using a surface-modified molecularly-imprinted polymer as an on-line pretreatment device. Anal. Bioanal. Chem. 381: 1193-1198. DOI: 10.1007/s00216-004-3031-1

1765. Watabe Y, Hosoya K, Tanaka N, Kubo T, Kondo T, Morita M. 2005. Novel surface modified molecularly imprinted polymer focused on the removal of interference in environmental water samples for chromatographic determination. J. Chromatogr. A 1073: 363-370. DOI: 10.1016/j.chroma.2004.09.016

1766. Xia Y, McGuffey JE, Bhattacharyya S, Sellergren B, Yilmaz E, Wang L, Bernert JT. 2005. Analysis of the Tobacco-Specific Nitrosamine 4(Methylnitrosamino)-1-(3-pyridyl)-1-butanol in Urine by Extraction on a Molecularly Imprinted Polymer Column and Liquid 
Chromatography/Atmospheric Pressure Ionization Tandem Mass Spectrometry. Anal. Chem. 77: 7639-7645. DOI: 10.1021/ac058027u

1767. Yang J, Zhu XL, Cai JB, De Sui Q, Gao Y, Zhang L. 2005. A new molecularly imprinted polymer for solid-phase extraction of cotinine from human urine. Chin. Chem. Lett. 16: 1503-1506.

1768. Yu JCC, Krushkova S, Lai EPC, Dabek-Zlotorzynska E. 2005. Molecularlyimprinted polypyrrole-modified stainless steel frits for selective solid phase preconcentration of ochratoxin A. Anal. Bioanal. Chem. 382: 1534-1540. DOI: $10.1007 / \mathrm{s} 00216-005-3342-x$

1769. Zhu XL, Yang J, Su QD, Cai JB, Gao Y. 2005. Selective solid-phase extraction using molecularly imprinted polymer for the analysis of polar organophosphorus pesticides in water and soil samples. J. Chromatogr. A 1092: 161-169. DOI: 10.1016/j.chroma.2005.07.037

1770. Zhu XL, Yang J, Su QD, Cai JB, Gao Y. 2005. Molecularly imprinted polymer for monocrotophos and its binding characteristics for organophosphorus pesticides. Annali Chim. 95: 877-885. DOI: 10.1002/adic.200590100

1771. Zurutuza A, Bayoudh S, Cormack PAG, Dambies L, Deere J, Bischoff R, Sherrington DC. 2005. Molecularly imprinted solid-phase extraction of cocaine metabolites from aqueous samples. Anal. Chim. Acta 542: 14-19. DOI: 10.1016/j.aca.2004.12.019

1772. Abdel-Rehim M, Andersson LI, Altun Z, Blomberg LG. 2006. Microextraction in Packed Syringe Online with Liquid Chromatography-Tandem Mass Spectrometry: Molecularly Imprinted Polymer as Packing Material for MEPS in Selective Extraction of Ropivacaine from Plasma. J. Liq. Chrom. Rel. Technol. 29: 1725-1736. DOI: 10.1080/10826070600716843 
1773. Breton F, Euzet P, Piletsky SA, Giardi MT, Rouillon R. 2006. Integration of photosynthetic biosensor with molecularly imprinted polymer-based solid phase extraction cartridge. Anal. Chim. Acta 569: 50-57. DOI: 10.1016/j.aca.2006.03.086

1774. Cacho C, Turiel E, Martin-Esteban A, Ayala D, Perez-Conde C. 2006. Semicovalent imprinted polymer using propazine methacrylate as template molecule for the clean-up of triazines in soil and vegetable samples. $J$. Chromatogr. A 1114: 255-262. DOI: 10.1016/j.chroma.2006.02.051

1775. Cao H, Xiao JB, Xu M. 2006. Evaluation of new selective molecularly imprinted polymers for the extraction of resveratrol from Polygonum cuspidatum. Macromol. Res. 14: 324-330. DOI: 10.1007/BF03219089

1776. Caro E, Marcé RM, Cormack PAG, Sherrington DC, Borrull F. 2006. Novel enrofloxacin imprinted polymer applied to the solid-phase extraction of fluorinated quinolones from urine and tissue samples. Anal. Chim. Acta 562: 145-151. DOI: 10.1016/j.aca.2006.01.080

1777. Caro E, Marcé RM, Cormack PAG, Sherrington DC, Borrull F. 2006. Direct determination of ciprofloxacin by mass spectrometry after a two-step solidphase extraction using a molecularly imprinted polymer. J. Sep. Sci. 29: 1230-1236. DOI: 10.1002/jssc.200500439

1778. Castell OK, Allender CJ, Barrow DA. 2006. Novel biphasic separations utilising highly selective molecularly imprinted polymers as biorecognition solvent extraction agents. Biosens. Bioelectron. 22: 526-533. DOI: 10.1016/j.bios.2006.07.017

1779. Chapuis F, Mullot JU, Pichon V, Tuffal G, Hennion MC. 2006. Molecularly imprinted polymers for the clean-up of a basic drug from environmental and biological samples. J. Chromatogr. A 1135: 127-134. DOI: 10.1016/j.chroma.2006.09.076 
1780. Ding L, Li H, Tang F, Yao SZ. 2006. Molecularly Imprinted Solid Phase Extraction of Epicatechin from Tea Beverage. Anal. Lett. 39: 2373-2385. DOI: $10.1080 / 00032710600748780$

1781. Farrington K, Magner E, Regan F. 2006. Predicting the performance of molecularly imprinted polymers: Selective extraction of caffeine by molecularly imprinted solid phase extraction. Anal. Chim. Acta 566: 60-68. DOI: 10.1016/j.aca.2006.02.057

1782. Harvey SD, Mong GM, Ozanich RM, Mclean JS, Goodwin SM, Valentine NB, Fredrickson JK. 2006. Preparation and evaluation of spore-specific affinity-augmented bio-imprinted beads. Anal. Bioanal. Chem. 386: 211-219. DOI: 10.1007/s00216-006-0622-z

1783. Jiang M, Zhang JH, Mei SR, Shi Y, Zou LJ, Zhu YX, Dai K, Lu B. 2006. Direct enrichment and high performance liquid chromatography analysis of ultra-trace Bisphenol $\mathrm{A}$ in water samples with narrowly dispersible Bisphenol A imprinted polymeric microspheres column. J. Chromatogr. A 1110: 27-34. DOI: 10.1016/j.chroma.2006.01.051

1784. Jin YZ, Row KH. 2006. Molecularly imprinted solid-phase extraction of caffeine from green tea. J. Ind. Eng. Chem. 12: 494-499.

1785. Karasová G, Lehotay J, Klodzinska E, Gadzala-Kopciuch R, Buszewski B. 2006. Comparison of several extraction methods for the isolation of benzoic acid derivatives from Melissa officinalis. J. Liq. Chrom. Rel. Technol. 29: 1633-1644. DOI: 10.1080/10826070600678365

1786. Kubo T, Nomachi M, Nemoto K, Sano T, Hosoya K, Tanaka N, Kaya K. 2006. Chromatographic separation for domoic acid using a fragment imprinted polymer. Anal. Chim. Acta 577: 1-7. DOI: 10.1016/j.aca.2006.06.028 
1787. Le Moullec S, Begos A, Pichon V, Bellier B. 2006. Selective extraction of organophosphorus nerve agent degradation products by molecularly imprinted solid-phase extraction. J. Chromatogr. A 1108: 7-13. DOI: 10.1016/j.chroma.2005.12.105

1788. Lin LQ, Zhang J, Fu Q, He LC, Li YC. 2006. Concentration and extraction of sinomenine from herb and plasma using a molecularly imprinted polymer as the stationary phase. Anal. Chim. Acta 561: 178-182. DOI: 10.1016/j.aca.2006.01.011

1789. Liu HY, Li SH, Yang GL. 2006. Solid-phase extraction of trace diniconazole on imprinted polymers stationary phase. Chem. J. Internet 8: Art. No.: 089056ne.

1790. Malitesta C, Picca RA, Ciccarella G, Sgobba V, Brattoli M. 2006. Synthesis of a Molecularly Imprinted Polymer for Dioxin. Sensors 6: 915-924. DOI: $10.3390 / \mathrm{s} 6080915$

1791. Méndez-Palacios I, López-Luna A, Bárzana E, Jiménez-Guzmán J, GarcíaGaribay M. 2006. Development of a Molecularly Imprinted Polymer (MIP) for the Recovery of Lactoferrin. In IUFoST World Congress: 13th World Congress of Food Science \& Technology, 1309-1313. DOI: 10.1051/IUFoST:20060639

1792. Ou JJ, Hu LH, Hu LG, Li X, Zou HF. 2006. Determination of phenolic compounds in river water with on-line coupling bisphenol A imprinted monolithic precolumn with high performance liquid chromatography. Talanta 69: 1001-1006. DOI: 10.1016/j.talanta.2005.12.003

1793. Pérez-Moral N, Mayes AG. 2006. Direct rapid synthesis of MIP beads in SPE cartridges. Biosens. Bioelectron. 21: 1798-1803. DOI: 10.1016/j.bios.2005.08.014 
1794. Pichon V, Haupt K. 2006. Affinity separations on molecularly imprinted polymers with special emphasis on solid-phase extraction. J. Liq. Chrom. Rel. Technol. 29: 989-1023. DOI: 10.1080/10826070600574739

1795. Schirmer C, Meisel H. 2006. Synthesis of a molecularly imprinted polymer for the selective solid-phase extraction of chloramphenicol from honey. $J$. Chromatogr. A 1132: 325-328. DOI: 10.1016/j.chroma.2006.09.032

1796. Shi Y, Zhang JH, Shi D, Jiang M, Zhu YX, Mei SR, Zhou YK, Dai K, Lu B. 2006. Selective solid-phase extraction of cholesterol using molecularly imprinted polymers and its application in different biological samples. $J$. Pharm. Biomed. Anal. 42: 549-555. DOI: 10.1016/j.jpba.2006.05.022

1797. Silva RGC, Augusto F. 2006. Sol-gel molecular imprinted ormosil for solidphase extraction of methylxanthines. J. Chromatogr. A 1114: 216-223. DOI: 10.1016/j.chroma.2006.03.073

1798. Suedee R, Seechamnanturakit V, Canyuk B, Ovatlarnporn C, Martin GP. 2006. Temperature sensitive dopamine-imprinted (N,N-methylene-bisacrylamide cross-linked) polymer and its potential application to the selective extraction of adrenergic drugs from urine. J. Chromatogr. A 1114: 239-249. DOI: 10.1016/j.chroma.2006.02.033

1799. Sun HW, Zhao XL. 2006. On-line solid-phase extraction of arsanilic acid from water with arsanilic acid imprinted monolith. Chem. J. Internet 8 :

1800. Theodoridis G, Lasáková M, Skeríková V, Tegou A, Giantsiou N, Jandera P. 2006. Molecular imprinting of natural flavonoid antioxidants: Application in solid-phase extraction for the sample pretreatment of natural products prior to HPLC analysis. J. Sep. Sci. 29: 2310-2321. DOI: 10.1002/jssc.200500492

1801. Urraca JL, Marazuela MD, Merino ER, Orellana G, Moreno-Bondi MC. 2006. Molecularly imprinted polymers with a streamlined mimic for zearalenone 
analysis. J. Chromatogr. A 1116: 127-134. DOI: 10.1016/j.chroma.2006.03.032

1802. Urraca JL, Marazuela MD, Moreno-Bondi MC. 2006. Molecularly imprinted polymers applied to the clean-up of zearalenone and $\alpha$-zearalenol from cereal and swine feed sample extracts. Anal. Bioanal. Chem. 385: 11551161. DOI: $10.1007 / \mathrm{s} 00216-006-0343-3$

1803. Watabe Y, Kubo T, Nishikawa T, Fujita T, Kaya K, Hosoya K. 2006. Fully automated liquid chromatography-mass spectrometry determination of $17 \alpha-$ estradiol in river water. J. Chromatogr. $A$ 1120: 252-259. DOI: 10.1016/j.chroma.2006.01.057

1804. Yang GL, Liu HY, Wang MM, Liu SB, Chen Y. 2006. Chromatographic characterization and solid-phase extraction on diniconazole-imprinted polymers stationary phase. Reac. Func. Polym. 66: 579-583. DOI: 10.1016/j.reactfunctpolym.2005.10.029

1805. Yang J, Hu Y, Cai JB, Zhu XL, Su QD. 2006. A new molecularly imprinted polymer for selective extraction of cotinine from urine samples by solidphase extraction. Anal. Bioanal. Chem. 384: 761-768. DOI: 10.1007/s00216005-0221-4

1806. Yin JF, Wang SM, Yang GQ, Yang GL, Chen Y. 2006. Molecularly imprinted solid-phase extraction for rapid screening of mycophenolic acid in human plasma. J. Chromatogr B 844: 142-147. DOI: 10.1016/j.jchromb.2006.07.009

1807. Yu JCC, Lai EPC. 2006. Molecularly imprinted polypyrrole modified carbon nanotubes on stainless steel frit for selective micro solid phase preconcentration of ochratoxin A. Reac. Func. Polym. 66: 702-711. DOI: 10.1016/j.reactfunctpolym.2005.10.021 
1808. Zhang JH, Jiang M, Zou LJ, Shi D, Mei SR, Zhu YX, Shi Y, Dai K, Lu B. 2006. Selective solid-phase extraction of bisphenol A using molecularly imprinted polymers and its application to biological and environmental samples. Anal. Bioanal. Chem. 385: 780-786. DOI: 10.1007/s00216-0060406-5

1809. Zhu XF, Cao Q, Hou NB, Wang GS, Ding ZT. 2006. The preparation and the recognition property of molecularly imprinted polymer of podophyllotoxin. Anal. Chim. Acta 561: 171-177. DOI: 10.1016/j.aca.2006.01.009

1810. Zhu XL, Cai JB, Yang J, Su QD, Gao Y. 2006. Films coated with molecular imprinted polymers for the selective stir bar sorption extraction of monocrotophos. J. Chromatogr. A 1131: 37-44. DOI: 10.1016/j.chroma.2006.07.041

1811. Appell M, Kendra DF, Kim EK, Maragos CM. 2007. Synthesis and evaluation of molecularly imprinted polymers as sorbents of moniliformin. Food Addit. Contam. 24: 43-52. DOI: 10.1080/02652030600887586

1812. Ariffin MM, Miller El, Cormack PAG, Anderson RA. 2007. Molecularly Imprinted Solid-Phase Extraction of Diazepam and Its Metabolites from Hair Samples. Anal. Chem. 79: 256-262. DOI: 10.1021/ac061062w

1813. Baggiani C, Baravalle P, Giraudi G, Tozzi C. 2007. Molecularly imprinted solid-phase extraction method for the high-performance liquid chromatographic analysis of fungicide pyrimethanil in wine. J. Chromatogr. A 1141: 158-164. DOI: 10.1016/j.chroma.2006.12.016

1814. Baydemir G, Andaç M, Bereli N, Say R, Denizli A. 2007. Selective Removal of Bilirubin from Human Plasma with Bilirubin-Imprinted Particles. Ind. Eng. Chem. Res. 46: 2843-2852. DOI: 10.1021/ie0611249

1815. Beltran A, Caro E, Marcé RM, Cormack PAG, Sherrington DC, Borrull F. 2007. Synthesis and application of a carbamazepine-imprinted polymer for 
solid-phase extraction from urine and wastewater. Anal. Chim. Acta 597: 611. DOI: $10.1016 /$ j.aca.2007.06.040

1816. Boyd B, Bjork H, Billing J, Shimelis O, Axelsson S, Leonora M, Yilmaz E. 2007. Development of an improved method for trace analysis of chloramphenicol using molecularly imprinted polymers. J. Chromatogr. A 1174: 63-71. DOI: 10.1016/j.chroma.2007.08.072

1817. Bravo JC, Garcinuño RM, Fernández P, Durand JS. 2007. A new molecularly imprinted polymer for the on-column solid-phase extraction of diethylstilbestrol from aqueous samples. Anal. Bioanal. Chem. 388: 10391045. DOI: 10.1007/s00216-007-1219-x

1818. Cobb Z, Sellergren B, Andersson LI. 2007. Water-compatible molecularly imprinted polymers for efficient direct injection on-line solid-phase extraction of ropivacaine and bupivacaine from human plasma. Analyst 132: 12621271. DOI: 10.1039/b711116a

1819. Djozan D, Baheri T, Azar MHP, Mahkam M. 2007. Preparation of new fibers on the basis of codeine imprinted polymer. Mater. Manufact. Proc. 22: 758763. DOI: $10.1080 / 10426910701385390$

1820. Djozan D, Baheri T. 2007. Preparation and evaluation of solid-phase microextraction fibers based on monolithic molecularly imprinted polymers for selective extraction of diacetylmorphine and analogous compounds. $J$. Chromatogr. A 1166: 16-23. DOI: 10.1016/j.chroma.2007.08.003

1821. Dzygiel P, O'Donnell E, Fraier D, Chassaing C, Cormack PAG. 2007. Evaluation of water-compatible molecularly imprinted polymers as solidphase extraction sorbents for the selective extraction of sildenafil and its desmethyl metabolite from plasma samples. J. Chromatogr B 853: 346-353. DOI: 10.1016/j.jchromb.2007.03.037 
1822. Fernández-Llano L, Blanco-López MC, Lobo-Castañón MJ, MirandaOrdieres AJ, Tuñón-Blanco P. 2007. Determination of Diclofenac in Urine Samples by Molecularly-Imprinted Solid-Phase Extraction and Adsorptive Differential Pulse Voltammetry. Electroanalysis 19: 1555-1561. DOI: 10.1002/elan.200703895

1823. Figueiredo EC, Tarley CRT, Kubota LT, Rath S, Arruda MAZ. 2007. On-line molecularly imprinted solid phase extraction for the selective spectrophotometric determination of catechol. Microchem. J. 85: 290-296. DOI: 10.1016/j.microc.2006.07.004

1824. Hu ML, Jiang M, Wang P, Mei SR, Lin YF, Hu XZ, Shi Y, Lu B, Dai K. 2007. Selective solid-phase extraction of tebuconazole in biological and environmental samples using molecularly imprinted polymers. Anal. Bioanal. Chem. 387: 1007-1016. DOI: 10.1007/s00216-006-1004-2

1825. Hu XG, Hu YL, Li GK. 2007. Development of novel molecularly imprinted solid-phase microextraction fiber and its application for the determination of triazines in complicated samples coupled with high-performance liquid chromatography. J. Chromatogr. A 1147: 1-9. DOI: 10.1016/j.chroma.2007.02.037

1826. Hu XG, Hu YL, Li GK. 2007. Preparation and characterization of prometryn molecularly imprinted solid-phase microextraction fibers. Anal. Lett. 40: 645660. DOI: 10.1080/00032710600966127

1827. Jiang XM, Tian W, Zhao CD, Zhang HX, Liu MC. 2007. A novel sol-gelmaterial prepared by a surface imprinting technique for the selective solidphase extraction of bisphenol A. Talanta 72: 119-125. DOI: 10.1016/j.talanta.2006.10.006

1828. Jin Y, Kyung HR. 2007. Solid-phase extraction of caffeine and catechin compounds from green tea by caffeine molecular imprinted polymer. Bull. Kor. Chem. Soc. 28: 276-280. 
1829. Kublickas R, Werner C, Jariene G, Voit B, Lasas L. 2007. Polyacrylamide gels containing ionized functional groups for the molecular imprinting of human growth hormone. Polym. Bull. 58: 611-617. DOI: 10.1007/s00289006-0694-0

1830. Lachová M, Lehotay J, Karasová G, Skacáni I, Armstrong DW. 2007. Isolation of L-theanine from plant material using a molecularly imprinted polymer. J. Liq. Chrom. Rel. Technol. 30: 2045-2058. DOI: 10.1080/10826070701435053

1831. Le Moullec S, Truong L, Montauban C, Begos A, Pichon V, Bellier B. 2007. Extraction of alkyl methylphosphonic acids from aqueous samples using a conventional polymeric solid-phase extraction sorbent and a molecularly imprinted polymer. J. Chromatogr. A 1139: 171-177. DOI: 10.1016/j.chroma.2006.11.022

1832. Le Noir M, Lepeuple AS, Guieysse B, Mattiasson B. 2007. Selective removal of $17 \alpha$-estradiol at trace concentration using a molecularly imprinted polymer. Water Res. 41: 2825-2831. DOI: 10.1016/j.watres.2007.03.023

1833. Le Noir M, Plieva F, Hey T, Guieysse B, Mattiasson B. 2007. Macroporous molecularly imprinted polymer/cryogel composite systems for the removal of endocrine disrupting trace contaminants. J. Chromatogr. A 1154: 158-164. DOI: 10.1016/j.chroma.2007.03.064

1834. Lulinski P, Maciejewska D, Bamburowicz-Klimkowska M, Szutowski M. 2007. Dopamine-imprinted polymers: Template-monomer interactions, analysis of template removal and application to solid phase extraction. Molecules 12: 2434-2449. DOI: 10.3390/12112434

1835. Lv YQ, Lin ZX, Feng W, Zhou X, Tan TW. 2007. Selective recognition and large enrichment of dimethoate from tea leaves by molecularly imprinted polymers. Biochem. Eng. J. 36: 221-229. DOI: 10.1016/j.bej.2007.02.023 
1836. Lv YQQ, Lin ZX, Feng W, Tan TW. 2007. Evaluation of the Polymerization and Recognition Mechanism for Phenol Imprinting SPE. Chromatographia 66: 339-347. DOI: 10.1365/s10337-007-0336-1

1837. Maury D, Couderc F, Garrigues JC, Poinsot V. 2007. Synthesis and evaluation of new lipomonosaccharide-imprinted polymers as MISPE supports. Talanta 73: 340-345. DOI: 10.1016/j.talanta.2007.03.051

1838. Mohamed R, Richoz-Payot J, Gremaud E, Mottier P, Yilmaz E, Tabet JC, Guy PA. 2007. Advantages of Molecularly Imprinted Polymers LC-ESIMS/MS for the Selective Extraction and Quantification of Chloramphenicol in Milk-Based Matrixes. Comparison with a Classical Sample Preparation. Anal. Chem. 79: 9557-9565. DOI: 10.1021/ac7019859

1839. Odabasi M, Say R, Denizli A. 2007. Molecular imprinted particles for lysozyme purification. Mater. Sci. Eng.: C 27: 90-99. DOI: 10.1016/j.msec.2006.03.002

1840. Panahi R, Vasheghani-Farahani E, Shojaosadati SA. 2007. Separation of Ilysine from dilute aqueous solution using molecular imprinting technique. Biochem. Eng. J. 35: 352-356. DOI: 10.1016/j.bej.2007.01.027

1841. Puoci F, Cirillo G, Curcio M, lemma F, Spizzirri UG, Picci N. 2007. Molecularly imprinted solid phase extraction for the selective HPLC determination of $\alpha$-tocopherol in bay leaves. Anal. Chim. Acta 593: 164-170. DOI: 10.1016/j.aca.2007.04.053

1842. Sambe H, Hoshina K, Haginaka J. 2007. Molecularly imprinted polymers for triazine herbicides prepared by multi-step swelling and polymerization method: Their application to the determination of methylthiotriazine herbicides in river water. J. Chromatogr. A 1152: 130-137. DOI: 10.1016/j.chroma.2006.09.003 
1843. Shi XZ, Wu AB, Zheng SL, Li RX, Zhang DB. 2007. Molecularly imprinted polymer microspheres for solid-phase extraction of chloramphenicol residues in foods. J. Chromatogr $B$ 850: 24-30. DOI: 10.1016/j.jchromb.2006.10.057

1844. Turiel E, Tadeo JL, Martin-Esteban A. 2007. Molecularly Imprinted Polymeric Fibers for Solid-Phase Microextraction. Anal. Chem. 79: 30993104. DOI: $10.1021 / \mathrm{ac} 062387 f$

1845. Turiel E, Martin-Esteban A, Tadeo JL. 2007. Molecular imprinting-based separation methods for selective analysis of fluoroquinolones in soils. $\mathrm{J}$. Chromatogr. A 1172: 97-104. DOI: 10.1016/j.chroma.2007.10.003

1846. Urraca JL, Moreno-Bondi MC, Hall AJ, Sellergren B. 2007. Direct Extraction of Penicillin $G$ and Derivatives from Aqueous Samples Using a Stoichiometrically Imprinted Polymer. Anal. Chem. 79: 695-701. DOI: 10.1021/ac061622r

1847. Wang GS, Cao QE, Ding ZT, Wang YG, Yang MH. 2007. Preparation and Characteristics of Esculin-Imprinted Polymers. Helv. Chim. Acta 90: 11791189. DOI: $10.1002 /$ hlca.200790117

1848. Wang S, Xu Z, Fang G, Duan Z, Zhang Y, Chen S. 2007. Synthesis and Characterization of a Molecularly Imprinted Silica Gel Sorbent for the OnLine Determination of Trace Sudan I in Chilli Powder through HighPerformance Liquid Chromatography. J. Agric. Food Chem. 55: 3869-3876. DOI: $10.1021 /$ ff070261t

1849. Wei Y, Qu LH, Owen C, Lai EPC. 2007. Encapsulation of quantum dots and carbon nanotubes with polypyrrole in a syringe needle for automated molecularly imprinted solid phase pre-concentration of ochratoxin $A$ in red wine analysis. Sens. Instrumen. Food Qual. 1: 133-141. DOI: 10.1007/s11694-007-9014-0 
1850. Wei Y, Qiu LH, Yu JCC, Lai EPC. 2007. Molecularly Imprinted Solid Phase Extraction in a Syringe Needle Packed with Polypyrrole-encapsulated Carbon Nanotubes for Determination of Ochratoxin A in Red Wine. Food Sci. Technol. Int. 13: 375-380. DOI: 10.1177/1082013207085914

1851. Widstrand C, Boyd B, Billing J, Rees A. 2007. Efficient extraction of toxic compounds from complex matrices using molecularly imprinted polymers. Am. Lab. 39: 23-24.

1852. Widstrand C, Boyd B, Shimelis O, Trinh A, Brandes H. 2007. Highly selectve trace level extraction using molecularly imprinted polymer solid-phase extraction. The Column 3: 28-34.

1853. Yan H, Qiao FX, Row KH. 2007. Molecularly Imprinted-Matrix Solid-Phase Dispersion for Selective Extraction of Five Fluoroquinolones in Eggs and Tissue. Anal. Chem. 79: 8242-8248. DOI: 10.1021/ac070644q

1854. Yan SL, Fang YJ, Yao W, Gao ZX. 2007. Characterization and quality assessment of binding properties of the monocrotophos molecularly imprinted microspheres prepared by precipitation polymerization in toluene. Polym. Eng. Sci. 47: 1302-1308. DOI: 10.1002/pen.20806

1855. Yang CY, Zhang ZJ, Chen SM, Yang F. 2007. Molecularly imprinted on-line solid-phase extraction combined with chemiluminescence for the determination of pazufloxacin mesilate. Microchim. Acta 159: 299-304. DOI: 10.1007/s00604-007-0758-3

1856. Yang J, Hu Y, Cai JB, Zhu XL, Su QD, Hu YQ, Liang FX. 2007. Selective hair analysis of nicotine by molecular imprinted solid-phase extraction: An application for evaluating tobacco smoke exposure. Food Chem. Toxicol. 45: 896-903. DOI: 10.1016/j.fct.2006.11.010 
1857. Yu JCC, Lai EPC. 2007. Determination of ochratoxin A in red wines by multiple pulsed elutions from molecularly imprinted polypyrrole. Food Chem. 105: 301-310. DOI: 10.1016/j.foodchem.2006.11.049

1858. Yu JCC, Hrdina A, Mancini C, Lai EPC. 2007. Molecularly imprinted polypyrrole encapsulated carbon nanotubes in stainless steel frit for micro solid phase extraction of estrogenic compounds. J. Nanosci. Nanotechnol. 7: 3095-3103. DOI: 10.1166/jnn.2007.666

1859. Zhu QH, He JF, Feng JY. 2007. Optimization of the process parameters of synthesis of vinblastine imprinted polymer. Eur. Polym. J. 43: 4043-4051. DOI: 10.1016/j.eurpolymj.2007.06.036

1860. Alizadeh T. 2008. Development of a molecularly imprinted polymer for pyridoxine using an ion-pair as template. Anal. Chim. Acta 623: 101-108. DOI: 10.1016/j.aca.2008.06.001

1861. Amalric L, Mouvet C, Pichon V, Bristeau S. 2008. Molecularly imprinted polymer applied to the determination of the residual mass of atrazine and metabolites within an agricultural catchment (Brevilles, France). J. Chromatogr. A 1206: 95-104. DOI: 10.1016/j.chroma.2008.08.034

1862. Anderson RA, Ariffin MM, Cormack PAG, Miller El. 2008. Comparison of molecularly imprinted solid-phase extraction (MISPE) with classical solidphase extraction (SPE) for the detection of benzodiazepines in post-mortem hair samples. Forensic Sci. Int. 174: 40-46. DOI: 10.1016/j.forsciint.2007.03.002

1863. Beltran A, Marcé RM, Cormack PAG, Sherrington DC, Borrull F. 2008. Selective solid-phase extraction of amoxicillin and cephalexin from urine samples using a molecularly imprinted polymer. J. Sep. Sci. 31: 2868-2874. DOI: 10.1002/jssc.200800085 
1864. Benito-Peña E, Urraca JL, Sellergren B, Moreno-Bondi MC. 2008. Solidphase extraction of fluoroquinolones from aqueous samples using a watercompatible stochiometrically imprinted polymer. J. Chromatogr. A 1208: 6270. DOI: 10.1016/j.chroma.2008.08.109

1865. Castell OK, Allender CJ, Barrow DA. 2008. Continuous molecular enrichment in microfluidic systems. Lab Chip 8: 1031-1033. DOI: 10.1039/b800521d

1866. Chen CY, Tsai WL, Wu HC, Syu MJ, Wu CC, Shiesh SC. 2008. Diagnostic role of biliary pancreatic elastase for cholangiocarcinoma in patients with cholestasis. Clin. Chim. Acta 390: 82-89. DOI: 10.1016/j.cca.2008.01.011

1867. Chen SM, Zhang ZJ. 2008. Molecularly imprinted solid-phase extraction combined with electrochemical oxidation fluorimetry for the determination of methotrexate in human serum and urine. Spectrochim. Acta, A 70: 36-41. DOI: 10.1016/j.saa.2007.07.009

1868. Claude B, Morin P, Lafosse M, Belmont AS, Haupt K. 2008. Selective solidphase extraction of a triterpene acid from a plant extract by molecularly imprinted polymer. Talanta 75: 344-350. DOI: 10.1016/j.talanta.2007.11.037

1869. Claude B, Morin P, Bayoudh S, de Ceaurriz J. 2008. Interest of molecularly imprinted polymers in the fight against doping: Extraction of tamoxifen and its main metabolite from urine followed by high-performance liquid chromatography with UV detection. J. Chromatogr. A 1196-1197: 81-88. DOI: 10.1016/j.chroma.2008.05.022

1870. Cummins W, Duggan P, McLoughlin P. 2008. Thermal desorption characterisation of molecularly imprinted polymers. Part I: a novel study using direct-probe GC-MS analysis. Anal. Bioanal. Chem. 391: 1237-1244. DOI: $10.1007 / \mathrm{s} 00216-008-1965-4$ 
1871. Dias ACB, Figueiredo EC, Grassi V, Zagatto EAG, Arruda MAZ. 2008. Molecularly imprinted polymer as a solid phase extractor in flow analysis. Talanta 76: 988-996. DOI: 10.1016/j.talanta.2008.05.040

1872. Djozan D, Ebrahimi B. 2008. Preparation of new solid phase micro extraction fiber on the basis of atrazine-molecular imprinted polymer: Application for $\mathrm{GC}$ and $\mathrm{GC} / \mathrm{MS}$ screening of triazine herbicides in water, rice and onion. Anal. Chim. Acta 616: 152-159. DOI: 10.1016/j.aca.2008.04.037

1873. Emgenbroich M, Borrelli C, Shinde S, Lazraq I, Vilela F, Hall AJ, Oxelbark J, De Lorenzi E, Courtois J, Simanova A, Verhage J, Irgum K, Karim K, Sellergren B. 2008. A Phosphotyrosine-Imprinted Polymer Receptor for the Recognition of Tyrosine Phosphorylated Peptides. Chem. Eur. J. 14: 95169529. DOI: 10.1002/chem.200801046

1874. Ersöz A, Diltemiz SE, Özcan AA, Denizli A, Say RI. 2008. Synergie between molecular imprinted polymer based on solid-phase extraction and quartz crystal microbalance technique for 8-OHdG sensing. Biosens. Bioelectron. 24: 742-747. DOI: 10.1016/j.bios.2008.06.058

1875. Feng QZ, Zhao LX, Yan W, Ji F, Wei YL, Lin JM. 2008. Molecularly imprinted solid-phase extraction and flow-injection chemiluminescence for trace analysis of 2,4-dichlorophenol in water samples. Anal. Bioanal. Chem. 391: 1073-1079. DOI: 10.1007/s00216-008-2059-z

1876. Frandsen H. 2008. Biomonitoring of urinary metabolites of 2-amino-1methyl-6-phenylimidazo[4,5-b]pyridine (PhIP) following human consumption of cooked chicken. Food Chem. Toxicol. 46: 3200-3205. DOI: 10.1016/j.fct.2008.07.008

1877. Garde-Cerdán T, Zalacain A, Lorenzo C, Alonso JL, Salinas MR. 2008. Molecularly Imprinted Polymer-Assisted Simple Clean-Up of 2,4,6Trichloroanisole and Ethylphenols from Aged Red Wines. Am. J. Enol. Vitic. 59: 396-400. 
1878. Gros M, Pizzolato TM, Petrovic M, de Alda MJL, Barceló D. 2008. Trace level determination of $\alpha$-blockers in waste waters by highly selective molecularly imprinted polymers extraction followed by liquid chromatography-quadrupole-linear ion trap mass spectrometry. J. Chromatogr. A 1189: 374-384. DOI: 10.1016/j.chroma.2007.10.052

1879. Guerreiro A, Soares A, Piletska E, Mattiasson B, Piletsky S. 2008. Preliminary evaluation of new polymer matrix for solid-phase extraction of nonylphenol from water samples. Anal. Chim. Acta 612: 99-104. DOI: 10.1016/j.aca.2008.02.010

1880. Guo LY, Jiang XM, Yang CL, Zhang HX. 2008. Analysis of sulfamerazine in pond water and several fishes by high-performance liquid chromatography using molecularly imprinted solid-phase extraction. Anal. Bioanal. Chem. 391: 2291-2298. DOI: 10.1007/s00216-008-2131-8

1881. Guo LY, Guan M, Zhao CD, Zhang HX. 2008. Molecularly imprinted matrix solid-phase dispersion for extraction of chloramphenicol in fish tissues coupled with high-performance liquid chromatography determination. Anal. Bioanal. Chem. 392: 1431-1438. DOI: 10.1007/s00216-008-2454-5

1882. Guo ZF, Guo TT, Guo MF. 2008. Preparation of molecularly imprinted adsorptive resin for trapping of ligustrazine from the traditional Chinese herb Ligusticum chuanxiong Hort. Anal. Chim. Acta 612: 136-143. DOI: 10.1016/j.aca.2008.02.039

1883. Guzzella L, Pozzoni F, Baggiani C. 2008. Synthesis and characterization of a propazine imprinted polymer for the extraction of triazines herbicides. Water Sci. Technol. 57: 139-144. DOI: 10.2166/wst.2008.812

1884. Han RF, Xing XC, Wang Y, Long Y, Sun Y, Zhao Z, Mi HF. 2008. Separation/enrichment of active natural low content protein using protein imprinted polymer. J. Chromatogr $B$ 873: 113-118. DOI: 10.1016/j.jchromb.2008.08.003 
1885. He JX, Wang S, Fang GZ, Zhu HP, Zhang Y. 2008. Molecularly Imprinted Polymer Online Solid-Phase Extraction Coupled with High-Performance Liquid Chromatography-UV for the Determination of Three Sulfonamides in Pork and Chicken. J. Agric. Food Chem. 56: 2919-2925. DOI: $10.1021 / \mathrm{jf} 703680 \mathrm{q}$

1886. Holland N, Owens E, Cummins W, Frisby J, Hughes H, McLoughlin P. 2008. Thermal desorption characterisation of molecularly imprinted polymers. Part II: Use of direct probe GC-MS analysis to study crosslinking effects. Anal. Bioanal. Chem. 391: 1245-1253. DOI: 10.1007/s00216-008-1974-3

1887. Holland N, Duggan P, Owens E, Cummins W, Frisby J, Hughes H, McLoughlin P. 2008. Thermal desorption characterisation of molecularly imprinted polymers. Part II: Use of direct probe GC-MS analysis to study crosslinking effects. Anal. Bioanal. Chem. 391: 2371. DOI: 10.1007/s00216008-2170-1

1888. Hu XG, Pan JL, Hu YL, Huo Y, Li GK. 2008. Preparation and evaluation of solid-phase microextraction fiber based on molecularly imprinted polymers for trace analysis of tetracyclines in complicated samples. J. Chromatogr. A 1188: 97-107. DOI: 10.1016/j.chroma.2008.02.062

1889. Hugon-Chapuis F, Mullot JU, Tuffal G, Hennion MC, Pichon V. 2008. Selective and automated sample pretreatment by molecularly imprinted polymer for the analysis of the basic drug alfuzosin from plasma. $J$. Chromatogr. A 1196-1197: 73-80. DOI: 10.1016/j.chroma.2008.04.038

1890. Jégourel D, Delépée R, Breton F, Rolland A, Vidal R, Agrofoglio LA. 2008. Molecularly imprinted polymer of 5-methyluridine for solid-phase extraction of pyrimidine nucleoside cancer markers in urine. Bioorg. Med. Chem. 16: 8932-8939. DOI: 10.1016/j.bmc.2008.08.063

1891. Jiang XM, Zhao CD, Jiang N, Zhang HX, Liu MC. 2008. Selective solidphase extraction using molecular imprinted polymer for the analysis of 
diethylstilbestrol. Food Chem. 108: 1061-1067. DOI: 10.1016/j.foodchem.2007.11.039

1892. Jin GY, Tang YW, Liu SZ, Wang SC, Xing RK. 2008. Preparation and Application of a Novel Silica-Supported Organic-Inorganic Hybrid Molecular Imprinting Polymer. Anal. Lett. 41: 1811-1817. DOI: $10.1080 / 00032710802162103$

1893. Khorrami AR, Mehrseresht S. 2008. Synthesis and evaluation of a selective molecularly imprinted polymer for the contraceptive drug levonorgestrel. J. Chromatogr B 867: 264-269. DOI: 10.1016/j.jchromb.2008.04.017

1894. Koohpaei AR, Shahtaheri SJ, Ganjali MR, Forushani AR, Golbabaei F. 2008. Molecular Imprinted Solid Phase Extraction for Determination of Atrazine in Environmental Samples. Iran. J. Environ. Health Sci. Eng. 5: 283-296.

1895. Kubo T, Tominaga Y, Watanabe F, Kaya K, Hosoya K. 2008. Selective Adsorption of Water-soluble lonic Compounds by an Interval Immobilization Technique Based on Molecular Imprinting. Anal. Sci. 24: 1633-1636. DOI: 10.2116/analsci.24.1633

1896. Li YH, Yang T, Qi XL, Qiao YW, Deng AP. 2008. Development of a group selective molecularly imprinted polymers based solid phase extraction of malachite green from fish water and fish feed samples. Anal. Chim. Acta 624: 317-325. DOI: 10.1016/j.aca.2008.07.004

1897. Liang FX, Zhu XL, Yang J, Su QD. 2008. Accelerated solvent extraction and matrix solid phase dispersion using molecularly imprinted polymer for the analysis of monocrotophos in soil. Asian J. Chem. 20: 3954-3960.

1898. Lin Y, Shi Y, Jiang M, Jin Y, Peng Y, Lu B, Dai K. 2008. Removal of phenolic estrogen pollutants from different sources of water using 
molecularly imprinted polymeric microspheres. Environ. Pollut. 153: 483491. DOI: 10.1016/j.envpol.2007.08.001

1899. Liu HM, Liu CH, Yang XJ, Zeng SJ, Xiong YQ, Xu WJ. 2008. Solid-phase extraction of ursolic acid from herb using $\beta$-cyclodextrin-based molecularly imprinted microspheres. J. Sep. Sci. 31: 3573-3580. DOI: $10.1002 /$ jssc.200800362

1900. Lu YK, Zhao N, Qin XY, Liu Y, Lu GD. 2008. Preparation and application of the sacrificial mesoporous silica imprinted polymers for the selective solidphase extraction of ofloxacin residues in chicken. Chem. J. Internet 10: Art. No. 106032pe.

1901. Luo W, Zhu LH, Yu C, Tang HQ, Yu HX, Li X, Zhang X. 2008. Synthesis of surface molecularly imprinted silica micro-particles in aqueous solution and the usage for selective off-line solid-phase extraction of 2,4-dinitrophenol from water matrixes. Anal. Chim. Acta 618: 147-156. DOI: 10.1016/j.aca.2008.04.051

1902. Michailof C, Manesiotis P, Panayiotou C. 2008. Synthesis of caffeic acid and p-hydroxybenzoic acid molecularly imprinted polymers and their application for the selective extraction of polyphenols from olive mill waste waters. $J$. Chromatogr. A 1182: 25-33. DOI: 10.1016/j.chroma.2008.01.001

1903. Mohajeri SA, Ebrahimi SA. 2008. Preparation and characterization of a lamotrigine imprinted polymer and its application for drug assay in human serum. J. Sep. Sci. 31: 3595-3602. DOI: 10.1002/jssc.200800377

1904. Mohamed R, Mottier P, Treguier L, Richoz-Payot J, Yilmaz E, Tabet JC, Guy PA. 2008. Use of Molecularly Imprinted Solid-Phase Extraction Sorbent for the Determination of Four 5-Nitroimidazoles and Three of Their Metabolites from Egg-Based Samples before Tandem LC-ESIMS/MS Analysis. J. Agric. Food Chem. 56: 3500-3508. DOI: 10.1021/jf072807h 
1905. Núñez L, Turiel E, Martín-Esteban A, Tadeo JL. 2008. Molecularly imprinted polymer for selective extraction of endocrine disrupters nonylphenol and its ethoxylated derivates from environmental solids. J. Sep. Sci. 31: 2492-2499. DOI: $10.1002 /$ jssc.200800146

1906. Okutucu B, Telefoncu A. 2008. Optimization of serotonin imprinted polymers and recognition study from platelet rich plasma. Talanta 76: 1153-1158. DOI: 10.1016/j.talanta.2008.05.033

1907. Pascale M, De Girolamo A, Visconti A, Magan N, Chianella I, Piletska EV, Piletsky SA. 2008. Use of itaconic acid-based polymers for solid-phase extraction of deoxynivalenol and application to pasta analysis. Anal. Chim. Acta 609: 131-138. DOI: 10.1016/j.aca.2008.01.004

1908. Pilau EJ, Silva RGC, Jardim ICFS, Augusto F. 2008. Molecularly imprinted sol-gel silica for solid phase extraction of phenobarbital. J. Braz. Chem. Soc. 19: 1136-1143. DOI: 10.1590/S0103-50532008000600012

1909. Puoci F, Curcio M, Cirillo G, lemma F, Spizzirri UG, Picci N. 2008. Molecularly imprinted solid-phase extraction for cholesterol determination in cheese products. Food Chem. 106: 836-842. DOI: 10.1016/j.foodchem.2007.06.043

1910. Qu GR, Wu AB, Shi XZ, Niu ZF, Xie W, Zhang DB. 2008. Improvement on Analyte Extraction by Molecularly Imprinted Polymer Microspheres toward Enrofloxacin. Anal. Lett. 41: 1443-1458. DOI: 10.1080/00032710802119566

1911. Rezaei B, Mallakpour S, Rahmanian O. 2008. A Selective Solid-Phase Extraction and Preconcentration Method with Using Molecularly Imprinted Polymer for Piroxicam in Pharmaceutical Sample. Anal. Lett. 41: 1818-1831. DOI: 10.1080/00032710802162129 
1912. Schirmer C, Meisel H. 2008. Molecularly imprinted polymers for the selective solid-phase extraction of chloramphenicol. Anal. Bioanal. Chem. 392: 223229. DOI: 10.1007/s00216-008-2269-4

1913. Shen XT, Zhu LH, Liu GX, Yu HW, Tang HQ. 2008. Enhanced Photocatalytic Degradation and Selective Removal of Nitrophenols by Using Surface Molecular Imprinted Titania. Environ. Sci. Technol. 42: 1687-1692. DOI: $10.1021 / \mathrm{es} 071788 p$

1914. Song SQ, Wu AB, Shi XZ, Li RX, Lin ZX, Zhang DB. 2008. Development and application of molecularly imprinted polymers as solid-phase sorbents for erythromycin extraction. Anal. Bioanal. Chem. 390: 2141-2150. DOI: $10.1007 / \mathrm{s} 00216-008-1985-0$

1915. Song SQ, Shi XZ, Li RX, Lin ZX, Wu AB, Zhang DB. 2008. Extraction of chlorpromazine with a new molecularly imprinted polymer from pig urine. Process Biochem. 43: 1209-1214. DOI: 10.1016/j.procbio.2008.06.015

1916. Sun HW, Qiao FX, Liu GY, Liang SX. 2008. Simultaneous isolation of six fluoroquinolones in serum samples by selective molecularly imprinted matrix solid-phase dispersion. Anal. Chim. Acta 625: 154-159. DOI: 10.1016/j.aca.2008.07.025

1917. Sun HW, Qiao FX. 2008. Recognition mechanism of water-compatible molecularly imprinted solid-phase extraction and determination of nine quinolones in urine by high performance liquid chromatography. $\mathrm{J}$. Chromatogr. A 1212: 1-9. DOI: 10.1016/j.chroma.2008.09.107

1918. Sun Z, Schüssler W, Sengl M, Niessner R, Knopp D. 2008. Selective trace analysis of diclofenac in surface and wastewater samples using solid-phase extraction with a new molecularly imprinted polymer. Anal. Chim. Acta 620: 73-81. DOI: 10.1016/j.aca.2008.05.020 
1919. Tang KJ, Chen SW, Gu XH, Wang HJ, Dai J, Tang J. 2008. Preparation of molecularly imprinted solid phase extraction using bensulfuron-methyl imprinted polymer and clean-up for the sulfonylurea-herbicides in soybean. Anal. Chim. Acta 614: 112-118. DOI: 10.1016/j.aca.2008.03.018

1920. Trammell SA, Zeinali M, Melde BJ, Charles PT, Velez FL, Dinderman MA, Kusterbeck A, Markowitz MA. 2008. Nanoporous Organosilicas as Preconcentration Materials for the Electrochemical Detection of Trinitrotoluene. Anal. Chem. 80: 4627-4633. DOI: 10.1021/ac702263t

1921. Wang S, Xu ZX, Fang GZ, Zhang Y, He JX. 2008. Separation and determination of estrone in environmental and drinking water using molecularly imprinted solid phase extraction coupled with HPLC. J. Sep. Sci. 31: 1181-1188. DOI: 10.1002/jssc.200700575

1922. Widstrand C, Kronauer S, Björk H, Wihlborg A-K, Trinh A. 2008. The simultaneous class-selective extraction and analysis of beta-blockers and $\beta$ agonists using molecularly imprinted polymer SPE. Am. Lab. 40: 6-7.

1923. Yan H, Row KH, Yang GL. 2008. Water-compatible molecularly imprinted polymers for selective extraction of ciprofloxacin from human urine. Talanta 75: 227-232. DOI: 10.1016/j.talanta.2007.11.002

1924. Yan H, Row KH. 2008. Novel molecularly imprinted monolithic column for selective on-line extraction of ciprofloxacin from human urine. Biomed. Chromatogr. 22: 487-493. DOI: 10.1002/bmc.957

1925. Yan H, Row KH. 2008. Molecularly imprinted solid-phase extraction for determination of enrofloxacin and ciprofloxacin in chicken muscle. Bull. Kor. Chem. Soc. 29: 1173-1178.

1926. Yan H, Tian M, Row KH. 2008. Determination of enrofloxacin and ciprofloxacin in milk using molecularly imprinted solid-phase extraction. $J$. Sep. Sci. 31: 3015-3020. DOI: 10.1002/jssc.200800315 
1927. Yang T, Li YH, Wei S, Li Y, Deng AP. 2008. Development of a selective molecularly imprinted polymer-based solid-phase extraction for indomethacin from water samples. Anal. Bioanal. Chem. 391: 2905-2914. DOI: 10.1007/s00216-008-2218-2

1928. Yao W, Fang YJ, Li GL, Gao ZX, Cheng YY. 2008. Adsorption of carbaryl using molecularly imprinted microspheres prepared by precipitation polymerization. Polym. Adv. Technol. 19: 812-816. DOI: 10.1002/pat.1039

1929. Yu Q, Deng SB, Yu G. 2008. Selective removal of perfluorooctane sulfonate from aqueous solution using chitosan-based molecularly imprinted polymer adsorbents. Water Res. 42: 3089-3097. DOI: 10.1016/j.watres.2008.02.024

1930. Zhang ZB, Hu JY. 2008. Selective removal of estrogenic compounds by molecular imprinted polymer (MIP). Water Res. 42: 4101-4108. DOI: 10.1016/j.watres.2008.07.006

1931. Zhu XL, Zhu QS. 2008. Molecular imprinted Nylon-6 stir bar as a novel extraction technique for enantioseparation of amino acids. J. Appl. Polym. Sci. 109: 2665-2670. DOI: 10.1002/app.27557

1932. Zorita S, Boyd B, Jönsson S, Yilmaz E, Svensson C, Mathiasson L, Bergström S. 2008. Selective determination of acidic pharmaceuticals in wastewater using molecularly imprinted solid-phase extraction. Anal. Chim. Acta 626: 147-154. DOI: 10.1016/j.aca.2008.07.051

1933. Al-Degs YS, Abu-Surrah AS, Ibrahim KA. 2009. Preparation of highly selective solid-phase extractants for Cibacron reactive dyes using molecularly imprinted polymers. Anal. Bioanal. Chem. 393: 1055-1062. DOI: $10.1007 / \mathrm{s} 00216-008-2502-1$

1934. Alizadeh T, Ganjali MR, Nourozi P, Zare M. 2009. Multivariate optimization of molecularly imprinted polymer solid-phase extraction applied to parathion 
determination in different water samples. Anal. Chim. Acta 638: 154-161. DOI: 10.1016/j.aca.2009.02.040

1935. Baggiani C, Anfossi L, Baravalle P, Giovannoli C, Giraudi G, Barolo C, Viscardi G. 2009. Determination of banned Sudan dyes in food samples by molecularly imprinted solid phase extraction-high performance liquid chromatography. J. Sep. Sci. 32: 3292-3300. DOI: 10.1002/jssc.200900126

1936. Beltran A, Marcé RM, Cormack PAG, Borrull F. 2009. Synthesis by precipitation polymerisation of molecularly imprinted polymer microspheres for the selective extraction of carbamazepine and oxcarbazepine from human urine. J. Chromatogr. $A$ 1216: 2248-2253. DOI: 10.1016/j.chroma.2009.01.024

1937. Beltran A, Fontanals N, Marcé RM, Cormack PAG, Borrull F. 2009. Molecularly imprinted solid-phase extraction of cephalexin from water-based matrices. J. Sep. Sci. 32: 3319-3326. DOI: 10.1002/jssc.200900268

1938. Borghard WG, Calabro DC, DiSanzo FP, Disko MM, Diehl JW, Fried JC, Markowitz MA, Zeinali M, Melde BJ, Riley AE. 2009. Characterization and Testing of Periodic Mesoporous Organosilicas as Potential Selective Benzene Adsorbents. Langmuir 25: 12661-12669. DOI: 10.1021/la901334z

1939. Bravo JC, Garcinuño RM, Fernández P, Durand JS. 2009. Selective solidphase extraction of ethynylestradiol from river water by molecularly imprinted polymer microcolumns. Anal. Bioanal. Chem. 393: 1763-1768. DOI: $10.1007 / \mathrm{s} 00216-008-2563-1$

1940. Cacho C, Turiel E, Pérez-Conde C. 2009. Molecularly imprinted polymers: An analytical tool for the determination of benzimidazole compounds in water samples. Talanta 78: 1029-1035. DOI: 10.1016/j.talanta.2009.01.007 
1941. Celiz MD, Aga DS, Colón LA. 2009. Evaluation of a molecularly imprinted polymer for the isolation/enrichment of $\alpha$-estradiol. Microchem. J. 92: 174179. DOI: 10.1016/j.microc.2009.03.007

1942. Chapuis-Hugon F, Cruz-Vera M, Savane R, Ali WH, Valcarcel M, Deveaux M, Pichon V. 2009. Selective sample pretreatment by molecularly imprinted polymer for the determination of LSD in biological fluids. J. Sep. Sci. 32 : 3301-3309. DOI: 10.1002/jssc.200900247

1943. Chen LG, Liu J, Zeng QL, Wang H, Yu AM, Zhang HQ, Ding L. 2009. Preparation of magnetic molecularly imprinted polymer for the separation of tetracycline antibiotics from egg and tissue samples. J. Chromatogr. A 1216: 3710-3719. DOI: 10.1016/j.chroma.2009.02.044

1944. Chen LG, Zhang XP, Sun L, Xu Y, Zeng QL, Wang H, Xu HY, Yu AM, Zhang HQ, Ding L. 2009. Fast and Selective Extraction of Sulfonamides from Honey Based on Magnetic Molecularly Imprinted Polymer. J. Agric. Food Chem. 57: 10073-10080. DOI: 10.1021/jf902257d

1945. De Smet D, Dubruel P, Van Peteghem C, Schacht E, De Saeger S. 2009. Molecularly imprinted solid-phase extraction of fumonisin $\mathrm{B}$ analogues in bell pepper, rice and corn flakes. Food Addit. Contam., Part A 26: 874-884. DOI: 10.1080/02652030902788920

1946. Díaz-Alvarez M, Turiel E, Martín-Esteban A. 2009. Selective sample preparation for the analysis of (fluoro)quinolones in baby food: molecularly imprinted polymers versus anion-exchange resins. Anal. Bioanal. Chem. 393: 899-905. DOI: 10.1007/s00216-008-2300-9

1947. Djozan D, Mahkam M, Ebrahimi B. 2009. Preparation and binding study of solid-phase microextraction fiber on the basis of ametryn-imprinted polymer: Application to the selective extraction of persistent triazine herbicides in tap water, rice, maize and onion. J. Chromatogr. A 1216: 2211-2219. DOI: 10.1016/j.chroma.2008.12.101 
1948. Ezhova NM, Polyakova IV, Pisarev OA. 2009. Sorption of lysine by molecularly imprinted carboxyl sorbents. Appl. Biochem. Microbiol. 45: 221225. DOI: 10.1134/S0003683809020185

1949. Fan J, Wei YF, Wang JJ, Wu CL, Shi HL. 2009. Study of molecularly imprinted solid-phase extraction of diphenylguanidine and its structural analogs. Anal. Chim. Acta 639: 42-50. DOI: 10.1016/j.aca.2009.02.045

1950. Feás X, Ye L, Regal P, Fente CA, Hosseini SV, Cepeda A. 2009. Application of dummy molecularly imprinted solid-phase extraction in the analysis of cyproheptadine in bovine urine. J. Sep. Sci. 32: 1740-1747. DOI: $10.1002 /$ jssc.200800726

1951. Feng QZ, Zhao LX, Yan W, Lin JM, Zheng ZX. 2009. Molecularly imprinted solid-phase extraction combined with high performance liquid chromatography for analysis of phenolic compounds from environmental water samples. J. Haz. Mater. 167: 282-288. DOI: 10.1016/j.jhazmat.2008.12.115

1952. Feng QZ, Zhao LX, Lin JM. 2009. Molecularly imprinted polymer as microsolid phase extraction combined with high performance liquid chromatography to determine phenolic compounds in environmental water samples. Anal. Chim. Acta 650: 70-76. DOI: 10.1016/j.aca.2009.04.016

1953. Fernández-Álvarez P, Le Noir M, Guieysse B. 2009. Removal and destruction of endocrine disrupting contaminants by adsorption with molecularly imprinted polymers followed by simultaneous extraction and phototreatment. J. Haz. Mater. 163: 1107-1112. DOI: 10.1016/j.jhazmat.2008.07.085

1954. Figueiredo EC, de Oliveira DM, de Siqueira MEPB, Arruda MAZ. 2009. Online molecularly imprinted solid-phase extraction for the selective spectrophotometric determination of nicotine in the urine of smokers. Anal. Chim. Acta 635: 102-107. DOI: 10.1016/j.aca.2008.12.045 
1955. Freitas MB, Costa PR, Brito JO, Vieira EP, Maia PP, Siqueira MEPB. 2009. Amitriptyline and Nortriptyline in Plasma: Solid-Phase Extraction in Molecularly Imprinted Polymers and Silica Octadecil for High Performance Liquid Chromatography Analysis. Latin Am. J. Pharm. 28: 70-79.

1956. Fu Q, He LC, Zhang QQ, Amut E, Fang Q, Chang C. 2009. Uniformly sized molecularly imprinted polymers for on-line concentration, purification, and measurement of nimodipine in plasma. J. Appl. Polym. Sci. 111: 2830-2836. DOI: 10.1002/app.29315

1957. Gadzala-Kopciuch R, Ricanyová J, Buszewski B. 2009. Isolation and detection of steroids from human urine by molecularly imprinted solid-phase extraction and liquid chromatography. J. Chromatogr B 877: 1177-1184. DOI: 10.1016/j.jchromb.2009.03.008

1958. Gadzala-Kopciuch R, Ricanyová J, Buszewski B. 2010. Corrigendum to "Isolation and detection of steroids from human urine by molecularly imprinted solid-phase extraction and liquid chromatography" [J. Chromatogr. B 877 (2009) 1177-1184]. J. Chromatogr B 878: 2046. DOI: 10.1016/j.jchromb.2010.05.026

1959. Tse Sum Bui B, Haupt K. 2009. Comment on "Isolation and detection of steroids from human urine by molecularly imprinted solid-phase extraction and liquid chromatography" by Gadzala-Kopciuch et al., J. Chromatogr. B 877 (2009), 1177-1184. J. Chromatogr B 877: 4180-4181. DOI: 10.1016/j.jchromb.2009.09.048

1960. Gadzala-Kopciuch R, Ricanyová J, Buszewski B. 2010. Reply to comment on "Isolation and detection of steroids from human urine by molecularly imprinted solid-phase extraction and liquid chromatography" by Tse Sum Bui and Haupt [J. Chromatogr. B 877 (2009) 4180-4181]. J. Chromatogr B 878: 2044-2045. DOI: 10.1016/j.jchromb.2010.05.025 
1961. Gallego-Gallegos M, Muños-Olivas R, Cámara C. 2009. Imprinted polymers as an analytical tool for organotin compounds speciation. J. Anal. Atom. Spectrosc. 24: 595-601. DOI: 10.1039/b818370h

1962. González-Mariño I, Quintana JB, Rodríguez I, Rodil R, González-Peñas J, Cela R. 2009. Comparison of molecularly imprinted, mixed-mode and hydrophilic balance sorbents performance in the solid-phase extraction of amphetamine drugs from wastewater samples for liquid chromatographytandem mass spectrometry determination. J. Chromatogr. A 1216: 84358441. DOI: 10.1016/j.chroma.2009.09.069

1963. He LM, Su YJ, Zheng YQ, Huang XH, Wu L, Liu YH, Zeng ZL, Chen ZL. 2009. Novel cyromazine imprinted polymer applied to the solid-phase extraction of melamine from feed and milk samples. J. Chromatogr. A 1216: 6196-6203. DOI: 10.1016/j.chroma.2009.06.081

1964. He LM, Su YQ, Shen XG, Zheng YQ, Guo HB, Zeng ZL. 2009. Solid-phase extraction of melamine from aqueous samples using water-compatible molecularly imprinted polymers. J. Sep. Sci. 32: 3310-3318. DOI: 10.1002/jssc.200900407

1965. Herrero-Hernández E, Carabias-Martínez R, Rodríguez-Gonzalo E. 2009. Use of a bisphenol-A imprinted polymer as a selective sorbent for the determination of phenols and phenoxyacids in honey by liquid chromatography with diode array and tandem mass spectrometric detection. Anal. Chim. Acta 650: 195-201. DOI: 10.1016/j.aca.2009.07.043

1966. Hu XG, Pan JL, Hu YL, Li GK. 2009. Preparation and evaluation of propranolol molecularly imprinted solid-phase microextraction fiber for trace analysis of $\alpha$-blockers in urine and plasma samples. J. Chromatogr. A 1216: 190-197. DOI: 10.1016/j.chroma.2008.11.064

1967. Hu YL, Liu RJ, Zhang Y, Li GK. 2009. Improvement of extraction capability of magnetic molecularly imprinted polymer beads in aqueous media via 
dual-phase solvent system. Talanta 79: 576-582. DOI: 10.1016/j.talanta.2009.04.029

1968. Hu YL, Wang YY, Hu YF, Li GK. 2009. Liquid-liquid-solid microextraction based on membrane-protected molecularly imprinted polymer fiber for trace analysis of triazines in complex aqueous samples. J. Chromatogr. A 1216: 8304-8311. DOI: 10.1016/j.chroma.2009.09.063

1969. Ibrahim MNM, Sipaut CS, Yusof NNM. 2009. Purification of vanillin by a molecular imprinting polymer technique. Sep. Purif. Technol. 66: 450-456. DOI: 10.1016/j.seppur.2009.02.010

1970. Javanbakht M, Shaabani N, Akbari-adergani B. 2009. Novel molecularly imprinted polymers for the selective extraction and determination of metoclopramide in human serum and urine samples using high-performance liquid chromatography. J. Chromatogr B 877: 2537-2544. DOI: 10.1016/j.jchromb.2009.06.030

1971. Javanbakht M, Namjumanesh MH, Akbari-adergani B. 2009. Molecularly imprinted solid-phase extraction for the selective determination of bromhexine in human serum and urine with high performance liquid chromatography. Talanta 80: 133-138. DOI: 10.1016/j.talanta.2009.06.033

1972. Javanbakht M, Shaabani N, Abdouss M, Ganjali MR, Mohammadi A, Norouzi P. 2009. Molecularly Imprinted Polymers for Selective Solid-Phase Extraction of Verapamil from Biological Fluids and Human Urine. Curr. Pharm. Anal. 5: 269-276.

1973. Ji YS, Yin JJ, Xu ZG, Zhao CD, Huang HY, Zhang HX, Wang CM. 2009. Preparation of magnetic molecularly imprinted polymer for rapid determination of bisphenol A in environmental water and milk samples. Anal. Bioanal. Chem. 395: 1125-1133. DOI: 10.1007/s00216-009-3020-5 
1974. Jiang TH, Zhao LX, Chu BL, Feng QH, Yan W, Lin JM. 2009. Molecularly imprinted solid-phase extraction for the selective determination of $17 \alpha$ estradiol in fishery samples with high performance liquid chromatography. Talanta 78: 442-447. DOI: 10.1016/j.talanta.2008.11.047

1975. Jin GY, Tang YW. 2009. Evaluation of a novel silica-supported sol-gel sorbent prepared by a surface molecular imprinting technique for the selective separation of estazolam from human plasma. Microchim. Acta 165: 143-149. DOI: 10.1007/s00604-008-0112-4

1976. Khorrami AR, Rashidpur A. 2009. Design of a new cartridge for selective solid phase extraction using molecularly imprinted polymers: Selective extraction of theophylline from human serum samples. Biosens. Bioelectron. 25: 647-651. DOI: 10.1016/j.bios.2008.11.033

1977. Kittlaus S, Lipinski J, Speer K. 2009. New Approaches for Determination of Glyphosate and Aminomethylphosphonic Acid from Different Tea SamplesProspects and Limits of Cleanup with Molecularly Imprinted Polymer and Titanium Dioxide. J. AOAC Int. 92: 703-714.

1978. Kobayashi T, Takeda K, Ohashi A, Makoto M, Sugiyama S. 2009. Selective Removal of Bisphenol A From Serum Using Molecular Imprinted Polymer Membranes. Therap. Apher. Dialysis 13: 19-26. DOI: 10.1111/j.17449987.2009.00651.x

1979. Koohpaei AR, Shahtaheri SJ, Ganjali MR, Forushani AR, Golbabaei F. 2009. Optimization of solid-phase extraction using developed modern sorbent for trace determination of ametryn in environmental matrices. $J$. Haz. Mater. 170: 1247-1255. DOI: 10.1016/j.jhazmat.2009.05.125

1980. Krupadam RJ, Bhagat B, Wate SR, Bodhe GL, Sellergren B, Anjaneyulu Y. 2009. Fluorescence Spectrophotometer Analysis of Polycyclic Aromatic Hydrocarbons in Environmental Samples Based on Solid Phase Extraction 
Using Molecularly Imprinted Polymer. Environ. Sci. Technol. 43: 2871-2877. DOI: $10.1021 / \mathrm{es} 802514 \mathrm{c}$

1981. Kueseng P, Noir ML, Mattiasson B, Thavarungkul P, Kanatharana P. 2009. Molecularly imprinted polymer for analysis of trace atrazine herbicide in water. J. Environ. Sci. Health, Part B 44: 772-780. DOI: $10.1080 / 03601230903238319$

1982. Lachová M, Lehotay J, Skacáni I, Cizmárik J. 2009. Isolation of Some Derivatives of Phenylcarbamic Acid from Human Plasma using Molecularly Imprinted Polymers. J. Liq. Chrom. Rel. Technol. 32: 167-181. DOI: $10.1080 / 10826070802602890$

1983. Lachová M, Lehotay J, Skacáni I, Cizmárik A. 2009. Molecularly Imprinted Solid-Phase Extraction of 1-Methyl-2-piperidinoethylesters of Alkoxyphenylcarbamic Acid from Human Plasma, Comparison with Classical Solid-Phase Extraction. J. Liq. Chrom. Rel. Technol. 32: 2293-2306. DOI: $10.1080 / 10826070903187684$

1984. Lasáková M, Thiébaut D, Jandera P, Pichon V. 2009. Molecularly imprinted polymer for solid-phase extraction of ephedrine and analogs from human plasma. J. Sep. Sci. 32: 1036-1042. DOI: 10.1002/jssc.200800684

1985. Le Noir M, Plieva FM, Mattiasson B. 2009. Removal of endocrine-disrupting compounds from water using macroporous molecularly imprinted cryogels in a moving-bed reactor. J. Sep. Sci. 32: 1471-1479. DOI: $10.1002 / j s s c .200800670$

1986. Li H, Li XY, Gong GL. 2009. Removal of $17 \alpha$-estradiol pollutants from contaminated water by molecularly imprinted polymers. In Proceedings of 2009 International Conference on Energy and Environment Technology, ICEET 2009, Vol. 3, IEEE: 683-686. DOI: 10.1109/ICEET.2009.403 
1987. Li MKY, Lei NY, Gong CB, Yu YJ, Lam KH, Lam MHW, Yu HX, Lam PKS. 2009. An organically modified silicate molecularly imprinted solid-phase microextraction device for the determination of polybrominated diphenyl ethers. Anal. Chim. Acta 633: 197-203. DOI: 10.1016/j.aca.2008.11.060

1988. Li Y, Li X, Li YQ, Qi JY, Bian J, Yuan YX. 2009. Selective removal of 2,4dichlorophenol from contaminated water using non-covalent imprinted microspheres. Environ. Pollut. 157: 1879-1885. DOI: 10.1016/j.envpol.2009.01.014

1989. Li Y, Li X, Dong CK, Li YQ, Jin PF, Qi JY. 2009. Selective recognition and removal of chlorophenols from aqueous solution using molecularly imprinted polymer prepared by reversible addition-fragmentation chain transfer polymerization. Biosens. Bioelectron. 25: 306-312. DOI: 10.1016/j.bios.2009.07.001

1990. Liu RG, Li X, Li YQ, Jin PF, Qin W, Qi JY. 2009. Effective removal of rhodamine $B$ from contaminated water using non-covalent imprinted microspheres designed by computational approach. Biosens. Bioelectron. 25: 629-634. DOI: 10.1016/j.bios.2009.01.039

1991. Liu XJ, Liu JZ, Huang YY, Zhao R, Liu GQ, Chen Y. 2009. Determination of methotrexate in human serum by high-performance liquid chromatography combined with pseudo template molecularly imprinted polymer. J. Chromatogr. A 1216: 7533-7538. DOI: 10.1016/j.chroma.2009.06.018

1992. Long CY, Mai ZB, Yang YF, Zhu BH, Xu XM, Lu L, Zou XY. 2009. Determination of multi-residue for malachite green, gentian violet and their metabolites in aquatic products by high-performance liquid chromatography coupled with molecularly imprinted solid-phase extraction. J. Chromatogr. A 1216: 2275-2281. DOI: 10.1016/j.chroma.2009.01.047

1993. Long CY, Mai ZB, Yang YF, Zhu BH, Xu XM, Lu L, Zou XY. 2009. Synthesis and characterization of a novel molecularly imprinted polymer for 
simultaneous extraction and determination of water-soluble and fat-soluble synthetic colorants in chilli products by solid phase extraction and high performance liquid chromatography. J. Chromatogr. A 1216: 8379-8385. DOI: 10.1016/j.chroma.2009.09.064

1994. Manesiotis P, Borrelli C, Aureliano CSA, Svensson C, Sellergren B. 2009. Water-compatible imprinted polymers for selective depletion of riboflavine from beverages. J. Mater. Chem. 19: 6185-6193. DOI: 10.1039/b906117g

1995. Mhaka B, Cukrowska E, Tse Sum Bui B, Ramström O, Haupt K, Tutu H, Chimuka L. 2009. Selective extraction of triazine herbicides from food samples based on a combination of a liquid membrane and molecularly imprinted polymers. J. Chromatogr. A 1216: 6796-6801. DOI: 10.1016/j.chroma.2009.08.003

1996. Mohammadi A, Alizadeh T, Dinarvand R, Ganjali MR, Walker RB. 2009. Synthesis of Molecularly Imprinted Polymer for Selective Solid-Phase Extraction of Salbutamol from Urine Samples. Asian J. Chem. 21: 28752880.

1997. Patachia S, Croitoru C, Moise G. 2009. Molecularly Imprinted Poly (Vinyl Alcohol) Films for the Selective Absorption of Glycyrrhizinic Acid from Aqueous Solutions. Environ. Eng. Manag. J. 8: 663-668.

1998. Pereira LA, Rath S. 2009. Molecularly imprinted solid-phase extraction for the determination of fenitrothion in tomatoes. Anal. Bioanal. Chem. 393: 1063-1072. DOI: 10.1007/s00216-008-2511-0

1999. Plesz K, Szajnecki L, Gawdzik B. 2009. Synthesis of Molecularly Imprinted Copolymer and its Application as a SPE Sorbent for Preconcentration of Metoprolol and Vitamin B6 from Water. J. Liq. Chrom. Rel. Technol. 32: 1831-1846. DOI: 10.1080/10826070903091365 
2000. Prasad BB, Tiwari K, Singh M, Sharma PS, Patel AK, Srivastava S. 2009. Zwitterionic molecularly imprinted polymer-based solid-phase microextraction coupled with molecularly imprinted polymer sensor for ultra-trace sensing of L-histidine. J. Sep. Sci. 32: 1096-1105. DOI: 10.1002/jssc.200800595

2001. Prasad BB, Tiwari K, Singh M, Sharma PS, Patel AK, Srivastava S. 2009. Ultratrace analysis of uracil and 5 -fluorouracil by molecularly imprinted polymer brushes grafted to silylated solid-phase microextraction fiber in combination with complementary molecularly imprinted polymer-based sensor. Biomed. Chromatogr. 23: 499-509. DOI: 10.1002/bmc.1145

2002. Prasad BB, Tiwari K, Singh M, Sharma PS, Patel AK, Srivastava S. 2009. Ultratrace Analysis of Dopamine Using a Combination of Imprinted PolymerBrush-Coated SPME and Imprinted Polymer Sensor Techniques. Chromatographia 69: 949-957. DOI: 10.1365/s10337-009-1039-6

2003. Rahiminejad M, Shahtaheri SJ, Ganjali MR, Forushani AR, Golbabaei F. 2009. Molecularly Imprinted Solid Phase Extraction for Trace Analysis of Diazinon in Drinking Water. Iran. J. Environ. Health Sci. Eng. 6: 97-106.

2004. Rejtharová M, Rejthar L. 2009. Determination of chloramphenicol in urine, feed water, milk and honey samples using molecular imprinted polymer clean-up. J. Chromatogr. A 1216: 8246-8253. DOI: 10.1016/j.chroma.2009.07.037

2005. Rezaei B, Mallakpour S, Majidi N. 2009. Solid-phase molecularly imprinted pre-concentration and spectrophotometric determination of isoxicam in pharmaceuticals and human serum. Talanta 78: 418-423. DOI: 10.1016/j.talanta.2008.11.042

2006. Rezaei B, Jafari MT, Khademi R. 2009. Selective separation and determination of primidone in pharmaceutical and human serum samples using molecular imprinted polymer-electrospray ionization ion mobility 
spectrometry (MIP-ESI-IMS). Talanta 79: 669-675. DOI: 10.1016/j.talanta.2009.04.046

2007. Schirmer C, Meisel H. 2009. Chromatographic evaluation of polymers imprinted with analogs of chloramphenicol and application to selective solidphase extraction. Anal. Bioanal. Chem. 394: 2249-2255. DOI: 10.1007/s00216-009-2898-2

2008. Shah KA, Halquist MS, Karnes HT. 2009. A modified method for the determination of tobacco specific nitrosamine 4-(methylnitrosamino)-1-(3pyridyl)-1-butanol in human urine by solid phase extraction using a molecularly imprinted polymer and liquid chromatography tandem mass spectrometry. J. Chromatogr B 877: 1575-1582. DOI: 10.1016/j.jchromb.2009.04.003

2009. Song XL, Li JH, Wang JT, Chen LX. 2009. Quercetin molecularly imprinted polymers: Preparation, recognition characteristics and properties as sorbent for solid-phase extraction. Talanta 80: 694-702. DOI: 10.1016/j.talanta.2009.07.051

2010. Sun XL, He XW, Zhang YK, Chen LX. 2009. Determination of tetracyclines in food samples by molecularly imprinted monolithic column coupling with high performance liquid chromatography. Talanta 79: 926-934. DOI: 10.1016/j.talanta.2009.05.033

2011. Tan F, Zhao HX, Li XN, Quan X, Chen JW, Xiang XM, Zhang X. 2009. Preparation and evaluation of molecularly imprinted solid-phase microextraction fibers for selective extraction of bisphenol $A$ in complex samples. J. Chromatogr. A 1216: 5647-5654. DOI: 10.1016/j.chroma.2009.06.007

2012. Tang JS, Zhang M, Cheng GH, Lu YT. 2009. Development and Application of Molecularly Imprinted Polymer as Solid Phase Extraction of Imidacloprid 
in Environmental Samples. J. Liq. Chrom. Rel. Technol. 32: 59-71. DOI: $10.1080 / 10826070802548622$

2013. Valtchev M, Palm BS, Schiller M, Steinfeld U. 2009. Development of sulfamethoxazole-imprinted polymers for the selective extraction from waters. J. Haz. Mater. 170: 722-728. DOI: 10.1016/j.jhazmat.2009.05.007

2014. Vonderheide AP, Boyd B, Ryberg A, Yilmaz E, Hieber TE, Kauffman PE, Garris ST, Morgan JN. 2009. Analysis of permethrin isomers in composite diet samples by molecularly imprinted solid-phase extraction and isotope dilution gas chromatography-ion trap mass spectrometry. J. Chromatogr. A 1216: 4633-4640. DOI: 10.1016/j.chroma.2009.03.077

2015. Wang DS, Wei Q, Zhang YJ, Zhao CS. 2009. Molecularly imprinted polyethersulfone microfibers for the binding and recognition of bisphenol $A$. J. Appl. Polym. Sci. 114: 4036-4041. DOI: 10.1002/app.31028

2016. Wang GS, Cao Q, Zhu XF, Yang XQ, Yang MH, Ding ZT. 2009. Molecular imprinted solid-phase extraction of huperzine A from Huperzia Serrata. J. Appl. Polym. Sci. 113: 3049-3058. DOI: 10.1002/app.30247

2017. Wang JP, Pan MF, Fang GZ, Wang S. 2009. Preparation of a novel molecularly imprinted polymer by a sol-gel process for on-line solid-phase extraction coupled with high performance liquid chromatography to detect trace enrofloxacin in fish and chicken samples. Microchim. Acta 166: 295302. DOI: $10.1007 / \mathrm{s} 00604-009-0205-8$

2018. Wang S, Liu L, Fang GZ, Zhang C, He JX. 2009. Molecularly imprinted polymer for the determination of trace ractopamine in pork using SPE followed by HPLC with fluorescence detection. J. Sep. Sci. 32: 1333-1339. DOI: $10.1002 /$ jssc.200800565

2019. Xia JJ, Long Y, Guo MJ, Wang Y, Mi HF. 2009. Separation/enrichment of the low-content high molecular weight natural protein using protein-imprinted 
polymers with ARPCs. Sci. China Ser. B Chem. 52: 1388-1393. DOI: 10.1007/s11426-009-0201-1

2020. Xu ZX, Chen SA, Huang W, Fang GZ, Pingzhu H, Wang S. 2009. Study on an on-line molecularly imprinted solid-phase extraction coupled to highperformance liquid chromatography for separation and determination of trace estrone in environment. Anal. Bioanal. Chem. 393: 1273-1279. DOI: $10.1007 / \mathrm{s} 00216-008-2544-4$

2021. Yan H, Tian M, Row KH. 2009. Selective Solid-Phase Extraction of Glabridin from Licorice Root using Molecularly Imprinted Polymer. Sep. Sci. Technol. 44: 359-369. DOI: 10.1080/01496390802437479

2022. Yan H, Qiao FX, Row K. 2009. Molecularly Imprinted Monolithic Column for Selective On-Line Extraction of Enrofloxacin and Ciprofloxacin from Urine. Chromatographia 70: 1087-1093. DOI: 10.1365/s10337-009-1244-3

2023. Yan HY, Tian ML, Row KH. 2009. Determination of Three Tanshinones from Radix Salvia Miltiorrhiza by Molecularly Imprinted Solid-phase Extraction. Chin. J. Chem. 27: 2212-2216. DOI: 10.1002/cjoc.200990371

2024. Yang HH, Zhou WH, Guo XC, Chen FR, Zhao HQ, Lin LM, Wang XR. 2009. Molecularly imprinted polymer as SPE sorbent for selective extraction of melamine in dairy products. Talanta 80: 821-825. DOI: 10.1016/j.talanta.2009.07.067

2025. Yang KG, Ma JJ, Zhou H, Li BQ, Yu BY, Zhao CS. 2009. Molecularly imprinted porous polysulfone particles for the binding and recognition of bisphenol A. Desalination 245: 232-245. DOI: 10.1016/j.desal.2008.07.010

2026. Zamora O, Paniagua EE, Cacho C, Vera-Avila LE, Perez-Conde C. 2009. Determination of benzimidazole fungicides in water samples by on-line MISPE-HPLC. Anal. Bioanal. Chem. 393: 1745-1753. DOI: $10.1007 / \mathrm{s} 00216-009-2631-1$ 
2027. Zhang QQ, Fu Q, Amut E, Fang Q, Zeng AG, Chang C. 2009. Preparation and Evaluation of Propranolol-Imprinted Monolithic Stationary Phase by In Situ Technique and Application in Analysis of Propranolol in Biological Samples. Anal. Lett. 42: 536-554. DOI: 10.1080/00032710802677084

2028. Zhang SJ, Yang GL, Zheng ZS, Chen Y. 2009. On-Line Preconcentration and Analysis of Metribuzin Residues in Corn Fields by Use of a Molecularly Imprinted Polymer. Chromatographia 69: 615-619. DOI: 10.1365/s10337008-0862-5

2029. Zhang SW, Xing J, Cai LS, Wu CY. 2009. Molecularly imprinted monolith intube solid-phase microextraction coupled with HPLC/UV detection for determination of 8-hydroxy-2'-deoxyguanosine in urine. Anal. Bioanal. Chem. 395: 479-487. DOI: 10.1007/s00216-009-2964-9

2030. Zhang ZH, Liu L, Li H, Yao SZ. 2009. Synthesis, characterization and evaluation of uniformly sized core-shell imprinted microspheres for the separation trans-resveratrol from giant knotweed. Appl. Surf. Sci. 255: 93279332. DOI: 10.1016/j.apsusc.2009.07.030

2031. Zhao CD, Ji YS, Shao YL, Jiang XM, Zhang HX. 2009. Novel molecularly imprinted polymer prepared by nanoattapulgite as matrix for selective solidphase extraction of diethylstilbestrol. J. Chromatogr. A 1216: 7546-7552. DOI: 10.1016/j.chroma.2009.06.011

2032. Zhao WF, Fang BH, Li N, Nie SQ, Wei Q, Zhao CS. 2009. Fabrication of pHresponsive molecularly imprinted polyethersulfone particles for bisphenol-A uptake. J. Appl. Polym. Sci. 113: 916-921. DOI: 10.1002/app.30014

2033. Zheng SL, Song SQ, Lan H, Qu GR, Li RX, Wu AB, Zhang DB. 2009. Newly Combined Method of Molecularly Imprinted Solid-Phase Extraction with ELISA for Rapid Detection of Clenbuterol in Animal-Tissue Samples. Anal. Lett. 42: 600-614. DOI: 10.1080/00032710802677134 
2034. Zhu QJ, Wang LP, Wu SF, Joseph W, Gu XH, Tang J. 2009. Selectivity of molecularly imprinted solid phase extraction for sterol compounds. Food Chem. 113: 608-615. DOI: 10.1016/j.foodchem.2008.07.044

2035. Xia JJ, Long Y, Guo MJ, Wang Y, Mi HF. 2009. Separation/enrichment of the low-content high molecular weight natural protein using protein-imprinted polymers with ARPCs. Sci. China Chem. 52: 1388-1393. DOI: 10.1007/s11426-009-0201-1

2036. Abdelkader MSA, Lockwood B, Sansongsak P. 2010. Uptake of nicotine from suspension culture of Nicotiana tabacum by molecularly imprinted polymers. J. Pharm. Pharmacol. 62: 633-637. DOI: 10.1211/jpp.62.05.0011

2037. Abu-Surrah AS, Al-Degs YS. 2010. A molecularly imprinted polymer via a salicylaldiminato-based cobalt(III) complex: A highly selective solid-phase extractant for anionic reactive dyes. J. Appl. Polym. Sci. 117: 2316-2323. DOI: 10.1002/app.32072

2038. Ali WH, Derrien D, Alix F, Pérollier C, Lépine O, Bayoudh S, Chapuis-Hugon F, Pichon V. 2010. Solid-phase extraction using molecularly imprinted polymers for selective extraction of a mycotoxin in cereals. J. Chromatogr. $A$ 1217: 6668-6673. DOI: 10.1016/j.chroma.2010.04.071

2039. Alizadeh T. 2010. Preparation of molecularly imprinted polymer containing selective cavities for urea molecule and its application for urea extraction. Anal. Chim. Acta 669: 94-101. DOI: 10.1016/j.aca.2010.04.044

2040. Arabzadeh N, Abdouss M. 2010. Synthesis and characterization of molecularly imprinted polymers for selective solid-phase extraction of pseudoephedrine. Colloid J. 72: 446-455. DOI: 10.1134/S1061933X10040022 
2041. Azodi-Deilami S, Abdouss M, Hasani SA. 2010. Preparation and utilization of a molecularly imprinted polymer for solid phase extraction of tramadol. Cent. Eur. J. Chem. 8: 861-869. DOI: 10.2478/s11532-010-0059-2

2042. Baggiani C, Baravalle P, Giovannoli C, Anfossi L, Giraudi G. 2010. Molecularly imprinted polymer/cryogel composites for solid-phase extraction of bisphenol A from river water and wine. Anal. Bioanal. Chem. 397: 815822. DOI: 10.1007/s00216-010-3591-1

2043. Beltran A, Marcé RM, Cormack PAG, Borrull F. 2010. Synthetic approaches to parabens molecularly imprinted polymers and their applications to the solid-phase extraction of river water samples. Anal. Chim. Acta 677: 72-78. DOI: 10.1016/j.aca.2010.07.021

2044. Bi W, Tian M, Zhou J, Row KH. 2010. Task-specific ionic liquid-assisted extraction and separation of astaxanthin from shrimp waste. J. Chromatogr B 878: 2243-2248. DOI: 10.1016/j.jchromb.2010.06.034

2045. Boonjob W, Yu YL, Miró M, Segundo MA, Wang JH, Cerdà V. 2010. Online Hyphenation of Multimodal Microsolid Phase Extraction Involving Renewable Molecularly Imprinted and Reversed-Phase Sorbents to Liquid Chromatography for Automatic Multiresidue Assays. Anal. Chem. 82: 30523060. DOI: 10.1021/ac100185s

2046. Buszewski B, Ricanyová J, Gadzala-Kopciuch R, Szumski M. 2010. Supramolecular recognition of estrogens via molecularly imprinted polymers. Anal. Bioanal. Chem. 397: 2977-2986. DOI: 10.1007/s00216-010-3859-5

2047. Byun HS, Youn YN, Yun YH, Yoon SD. 2010. Selective separation of aspirin using molecularly imprinted polymers. Sep. Purif. Technol. 74: 144-153. DOI: 10.1016/j.seppur.2010.05.017

2048. Canale F, Cordero C, Baggiani C, Baravalle P, Giovannoli C, Bicchi C. 2010. Development of a molecularly imprinted polymer for selective 
extraction of bisphenol A in water samples. J. Sep. Sci. 33: 1644-1651. DOI: $10.1002 /$ jssc. 201000013

2049. Chang YH, Zhang L, Ying HJ, Li ZJ, Lv H, Ouyang PK. 2010. Desulfurization of Gasoline using Molecularly Imprinted Chitosan as Selective Adsorbents. Appl. Biochem. Biotechnol. 160: 593-603. DOI: 10.1007/s12010-008-8441-7

2050. Chen AH, Huang YY. 2010. Adsorption of Remazol Black 5 from aqueous solution by the templated crosslinked-chitosans. J. Haz. Mater. 177: 668675. DOI: 10.1016/j.jhazmat.2009.12.083

2051. Chen LG, Zhang XP, Xu Y, Du XB, Sun X, Sun L, Wang H, Zhao Q, Yu AM, Zhang $H Q$, Ding L. 2010. Determination of fluoroquinolone antibiotics in environmental water samples based on magnetic molecularly imprinted polymer extraction followed by liquid chromatography-tandem mass spectrometry. Anal. Chim. Acta 662: 31-38. DOI: 10.1016/j.aca.2010.01.001

2052. Chen LN, Jia XJ, Lu Q, Peng Y, Du SH, Chen Q. 2010. Highly efficient and selective enrichment of puerarin from Radix Puerariae by molecularly imprinted solid-phase extraction. Sep. Purif. Technol. 71: 324-330. DOI: 10.1016/j.seppur.2009.12.018

2053. Claude B, Viron-Lamy C, Haupt K, Morin P. 2010. Synthesis of a molecularly imprinted polymer for the solid-phase extraction of betulin and betulinic acid from plane bark. Phytochem. Anal. 21: 180-185. DOI: 10.1002/pca. 1175

2054. Curcio M, Puoci F, Cirillo G, lemma F, Spizzirri UG, Picci N. 2010. Selective Determination of Melamine in Aqueous Medium by Molecularly Imprinted Solid Phase Extraction. J. Agric. Food Chem. 58: 11883-11887. DOI: $10.1021 / \mathrm{j} 102936 \mathrm{p}$

2055. Dai CM, Geissen SU, Zhang YL, Zhang YJ, Zhou XF. 2010. Performance evaluation and application of molecularly imprinted polymer for separation of 
carbamazepine in aqueous solution. J. Haz. Mater. 184: 156-163. DOI: 10.1016/j.jhazmat.2010.08.018

2056. de Barros LA, Martins I, Rath S. 2010. A selective molecularly imprinted polymer-solid phase extraction for the determination of fenitrothion in tomatoes. Anal. Bioanal. Chem. 397: 1355-1361. DOI: 10.1007/s00216-0103629-4

2057. De Smet D, Monbaliu S, Dubruel P, Van Peteghem C, Schacht E, De Saeger S. 2010. Synthesis and application of a T-2 toxin imprinted polymer. J. Chromatogr. A 1217: 2879-2886. DOI: 10.1016/j.chroma.2010.02.068

2058. Demeestere K, Petrovic M, Gros M, Dewulf J, Van Langenhove H, Barceló D. 2010. Trace analysis of antidepressants in environmental waters by molecularly imprinted polymer-based solid-phase extraction followed by ultra-performance liquid chromatography coupled to triple quadrupole mass spectrometry. Anal. Bioanal. Chem. 396: 825-837. DOI: 10.1007/s00216009-3270-2

2059. Dinçer A, Zihnioglu F. 2010. Preparation of glutathione imprinted polymer. Prep. Biochem. Biotechnol. 40: 188-197. DOI: 10.1080/10826068.2010.488515

2060. Divya MP, Rajput YS, Sharma R. 2010. Synthesis and Application of Tetracycline Imprinted Polymer. Anal. Lett. 43: 919-928. DOI: $10.1080 / 00032710903491039$

2061. Djozan D, Ebrahimi B, Mahkam M, Farajzadeh MA. 2010. Evaluation of a new method for chemical coating of aluminum wire with molecularly imprinted polymer layer. Application for the fabrication of triazines selective solid-phase microextraction fiber. Anal. Chim. Acta 674: 40-48. DOI: 10.1016/j.aca.2010.06.006 
2062. Ebrahimzadeh H, Abedi H, Yamini Y, Adlnasab L. 2010. Molecular-imprinted polymer extraction combined with dispersive liquid-liquid micro-extraction for ultra-preconcentration of mononitrotoluene. J. Sep. Sci. 33: 3759-3766. DOI: $10.1002 /$ jssc.201000376

2063. El-Sheikh AH, Al-Quse RW, El-Barghouthi MI, Al-Masri FS. 2010. Derivatization of 2-chlorophenol with 4-amino-anti-pyrine: A novel method for improving the selectivity of molecularly imprinted solid phase extraction of 2-chlorophenol from water. Talanta 83: 667-673. DOI: 10.1016/j.talanta.2010.10.022

2064. Farhadi K, Matin AA, Hashemi P. 2010. Removal of malachite green from aqueous solutions using molecularly imprinted polymer. Desal. Water Treat. 24: 20-27. DOI: 10.5004/dwt.2010.1125

2065. Figueiredo EC, Sanvido GB, Arruda MAZ, Eberlin MN. 2010. Molecularly imprinted polymers as analyte sequesters and selective surfaces for easy ambient sonic-spray ionization. Analyst 135: 726-730. DOI: $10.1039 / b 923289 c$

2066. Gai QQ, Qu F, Liu ZJ, Dai RJ, Zhang YK. 2010. Superparamagnetic lysozyme surface-imprinted polymer prepared by atom transfer radical polymerization and its application for protein separation. J. Chromatogr. A 1217: 5035-5042. DOI: 10.1016/j.chroma.2010.06.001

2067. Gao RX, Zhang JJ, He XW, Chen LX, Zhang YK. 2010. Selective extraction of sulfonamides from food by use of silica-coated molecularly imprinted polymer nanospheres. Anal. Bioanal. Chem. 398: 451-461. DOI: 10.1007/s00216-010-3909-z

2068. Gao RX, Kong X, Su FH, He XW, Chen LX, Zhang YK. 2010. Synthesis and evaluation of molecularly imprinted core-shell carbon nanotubes for the determination of triclosan in environmental water samples. J. Chromatogr. A 1217: 8095-8102. DOI: 10.1016/j.chroma.2010.10.121 
2069. García-Galán MJ, Díaz-Cruz MS, Barceló D. 2010. Determination of triazines and their metabolites in environmental samples using molecularly imprinted polymer extraction, pressurized liquid extraction and LC-tandem mass spectrometry. J. Hydrol. 383: 30-38. DOI: 10.1016/j.jhydrol.2009.09.025

2070. Ge SG, Yan M, Cheng XL, Zhang CC, Yu JH, Zhao PN, Gao WQ. 2010. Online molecular imprinted solid-phase extraction flow-injection fluorescence sensor for determination of florfenicol in animal tissues. J. Pharm. Biomed. Anal. 52: 615-619. DOI: 10.1016/j.jpba.2010.01.030

2071. Gholivand MB, Khodadadian M, Ahmadi F. 2010. Computer aided-molecular design and synthesis of a high selective molecularly imprinted polymer for solid-phase extraction of furosemide from human plasma. Anal. Chim. Acta 658: 225-232. DOI: 10.1016/j.aca.2009.11.019

2072. Gu XH, Xu R, Yuan GL, Lu H, Gu BiR, Xie HP. 2010. Preparation of chlorogenic acid surface-imprinted magnetic nanoparticles and their usage in separation of Traditional Chinese Medicine. Anal. Chim. Acta 675: 64-70. DOI: 10.1016/j.aca.2010.06.033

2073. Guo BY, Wang SX, Ren BZ, Li X, Qin F, Li JZ. 2010. Citrinin selective molecularly imprinted polymers for SPE. J. Sep. Sci. 33: 1156-1160. DOI: 10.1002/jssc.200900652

2074. Han DM, Jia WP, Liang HD. 2010. Selective removal of 2,4dichlorophenoxyacetic acid from water by molecularly-imprinted aminofunctionalized silica gel sorbent. J. Environ. Sci. 22: 237-241. DOI: 10.1016/S1001-0742(09)60099-1

2075. Harun N, Anderson RA, Cormack PAG. 2010. Analysis of ketamine and norketamine in hair samples using molecularly imprinted solid-phase extraction (MISPE) and liquid chromatography-tandem mass spectrometry 
(LC-MS/MS). Anal. Bioanal. Chem. 396: 2449-2459. DOI: 10.1007/s00216009-3404-6

2076. He JA, Lv RH, Cheng J, Li YX, Xue JF, Lu K, Wang FC. 2010. Preparation and characterization of molecularly imprinted microspheres for dibutyl phthalate recognition in aqueous environment. J. Sep. Sci. 33: 3409-3414. DOI: $10.1002 /$ jssc.201000301

2077. He JA, Lv RH, Zhan HJ, Wang HZ, Cheng J, Lu K, Wang FC. 2010. Preparation and evaluation of molecularly imprinted solid-phase microextraction fibers for selective extraction of phthalates in an aqueous sample. Anal. Chim. Acta 674: 53-58. DOI: 10.1016/j.aca.2010.06.018

2078. He JA, Lv RH, Zhu J, Lu K. 2010. Selective solid-phase extraction of dibutyl phthalate from soybean milk using molecular imprinted polymers. Anal. Chim. Acta 661: 215-221. DOI: 10.1016/j.aca.2009.12.029

2079. Ho WL, Lin TC, Liu YY, Chen JA. 2010. Analysis of smoke PAHs from selected Taiwanese cigarettes by using molecular imprinting polymers. J. Environ. Sci. Health, Part $A$ 45: 211-223. DOI: $10.1080 / 10934520903429907$

2080. Hu TP, Zhang YM, Zheng LH, Fan GZ. 2010. Molecular recognition and adsorption performance of benzothiophene imprinted polymer on silica gel surface. Journal of Fuel Chemistry and Technology 38: 722-729. DOI: 10.1016/S1872-5813(11)60006-6

2081. Hu XG, Dai GM, Huang JJ, Ye TT, Fan HJ, Youwen T, Yu Y, Liang Y. 2010. Molecularly imprinted polymer coated on stainless steel fiber for solid-phase microextraction of chloroacetanilide herbicides in soybean and corn. $J$. Chromatogr. A 1217: 5875-5882. DOI: 10.1016/j.chroma.2010.07.011

2082. Hu YL, Wang YY, Chen XG, Hu YF, Li GK. 2010. A novel molecularly imprinted solid-phase microextraction fiber coupled with high performance 
liquid chromatography for analysis of trace estrogens in fishery samples. Talanta 80: 2099-2105. DOI: 10.1016/j.talanta.2009.11.015

2083. Hu YL, Li JW, Hu YF, Li GK. 2010. Development of selective and chemically stable coating for stir bar sorptive extraction by molecularly imprinted technique. Talanta 82: 464-470. DOI: 10.1016/j.talanta.2010.04.057

2084. Hu YL, Liu RJ, Li YW, Li GK. 2010. Investigation of ractopamine-imprinted polymer for dispersive solid-phase extraction of trace $\alpha$-agonists in pig tissues. J. Sep. Sci. 33: 2017-2025. DOI: 10.1002/jssc.201000063

2085. Huang WH, Zhou W, Xu WZ, Xu PP, Xu XJ, Yan YS. 2010. Preparation, Characterization and Performance of a Novel Surfaceimprinting Polymer for the Adsorption of Dibenzothiophene. Adsorpt. Sci. Technol. 28: 629-640. DOI: 10.1260/0263-6174.28.7.629

2086. Jackson R, Petrikovics I, Lai EPC, Yu JCC. 2010. Molecularly imprinted polymer stir bar sorption extraction and electrospray ionization tandem mass spectrometry for determination of 2-aminothiazoline-4-carboxylic acid as a marker for cyanide exposure in forensic urine analysis. Anal. Methods 2: 552-557. DOI: 10.1039/b9ay00251k

2087. Javanbakht M, Attaran AM, Namjumanesh MH, Esfandyari-Manesh M, Akbari-adergani B. 2010. Solid-phase extraction of tramadol from plasma and urine samples using a novel water-compatible molecularly imprinted polymer. J. Chromatogr B 878: 1700-1706. DOI: 10.1016/j.jchromb.2010.04.006

2088. Jing T, Du HR, Dai Q, Xia HA, Niu JW, Hao QL, Mei SR, Zhou YK. 2010. Magnetic molecularly imprinted nanoparticles for recognition of lysozyme. Biosens. Bioelectron. 26: 301-306. DOI: 10.1016/j.bios.2010.08.044

2089. Jing T, Wang Y, Dai Q, Xia HA, Niu JW, Hao QL, Mei SR, Zhou YK. 2010. Preparation of mixed-templates molecularly imprinted polymers and 
investigation of the recognition ability for tetracycline antibiotics. Biosens. Bioelectron. 25: 2218-2224. DOI: 10.1016/j.bios.2010.02.023

2090. Khodadadian M, Ahmadi F. 2010. Computer-assisted design and synthesis of molecularly imprinted polymers for selective extraction of acetazolamide from human plasma prior to its voltammetric determination. Talanta 81: 1446-1453. DOI: 10.1016/j.talanta.2010.02.049

2091. Khorrami AR, Edrisi M. 2010. Synthesis and Evaluation of a Molecularly Imprinted Polymer for Solid Phase Extraction of Ethopabate from Chicken Tissue. Sep. Sci. Technol. 45: 404-412. DOI: 10.1080/01496390903409567

2092. Krupadam RJ, Bhagat B, Khan MS. 2010. Highly sensitive determination of polycyclic aromatic hydrocarbons in ambient air dust by gas chromatography-mass spectrometry after molecularly imprinted polymer extraction. Anal. Bioanal. Chem. 397: 3097-3106. DOI: 10.1007/s00216010-3858-6

2093. Krupadam RJ, Khan MS, Wate SR. 2010. Removal of probable human carcinogenic polycyclic aromatic hydrocarbons from contaminated water using molecularly imprinted polymer. Water Res. 44: 681-688. DOI: 10.1016/j.watres.2009.09.044

2094. Lai EPC, De Maleki Z, Wu S. 2010. Characterization of molecularly imprinted and nonimprinted polymer submicron particles specifically tailored for removal of trace $17 \alpha$-estradiol in water treatment. J. Appl. Polym. Sci. 116: 1499-1508. DOI: 10.1002/app.31698

2095. Lee MH, Chen YC, Ho MH, Lin HY. 2010. Optical recognition of salivary proteins by use of molecularly imprinted poly(ethylene-co-vinyl alcohol)/quantum dot composite nanoparticles. Anal. Bioanal. Chem. 397: 1457-1466. DOI: 10.1007/s00216-010-3631-x 
2096. Lee MH, Thomas JL, Ho MH, Yuan C, Lin HY. 2010. Synthesis of Magnetic Molecularly Imprinted Poly(ethylene-co-vinyl alcohol) Nanoparticles and Their Uses in the Extraction and Sensing of Target Molecules in Urine. ACS Appl. Mater. Interf. 2: 1729-1736. DOI: 10.1021/am100227r

2097. Li M, Zhang LY, Meng ZH, Wang ZY, Wu H. 2010. Molecularly-imprinted microspheres for selective extraction and determination of melamine in milk and feed using gas chromatography-mass spectrometry. J. Chromatogr $B$ 878: 2333-2338. DOI: 10.1016/j.jchromb.2010.07.003

2098. Li Y, Li X, Chu J, Dong CK, Qi JY, Yuan YX. 2010. Synthesis of core-shell magnetic molecular imprinted polymer by the surface RAFT polymerization for the fast and selective removal of endocrine disrupting chemicals from aqueous solutions. Environ. Pollut. 158: 2317-2323. DOI: 10.1016/j.envpol.2010.02.007

2099. Liu J, Yang M, Huai LF. 2010. Molecularly imprinted solid-phase extraction for chlorpyrifos determination in water samples. In Environment Materials and Environment Management, Advanced Materials Research, Vol. 113116, Du ZY, Sun XB (eds). Scientific.net: 947-951. DOI: 10.4028/www.scientific.net/AMR.113-116.947

2100. Liu J, Yang M, Huai LF, Chen YT. 2010. Removal of chlorpyrifos from contaminated water using molecularly imprinted polymeric microspheres. In 4th International Conference on Bioinformatics and Biomedical Engineering (iCBBE), 2010, IEEE: Art. No. 5514935. DOI: 10.1109/ICBBE.2010.5514935

2101. Liu MH, Li MJ, Qiu B, Chen X, Chen GN. 2010. Synthesis and applications of diethylstilbestrol-based molecularly imprinted polymer-coated hollow fiber tube. Anal. Chim. Acta 663: 33-38. DOI: 10.1016/j.aca.2010.01.027

2102. Liu PY, Shen J, Gao L, Liu L, Li R, Li QA. 2010. Determination of Fluoroquinolones in Milk by High-Performance Liquid Chromatography 
Using Mixed-Templates Imprinted Polymer Extraction. Asian J. Chem. 22: 6275-6288.

2103. Liu Z, Bucknall DG, Allen MG. 2010. Absorption performance of iodixanolimprinted polymers in aqueous and blood plasma media. Acta Biomater. 6: 2003-2012. DOI: 10.1016/j.actbio.2009.11.007

2104. Lombardo-Agüí M, García-Campaña AM, Gámiz-Gracia L, Cruces Blanco C. 2010. Laser induced fluorescence coupled to capillary electrophoresis for the determination of fluoroquinolones in foods of animal origin using molecularly imprinted polymers. J. Chromatogr. A 1217: 2237-2242. DOI: 10.1016/j.chroma.2010.02.016

2105. Lordel S, Chapuis-Hugon F, Eudes V, Pichon V. 2010. Development of imprinted materials for the selective extraction of nitroaromatic explosives. $J$. Chromatogr. A 1217: 6674-6680. DOI: 10.1016/j.chroma.2010.04.081

2106. Lu Q, Chen XM, Nie L, Luo J, Jiang HJ, Chen LN, Hu Q, Du SH, Zhang ZP. 2010. Tuning of the vinyl groups' spacing at surface of modified silica in preparation of high density imprinted layer-coated silica nanoparticles: A dispersive solid-phase extraction materials for chlorpyrifos. Talanta 81: 959966. DOI: 10.1016/j.talanta.2010.01.044

2107. Lucci P, Derrien D, Alix F, Pérollier C, Bayoudh S. 2010. Molecularly imprinted polymer solid-phase extraction for detection of zearalenone in cereal sample extracts. Anal. Chim. Acta 672: 15-19. DOI: 10.1016/j.aca.2010.03.010

2108. Lv RH, Xu L, Huang $X H$, Wang $Y Q$, Zhang J. 2010. Preparation and evaluation of a molecularly imprinted polymer for tolazoline. J. Appl. Polym. Sci. 115: 198-203. DOI: 10.1002/app.31037

2109. Martínez Bueno MJ, Herrera S, Uclés A, Agüera A, Hernando MD, Shimelis O, Rudolfsson M, Fernández-Alba AR. 2010. Determination of malachite 
green residues in fish using molecularly imprinted solid-phase extraction followed by liquid chromatography-linear ion trap mass spectrometry. Anal. Chim. Acta 665: 47-54. DOI: 10.1016/j.aca.2010.03.001

2110. Mohajeri SA, Karimi G, Khansari MR. 2010. Clozapine imprinted polymers: Synthesis, characterization and application for drug assay in human serum. Anal. Chim. Acta 683: 143-148. DOI: 10.1016/j.aca.2010.10.023

2111. Mohajeri SA, Hosseinzadeh H, Keyhanfar F, Aghamohammadian J. 2010. Extraction of crocin from saffron (Crocus sativus) using molecularly imprinted polymer solid-phase extraction. J. Sep. Sci. 33: 2302-2309. DOI: $10.1002 /$ jssc.201000183

2112. Möller K, Davies R, Fred C, Törnqvist M, Nilsson U. 2010. Evaluation of molecularly imprinted solid-phase extraction for a 1,2:3,4-diepoxybutane adduct to valine. J. Chromatogr $B$ 878: 2497-2501. DOI: 10.1016/j.jchromb.2010.02.012

2113. Núñez L, Turiel E, Martin-Esteban A, Tadeo JL. 2010. Molecularly imprinted polymer for the extraction of parabens from environmental solid samples prior to their determination by high performance liquid chromatographyultraviolet detection. Talanta 80: 1782-1788. DOI: 10.1016/j.talanta.2009.10.023

2114. Okutucu B, Telefoncu A, Haupt K. 2010. Molecularly Imprinted Polymer for Serotonin Recognition. Hacettepe J. Biol. \& Chem. 38: 79-84.

2115. Okutucu B, Akkaya A, Pazarlioglu NK. 2010. Molecularly Imprinted Polymers for Some Reactive Dyes. Prep. Biochem. Biotechnol. 40: 366-376. DOI: 10.1080/10826068.2010.525428

2116. Oliveira HM, Segundo MA, Lima JLFC, Miró M, Cerdà V. 2010. Exploiting automatic on-line renewable molecularly imprinted solid-phase extraction in lab-on-valve format as front end to liquid chromatography: application to the 
determination of riboflavin in foodstuffs. Anal. Bioanal. Chem. 397: 77-86. DOI: 10.1007/s00216-010-3522-1

2117. Ouyang RZ, Lei JP, Ju HX. 2010. Artificial receptor-functionalized nanoshell: facile preparation, fast separation and specific protein recognition. Nanotechnology 21: Art. No. 185502. DOI: 10.1088/0957$4484 / 21 / 18 / 185502$

2118. Özkütük EB, Özalp E, Ersöz A, Açikkalp E, Say R. 2010. Thiocyanate separation by imprinted polymeric systems. Microchim. Acta 169: 129-135. DOI: $10.1007 / \mathrm{s} 00604-010-0319-z$

2119. Pan JM, Zou XH, Wang X, Guan W, Yan YS, Han JA. 2010. Selective recognition of 2,4-dichlorophenol from aqueous solution by uniformly sized molecularly imprinted microspheres with $\alpha$-cyclodextrin/attapulgite composites as support. Chem. Eng. J. 162: 910-918. DOI: 10.1016/j.cej.2010.06.039

2120. Pan MF, Wang JP, Fang GZ, Tang WW, Wang S. 2010. Synthesis and characterization of a molecularly imprinted polymer and its application as SPE enrichment sorbent for determination of trace methimazole in pig samples using HPLC-UV. J. Chromatogr $B$ 878: 1531-1536. DOI: 10.1016/j.jchromb.2010.04.004

2121. Parisi OI, Cirillo G, Curcio M, Puoci F, lemma F, Spizzirri UG, Picci N. 2010. Surface modifications of molecularly imprinted polymers for improved template recognition in water media. J. Polym. Res. 17: 355-362. DOI: 10.1007/s10965-009-9322-7

2122. Peng Y, Xie Y, Luo J, Nie L, Chen Y, Chen LN, Du SH, Zhang ZP. 2010. Molecularly imprinted polymer layer-coated silica nanoparticles toward dispersive solid-phase extraction of trace sulfonylurea herbicides from soil and crop samples. Anal. Chim. Acta 674: 190-200. DOI: 10.1016/j.aca.2010.06.022 
2123. Prasad BB, Tiwari MP, Madhuri R, Sharma PS. 2010. Development of a highly sensitive and selective hyphenated technique (molecularly imprinted micro-solid phase extraction fiber-molecularly imprinted polymer fiber sensor) for ultratrace analysis of folic acid. Anal. Chim. Acta 662: 14-22. DOI: 10.1016/j.aca.2009.12.037

2124. Prasad BB, Tiwari MP, Madhuri R, Sharma PS. 2010. Enantioselective quantitative separation of $d$ - and I-thyroxine by molecularly imprinted microsolid phase extraction silver fiber coupled with complementary molecularly imprinted polymer-sensor. J. Chromatogr. A 1217: 4255-4266. DOI: 10.1016/j.chroma.2010.04.055

2125. Qi PP, Wang JC, Jin J, Su F, Chen JP. 2010. 2,4-Dimethylphenol imprinted polymers as a solid-phase extraction sorbent for class-selective extraction of phenolic compounds from environmental water. Talanta 81: 1630-1635. DOI: 10.1016/j.talanta.2010.03.015

2126. Qiao FX, Sun HW. 2010. Simultaneous extraction of enrofloxacin and ciprofloxacin from chicken tissue by molecularly imprinted matrix solid-phase dispersion. J. Pharm. Biomed. Anal. 53: 795-798. DOI: 10.1016/j.jpba.2010.06.008

2127. Qiu LJ, Liu W, Huang M, Zhang L. 2010. Preparation and application of solid-phase microextraction fiber based on molecularly imprinted polymer for determination of anabolic steroids in complicated samples. J. Chromatogr. A 1217: 7461-7470. DOI: 10.1016/j.chroma.2010.08.056

2128. Qu SS, Wang XB, Tong CL, Wu JM. 2010. Metal ion mediated molecularly imprinted polymer for selective capturing antibiotics containing $\beta$-diketone structure. J. Chromatogr. A 1217: 8205-8211. DOI: 10.1016/j.chroma.2010.10.097

2129. Rahiminezhad M, Shahtaheri SJ, Ganjali MR, Koohpaei AR, Rahimi Forushani A, Golbabaei F. 2010. An experimental investigation of the 
molecularly imprinted polymers as tailor-made sorbents of diazinon. J. Anal. Chem. 65: 694-698. DOI: 10.1134/S1061934810070063

2130. Rahiminezhad M, Shahtaheri SJ, Ganjali MR, Koohpaei AR, Forushani AR, Golbabaei F. 2010. Synthesis of Molecularly Imprinted Polymer as A Solid Phase Sorbent for Pesticide Dursban. Int. J. Occup. Hygiene 2: 51-56.

2131. Rezaei B, Mallakpour S, Rahmanian O. 2010. Application of Molecularly Imprinted Polymer for Solid Phase Extraction and Preconcentration of Hydrochlorothiazide in Pharmaceutical and Serum Sample Analysis. J. Iranian Chem. Soc. 7: 1004-1011. DOI: 10.1007/BF03246097

2132. Ricanyová J, Gadzala-Kopciuch R, Reiffova K, Bazel Y, Buszewski B. 2010. Molecularly imprinted adsorbents for preconcentration and isolation of progesterone and testosterone by solid phase extraction combined with HPLC. Adsorption 16: 473-483. DOI: 10.1007/s10450-010-9265-7

2133. Saifuddin N, Yasumira AAN. 2010. Microwave Enhanced Synthesis of Chitosan-graft-Polyacrylamide Molecularly Imprinted Polymer (MIP) for Selective Extraction of Antioxidants. E-J. Chem. 7: 1362-1374.

2134. Samatya S, Mizuki H, Ito Y, Kawakita H, Uezu K. 2010. The effect of polystyrene as a porogen on the fluoride ion adsorption of $\mathrm{Zr}(\mathrm{IV})$ surfaceimmobilized resin. Reac. Func. Polym. 70: 63-68. DOI: 10.1016/j.reactfunctpolym.2009.10.004

2135. Scorrano S, Longo L, Vasapollo G. 2010. Molecularly imprinted polymers for solid-phase extraction of 1-methyladenosine from human urine. Anal. Chim. Acta 659: 167-171. DOI: 10.1016/j.aca.2009.11.046

2136. See HH, Marsin Sanagi M, Ibrahim WAW, Naim AA. 2010. Determination of triazine herbicides using membrane-protected carbon nanotubes solid phase membrane tip extraction prior to micro-liquid chromatography. $\mathrm{J}$. Chromatogr. A 1217: 1767-1772. DOI: 10.1016/j.chroma.2010.01.053 
2137. She YX, Cao WQ, Shi XM, Lv XL, Liu JJ, Wang RY, Jin F, Wang J, Xiao H. 2010. Class-specific molecularly imprinted polymers for the selective extraction and determination of sulfonylurea herbicides in maize samples by high-performance liquid chromatography-tandem mass spectrometry. $\mathrm{J}$. Chromatogr B 878: 2047-2053. DOI: 10.1016/j.jchromb.2010.05.038

2138. Shi XZ, Song SQ, Sun AL, Li DX, Wu AB, Zhang DB. 2010. Determination of Chloramphenicol Residues in Foods by ELISA and LC-MS/MS Coupled with Molecularly Imprinted Solid Phase Extraction. Anal. Lett. 43: 2798-2807. DOI: $10.1080 / 00032711003763616$

2139. Shi XZ, Song SQ, Qu GR, Zheng SL, Wu AB, Zhang DB. 2010. Water Compatible Molecularly Imprinted Polymer Microspheres for Extraction of Ampicillin in Foods. Anal. Lett. 43: 757-767. DOI: $10.1080 / 00032710903486286$

2140. Shimelis O, Stenerson K, Vitkuske D, Beyowich S, Boyd B. 2010. Extraction and Analysis of PAHs in Olive Oil using Molecularly Imprinted Polymer SPE and GC-MS. The Reporter 27: 12-13.

2141. Shimelis O, Wihlborg A-K, Rudolfsson M, Boyd B, Trinh A. 2010. Extraction of Nitroimidazoles from Milk and Eggs using Molecularly Imprinted Polymers. The Reporter 27: 15-17.

2142. Silva RGC, Vigna CRM, Bottoli CBG, Collins CH, Augusto F. 2010. Molecularly imprinted silica as a selective SPE sorbent for triazine herbicides. J. Sep. Sci. 33: 1319-1324. DOI: 10.1002/jssc.200900785

2143. Sun XL, He J, Cai GR, Lin AQ, Zheng WJ, Liu X, Chen LX, He XW, Zhang YK. 2010. Room temperature ionic liquid-mediated molecularly imprinted polymer monolith for the selective recognition of quinolones in pork samples. J. Sep. Sci. 33: 3786-3793. DOI: 10.1002/jssc.201000337 
2144. Tang PP, Luo ZF, Cai JB, Su QD. 2010. A novel indirect inhibitive immunoassay for sulfamethoxazole. Chin. Chem. Lett. 21: 955-958. DOI: 10.1016/j.cclet.2010.03.029

2145. Tehrani MS, Vardini MT, Azar PA, Husain SW. 2010. Solid-Phase Extraction of Metoprolol onto (Methacrylic acid- ethylene glycol dimethacrylate)-based Molecularly Imprinted Polymer and Its Spectrophotometric Determination. Chin. J. Chem. 28: 647-655. DOI: 10.1002/cjoc.201090127

2146. Thongchai W, Liawruangath B, Liawruangrath S, Greenway GM. 2010. A microflow chemiluminescence system for determination of chloramphenicol in honey with preconcentration using a molecularly imprinted polymer. Talanta 82: 560-566. DOI: 10.1016/j.talanta.2010.05.007

2147. Tse Sum Bui B, Merlier F, Haupt K. 2010. Toward the Use of a Molecularly Imprinted Polymer in Doping Analysis: Selective Preconcentration and Analysis of Testosterone and Epitestosterone in Human Urine. Anal. Chem. 82: 4420-4427. DOI: 10.1021/ac100128c

2148. van Biesen G, Wiseman JM, Li J, Bottaro CS. 2010. Desorption electrospray ionization-mass spectrometry for the detection of analytes extracted by thinfilm molecularly imprinted polymers. Analyst 135: 2237-2240. DOI: 10.1039/c0an00331j

2149. Verma KS, Xia K. 2010. Analysis of triclosan and triclocarban in soil and biosolids using molecularly imprinted solid phase extraction coupled with HPLC-UV. J. AOAC Int. 93: 1313-1321. DOI: 10.5555/jaoi.93.4.1313

2150. Wichner NM, Ghavtadze N, Würthwein EU, Koller H. 2010. Imprinting With Phenyl Group Interactions: A Case Study of the Hybrid Sol-Gel Encapsulation of the Complex $\{\mathrm{Na}[\mathrm{Ph} 2 \mathrm{P}(\mathrm{O})-\mathrm{CH} 2-\mathrm{P}(\mathrm{O}) \mathrm{Ph} 2] 3\}+$. J. Phys. Chem. C 114: 22590-22596. DOI: 10.1021/jp107581g 
2151. Wihlborg A-K, Shimelis O, Trinh A. 2010. Molecularly Imprinted Polymer SPE for the Highly Selective Extraction of Fluoroquinolones from Bovine Kidney. The Reporter 27: 18-20.

2152. Xia JJ, Long Y, Guo MJ, Liu HJ, Wang Y, Mi HF. 2010. Using Proteinimprinted Polymers as Artificial Antibodies to Isolate Immunoglobulin Binding Protein (BiP) and Study Protein-Protein Interactions. Chem. Lett. 39: 475-477. DOI: 10.1246/cl.2010.475

2153. Xu L, Lv RH, Wang YQ, Gao JZ. 2010. Selective solid-phase extraction of naphazoline using imprinted polymers as matrix prepared by precipitation polymerization. J. Appl. Polym. Sci. 118: 881-886. DOI: 10.1002/app.32352

2154. Xu PP, Xu WZ, Zhang XJ, Yan YS. 2010. A surface-imprinted polymer for removing dibenzothiophene from gasoline. Microchim. Acta 171: 441-449. DOI: 10.1007/s00604-010-0462-6

2155. Xu WJ, Su SF, Jiang P, Wang HS, Dong XC, Zhang M. 2010. Determination of sulfonamides in bovine milk with column-switching high performance liquid chromatography using surface imprinted silica with hydrophilic external layer as restricted access and selective extraction material. $J$. Chromatogr. A 1217: 7198-7207. DOI: 10.1016/j.chroma.2010.09.035

2156. Xu ZG, Hu YF, Hu YL, Li GK. 2010. Investigation of ractopamine molecularly imprinted stir bar sorptive extraction and its application for trace analysis of a2-agonists in complex samples. J. Chromatogr. A 1217: 3612-3618. DOI: 10.1016/j.chroma.2010.03.046

2157. Xu ZX, Zhou J, Zhao DY, Qiao XG, Yang JM. 2010. Determination of Trace Para Red Residues in Foods through On-Line Molecularly Imprinted Solid Phase Extraction Coupled with High-Performance Liquid Chromatography. J. Food Sci. 75: C49-C54. DOI: 10.1111/j.1750-3841.2009.01416.x 
2158. Xu ZX, Fang GZ, Wang S. 2010. Molecularly imprinted solid phase extraction coupled to high-performance liquid chromatography for determination of trace dichlorvos residues in vegetables. Food Chem. 119: 845-850. DOI: 10.1016/j.foodchem.2009.08.047

2159. Yang LQ, Zhao XM, Zhou J. 2010. Selective enrichment and determination of nicosulfuron in water and soil by a stir bar based on molecularly imprinted polymer coatings. Anal. Chim. Acta 670: 72-77. DOI: 10.1016/j.aca.2010.04.041

2160. Yang ML, Gu WC, Sun L, Zhang F, Ling Y, Chu XG, Wang DN. 2010. Study on the molecularly imprinted polymers with methyl-testosterone as the template. Talanta 81: 156-161. DOI: 10.1016/j.talanta.2009.11.051

2161. Yin JF, Meng ZH, Du MJ, Liu C, Song MY, Wang HL. 2010. Pseudotemplate molecularly imprinted polymer for selective screening of trace $\alpha$ lactam antibiotics in river and tap water. J. Chromatogr. A 1217: 5420-5426. DOI: 10.1016/j.chroma.2010.06.044

2162. Yin $X Y$, Shan $L Q$, Han $X L$, Luo YM. 2010. Preparation and recognition properties of andrographolide molecularly imprinted ploymer microspheres. In Materials Science and Engineering Applications, Advanced Materials Research, Vol. 160-162, Zhang GJ, Xu J (eds). Scientific.net: 777-782. DOI: 10.4028/www.scientific.net/AMR.160-162.777

2163. Zhang J, Yue XF, Liang PP. 2010. Preparation of molecular imprinted monolithic extraction column and its application to strychnine analysis in Wan Tong Jin Gu troche by HPLC. J. Liq. Chrom. Rel. Technol. 33: 770777. DOI: 10.1080/10826071003684133

2164. Zhang XP, Chen LG, Xu Y, Wang $H$, Zeng $Q L$, Zhao Q, Ren NQ, Ding L. 2010. Determination of $\alpha$-lactam antibiotics in milk based on magnetic molecularly imprinted polymer extraction coupled with liquid 
chromatography-tandem mass spectrometry. J. Chromatogr B 878: 3421 3426. DOI: 10.1016/j.jchromb.2010.10.030

2165. Zhang Y, Li YW, Hu YL, Li GK, Chen YQ. 2010. Preparation of magnetic indole-3-acetic acid imprinted polymer beads with 4-vinylpyridine and $\alpha$ cyclodextrin as binary monomer via microwave heating initiated polymerization and their application to trace analysis of auxins in plant tissues. J. Chromatogr. A 1217: 7337-7344. DOI: 10.1016/j.chroma.2010.09.059

2166. Zhang ZH, Liu L, Li H, Yao SZ. 2010. Preparation and Evaluation of Uniform-Sized Sol-Gel Theophylline Imprinted Microspheres as Solid Phase Extraction Sorbent. Anal. Lett. 43: 2318-2330. DOI: $10.1080 / 00032711003717364$

2167. Zhang ZH, Zhang ML, Luo LJ, Yang X, Hu YF, Zhang HB, Yao SZ. 2010. Synthesis and application of core-shell complex-imprinted polymer for the solid-phase extraction of melamine from dairy products. J. Sep. Sci. 33: 2854-2861. DOI: 10.1002/jssc.201000264

2168. Zhang ZH, Zhang HB, Hu YF, Yao SZ. 2010. Synthesis and application of multi-walled carbon nanotubes-molecularly imprinted sol-gel composite material for on-line solid-phase extraction and high-performance liquid chromatography determination of trace Sudan IV. Anal. Chim. Acta 661: 173-180. DOI: 10.1016/j.aca.2009.12.024

2169. Zhao CD, Zhao T, Liu XY, Zhang HX. 2010. A novel molecularly imprinted polymer for simultaneous extraction and determination of sudan dyes by online solid phase extraction and high performance liquid chromatography. $\mathrm{J}$. Chromatogr. A 1217: 6995-7002. DOI: 10.1016/j.chroma.2010.09.005

2170. Zhao WH, Sheng N, Zhu R, Wei FD, Cai Z, Zhai MJ, Du SH, Hu Q. 2010. Preparation of dummy template imprinted polymers at surface of silica 
microparticles for the selective extraction of trace bisphenol A from water samples. J. Haz. Mater. 179: 223-229. DOI: 10.1016/j.jhazmat.2010.02.083

2171. Zheng MM, Gong R, Zhao X, Feng YQ. 2010. Selective sample pretreatment by molecularly imprinted polymer monolith for the analysis of fluoroquinolones from milk samples. J. Chromatogr. A 1217: 2075-2081. DOI: 10.1016/j.chroma.2010.02.011

2172. Zhou JF, Ma C, Zhou S, Ma PL, Chen FR, Qi Y, Chen HX. 2010. Preparation, evaluation and application of molecularly imprinted solid-phase microextraction monolith for selective extraction of pirimicarb in tomato and pear. J. Chromatogr. A 1217: 7478-7483. DOI: 10.1016/j.chroma.2010.09.079

2173. Zhou WH, Lu CH, Guo XC, Chen FR, Yang HH, Wang XR. 2010. Musselinspired molecularly imprinted polymer coating superparamagnetic nanoparticles for protein recognition. J. Mater. Chem. 20: 880-883. DOI: 10.1039/b916619j

2174. Zhu QH, Huang DD, Li L, Yin YG. 2010. Synthesis of molecularly imprinted polymers for the application of selective clean-up vinblastine from Catharanthus roseus extract. Sci. China Chem. 53: 2587-2592. DOI: 10.1007/s11426-010-4007-y

2175. Zhu R, Zhao WH, Zhai MJ, Wei FD, Cai Z, Sheng N, Hu Q. 2010. Molecularly imprinted layer-coated silica nanoparticles for selective solidphase extraction of bisphenol $A$ from chemical cleansing and cosmetics samples. Anal. Chim. Acta 658: 209-216. DOI: 10.1016/j.aca.2009.11.008

2176. Ameli A, Alizadeh N. 2011. Nanostructured conducting molecularly imprinted polymer for selective extraction of salicylate from urine and serum samples by electrochemically controlled solid-phase micro-extraction. Anal. Chim. Acta 707: 62-68. DOI: 10.1016/j.aca.2011.09.005 
2177. Ashri NY, Abdel-Rehim M. 2011. Sample treatment based on extraction techniques in biological matrices. Bioanalysis 3: 2003-2018. DOI: 10.4155/bio.11.201

2178. Asman S, Yusof NA, Abdullah AH, Haron MJ. 2011. Synthesis and characterization of a molecularly imprinted polymer for methylene blue. Asian J. Chem. 23: 4786-4794.

2179. Aufartová J, Mahugo-Santana C, Sosa-Ferrera Z, Santana-Rodríguez JJ, Nováková L, Solich P. 2011. Determination of steroid hormones in biological and environmental samples using green microextraction techniques: An overview. Anal. Chim. Acta 704: 33-46. DOI: 10.1016/j.aca.2011.07.030

2180. Bahraini N, Lai EPC, Li CS, Sadi BB, Kramer GH. 2011. Molecularly Imprinted Polymers for 90Sr Urine Bioassay. Health Physics 101: 128-135. DOI: 10.1097/HP.0b013e318213a5ef

2181. Barahona F, Turiel E, Cormack PAG, Martín-Esteban A. 2011. Synthesis of core-shell molecularly imprinted polymer microspheres by precipitation polymerization for the inline molecularly imprinted solid-phase extraction of thiabendazole from citrus fruits and orange juice samples. J. Sep. Sci. 34: 217-224. DOI: 10.1002/jssc.201000277

2182. Beltran A, Borrull F, Cormack PAG, Marcé RM. 2011. Molecularly imprinted polymer with high-fidelity binding sites for the selective extraction of barbiturates from human urine. J. Chromatogr. A 1218: 4612-4618. DOI: 10.1016/j.chroma.2011.05.049

2183. Borrelli C, Barsanti S, Silvestri D, Manesiotis P, Ciardelli G, Sellergren B. 2011. Selective Depletion of Riboflavine from Beer Using Membranes Incorporating Imprinted Polymer Particles. J. Food Process. Preserv. 35: 112-128. DOI: 10.1111/j.1745-4549.2009.00464.x 
2184. Chang HC, Han GX, Bai K, Gao WB. 2011. Studies of theophylline imprinted phenolic resin sorbent. Acta Polym. Sin. 340-346. DOI: 10.3724/SP.J.1105.2011.10075

2185. Chen CB, Yang LQ, Zhou J. 2011. Trace bensulfuron-methyl analysis in tap water, soil, and soybean samples by a combination of molecularly imprinted stir bar sorption extraction and HPLC-UV. J. Appl. Polym. Sci. 122: 11981205. DOI: 10.1002/app.34256

2186. Chen FF, Wang GY, Shi YP. 2011. Molecularly imprinted polymer microspheres for solid-phase extraction of protocatechuic acid in Rhizoma homalomenae. J. Sep. Sci. 34: 2602-2610. DOI: 10.1002/jssc.201100463

2187. Chigome S, Darko G, Torto N. 2011. Electrospun nanofibers as sorbent material for solid phase extraction. Analyst 136: 2879-2889.

2188. Chimuka L, van Pinxteren M, Billing J, Yilmaz E, Jönsson JÅ. 2011. Selective extraction of triazine herbicides based on a combination of membrane assisted solvent extraction and molecularly imprinted solid phase extraction. J. Chromatogr. A 1218: 647-653. DOI: 10.1016/j.chroma.2010.12.019

2189. Cirillo G, Curcio M, Parisi OI, Puoci F, lemma F, Spizzirri UG, Restuccia D, Picci N. 2011. Molecularly imprinted polymers for the selective extraction of glycyrrhizic acid from liquorice roots. Food Chem. 125: 1058-1063. DOI: 10.1016/j.foodchem.2010.09.077

2190. Cizmárik A, Hrobonová K, Lehotay J, Spevak A. 2011. Examination of the chemical composition of propolis VII: Use of molecularly imprinted polymers for SPE of umbelliferone from propolis. Farmaceuticky Obzor 80: 251-256.

2191. Claude B, Nehmé R, Morin P. 2011. Analysis of urinary neurotransmitters by capillary electrophoresis: Sensitivity enhancement using field-amplified 
sample injection and molecular imprinted polymer solid phase extraction. Anal. Chim. Acta 699: 242-248. DOI: 10.1016/j.aca.2011.05.014

2192. Dai CM, Geissen SU, Zhang YL, Zhang YJ, Zhou XF. 2011. Selective removal of diclofenac from contaminated water using molecularly imprinted polymer microspheres. Environ. Pollut. 159: 1660-1666. DOI: 10.1016/j.envpol.2011.02.041

2193. Dai CM, Zhou XF, Zhang YL, Liu SG, Zhang J. 2011. Synthesis by precipitation polymerization of molecularly imprinted polymer for the selective extraction of diclofenac from water samples. J. Haz. Mater. 198: 175-181. DOI: 10.1016/j.jhazmat.2011.10.027

2194. De Smet D, Kodeck V, Dubruel P, Van Peteghem C, Schacht E, De Saeger S. 2011. Design of an imprinted clean-up method for mycophenolic acid in maize. J. Chromatogr. A 1218: 1122-1130. DOI: 10.1016/j.chroma.2010.12.085

2195. De Smet D, Dubruel P, Van Peteghem C, De Saeger S. 2011. Development of a molecularly imprinted polymer for patulin in apple juice. World Mycotoxin J. 4: 375-383. DOI: 10.3920/WMJ2010.1276

2196. del Sole R, Scardino A, Lazzoi MR, Vasapollo G. 2011. Molecularly imprinted polymer for solid phase extraction of nicotinamide in pork liver samples. J. Appl. Polym. Sci. 120: 1634-1641. DOI: 10.1002/app.33267

2197. Ding MJ, Wu XL, Yuan LH, Wang S, Li Y, Wang RY, Wen TT, Du SH, Zhou XM. 2011. Synthesis of core-shell magnetic molecularly imprinted polymers and detection of sildenafil and vardenafil in herbal dietary supplements. $J$. Haz. Mater. 191: 177-183. DOI: 10.1016/j.jhazmat.2011.04.058

2198. Dopico-García MS, Cela-Pérez C, López-Vilariño JM, González-Rodríguez MV, Barral-Losada LF. 2011. An approach to imprint irganox 1076: Potential 
application to the specific migration test in olive oil. J. Appl. Polym. Sci. 119: 2866-2874. DOI: 10.1002/app.32964

2199. Djozan D, Farajzadeh MA, Sorouraddin SM, Baheri T. 2011. Synthesis and Application of High Selective Monolithic Fibers Based on Molecularly Imprinted Polymer for SPME of Trace Methamphetamine. Chromatographia 73: 975-983. DOI: 10.1007/s10337-011-1984-8

2200. Duan ZJ, Fan LP, Fang GZ, Yi JH, Wang S. 2011. Novel surface molecularly imprinted sol-gel polymer applied to the online solid phase extraction of methyl-3-quinoxaline-2-carboxylic acid and quinoxaline-2carboxylic acid from pork muscle. Anal. Bioanal. Chem. 401: 2291-2299. DOI: $10.1007 / \mathrm{s} 00216-011-5329-0$

2201. Fu QA, Fang Q, Feng BL, Sun SJ, Du W, Amut EJ, Xiao AP, Chang C. 2011. Matrine-imprinted monolithic stationary phase for extraction and purification of matrine from Sophorae flavescentis Ait. J. Chromatogr B 879: 894-900. DOI: 10.1016/j.jchromb.2011.02.041

2202. Gadzala-Kopciuch R, Cendrowski K, Cesarz A, Kielbasa P, Buszewski B. 2011. Determination of zearalenone and its metabolites in endometrial cancer by coupled separation techniques. Anal. Bioanal. Chem. 401: 20692078. DOI: $10.1007 / \mathrm{s} 00216-011-5206-x$

2203. Gomez-Caballero A, Guerreiro A, Karim K, Piletsky S, Goicolea MA, Barrio RJ. 2011. Chiral imprinted polymers as enantiospecific coatings of stir bar sorptive extraction devices. Biosens. Bioelectron. 28: 25-32. DOI: 10.1016/j.bios.2011.06.048

2204. Guo HB, Liu KY, Liu YH, Fang BH, Liu M, He LM, Zeng ZL. 2011. Molecularly imprinted solid-phase extraction for the selective determination of valnemulin in feeds with high performance liquid chromatography. $J$. Chromatogr B 879: 181-185. DOI: 10.1016/j.jchromb.2010.11.041 
2205. Guo L, Deng QL, Fang GZ, Gao W, Wang S. 2011. Preparation and evaluation of molecularly imprinted ionic liquids polymer as sorbent for online solid-phase extraction of chlorsulfuron in environmental water samples. J. Chromatogr. A 1218: 6271-6277. DOI: 10.1016/j.chroma.2011.07.016

2206. Guo LQ, Zeng YB, Guan AH, Chen GN. 2011. Preparation and characterization of molecularly imprinted silica particles for selective adsorption of naphthalene. Reac. Func. Polym. 71: 1172-1176. DOI: 10.1016/j.reactfunctpolym.2011.08.005

2207. Guo WL, Hu W, Pan JM, Zhou HC, Guan W, Wang X, Dai JD, Xu LC. 2011. Selective adsorption and separation of BPA from aqueous solution using novel molecularly imprinted polymers based on kaolinite/Fe3O4 composites. Chem. Eng. J. 171: 603-611. DOI: 10.1016/j.cej.2011.04.036

2208. Guo ZF, Zhang LX, Song CY, Zhang XX. 2011. Molecularly imprinted solidphase extraction of matrine from radix Sophorae tonkinensis. Analyst 136: 3016-3022. DOI: 10.1039/C1AN15281E

2209. Guo ZY, Gai PP, Hao TT, Duan J, Wang S. 2011. Determination of Malachite Green Residues in Fish Using a Highly Sensitive Electrochemiluminescence Method Combined with Molecularly Imprinted Solid Phase Extraction. J. Agric. Food Chem. 59: 5257-5262. DOI: $10.1021 / \mathrm{jf} 2008502$

2210. He J, Shen YZ, Chen S, Wei HL, Zhu J, You LQ, Lu K. 2011. Preparation and evaluation of molecularly imprinted microspheres for solid-phase extraction of 1,4-hydroxybenzoic acid esters in soy. J. Sep. Sci. 34: 27392744. DOI: $10.1002 /$ jssc.201100452

2211. Helling S, Shinde S, Brosseron F, Schnabel A, Müller T, Meyer HE, Marcus K, Sellergren B. 2011. Ultratrace Enrichment of Tyrosine Phosphorylated Peptides on an Imprinted Polymer. Anal. Chem. 83: 1862-1865. DOI: $10.1021 / \mathrm{ac} 103086 \mathrm{v}$ 
2212. Herrero-Hernández E, Carabias-Martínez R, Rodríguez-Gonzalo E. 2011. Behavior of Phenols and Phenoxyacids on a Bisphenol-A Imprinted Polymer. Application for Selective Solid-Phase Extraction from Water and Urine Samples. Int. J. Mol. Sci. 12: 3322-3339. DOI: 10.3390/ijms12053322

2213. Ho WL, Liu YY, Lin TC. 2011. Development of Molecular Imprinted Polymer for Selective Adsorption of Benz[a]pyrene Among Airborne Polycyclic Aromatic Hydrocarbon Compounds. Environ. Eng. Sci. 28: 421-434. DOI: 10.1089/ees.2010.0268

2214. Hoshina K, Horiyama S, Matsunaga H, Haginaka J. 2011. Simultaneous determination of non-steroidal anti-inflammatory drugs in river water samples by liquid chromatography-tandem mass spectrometry using molecularly imprinted polymers as a pretreatment column. J. Pharm. Biomed. Anal. 55: 916-922. DOI: 10.1016/j.jpba.2011.03.014

2215. Hu XG, Ye TT, Yu Y, Cao YJ, Guo CJ. 2011. Novel liquid-liquid-solid microextraction method with molecularly imprinted polymer-coated stainless steel fiber for aqueous sample pretreatment. J. Chromatogr. A 1218: 39353939. DOI: 10.1016/j.chroma.2011.04.069

2216. Hu XG, Dai GM, Huang JJ, Jin HJ, Yu Y, Liang Y. 2011. Preparation and Characterization of Metolachlor Molecularly Imprinted Polymer Coating on Stainless Steel Fibers for Solid-Phase Microextraction. Anal. Lett. 44: 13581370. DOI: $10.1080 / 00032719.2010 .511743$

2217. Hu XG, Pan JL, Hu YL, Li GK. 2011. Preparation of molecularly imprinted polymer coatings with the multiple bulk copolymerization method for solidphase microextraction. J. Appl. Polym. Sci. 120: 1266-1277. DOI: 10.1002/app.33129

2218. Hu YL, Li YW, Zhang Y, Li GK, Chen YQ. 2011. Development of sample preparation method for auxin analysis in plants by vacuum microwave- 
assisted extraction combined with molecularly imprinted clean-up procedure. Anal. Bioanal. Chem. 399: 3367-3374. DOI: 10.1007/s00216-010-4257-8

2219. Hu YL, Li YW, Liu RJ, Tan W, Li GK. 2011. Magnetic molecularly imprinted polymer beads prepared by microwave heating for selective enrichment of $\alpha$-agonists in pork and pig liver samples. Talanta 84: 462-470. DOI: 10.1016/j.talanta.2011.01.045

2220. Hu YL, Li JW, Li GK. 2011. Synthesis and application of a novel molecularly imprinted polymer-coated stir bar for microextraction of triazole fungicides in soil. J. Sep. Sci. 34: 1190-1197. DOI: 10.1002/jssc.201100068

2221. Hua KC, Zhang L, Zhang ZH, Guo Y, Guo TY. 2011. Surface hydrophilic modification with a sugar moiety for a uniform-sized polymer molecularly imprinted for phenobarbital in serum. Acta Biomater. 7: 3086-3093. DOI: 10.1016/j.actbio.2011.05.006

2222. Huang JX, Hu YF, Hu YL, Li GK. 2011. Development of metal complex imprinted solid-phase microextraction fiber for 2,2'-dipyridine recognition in aqueous medium. Talanta 83: 1721-1729. DOI: 10.1016/j.talanta.2010.12.001

2223. Jafari MT, Rezaei B, Javaheri M. 2011. A new method based on electrospray ionisation ion mobility spectrometry (ESI-IMS) for simultaneous determination of caffeine and theophylline. Food Chem. 126: 1964-1970. DOI: 10.1016/j.foodchem.2010.12.054

2224. Jin GY, Zhang BF, Tang YW, Zuo XJ, Wang SC, Tang JY. 2011. Imprinted functionalized silica sol-gel for solid-phase extraction of triazolamin. Talanta 84: 644-650. DOI: 10.1016/j.talanta.2011.01.035

2225. Jin $Y$, Xuan $Y H$, Jin YS, Row KH. 2011. Multi-SPE of caffeine and catechin compounds from green tea by caffeine and (+) catechin MIPS. J. Liq. Chrom. Rel. Technol. 34: 1604-1616. DOI: 10.1080/10826076.2011.576296 
2226. Jing T, Niu JW, Xia HA, Dai Q, Zheng HY, Hao QL, Mei SR, Zhou YK. 2011. Online coupling of molecularly imprinted solid-phase extraction to HPLC for determination of trace tetracycline antibiotic residues in egg samples. $J$. Sep. Sci. 34: 1469-1476. DOI: 10.1002/jssc.201100030

2227. Jing T, Xia HA, Guan Q, Lu WH, Dai Q, Niu JW, Lim JM, Hao QL, Lee YI, Zhou YK, Mei SR. 2011. Rapid and selective determination of urinary lysozyme based on magnetic molecularly imprinted polymers extraction followed by chemiluminescence detection. Anal. Chim. Acta 692: 73-79. DOI: 10.1016/j.aca.2011.02.057

2228. Khorrami AR, Taherkhani M. 2011. Synthesis and Evaluation of a Molecularly Imprinted Polymer for Pre-concentration of Patulin from Apple Juice. Chromatographia 73: S151-S156. DOI: 10.1007/s10337-010-1892-3

2229. Koç I, Baydemir G, Bayram E, Yavuz H, Denizli A. 2011. Selective removal of $17 \alpha$-estradiol with molecularly imprinted particle-embedded cryogel systems. J. Haz. Mater. 192: 1819-1826. DOI: 10.1016/j.jhazmat.2011.07.017

2230. Kowalsky D, Pobozy E, Trojanowicz M. 2011. Flow-Injection Preconcentration of Chloramphenicol Using Molecularly Imprinted Polymer for HPLC Determination in Environmental Samples. J. Automat. Meth. Management Chem. 2011: Art. No. 143416. DOI: 10.1155/2011/143416

2231. Lagha A, Adhoum N, Monser L. 2011. A Molecularly Imprinted Polymer for the Selective Solid-Phase Extraction of Ibuprofen from Urine Samples. The Open Chemical and Biomedical Methods Journal 4: 7-13. DOI: $10.2174 / 1875038901004010007$

2232. Lakka A, Mylonis I, Bonanou S, Simos G, Tsakalof A. 2011. Isolation of hypoxia-inducible factor 1 (HIF-1) inhibitors from frankincense using a molecularly imprinted polymer. Investigational New Drugs 29: 1081-1089. DOI: 10.1007/s10637-010-9440-4 
2233. Liu QS, Liu R, He HM, Cui J, Pang ZR, Yin XY. 2011. Preparation and Application of Picroside II Molecularly Imprinted Ploymer for TCM Researching. In Applications of Engineering Materials, Advanced Materials Research, Vol. 287-290, Bu JL, Wang PC, Ai LQ, Sang XM, Li YG (eds). Trans Tech Publications: 1987-1990. DOI: 10.4028/www.scientific.net/AMR.287-290.1987

2234. Liu X, Zhang ZH, Zhang HB, Hu YF, Yang X, Nie LH. 2011. Solid Phase Extraction of Ursolic Acid Using Imprinted Polymer Modified Multi-walled Carbon Nanotubes. Chin. J. Anal. Chem. 39: 839-845. DOI: 10.1016/S18722040(10)60443-4

2235. Lopez C, Claude B, Morin P, Pelissou M, Pena R, Max JP, Ribet JP. 2011. Synthesis and study of a molecularly imprinted polymer for specific solidphase extraction of vinflunine and its metabolite from biological fluids. $J$. Sep. Sci. 34: 1902-1909. DOI: 10.1002/jssc.201100015

2236. Lopez C, Claude B, Morin P, Max JP, Pena R, Ribet JP. 2011. Synthesis and study of a molecularly imprinted polymer for the specific extraction of indole alkaloids from Catharanthus roseus extracts. Anal. Chim. Acta 683: 198-205. DOI: 10.1016/j.aca.2010.09.051

2237. Lordel S, Chapuis-Hugon S, Eudes V, Pichon V. 2011. Selective extraction of nitroaromatic explosives by using molecularly imprinted silica sorbents. Anal. Bioanal. Chem. 399: 449-458. DOI: 10.1007/s00216-010-4346-8

2238. Lucci $P$, Núñez O, Galceran MT. 2011. Solid-phase extraction using molecularly imprinted polymer for selective extraction of natural and synthetic estrogens from aqueous samples. J. Chromatogr. A 1218: 48284833. DOI: 10.1016/j.chroma.2011.02.007

2239. Luo XB, Zhan YC, Tu XM, Huang YN, Luo SL, Yan LS. 2011. Novel molecularly imprinted polymer using 1-( $\alpha$-methyl acrylate)-3methylimidazolium bromide as functional monomer for simultaneous 
extraction and determination of water-soluble acid dyes in wastewater and soft drink by solid phase extraction and high performance liquid chromatography. J. Chromatogr. A 1218: 1115-1121. DOI: 10.1016/j.chroma.2010.12.081

2240. Luo XB, Zhan YC, Huang YN, Yang LX, Tu XM, Luo SL. 2011. Removal of water-soluble acid dyes from water environment using a novel magnetic molecularly imprinted polymer. J. Haz. Mater. 187: 274-282. DOI: 10.1016/j.jhazmat.2011.01.009

2241. Luo YF, Huang P, Fu Q, Du W, Sun SJ, Li Y, Liu M, Chang C. 2011. Preparation of Monolithic Imprinted Stationary Phase for Clenbuterol by In Situ Polymerization and Application in Biological Samples Pretreatment. Chromatographia 74: 693-701. DOI: 10.1007/s10337-011-2129-9

2242. Lv JC, Huo SX, Yong KL. 2011. Synthesis of molecularly imprinted polymer of loureirin B and its application in selective extraction of functional components from dragon's blood. J. Liq. Chrom. Rel. Technol. 34: 23732382. DOI: $10.1080 / 10826076.2011 .591019$

2243. Mausia T, De Smet D, Guorun Q, Van Peteghem C, Zhang DB, Wu AB, De Saeger S. 2011. Molecularly Imprinted Polymers as Specific Adsorbents for Zearalenone Produced by Precipitation Polymerization and Applied to Mycotoxin Production. Anal. Lett. 44: 2633-2643. DOI: $10.1080 / 00032719.2011 .553009$

2244. Mei SR, Wu D, Jiang M, Lu B, Lim JM, Zhou YK, Lee YI. 2011. Determination of trace bisphenol $A$ in complex samples using selective molecularly imprinted solid-phase extraction coupled with capillary electrophoresis. Microchem. J. 98: 150-155. DOI: 10.1016/j.microc.2011.01.003

2245. Meng L, Qiao XG, Song JM, Xu ZX, Xin JH, Zhang Y. 2011. Study of an Online Molecularly Imprinted Solid Phase Extraction Coupled to 
Chemiluminescence Sensor for the Determination of Trichlorfon in Vegetables. J. Agric. Food Chem. 59: 12745-12751. DOI: $10.1021 / \mathrm{jf} 203801 \mathrm{n}$

2246. Mirmahdieh S, Mardihallaj A, Hashemian Z, Razavizadeh J, Ghaziaskar H, Khayamian T. 2011. Analysis of testosterone in human urine using molecularly imprinted solid-phase extraction and corona discharge ion mobility spectrometry. J. Sep. Sci. 34: 107-112. DOI: 10.1002/jssc.201000583

2247. Moein MM, Javanbakht M, Akbari-adergani B. 2011. Molecularly imprinted polymer cartridges coupled on-line with high performance liquid chromatography for simple and rapid analysis of dextromethorphan in human plasma samples. J. Chromatogr B 879: 777-782. DOI: 10.1016/j.jchromb.2011.02.031

2248. Peighambarzadeh SZ, Safi S, Shahtaheri SJ, Javanbakht M, Rahimi Forushani A. 2011. Presence of atrazine in the biological samples of cattle and its consequence adversity in human health. Iran J. Pub. Health 40: 112121.

2249. Peng L, Wang YZ, Zeng H, Yuan Y. 2011. Molecularly imprinted polymer for solid-phase extraction of rutin in complicated traditional Chinese medicines. Analyst 136: 756-763. DOI: 10.1039/COAN00798F

2250. Popov SA, Chumichkina YuA, Shapovalova EN, Dmitrienko SG, Zolotov YuA. 2011. Preconcentration of 2,4-dichlorophenoxyacetic acid on molecularly imprinted polymers and its subsequent determination by high performance liquid chromatography. J. Anal. Chem. 66: 6-10. DOI: $10.1134 /$ S1061934811010126

2251. Prieto A, Vallejo A, Zuloaga O, Paschke A, Sellergren B, Schillinger E, Schrader S, Möder M. 2011. Selective determination of estrogenic compounds in water by microextraction by packed sorbents and a 
molecularly imprinted polymer coupled with large volume injection-in-portderivatization gas chromatography-mass spectrometry. Anal. Chim. Acta 703: 41-51. DOI: 10.1016/j.aca.2011.07.007

2252. Prieto A, Schrader S, Bauer C, Möder M. 2011. Synthesis of a molecularly imprinted polymer and its application for microextraction by packed sorbent for the determination of fluoroquinolone related compounds in water. Anal. Chim. Acta 685: 146-152. DOI: 10.1016/j.aca.2010.11.038

2253. Qi PP, Wang JC, Li Y, Su F, Jin J, Chen JP. 2011. Molecularly imprinted solid-phase extraction coupled with HPLC for the selective determination of monobutyl phthalate in bottled water. J. Sep. Sci. 34: 2712-2718. DOI: $10.1002 /$ jssc.201100264

2254. Qiao FX, Geng YR, He CQ, Wu YP, Pan PY. 2011. Molecularly imprinted microspheres as SPE sorbent for selective extraction of four Sudan dyes in catsup products. J. Chromatogr $B$ 879: 2891-2896. DOI: 10.1016/j.jchromb.2011.08.019

2255. Qiao FX, Yan HY. 2011. Simultaneous analysis of fluoroquinolones and xanthine derivatives in serum by molecularly imprinted matrix solid-phase dispersion coupled with liquid chromatography. J. Chromatogr B 879: 3551 3555. DOI: 10.1016/j.jchromb.2011.09.040

2256. Qiao FX, Yan HY. 2011. Validation of the Pseudo Imprinted Microspheres for Solid-Phase Extraction of Acyclovir from Swine Tissue. In Biotechnology, Chemical and Materials Engineering, Vol. 393-395, Chen R, Sung WP (eds). Scientific.net: 1591-1594. DOI: 10.4028/www.scientific.net/AMR.393395.1591

2257. Qiao JD, Yan HY, Wang H, Lv YK. 2011. Determination of ofloxacin and lomefloxacin in chicken muscle using molecularly imprinted solid-phase extraction coupled with liquid chromatography. J. Sep. Sci. 34: 2668-2673. DOI: $10.1002 /$ jssc.201100209 
2258. Qiao JD, Yan HY, Wang H, Wu YP, Pan PY, Geng YR. 2011. Simultaneous Determination of Four Sudan Dyes in Egg Yolks by Molecularly Imprinted SPE Coupled with LC-UV Detection. Chromatographia 73: 227-233. DOI: 10.1007/s10337-010-1872-7

2259. Rajabi Khorrami A, Narouenezhad E. 2011. Synthesis of molecularly imprinted monolithic fibers for solid-phase microextraction of acetaldehyde from head-space of beverages stored in PET bottles. Talanta 86: 58-63. DOI: 10.1016/j.talanta.2011.08.002

2260. Rao RN, Maurya PK, Khalid S. 2011. Development of a molecularly imprinted polymer for selective extraction followed by liquid chromatographic determination of sitagliptin in rat plasma and urine. Talanta 85: 950-957. DOI: $10.1016 /$ j.talanta.2011.05.002

2261. Rao RN, Maurya PK, Kuntamukkala R, Vitthal WD, Talluri MVNK. 2011. Molecularly imprinted polymer for selective extraction of 3-methylflavone-8carboxylic acid from human urine followed by its determination using zwitterionic hydrophilic interaction liquid chromatography. J. Sep. Sci. 34: 3265-3271. DOI: $10.1002 /$ jssc.201100560

2262. Rezaei B, Jafari MT, Rahmanian O. 2011. Selective pretreatment and determination of phenazopyridine using an imprinted polymer-electrospray ionization ion mobility spectrometry system. Talanta 83: 765-769. DOI: 10.1016/j.talanta.2010.10.041

2263. Rodriguez E, Moreno-Bondi MC, Marazuela MD. 2011. Multiresidue determination of fluoroquinolone antimicrobials in baby foods by liquid chromatography. Food Chem. 127: 1354-1360. DOI: 10.1016/j.foodchem.2011.01.098

2264. Rodríguez E, Navarro-Villoslada F, Benito-Peña E, Marazuela MD, MorenoBondi MC. 2011. Multiresidue Determination of Ultratrace Levels of Fluoroquinolone Antimicrobials in Drinking and Aquaculture Water Samples 
by Automated Online Molecularly Imprinted Solid Phase Extraction and Liquid Chromatography. Anal. Chem. 83: 2046-2055. DOI: 10.1021/ac102839n

2265. Sanagi MM, Salleh S, Ibrahim WAW, Naim AA. 2011. Determination of organophosphorus pesticides using molecularly imprinted polymer solid phase extraction. The Malaysian Journal of Analytical Sciences 15: 175-183.

2266. Schwarz LJ, Danylec B, Yang YZ, Harris SJ, Boysen RI, Hearn MTW. 2011. Enrichment of (E)-Resveratrol from Peanut Byproduct with Molecularly Imprinted Polymers. J. Agric. Food Chem. 59: 3539-3543. DOI: $10.1021 /$ jf $104230 f$

2267. Schwarz LJ, Danylec B, Harris SJ, Boysen RI, Hearn MTW. 2011. Preparation of molecularly imprinted polymers for the selective recognition of the bioactive polyphenol, (E)-resveratrol. J. Chromatogr. A 1218: 21892195. DOI: 10.1016/j.chroma.2011.02.043

2268. Shah KA, Peoples MC, Halquist MS, Rutan SC, Karnes HT. 2011. Microfluidic direct injection method for analysis of urinary 4(methylnitrosamino)-1-(3-pyridyl)-1-butanol (NNAL) using molecularly imprinted polymers coupled on-line with LC-MS/MS. J. Pharm. Biomed. Anal. 54: 368-378. DOI: 10.1016/j.jpba.2010.09.001

2269. Shen ZL, Yuan D, Su QD, Zhang H, Wang J, Zhu JH, Liu YM. 2011. Selective Solid-Phase Extraction Using Molecularly Imprinted Polymer for Analysis of Methamidophos in Water and Soil Samples. Bioscience Biotechnology and Biochemistry 75: 473-479. DOI: 10.1271/bbb.100668

2270. Shi Y, Peng DD, Shi CH, Zhang X, Xie YT, Lu B. 2011. Selective determination of trace $17 \alpha$-estradiol in dairy and meat samples by molecularly imprinted solid-phase extraction and HPLC. Food Chem. 126: 1916-1925. DOI: 10.1016/j.foodchem.2010.12.020 
2271. Si BJ, Zhou J. 2011. Non-hydrolytic Sol-gel Methodology to Prepare a Molecularly Imprinted, Organic-silica Hybrid-based Stir Bar for Recognition of Sulfonylurea Herbicides. Chin. J. Chem. 29: 2487-2494. DOI: 10.1002/cjoc.201180421

2272. Siewing A. 2011. The Extraction of Acid NSAIDs from Muscle Tissue using Molecularly Imprinted Polymer SPE. The Reporter 29: 18-19.

2273. Siewing A. 2011. The Extraction of Acid NSAIDs from Muscle Tissue Using Molecularly Imprinted Polymer SPE. The Reporter 46: 16-17.

2274. Son LT, Takaomi K. 2011. Hollow-fiber membrane absorbents embedded molecularly imprinted polymeric spheres for bisphenol A target. J. Membr. Sci. 384: 117-125. DOI: 10.1016/j.memsci.2011.09.013

2275. Son LT, Katagawa K, Kobayashi T. 2011. Using molecularly imprinted polymeric spheres for hybrid membranes with selective adsorption of bisphenol A derivatives. J. Membr. Sci. 375: 295-303. DOI: 10.1016/j.memsci.2011.03.054

2276. Song JM, Qiao XG, Chen HH, Zhao DY, Zhang Y, Xu ZX. 2011. Molecularly imprinted solid-phase extraction combined with high-performance liquid chromatography for analysis of trace olaquindox residues in chick feeds. $\mathrm{J}$. Sci. Food Agric. 91: 2378-2385. DOI: 10.1002/jsfa.4471

2277. Su QL, Zhang BF, Tang YW, Jin GY, Liang YP. 2011. Evaluation of novel solid-phase extraction microspheres prepared by a surface imprinting technique and its application to the separation of diethylstilbestrol from chicken samples. J. Liq. Chrom. Rel. Technol. 34: 2063-2073. DOI: $10.1080 / 10826076.2011 .583374$

2278. Tan F, Deng MJ, Liu X, Zhao HX, Li XN, Quan X, Chen JW. 2011. Evaluation of a novel microextraction technique for aqueous samples: Porous membrane envelope filled with multiwalled carbon nanotubes coated 
with molecularly imprinted polymer. J. Sep. Sci. 34: 707-715. DOI: $10.1002 /$ jssc.201000791

2279. Tang YW, Fang GZ, Wang S, Li JL. 2011. Covalent imprinted polymer for selective and rapid enrichment of ractopamine by a noncovalent approach. Anal. Bioanal. Chem. 401: 2275-2282. DOI: 10.1007/s00216-011-5280-0

2280. Tian M, Han D, Row KH. 2011. Preparation of Molecularly Imprinted Polymer for Extracting Flavones from Chamaecyparis Obtusa. Anal. Lett. 44: 737-746. DOI: 10.1080/00032711003783176

2281. Tian M, Row KH. 2011. SPE of Tanshinones from Salvia miltiorrhiza Bunge by using Imprinted Functionalized Ionic Liquid-Modified Silica. Chromatographia 73: 25-31. DOI: 10.1007/s10337-010-1836-y

2282. Tong YJ, Xin Y, Yang HL, Zhang L, Zhang YR, Chen Y, Xia XL, Wang W. 2011. Preparation and Performance Research on Glutathione Molecularly Imprinted Polymers. Chromatographia 74: 443-450. DOI: 10.1007/s10337011-2089-0

2283. Tsai HA, Syu MJ. 2011. Preparation of imprinted poly(tetraethoxysilanol) sol-gel for the specific uptake of creatinine. Chem. Eng. J. 168: 1369-1376. DOI: 10.1016/j.cej.2011.02.047

2284. Urraca JL, Aureliano CSA, Schillinger E, Esselmann H, Wiltfang J, Sellergren B. 2011. Polymeric Complements to the Alzheimer's Disease Biomarker $\alpha$-Amyloid Isoforms $A \alpha 1-40$ and A $\alpha 1-42$ for Blood Serum Analysis under Denaturing Conditions. J. Am. Chem. Soc. 133: 9220-9223. DOI: 10.1021/ja202908z

2285. Vitor RV, Martins MCG, Figueiredo EC, Martins I. 2011. Application of molecularly imprinted polymer solid-phase extraction for salivary cotinine. Anal. Bioanal. Chem. 400: 2109-2117. DOI: 10.1007/s00216-011-4870-1 
2286. Wang $B$, Wang $Y Z$, Yang $H$, Wang JQ, Deng AP. 2011. Preparation and characterization of molecularly imprinted microspheres for selective extraction of trace melamine from milk samples. Microchim. Acta 174: 191199. DOI: $10.1007 / \mathrm{s} 00604-011-0613-4$

2287. Wang H, Yan HY, Qiu MD, Qiao JD, Yang GL. 2011. Determination of dicofol in aquatic products using molecularly imprinted solid-phase extraction coupled with GC-ECD detection. Talanta 85: 2100-2105. DOI: 10.1016/j.talanta.2011.07.061

2288. Wang S, Li Y, Wu XL, Ding MJ, Yuan LH, Wang RY, Wen TT, Zhang J, Chen LN, Zhou XM, Li F. 2011. Construction of uniformly sized pseudo template imprinted polymers coupled with HPLC-UV for the selective extraction and determination of trace estrogens in chicken tissue samples. $J$. Haz. Mater. 186: 1513-1519. DOI: 10.1016/j.jhazmat.2010.12.026

2289. Wang S, Li Y, Ding MJ, Wu XL, Xu JH, Wang RY, Wen TT, Huang WY, Zhou P, Ma KF, Zhou XM, Du SH. 2011. Self-assembly molecularly imprinted polymers of $17 \alpha$-estradiol on the surface of magnetic nanoparticles for selective separation and detection of estrogenic hormones in feeds. J. Chromatogr $B$ 879: 2595-2600. DOI: 10.1016/j.jchromb.2011.07.017

2290. Wang T, Tong J, Sun ML, Chen LG. 2011. Fast and selective extraction of chloramphenicol from soil by matrix solid-phase dispersion using molecularly imprinted polymer as dispersant. J. Sep. Sci. 34: 1886-1892. DOI: $10.1002 /$ jssc.201100046

2291. Wang X, Mao HP, Huang WH, Guan W, Zou XH, Pan JM, Yan YS. 2011. Preparation of magnetic imprinted polymer particles via microwave heating initiated polymerization for selective enrichment of 2-amino-4-nitrophenol from aqueous solution. Chem. Eng. J. 178: 85-92. DOI: 10.1016/j.cej.2011.10.015 
2292. Wang X, Pan JM, Guan W, Dai JD, Zou XH, Yan YS, Li CX, Hu W. 2011. Selective Removal of 3-Chlorophenol from Aqueous Solution Using Surface Molecularly Imprinted Microspheres. J. Chem. Eng. Data 56: 2793-2801. DOI: $10.1021 / \mathrm{je} 101275 \mathrm{e}$

2293. Wang XH, Chen LR, Xu XJ, Li YZ. 2011. Synthesis of molecularly imprinted polymers via ring-opening metathesis polymerization for solid-phase extraction of bisphenol A. Anal. Bioanal. Chem. 401: 1423-1432. DOI: $10.1007 / \mathrm{s} 00216-011-5178-x$

2294. Wu SQ, Xu ZG, Yuan QH, Tang YW, Zuo XJ, Lai JP. 2011. Recognition characteristics of molecularly imprinted microspheres for triazine herbicides using hydrogen-bond array strategy and their analytical applications for corn and soil samples. J. Chromatogr. A 1218: 1340-1346. DOI: 10.1016/j.chroma.2011.01.008

2295. Xin JH, Zhao DY, Zhang LM, Xu ZX, Zhou J, Qu DJ. 2011. Determination of Trace Sudan IV Residues in Foods Through Molecularly Imprinted SPE Coupled with LC. Chromatographia 73: 235-242. DOI: 10.1007/s10337-010$1889-y$

2296. Xu SF, Li JH, Chen LX. 2011. Molecularly imprinted core-shell nanoparticles for determination of trace atrazine by reversible addition-fragmentation chain transfer surface imprinting. J. Mater. Chem. 21: 4346-4351.

2297. Xu SF, Li JH, Chen LX. 2011. Molecularly imprinted polymers by reversible addition-fragmentation chain transfer precipitation polymerization for preconcentration of atrazine in food matrices. Talanta 85: 282-289. DOI: 10.1016/j.talanta.2011.03.060

2298. Xu SF, Chen LX, Li JH, Qin W, Ma JP. 2011. Preparation of hollow porous molecularly imprinted polymers and their applications to solid-phase extraction of triazines in soil samples. J. Mater. Chem. 21: 12047-12053. DOI: 10.1039/C1JM10905G 
2299. Xu WZ, Zhou W, Huang WH, Pan JM, Li H, Wu XY, Yan YS. 2011. Preparation and evaluation of a novel surface-imprinted polymer for selective adsorption of dibenzothiophene. Microchim. Acta 175: 167-175. DOI: 10.1007/s00604-011-0661-9

2300. Xu WZ, Zhou W, Bian LH, Huang WH, Wu XY. 2011. Preparation of molecularly imprinted polymer by surface imprinting technique and its performance for adsorption of dibenzothiophene. J. Sep. Sci. 34: 17461753. DOI: $10.1002 /$ jssc.201100147

2301. Xu Z, Ding L, Long YJ, Xu LG, Wang LB, Xu CL. 2011. Preparation and evaluation of superparamagnetic surface molecularly imprinted polymer nanoparticles for selective extraction of bisphenol A in packed food. Anal. Methods 3: 1737-1744. DOI: 10.1039/C1AY05206C

2302. Xu ZG, Song CY, Hu YL, Li GK. 2011. Molecularly imprinted stir bar sorptive extraction coupled with high performance liquid chromatography for trace analysis of sulfa drugs in complex samples. Talanta 85: 97-103. DOI: 10.1016/j.talanta.2011.03.041

2303. Yan HY, Wang H, Qiao JD, Yang GL. 2011. Molecularly imprinted matrix solid-phase dispersion combined with dispersive liquid-liquid microextraction for the determination of four Sudan dyes in egg yolk. J. Chromatogr. A 1218: 2182-2188. DOI: 10.1016/j.chroma.2011.02.042

2304. Yan HY, Qiao JD, Wang H, Yang GL, Row KH. 2011. Molecularly imprinted solid-phase extraction combined with ultrasound-assisted dispersive liquidliquid microextraction for the determination of four Sudan dyes in sausage samples. Analyst 136: 2629-2634. DOI: 10.1039/C0AN00951B

2305. Yang M, Chen YT, Liu J, Ma J, Huai LF. 2011. Determination of trace rotenone by UV-Vis spectrophotometry using molecularly imprinted polymer microspheres. J. Anal. Chem. 66: 820-823. DOI: $10.1134 /$ S1061934811090097 
2306. Yang YZ, Liu XG, Guo MC, Li S, Liu WF, Xu BS. 2011. Molecularly imprinted polymer on carbon microsphere surfaces for adsorbing dibenzothiophene. Colloids Surf., $A$ 377: 379-385. DOI: 10.1016/j.colsurfa.2011.01.032

2307. Yin JF, Meng ZH, Zhu YH, Song MY, Wang HL. 2011. Dummy molecularly imprinted polymer for selective screening of trace bisphenols in river water. Anal. Methods 3: 173-180. DOI: 10.1039/COAY00540A

2308. Yin XY, Liu QS, Jiang YF, Luo YM. 2011. Development of andrographolide molecularly imprinted polymer for solid-phase extraction. Spectrochim. Acta, A 79: 191-196. DOI: 10.1016/j.saa.2011.02.034

2309. Yuan Y, Wang YZ, Huang MD, Xu R, Zeng HA, Nie C, Kong JH. 2011. Development and characterization of molecularly imprinted polymers for the selective enrichment of podophyllotoxin from traditional Chinese medicines. Anal. Chim. Acta 695: 63-72. DOI: 10.1016/j.aca.2011.04.007

2310. Zakaria ND, Yusof NA, Haron MJ, Abdullah AH. 2011. Synthesis and Characterization of a Molecularly Imprinted Polymer for 2,4-Dinitrophenol Uptake Using 4-Vinylbenzoic Acid as the Complexing Monomer. Asian J. Chem. 23: 2456-2460.

2311. Zhang HB, Zhang ZH, Hu YF, Yang X, Yao SZ. 2011. Synthesis of a Novel Composite Imprinted Material Based on Multiwalled Carbon Nanotubes as a Selective Melamine Absorbent. J. Agric. Food Chem. 59: 1063-1071. DOI: 10.1021/jf104064y

2312. Zhang KC, Zhao SY, Yang M. 2011. Removal of Rotenone from Contaminated Water Using Molecularly Imprinted Polymeric Microspheres. In Frontiers of Green Building, Materials and Civil Engineering, Vol. 71-78, Sun DY, Sung WP, Chen R (eds). Trans Tech Publications: Switzerland; 1425-1428. DOI: 10.4028/www.scientific.net/AMM.71-78.1425 
2313. Zhang QJ, Su YJ, He QQ, Shen XG, He LM, Zhang N, Zeng ZL. 2011. Molecularly imprinted solid-phase extraction for the selective HPLC determination of ractopamine in pig urine. J. Sep. Sci. 34: 3399-3409. DOI: 10.1002/jssc.201100655

2314. Zhang ZM, Tan W, Hu YL, Li GK. 2011. Simultaneous determination of trace sterols in complicated biological samples by gas chromatography-mass spectrometry coupled with extraction using $\alpha$-sitosterol magnetic molecularly imprinted polymer beads. J. Chromatogr. A 1218: 4275-4283. DOI: 10.1016/j.chroma.2011.05.022

2315. Zhao L, Ma ZH, Pan LG, Wag JH. 2011. MISPE Combined with GCMS for Analysis of Organophosphorus Pesticides from Environmental Water Sample. In Advanced Materials, Advanced Materials Research, Vol. 239 242, Cao Z, Cao XQ, Sun LX, He YH (eds). Scientific.net: 3216-3220. DOI: 10.4028/www.scientific.net/AMR.239-242.3216

2316. Zheng YQ, Liu YH, Guo HB, He LM, Fang BH, Zeng ZL. 2011. Molecularly imprinted solid-phase extraction for determination of tilmicosin in feed using high performance liquid chromatography. Anal. Chim. Acta 690: 269-274. DOI: 10.1016/j.aca.2011.02.029

2317. Zhou WH, Guo XC, Zhao HQ, Wu SX, Yang HH, Wang XR. 2011. Molecularly imprinted polymer for selective extraction of domoic acid from seafood coupled with high-performance liquid chromatographic determination. Talanta 84: 777-782. DOI: 10.1016/j.talanta.2011.02.004

2318. Zhu GF, Fan J, Gao YB, Gao X, Wang JJ. 2011. Synthesis of surface molecularly imprinted polymer and the selective solid phase extraction of imidazole from its structural analogs. Talanta 84: 1124-1132. DOI: 10.1016/j.talanta.2011.03.015

2319. Zhu HB, Wang YZ, Yuan Y, Zeng H. 2011. Development and characterization of molecularly imprinted polymer microspheres for the 
selective detection of kaempferol in traditional Chinese medicines. Anal. Methods 3: 348-355. DOI: 10.1039/COAY00578A

2320. Zhu HP, Ma LG, Fang GZ, Pan MF, Lu JP, Wang XN, Wang S. 2011. Preparation of a molecularly imprinted polymer using TMB as a dummy template and its application as SPE sorbent for determination of six PBBs in water and fish samples. Anal. Methods 3: 393-399. DOI: 10.1039/COAY00479K

2321. Zhu T, Li S, Row KH. 2011. Molecularly imprinted monolithic material for the extraction of three organic acids from Salicornia herbacea L. J. Appl. Polym. Sci. 121: 1691-1696. DOI: 10.1002/app.33755

2322. Zhu T, Yoon CW, Row KH. 2011. Solid-phase Extraction of $\alpha$-Sitosterol from Oldenlandia diffusa Using Molecular Imprinting Polymer. Chin. J. Chem. 29: 1246-1250. DOI: 10.1002/cjoc.201190231

2323. Zhao L, Ping H, Xiang L, Han P, Wang JH, Pan LG. 2011. Application of Molecular Imprinting Technique in Organophosphorus Pesticides Detection. In Computer and Computing Technologies in Agriculture IV, IFIP Advances in Information and Communication Technology, Vol. 344, Li DL, Liu YD, Chen YY (eds). Springer: Boston; 290-295. DOI: 10.1007/978-3-642-183331 34

2324. Zhang ZH, Yang X, Chen X, Zhang ML, Luo LJ, Peng MJ, Yao SZ. 2011. Novel magnetic bovine serum albumin imprinted polymers with a matrix of carbon nanotubes, and their application to protein separation. Anal. Bioanal. Chem. 401: 2855-2863. DOI: 10.1007/s00216-011-5373-9

2325. Zhang ZH, Yang X, Zhang HB, Zhang ML, Luo LJ, Hu YF, Yao SZ. 2011. Novel molecularly imprinted polymers based on multi-walled carbon nanotubes with binary functional monomer for the solid-phase extraction of erythromycin from chicken muscle. J. Chromatogr B 879: 1617-1624. DOI: 10.1016/j.jchromb.2011.03.054 
2326. Mullett WM, Walles M, Levsen K, Borlak J, Pawliszyn J. 2004. Multidimensional on-line sample preparation of verapamil and its metabolites by a molecularly imprinted polymer coupled to liquid chromatography-mass spectrometry. J. Chromatogr. B 801: 297-306. DOI: 10.1016/j.jchromb.2003.11.041

2327. Figueiredo EC, Sparrapan R, Sanvido GB, Santos MG, Zezzi Arruda MA, Eberlin MN. 2011. Quantitation of drugs via molecularly imprinted polymer solid phase extraction and electrospray ionization mass spectrometry: benzodiazepines in human plasma. Analyst 136: 3753-3757. DOI: 10.1039/C1AN15198C

2328. Weetall HH, Hatchett DW, Rogers KR. 2005. Electrochemically deposited polymer-coated gold electrodes selective for 2,4-dichlorophenoxyacetic acid. Electroanalysis 17: 1789-1794. DOI: 10.1002/elan.200503304

2329. Gallego-Gallegos M, Liva M, Olivas RM, Cámara C. 2006. Focused ultrasound and molecularly imprinted polymers: A new approach to organotin analysis in environmental samples. J. Chromatogr. A 1114: 82-88. DOI: 10.1016/j.chroma.2006.02.040

2330. Hoshina K, Horiyama S, Matsunaga H, Haginaka J. 2009. Molecularly imprinted polymers for simultaneous determination of antiepileptics in river water samples by liquid chromatography-tandem mass spectrometry. $\mathrm{J}$. Chromatogr. A 1216: 4957-4962. DOI: 10.1016/j.chroma.2009.04.071

2331. Maier NM, Buttinger G, Welhartizki S, Gavioli E, Lindner W. 2004. Molecularly imprinted polymer-assisted sample clean-up of ochratoxin $A$ from red wine: merits and limitations. J. Chromatogr. B 804: 103-111. DOI: 10.1016/j.jchromb.2004.01.014

2332. Aburto J, Mendez-Orozco A, Le Borgne S. 2004. Hydrogels as adsorbents of organosulphur compounds currently found in diesel. Chem. Eng. Proc. 43: 1587-1595. DOI: 10.1016/j.cep.2004.02.006 
2333. Andaç M, Say R, Denizli A. 2004. Molecular recognition based cadmium removal from human plasma. J. Chromatogr $B$ 811: 119-126. DOI: 10.1016/j.jchromb.2004.08.024

2334. Blanco-López MC, Lobo-Castañón MJ, Miranda-Ordieres AJ, Tuñón-Blanco P. 2004. Electrochemical sensors based on molecularly imprinted polymers. TrAC, Trends Anal. Chem. 23: 36-48. DOI: 10.1016/S0165-9936(04)001025

2335. Ersöz A, Say R, Denizli A. 2004. Ni(II) ion-imprinted solid-phase extraction and preconcentration in aqueous solutions by packed-bed columns. Anal. Chim. Acta 502: 91-97. DOI: 10.1016/j.aca.2003.09.059

2336. Gladis JM, Rao TP. 2004. Effect of porogen type on the synthesis of uranium ion imprinted polymer materials for the preconcentration/separation of traces of uranium. Microchim. Acta 146: 251-258. DOI: 10.1007/s00604004-0207-5

2337. Huan SY, Jiao C, Shen Q, Jiang J, Zeng GM, Huang GH, Shen GL, Yu RQ. 2004. Determination of heavy metal ions in mixed solution by imprinted SAMs. Electrochim. Acta 49: 4273-4280. DOI: 10.1016/j.electacta.2004.03.035

2338. Kala R, Gladis JM, Rao TP. 2004. Preconcentrative separation of erbium from Y, Dy, Ho, Tb and Tm by using ion imprinted polymer particles via solid phase extraction. Anal. Chim. Acta 518: 143-150. DOI: 10.1016/j.aca.2004.05.029

2339. Kanazawa R, Yoshida T, Gotoh T, Sakohara S. 2004. Preparation of molecular imprinted thermosensitive gel adsorbents and adsorption/desorption properties of heavy metal ions by temperature swing. J. Chem. Eng. Jpn. 37: 59-66. DOI: 10.1252/jcej.37.59 
2340. Liu YW, Chang XJ, Wang S, Guo Y, Din BJ, Meng SM. 2004. Solid-phase extraction and preconcentration of cadmium(II) in aqueous solution with Cd(II)-imprinted resin (poly-Cd(II)-DAAB-VP) packed columns. Anal. Chim. Acta 519: 173-179. DOI: 10.1016/j.aca.2004.06.017

2341. Metilda P, Gladis JM, Rao TP. 2004. Influence of binary/ternary complex of imprint ion on the preconcentration of uranium $(\mathrm{VI})$ using ion imprinted polymer materials. Anal. Chim. Acta 512: 63-73. DOI: 10.1016/j.aca.2004.02.041

2342. Say R, Ersöz A, Turk H, Denizli A. 2004. Selective separation and preconcentration of cyanide by a column packed with cyanide-imprinted polymeric microbeads. Sep. Purif. Technol. 40: 9-14. DOI: 10.1016/j.seppur.2003.12.021

2343. Uezu K, Kuwabara T, Yoshida M, Goto M, Furusaki S. 2004. Lanthanoid element recognition on surface-imprinted polymers containing dioleylphosphoric acid as a functional host. Anal. Sci. 20: 1593-1597. DOI: 10.2116/analsci.20.1593

2344. Asir S, Uzun L, Türkmen D, Say R, Denizli A. 2005. Ion-selective imprinted superporous monolith for cadmium removal from human plasma. Sep. Sci. Technol. 40: 3167-3185. DOI: 10.1080/01496390500385376

2345. Büyüktiryaki S, Say RI, Ersöz A, Birlik E, Denizli A. 2005. Selective preconcentration of thorium in the presence of $\mathrm{UO}_{2}{ }^{2+}, \mathrm{Ce}^{3+}$ and $\mathrm{La}^{3+}$ using Th(IV)-imprinted polymer. Talanta 67: 640-645. DOI: 10.1016/j.talanta.2005.03.016

2346. Daniel S, Babu PEJ, Rao TP. 2005. Preconcentrative separation of palladium(II) using palladium(II) ion-imprinted polymer particles formed with different quinoline derivatives and evaluation of binding parameters based on adsorption isotherm models. Talanta 65: 441-452. DOI: 10.1016/j.talanta.2004.06.024 
2347. Fang GZ, Tan J, Yan MP. 2005. An ion-imprinted functionalized silica gel sorbent prepared by a surface imprinting technique combined with a sol-gel process for selective solid-phase extraction of cadmium(II). Anal. Chem. 77: 1734-1739. DOI: 10.1021/ac048570v

2348. Fang GZ, Tan J, Yan XP. 2005. Synthesis and evaluation of an ionimprinted functionalized sorbent for selective separation of cadmium ion. Sep. Sci. Technol. 40: 1597-1608. DOI: 10.1081/SS-200054587

2349. Kala R, Biju VM, Rao TP. 2005. Synthesis, characterization, and analytical applications of erbium(III) ion imprinted polymer particles prepared via $\gamma$ irradiation with different functional and crosslinking monomers. Anal. Chim. Acta 549: 51-58. DOI: 10.1016/j.aca.2005.06.024

2350. Krishna PG, Gladis JM, Rao TP, Naidu GR. 2005. Selective recognition of neodymium(III) using ion imprinted polymer particles. J. Mol. Recognit. 18: 109-116. DOI: $10.1002 / \mathrm{jmr} .720$

2351. Liu YW, Chang XJ, Yang D, Guo Y, Meng SM. 2005. Highly selective determination of inorganic mercury(II) after preconcentration with $\mathrm{Hg}$ (II)imprinted diazoaminobenzene-vinylpyridine copolymers. Anal. Chim. Acta 538: 85-91. DOI: 10.1016/j.aca.2005.02.017

2352. Owens GS, Southard GE, Van Houten KA, Murray GM. 2005. Molecularly imprinted ion-exchange resin for $\mathrm{Fe}^{3+}$. Sep. Sci. Technol. 40: 2205-2211. DOI: 10.1080/01496390500201177

2353. Su HJ, Wang ZX, Tan TW. 2005. Preparation of a surface molecularimprinted adsorbent for $\mathrm{Ni}^{2+}$ based on Penicillium chrysogenum. J. Chem. Technol. Biotechnol. 80: 439-444. DOI: 10.1002/jctb.1206

2354. Yavuz H, Say RI, Denizli A. 2005. Iron removal from human plasma based on molecular recognition using imprinted beads. Mater. Sci. Eng.: C 25: 521528. DOI: 10.1016/j.msec.2005.04.005 
2355. Zuo X, Mosha D, Archibald SJ, McCasland AK, Hassan AM, Givens RS, Busch DH. 2005. Toward the soil poultice and a new separations methodology: Rebinding of macrocyclic metal complexes to molecularly imprinted polymers specifically templated via noncovalent interactions. $J$. Coord. Chem. 58: 21-39. DOI: 10.1080/00958970512331327357

2356. Andaç M, Özyapi E, Senel S, Say R, Denizli A. 2006. Ion-selective imprinted beads for aluminum removal from aqueous solutions. Ind. Eng. Chem. Res. 45: 1780-1786. DOI: 10.1021/ie0512338

2357. Birlik E, Ersöz A, Denizli A, Say RI. 2006. Preconcentration of copper using double-imprinted polymer via solid phase extraction. Anal. Chim. Acta 565: 145-151. DOI: 10.1016/j.aca.2006.02.051

2358. Birlik E, Büyüktiryaki S, Ersöz A, Denizli A, Say R. 2006. Selective separation of thorium using ion imprinted chitosan-phthalate particles via solid phase extraction. Sep. Sci. Technol. 41: 3109-3121. DOI: $10.1080 / 01496390600851400$

2359. Chauvin AS, Bünzli JCG, Bochud F, Scopelliti R, Froidevaux P. 2006. Use of Dipicolinate-Based Complexes for Producing Ion-Imprinted Polystyrene Resins for the Extraction of Yttrium-90 and Heavy Lanthanide Cations. Chem. Eur. J. 12: 6852-6864. DOI: 10.1002/chem.200501370

2360. Fan ZF. 2006. $\mathrm{Hg}(\mathrm{II})$-imprinted thiol-functionalized mesoporous sorbent micro-column preconcentration of trace mercury and determination by inductively coupled plasma optical emission spectrometry. Talanta 70: 11641169. DOI: 10.1016/j.talanta.2006.03.021

2361. Froidevaux P, Happel S, Chauvin AS. 2006. Ion-imprinted polymer concept for selective extraction of $\mathrm{Y}-90$ and Eu-152 for medical applications and nuclear power plant monitoring. Chimia 60: 203-206. DOI: $10.2533 / 000942906777674895$ 
2362. Jiang N, Chang XJ, Zheng $\mathrm{H}, \mathrm{He} Q$, Hu Z. 2006. Selective solid-phase extraction of nickel(II) using a surface-imprinted silica gel sorbent. Anal. Chim. Acta 577: 225-231. DOI: 10.1016/j.aca.2006.06.049

2363. Kala R, Rao TP. 2006. Ion imprinted polymer particles for separation of yttrium from selected lanthanides. J. Sep. Sci. 29: 1281-1287. DOI: $10.1002 /$ jssc.200600008

2364. Li F, Li XM, Zhang SS. 2006. One-pot preparation of silica-supported hybrid immobilized metal affinity adsorbent with macroporous surface based on surface imprinting coating technique combined with polysaccharide incorporated sol-gel process. J. Chromatogr. A 1129: 223-230. DOI: 10.1016/j.chroma.2006.07.009

2365. Liu YW, Zai YH, Chang XJ, Guo Y, Meng SM, Feng F. 2006. Highly selective determination of methylmercury with methylmercury-imprinted polymers. Anal. Chim. Acta 575: 159-165. DOI: 10.1016/j.aca.2006.05.081

2366. Pan JY, Wang S, Zhang RF. 2006. A novel Pb(II)-imprinted IPN for selective preconcentration of lead from water and sediments. Int. J. Environ. Anal. Chem. 86: 855-865. DOI: 10.1080/03067310600665654

2367. Preetha CR, Gladis JM, Rao TP, Venkateswaran G. 2006. Removal of toxic Uranium from synthetic nuclear power reactor effluents using uranyl ion imprinted polymer particles. Environ. Sci. Technol. 40: 3070-3074. DOI: 10.1021/es052195m

2368. Ramakrishnan K, Rao TP. 2006. Ion imprinted polymer solid phase extraction (IIP-SPE) for preconcentrative separation of erbium(III) from adjacent lanthanides and yttrium. Sep. Sci. Technol. 41: 233-246. DOI: 10.1080/01496390500446327 
2369. Saatçilar Ö, Satiroglu N, Say R, Bektas S, Denizli A. 2006. Binding behavior of $\mathrm{Fe} 3+$ ions on ion-imprinted polymeric beads for analytical applications. $J$. Appl. Polym. Sci. 101: 3520-3528. DOI: 10.1002/app.24591

2370. Su HJ, Zhao Y, Li J, Tan TW. 2006. Biosorption of Ni2+ by the surface molecular imprinting adsorbent. Process Biochem. 41: 1422-1426. DOI: 10.1016/j.biomaterials.2006.03.020

2371. Wang S, Zhang RF. 2006. Selective solid-phase extraction of trace copper ions in aqueous solution with a $\mathrm{Cu}(\mathrm{II})$-imprinted interpenetrating polymer network gel prepared by ionic imprinted polymer (IIP) technique. Microchim. Acta 154: 73-80. DOI: 10.1007/s00604-006-0484-2

2372. Andaç M, Mirel S, Senel S, Say R, Ersöz A, Denizli A. 2007. Ion-imprinted beads for molecular recognition based mercury removal from human serum. Int. J. Biol. Macromol. 40: 159-166. DOI: 10.1016/j.ijbiomac.2006.07.002

2373. Birlik E, Ersöz A, Açikkalp E, Denizli A, Say R. 2007. Cr(III)-imprinted polymeric beads: Sorption and preconcentration studies. J. Haz. Mater. 140: 110-116. DOI: 10.1016/j.jhazmat.2006.06.141

2374. Büyüktiryaki S, Say R, Denizli A, Ersöz A. 2007. Mimicking receptor for methylmercury preconcentration based on ion-imprinting. Talanta 71: 699705. DOI: $10.1016 /$ j.talanta.2006.05.026

2375. Chang XJ, Jiang N, Zheng H, He Q, Hu Z, Zhai YH, Cui YM. 2007. Solidphase extraction of iron(III) with an ion-imprinted functionalized silica gel sorbent prepared by a surface imprinting technique. Talanta 71: 38-43. DOI: 10.1016/j.talanta.2006.03.012

2376. Dakova I, Karadjova I, Ivanov I, Georgieva V, Evtimova B, Georgiev G. 2007. Solid phase selective separation and preconcentration of $\mathrm{Cu}(\mathrm{II})$ by $\mathrm{Cu}(\mathrm{II})$-imprinted polymethacrylic microbeads. Anal. Chim. Acta 584: 196203. DOI: 10.1016/j.aca.2006.10.050 
2377. He Q, Chang XJ, Wu Q, Huang XP, Hu Z, Zhai YH. 2007. Synthesis and applications of surface-grafted Th(IV)-imprinted polymers for selective solidphase extraction of thorium(IV). Anal. Chim. Acta 605: 192-197. DOI: 10.1016/j.aca.2007.10.026

2378. Khajeh M, Yamini Y, Ghasemi E, Fasihi J, Shamsipur M. 2007. Imprinted polymer particles for selenium uptake: Synthesis, characterization and analytical applications. Anal. Chim. Acta 581: 208-213. DOI: 10.1016/j.aca.2006.08.037

2379. Li F, Du P, Chen W, Zhang SS. 2007. Preparation of silica-supported porous sorbent for heavy metal ions removal in wastewater treatment by organicinorganic hybridization combined with sucrose and polyethylene glycol imprinting. Anal. Chim. Acta 585: 211-218. DOI: 10.1016/j.aca.2006.12.047

2380. Li F, Jiang HQ, Zhang SS. 2007. An ion-imprinted silica-supported organicinorganic hybrid sorbent prepared by a surface imprinting technique combined with a polysaccharide incorporated sol-gel process for selective separation of cadmium(II) from aqueous solution. Talanta 71: 1487-1493. DOI: $10.1016 /$ j.talanta.2006.07.023

2381. Li Q, Su HJ, Li J, Tan TW. 2007. Application of surface molecular imprinting adsorbent in expanded bed for the adsorption of $\mathrm{Ni2}+$ and adsorption model. J. Environ. Manag. 85: 900-907. DOI: 10.1016/j.jenvman.2006.10.023

2382. Liu YC, Xu W, Xu WJ, Liu HM, Zhang XW. 2007. Comparison between two commercial uranium resins and a uranyl sulphate imprinted resin based on self-assembling MIT. Frontiers Chem. Eng. Chin. 1: 327-331. DOI: 10.1007/s11705-007-0059-8

2383. Metilda P, Gladis JM, Venkateswaran G, Prasada Rao T. 2007. Investigation of the role of chelating ligand in the synthesis of ion-imprinted polymeric resins on the selective enrichment of uranium(VI). Anal. Chim. Acta 587: 263-271. DOI: 10.1016/j.aca.2007.01.039 
2384. Randhawa M, Gartner I, Becker C, Student J, Chai MH, Mueller A. 2007. Imprinted polymers for water purification. J. Appl. Polym. Sci. 106: 3321 3326. DOI: 10.1002/app.26873

2385. Sadeghi S, Mofrad AA. 2007. Synthesis of a new ion imprinted polymer material for separation and preconcentration of traces of uranyl ions. Reac. Func. Polym. 67: 966-976. DOI: 10.1016/j.reactfunctpolym.2007.05.020

2386. Shamsipur M, Fasihi J, Ashtari K. 2007. Grafting of lon-Imprinted Polymers on the Surface of Silica Gel Particles through Covalently Surface-Bound Initiators: A Selective Sorbent for Uranyl Ion. Anal. Chem. 79: 7116-7123. DOI: $10.1021 / \mathrm{ac} 070968 \mathrm{e}$

2387. Shamsipur M, Fasihi J, Khanchi A, Hassani R, Alizadeh K, Shamsipur H. 2007. A stoichiometric imprinted chelating resin for selective recognition of copper(II) ions in aqueous media. Anal. Chim. Acta 599: 294-301. DOI: 10.1016/j.aca.2007.08.013

2388. Wu GH, Wang ZQ, Wang J, He CY. 2007. Hierarchically imprinted organicinorganic hybrid sorbent for selective separation of mercury ion from aqueous solution. Anal. Chim. Acta 582: 304-310. DOI: 10.1016/j.aca.2006.09.034

2389. Zhai YH, Liu YW, Chang XJ, Chen SB, Huang XP. 2007. Selective solidphase extraction of trace cadmium(II) with an ionic imprinted polymer prepared from a dual-ligand monomer. Anal. Chim. Acta 593: 123-128. DOI: 10.1016/j.aca.2007.04.040

2390. Zhang N, Hu B, Huang CZ. 2007. A new ion-imprinted silica gel sorbent for on-line selective solid-phase extraction of dysprosium(III) with detection by inductively coupled plasma-atomic emission spectrometry. Anal. Chim. Acta 597: 12-18. DOI: 10.1016/j.aca.2007.06.045 
2391. Zhao JC, Han B, Zhang YF, Wang DD. 2007. Synthesis of $\mathrm{Zn}(\mathrm{II})$ ionimprinted solid-phase extraction material and its analytical application. Anal. Chim. Acta 603: 87-92. DOI: 10.1016/j.aca.2007.09.024

2392. Zheng H, Zhang D, Wang WY, Fan YQ, Li J, Han HP. 2007. Highly selective determination of palladium(II) after preconcentration using $\mathrm{Pd}(\mathrm{II})$-imprinted functionalized silica gel sorbent prepared by a surface imprinting technique. Microchim. Acta 157: 7-11. DOI: 10.1007/s00604-006-0649-z

2393. Chang XJ, Wang XY, Jiang N, He Q, Zhai YH, Zhu XB, Hu Z. 2008. Silica gel surface-imprinted solid-phase extraction of $\mathrm{Zr}(\mathrm{IV})$ from aqueous solutions. Microchim. Acta 162: 113-119. DOI: 10.1007/s00604-007-0870-4

2394. He Q, Chang XJ, Zheng H, Jiang N, Wang XY. 2008. Determination of chromium(III) and total chromium in natural waters using a surface ionimprinted silica gel as selective adsorbent. Int. J. Environ. Anal. Chem. 88: 373-384. DOI: 10.1080/03067310701710870

2395. Kugimiya A, Takei H. 2008. Selective Recovery of Phosphate from River Water Using Molecularly Imprinted Polymers. Anal. Lett. 41: 302-311. DOI: 10.1080/00032710701792919

2396. Otero-Romaní J, Moreda-Piñeiro A, Bermejo-Barrera P, Martin-Esteban A. 2008. Synthesis, characterization and evaluation of ionic-imprinted polymers for solid-phase extraction of nickel from seawater. Anal. Chim. Acta 630: 19. DOI: 10.1016/j.aca.2008.09.049

2397. Özkara S, Say R, Denizli A. 2008. An Ion-Imprinted Monolith for in Vitro Removal of Iron out of Human Plasma with B Thalassemia. Ind. Eng. Chem. Res. 47: 7849-7856. DOI: 10.1021/ie071471y

2398. Özkütük EB, Ersöz A, Denizli A, Say RI. 2008. Preconcentration of phosphate ion onto ion-imprinted polymer. J. Haz. Mater. 157: 130-136. DOI: 10.1016/j.jhazmat.2007.12.118 
2399. Ren YM, Zhang ML, Zhao D. 2008. Synthesis and properties of magnetic $\mathrm{Cu}$ (II) ion imprinted composite adsorbent for selective removal of copper. Desalination 228: 135-149. DOI: 10.1016/j.desal.2007.08.013

2400. Ren YM, Wei XZ, Zhang ML. 2008. Adsorption character for removal Cu(II) by magnetic $\mathrm{Cu}(\mathrm{II})$ ion imprinted composite adsorbent. J. Haz. Mater. 158: 14-22. DOI: 10.1016/j.jhazmat.2008.01.044

2401. Shirvani-Arani S, Ahmadi SJ, Bahrami-Samani A, Ghannadi-Maragheh M. 2008. Synthesis of nano-pore samarium (III)-imprinted polymer for preconcentrative separation of samarium ions from other lanthanide ions via solid phase extraction. Anal. Chim. Acta 623: 82-88. DOI: 10.1016/j.aca.2008.05.071

2402. Walas S, Tobiasz A, Gawin M, Trzewik B, Strojny M, Mrowiec H. 2008. Application of a metal ion-imprinted polymer based on salen-Cu complex to flow injection preconcentration and FAAS determination of copper. Talanta 76: 96-101. DOI: 10.1016/j.talanta.2008.02.008

2403. Zhai YH, Yang D, Chang XJ, Liu YW, He Q. 2008. Selective enrichment of trace copper(II) from biological and natural water samples by SPE using ionimprinted polymer. J. Sep. Sci. 31: 1195-1200. DOI: $10.1002 /$ jssc.200700392

2404. Zhang N, Suleiman JS, He M, Hu B. 2008. Chromium(III)-imprinted silica gel for speciation analysis of chromium in environmental water samples with ICP-MS detection. Talanta 75: 536-543. DOI: 10.1016/j.talanta.2007.11.059

2405. Zhong SA, Yuan ZL, Qiao R, Li W. 2008. Preparation and adsorption behaviors of $\mathrm{Cu}(\mathrm{II})$ ion-imprinted polymers. J. Chongqing Univ. (Eng. Ed.) 23-27. 
2406. An FQ, Gao BJ, Feng XQ. 2009. Adsorption and recognition properties of ionic imprinted polyamine IIP-PEI/SiO2 towards $\mathrm{Pb2}+$ ion. J. Appl. Polym. Sci. 112: 2241-2246. DOI: 10.1002/app.29751

2407. An FQ, Gao BJ. 2009. Adsorption characteristics of $\mathrm{Cr}(\mathrm{III})$ ionic imprinting polyamine on silica gel surface. Desalination 249: 1390-1396. DOI: 10.1016/j.desal.2009.04.004

2408. Bhaskarapillai A, Sevilimedu NV, Sellergren B. 2009. Synthesis and Characterization of Imprinted Polymers for Radioactive Waste Reduction. Ind. Eng. Chem. Res. 48: 3730-3737. DOI: 10.1021/ie801640b

2409. Candan N, Tüzmen N, Andaç M, Andaç CA, Say RI, Denizli A. 2009. Cadmium removal out of human plasma using ion-imprinted beads in a magnetic column. Mater. Sci. Eng.: C 29: 144-152. DOI: 10.1016/j.msec.2008.06.002

2410. Chen AH, Yang CY, Chen CY, Chen CY, Chen CW. 2009. The chemically crosslinked metal-complexed chitosans for comparative adsorptions of $\mathrm{Cu}(\mathrm{II}), \mathrm{Zn}(\mathrm{II}), \mathrm{Ni}(\mathrm{II})$ and $\mathrm{Pb}(\mathrm{II})$ ions in aqueous medium. J. Haz. Mater. 163: 1068-1075. DOI: 10.1016/j.jhazmat.2008.07.073

2411. Dakova I, Karadjova I, Georgieva V, Georgiev G. 2009. Ion-imprinted polymethacrylic microbeads as new sorbent for preconcentration and speciation of mercury. Talanta 78: 523-529. DOI: 10.1016/j.talanta.2008.12.005

2412. Dam HA, Kim D. 2009. Selective Copper(II) Sorption Behavior of SurfaceImprinted Core-Shell-Type Polymethacrylate Microspheres. Ind. Eng. Chem. Res. 48: 5679-5685. DOI: 10.1021/ie801321d

2413. Demircelik AH, Andaç M, Andaç CA, Say R, Denizli A. 2009. Molecular Recognition-Based Detoxification of Aluminum in Human Plasma. J. Biomater. Sci. Polym. Ed. 20: 1235-1258. DOI: 10.1163/156856209X452971 
2414. Dey RK, Jha U, Patnaik T, Singh AC, Singh VK. 2009. Removal of Toxic/Heavy Metal lons Using Ion-Imprinted Aminofunctionalized Silica Gel. Sep. Sci. Technol. 44: 1829-1850. DOI: 10.1080/01496390902880115

2415. Esen C, Andaç M, Bereli N, Say RI, Henden E, Denizli A. 2009. Highly selective ion-imprinted particles for solid-phase extraction of $\mathrm{Pb} 2+$ ions. Mater. Sci. Eng.: C 29: 2464-2470. DOI: 10.1016/j.msec.2009.07.012

2416. García-Otero N, Teijeiro-Valiño C, Otero-Romaní J, Peña-Vázquez E, Moreda-Piñeiro A, Bermejo-Barrera P. 2009. On-line ionic imprinted polymer selective solid-phase extraction of nickel and lead from seawater and their determination by inductively coupled plasma-optical emission spectrometry. Anal. Bioanal. Chem. 395: 1107-1115. DOI: 10.1007/s00216-009-3044-x

2417. Guo JJ, Su QD, Gan WE. 2009. On-line Selective Solid-Phase Extraction of Copper with a Surface Ion Imprinted Silica Gel Sorbent. J. Chin. Chem. Soc. 56: 763-770.

2418. Hoai NT, Kim D. 2009. Synthesis, structure, and selective separation behavior of copper-imprinted microporous polymethacrylate beads. AIChE J 55: 3248-3254. DOI: 10.1002/aic.11943

2419. Huo HY, Su HJ, Tan TW. 2009. Adsorption of Ag+ by a surface molecularimprinted biosorbent. Chem. Eng. J. 150: 139-144. DOI: 10.1016/j.cej.2008.12.014

2420. James D, Venkateswaran G, Rao TP. 2009. Removal of uranium from mining industry feed simulant solutions using trapped amidoxime functionality within a mesoporous imprinted polymer material. Microporous Mesoporous Mater. 119: 165-170. DOI: 10.1016/j.micromeso.2008.10.011

2421. Khajeh M, Bohlooli M, Hashemi H. 2009. Imprinted Polymer Particles for Aluminum Uptake: Synthesis and Analytical Applications. J. Macromol. Sci. A 46: 526-532. DOI: 10.1080/10601320902797806 
2422. Khajeh M, Kaykhaii M, Mirmoghaddam M, Hashemi H. 2009. Separation of zinc from aqueous samples using a molecular imprinting technique. Int. J. Environ. Anal. Chem. 89: 981-992. DOI: 10.1080/03067310902719159

2423. Khajeh M, Kaykhaii M, Hashemi H, Mirmoghaddam M. 2009. Imprinted polymer particles for iron uptake: Synthesis, characterization and analytical applications. Polym. Sci. Ser. B 51: 344-351. DOI: $10.1134 / \mathrm{S} 1560090409090048$

2424. Li CX, Pan JM, Gao J, Yan YS, Zhao GQ. 2009. An ion-imprinted polymer supported by attapulgite with a chitosan incorporated sol-gel process for selective separation of $\mathrm{Ce}$ (III). Chin. Chem. Lett. 20: 985-989. DOI: 10.1016/j.cclet.2009.03.020

2425. Li CX, Gao J, Pan JM, Zhang ZL, Yan YS. 2009. Synthesis, characterization, and adsorption performance of $\mathrm{Pb}(\mathrm{II})$-imprinted polymer in nano-TiO2 matrix. J. Environ. Sci. 21: 1722-1729. DOI: 10.1016/S10010742(08)62479-1

2426. Li CX, Zhang XJ, Pan JM, Xu PP, Liu Y, Yan YS, Zhang ZL. 2009. Strontium(II) ion surface-imprinted polymers supported by potassium tetratitanate whiskers: Synthesis, characterization and adsorption behaviours. Adsorpt. Sci. Technol. 27: 845-859. DOI: 10.1260/02636174.27.9.845

2427. Otero-Romaní J, Moreda-Piñeiro A, Bermejo-Barrera P, Martin-Esteban A. 2009. Inductively coupled plasma-optical emission spectrometry/mass spectrometry for the determination of $\mathrm{Cu}, \mathrm{Ni}, \mathrm{Pb}$ and $\mathrm{Zn}$ in seawater after ionic imprinted polymer based solid phase extraction. Talanta 79: 723-729. DOI: 10.1016/j.talanta.2009.04.066

2428. Otero-Romaní J, Moreda-Piñeiro A, Bermejo-Barrera P, Martin-Esteban A. 2009. Ionic imprinted polymer for nickel recognition by using the bifunctionalized 5-vinyl-8-hydroxyquinoline as a monomer: Application as a 
new solid phase extraction support. Microchem. J. 93: 225-231. DOI: 10.1016/j.microc.2009.07.011

2429. Qu GR, Zheng SL, Liu YM, Xie W, Wu AB, Zhang DB. 2009. Metal ion mediated synthesis of molecularly imprinted polymers targeting tetracyclines in aqueous samples. J. Chromatogr $B$ 877: 3187-3193. DOI: 10.1016/j.jchromb.2009.08.012

2430. Quirarte-Escalante CA, Soto V, de la Cruz W, Porras GR, Manríquez R, Gomez-Salazar S. 2009. Synthesis of Hybrid Adsorbents Combining Sol-Gel Processing and Molecular Imprinting Applied to Lead Removal from Aqueous Streams. Chem. Mater. 21: 1439-1450. DOI: 10.1021/cm801480v

2431. Saraji M, Yousefi H, Meghdadi S. 2009. Preparation and evaluation of an ion imprinted sol-gel material for selective solid-phase extraction of $\mathrm{Ni}(\mathrm{II})$. Int. J. Environ. Anal. Chem. 89: 305-317. DOI: 10.1080/03067310802610268

2432. Saraji M, Yousefi H. 2009. Selective solid-phase extraction of $\mathrm{Ni}(\mathrm{II})$ by an ion-imprinted polymer from water samples. J. Haz. Mater. 167: 1152-1157. DOI: 10.1016/j.jhazmat.2009.01.111

2433. Singh DK, Mishra S. 2009. Synthesis, characterization and removal of Cd(II) using Cd(II)-ion imprinted polymer. J. Haz. Mater. 164: 1547-1551. DOI: 10.1016/j.jhazmat.2008.09.112

2434. Singh DK, Mishra S. 2009. Synthesis and characterization of UO22+-ion imprinted polymer for selective extraction of UO22+. Anal. Chim. Acta 644: 42-47. DOI: 10.1016/j.aca.2009.04.020

2435. Singh DK, Mishra S. 2009. Synthesis of a New Cu(II)-Ion Imprinted Polymer for Solid Phase Extraction and Preconcentration of $\mathrm{Cu}(\mathrm{II})$. Chromatographia 70: 1539-1545. DOI: 10.1365/s10337-009-1379-2

2436. Tobiasz A, Walas S, Trzewik B, Grzybek P, Zaitz MM, Gawin M, Mrowiec H. 2009. $\mathrm{Cu}(\mathrm{II})$-imprinted styrene-divinylbenzene beads as a new sorbent for 
flow injection-flame atomic absorption determination of copper. Microchem. J. 93: 87-92. DOI: 10.1016/j.microc.2009.05.002

2437. Wang LM, Zhou MH, Jing ZJ, Zhong AF. 2009. Selective separation of lead from aqueous solution with a novel $\mathrm{Pb}$ (II) surface ion-imprinted sol-gel sorbent. Microchim. Acta 165: 367-372. DOI: 10.1007/s00604-009-0146-2

2438. Wang LM, Xue G, Zhou MH, Chen YB, Yuan JC. 2009. Selective Separation of $\mathrm{Cd}$ (II) Ion from Aqueous Solution by $\mathrm{Cd}(\mathrm{II})$-Imprinted Polymers. In Bioinformatics and Biomedical Engineering, 2009. ICBBE 2009. 3rd International Conference on, IEEE: 1-4. DOI: 10.1109/ICBBE.2009.5163376

2439. Wang XW, Zhang L, Ma CL, Song RY, Hou HB, Li DL. 2009. Enrichment and separation of silver from waste solutions by metal ion imprinted membrane. Hydrometallurgy 100: 82-86. DOI: 10.1016/j.hydromet.2009.10.006

2440. Wang ZQ, Wu GH, He CY. 2009. Ion-imprinted thiol-functionalized silica gel sorbent for selective separation of mercury ions. Microchim. Acta 165: 151157. DOI: 10.1007/s00604-008-0113-3

2441. Wang $Z Q$, Wu GH, Wang M, He CY. 2009. An imprinted organic-inorganic hybrid sorbent for selective separation of copper ion from aqueous solution. J. Mater. Sci. 44: 2694-2699. DOI: 10.1007/s10853-009-3353-7

2442. Yusof NA, Beyan A, Haron MJ, Ibrahim NA. 2009. Synthesis and Evaluation of a Molecularly Imprinted Polymer for Pb(II) Ion Uptake. Pernanika J. Sci. Technol. 17: 155-161.

2443. Zhu XB, Cui YM, Chang XJ, Zou XJ, Li ZH. 2009. Selective solid-phase extraction of lead(II) from biological and natural water samples using surface-grafted lead(II)-imprinted polymers. Microchim. Acta 164: 125-132. DOI: 10.1007/s00604-008-0045-y 
2444. Li CX, Pan JM, Gao J, Yan YS. 2009. A novel Celll-imprinted polymer supported by attapulgite: synthesis, characterisation and adsorption behaviours towards Celll in aqueous solution. Int. J. Mater. Struct. Integr. 3: 294-308. DOI: 10.1504/IJMSI.2009.029337

2445. Ahmadi SJ, Noori-Kalkhoran O, Shirvani-Arani S. 2010. Synthesis and characterization of new ion-imprinted polymer for separation and preconcentration of uranyl (UO22+) ions. J. Haz. Mater. 175: 193-197. DOI: 10.1016/j.jhazmat.2009.09.148

2446. Asliyüce S, Bereli N, Uzun L, Onur MA, Say RI, Denizli A. 2010. Ionimprinted supermacroporous cryogel, for in vitro removal of iron out of human plasma with $\beta$ thalassemia. Sep. Purif. Technol. 73: 243-249. DOI: 10.1016/j.seppur.2010.04.007

2447. Bhaskarapillai A, Sevilimedu NV, Sellergren B. 2010. One in a billion. Nuclear Engineering International 55: 22-24.

2448. Buhani, Suharso, Sumadi. 2010. Adsorption kinetics and isotherm of $\mathrm{Cd}(\mathrm{II})$ ion on Nannochloropsis sp biomass imprinted ionic polymer. Desalination 259: 140-146. DOI: 10.1016/j.desal.2010.04.019

2449. Buhani, Narsito, Nuryono, Kunarti ES. 2010. Production of metal ion imprinted polymer from mercapto-silica through sol-gel process as selective adsorbent of cadmium. Desalination 251: 83-89. DOI: 10.1016/j.desal.2009.09.139

2450. Chen JH, Li GP, Liu QL, Ni JC, Wu WB, Lin JM. 2010. Cr(III) ionic imprinted polyvinyl alcohol/sodium alginate (PVA/SA) porous composite membranes for selective adsorption of $\mathrm{Cr}$ (III) ions. Chem. Eng. J. 165: 465-473. DOI: 10.1016/j.cej.2010.09.034

2451. de Avila TC, Segatelli MG, Beijo LA, Tarley CRT. 2010. Employ of Silica Gel Organically Modified and Ionically Imprinted for Selective On-Line 
Preconcentration of Copper lons. Quimica Nova 33: 301-308. DOI: $10.1590 /$ S0100-40422010000200014

2452. Demiralay EÇ, Andac M, Say R, Alsancak G, Denizli A. 2010. Nickel(II)imprinted monolithic columns for selective nickel recognition. J. Appl. Polym. Sci. 117: 3704-3714. DOI: 10.1002/app.32269

2453. El-Arnaouty MB. 2010. Radiation Synthesis and Characterization Study of Imprinted Hydrogels for Metal Ion Adsorption. Polym. -Plast. Technol. Eng. 49: 963-971. DOI: 10.1080/03602559.2010.482071

2454. Gawin M, Konefal J, Trzewik B, Walas S, Tobiasz A, Mrowiec H, Witek E. 2010. Preparation of a new $\mathrm{Cd}(\mathrm{II})$-imprinted polymer and its application to determination of cadmium(II) via flow-injection-flame atomic absorption spectrometry. Talanta 80: 1305-1310. DOI: 10.1016/j.talanta.2009.09.021

2455. Godlewska-Zylkiewicz B, Lesniewska B, Wawreniuk I. 2010. Assessment of ion imprinted polymers based on $\mathrm{Pd}(\mathrm{II})$ chelate complexes for preconcentration and FAAS determination of palladium. Talanta 83: 596604. DOI: 10.1016/j.talanta.2010.10.005

2456. Hajizadeh S, Kirsebom H, Galaev IY, Mattiasson B. 2010. Evaluation of selective composite cryogel for bromate removal from drinking water. $\mathrm{J}$. Sep. Sci. 33: 1752-1759. DOI: 10.1002/jssc.201000019

2457. Hoai NT, Yoo DK, Kim D. 2010. Batch and column separation characteristics of copper-imprinted porous polymer micro-beads synthesized by a direct imprinting method. J. Haz. Mater. 173: 462-467. DOI: 10.1016/j.jhazmat.2009.08.107

2458. Jiang W, Su HJ, Huo HY, Tan TW. 2010. Synthesis and Properties of Surface Molecular Imprinting Adsorbent for Removal of Pb2+. Appl. Biochem. Biotechnol. 160: 467-476. DOI: 10.1007/s12010-008-8366-1 
2459. Jo SH, Lee SY, Park KM, Yi SC, Kim D, Mun S. 2010. Continuous separation of copper ions from a mixture of heavy metal ions using a threezone carousel process packed with metal ion-imprinted polymer. J. Chromatogr. A 1217: 7100-7108. DOI: 10.1016/j.chroma.2010.09.014

2460. Karabörk M, Özkütük EB, Ersöz A, Say R. 2010. Selective Preconcentration of Fe3+ Using lon-Imprinted Thermosensitive Particles. Hacettepe J. Biol. \& Chem. 38: 27-39.

2461. Khajeh M, Sanchooli E. 2010. Development of a Selective Molecularly Imprinted Polymer-Based Solid-Phase Extraction for Copper from Food Samples. Biol. Trace Elem. Res. 135: 325-333. DOI: 10.1007/s12011-009$8481-z$

2462. Li CX, Zhang ZL, Pan JM, Gao J, Zhao GQ, Guan WS, Yan YS. 2010. Synthesis and characterisation of sodium trititanate whisker surface Cdll ion-imprinted polymer and selective solid-phase extraction of cadmium. Int. J. Mater. Struct. Integr. 4: 291-307. DOI: 10.1504/IJMSI.2010.035211

2463. Li QA, Liu HN, Liu TY, Guo M, Qing BJ, Ye XS, Wu ZJ. 2010. Strontium and calcium ion adsorption by molecularly imprinted hybrid gel. Chem. Eng. J. 157: 401-407. DOI: 10.1016/j.cej.2009.11.029

2464. Lin CR, Wang HQ, Wang YY, Cheng ZQ. 2010. Selective solid-phase extraction of trace thorium(IV) using surface-grafted Th(IV)-imprinted polymers with pyrazole derivative. Talanta 81: 30-36. DOI: 10.1016/j.talanta.2009.11.032

2465. Liu Y, Gao J, Zhang ZL, Dai JD, Xie JM, Yan YS. 2010. A New Sr(II) Ionimprinted Polymer Grafted onto Potassium Titanate Whiskers: Synthesis and Adsorption Performance for the Selective Separation of Strontium lons. Adsorpt. Sci. Technol. 28: 23-37. DOI: 10.1260/0263-6174.28.1.23 
2466. Liu Y, Gao J, Li CX, Pan JM, Yan YS, Xie JM. 2010. Synthesis and Adsorption Performance of Surface-Grafted Co(II)-Imprinted Polymer for Selective Removal of Cobalt. Chin. J. Chem. 28: 548-554. DOI: 10.1002/cjoc.201090110

2467. Liu YH, Cao XH, Le ZG, Luo MB, Xu WY, Huang GL. 2010. PreConcentration and Determination of Trace Uranium (VI) in Environments using Ion-imprinted Chitosan Resin via Solid Phase Extraction. J. Braz. Chem. Soc. 21: 533-540.

2468. Liu YH, Cao XH, Hua R, Wang YQ, Liu YT, Pang C, Wang Y. 2010. Selective adsorption of uranyl ion on ion-imprinted chitosan/PVA crosslinked hydrogel. Hydrometallurgy 104: 150-155. DOI: 10.1016/j.hydromet.2010.05.009

2469. Nacano LR, Segatelli MG, Tarley CRT. 2010. Selective Sorbent Enrichment of Nickel lons from Aqueous Solutions using a Hierarchically Hybrid Organic-Inorganic Polymer Based on Double Imprinting Concept. J. Braz. Chem. Soc. 21: 419-430. DOI: 10.1590/S0103-50532010000300004

2470. Özkütük EB, Özalp E, Isler G, Diltemiz SE, Ersöz A. 2010. Selective SolidPhase Extraction of $\mathrm{Cd}(\mathrm{II})$ Using Double Imprinting Strategy. Gazi Univ. J. Sci. 23: $19-26$.

2471. Özkütük EB, Özalp E, Isler G. 2010. Single and double imprinted polymer for selective recognition of $\mathrm{Cd}(\mathrm{II})$ ions in aqueous media. Anadolu Univ. J. Sci. Technol. A Appl. Sci. Eng. 11: 149-161.

2472. Pan JM, Zou XH, Yan YS, Wang X, Guan W, Han JA, Wu XY. 2010. An ionimprinted polymer based on palygorskite as a sacrificial support for selective removal of strontium(II). Appl. Clay Sci. 50: 260-265. DOI: 10.1016/j.clay.2010.08.007 
2473. Pan JM, Guan W, Zhang ZL, Wang X, Li CX, Yan YS. 2010. Selective Adsorption of $\mathrm{Co}$ (II) lons by Whisker Surface Ion-Imprinted Polymer: Equilibrium and Kinetics Modeling. Chin. J. Chem. 28: 2483-2488. DOI: 10.1002/cjoc.201190026

2474. Pan JM, Zou XH, Li CX, Liu Y, Yan YS, Han JA. 2010. Synthesis and applications of $\mathrm{Ce}$ (III)-imprinted polymer based on attapulgite as the sacrificial support material for selective separation of cerium(III) ions. Microchim. Acta 171: 151-160. DOI: 10.1007/s00604-010-0416-z

2475. Segatelli MG, Santos VS, Presotto ABT, Yoshida IVP, Tarley CRT. 2010. Cadmium ion-selective sorbent preconcentration method using ion imprinted poly(ethylene glycol dimethacrylate-co-vinylimidazole). Reac. Func. Polym. 70: 325-333. DOI: 10.1016/j.reactfunctpolym.2010.02.006

2476. Shamsipur M, Besharati-Seidani A, Fasihi J, Sharghi H. 2010. Synthesis and characterization of novel ion-imprinted polymeric nanoparticles for very fast and highly selective recognition of copper(II) ions. Talanta 83: 674-681. DOI: $10.1016 /$ j.talanta.2010.10.021

2477. Singh DK, Mishra S. 2010. Synthesis and characterization of $\mathrm{Fe}(\mathrm{III})$-ion imprinted polymer for recovery of Fe(III) from water samples. J. Sci. Ind. Res. 69: 767-772.

2478. Singh DK, Mishra S. 2010. Synthesis, characterization and analytical applications of Ni(II)-ion imprinted polymer. Appl. Surf. Sci. 256: 7632-7637. DOI: 10.1016/j.apsusc.2010.06.018

2479. Suspène C, Brandès S, Guilard R. 2010. Reversible Coordination of Dioxygen by Tripodal Tetraamine Copper Complexes Incorporated in a Porous Silica Framework. Chem. Eur. J. 16: 6352-6364. DOI: 10.1002/chem.200903148 
2480. Tajodini N, Moghimi A. 2010. Preconcentration and Determination of Ultra Trace Cobalt(II) in Water Samples Using Co(II)-Imprinted Diazoaminobenzene-Vinylpyridine Copolymers. Asian J. Chem. 22: 33353344.

2481. Wang ZQ, Wang M, Wu GH, Shen YY, He CY. 2010. Ion imprinted sol-gel nanotubes membrane for selective separation of copper ion from aqueous solution. Microchim. Acta 169: 195-200. DOI: 10.1007/s00604-010-0332-2

2482. Wu GH, Song GC, Wu DY, Shen YY, Wang ZQ, He CY. 2010. Synthesis of ion-imprinted mesoporous silica gel sorbent for selective adsorption of copper ions in aqueous media. Microchim. Acta 171: 203-209. DOI: 10.1007/s00604-010-0414-1

2483. Xu WZ, Yang WM, Zhou W. 2010. Selective Solid-phase Extraction of Pb(II) lons Using an Ion-imprinted Functionalized Sodium Trititanate Whisker Adsorbent Prepared by a Surface-imprinting Technique. Adsorpt. Sci. Technol. 28: 509-519. DOI: 10.1260/0263-6174.28.6.509

2484. Xu WZ, Xu PP, Zhou W, Yan YS. 2010. Synthesis, characterization, evaluation of potassium tertatitanium whisker surface $\mathrm{Ni}(\mathrm{II})$ ion-imprinted polymers and selective adsorption of nickel. In Environment Materials and Environment Management, Advanced Materials Research, Vol. 113-116, Du ZY, Sun XB (eds). Scientific.net: 644-650. DOI: 10.4028/www.scientific.net/AMR.113-116.644

2485. Yusof NA, Beyan A, Haron MJ, Ibrahim NA. 2010. Synthesis and Characterization of a Molecularly Imprinted Polymer for Pb2+ Uptake Using 2-vinylpyridine as the Complexing Monomer. Sains Malaysiana 39: 829-835.

2486. Zhang XJ, Li CX, Yan YS, Pan JM, Xu PP, Zhao XH. 2010. A Ce3+imprinted functionalized potassium tetratitanate whisker sorbent prepared by surface molecularly imprinting technique for selective separation and 
determination of Ce3+. Microchim. Acta 169: 289-296. DOI: $10.1007 / s 00604-010-0352-y$

2487. Zhang ZH, Zhang HB, Hu YF, Yang X, Yao SZ. 2010. Novel surface molecularly imprinted material modified multi-walled carbon nanotubes as solid-phase extraction sorbent for selective extraction gallium ion from fly ash. Talanta 82: 304-311. DOI: 10.1016/j.talanta.2010.04.038

2488. Arbab-Zavar MH, Chamsaz M, Zohuri G, Darroudi A. 2011. Synthesis and characterization of nano-pore thallium (III) ion-imprinted polymer as a new sorbent for separation and preconcentration of thallium. J. Haz. Mater. 185: 38-43. DOI: 10.1016/j.jhazmat.2010.08.093

2489. Alizadeh T. 2011. A novel imprinted polymer for removal of Cd+2 from water samples: optimization of adsorption and recovery steps by experimental design. Chinese Journal of Polymer Science 29: 658-669. DOI: 10.1007/s10118-011-1082-2

2490. Ashraf S, Cluley A, Mercado C, Mueller A. 2011. Imprinted polymers for the removal of heavy metal ions from water. Water Sci. Technol. 64: 1325-1332. DOI: $10.2166 /$ wst.2011.423

2491. Baba Y, Oshima T, Kanemaru S. 2011. A Quantitative Consideration for Template Effect of Palladium(II) Using $N$-[Pyridylmethyl] Chitosan. Solv. Extract. Ion Exch. 29: 509-517. DOI: 10.1080/07366299.2011.573452

2492. Bayramoglu G, Arica MY. 2011. Synthesis of $\mathrm{Cr}(\mathrm{VI})$-imprinted poly(4-vinyl pyridine-co-hydroxyethyl methacrylate) particles: Its adsorption propensity to Cr(VI). J. Haz. Mater. 187: 213-221. DOI: 10.1016/j.jhazmat.2011.01.022

2493. Chen CY, Yang CY, Chen AH. 2011. Biosorption of $\mathrm{Cu}(\mathrm{II}), \mathrm{Zn}(\mathrm{II}), \mathrm{Ni}(\mathrm{II})$ and $\mathrm{Pb}$ (II) ions by cross-linked metal-imprinted chitosans with epichlorohydrin. $J$. Environ. Manag. 92: 796-802. DOI: 10.1016/j.jenvman.2010.10.029 
2494. Chen JH, Lin H, Luo ZH, He YS, Li GP. 2011. Cu(II)-imprinted porous film adsorbent Cu-PVA-SA has high uptake capacity for removal of $\mathrm{Cu}(\mathrm{II})$ ions from aqueous solution. Desalination 277: 265-273. DOI: 10.1016/j.desal.2011.04.040

2495. Chen QY, Zhou DF, Chen H, Wang J. 2011. Selective adsorption of Mn(II) ions using $\mathrm{Mn}$ (II) ion imprinted silica modified by di(2-picolyl)amine. In Proceeding of the 2011 International Conference on Remote Sensing, Environment and Transportation Engineering (RSETE), IEEE: 7811-7814. DOI: 10.1109/RSETE.2011.5966187

2496. Cheng ZQ, Wang HQ, Wang YY, He FF, Zhang HS, Yang SQ. 2011. Synthesis and characterization of an ion-imprinted polymer for selective solid phase extraction of thorium(IV). Microchim. Acta 173: 423-431. DOI: 10.1007/s00604-011-0576-5

2497. Dakova I, Karadjova I, Georgieva V, Georgiev G. 2011. Synthesis and characterization of the $\mathrm{Hg}(\mathrm{II})$-ion imprinted microbeads and membranes for enrichment of inorganic $\mathrm{Hg}(\mathrm{II})$. Annuaire de l'Université de Sofia 'St. Kliment Ohridski' Faculte de Chimie 102-103: 165-175.

2498. Fan LL, Luo CN, Lv Z, Lu FG, Qiu HM. 2011. Removal of Ag+ from water environment using a novel magnetic thiourea-chitosan imprinted $\mathrm{Ag}+\mathrm{J}$. Haz. Mater. 194: 193-201. DOI: 10.1016/j.jhazmat.2011.07.080

2499. Fasihi J, Ammari Alahyari S, Shamsipur M, Sharghi H, Charkhi A. 2011. Adsorption of uranyl ion onto an anthraquinone based ion-imprinted copolymer. Reac. Func. Polym. 71: 803-808. DOI: 10.1016/j.reactfunctpolym.2011.03.014

2500. Fu XC, Chen X, Guo Z, Xie CG, Kong LT, Liu JH, Huang XJ. 2011. Stripping voltammetric detection of mercury(II) based on a surface ion imprinting strategy in electropolymerized microporous poly(2-mercaptobenzothiazole) 
films modified glassy carbon electrode. Anal. Chim. Acta 685: 21-28. DOI: 10.1016/j.aca.2010.11.020

2501. Huang H, Huang ZJ, Wang XG. 2011. Selective solid-phase extraction of $\mathrm{Ni}(\mathrm{II})$ by an ion-imprinted polymer from biological samples. Asian J. Chem. 23: $1527-1530$.

2502. Khajeh M, Sanchooli E. 2011. Imprinted polymer particles for preconcentration of copper from water and biological samples. Environ. Chem. Lett. 9: 177-183. DOI: 10.1007/s10311-009-0260-8

2503. Khajeh M, Sanchooli E. 2011. Synthesis of ion-selective imprinted polymer for manganese removal from environmental water. Polym. Bull. 67: 413-425. DOI: 10.1007/s00289-010-0389-4

2504. Khajeh M, Heidari ZS, Sanchooli E. 2011. Synthesis, characterization and removal of lead from water samples using lead-ion imprinted polymer. Chem. Eng. J. 166: 1158-1163. DOI: 10.1016/j.cej.2010.12.018

2505. Lesniewska B, Kosinska M, Godlewska-Zylkiewicz B, Zambrzycka E, Wilczewska AZ. 2011. Selective solid phase extraction of platinum on an ion imprinted polymers for its electrothermal atomic absorption spectrometric determination in environmental samples. Microchim. Acta 175: 273-282. DOI: 10.1007/s00604-011-0685-1

2506. Li CX, Pan JM, Zou XH, Gao J, Xie JM, Yongsheng Y. 2011. Synthesis and applications of novel attapulgite-supported $\mathrm{Co}(\mathrm{II})$-imprinted polymers for selective solid-phase extraction of cobalt(II) from aqueous solutions. Int. J. Environ. Anal. Chem. 91: 1035-1049. DOI: 10.1080/03067310903502752

2507. Li TY, Chen SX, Li HC, Li QH, Wu L. 2011. Preparation of an lon-Imprinted Fiber for the Selective Removal of Cu2+. Langmuir 27: 6753-6758. DOI: $10.1021 / \mathrm{la200986v}$ 
2508. Li ZC, Fan HT, Sun T. 2011. Application of Imprinted Functionalized Silica Gel Sorbent for Selective Removal of Cadmium (II) from Industial Wastewaters. In Advanced Materials Research, Advanced Materials Research, Vol. 213, Yushu Z (ed). Scientific.net: 441-444. DOI: 10.4028/www.scientific.net/AMR.213.441

2509. Li ZC, Fan HT, Zhang Y, Chen MX, Yu ZY, Cao XQ, Sun T. 2011. Cd(II)imprinted polymer sorbents prepared by combination of surface imprinting technique with hydrothermal assisted sol-gel process for selective removal of cadmium(II) from aqueous solution. Chem. Eng. J. 171: 703-710. DOI: 10.1016/j.cej.2011.05.023

2510. Lin CR, Wang HQ, Wang YY, Zhou L, Liang J. 2011. Selective preconcentration of trace thorium from aqueous solutions with Th(IV)imprinted polymers prepared by a surface-grafted technique. Int. J. Environ. Anal. Chem. 91: 1050-1061. DOI: 10.1080/03067311003629677

2511. Lin CX, Liu MH, Zhan HY. 2011. Pb(II)-Imprinted Polymer Prepared by Graft Copolymerization of Acrylic Acid onto Cellulose. In Manufacturing Science and Technology, Advanced Materials Research, Vol. 295-297, Wang PC, Ai LQ, Li YG, Sang XM, Bu JL (eds). Trans Tech Publications: 2045-2048. DOI: 10.4028/www.scientific.net/AMR.295-297.2045

2512. Liu BH, Han MY, Guan GJ, Wang SH, Liu RY, Zhang ZP. 2011. HighlyControllable Molecular Imprinting at Superparamagnetic Iron Oxide Nanoparticles for Ultrafast Enrichment and Separation. J. Phys. Chem. C 115: 17320-17327. DOI: 10.1021/jp205327q

2513. Liu BJ, Wang DF, Xu Y, Huang GQ. 2011. Adsorption properties of Cd(II)imprinted chitosan resin. J. Mater. Sci. 46: 1535-1541. DOI: 10.1007/s10853-010-4958-6

2514. Liu BJ, Wang DF, Li HY, Xu Y, Zhang L. 2011. As(III) removal from aqueous solution using $\alpha$-Fe2O3 impregnated chitosan beads with $\mathrm{As}(\mathrm{III})$ as 
imprinted ions. Desalination 272: 286-292. DOI: 10.1016/j.desal.2011.01.034

2515. Liu BJ, Wang DF, Gao X, Zhang L, Xu Y, Li YJ. 2011. Removal of arsenic from Laminaria japonica Aresch juice using As(III)-imprinted chitosan resin. Eur. Food Res. Technol. 232: 911-917. DOI: 10.1007/s00217-011-1460-6

2516. Liu HJ, Yang F, Zheng YM, Kang J, Qu JH, Chen JP. 2011. Improvement of metal adsorption onto chitosan/Sargassum sp. composite sorbent by an innovative ion-imprint technology. Water Res. 45: 145-154. DOI: 10.1016/j.watres.2010.08.017

2517. Liu QP, Li HZ, Zhuang HY, Pei MS. 2011. Synthesis of a New lon Imprinted Polymer Material for Separation and Preconcentration of Traces of Neodymium Ions. In Emerging Focus on Advanced Materials, Advanced Materials Research, Vol. 306-307, Liu SQ, Zuo M (eds). Scientific.net: 705708. DOI: 10.4028/www.scientific.net/AMR.306-307.705

2518. Liu Y, Liu ZC, Wang Y, Dai JD, Gao J, Xie JM, Yan YS. 2011. A surface ionimprinted mesoporous sorbent for separation and determination of $\mathrm{Pb}(\mathrm{II})$ ion by flame atomic absorption spectrometry. Microchim. Acta 172: 309-317. DOI: $10.1007 / \mathrm{s} 00604-010-0491-1$

2519. Liu Y, Liu ZC, Gao J, Dai JD, Han J, Wang Y, Xie JM, Yan YS. 2011. Selective adsorption behavior of $\mathrm{Pb}(\mathrm{II})$ by mesoporous silica SBA-15supported $\mathrm{Pb}(\mathrm{II})$-imprinted polymer based on surface molecularly imprinting technique. J. Haz. Mater. 186: 197-205. DOI: 10.1016/j.jhazmat.2010.10.105

2520. Milja TE, Prathish KP, Prasada Rao T. 2011. Synthesis of surface imprinted nanospheres for selective removal of uranium from simulants of Sambhar salt lake and ground water. J. Haz. Mater. 188: 384-390. DOI: 10.1016/j.jhazmat.2011.01.121 
2521. Mizuki H, Ito Y, Samatya S, Harada H, Kawakita H, Uezu K. 2011. Zr(IV)Immobilized Resin Prepared by Surface Template Polymerization for Fluoride Ion Removal. Solv. Extract. Ion Exch. 29: 146-156. DOI: $10.1080 / 07366299.2011 .539417$

2522. Özkahraman B, Acar I, Güçlü K, Güçlü G. 2011. Synthesis of Zn(II) IonImprinted Polymeric Adsorbent for Selective Removal of Zinc from Aqueous Solutions. Polym. -Plast. Technol. Eng. 50: 216-219. DOI: $10.1080 / 03602559.2010 .531434$

2523. Özkara S, Andaç M, Karakoç V, Say R, Denizli A. 2011. Ion-imprinted PHEMA based monolith for the removal of $\mathrm{Fe} 3+$ ions from aqueous solutions. J. Appl. Polym. Sci. 120: 1829-1836. DOI: 10.1002/app.33400

2524. Pakade V, Cukrowska E, Darkwa J, Torto N, Chimuka L. 2011. Selective removal of chromium ( $\mathrm{VI})$ from sulphates and other metal anions using an ion-imprinted polymer. Water SA 37: 529-537.

2525. Rammika M, Darko G, Tshentu Z, Sewry J, Torto N. 2011. Dimethylglyoxime based ion-imprinted polymer for the determination of $\mathrm{Ni}(\mathrm{II})$ ions from aqueous samples. Water SA 37: 321-329.

2526. Rammika M, Darko G, Torto N. 2011. Incorporation of Ni(II)dimethylglyoxime ion-imprinted polymer into electrospun polysulphone nanofibre for the determination of $\mathrm{Ni}(\mathrm{II})$ ions from aqueous samples. Water SA 37: 539-546.

2527. Zambrzycka E, Roszko D, Lesniewska B, Wilczewska AZ, GodlewskaZylkiewicz B. 2011. Studies of ion-imprinted polymers for solid-phase extraction of ruthenium from environmental samples before its determination by electrothermal atomic absorption spectrometry. Spectrochim. Acta, B 66: 508-516. DOI: 10.1016/j.sab.2011.03.006 
2528. Zhang ML, Zhang ZH, Liu YN, Yang X, Luo LJ, Chen JT, Yao SZ. 2011. Preparation of core-shell magnetic ion-imprinted polymer for selective extraction of $\mathrm{Pb}$ (II) from environmental samples. Chem. Eng. J. 178: 443450. DOI: 10.1016/j.cej.2011.10.035

2529. Zheng F, Zhang N, Hu B. 2011. Mn(II) imprinted 3mercaptopropyltrimethoxysilane (MPTS)-silica coated capillary microextraction on-line hyphenated with inductively coupled plasma mass spectrometry for the determination of trace $\mathrm{Mn}$ (II) in biological samples. $\mathrm{J}$. Anal. Atom. Spectrosc. 26: 1521-1525. DOI: 10.1039/C1JA10025D

2530. Zheng H, Yang SL, Wang JC, Sun WF. 2011. Highly selective determination of rhodium(III) using silica gel surface-imprinted solid-phase extraction. Int. J. Environ. Anal. Chem. 91: 1013-1023. DOI: 10.1080/03067310903402441

2531. Zhuang HY, Li HZ, Xue Y. 2011. Synthesis, Characterization and Selective Recognition of Lead Using a New Ion Imprinted Polymer Material. In Emerging Focus on Advanced Materials, Advanced Materials Research, Vol. 306-307, Liu SQ, Zuo M (eds). Scientific.net: 688-691. DOI: 10.4028/www.scientific.net/AMR.306-307.688

2532. Wang LM, Li YH, Wei W. 2011. A Double Imprinted Organic-Inorganic Hybrid Sorbent for Selective Separation of Lead from Aqueous Solution. In Advanced Materials and Processes, Advanced Materials Research, Vol. 311-313, Guo ZN (ed). Scientific.net: 1491-1495. DOI: 10.4028/www.scientific.net/AMR.311-313.1491

2533. Lu YK, Yan XP. 2004. An imprinted organic-inorganic hybrid sorbent for selective separation of cadmium from aqueous solution. Anal. Chem. 76: 453-457. DOI: $10.1021 / \mathrm{ac} 0347718$

2534. Jahantigh N. 2008. Molecular Imprinted Polymer (MIP) for arsenic extraction from environmental water and biological samples. In Metal lons in Biology and Medicine, Metal Ions in Biology and Medicine, Vol. 10, Collery P, 
Maynard I, Theophanides T, Khassanova L, Collery T (eds). John Libby Eurotext: Montrouge, France; 247-251.

2535. Tarley CRT, Fernandes FF, Luccas PO, Segatelli MG. 2011. Enhanced Selectivity and Sensitivity for Flow Injection Spectrophotometric Determination of Cobalt Using Solid Phase Extraction with a 2D lonImprinted Adsorbent. Anal. Lett. 44: 216-231. DOI: $10.1080 / 00032719.2010 .500756$

2536. Bossi A, Castelletti L, Piletsky SA, Turner APF, Righetti PG. 2004. Properties of poly-aminophenylboronate coatings in capillary electrophoresis for the selective separation of diastereoisomers and glycoproteins. $J$. Chromatogr. A 1023: 297-303. DOI: 10.1016/j.chroma.2003.10.023

2537. Guihen E, Glennon JD. 2004. Recent highlights in stationary phase design for open-tubular capillary electrochromatography. J. Chromatogr. A 1044: 67-81. DOI: 10.1016/j.chroma.2004.05.107

2538. Huang YC, Lin CC, Liu CY. 2004. Preparation and evaluation of molecularly imprinted polymers based on 9-ethyladenine for the recognition of nucleotide bases in capillary electrochromatography. Electrophoresis 25: 554-561. DOI: 10.1002/elps.200305735

2539. Liu ZS, Xu YL, Wang HF, Yan C, Gao RY. 2004. Chiral separation of binaphthol enantiomers on molecularly imprinted polymer monolith by capillary electrochromatography. Anal. Sci. 20: 673-678. DOI: 10.2116/analsci.20.673

2540. Liu ZS, Xu YL, Yan C, Gao RY. 2004. Preparation and characterization of molecularly imprinted monolithic column based on 4-hydroxybenzoic acid for the molecular recognition in capillary electrochromatography. Anal. Chim. Acta 523: 243-250. DOI: 10.1016/j.aca.2004.07.001 
2541. Liu ZS, Xu YL, Yan C, Gao RY. 2004. Capillary electrochromatography of molecularly imprinted monolithic column using p-hydroxybenzoic acid as templates. Chin. Chem. Lett. 15: 1469-1472.

2542. Palmer CP, McCarney JP. 2004. Developments in the use of soluble ionic polymers as pseudo-stationary phases for electrokinetic chromatography and stationary phases for electrochromatography. J. Chromatogr. A 1044: 159-176. DOI: 10.1016/j.chroma.2004.04.068

2543. Quaglia M, Sellergren B, De Lorenzi E. 2004. Approaches to imprinted stationary phases for affinity capillary electrochromatography. $J$. Chromatogr. A 1044: 53-66. DOI: 10.1016/j.chroma.2004.05.055

2544. Szumski M, Buszewski B. 2004. Molecularly imprinted polymers: A new tool for separation of steroid isomers. J. Sep. Sci. 27: 837-842. DOI: 10.1002/jssc.200401799

2545. Deng QL, Lun ZH, Shao H, Yan C, Gao RY. 2005. Molecularly imprinted polymers as tools for the screening of felodipine from dihydropyridine calcium antagonists by pressurized capillary electrochromatography. Anal. Bioanal. Chem. 382: 51-58. DOI: 10.1007/s00216-005-3152-1

2546. Liu ZS, Xu YL, Yan C, Gao RY. 2005. Mechanism of molecular recognition on molecular imprinted monolith by capillary electrochromatography. $\mathrm{J}$. Chromatogr. A 1087: 20-28. DOI: 10.1016/j.chroma.2005.05.107

2547. Xu YL, Liu ZS, Wang HF, Yan C, Gao RY. 2005. Chiral recognition ability of an (S)-naproxen-imprinted monolith by capillary electrochromatography. Electrophoresis 26: 804-811. DOI: 10.1002/elps.200410171

2548. Bacskay I, Takátsy A, Végvári A, Elfwing E, Ballagi-Pordány A, Kilár F, Hjertén S. 2006. Universal method for synthesis of artificial gel antibodies by the imprinting approach combined with a unique electrophoresis technique for detection of minute structural differences of proteins, viruses, and cells 
(bacteria). III: Gel antibodies against cells (bacteria). Electrophoresis 27: 4682-4687. DOI: 10.1002/elps.200600192

2549. Deng QL, Lun ZH, Gao RY, Zhang LH, Zhang WB, Zhang YK. 2006. SIbuprofen-imprinted polymers incorporating $\gamma$-methacryloxypropyltrimethoxysilane for CEC separation of ibuprofen enantiomers. Electrophoresis 27: 4351-4358. DOI: 10.1002/elps.200600180

2550. Klodzinska E, Moravcova D, Jandera P, Buszewski B. 2006. Monolithic continuous beds as a new generation of stationary phase for chromatographic and electro-driven separations. J. Chromatogr. A 1109: 5159. DOI: 10.1016/j.chroma.2005.12.024

2551. Lin CC, Wang GR, Liu CY. 2006. A novel monolithic column for capillary electrochromatographic separation of oligopeptides. Anal. Chim. Acta $\mathbf{5 7 2}$ 197-204. DOI: 10.1016/j.aca.2006.05.028

2552. Lun ZH, Deng QL, Yan C, Gao RY. 2006. Molecularly imprinted polymer monolithic column separation of isomers and analogues of vanillin by capillary electrochromatography. Chin. J. Chem. 24: 442-444. DOI: 10.1002/cjoc.200690085

2553. Nilsson C, Nilsson S. 2006. Nanoparticle-based pseudostationary phases in capillary electrochromatography. Electrophoresis 27: 76-83. DOI: 10.1002/elps.200500535

2554. Ogiso M, Minoura N, Shinbo T, Shimizu T. 2006. Detection of a specific DNA sequence by electrophoresis through a molecularly imprinted polymer. Biomaterials 27: 4177-4182. DOI: 10.1016/j.biomaterials.2006.03.020

2555. Qin F, Xie CH, Yu ZY, Kong L, Ye ML, Zou HF. 2006. Monolithic enantiomer-selective stationary phases for capillary electrochromatography. J. Sep. Sci. 29: 1332-1343. DOI: 10.1002/jssc.200600030 
2556. Takátsy A, Kilár A, Kilár F, Hjertén S. 2006. Universal method for synthesis of artificial gel antibodies by the imprinting approach combined with a unique electrophoresis technique for detection of minute structural differences of proteins, viruses, and cells (bacteria): la. Gel antibodies against proteins (transferrins). J. Sep. Sci. 29: 2802-2809. DOI: 10.1002/jssc.200600211

2557. Ogiso M, Minoura N, Shinbo T, Shimizu T. 2007. DNA detection system using molecularly imprinted polymer as the gel matrix in electrophoresis. Biosens. Bioelectron. 22: 1974-1981. DOI: 10.1016/j.bios.2006.08.026

2558. Ou JJ, Li X, Feng S, Dong J, Dong XL, Kong L, Ye ML, Zou HF. 2007. Preparation and Evaluation of a Molecularly Imprinted Polymer Derivatized Silica Monolithic Column for Capillary Electrochromatography and Capillary Liquid Chromatography. Anal. Chem. 79: 639-646. DOI: 10.1021/ac061475x

2559. Ou JJ, Dong J, Tian TJ, Hu JW, Ye ML, Zou HF. 2007. Enantioseparation of tetrahydropalmatine and Tröger's base by molecularly imprinted monolith in capillary electrochromatography. J. Biochem. Biophys. Meth. 70: 71-76. DOI: 10.1016/j.jbbm.2006.07.003

2560. Zhang YP, Zuo GQ, Gong WJ, Deng YE, Li QM. 2007. Novel preparation of monolithic imprinted columns for electrochromatographic separation by photopolymerization. Chin. Chem. Lett. 18: 734-737. DOI: 10.1016/j.cclet.2007.04.029

2561. Zheng C, Liu ZS, Gao RY, Zhang LH, Zhang YK. 2007. Recognition of oxytocin by capillary electrochromatography with monolithic tetrapeptideimprinted polymer used as the stationary phase. Anal. Bioanal. Chem. 388: 1137-1145. DOI: 10.1007/s00216-007-1325-9

2562. Cacho C, Schweitz L, Turiel E, Pérez-Conde C. 2008. Molecularly imprinted capillary electrochromatography for selective determination of thiabendazole in citrus samples. J. Chromatogr. A 1179: 216-223. DOI: 10.1016/j.chroma.2007.11.097 
2563. Lara FJ, Lynen F, Sandra P, García-Campaña AM, Alés-Barrero F. 2008. Evaluation of a molecularly imprinted polymer as in-line concentrator in capillary electrophoresis. Electrophoresis 29: 3834-3841. DOI: 10.1002/elps.200700889

2564. Priego-Capote F, Ye L, Shakil S, Shamsi SA, Nilsson S. 2008. Monoclonal Behavior of Molecularly Imprinted Polymer Nanoparticles in Capillary Electrochromatography. Anal. Chem. 80: 2881-2887. DOI: $10.1021 / \mathrm{ac} 070038 \mathrm{v}$

2565. Wang HF, Zhu YZ, Lin JP, Yan XP. 2008. Fabrication of molecularly imprinted hybrid monoliths via a room temperature ionic liquid-mediated nonhydrolytic sol-gel route for chiral separation of zolmitriptan by capillary electrochromatography. Electrophoresis 29: 952-959. DOI: 10.1002/elps.200700402

2566. Zheng C, Liu ZS, Gao RY, Zhang YK. 2008. Mechanism of Molecular Recognition on Tetrapetide-imprinted Monolith by Capillary Electrochromatography. Chin. J. Chem. 26: 1857-1862. DOI: $10.1002 /$ cjoc.200890335

2567. Li M, Lin XC, Xie ZH. 2009. Investigation of enantiomer recognition of molecularly imprinted polymeric monoliths in pressurized capillary electrochromatography screening the amino acids and their derivatives. $J$. Chromatogr. A 1216: 5320-5326. DOI: 10.1016/j.chroma.2009.05.012

2568. Spégel P, Schweitz L, Andersson LI, Nilsson S. 2009. Novel Vinylpyridine Based Cationic MIP Monoliths for Enantiomer Separation in CEC. Chromatographia 69: 277-285. DOI: 10.1365/s10337-008-0911-0

2569. Zaidi SA, Han KM, Kim SS, Hwang DG, Cheong WJ. 2009. Open tubular layer of S-ofloxacin imprinted polymer fabricated in silica capillary for chiral CEC separation. J. Sep. Sci. 32: 996-1001. DOI: 10.1002/jssc.200800631 
2570. Zaidi SA, Cheong WJ. 2009. Long open tubular molecule imprinted polymer capillary columns with excellent separation efficiencies in chiral and nonchiral separation by capillary electrochromatography. Electrophoresis $\mathbf{3 0}$ : 1603-1607. DOI: 10.1002/elps.200800541

2571. DeMaleki Z, Lai EPC, Dabek-Zlotorzynska E. 2010. Capillary electrophoresis characterization of molecularly imprinted polymer particles in fast binding with $17 \alpha$-estradiol. J. Sep. Sci. 33: 2796-2803. DOI: $10.1002 / j s s c .201000257$

2572. He JX, Fang GZ, Yao YC, Wang S. 2010. Preparation and characterization of molecularly imprinted silica monolith for screening sulfamethazine. J. Sep. Sci. 33: 3263-3271. DOI: 10.1002/jssc.200900650

2573. Liao SL, Wang XC, Lin XC, Wu XP, Xie Z. 2010. A molecularly imprinted monolith for the fast chiral separation of antiparasitic drugs by pressurized CEC. J. Sep. Sci. 33: 2123-2130. DOI: 10.1002/jssc.200900855

2574. Liao SL, Wang XC, Lin XC, Xie ZH. 2010. Preparation and characterization of a molecularly imprinted monolithic column for pressure-assisted CEC separation of nitroimidazole drugs. Electrophoresis 31: 2822-2830. DOI: 10.1002/elps.201000035

2575. Wu X, Wei ZH, Huang YP, Liu ZS. 2010. Preparation of Molecularly Imprinted Coatings with Ternary Porogen for CEC. Chromatographia 72: 101-109. DOI: 10.1365/s10337-010-1627-5

2576. Zaidi SA, Lee SM, Lee JY, Cheong WJ. 2010. Comparison of enantioselective CEC separation of OT-MIP capillary columns with templates of various camphor derivatives made by the pre-established general preparation protocol. Bull. Kor. Chem. Soc. 31: 2934-2938.

2577. Zaidi SA, Han KM, Hwang DG, Cheong WJ. 2010. Preparation of open tubular molecule imprinted polymer capillary columns with various templates 
by a generalized procedure and their chiral and non-chiral separation performance in CEC. Electrophoresis 31: 1019-1028. DOI: 10.1002/elps.200900457

2578. Zhang SW, Zou CJ, Luo N, Weng QF, Cai LS, Wu CY, Xing J. 2010. Determination of urinary 8-hydroxy-2'-deoxyguanosine by capillary electrophoresis with molecularly imprinted monolith in-tube solid phase microextraction. Chin. Chem. Lett. 21: 85-88. DOI: 10.1016/j.cclet.2009.08.010

2579. Huang BY, Chen YC, Liu CY. 2011. An insight into the mechanism of CEC separation of template analogues on a norepinephrine-imprinted monolith. $J$. Sep. Sci. 34: 2293-2300. DOI: 10.1002/jssc.201000918

2580. Huang BY, Chen YC, Wang GR, Liu CY. 2011. Preparation and evaluation of a monolithic molecularly imprinted polymer for the chiral separation of neurotransmitters and their analogues by capillary electrochromatography. J. Chromatogr. A 1218: 849-855. DOI: 10.1016/j.chroma.2010.12.054

2581. Iqbal Z, Alsudir S, Miah M, Lai EPC. 2011. Rapid CE-UV binding tests of environmentally hazardous compounds with polymer-modified magnetic nanoparticles. Electrophoresis 32: 2181-2187. DOI: 10.1002/elps.201100106

2582. Jang R, Kim KH, Zaidi SA, Cheong WJ, Moon MH. 2011. Analysis of phospholipids using an open-tubular capillary column with a monolithic layer of molecularly imprinted polymer in capillary electrochromatographyelectrospray ionization-tandem mass spectrometry. Electrophoresis 32: 2167-2173. DOI: 10.1002/elps.201100205

2583. Lombardo-Agüí M, Gámiz-Gracia L, Cruces-Blanco C, García-Campaña AM. 2011. Comparison of different sample treatments for the analysis of quinolones in milk by capillary-liquid chromatography with laser induced 
fluorescence detection. J. Chromatogr. A 1218: 4966-4971. DOI: 10.1016/j.chroma.2011.01.007

2584. Qu P, Lei JP, Sheng J, Zhang L, Ju HX. 2011. Simultaneous multiple enantioseparation with a one-pot imprinted microfluidic channel by microchip capillary electrochromatography. Analyst 136: 920-926. DOI: 10.1039/COAN00559B

2585. Shi XX, Xu L, Duan HQ, Huang YP, Liu ZS. 2011. CEC separation of ofloxacin enantiomers using imprinted microparticles prepared in molecular crowding conditions. Electrophoresis 32: 1348-1356. DOI: 10.1002/elps.201000515

2586. Wei ZH, Wu X, Zhang B, Li R, Huang YP, Liu ZS. 2011. Coatings of one monomer molecularly imprinted polymers for open tubular capillary electrochromatography. J. Chromatogr. A 1218: 6498-6504. DOI: 10.1016/j.chroma.2011.07.012

2587. Zaidi SA, Lee SM, AL Othman ZA, AL Majid AM, Cheong WJ. 2011. Examination of Template Structural Effects on CEC Chiral Separation Performance of Molecule Imprinted Polymers Made by a Generalized Preparation Protocol. Chromatographia 73: 517-525. DOI: 10.1007/s10337011-1934-5

2588. Zaidi SA, Lee SM, Cheong WJ. 2011. Open tubular capillary columns with basic templates made by the generalized preparation protocol in capillary electrochromatography chiral separation and template structural effects on chiral separation capability. J. Chromatogr. A 1218: 1291-1299. DOI: 10.1016/j.chroma.2010.12.117

2589. Klabunovskii E, Balandin AA, Godunova LF. 1961. Chromatographic isolation of menthol. Izv. Akad. Nauk SSSR, Otd. Khim. Nauk 2243-2244. 
2590. Asanuma H, Hishiya T, Komiyama M. 2004. Efficient separation of hydrophobic molecules by molecularly imprinted cyclodextrin polymers. $J$. Inclusion Phenom. Macrocyl. Chem. 50: 51-55. DOI: 10.1007/s10847-0038838-4

2591. Buszewski B, Welerowicz T. 2004. Stationary phases with special structural properties for high-throughput separation techniques: Preparation, characterization and applications. Comb. Chem. High Throughput Screening 7: 291-312.

2592. Fairhurst RE, Chassaing C, Venn RF, Mayes AG. 2004. A direct comparison of the performance of ground, beaded and silica-grafted MIPs in HPLC and turbulent flow chromatography applications. Biosens. Bioelectron. 20: 10981105. DOI: 10.1016/j.bios.2004.01.020

2593. Haginaka J, Kagawa C. 2004. Retentivity and enantioselectivity of uniformly sized molecularly imprinted polymers for $d$-chlorpheniramine and brompheniramine in hydro-organic mobile phases. J. Chromatogr. B 804: 19-24. DOI: 10.1016/j.jchromb.2003.12.037

2594. Hosoya K, Watabe Y, Ikegami T, Tanaka N, Kubo T, Sano T, Kaya K. 2004. A molecular recognition strategy towards tetra-chlorinated dibenzo- $p$ dioxins, TCDDs. Biosens. Bioelectron. 20: 1185-1189. DOI: 10.1016/j.bios.2003.11.032

2595. Huang XD, Qin F, Chen XM, Liu YQ, Zou HF. 2004. Short columns with molecularly imprinted monolithic stationary phases for rapid separation of diastereomers and enantiomers. J. Chromatogr. B 804: 13-18. DOI: 10.1016/j.jchromb.2004.01.015

2596. Hwang CC. 2004. Separation of the enantiomers of mandelic acid by liquid chromatography using molecularly imprinted polymer as the stationary phase. Asian Pacific Confederation of Chemical Engineering congress 
program and abstracts, Vol. 2004, The Society of Chemical Engineers, Japan: 172-181.

2597. Ikegami T, Lee WS, Nariai H, Takeuchi T. 2004. Covalent molecular imprinting of bisphenol $A$ using its diesters followed by the reductive cleavage with $\mathrm{LiAlH}_{4}$. J. Chromatogr. $B$ 804: 197-201. DOI: 10.1016/j.jchromb.2003.12.031

2598. Kubo H, Player TN, Shinoda S, Tsukube H, Nariai H, Takeuchi T. 2004. Chiral recognition of octadentate $\mathrm{Na}^{+}$complex with tetra-armed cyclen by molecularly imprinted polymers. Anal. Chim. Acta 504: 137-140. DOI: 10.1016/S0003-2670(03)00952-8

2599. Kubo T, Tanaka N, Hosoya K. 2004. Target-selective ion-exchange media for highly hydrophilic compounds: a possible solution by use of the "interval immobilization technique". Anal. Bioanal. Chem. 378: 84-88. DOI: $10.1007 / \mathrm{s} 00216-003-2320-4$

2600. Kubo T, Hosoya K, Watabe Y, Tanaka N, Takagi H, Sano T, Kaya K. 2004. Interval immobilization technique for recognition toward a highly hydrophilic cyanobacterium toxin. J. Chromatogr. $B$ 806: 229-235. DOI: 10.1016/j.jchromb.2004.03.064

2601. Kubo T, Hosoya K, Watabe Y, Tanaka N, Sano T, Kaya K. 2004. Toxicity recognition of hepatotoxin, homologues of microcystin with artificial trapping devices. J. Environ. Sci. Health, Part A 39: 2597-2614. DOI: 10.1081/ESE200027015

2602. Machtejevas E, Sellergren B, Martynaitis V, Owens PK, Maruska A. 2004. Screening of oxazepine indole enantiomers by means of high performance liquid chromatography with imprinted polymer stationary phase. J. Sep. Sci. 27: 547-551. DOI: 10.1002/jssc.200301675 
2603. Rachkov A, Hu MJ, Bulgarevich E, Matsumoto T, Minoura N. 2004. Molecularly imprinted polymers prepared in aqueous solution selective for [Sar(1),Ala(8)]angiotensin II. Anal. Chim. Acta 504: 191-197. DOI: 10.1016/S0003-2670(03)00764-5

2604. Shim YH, Yilmaz E, Solange LA, Haupt K. 2004. Chiral recognition and separation of $\beta^{2}$-amino acids using noncovalently molecularly imprinted polymers. Analyst 129: 1211-1215. DOI: 10.1039/b409836f

2605. Szabelski P. 2004. A simple model of enantioselective adsorption on chiral stationary phases. Appl. Surf. Sci. 227: 94-103. DOI: 10.1016/j.apsusc.2003.11.048

2606. Wang DX, Hong SP, Row KH. 2004. Chromatographic separation of xanthine derivatives on single and mixed-template imprinted polymers. Bull. Kor. Chem. Soc. 25: 357-360.

2607. Watabe Y, Kondo T, Morita M, Tanaka N, Haginaka J, Hosoya K. 2004. Determination of bisphenol $A$ in environmental water at ultra-low level by high-performance liquid chromatography with an effective on-line pretreatment device. J. Chromatogr. A 1032: 45-49. DOI: 10.1016/j.chroma.2003.11.079

2608. Watabe Y, Kondo T, Imai H, Morita M, Tanaka N, Hosoya K. 2004. Reducing bisphenol A contamination from analytical procedures to determine ultralow levels in environmental samples using automated HPLC microanalysis. Anal. Chem. 76: 105-109. DOI: 10.1021/ac0301595

2609. Yang GL, Yin JF, Li ZW, Liu HY, Cai LP, Wang DX, Chen Y. 2004. Chiral separation of nateglinide and its $(L)$ enantiomer on a molecularly imprinted polymer-based stationary phase. Chromatographia 59: 705-708. DOI: 10.1365/s10337-004-0276-y 
2610. Yang ML, Li YZ. 2004. Molecularly imprinted polymers with $p$ acetaminophenol and its positional isomers as templates. Anal. Lett. 37: 2043-2052. DOI: 10.1081/AL-200026668

2611. Zheng N, Li YZ, Wen MJ. 2004. Sulfamethoxazole-imprinted polymer for selective determination of sulfamethoxazole in tablets. J. Chromatogr. A 1033: 179-182. DOI: 10.1016/j.chroma.2003.10.010

2612. Baggiani C, Baravalle P, Anfossi L, Tozzi C. 2005. Comparison of pyrimethanil-imprinted beads and bulk polymer as stationary phase by nonlinear chromatography. Anal. Chim. Acta 542: 125-134. DOI: 10.1016/j.aca.2004.10.088

2613. Deng QL, Lun ZH, Yan C, Gao RY. 2005. Preparation and evaluation of molecularly imprinted monolithic column for felodipine in micro-liquid chromatography. Chin. Chem. Lett. 16: 955-958.

2614. Guo TY, Xia YQ, Wang J, Song MD, Zhang BH. 2005. Chitosan beads as molecularly imprinted polymer matrix for selective separation of proteins. Biomaterials 26: 5737-5745. DOI: 10.1016/j.biomaterials.2005.02.017

2615. Hosoya K, Aoki H, Kubo T, Teramachi M, Tanaka N, Haginaka J. 2005. Selective retention of some polyaromatic hydrocarbons by highly crosslinked polymer networks. J. Polym. Sci. A,Polym. Chem. 43: 2556-2566. DOI: 10.1002/pola.20730

2616. Huang JT, Zhang J, Zhang JQ, Zheng SH. 2005. Template imprinting amphoteric polymer for the recognition of proteins. J. Appl. Polym. Sci. 95: 358-361. DOI: 10.1002/app.21262

2617. Hung $\mathrm{CY}$, Hwang CC, Huang HH. 2005. Chiral separations of mandelic acid by HPLC using molecularly imprinted polymers. Ecléctica Química 30: 6773. DOI: 10.1590/S0100-46702005000400009 
2618. Kim H, Guiochon G. 2005. Comparison of the thermodynamic properties of particulate and monolithic columns of molecularly imprinted copolymers. Anal. Chem. 77: 93-102. DOI: 10.1021/ac0401218

2619. Kim K, Kim D. 2005. High-performance liquid chromatography separation characteristics of molecular-imprinted poly(methacrylic acid) microparticles prepared by suspension polymerization. J. Appl. Polym. Sci. 96: 200-212. DOI: 10.1002/app.21419

2620. Kubo T, Hosoya K, Sano T, Nomachi M, Tanaka N, Kaya K. 2005. Selective separation of brominated bisphenol A homologues using a polymer-based medium prepared by the fragment imprinting technique. Anal. Chim. Acta 549: 45-50. DOI: 10.1016/j.aca.2005.06.015

2621. Li H, Liu YJ, Zhang ZH, Liao HP, Nie LH, Yao SZ. 2005. Separation and purification of chlorogenic acid by molecularly imprinted polymer monolithic stationary phase. J. Chromatogr. A 1098: 66-74. DOI: 10.1016/j.chroma.2005.08.046

2622. Li ZW, Yang GL, Liu SB, Chen Y. 2005. Adsorption isotherms on nicotinamide-imprinted polymer stationary phase. J. Chromatogr. Sci. 43: 362-366.

2623. Liu HY, Row KH, Yan GL. 2005. Monolithic molecularly imprinted columns for chromatographic separation. Chromatographia 61: 429-432. DOI: 10.1365/s10337-005-0531-x

2624. Liu Y, Ding GS, Wang JD. 2005. Recognition interactions of metalcomplexing imprinted polymer. Chin. Chem. Lett. 16: 797-800.

2625. Ogawa T, Hoshina K, Haginaka J, Honda C, Moto TT, Uchida T. 2005. Screening of bitterness-suppressing agents for quinine: The use of molecularly imprinted polymers. J. Pharm. Sci. 94: 353-362. DOI: 10.1002/jps.20248 
2626. Ou JJ, Tang SW, Zou HF. 2005. Chiral separation of 1,1'-bi-2-naphthol and its analogue on molecular imprinting monolithic columns by HPLC. J. Sep. Sci. 28: 2282-2287. DOI: 10.1002/jssc.200500165

2627. Piletska EV, Romero-Guerra M, Chianella I, Karim K, Turner APF, Piletsky SA. 2005. Towards the development of multisensor for drugs of abuse based on molecular imprinted polymers. Anal. Chim. Acta 542: 111-117. DOI: 10.1016/j.aca.2005.03.067

2628. Robak AJ, Branchaud BP. 2005. First example of a molecularly imprinted polymer incorporating a difunctionalized alloxazine flavin isomer. Tetrahedron Lett. 46: 5651-5654. DOI: 10.1016/j.tetlet.2005.06.102

2629. Sambe H, Hoshina K, Hosoya K, Haginaka J. 2005. Direct injection analysis of bisphenol $A$ in serum by combination of isotope imprinting with liquid chromatography-mass spectrometry. Analyst 130: 38-40. DOI: 10.1039/b412641f

2630. Sambe H, Hoshina K, Haginaka J. 2005. Retentivity and enantioselectivity of uniformly-sized molecularly imprinted polymers for (S)-nilvadipine in aqueous and non-aqueous mobile phases. Anal. Sci. 21: 391-395. DOI: 10.2116/analsci.21.391

2631. Simon R, Houck S, Spivak DA. 2005. Comparison of particle size and flow rate optimization for chromatography using one-monomer molecularly imprinted polymers versus traditional non-covalent molecularly imprinted polymers. Anal. Chim. Acta 542: 104-110. DOI: 10.1016/j.aca.2005.03.048

2632. Tamayo FG, Martin-Esteban A. 2005. Selective high performance liquid chromatography imprinted-stationary phases for the screening of phenylurea herbicides in vegetable samples. J. Chromatogr. A 1098: 116-122. DOI: 10.1016/j.chroma.2005.08.068 
2633. Tamayo FG, Titirici MM, Martin-Esteban A, Sellergren B. 2005. Synthesis and evaluation of new propazine-imprinted polymer formats for use as stationary phases in liquid chromatography. Anal. Chim. Acta 542: 38-46. DOI: 10.1016/j.aca.2004.12.063

2634. Tóth B, László K, Horvai G. 2005. Chromatographic behavior of silicapolymer composite molecularly imprinted materials. J. Chromatogr. A 1100: 60-67. DOI: 10.1016/j.chroma.2005.09.015

2635. Turiel E, Tadeo JL, Cormack PAG, Martin-Esteban A. 2005. HPLC imprinted-stationary phase prepared by precipitation polymerisation for the determination of thiabendazole in fruit. Analyst 130: 1601-1607. DOI: 10.1039/b511031a

2636. Varilova T. 2005. Stationary phases for affinity chromatography. Chem. Listy 99: 570-577.

2637. Yin JF, Yang GL, Chen Y. 2005. Rapid and efficient chiral separation of nateglinide and its I-enantiomer on monolithic molecularly imprinted polymers. J. Chromatogr. A 1090: 68-75. DOI: 10.1016/j.chroma.2005.08.020

2638. Zhang J, He L, Fu Q. 2005. Chromatographic features and molecular recognition mechanism of a strychnine monolithic molecularly imprinted polymer. Chromatographia 62: 319-323. DOI: 10.1365/s10337-005-0614-8

2639. Hantash J, Bartlett $A$, Oldfield $P$, Dénès $G$, O'Rielly R, Roudiere $D$, Menduni S. 2006. Use of an on-line imprinted polymer pre-column, for the liquid chromatographic-UV absorbance determination of carbaryl and its metabolite in complex matrices. J. Chromatogr. A 1125: 104-111. DOI: 10.1016/j.chroma.2006.05.054 
2640. Hung CY, Huang YT, Huang HH, Hwang CC. 2006. HPLC separation of ibuprofen and mefenamic acid using molecularly imprinted polymer as stationary phase. E-Polymers Art. No. 065.

2641. Hung CY, Huang YT, Huang HH, Hwang CC. 2006. Synthesis and molecular recognition of molecularly imprinted polymer with ibuprofen as template. J. Chin. Chem. Soc. 53: 1173-1180.

2642. Ou JJ, Kong L, Pan CS, Su XY, Lei XY, Zou HF. 2006. Determination of dltetrahydropalmatine in Corydalis yanhusuo by I-tetrahydropalmatine imprinted monolithic column coupling with reversed-phase high performance liquid chromatography. J. Chromatogr. A 1117: 163-169. DOI: 10.1016/j.chroma.2006.03.084

2643. Sambe H, Hoshina K, Hosoya K, Haginaka J. 2006. Simultaneous determination of bisphenol $A$ and its halogenated derivatives in river water by combination of isotope imprinting and liquid chromatography-mass spectrometry. J. Chromatogr. A 1134: 16-23. DOI: 10.1016/j.chroma.2006.08.072

2644. Sun HW, Qiao FX, Liu GY. 2006. Characteristic of theophylline imprinted monolithic column and its application for determination of xanthine derivatives caffeine and theophylline in green tea. J. Chromatogr. A 1134: 194-200. DOI: 10.1016/j.chroma.2006.09.004

2645. Wang XD, Yao JS, Wei MX, Wu G, Luo C. 2006. Enantioselection and development in chiral stationary phase based on amino acid. Chin. J. Org. Chem. 26: 912-921.

2646. Xia YQ, Guo TY, Song MD, Zhang BH, Zhang BL. 2006. Selective separation of quercetin by molecular imprinting using chitosan beads as functional matrix. Reac. Func. Polym. 66: 1734-1740. DOI: 10.1016/j.reactfunctpolym.2006.08.001 
2647. Yamamoto C, Yamada K, Motoya K, Kamiya Y, Kamigaito M, Okamoto Y, Aratani T. 2006. Preparation of HPLC chiral packing materials using cellulose tris(4-methylbenzoate) for the separation of chrysanthemate isomers. J. Polym. Sci. A,Polym. Chem. 44: 5087-5097. DOI: 10.1002/pola. 21620

2648. Yan H, Row KH. 2006. Molecularly imprinted monolithic stationary phases for liquid chromatographic separation of tryptophan and N-CBZphenylalanine enantiomers. Biotechnol. Bioprocess Eng. 11: 357-363. DOI: 10.1007/BF03026253

2649. Baggiani C, Anfossi L, Baravalle P, Giovannoli C, Giraudi G. 2007. Molecular recognition of polycyclic aromatic hydrocarbons by pyreneimprinted microspheres. Anal. Bioanal. Chem. 389: 413-422. DOI: 10.1007/s00216-007-1318-8

2650. Haginaka J, Tabo H, Ichitani M, Takihara T, Sugimoto A, Sambe H. 2007. Uniformly-sized, molecularly imprinted polymers for (-)-epigallocatechin gallate, -epicatechin gallate and -gallocatechin gallate by multi-step swelling and polymerization method. J. Chromatogr. A 1156: 45-50. DOI: 10.1016/j.chroma.2006.10.026

2651. Hung CY, Hwang CC. 2007. HPLC behavior of sulfonamides oil molecularly imprinted polymeric stationary phases. Acta Chromatogr. 18: 106-115.

2652. Hung CY, Huang YT, Huang HH, Hwang CC. 2007. Sulfamethazine and sulfadimethoxine separation strategies based on molecularly imprinted adsorbents. Anal. Lett. 40: 3232-3244. DOI: 10.1080/00032710701672707

2653. Kubo T, Matsumoto H, Shiraishi F, Nomachi M, Nemoto K, Hosoya K, Kaya K. 2007. Selective separation of hydroxy polychlorinated biphenyls (HOPCBs) by the structural recognition on the molecularly imprinted polymers: Direct separation of the thyroid hormone active analogues from mixtures. Anal. Chim. Acta 589: 180-185. DOI: 10.1016/j.aca.2007.02.070 
2654. Lai JP, Yang ML, Niessner R, Knopp D. 2007. Molecularly imprinted microspheres and nanospheres for di(2-ethylhexyl)phthalate prepared by precipitation polymerization. Anal. Bioanal. Chem. 389: 405-412. DOI: 10.1007/s00216-007-1321-0

2655. Legido-Quigley C, Oxelbark J, De Lorenzi E, Zurutuza-Elorza A, Cormack PAG. 2007. Chromatographic characterisation, under highly aqueous conditions, of a molecularly imprinted polymer binding the herbicide 2,4dichlorophenoxyacetic acid. Anal. Chim. Acta 591: 22-28. DOI: 10.1016/j.aca.2007.01.022

2656. Li ZY, Liu ZS, Zhang QW, Duan HQ. 2007. Chiral separation by (S)naproxen imprinted monolithic column with mixed functional monomers. Chin. Chem. Lett. 18: 322-324. DOI: 10.1016/j.cclet.2007.01.037

2657. Lin YC, Pan HH, Hwang CC, Lee WC. 2007. Side chain functionality dominated the chromatography of $\mathrm{N}$-protected amino acid on molecularly imprinted polymer. J. Appl. Polym. Sci. 105: 3519-3524. DOI: 10.1002/app.26621

2658. Luo Y, Liu L, Li LH, Deng QY. 2007. Chiral Resolution of Racemic 4Phenyl(benzyl)-2-Oxazolidone by Use of Molecularly Imprinted Polymers. Chromatographia 65: 675-679. DOI: 10.1365/s10337-007-0209-7

2659. Matsui J, Goji S, Murashima T, Miyoshi D, Komai S, Shigeyasu A, Kushida T, Miyazawa T, Yamada T, Tamaki K, Sugimoto N. 2007. Molecular Imprinting under Molecular Crowding Conditions: An Aid to the Synthesis of a High-Capacity Polymeric Sorbent for Triazine Herbicides. Anal. Chem. 79: 1749-1757. DOI: 10.1021/ac060441m

2660. Oxelbark J, Legido-Quigley C, Aureliano CSA, Titirici MM, Schillinger E, Sellergren B, Courtois J, Irgum K, Dambies L, Cormack PAG, Sherrington DC, De Lorenzi E. 2007. Chromatographic comparison of bupivacaine imprinted polymers prepared in crushed monolith, microsphere, silica-based 
composite and capillary monolith formats. J. Chromatogr. A 1160: 215-226. DOI: 10.1016/j.chroma.2007.05.057

2661. Seebach A, Seidel-Morgenstern A. 2007. Enantioseparation on molecularly imprinted monoliths--Preparation and adsorption isotherms. Anal. Chim. Acta 591: 57-62. DOI: 10.1016/j.aca.2007.02.059

2662. Shi XZ, Wu AB, Qu GR, Li RX, Zhang DB. 2007. Development and characterisation of molecularly imprinted polymers based on methacrylic acid for selective recognition of drugs. Biomaterials 28: 3741-3749. DOI: 10.1016/j.biomaterials.2007.04.036

2663. Shi XZ, Wu AB, Qu GR, Li RX, Zhang DB. 2007. Corrigendum to "Development and characterisation of molecularly imprinted polymers based on methacrylic acid for selective recognition of drugs": [Biomaterials 28 (2007) 3741-3749]. Biomaterials 28: 4330. DOI: 10.1016/j.biomaterials.2007.06.021

2664. Sirc J, Bosakova Z, Coufal P, Michalek J, Pradny M, Hobzova R, Hradil J. 2007. Morphological and chromatographic characterization of molecularly imprinted monolithic columns. E-Polymers Art. No. 117.

2665. Song SH, Shirasaka K, Katayama M, Nagaoka S, Yoshihara S, Osawa T, Sumaoka J, Asanuma H, Komiyama M. 2007. Recognition of Solution Structures of Peptides by Molecularly Imprinted Cyclodextrin Polymers. Macromolecules 40: 3530-3532. DOI: 10.1021/ma070348f

2666. Wei XL, Husson SM. 2007. Surface-Grafted, Molecularly Imprinted Polymers Grown from Silica Gel for Chromatographic Separations. Ind. Eng. Chem. Res. 46: 2117-2124. DOI: 10.1021/ie0606284

2667. Wu LQ, Gao YH, Wang J. 2007. Synthesis, application, and molecular recognition mechanism study of phenylalanine molecularly imprinted polymer. Anal. Lett. 40: 3129-3147. DOI: 10.1080/00032710701603835 
2668. Yan H, Row KH. 2007. Characteristics of a monolithic molecularly imprinted column and its application for chromatographic separation. J. Ind. Eng. Chem. 13: 552-557.

2669. Zhang HT, Song T, Zhang W, Hua W, Pan CP. 2007. Retention behavior of phenoxyacetic herbicides on a molecularly imprinted polymer with phenoxyacetic acid as a dummy template molecule. Bioorg. Med. Chem. 15: 6089-6095. DOI: 10.1016/j.bmc.2007.06.039

2670. Zhang RW, Wei ST, Mizaikoff B. 2007. Selective recognition of bile acids by molecular imprints. Vol. 1-3, IEEE: 1048-1051. DOI: 10.1109/ICSENS.2007.4388585

2671. Zhu QJ, Tang J, Dai J, Gu XH, Chen SW. 2007. Synthesis and characteristics of imprinted 17- $\alpha$-estradiol microparticle and nanoparticle with TFMAA as functional monomer. J. Appl. Polym. Sci. 104: 1551-1558. DOI: 10.1002/app.25564

2672. Alexiadou DK, Maragou NC, Thomaidis NS, Theodoridis GA, Koupparis MA. 2008. Molecularly imprinted polymers for bisphenol A for HPLC and SPE from water and milk. J. Sep. Sci. 31: 2272-2282. DOI: $10.1002 /$ jssc. 200700643

2673. Haginaka J, Futagami A. 2008. Addition of N-carbobenzyloxy-I-tryptophan as a co-template molecule to molecularly imprinted polymer monoliths for (+)-nilvadipine. J. Chromatogr. A 1185: 258-262. DOI: 10.1016/j.chroma.2008.01.059

2674. Hung CY, Huang YT, Hwang CC. 2008. Molecular recognition and identification of sulfapyridine and sulfadimethoxine by high performance liquid chromatography with molecularly imprinted polymer as the stationary phase. J. Food Drug Anal. 16: 8-14. 
2675. Hung CY, Hwang CC. 2008. Analysis of ketoprofen and mefenamic acid by high-performance liquid chromatography with molecularly imprinted polymer as the stationary phase. J. Chromatogr. Sci. 46: 813-818.

2676. Khan H, Khan T, Park JK. 2008. Separation of phenylalanine racemates using d-phenylalanine imprinted microbeads as HPLC stationary phase. Sep. Purif. Technol. 62: 363-369. DOI: 10.1016/j.seppur.2008.02.011

2677. Lee WC, Hung CY, Hwang CC. 2008. Separation of cholesterol from other steroids using molecularly imprinted Polymer prepared by seeded suspension polymerization. Chem. Biochem. Eng. Q. 22: 151-156.

2678. Liu HN, Zhuang XL, Turson M, Zhang M, Dong XC. 2008. Enrofloxacinimprinted monolithic columns synthesized using reversible additionfragmentation chain transfer polymerization. J. Sep. Sci. 31: 1694-1701. DOI: $10.1002 /$ jssc.200700602

2679. Maciuk A, Moaddel R, Haginaka J, Wainer IW. 2008. Screening of tobacco smoke condensate for nicotinic acetylcholine receptor ligands using cellular membrane affinity chromatography columns and missing peak chromatography. J. Pharm. Biomed. Anal. 48: 238-246. DOI: 10.1016/j.jpba.2007.11.024

2680. Nagaoka S, Song S, Sumaoka J, Komiyama M. 2008. Reconfirmation of Recognition Site in Composites of Imprinted $\beta$-Cyclodextrin Polymer/Solid Support as Stationary Phase of HPLC. Chem. Lett. 37: 1148-1149. DOI: $10.1246 / \mathrm{cl} .2008 .1148$

2681. Qin L, He XW, Li WY, Zhang YK. 2008. Molecularly imprinted polymer prepared with bonded $\alpha$-cyclodextrin and acrylamide on functionalized silica gel for selective recognition of tryptophan in aqueous media. J. Chromatogr. A 1187: 94-102. DOI: 10.1016/j.chroma.2008.02.004 
2682. Su SF, Zhang M, Li BL, Zhang HY, Dong XC. 2008. HPLC determination of sulfamethazine in milk using surface-imprinted silica synthesized with iniferter technique. Talanta 76: 1141-1146. DOI: 10.1016/j.talanta.2008.05.015

2683. Xu ZY, Wan JF, Liang S, Cao.X.J. 2008. Separation of ursodeoxycholic acid from its isomeric mixture using core-shell molecular imprinting polymer. Biochem. Eng. J. 41: 280-287. DOI: 10.1016/j.bej.2008.05.019

2684. Zaidi SA, Cheong WJ. 2008. Robust open tubular layer of S-ketoprofen imprinted polymer for chiral LC separation. J. Sep. Sci. 31: 2962-2970. DOI: $10.1002 /$ jssc.200800160

2685. Zhuang XL, Dong XC, Ma SJ, Zhang T. 2008. Selective on-line extraction of trans-resveratrol and emodin from Polygonum cuspidatum using molecularly imprinted polymer. J. Chromatogr. Sci. 46: 739-742.

2686. Cheng TC, Huang YT, Chang CY, Yao KS, Hwang CC. 2009. Molecular Recognition of Sulfaquinoxaline and Sulfapyridine with Molecularly Imprinted Polymer. J. Chil. Chem. Soc. 54: 295-298. DOI: 10.4067/S071797072009000300019

2687. Duy SV, Lefebvre-Tournier I, Pichon V, Hugon-Chapuis F, Puy JY, Périgaud C. 2009. Molecularly imprinted polymer for analysis of zidovudine and stavudine in human serum by liquid chromatography-mass spectrometry. $\mathrm{J}$. Chromatogr B 877: 1101-1108. DOI: 10.1016/j.jchromb.2009.02.068

2688. Jiang M, Shi Y, Zhang RL, Shi CH, Peng Y, Huang Z, Lu B. 2009. Selective molecularly imprinted stationary phases for Bisphenol $A$ analysis prepared by modified precipitation polymerization. J. Sep. Sci. 32: 3265-3273A. DOI: 10.1002/jssc.200900207

2689. Jing $T$, Gao XD, Wang $P$, Wang $Y$, Lin YF, Hu XZ, Hao QL, Zhou YK, Mei SR. 2009. Determination of trace tetracycline antibiotics in foodstuffs by 
liquid chromatography-tandem mass spectrometry coupled with selective molecular-imprinted solid-phase extraction. Anal. Bioanal. Chem. 393: 20092018. DOI: 10.1007/s00216-009-2641-z

2690. Lee SC, Peng CL, Chen H. 2009. Studies on the Preparation and Properties of Sol-Gel Molecularly Imprinted Polymer Based on Tetramethoxysilane and Methyltrimethoxysilane for Recognized Sulfonamides. Polym. J. 41: 10921097. DOI: 10.1295/polymj.PJ2009147

2691. Lee TT, Huang IB, Hwang CC. 2009. Analysis of Sulfachloropyridazine and Sulfapyridine by High-Performance Liquid Chromatography with Molecularly Imprinted Polymer As the Stationary Phase. Biomed. Eng. Appl. Basis Commun. 21: 457-460. DOI: 10.4015/S1016237209001647

2692. Li H, Nie LH, Li YN, Zhang ZH, Shi H, Hu WB, Zhang YK. 2009. Application of Molecularly Imprinted Column for Separation and Purification of Bioactive Compound from Cirsium segetum Bunge. Sep. Sci. Technol. 44: 370-385. DOI: $10.1080 / 01496390802437370$

2693. Lin Z, Yang F, He XW, Zhao XM, Zhang YK. 2009. Preparation and evaluation of a macroporous molecularly imprinted hybrid silica monolithic column for recognition of proteins by high performance liquid chromatography. J. Chromatogr. A 1216: 8612-8622. DOI: 10.1016/j.chroma.2009.10.025

2694. Matsui J, Sodeyama T, Saiki Y, Miyazawa T, Yamada T, Tamaki K, Murashima T. 2009. Face-to-face porphyrin moieties assembled with spacing for pyrazine recognition in molecularly imprinted polymers. Biosens. Bioelectron. 25: 635-639. DOI: 10.1016/j.bios.2009.01.047

2695. Pradhan S, Boopathi M, Kumar O, Baghel A, Pandey P, Mahato TH, Singh B, Vijayaraghavan R. 2009. Molecularly imprinted nanopatterns for the recognition of biological warfare agent ricin. Biosens. Bioelectron. 25: 592598. DOI: 10.1016/j.bios.2009.03.041 
2696. Qin L, He XW, Zhang W, Li WY, Zhang YK. 2009. Surface-modified polystyrene beads as photografting imprinted polymer matrix for chromatographic separation of proteins. J. Chromatogr. A 1216: 807-814. DOI: 10.1016/j.chroma.2008.12.007

2697. Schirmer C, Meisel H. 2009. Synthesis and Evaluation of Molecularly Imprinted Polymers (MIP) with Affinity for the Polypeptide Nisin. Food Anal. Meth. 2: 257-263. DOI: 10.1007/s12161-008-9061-0

2698. Song XL, Wang JT, Zhu J. 2009. Effect of Porogenic Solvent on Selective Performance of Molecularly Imprinted Polymer for Quercetin. Mater. Res. Ibero-Am. J. Mater. 12: 299-304. DOI: 10.1590/S1516-14392009000300009

2699. Yilmaz E, Billing J, Boyd B, Möller P, Rees A. 2009. Studies towards enantioselective surface imprinted polymers. J. Sep. Sci. 32: 3274-3277. DOI: $10.1002 /$ jssc.200900236

2700. Amut E, Fu QA, Fang Q, Liu R, Xiao AP, Zeng AG, Chang C. 2010. In situ polymerization preparation of chiral molecular imprinting polymers monolithic column for amlodipine and its recognition properties study. $J$. Polym. Res. 17: 401-409. DOI: 10.1007/s10965-009-9326-3

2701. da Silva MS, Vão ER, Temtem M, Mafra L, Caldeira J, Aguiar-Ricardo A, Casimiro T. 2010. Clean synthesis of molecular recognition polymeric materials with chiral sensing capability using supercritical fluid technology. Application as HPLC stationary phases. Biosens. Bioelectron. 25: 17421747. DOI: 10.1016/j.bios.2009.12.023

2702. Dong HX, Tong F, Li JQ, Wang ZX, Wang YH, Shen XD. 2010. Chiral resolution of racemic ( \pm )-1,1'-Bi (2-naphthol) by use of molecularly imprinted polymers. In Manufacturing Processes and Systems, Advanced Materials Research, Vol. 150-151, Liu XH, Jiang ZY, Han JT (eds). Scientific.net: 150159. DOI: 10.4028/www.scientific.net/AMR.150-151.150 
2703. Gallego-Gallegos M, Garrido ML, Olivas RM, Baravalle P, Baggiani C, Cámara C. 2010. A new application of imprinted polymers: Speciation of organotin compounds. J. Chromatogr. A 1217: 3400-3407. DOI: 10.1016/j.chroma.2010.03.007

2704. Kitahara KI, Yoshihama I, Hanada T, Kokuba H, Arai S. 2010. Synthesis of monodispersed molecularly imprinted polymer particles for highperformance liquid chromatographic separation of cholesterol using templating polymerization in porous silica gel bound with cholesterol molecules on its surface. J. Chromatogr. $A$ 1217: 7249-7254. DOI: 10.1016/j.chroma.2010.09.041

2705. Monier M, Ayad DM, Wei Y, Sarhan AA. 2010. Preparation of cross-linked chitosan/glyoxal molecularly imprinted resin for efficient chiral resolution of aspartic acid isomers. Biochem. Eng. J. 51: 140-146. DOI: 10.1016/j.bej.2010.06.007

2706. Monier M, El-Sokkary AMA. 2010. Preparation of molecularly imprinted cross-linked chitosan/glutaraldehyde resin for enantioselective separation of I-glutamic acid. Int. J. Biol. Macromol. 47: 207-213. DOI: 10.1016/j.ijbiomac.2010.04.020

2707. Qi PP, Wang JC, Wang LD, Li Y, Jin J, Su F, Tian YZ, Chen JP. 2010. Molecularly imprinted polymers synthesized via semi-covalent imprinting with sacrificial spacer for imprinting phenols. Polymer 51: 5417-5423. DOI: 10.1016/j.polymer.2010.09.037

2708. Qian K, Fang GZ, He JX, Pan MF, Wang S. 2010. Preparation and application of a molecularly imprinted polymer for the determination of trace metolcarb in food matrices by high performance liquid chromatography. $\mathrm{J}$. Sep. Sci. 33: 2079-2085. DOI: 10.1002/jssc.200900877

2709. Qu P, Lei JP, Zhang L, Ouyang RZ, Ju HX. 2010. Molecularly imprinted magnetic nanoparticles as tunable stationary phase located in microfluidic 
channel for enantioseparation. J. Chromatogr. A 1217: 6115-6121. DOI: 10.1016/j.chroma.2010.07.063

2710. Tom LA, Foster N. 2010. Development of a molecularly imprinted polymer for the analysis of avermectin. Anal. Chim. Acta 680: 79-85. DOI: 10.1016/j.aca.2010.09.015

2711. Yang ML, Chu XG. 2010. Molecularly Imprinted Polymers with Mandelic Acid as Template. Anal. Lett. 43: 2390-2398. DOI: $10.1080 / 00032711003717380$

2712. Ambrosini S, Serra M, Shinde S, Sellergren B, De Lorenzi E. 2011. Synthesis and chromatographic evaluation of molecularly imprinted polymers prepared by the substructure approach for the class-selective recognition of glucuronides. J. Chromatogr. A 1218: 6961-6969. DOI: 10.1016/j.chroma.2011.07.104

2713. Chen DM, Fu Q, Du W, Sun SJ, Huang P, Chang C. 2011. Preparation and evaluation of monolithic molecularly imprinted stationary phase for $\mathrm{S}$ naproxen. J. Pharmaceut. Anal. 1: 26-31.

2714. Gu JY, Zhang H, Yuan G, Chen LR, Xu XJ. 2011. Surface-initiated molecularly imprinted polymeric column: In situ synthesis and application for semi-preparative separation by high performance liquid chromatography. $\mathrm{J}$. Chromatogr. A 1218: 8150-8155. DOI: 10.1016/j.chroma.2011.09.019

2715. Guan W, Pan JM, Wang X, Hu W, Xu LC, Zou XH, Li CX. 2011. Selective recognition of 4-nitrophenol from aqueous solution by molecularly imprinted polymers with functionalized tetratitanate whisker composites as support. $J$. Sep. Sci. 34: 1244-1252. DOI: 10.1002/jssc.201100032

2716. Hsu JJ, Huang IB, Hwang CC, Wu MC. 2011. Rapid chromatographic separation of sulfa drugs by used molecularly imprinted polymer. Indian $\mathrm{J}$. Chem. Technol. 18: 7-12. 
2717. Lv YK, Zhao MG, Zhang D, Yan HY. 2011. Enrofloxacin-imprinted monolithic HPLC columns synthesized by in situ copolymerization for chromatographic separation. J. Liq. Chrom. Rel. Technol. 34: 705-718. DOI: 10.1080/10826076.2011.562592

2718. Su LQ, Wang Y, Chu HT. 2011. Chiral Separation of Amino Acid Derivatives by Molecular Imprinting Technique. In Advanced Materials, Advanced Materials Research, Vol. 239 - 242, Cao Z, Cao XQ, Sun LX, He YH (eds). Scientific.net: 2545-2548. DOI: 10.4028/www.scientific.net/AMR.239242.2545

2719. Valero-Navarro A, Gómez-Romero $M$, Fernández-Sánchez JF, Cormack PAG, Segura-Carretero A, Fernández-Gutiérrez A. 2011. Synthesis of caffeic acid molecularly imprinted polymer microspheres and highperformance liquid chromatography evaluation of their sorption properties. $J$. Chromatogr. A 1218: 7289-7296. DOI: 10.1016/j.chroma.2011.08.043

2720. Wang SS, Li DM, Hua ZD, Zhao MP. 2011. Molecularly imprinted monolith coupled on-line with high performance liquid chromatography for simultaneous quantitative determination of cyromazine and melamine. Analyst 136: 3672-3679.

2721. Lei JD, Tan TW. 2004. Enantioselective separation of racemic ketoprofen using molecular imprinting. Tong ZF, Kim SH (eds). World Scientific: 735740 .

2722. Kareuhanon W, Lee VS, Nimmanpipug P, Tayapiwatana C, Pattarawarapan M. 2009. Synthesis of Molecularly Imprinted Polymers for Nevirapine by Dummy Template Imprinting Approach. Chromatographia 70: 1531-1537. DOI: 10.1365/s10337-009-1385-4

2723. Aoki S, Jikiba A, Takeda K, Kimura E. 2004. A zinc(II) complex-conjugated polymer for selective recognition and separation of phosphates. J. Phys. Org. Chem. 17: 489-497. DOI: 10.1002/poc.773 
2724. Greene NT, Morgan SL, Shimizu KE. 2004. Molecularly imprinted polymer sensor arrays. Chem. Commun. 1172-1173. DOI: 10.1039/b401677g

2725. Kubo T, Hosoya K, Watabe Y, Tanaka N, Sano T, Kaya K. 2004. Recognition of hepatotoxic homologues of Microcystin using a combination of selective adsorption media. J. Sep. Sci. 27: 316-324. DOI: 10.1002/jssc.200301707

2726. Lavignac N, Allender CJ, Brain KR. 2004. 4-(3-Aminopropylene)-7nitrobenzofurazan: a new polymerisable monomer for use in homogeneous molecularly imprinted sorbent fluoroassays. Tetrahedron Lett. 45: 36253627. DOI: 10.1016/j.tetlet.2004.03.053

2727. Liu JQ, Zhang K, Ren XJ, Luo GM, Shen JC. 2004. Bioimprinted protein exhibits glutathione peroxidase activity. Anal. Chim. Acta 504: 185-189. DOI: 10.1016/S0003-2670(03)00763-3

2728. Manesiotis $P$, Hall AJ, Emgenbroich M, Quaglia M, De Lorenzi E, Sellergren B. 2004. An enantioselective imprinted receptor for Z-glutamate exhibiting a binding induced color change. Chem. Commun. 2278-2279. DOI: 10.1039/b407870e

2729. Mohr GJ. 2004. Chromo- and fluororeactands: Indicators for detection of neutral analytes by using reversible covalent-bond chemistry. Chem. Eur. J. 10: 1082-1090. DOI: 10.1002/chem.200305524

2730. Theodoridis G, Konsta G, Bagia C. 2004. Synthesis and evaluation of molecularly imprinted polymers for enalapril and lisinopril, two synthetic peptide anti-hypertensive drugs. J. Chromatogr. B 804: 43-51. DOI: 10.1016/j.jchromb.2003.12.035

2731. Wu LQ, Li YZ. 2004. Metal ion-mediated molecular-imprinting polymer for indirect recognition of formate, acetate and propionate. Anal. Chim. Acta 517: 145-151. DOI: 10.1016/j.aca.2004.05.015 
2732. Kim H, Guiochon G. 2005. Thermodynamic studies of the solvent effects in chromatography on molecularly imprinted polymers. 3. Nature of the organic mobile phase. Anal. Chem. 77: 2496-2504. DOI: 10.1021/ac040171c

2733. Daniel S, Praveen RS, Rao TP. 2006. Ternary ion-association complex based ion imprinted polymers (IIPs) for trace determination of palladium(II) in environmental samples. Anal. Chim. Acta 570: 79-87. DOI: 10.1016/j.aca.2006.04.007

2734. Hoshino F, Ikawa T, Mouri M, Narita M, Watanabe O, Ueno Y, Tsuda T, Osawa T. 2006. Antibody microarray using molecular-shape imprinting on a polymer containing azo dye. Polym. Prepr. 47: 1072-1073.

2735. Hunt CE, Pasetto P, Ansell RJ, Haupt K. 2006. A fluorescence polarisation molecular imprint sorbent assay for 2,4-D: a non-separation pseudoimmunoassay. Chem. Commun. 1754-1756. DOI: 10.1039/b516194k

2736. Hunt CE, Ansell RJ. 2006. Use of fluorescence shift and fluorescence anisotropy to evaluate the re-binding of template to (S)-propranolol imprinted polymers. Analyst 131: 678-683. DOI: 10.1039/b518248d

2737. Nicholls C, Karim K, Piletsky S, Saini S, Setford S. 2006. Displacement imprinted polymer receptor analysis (DIPRA) for chlorophenolic contaminants in drinking water and packaging materials. Biosens. Bioelectron. 21: 1171-1177. DOI: 10.1016/j.bios.2005.05.002

2738. Rong F, Feng XG, Yuan CW, Fu DG, Li P. 2006. Chiral Separation of Mandelic Acid and Its Derivatives by Thin-Layer Chromatography Using Molecularly Imprinted Stationary Phases. J. Liq. Chrom. Rel. Technol. 29: 2593-2602. DOI: 10.1080/10826070600915213

2739. Tai DF, Lin CY, Wu TZ, Huang JH, Shu PY. 2006. Artificial receptors in serologic tests for the early diagnosis of dengue virus infection. Clin. Chem. 52: 1486-1491. DOI: 10.1373/clinchem.2005.064501 
2740. Yoshimatsu K, Reimhult K, Krozer A, Mosbach K, Sode K, Ye L. 2007. Uniform molecularly imprinted microspheres and nanoparticles prepared by precipitation polymerization: The control of particle size suitable for different analytical applications. Anal. Chim. Acta 584: 112-121. DOI: 10.1016/j.aca.2006.11.004

2741. Philip JYN, Buchweishaija J, Mkayula LL, Ye L. 2007. Preparation of Molecularly Imprinted Polymers Using Anacardic Acid Monomers Derived from Cashew Nut Shell Liquid. J. Agric. Food Chem. 55: 8870-8876.

2742. Lu CH, Zhou WH, Han B, Yang HH, Chen X, Wang XR. 2007. SurfaceImprinted Core-Shell Nanoparticles for Sorbent Assays. Anal. Chem. 79: 5457-5461. DOI: 10.1021/ac070282m

2743. Malosse L, Buvat $P$, Ades D, Siove A. 2008. Detection of degradation products of chemical warfare agents by highly porous molecularly imprinted microspheres. Analyst 133: 588-595. DOI: 10.1039/b713713c

2744. Tse Sum Bui B, Belmont AS, Witters H, Haupt K. 2008. Molecular recognition of endocrine disruptors by synthetic and natural $17 \alpha$-estradiol receptors: a comparative study. Anal. Bioanal. Chem. 390: 2081-2088. DOI: 10.1007/s00216-008-1949-4

2745. Wang L, Zhang ZJ, Huang LG. 2008. Molecularly imprinted polymer based on chemiluminescence imaging for the chiral recognition of dansylphenylalanine. Anal. Bioanal. Chem. 390: 1431-1436. DOI: 10.1007/s00216007-1795-9

2746. Yoshimatsu K, Ye L, Stenlund P, Chronakis IS. 2008. A simple method for preparation of molecularly imprinted nanofiber materials with signal transduction ability. Chem. Commun. 2022-2024. DOI: 10.1039/b719586a 
2747. Zhang JK, Wang H, Liu WS, Bai LR, Ma N, Lu JH. 2008. Synthesis of Molecularly Imprinted Polymer for Sensitive Penicillin Determination in Milk. Anal. Lett. 41: 3411-3419. DOI: 10.1080/00032710802568671

2748. Feás X, Fente CA, Hosseini SV, Seijas JA, Vázquez BI, Franco CM, Cepeda A. 2009. Use of acrylic acid in the synthesis of molecularly imprinted polymers for the analysis of cyproheptadine. Mater. Sci. Eng.: C 29: 398-404. DOI: 10.1016/j.msec.2008.08.011

2749. Wang S, Xu ZX, Fang GZ, Zhang Y, Liu B, Zhu HP. 2009. Development of a Biomimetic Enzyme-Linked Immunosorbent Assay Method for the Determination of Estrone in Environmental Water using Novel Molecularly Imprinted Films of Controlled Thickness as Artificial Antibodies. J. Agric. Food Chem. 57: 4528-4534. DOI: 10.1021/jf900505k

2750. Zhao YJ, Zhao XW, Hu J, Li J, Xu.W.Y., Gu ZZ. 2009. Multiplex Label-Free Detection of Biomolecules with an Imprinted Suspension Array. Angew. Chem. Int. Edit. 48: 7350-7352. DOI: 10.1002/anie.200903472

2751. Fang GZ, Lu JP, Pan MF, Li W, Ren L, Wang S. 2011. Substitution of Antibody with Molecularly Imprinted Film in Enzyme-Linked Immunosorbent Assay for Determination of Trace Ractopamine in Urine and Pork Samples. Food Anal. Meth. 4: 590-597. DOI: 10.1007/s12161-011-9206-4

2752. Kim E, Kim HC, Lee SG, Lee SJ, Go TJ, Baek CS, Jeong SW. 2011. CReactive protein-directed immobilization of phosphocholine ligands on a solid surface. Chem. Commun. 47: 11900-11902. DOI: 10.1039/C1CC15079K

2753. Li JP, Jiang FY, Li YP, Chen ZQ. 2011. Fabrication of an oxytetracycline molecular-imprinted sensor based on the competition reaction via a GODenzymatic amplifier. Biosens. Bioelectron. 26: 2097-2101. DOI: 10.1016/j.bios.2010.09.013 
2754. Meng SM, Jing BQ, Fan YQ, Liu YW, Guo Y. 2011. Spectrophotometric determination of trace cadmium in vegetables with 3,5-bis(4phenylazophenylaminodiazo)benzoic acid. J. Anal. Chem. 66: 31-36. DOI: $10.1134 /$ S1061934811010138

2755. Rosengren AM, Karlsson BCG, Näslund I, Andersson PO, Nicholls IA. 2011. In situ detection of warfarin using time-correlated single-photon counting. Biochem. Biophys. Res. Commun. 407: 60-62. DOI: 10.1016/j.bbrc.2011.02.103

2756. Stringer RC, Gangopadhyay S, Grant SA. 2011. Comparison of molecular imprinted particles prepared using precipitation polymerization in water and chloroform for fluorescent detection of nitroaromatics. Anal. Chim. Acta 703: 239-244. DOI: 10.1016/j.aca.2011.07.034

2757. Sun Q, Yao QQ, Sun ZL, Zhou TS, Nie DX, Shi GY, Jin LT. 2011. Determination of Parathion-methyl in Vegetables by Fluorescent-Labeled Molecular Imprinted Polymer. Chin. J. Chem. 29: 2134-2140. DOI: 10.1002/cjoc.201180370

2758. Tai DF, Ho YF, Wu CH, Lin TC, Lu KH, Lin KS. 2011. Artificial-epitope mapping for CK-MB assay. Analyst 136: 2230-2233. DOI: 10.1039/COAN00919A

2759. Tang JS, Xiang L. 2010. Development of a competitive format sorbent assay for the determination of parathion in water using molecular imprinted polymer as specific sorbent carrier. Chin. Chem. Lett. 21: 1361-1365. DOI: 10.1016/j.cclet.2010.05.014

2760. Tarley CRT, Andrade FN, de Oliveira FM, Corazza MZ, de Azevedo LFM, Segatelli MG. 2011. Synthesis and application of imprinted polyvinylimidazole-silica hybrid copolymer for $\mathrm{Pb} 2+$ determination by flowinjection thermospray flame furnace atomic absorption spectrometry. Anal. Chim. Acta 703: 145-151. DOI: 10.1016/j.aca.2011.07.029 
2761. Tse Sum Bui B, Haupt K. 2011. Preparation and evaluation of a molecularly imprinted polymer for the selective recognition of testosterone - application to molecularly imprinted sorbent assays. J. Mol. Recognit. 24: 1123-1129. DOI: $10.1002 / j m r .1162$

2762. Wang JP, Tang WW, Fang GZ, Pan MF, Wang S. 2011. Development of a Biomimetic Enzyme-linked Immunosorbent Assay Method for the Determination of Methimazole in Urine Sample. J. Chin. Chem. Soc. 58: 463-469. DOI: $10.1002 / j c c s .201190007$

2763. Zang DJ, Ge L, Zhao PN, Yu JH, Huang JD. 2011. A High Throughput and High Selective Chemiluminescence Method for Quantification of Bifenthrin Based on Molecularly Imprinted Film. In Emerging Focus on Advanced Materials, Advanced Materials Research, Vol. 306-307, Liu SQ, Zuo M (eds). Scientific.net: 663-666. DOI: 10.4028/www.scientific.net/AMR.306307.663

2764. Dong H, Tong AJ. 2001. Recognition of Histamine by a Molecularly Imprinted Polymer Based on Zinc(II)-Protoporphyrin as the Fluorescent Functional Monomer. Anal. Sci. 17: a295-a298.

2765. Anderson J, Nelson J, Reynolds C, Ringelberg D, Tepper G, Pestov D. 2004. Steady-state and frequency-domain lifetime measurements of an activated molecular imprinted polymer imprinted to dipicolinic acid. $J$. Fluoresc. 14: 269-274. DOI: 10.1023/B:JOFL.0000024558.68095.27

2766. Chen YC, Brazier JJ, Yan MD, Bargo PR, Prahl SA. 2004. Fluorescencebased optical sensor design for molecularly imprinted polymers. Sens. Actuators B 102: 107-116. DOI: 10.1016/j.snb.2004.02.044

2767. Chen YC, Wang ZM, Yan MD, Prahl SA. 2004. Steady-state fluorescence anisotropy studies of molecularly imprinted polymer sensors. In Molecularly Imprinted Materials-2003, MRS Symposium Proceedings, Vol. 787, Kofinas 
P, Sellergren B, Roberts MJ (eds). Materials Research Society: Warrendale; 35-39. DOI: 10.1557/PROC-787-G3.3

2768. Chen YC, Brazier JJ, Yan MD, Prahl SA. 2004. Evaluation of molecularly imprinted polyurethane as an optical waveguide for $\mathrm{PAH}$ sensing. In Nanosensing: Materials And Devices, Proceedings of SPIE, Vol. 5593, Islam MS, Dutta AK (eds). The International Society for Optical Engineering: 513520. DOI: $10.1117 / 12.570539$

2769. Fernández-González A, Laíño RB, Diaz-García ME, Guardia L, Viale A. 2004. Assessment of molecularly imprinted sol-gel materials for selective room temperature phosphorescence recognition of nafcillin. J. Chromatogr. B 804: 247-254. DOI: 10.1016/j.jchromb.2003.12.030

2770. Huang HC, Lin Cl, Joseph AK, Lee YD. 2004. Photo-lithographically impregnated and molecularly imprinted polymer thin film for biosensor applications. J. Chromatogr. A 1027: 263-268. DOI: 10.1016/j.chroma.2003.08.106

2771. Lin Cl, Joseph AK, Chang CK, Lee YD. 2004. Synthesis and photoluminescence study of molecularly imprinted polymers appended onto CdSe/ZnS core-shells. Biosens. Bioelectron. 20: 127-131. DOI: 10.1016/j.bios.2003.10.017

2772. Matsui J, Akamatsu K, Nishiguchi S, Miyoshi D, Nawafune H, Tamaki K, Sugimoto N. 2004. Composite of Au nanoparticles and molecularly imprinted polymer as a sensing material. Anal. Chem. 76: 1310-1315. DOI: $10.1021 / a c 034788 q$

2773. Murray GM, Southard GE. 2004. Optical transduction schemes for molecularly imprinted polymer sensors. In Molecularly Imprinted Materials2003, MRS Symposium Proceedings, Vol. 787, Kofinas P, Sellergren B, Roberts MJ (eds). Materials Research Society: Warrendale; 61-70. DOI: 10.1557/PROC-787-G5.1 
2774. Oral E, Peppas NA. 2004. Dynamic studies of molecular imprinting polymerizations. Polymer 45: 6163-6173. DOI: 10.1016/j.polymer.2004.06.059

2775. Pestov D, Anderson J, Nelson J, Tepper G. 2004. Chemical point detection using differential fluorescence from molecularly imprinted polymers. In Chemical And Biological Point Sensors For Homeland Defense II, Proceedings of SPIE, Vol. 5585, Sedlacek AJ, Christesen SD, Vo-Dinh T, Combs RJ (eds). The International Society for Optical Engineering: 109-112. DOI: $10.1117 / 12.571411$

2776. Pogorelova SP, Kharitonov AB, Willner I, Sukenik CN, Pizem H, Bayer T. 2004. Development of ion-sensitive field-effect transistor-based sensors for benzylphosphonic acids and thiophenols using molecularly imprinted $\mathrm{TiO}_{2}$ films. Anal. Chim. Acta 504: 113-122. DOI: 10.1016/S0003-2670(03)005324

2777. Zhou HJ, Zhang ZJ, He DY, Hu YF, Huang Y, Chen DL. 2004. Flow chemiluminescence sensor for determination of clenbuterol based on molecularly imprinted polymer. Anal. Chim. Acta 523: 237-242. DOI: 10.1016/j.aca.2004.06.068

2778. Lotierzo M, Henry OYF, Piletsky S, Tothill I, Cullen D, Kania M, Hock B, Turner APF. 2004. Surface plasmon resonance sensor for domoic acid based on grafted imprinted polymer. Biosens. Bioelectron. 20: 145-152. DOI: 10.1016/j.bios.2004.01.032

2779. Björk P, Persson NK, Peter K, Nilsson R, Åsberg P, Inganäs O. 2005. Dynamics of complex formation between biological and luminescent conjugated polyelectrolytes - A surface plasmon resonance study. Biosens. Bioelectron. 20: 1764-1771. DOI: 10.1016/j.bios.2004.07.001 
2780. Bravo JC, Fernandez P, Durand JS. 2005. Flow injection fluorimetric determination of $\beta$-estradiol using a molecularly imprinted polymer. Analyst 130: 1404-1409. DOI: 10.1039/b509618a

2781. Devanathan S, Salamon Z, Nagar A, Narang S, Schleich D, Darman P, Hruby V, Tollin G. 2005. Subpicomolar sensing of $\delta$-opioid receptor ligands by molecular-imprinted polymers using plasmon-waveguide resonance spectroscopy. Anal. Chem. 77: 2569-2574. DOI: 10.1021/ac048476e

2782. Du XZ, Hlady V, Britt D. 2005. Langmuir monolayer approaches to protein recognition through molecular imprinting. Biosens. Bioelectron. 20: 20532060. DOI: 10.1016/j.bios.2004.08.044

2783. Feng LA, Liu YJ, Zhou XD, Hu JM. 2005. The fabrication and characterization of a formaldehyde odor sensor using molecularly imprinted polymers. J. Colloid Interface Sci. 284: 378-382. DOI: 10.1016/j.jcis.2004.10.054

2784. Greene NT, Shimizu KD. 2005. Colorimetric molecularly imprinted polymer sensor array using dye displacement. J. Am. Chem. Soc. 127: 5695-5700. DOI: $10.1021 /$ ja0468022

2785. He YH, Lu JR, Zhang HG, Du JX. 2005. Molecular imprintingchemiluminescence determination of norfloxacin using a norfloxacinimprinted polymer as the recognition material. Microchim. Acta 149: 239244. DOI: 10.1007/s00604-004-0305-4

2786. He YH, Lu JR, Liu M, Du JX. 2005. Molecular imprinting-chemiluminescence determination of trimethoprim using trimethoprim-imprinted polymer as recognition material. Analyst 130: 1032-1037. DOI: 10.1039/b418460b

2787. He YH, Lu JR, Liu M, Du JX, Nie F. 2005. Determination of morphine by molecular imprinting-chemiluminescence method. J. Anal. Toxicol. 29: 528532. 
2788. Jenkins AL, Bae SY. 2005. Molecularly imprinted polymers for chemical agent detection in multiple water matrices. Anal. Chim. Acta 542: 32-37. DOI: 10.1016/j.aca.2004.12.088

2789. Kubo H, Yoshioka N, Takeuchi T. 2005. Fluorescent imprinted polymers prepared with 2-acrylamidoquinoline as a signaling monomer. Org. Lett. 7: 359-362. DOI: 10.1021/0I0479920

2790. Li X, Husson SM. 2005. Adsorption of dansylated amino acids on molecularly imprinted surfaces: A surface plasmon resonance study. In Proceedings of the 2005 AiChE Annual Meeting and Fall Showcase, 50755086.

2791. Liu M, Lu JR, He YH, Du JX. 2005. Molecular imprinting-chemiluminescence sensor for the determination of brucine. Anal. Chim. Acta 541: 97-102. DOI: 10.1016/j.aca.2004.11.003

2792. Matsui J, Akamatsu K, Hara N, Miyoshi D, Nawafune H, Tamaki K, Sugimoto N. 2005. SPR sensor chip for detection of small molecules using molecularly imprinted polymer with embedded gold nanoparticles. Anal. Chem. 77: 4282-4285. DOI: 10.1021/ac050227i

2793. Mohr GJ. 2005. Covalent bond formation as an analytical tool to optically detect neutral and anionic analytes. Sens. Actuators B 107: 2-13. DOI: 10.1016/j.snb.2004.06.039

2794. Nie F, Lu JR, He YH, Du JX. 2005. Determination of indomethacin in urine using molecule imprinting-chemiluminescence method. Talanta 66: 728-733. DOI: 10.1016/j.talanta.2004.12.023

2795. Nie F, Lu JR, Niu WF. 2005. Chemiluminescence determination of indapamide using indapamide-imprinted polymer as recognition material. Anal. Chim. Acta 545: 129-136. DOI: 10.1016/j.aca.2005.04.077 
2796. Nie F, Lu JR, He YH, Du JX. 2005. Use of molecule imprintingchemiluminescence method for the determination of tamoxifen in breast cancer sufferers' urine. Luminescence 20: 315-320. DOI: 10.1002/bio.837

2797. Salinas-Castillo A, Sánchez-Barragán I, Costa-Fernández JM, Pereiro $R$, Ballesteros A, González JM, Segura-Carretero A, Fernández-Gutiérrez A, Sanz-Medel A. 2005. lodinated molecularly imprinted polymer for room temperature phosphorescence optosensing of fluoranthene. Chem. Commun. 3224-3226. DOI: 10.1039/b502706c

2798. Sánchez-Barragán I, Costa-Fernández JM, Pereiro R, Sanz-Medel A, Salinas A, Segura A, Fernández-Gutiérrez A, Ballesteros A, González JM. 2005. Molecularly imprinted polymers based on iodinated monomers for selective room-temperature phosphorescence optosensing of fluoranthene in water. Anal. Chem. 77: 7005-7011. DOI: 10.1021/ac050400a

2799. Takeuchi T, Seko A, Mukawa T. 2005. Molecularly imprinted polymers with signaling function based on the UV-Vis spectral change by diastereoselective binding events. Bull. Chem. Soc. Jpn. 78: 356-360. DOI: $10.1246 /$ bcsj. 78.356

2800. Tao Z, Tehan EC, Bukowski RM, Tang Y, Shughart EL, Holthoff WG, Cartwright AN, Titus AH, Bright FV. 2005. Biomolecule-less sensors for biomolecules based on templated xerogel platforms. In Progress in Biomedical Optics and Imaging, Proceedings of the SPIE, Vol. 5969, Chan WCW, Yu K, Krull UJ, Hornsey RI, Wilson BC, Weersink RA (eds). International Soceity for Optical Engineering: Art. No.-59690F. DOI: $10.1117 / 12.629169$

2801. Yu JCC, Lai EPC. 2005. Interaction of ochratoxin A with molecularly imprinted polypyrrole film on surface plasmon resonance sensor. Reac. Func. Polym. 63: 171-176. DOI: 10.1016/j.reactfunctpolym.2005.03.001 
2802. Zhang ZJ, He DY, Liu W, Lv Y. 2005. Chemiluminescence micro-flowinjection analysis on a chip. Luminescence 20: 377-381. DOI: 10.1002/bio.846

2803. Zhou HJ, Zhang ZJ, He DY, Xiong Y. 2005. Flow through chemiluminescence sensor using molecularly imprinted polymer as recognition elements for detection of salbutamol. Sens. Actuators $B$ 107: 798-804. DOI: 10.1016/j.snb.2004.12.020

2804. Suah FBM, Tajri N, Noor SFM, Jani AMM, Taib MN. 2005. Preliminary study towards the fabrication of the nanoscale optical $\mathrm{pH}$ sensor with tailored pK/sub a/ value by using molecularly imprinting technique. In 2005 Asian Conference on Sensors and the International Conference on new Techniques in Pharmaceutical and Biomedical Research Proceedings, Ghodgaonkar DK, Ahmad M, Heng LY, Habash RW, Wui WT, Taib MN (eds). IEEE: 61-63. DOI: 10.1109/ASENSE.2005.1564506

2805. Al-Kindy SMZ, Suliman FEO, Pillay AE. 2006. Fluorimetric determination of aluminium using Sequential Injection Analysis (SIA): State of our art and future developments. Instr. Sci. Technol. 34: 619-633. DOI: $10.1080 / 10739140600963830$

2806. Altschuh D, Oncul S, Demchenko AP. 2006. Fluorescence sensing of intermolecular interactions and development of direct molecular biosensors. J. Mol. Recognit. 19: 459-477. DOI: 10.1002/jmr.807

2807. Banerji S, Peng W, Kim YC, Booksh KS. 2006. Molecularly imprinted polymerization based surface plasmon resonance sensing for glucose detection in human urine. In Smart Medical and Biomedical Sensor Technology IV, Proceedings of the SPIE, Vol. 6380, Cullum BM, Carter JC (eds). SPIE: U235-U245. DOI: 10.1117/12.686483

2808. Benito-Peña E, Moreno-Bondi MC, Aparicio S, Orellana G, Cederfur J, Kempe M. 2006. Molecular engineering of fluorescent penicillins for 
molecularly imprinted polymer assays. Anal. Chem. 78: 2019-2027. DOI: 10.1021/ac051939b

2809. Carlson CA, Lloyd JA, Dean SL, Walker NR, Edmiston PL. 2006. Sensor for fluorene based on the incorporation of an environmentally sensitive fluorophore proximal to a molecularly imprinted binding site. Anal. Chem. 78: 3537-3542. DOI: 10.1021/ac051375b

2810. Chyou JJ, Chu CS, Chien FC, Lin CY, Yeh TL, Hsu RC, Chen SJ. 2006. Precise determination of the dielectric constant and thickness of a nanolayer by use of surface plasmon resonance sensing and multiexperiment linear data analysis. Appl. Optics 45: 6038-6044.

2811. Gong CB, Lam MHW, Yu HX. 2006. The Fabrication of a Photoresponsive Molecularly Imprinted Polymer for the Photoregulated Uptake and Release of Caffeine. Adv. Funct. Mater. 16: 1759-1767. DOI: 10.1002/adfm.200500907

2812. Gräfe A, Haupt K, Mohr GJ. 2006. Optical sensor materials for the detection of amines in organic solvents. Anal. Chim. Acta 565: 42-47. DOI: 10.1016/j.aca.2006.02.034

2813. Guardia L, Badía R, Díaz-García ME. 2006. Molecular imprinted ormosils for nafcillin recognition by room temperature phosphorescence optosensing. Biosens. Bioelectron. 21: 1822-1829. DOI: 10.1016/j.bios.2005.09.016

2814. He C, Zhang ZJ, He DY, Xiong Y. 2006. Chemiluminescence determination of metformin based on hydroxyl radical reaction and molecularly imprinted polymer on-line enrichment. Anal. Bioanal. Chem. 385: 128-133. DOI: $10.1007 / \mathrm{s} 00216-006-0378-5$

2815. He DY, Zhang ZJ, Zhou HJ, Huang Y. 2006. Micro flow sensor on a chip for the determination of terbutaline in human serum based on 
chemiluminescence and a molecularly imprinted polymer. Talanta 69: 12151220. DOI: 10.1016/j.talanta.2005.12.043

2816. Hu XB, An Q, Li GT, Tao SY, Liu J. 2006. Imprinted Photonic Polymers for Chiral Recognition. Angew. Chem. Int. Edit. 45: 8145-8148. DOI: 10.1002/anie.200601849

2817. Huang SC, Lee GB, Chien FC, Chen SJ, Chen WJ, Yang MC. 2006. A microfluidic system with integrated molecular imprinting polymer films for surface plasmon resonance detection. J. Micromech. Microeng. 16: 1251 1257. DOI: $10.1088 / 0960-1317 / 16 / 7 / 019$

2818. Lee KH, Su YD, Chen SJ, Lee GB. 2006. 2-dimensional SPR detection system integrated with molecular imprinting polymer microarrays using microfluidic technology. In Proceedings of the 19th IEEE International conference on Micro Electro Mechanical Systems (MEMS), Vol. 2006, IEEE: 430-433.

2819. Li X, Husson SM. 2006. Two-Dimensional Molecular Imprinting Approach to Produce Optical Biosensor Recognition Elements. Langmuir 22: 9658-9663. DOI: 10.1021/la0612163

2820. Nesterov EE, Li JH. 2006. Fluorescent sensors based on molecularly imprinted cross-linked conjugated polymers: increasing selectivity through shape recognition. Polym. Prepr. 47: 392-393.

2821. Ng SM, Narayanaswamy R. 2006. Fluorescence sensor using a molecularly imprinted polymer as a recognition receptor for the detection of aluminium ions in aqueous media. Anal. Bioanal. Chem. 386: 1235-1244. DOI: 10.1007/s00216-006-0736-3

2822. Niu WF, Feng N, Nie F, Lu JR. 2006. Investigating the postchemiluminescence behavior of phenothiazine medications in the luminolpotassium ferricyanide system: molecular imprinting-post- 
chemiluminescence method for the determination of chlorpromazine hydrochloride. Anal. Bioanal. Chem. 385: 153-160. DOI: 10.1007/s00216006-0365-x

2823. Pestov D, Anderson J, Tepper G. 2006. Differential fluorescence from molecularly imprinted polymers containing europium ions as a transducer element. In Chemical and Biological Sensors for Industrial and Environmental Monitoring II, Proceedings of SPIE, Christensen SD, Sedlacek AJ, Gillespie JB, Ewing KJ (eds). SPIE: U221-U225. DOI: $10.1117 / 12.686219$

2824. Shughart EL, Ahsan K, Detty MR, Bright FV. 2006. Site selectively templated and tagged xerogels for chemical sensors. Anal. Chem. 78: 31653170. DOI: 10.1021/ac060113m

2825. Smith C, Tatineni B, Anderson J, Tepper G. 2006. Optical bar code recognition of methyl salicylate (MES) for environmental monitoring using fluorescence resonance energy transfer (FRET) on thin films. In Chemical and Biological Sensors for Industrial and Environmental Monitoring II, Proceedings of SPIE, Christensen SD, Sedlacek AJ, Gillespie JB, Ewing KJ (eds). SPIE: U231-U235. DOI: 10.1117/12.684416

2826. Sreenivasan K. 2006. Detection of creatinine enriched on a surface imprinted polystyrene film using FT-ATR-IR. J. Mol. Recognit. 19: 408-412. DOI: 10.1002/jmr.797

2827. Stephenson CJ, Shimizu KD. 2006. Colormetric imprinted polymers as sensors. Polym. Prepr. 47: 7.

2828. Stephenson CJ, Shimizu KD. 2006. Formation of multi-channelcolorimetric polymers as a sensor for biogenic amines. Polym. Prepr. 47: 1084.

2829. Tao ZY, Tehan EC, Bukowski RM, Tang Y, Shughart EL, Holthoff WG, Cartwright AN, Titus AH, Bright FV. 2006. Templated xerogels as platforms 
for biomolecule-less biomolecule sensors. Anal. Chim. Acta 564: 59-65. DOI: 10.1016/j.aca.2006.01.076

2830. Tokareva I, Tokarev I, Minko S, Hutter E, Fendler JH. 2006. Ultrathin molecularly imprinted polymer sensors employing enhanced transmission surface plasmon resonance spectroscopy. Chem. Commun. 3343-3345. DOI: 10.1039/b604841b

2831. Xiong Y, Zhou HJ, Zhang ZJ, He DY, He C. 2006. Molecularly imprinted online solid-phase extraction combined with flow-injection chemiluminescence for the determination of tetracycline. Analyst 131: 829-834. DOI: $10.1039 / b 606779 b$

2832. Yan X, Zhou HJ, Zhang ZJ, He DY, He C. 2006. Determination of hydralazine with flow injection chemiluminescence sensor using molecularly imprinted polymer as recognition element. J. Pharm. Biomed. Anal. 41: 694700. DOI: 10.1016/j.jpba.2006.01.008

2833. Belmont AS, Jaeger S, Knopp D, Niessner R, Gauglitz G, Haupt K. 2007. Molecularly imprinted polymer films for reflectometric interference spectroscopic sensors. Biosens. Bioelectron. 22: 3267-3272. DOI: 10.1016/j.bios.2007.01.023

2834. Cywinski P, Sadowska M, Danel A, Buma WJ, Brouwer AM, Wandelt B. 2007. Fluorescent, molecularly imprinted thin-layer films based on a common polymer. J. Appl. Polym. Sci. 105: 229-235. DOI: 10.1002/app.26107

2835. Guardia L, Badía R, Díaz-García ME. 2007. Molecularly Imprinted Sol-Gels for Nafcillin Determination in Milk-Based Products. J. Agric. Food Chem. 55: 566-570. DOI: 10.1021/jf062058o 
2836. Hu XB, Li GT, Huang J, Zhang D, Qiu Y. 2007. Construction of SelfReporting Specific Chemical Sensors with High Sensitivity. Adv. Mater. 19: 4327-4332. DOI: 10.1002/adma.200701084

2837. Johnson-White $B$, Zeinali $M$, Shaffer KM, Patterson $\mathrm{CH}$, Charles PT, Markowitz MA. 2007. Detection of organics using porphyrin embedded nanoporous organosilicas. Biosens. Bioelectron. 22: 1154-1162. DOI: 10.1016/j.bios.2006.07.040

2838. Kobayashi T, Kusunoki T, Zhang Q, Takeda K. 2007. Bile acid imprinting polymers prepared by covalent-ester monomer-template technique: Synthesis, characterization and fluorescence application for BA recognition. J. Chem. Eng. Jpn. 40: 516-522. DOI: 10.1252/jcej.40.516

2839. Lavine BK, Westover DJ, Kaval N, Mirjankar N, Oxenford L, Mwangi GK. 2007. Swellable molecularly imprinted polyN-(N-propyl)acrylamide particles for detection of emerging organic contaminants using surface plasmon resonance spectroscopy. Talanta 72: 1042-1048. DOI: 10.1016/j.talanta.2006.12.046

2840. Li J, Kendig CE, Nesterov EE. 2007. Chemosensory Performance of Molecularly Imprinted Fluorescent Conjugated Polymer Materials. J. Am. Chem. Soc. 129: 15911-15918. DOI: 10.1021/ja0748027

2841. Li YH, Niu WF, Lu JR. 2007. Sensitive determination of phenothiazines in pharmaceutical preparation and biological fluid by flow injection chemiluminescence method using luminol-KMnO4 system. Talanta 71: 1124-1129. DOI: 10.1016/j.talanta.2006.06.007

2842. Li ZM, Liu JM, Liu ZB, Liu QY, Lin X, Li FM, Yang ML, Zhu GH, Huang XM. 2007. Preparation for nitrocellulose membrane-poly (vinyl alcohol)-ionic imprinting and its application to determine trace copper by room temperature phosphorimetry. Anal. Chim. Acta 589: 44-50. DOI: 10.1016/j.aca.2007.02.044 
2843. Matsunaga T, Hishiya T, Takeuchi T. 2007. Surface plasmon resonance sensor for lysozyme based on molecularly imprinted thin films. Anal. Chim. Acta 591: 63-67. DOI: 10.1016/j.aca.2007.02.072

2844. Moreno-Bondi MC, Urraca JL, Benito-Peña E, Navarro-Villoslada F, Martins FA, Orellana G, Sellergren B. 2007. Molecularly imprinted polymers as biomimetic receptors for fluorescence-based optical sensors. In Proceedings of the Third European Workshop on Optical Fibre Sensors, Proceedings of SPIE, Cutolo A, Culshaw B, López-Higuera JM (eds). Art. No. 66190C. DOI: $10.1117 / 12.738347$

2845. Navarro-Villoslada F, Urraca JL, Moreno-Bondi MC, Orellana G. 2007. Zearalenone sensing with molecularly imprinted polymers and tailored fluorescent probes. Sens. Actuators B 121: 67-73. DOI: 10.1016/j.snb.2006.09.042

2846. Sánchez-Barragán I, Karim K, Costa-Fernández JM, Piletsky SA, SanzMedel A. 2007. A molecularly imprinted polymer for carbaryl determination in water. Sens. Actuators B 123: 798-804. DOI: 10.1016/j.snb.2006.10.026

2847. Southard GE, Van Houten KA, Ott EW, Murray GM. 2007. Luminescent sensing of organophosphates using europium(III) containing imprinted polymers prepared by RAFT polymerization. Anal. Chim. Acta 581: 202-207. DOI: 10.1016/j.aca.2006.08.027

2848. Southard GE, Van Houten KA, Murray GM. 2007. Soluble and Processable Phosphonate Sensing Star Molecularly Imprinted Polymers. Macromolecules 40: 1395-1400. DOI: 10.1021/ma062443e

2849. Sreenivasan K. 2007. Identification of salicylic acid using surface modified polyurethane film using an imprinted layer of polyaniline. Anal. Chim. Acta 583: 284-288. DOI: 10.1016/j.aca.2006.10.019 
2850. Tappura K, Vikholm-Lundin I, Albers WM. 2007. Lipoate-based imprinted self-assembled molecular thin films for biosensor applications. Biosens. Bioelectron. 22: 912-919. DOI: 10.1016/j.bios.2006.03.014

2851. Tatemichi M, Sakamoto M, Mizuhata M, Deki S, Takeuchi T. 2007. ProteinTemplated Organic/Inorganic Hybrid Materials Prepared by Liquid-Phase Deposition. J. Am. Chem. Soc. 129: 10906-10910. DOI: 10.1021/ja071890m

2852. Traviesa-Alvarez JM, Sánchez-Barragán I, Costa-Fernández JM, Pereiro R, Sanz-Medel A. 2007. Room temperature phosphorescence optosensing of benzo[a]pyrene in water using halogenated molecularly imprinted polymers. Analyst 132: 218-223. DOI: 10.1039/b616919h

2853. Urraca JL, Moreno-Bondi MC, Orellana G, Sellergren B, Hall AJ. 2007. Molecularly Imprinted Polymers as Antibody Mimics in Automated On-Line Fluorescent Competitive Assays. Anal. Chem. 79: 4915-4923. DOI: $10.1021 / \mathrm{ac} 070277 \mathrm{i}$

2854. Walker NR, Linman MJ, Timmers MM, Dean SL, Burkett CM, Lloyd JA, Keelor JD, Baughman BM, Edmiston PL. 2007. Selective detection of gasphase TNT by integrated optical waveguide spectrometry using molecularly imprinted sol-gel sensing films. Anal. Chim. Acta 593: 82-91. DOI: 10.1016/j.aca.2007.04.034

2855. Wang L, Zhang ZJ. 2007. Molecular imprinted polymer-based chemiluminescence imaging sensor for the detection of trans-resveratrol. Anal. Chim. Acta 592: 115-120. DOI: 10.1016/j.aca.2007.04.007

2856. Xiong Y, Zhou HJ, Zhang ZJ, He DY, He C. 2007. Flow-injection chemiluminescence sensor for determination of isoniazid in urine sample based on molecularly imprinted polymer. Spectrochim. Acta, A 66: 341-346. DOI: 10.1016/j.saa.2006.03.001 
2857. Zattoni A, Reschiglian P, Montalti M, Zaccheroni N, Prodi L, Picca RA, Malitesta C. 2007. Characterization of titanium dioxide nanoparticles imprinted for tyrosine by flow field-flow fractionation and spectrofluorimetric analysis. Inorg. Chim. Acta 360: 1063-1071. DOI: 10.1016/j.ica.2006.07.088

2858. Andreetta HA, Bruzzone L. 2008. Fluorescence Detection of Atenolol Using a Molecular Imprinted Polymer. Anal. Lett. 41: 36-45. DOI: 10.1080/00032710701748465

2859. Diltemiz SE, Denizli A, Ersöz A, Say RI. 2008. Molecularly imprinted ligandexchange recognition assay of DNA by SPR system using guanosine and guanine recognition sites of DNA. Sens. Actuators B 133: 484-488. DOI: 10.1016/j.snb.2008.03.007

2860. Diltemiz SE, Say RI, Büyüktiryaki S, Hür D, Denizli A, Ersöz A. 2008. Quantum dot nanocrystals having guanosine imprinted nanoshell for DNA recognition. Talanta 75: 890-896. DOI: 10.1016/j.talanta.2007.12.036

2861. Holthoff EL, Bright FV. 2008. Photophysics of 9,10-anthracenediol and a bifunctional sacrificial template in solution and xerogels. Appl. Spectros. 62: 345-352. DOI: 10.1366/000370208784046740

2862. Hu XB, Li GT, Li MH, Huang J, Li Y, Gao YB, Zhang YH. 2008. Ultrasensitive Specific Stimulant Assay Based on Molecularly Imprinted Photonic Hydrogels. Adv. Funct. Mater. 18: 575-583. DOI: 10.1002/adfm.200700527

2863. James D, Gladis JM, Pandey AK, Naidu GRK, Rao TP. 2008. Design of twodimensional biomimetic uranyl optrode and its application to the analysis of natural waters. Talanta 74: 1420-1427. DOI: 10.1016/j.talanta.2007.09.019

2864. Lieberzeit PA, Halikias K, Afzal A, Dickert FL. 2008. Polymers imprinted with PAH mixtures-comparing fluorescence and QCM sensors. Anal. Bioanal. Chem. 392: 1405-1410. DOI: 10.1007/s00216-008-2413-1 
2865. Paniagua-González G, Fernandéz-Hernando P, Durand-Alegría JS. 2008. Determination of digoxin in serum samples using a flow-through fluorosensor based on a molecularly imprinted polymer. Biosens. Bioelectron. 23: 1754-1758. DOI: 10.1016/j.bios.2008.01.018

2866. Sadowska M, Wandelt B. 2008. Molecularly Imprinted Thin Polymeric Film as a Fluorescent Sensor for Nucleotides. Mol. Cryst. Liq. Cryst. 486: 203212. DOI: $10.1080 / 15421400801918005$

2867. Smith CB, Anderson JE, Massaro RD, Tatineni B, Kam KC, Tepper GC. 2008. Fluorescence resonance energy transfer based MCM-EDTA-Tb3+MES sensor. Appl. Spectros. 62: 604-610. DOI: 10.1366/000370208784658101

2868. Vikholm-Lundin I, Pulli T, Albers WM, Tappura K. 2008. A comparative evaluation of molecular recognition by monolayers composed of synthetic receptors or oriented antibodies. Biosens. Bioelectron. 24: 1036-1038. DOI: 10.1016/j.bios.2008.06.049

2869. Wandelt B, Sadowska M, Cywinski P, Hachulka K. 2008. Polymeric Sensory Systems Based on Molecular Imprinting for Identification and Separation of Molecules and Bigger Biological Objects. Mol. Cryst. Liq. Cryst. 486: 257270. DOI: $10.1080 / 15421400801921728$

2870. Wang L, Zhang ZJ. 2008. Chemiluminescence imaging assay dipyridamole based on molecular imprinted polymer as recognition material. Sens. Actuators B 133: 40-45. DOI: 10.1016/j.snb.2008.01.051

2871. Wang L, Zhang ZJ. 2008. The study of oxidization fluorescence sensor with molecular imprinting polymer and its application for 6-mercaptopurine (6MP) determination. Talanta 76: 768-771. DOI: 10.1016/j.talanta.2008.04.024

2872. Wu Z, Tao CA, Lin CX, Shen DZ, Li GT. 2008. Label-Free Colorimetric Detection of Trace Atrazine in Aqueous Solution by Using Molecularly 
Imprinted Photonic Polymers. Chem. Eur. J. 14: 11358-11368. DOI: 10.1002/chem.200801250

2873. Yoshikawa M, Guiver MD, Robertson GP. 2008. Surface plasmon resonance studies on molecularly imprinted films. J. Appl. Polym. Sci. 110: 2826-2832. DOI: 10.1002/app.28686

2874. Alvarez-Diaz A, Costa JM, Pereiro R, Sanz-Medel A. 2009. Halogenated molecularly imprinted polymers for selective determination of carbaryl by phosphorescence measurements. Anal. Bioanal. Chem. 394: 1569-1576. DOI: $10.1007 /$ s00216-009-2725-9

2875. Choi SW, Chang HJ, Lee N, Kim JH, Chun HS. 2009. Detection of Mycoestrogen Zearalenone by a Molecularly Imprinted Polypyrrole-Based Surface Plasmon Resonance (SPR) Sensor. J. Agric. Food Chem. 57: 11131118. DOI: $10.1021 / j f 804022 p$

2876. Fang YJ, Yan SL, Ning BA, Liu N, Gao ZX, Chao FH. 2009. Flow injection chemiluminescence sensor using molecularly imprinted polymers as recognition element for determination of maleic hydrazide. Biosens. Bioelectron. 24: 2323-2327. DOI: 10.1016/j.bios.2008.10.034

2877. González GP, Hernando PF, Alegría JSD. 2009. An optical sensor for the determination of digoxin in serum samples based on a molecularly imprinted polymer membrane. Anal. Chim. Acta 638: 209-212. DOI: 10.1016/j.aca.2009.02.023

2878. Gültekin A, Ersöz A, Hür D, Sariözlü NY, Denizli A, Say RI. 2009. Gold nanoparticles having dipicolinic acid imprinted nanoshell for Bacillus cereus spores recognition. Appl. Surf. Sci. 256: 142-148. DOI: 10.1016/j.apsusc.2009.07.097

2879. Kantarovich K, Tsarfati I, Gheber LA, Haupt K, Bar I. 2009. Writing Droplets of Molecularly Imprinted Polymers by Nano Fountain Pen and Detecting 
Their Molecular Interactions by Surface-Enhanced Raman Scattering. Anal. Chem. 81: 5686-5690. DOI: 10.1021/ac900418x

2880. Lin HY, Ho MS, Lee MH. 2009. Instant formation of molecularly imprinted poly(ethylene-co-vinyl alcohol)/quantum dot composite nanoparticles and their use in one-pot urinalysis. Biosens. Bioelectron. 25: 579-586. DOI: 10.1016/j.bios.2009.03.039

2881. Ng SM, Narayanaswamy R. 2009. Molecularly imprinted $\alpha$-cyclodextrin polymer as potential optical receptor for the detection of organic compound. Sens. Actuators B 139: 156-165. DOI: 10.1016/j.snb.2008.10.035

2882. Nguyen TH, Ansell RJ. 2009. Fluorescent imprinted polymer sensors for chiral amines. Org. Biomol. Chem. 7: 1211-1220. DOI: 10.1039/b816733h

2883. Paniagua-González G, Fernández-Hernando P, Durand-Alegría JS. 2009. A MIP-based flow-through fluoroimmunosensor as an alternative to immunosensors for the determination of digoxin in serum samples. Anal. Bioanal. Chem. 394: 963-970. DOI: 10.1007/s00216-008-2584-9

2884. Riskin M, Tel-Vered R, Lioubashevski O, Willner I. 2009. Ultrasensitive Surface Plasmon Resonance Detection of Trinitrotoluene by a Bis-anilineCross-Linked Au Nanoparticles Composite. J. Am. Chem. Soc. 131: 73687378. DOI: $10.1021 / \mathrm{ja9} 001212$

2885. Roche PJR, Ng SM, Narayanaswamy R, Goddard N, Page KM. 2009. Multiple surface plasmon resonance quantification of dextromethorphan using a molecularly imprinted $\alpha$-cyclodextrin polymer: A potential probe for drug-drug interactions. Sens. Actuators $B$ 139: 22-29. DOI: 10.1016/j.snb.2008.07.029

2886. Stringer RC, Gangopadhyay S, Grant SA. 2009. Fluorescent imprinted polymers for detection of explosive nitroaromatic compounds. Vol. 7303, Art. No. 73031R. DOI: 10.1117/12.818768 
2887. Tan J, Wang HF, Yan XP. 2009. A fluorescent sensor array based on ion imprinted mesoporous silica. Biosens. Bioelectron. 24: 3316-3321. DOI: 10.1016/j.bios.2009.04.024

2888. Tan J, Wang HF, Yan XP. 2009. Discrimination of Saccharides with a Fluorescent Molecular Imprinting Sensor Array Based on Phenylboronic Acid Functionalized Mesoporous Silica. Anal. Chem. 81: 5273-5280. DOI: 10.1021/ac900484x

2889. Tierney S, Stokke BT. 2009. Development of an Oligonucleotide Functionalized Hydrogel Integrated on a High Resolution Interferometric Readout Platform as a Label-Free Macromolecule Sensing Device. Biomacromolecules 10: 1619-1626. DOI: 10.1021/bm900218c

2890. Uzun L, Say RI, Ünal S, Denizli A. 2009. Production of surface plasmon resonance based assay kit for hepatitis diagnosis. Biosens. Bioelectron. 24: 2878-2884. DOI: 10.1016/j.bios.2009.02.021

2891. Valero-Navarro A, Damiani PC, Fernández-Sánchez JF, Segura-Carretero A, Fernández-Gutiérrez A. 2009. Chemometric-assisted MIP-optosensing system for the simultaneous determination of monoamine naphthalenes in drinking waters. Talanta 78: 57-65. DOI: 10.1016/j.talanta.2008.10.045

2892. Valero-Navarro A, Salinas-Castillo A, Fernández-Sánchez JF, SeguraCarretero A, Mallavia R, Fernández-Gutiérrez A. 2009. The development of a MIP-optosensor for the detection of monoamine naphthalenes in drinking water. Biosens. Bioelectron. 24: 2305-2311. DOI: 10.1016/j.bios.2008.11.022

2893. Wang HF, He Y, Ji TR, Yan XP. 2009. Surface Molecular Imprinting on MnDoped ZnS Quantum Dots for Room-Temperature Phosphorescence Optosensing of Pentachlorophenol in Water. Anal. Chem. 81: 1615-1621. DOI: $10.1021 / a c 802375 a$ 
2894. Wu N, Feng L, Tan YY, Hu JM. 2009. An optical reflected device using a molecularly imprinted polymer film sensor. Anal. Chim. Acta 653: 103-108. DOI: 10.1016/j.aca.2009.08.043

2895. Yu FS, Gao YW, Wei J, Chen F, Ding YB. 2009. Molecular ImprintingChemiluminescence Sensor for the Determination of Prulifloxacin. Anal. Lett. 42: 381-392. DOI: 10.1080/00032710802508156

2896. Yu JH, Wan FW, Dai P, Ge SG, Li B, Huang JD. 2009. Flow Injection Chemiluminescence Sensor with Novel Rhodanine Ramification for Determination of Fenfluramine Based on Molecularly Imprinted Polymer. Anal. Lett. 42: 746-757. DOI: 10.1080/00032710902722004

2897. Yu JH, Dai P, Wan FW, Li B, Ge SG. 2009. Quantification of fenfluramine with a molecularly imprinted chemiluminescence sensor and sulfonophenylazo rhodanine. J. Sep. Sci. 32: 2170-2179. DOI: 10.1002/jssc.200800761

2898. Yu JH, Zhang CC, Dai P, Ge SG. 2009. Highly selective molecular recognition and high throughput detection of melamine based on molecularly imprinted sol-gel film. Anal. Chim. Acta 651: 209-214. DOI: 10.1016/j.aca.2009.08.018

2899. Yu JH, Zhang CC, Ge L, Dai P, Ge SG. 2009. Quantification of Sorbic Acid Using the Molecularly Imprinted Chemiluminescence Method with Rhodanine Derivative. Anal. Sci. 25: 1351-1356. DOI: 10.2116/analsci.25.1351

2900. Yu JH, Ge L, Dai P, Zhang CC, Ge SG, Huang JD. 2009. Highly selective determination of phenolphthalein by flow injection chemiluminescence method based on a molecular imprinting polymer. Luminescence 24: 444447. DOI: 10.1002/bio. 1135 
2901. Zheng HF, Du XZ. 2009. Enhanced Binding and Biosensing of Carbohydrate-Functionalized Monolayers to Target Proteins by Surface Molecular Imprinting. J. Phys. Chem. B 113: 11330-11337. DOI: 10.1021/jp9060279

2902. Kim E, Lee SJ, Kim HC, Lee SG, Go TJ, Baek CS, Jeong SW. 2009. Selective detection of estradiol using a molecularly imprinted self-assembled monolayer on gold surface. Toxicol. Environ. Health Sci. 1: 117-121. DOI: 10.1007/BF03216472

2903. Banerji S, Peng W, Kim YC, Booksh KS. 2010. Chemically responsive hydrogel with nanoparticle enhanced detection for small biomolecules. In Smart Biomedical and Physiological Sensor Technologies VII, Proceedings of the SPIE, Vol. 7674, Cullum BM, Porterfield DM, Booksh KS (eds). SPIE: Orlando, Florida, USA; Art. No. 76740A. DOI: 10.1117/12.854610

2904. Ben-Amram Y, Riskin M, Willner I. 2010. Selective and enantioselective analysis of mono- and disaccharides using surface plasmon resonance spectroscopy and imprinted boronic acid-functionalized $A u$ nanoparticle composites. Analyst 135: 2952-2959. DOI: 10.1039/COAN00268B

2905. Bompart M, De Wilde Y, Haupt K. 2010. Chemical Nanosensors Based on Composite Molecularly Imprinted Polymer Particles and Surface-Enhanced Raman Scattering. Adv. Mater. 22: 2343-2348. DOI: 10.1002/adma.200904442

2906. Bright FV, Holthoff EL. 2010. Dynamics Within Site Selectively Templated and Tagged Xerogel Sensor Platforms. Appl. Spectros. 64: 1073-1077. DOI: 10.1366/000370210792973569

2907. Chang PP, Zhang ZJ, Yang CY. 2010. Molecularly imprinted polymer-based chemiluminescence array sensor for the detection of proline. Anal. Chim. Acta 666: 70-75. DOI: 10.1016/j.aca.2010.03.031 
2908. Cywinski PJ, Sadowska M, Wandelt B, Buma WJ, Brouwe AM. 2010. Thinlayer molecularly imprinted sensors studied by fluorescence microscopy. eJ. Surf. Sci. Nanotechnol. 8: 293-297. DOI: 10.1380/ejssnt.2010.293

2909. Edmiston PL, Campbell DP, Gottfried DS, Baughman J, Timmers MM. 2010. Detection of vapor phase trinitrotoluene in the parts-per-trillion range using waveguide interferometry. Sens. Actuators B 143: 574-582. DOI: 10.1016/j.snb.2009.09.059

2910. Frasconi M, Tel-Vered R, Riskin M, Willner I. 2010. Electrified Selective "Sponges" Made of Au Nanoparticles. J. Am. Chem. Soc. 132: 9373-9382. DOI: 10.1021/ja102153f

2911. Frasconi M, Tel-Vered R, Riskin M, Willner I. 2010. Surface Plasmon Resonance Analysis of Antibiotics Using Imprinted Boronic AcidFunctionalized Au Nanoparticle Composites. Anal. Chem. 82: 2512-2519. DOI: $10.1021 /$ ac902944k

2912. Ge L, Dai P, Ge SG, Yu JH. 2010. High selectivity chemiluminescence sensor for determination of puerarin in diet foods/weight loss promoters based on novel rhodanine and monodisperse molecularly imprinted microspheres. Anal. Methods 2: 1506-1513.

2913. Gupta G, Singh PK, Boopathi M, Kamboj DV, Singh B, Vijayaraghavan R. 2010. Molecularly imprinted polymer for the recognition of biological warfare agent staphylococcal enterotoxin B based on Surface Plasmon Resonance. Thin Solid Films 519: 1115-1121. DOI: 10.1016/j.tsf.2010.08.054

2914. Gültekin A, Ersöz A, Sariözlü NY, Denizli A, Say RI. 2010. Nanosensors having dipicolinic acid imprinted nanoshell for Bacillus cereus spores detection. J. Nanopart. Res. 12: 2069-2079. DOI: 10.1007/s11051-0099766-z 
2915. Güney O, Cebeci FÇ. 2010. Molecularly imprinted fluorescent polymers as chemosensors for the detection of mercury ions in aqueous media. J. Appl. Polym. Sci. 117: 2373-2379. DOI: 10.1002/app.32077

2916. Halvorson B, Whelton A, Vikesland P. 2010. Transitioning molecular imprinted polymers and SERS to the water industry: Practical significance and progress. In Proceedings: Water Quality Technology Conference and Exposition 2010, 108-111.

2917. Holthoff EL, Bright FV. 2010. Photophysics associated with site selectively templated and tagged xerogel sensor platforms. Appl. Spectros. 64: 714719. DOI: $10.1366 / 000370210791666408$

2918. Holthoff EL, Stratis-Cullum DN, Hankus ME. 2010. Xerogel-based molecularly imprinted polymers for explosives detection. In Explosives Detection III, Proceedings of the SPIE, Vol. 7665, Fountain AWI, Gardner PJ (eds). SPIE: Orlando, Florida, USA; Art. No. 76650W. DOI: $10.1117 / 12.850129$

2919. Hong CC, Chang PH, Lin CC, Hong CL. 2010. A disposable microfluidic biochip with on-chip molecularly imprinted biosensors for optical detection of anesthetic propofol. Biosens. Bioelectron. 25: 2058-2064. DOI: 10.1016/j.bios.2010.01.037

2920. Kantarovich K, Tsarfati I, Gheber LA, Haupt K, Bar I. 2010. Reading microdots of a molecularly imprinted polymer by surface-enhanced Raman spectroscopy. Biosens. Bioelectron. 26: 809-814. DOI: 10.1016/j.bios.2010.06.018

2921. Li HB, Li YL, Cheng J. 2010. Molecularly Imprinted Silica Nanospheres Embedded CdSe Quantum Dots for Highly Selective and Sensitive Optosensing of Pyrethroids. Chem. Mater. 22: 2451-2457. DOI: $10.1021 / \mathrm{cm} 902856 \mathrm{y}$ 
2922. Liu JX, Chen H, Lin Z, Lin JM. 2010. Preparation of Surface Imprinting Polymer Capped Mn-Doped ZnS Quantum Dots and Their Application for Chemiluminescence Detection of 4-Nitrophenol in Tap Water. Anal. Chem. 82: 7380-7386. DOI: 10.1021/ac101510b

2923. Manju S, Hari PR, Sreenivasan K. 2010. Fluorescent molecularly imprinted polymer film binds glucose with a concomitant changes in fluorescence. Biosens. Bioelectron. 26: 894-897. DOI: 10.1016/j.bios.2010.07.025

2924. Medina-Castillo AL, Mistlberger G, Fernandez-Sanchez JF, SeguraCarretero A, Klimant I, Fernandez-Gutierrez A. 2010. Novel Strategy To Design Magnetic, Molecular Imprinted Polymers with Well-Controlled Structure for the Application in Optical Sensors. Macromolecules 43: 55-61. DOI: 10.1021/ma902095s

2925. Ng SM, Narayanaswamy R. 2010. Demonstration of a simple, economical and practical technique utilising an imprinted polymer for metal ion sensing. Microchim. Acta 169: 303-311. DOI: 10.1007/s00604-010-0357-6

2926. Nguyen TH, Sun T, Grattan KTV, Hardwick SA. 2010. A fibre optic chemical sensor for the detection of cocaine. In Fourth European Workshop on Optical Fibre Sensors, Vol. 7653, Santos JL, Culshaw B, Lopez-Higuera JM, MacPherson WN (eds). SPIE: Porto, Portugal; Art. No. 76531V. DOI: $10.1117 / 12.866496$

2927. Pernites RB, Ponnapati RR, Advincula RC. 2010. Surface Plasmon Resonance (SPR) Detection of Theophylline via Electropolymerized Molecularly Imprinted Polythiophenes. Macromolecules 43: 9724-9735. DOI: 10.1021/ma101868y

2928. Queirós RB, Silva SO, Sales MGF, Noronha JP, Frazao O, Jorge PAS, Aguilar GG. 2010. Optical cavity fibre sensor for detection of microcystin-LR in water. Vol. 7653, Santos JL, Culshaw B, Lopez-Higuera JM, MacPherson WN (eds). SPIE: Porto, Portugal; Art. No. 76531N. DOI: 10.1117/12.865004 
2929. Riskin M, Tel-Vered R, Willner I. 2010. Imprinted Au-Nanoparticle Composites for the Ultrasensitive Surface Plasmon Resonance Detection of Hexahydro-1,3,5-trinitro-1,3,5-triazine (RDX). Adv. Mater. 22: 1387-1391. DOI: $10.1002 /$ adma.200903007

2930. Riskin M, Tel-Vered R, Frasconi M, Yavo N, Willner I. 2010. Stereoselective and Chiroselective Surface Plasmon Resonance (SPR) Analysis of Amino Acids by Molecularly Imprinted Au-Nanoparticle Composites. Chem. Eur. J. 16: 7114-7120. DOI: 10.1002/chem.200903215

2931. Sergeyeva TA, Gorbach LA, Slinchenko OA, Goncharova LA, Piletska OV, Brovko OO, Sergeeva LM, Elska GV. 2010. Towards development of colorimetric test-systems for phenols detection based on computationallydesigned molecularly imprinted polymer membranes. Mater. Sci. Eng.: C 30: 431-436. DOI: 10.1016/j.msec.2009.12.012

2932. Stringer RC, Gangopadhyay S, Grant SA. 2010. Detection of Nitroaromatic Explosives Using a Fluorescent-Labeled Imprinted Polymer. Anal. Chem. 82: 4015-4019. DOI: 10.1021/ac902838c

2933. Stringer RC, Gangopadhyay S, Grant SA. 2010. Use of dot-labeled imprinted polymer microparticles for detection of nitroaromatic compounds. In Advanced Environmental, Chemical, and Biological Sensing Technologies VII, Proceedings of the SPIE, Vol. 7673, Vo-Dinh T, Lieberman RA, Gauglitz G (eds). SPIE: Orlando, Florida, USA; 767304-767306. DOI: $10.1117 / 12.852913$

2934. Sunayama H, Ooya T, Takeuchi T. 2010. Fluorescent protein recognition polymer thin films capable of selective signal transduction of target binding events prepared by molecular imprinting with a post-imprinting treatment. Biosens. Bioelectron. 26: 458-462. DOI: 10.1016/j.bios.2010.07.091 
2935. Syu MJ, Hsu TJ, Lin ZK. 2010. Synthesis of Recognition Matrix from 4Methylamino-N-Allylnaphthal-Imide with Fluorescent Effect for the Imprinting of Creatinine. Anal. Chem. 82: 8821-8829. DOI: 10.1021/ac1011848

2936. Wan FW, Yu JH, Ge SG, Yan M, Yu KX, Zhang M. 2010. A high throughput chemiluminescence method based on molecularly imprinted sol-gel films for determination of sibutramine. Adv. Mat. Lett. 1: 164-169. DOI: 10.5185/amlett.2010.7138

2937. Wan FW, Yu JH, Dai P, Ge SG. 2010. Molecular ImprintingChemiluminescence Sensor for the Determination of Amoxicillin. Anal. Lett. 43: 1033-1045. DOI: 10.1080/00032710903491104

2938. Wu P, Yan XP. 2010. A simple chemical etching strategy to generate "ionimprinted" sites on the surface of quantum dots for selective fluorescence turn-on detecting of metal ions. Chem. Commun. 7046-7048. DOI: 10.1039/COCC01762K

2939. Xie CG, Zhou HK, Gao S, Li HF. 2010. Molecular imprinting method for online enrichment and chemiluminescent detection of the organophosphate pesticide triazophos. Microchim. Acta 171: 355-362. DOI: 10.1007/s00604010-0447-5

2940. Yang LB, Ma L, Chen GY, Liu JH, Tian ZQ. 2010. Ultrasensitive SERS Detection of TNT by Imprinting Molecular Recognition Using a New Type of Stable Substrate. Chem. Eur. J. 16: 12683-12693. DOI: 10.1002/chem.201001053

2941. Yang Y, Lai EPC. 2010. An investigation of porous structure in molecularly imprinted polymer for sensor development: Non-linear fluorescence quenching of $17 \alpha$-estradiol bound inside MIP submicron particles by sodium nitrite and methacrylamide. J. Photochem. Photobiol. A 213: 123-128. DOI: 10.1016/j.jphotochem.2010.05.010 
2942. Yu JH, Wan FW, Zhang CC, Yan M, Zhang XN, Wang SW. 2010. Molecularly imprinted polymeric microspheres for determination of bovine serum albumin based on flow injection chemiluminescence sensor. Biosens. Bioelectron. 26: 632-637. DOI: 10.1016/j.bios.2010.07.009

2943. Casey CN, Campbell SE, Gibson UJ. 2010. Phenylalanine detection using matrix assisted pulsed laser evaporation of molecularly imprinted amphiphilic block copolymer films. Biosens. Bioelectron. 26: 703-709. DOI: 10.1016/j.bios.2010.06.060

2944. Barrios CA, Zhenhe C, Navarro-Villoslada F, López-Romero D, MorenoBondi MC. 2011. Molecularly imprinted polymer diffraction grating as labelfree optical bio(mimetic)sensor. Biosens. Bioelectron. 26: 2801-2804. DOI: 10.1016/j.bios.2010.11.009

2945. Choi SW, Chang HJ, Lee N, Chun HS. 2011. A Surface Plasmon Resonance Sensor for the Detection of Deoxynivalenol Using a Molecularly Imprinted Polymer. Sensors 11: 8654-8664. DOI: 10.3390/s110908654

2946. Ertürk G, Uzun L, Tümer MA, Say RI, Denizli A. 2011. Fab fragments imprinted SPR biosensor for real-time human immunoglobulin $G$ detection. Biosens. Bioelectron. 28: 97-104. DOI: 10.1016/j.bios.2011.07.004

2947. Ge SG, Lu JJ, Ge L, Yan M, Yu JH. 2011. Development of a novel deltamethrin sensor based on molecularly imprinted silica nanospheres embedded CdTe quantum dots. Spectrochim. Acta, A 79: 1704-1709. DOI: 10.1016/j.saa.2011.05.040

2948. Ge SG, Zhang CC, Yu F, Yan M, Yu JH. 2011. Layer-by-layer self-assembly CdTe quantum dots and molecularly imprinted polymers modified chemiluminescence sensor for deltamethrin detection. Sens. Actuators $B$ 156: 222-227. DOI: 10.1016/j.snb.2011.04.024 
2949. Griffete N, Frederich H, Maître A, Schwob C, Ravaine S, Carbonnier B, Chehimi MM, Mangeney C. 2011. Introduction of a planar defect in a molecularly imprinted photonic crystal sensor for the detection of bisphenol A. J. Colloid Interface Sci. 364: 18-23. DOI: 10.1016/j.jcis.2011.07.028

2950. Gupta G, Bhaskar ASB, Tripathi BK, Pandey P, Boopathi M, Rao PVL, Singh B, Vijayaraghavan R. 2011. Supersensitive detection of T-2 toxin by the in situ synthesized $\pi$-conjugated molecularly imprinted nanopatterns. An in situ investigation by surface plasmon resonance combined with electrochemistry. Biosens. Bioelectron. 26: 2534-2540. DOI: 10.1016/j.bios.2010.10.050

2951. Hao HX, Zhou H, Chang J, Zhu J, Wei TX. 2011. Molecularly imprinted polymers for highly sensitive detection of morphine using surface plasmon resonance spectroscopy. Chin. Chem. Lett. 22: 477-480. DOI: 10.1016/j.cclet.2010.11.004

2952. Harz S, Schimmelpfennig M, Tse Sum Bui B, Marchyk N, Haupt K, Feller $\mathrm{KH}$. 2011. Fluorescence optical spectrally resolved sensor based on molecularly imprinted polymers and microfluidics. Engineering in Life Sciences 11: 559-565. DOI: 10.1002/elsc.201000222

2953. Holthoff EL, Stratis-Cullum DN, Hankus ME. 2011. A Nanosensor for TNT Detection Based on Molecularly Imprinted Polymers and Surface Enhanced Raman Scattering. Sensors 11: 2700-2714. DOI: 10.3390/s110302700

2954. Hong CC, Lin CC, Hong CL, Chang PH. 2011. Low cost and high specific anesthetic biosensors with functionally imprinted nanocavities on polymer films. In Proceedings of the 2011 16th International Solid-State Sensors, Actuators and Microsystems Conference (TRANSDUCERS), 1196-1199. DOI: 10.1109/TRANSDUCERS.2011.5969326 
2955. Inoue J, Ooya T, Takeuchi T. 2011. Protein imprinted TiO2-coated quantum dots for fluorescent protein sensing prepared by liquid phase deposition. Soft Matter 7: 9681-9684. DOI: 10.1039/C1SM05088E

2956. Krupadam RJ. 2011. An efficient fluorescent polymer sensing material for detection of traces of benzo[a]pyrene in environmental samples. Environ. Chem. Lett. 9: 389-395. DOI: 10.1007/s10311-010-0291-1

2957. Lai EPC, Yang Y. 2011. Optimization of Molecularly Imprinted Polymer Method for Rapid Screening of $17 \alpha$-Estradiol in Water by Fluorescence Quenching. Int. J. Anal. Chem. 2011: Article ID 214747. DOI: $10.1155 / 2011 / 214747$

2958. Li JH, Zhang Z, Xu SF, Chen LX, Zhou N, Xiong H, Peng HL. 2011. Labelfree colorimetric detection of trace cholesterol based on molecularly imprinted photonic hydrogels. J. Mater. Chem. 21: 19267-19274. DOI: 10.1039/C1JM14230E

2959. Liu P, Liu RY, Guan GJ, Jiang CL, Wang SH, Zhang ZP. 2011. Surfaceenhanced Raman scattering sensor for theophylline determination by molecular imprinting on silver nanoparticles. Analyst 136: 4152-4158. DOI: 10.1039/C1AN15318H

2960. Liu RY, Guan GJ, Wang SH, Zhang ZP. 2011. Core-shell nanostructured molecular imprinting fluorescent chemosensor for selective detection of atrazine herbicide. Analyst 136: 184-190. DOI: 10.1039/COAN00447B

2961. Ng SM, Narayanaswamy R. 2011. Molecularly imprinted polymers as optical sensing receptors: Correlation between analytical signals and binding isotherms. Anal. Chim. Acta 703: 226-233. DOI: 10.1016/j.aca.2011.07.032

2962. Pernites R, Ponnapati R, Felipe MJ, Advincula R. 2011. Electropolymerization molecularly imprinted polymer (E-MIP) SPR sensing of drug molecules: Pre-polymerization complexed terthiophene and 
carbazole electroactive monomers. Biosens. Bioelectron. 26: 2766-2771. DOI: 10.1016/j.bios.2010.10.027

2963. Queirós RB, Silva SO, Noronha JP, Fraz $\pi$ O O, Jorge P, Aguilar G, Marques PVS, Sales MGF. 2011. Microcystin-LR detection in water by the FabryPérot interferometer using an optical fibre coated with a sol-gel imprinted sensing membrane. Biosens. Bioelectron. 26: 3932-3937. DOI: 10.1016/j.bios.2011.03.015

2964. Riskin M, Ben-Amram Y, Tel-Vered R, Chegel V, Almog J, Willner I. 2011. Molecularly Imprinted Au Nanoparticles Composites on Au Surfaces for the Surface Plasmon Resonance Detection of Pentaerythritol Tetranitrate, Nitroglycerin, and Ethylene Glycol Dinitrate. Anal. Chem. 83: 3082-3088. DOI: $10.1021 / \mathrm{ac} 1033424$

2965. Sainz-Gonzalo FJ, Medina-Castillo AL, Fernández-Sánchez JF, FernándezGutiérrez A. 2011. Synthesis and characterization of a molecularly imprinted polymer optosensor for TEXs-screening in drinking water. Biosens. Bioelectron. 26: 3331-3338. DOI: 10.1016/j.bios.2011.01.008

2966. Sainz-Gonzalo FJ, Fernandez-Sanchez JF, Fernandez-Gutierrez A. 2011. The development of a screening molecularly imprinted polymer optosensor for detecting xylenes in water samples. Microchem. J. 99: 278-282. DOI: 10.1016/j.microc.2011.05.017

2967. Sener G, Uzun L, Say RI, Denizli A. 2011. Use of molecular imprinted nanoparticles as biorecognition element on surface plasmon resonance sensor. Sens. Actuators B 160: 791-799. DOI: 10.1016/j.snb.2011.08.064

2968. Smith CB, Anderson JE, Edwards JD, Kam KC. 2011. In Situ SurfaceEtched Bacterial Spore Detection Using Dipicolinic Acid - Europium - Silica Nanoparticle Bioreporters. Appl. Spectros. 65: 866-875. 
2969. Valero-Navarro A, Medina-Castillo AL, Fernandez-Sanchez JF, FernándezGutiérrez A. 2011. Synthesis of a novel polyurethane-based-magnetic imprinted polymer for the selective optical detection of 1-naphthylamine in drinking water. Biosens. Bioelectron. 26: 4520-4525. DOI: 10.1016/j.bios.2011.05.013

2970. Wang J, Banerji S, Menegazzo N, Peng W, Zou QJ, Booksh KS. 2011. Glucose detection with surface plasmon resonance spectroscopy and molecularly imprinted hydrogel coatings. Talanta 86: 133-141. DOI: 10.1016/j.talanta.2011.08.046

2971. Wei CP, Zhou HQ, Chen CB, Li ZC, Zhou J. 2011. On-Line Monitoring 1HIndole-3-Acetic Acid in Plant Tissues Using Molecular Imprinting Monolayer Techniques on a Surface Plasmon Resonance Sensor. Anal. Lett. 44: 29112921. DOI: $10.1080 / 00032719.2011 .582552$

2972. Wei CP, Zhou HQ, Zhou J. 2011. Ultrasensitively sensing acephate using molecular imprinting techniques on a surface plasmon resonance sensor. Talanta 83: 1422-1427. DOI: 10.1016/j.talanta.2010.11.034

2973. Wei QQ, Wei TX. 2011. A novel method to prepare SPR sensor chips based on photografting molecularly imprinted polymer. Chin. Chem. Lett. 22: 721724. DOI: 10.1016/j.cclet.2010.11.024

2974. Wei QQ, Wei TX, Wu XG. 2011. Ultrasensitive and Highly Selective Detection of Testosterone Using a Surface Plasmon Resonance Sensor Combined with Molecularly Imprinted Films. Chem. Lett. 40: 132-133. DOI: 10.1246/cl.2011.132

2975. Wu J, Fu XC, Xie CG, Yang M, Fang W, Gao S. 2011. TiO2 nanoparticlesenhanced luminol chemiluminescence and its analytical applications in organophosphate pesticide imprinting. Sens. Actuators B 160: 511-516. DOI: 10.1016/j.snb.2011.08.019 
2976. Xie CG, Gao S, Zhou HK, Li HF. 2011. Chemiluminescence sensor for sulfonylurea herbicide using molecular imprinted microspheres as recognition element. Luminescence 26: 271-279. DOI: 10.1002/bio.1224

2977. Xie CG, Li HF, Li SQ, Gao S. 2011. Surface molecular imprinting for chemiluminescence detection of the organophosphate pesticide chlorpyrifos. Microchim. Acta 174: 311-320. DOI: 10.1007/s00604-011-0626-z

2978. Yang LZ, Chen ZL. 2011. Direct Evaluation of Ligand-Receptor Interaction by a Novel Imaging Model Based on Fluorescent Silica Nanoparticle as Ligand Probe and Molecular Imprinting Polymer as Artificial Receptor. Anal. Lett. 44: 687-697. DOI: 10.1080/00032711003783085

2979. Zhang W, He XW, Chen Y, Li WY, Zhang YK. 2011. Composite of CdTe quantum dots and molecularly imprinted polymer as a sensing material for cytochrome c. Biosens. Bioelectron. 26: 2553-2558. DOI: 10.1016/j.bios.2010.11.004

2980. Zhao PN, Liu SQ, Yu JH. 2011. A Novel Molecularly Imprinted Core-Shell Chemiluminescence Sensor: Preparation and Pendimethalin Recognition. J. Inorg. Organomet. Polym. Mater. 21: 846-851. DOI: 10.1007/s10904-0119599-y

2981. Zhao PN, Yan M, Zhang CC, Peng RX, Ma DS, Yu JH. 2011. Determination of glyphosate in foodstuff by one novel chemiluminescence-molecular imprinting sensor. Spectrochim. Acta, A 78: 1482-1486. DOI: 10.1016/j.saa.2011.01.037

2982. Zhao WT, Chen ZH, Xue B, Sun LQ, Luo AQ. 2011. A Biomimetic Sensor for Fast Lysozyme Detection. In Advanced Materials, Advanced Materials Research, Vol. 239 - 242, Cao Z, Cao XQ, Sun LX, He YH (eds). Scientific.net: 283-287. DOI: 10.4028/www.scientific.net/AMR.239-242.283 
2983. Zheng R, Cameron BD. 2011. Development of a molecularly imprinted polymer based surface plasmon resonance sensor for theophylline monitoring. In Plasmonics in Biology and Medicine VIII, Proceedings of the SPIE, Vol. 7911, Vo-Dinh T, Lakowicz JR (eds). SPIE: Article No. 79110F. DOI: $10.1117 / 12.875312$

2984. Zhu W, Tao SY, Tao CA, Li WN, Lin CX, Li M, Wen YQ, Li GT. 2011. Hierarchically Imprinted Porous Films for Rapid and Selective Detection of Explosives. Langmuir 27: 8451-8457. DOI: 10.1021/la201055b

2985. Dutta P, Pernites RB, Danda C, Advincula RC. 2011. SPR Detection of Dopamine Using Cathodically Electropolymerized, Molecularly Imprinted Poly-p-aminostyrene Thin Films. Macromol. Chem. Phys. 212: 2439-2451. DOI: 10.1002/macp.201100365

2986. Lee GB, Chen SJ, Huang SC, Chien FC, Chen WJ. 2005. Microfluidic systems using localized molecular imprinting polymers for detection of nanoscale bio-molecules based on surface plasmon resonance. In Proceedings of 2005 5th IEEE Conference on Nanotechnology, IEEE: New York; 841844. DOI: 10.1109/NANO.2005.1500664

2987. González J, Campo JC, Valledor M, Ferrero FJ, Traviesa JM, Costa JM, Pereiro R, Sanz-Medel A. 2008. Measurement of polycyclic aromatic hydrocarbons by using molecularly imprinted polymers. In Instrumentation and Measurement Technology Conference Proceedings, IEEE: 1194-1197. DOI: 10.1109/IMTC.2008.4547221

2988. Lin Cl, Joseph AK, Chang CK, Lee YD. 2004. Molecularly imprinted polymeric film on semiconductor nanoparticles - Analyte detection by quantum dot photoluminescence. J. Chromatogr. A 1027: 259-262. DOI: 10.1016/j.chroma.2003.10.037

2989. Huang SC, Chien FC, Lin JS, Chen SJ, Chen WJ, Lee GB. 2005. A microfluidic chip integrated with molecular imprinting polymers for surface 
plasmon resonance detection. In TRANSDUCERS '05 Digest of Technical Papers, IEEE: NEW YORK; 1776-1779.

2990. Li X, Husson SM. 2006. Adsorption of dansylated amino acids on molecularly imprinted surfaces: A surface plasmon resonance study. Biosens. Bioelectron. 22: 336-348. DOI: 10.1016/j.bios.2006.04.016

2991. Niu WF, Nie F, Lu JR. 2006. Chemiluminescence sensor for the determination of perphenazine based on a molecular imprinted polymer. Anal. Sci. 22: 971-975. DOI: 10.2116/analsci.22.971

2992. Matsui J, Megumi T, Akamatsu T, Nawafune H, Tamaki K, Sugimoto N. 2009. Molecularly imprinted nanocomposites for highly sensitive SPR detection of a non-aqueous atrazine sample. Analyst 134: 80-86. DOI: 10.1039/b803350a

2993. Nie F, Hao L, Gao M, Wu YC, Li XS, Yu S. 2011. Cu2+-imprinted crosslinked chitosan resin as micro-column packing materials for online chemiluminescence determination of trace copper. Luminescence 26: 289295. DOI: $10.1002 /$ bio. 1226

2994. Raitman OA, Chegel VI, Kharitonov AB, Zayats M, Katz E, Willner I. 2004. Analysis of $\mathrm{NAD}(\mathrm{P})^{+}$and $\mathrm{NAD}(\mathrm{P}) \mathrm{H}$ cofactors by means of imprinted polymers associated with Au surfaces: A surface plasmon resonance study. Anal. Chim. Acta 504: 101-111. DOI: 10.1016/S0003-2670(03)00511-7

2995. Wu Z, Hu X, Tao CA, Li Y, Liu J, Yang C, Shen D, Li G. 2008. Direct and label-free detection of cholic acid based on molecularly imprinted photonic hydrogels. J. Mater. Chem. 18: 5452-5458. DOI: 10.1039/b811189h

2996. Kriz D, Kempe M, Mosbach K. 1995. Introduction Of Molecularly Imprinted Polymers As Recognition Elements In Conductometric Chemical Sensors. In Proceedings of the 8th International Conference on Solid-state Sensors and Actuators, and Eurosensors IX, Vol. 1, 878-881. 
2997. Mirsky VM, Hirsch T, Piletsky SA, Wolfbeis OS. 1999. A spreader-bar approach to molecular architecture: formation of stable artificial chemoreceptors. Angew. Chem. Int. Edit. 38: 1108-1110. DOI: 10.1002/(SICI)1521-3773(19990419)38:8<1108::AID-ANIE1108>3.0.CO;2C

2998. Shinohara H, Aizawa M, Shirakawa H. 1986. lon-sieving of electrosynthesized polypyrrole films. J. Chem. Soc. Chem. Commun. 87-88. DOI: $10.1039 / \mathrm{C} 39860000087$

2999. Blanco-López MC, Gutiérrez-Fernández S, Lobo-Castañón MJ, MirandaOrdieres AJ, Tuñón-Blanco P. 2004. Electrochemical sensing with electrodes modified with molecularly imprinted polymer films. Anal. Bioanal. Chem. 378: 1922-1928. DOI: 10.1007/s00216-003-2330-2

3000. Blanco-López MC, Fernández-Llano L, Lobo-Castañón MJ, MirandaOrdieres AJ, Tuñón-Blanco P. 2004. Voltammetry of diclofenac at graphite, carbon composites, and molecularly imprinted polymer-composite electrodes. Anal. Lett. 37: 915-927. DOI: 10.1081/AL-120030287

3001. Chmurski K, Temeriusz A, Bilewicz R. 2004. Measurement of ibuprofen binding to mixed monolayers containing $\beta$-cyclodextrin active sites. $J$. Inclusion Phenom. Macrocyl. Chem. 49: 187-191. DOI: 10.1023/B:JIPH.0000031134.27231.e5

3002. Feng L, Liu Y, Tan Y, Hu J. 2004. Biosensor for the determination of sorbitol based on molecularly imprinted electro synthesized polymers. Biosens. Bioelectron. 19: 1513-1519. DOI: 10.1016/j.bios.2003.12.007

3003. Gong JL, Gong FC, Kuang Y, Zeng GM, Shen GL, Yu RQ. 2004. Capacitive chemical sensor for fenvalerate assay based on electropolymerized molecularly imprinted polymer as the sensitive layer. Anal. Bioanal. Chem. 379: 302-307. DOI: 10.1007/s00216-004-2568-3 
3004. Guan JG, Miao YQ, Zhang QJ. 2004. Impedimetric biosensors. J. Biosci. Bioeng. 97: 219-226. DOI: 10.1016/S1389-1723(04)70195-4

3005. Hattori K, Yoshimi Y, Ito T, Hirano K, Kohori F, Sakai K. 2004. Effect of electrostatic interactions on gate effect in molecularly imprinted polymers. Electrochemistry 72: 508-510.

3006. Huan SY, Chu H, Jiao CX, Zeng GM, Huang GH, Shen GL, Yu RQ. 2004. Selective electrochemical molecular recognition of benzenediol isomers using molecularly imprinted $\mathrm{TiO}_{2}$ film electrodes. Anal. Chim. Acta 506: 31 39. DOI: 10.1016/j.aca.2003.10.082

3007. Huan SY, Shen GL, Yu RQ. 2004. Enantioselective recognition of amino acid by differential pulse voltammetry in molecularly imprinted monolayers assembled on $\mathrm{Au}$ electrodes. Electroanalysis 16: 1019-1023. DOI: 10.1002/elan.200302879

3008. Kitade T, Kitamura K, Konishi T, Takegami S, Okuno T, Ishikawa M, Wakabayashi M, Nishikawa K, Muramatsu Y. 2004. Potentiometric immunosensor using artificial antibody based on molecularly imprinted polymers. Anal. Chem. 76: 6802-6807. DOI: 10.1021/ac040098q

3009. Liao HP, Zhang ZH, Li H, Nie LH, Yao SZ. 2004. Preparation of the molecularly imprinted polymers-based capacitive sensor specific for tegafur and its characterization by electrochemical impedance and piezoelectric quartz crystal microbalance. Electrochim. Acta 49: 4101-4107. DOI: 10.1016/j.electacta.2004.04.003

3010. Parmpi P, Kofinas P. 2004. Biomimetic glucose recognition using molecularly imprinted polymer hydrogels. Biomaterials 25: 1969-1973. DOI: 10.1016/j.biomaterials.2003.08.025

3011. Piletsky S, Piletska E, Karim K, Foster G, Legge C, Turner A. 2004. Custom synthesis of molecular imprinted polymers for biotechnological application - 
Preparation of a polymer selective for tylosin. Anal. Chim. Acta 504: 123130. DOI: 10.1016/S0003-2670(03)00814-6

3012. Ramanaviciene A, Ramanavicius A. 2004. Molecularly imprinted polypyrrole-based synthetic receptor for direct detection of bovine leukemia virus glycoproteins. Biosens. Bioelectron. 20: 1076-1082. DOI: 10.1016/j.bios.2004.05.014

3013. Ramanaviciene A, Finkelsteinas A, Ramanavicius A. 2004. Molecularly Imprinted Polypyrrole for Sensor Design. Mater. Sci. 10: 18-23.

3014. Weetall HH, Rogers KR. 2004. Preparation and characterization of molecularly imprinted electropolymerized carbon electrodes. Talanta 62: 329-335. DOI: 10.1016/j.talanta.2003.07.014

3015. Yang L, Wei WZ, Xia JJ, Tao H. 2004. Artificial receptor layer for herbicide detection based on electrosynthesized molecular imprinting technique and capacitive transduction. Anal. Lett. 37: 2303-2319. DOI: 10.1081/AL200028089

3016. Yin F. 2004. Capacitive sensors using electropolymerized ophenylenediamine film doped with ion-pair complex as selective elements for the determination of pentoxyverine. Talanta 63: 641-646. DOI: 10.1016/j.talanta.2003.12.004

3017. Yin F, Xu XX. 2004. Construction and analytical application of a novel ionselective capacitive sensor for determination of cinchonine. Anal. Lett. 37: 3129-3147. DOI: 10.1081/AL-200040283

3018. Zhou YX, Yu B, Shiu E, Levon K. 2004. Potentiometric sensing of chemical warfare agents: Surface imprinted polymer integrated with an indium tin oxide electrode. Anal. Chem. 76: 2689-2693. DOI: 10.1021/ac035072y 
3019. Chou LCS, Liu CC. 2005. Development of a molecular imprinting thick film electrochemical sensor for cholesterol detection. Sens. Actuators B 110: 204-208. DOI: 10.1016/j.snb.2005.01.031

3020. Gómez-Caballero A, Goicolea MA, Barrio RJ. 2005. Paracetamol voltammetric microsensors based on electrocopolymerized-molecularly imprinted film modified carbon fiber microelectrodes. Analyst 130: 10121018. DOI: $10.1039 / \mathrm{b} 502827 \mathrm{~b}$

3021. Ho KC, Yeh WM, Tung TS, Liao JY. 2005. Amperometric detection of morphine based on poly(3,4-ethylenedioxythiophene) immobilized molecularly imprinted polymer particles prepared by precipitation polymerization. Anal. Chim. Acta 542: 90-96. DOI: 10.1016/j.aca.2005.02.036

3022. Kindschy LM, Alocilja EC. 2005. A molecularly imprinted polymer on indium tin oxide and silicon. Biosens. Bioelectron. 20: 2163-2167. DOI: 10.1016/j.bios.2004.08.028

3023. Kirsch N, Honeychurch KC, Hart JP, Whitcombe MJ. 2005. Voltammetric determination of urinary 1-hydroxypyrene using molecularly imprinted polymer-modified screen-printed carbon electrodes. Electroanalysis 17: 571578. DOI: 10.1002/elan.200403131

3024. Li CY, Wang CF, Guan B, Zhang YY, Hu SS. 2005. Electrochemical sensor for the determination of parathion based on p-tert-butylcalix[6]arene-1,4crown-4 sol-gel film and its characterization by electrochemical methods. Sens. Actuators B 107: 411-417. DOI: 10.1016/j.snb.2004.10.037

3025. Li CY, Wang CF, Wang CH, Hu SS. 2005. Construction of a novel molecularly imprinted sensor for the determination of O,O-dimethyl-(2,4dichlorophenoxyacetoxyl) (3'-nitrophenyl)methinephosphonate. Anal. Chim. Acta 545: 122-128. DOI: 10.1016/j.aca.2005.04.085 
3026. Liang HJ, Ling TR, Rick JF, Chou TC. 2005. Molecularly imprinted electrochemical sensor able to enantroselectivly recognize $\mathrm{d}$ and I-tyrosine. Anal. Chim. Acta 542: 83-89. DOI: 10.1016/j.aca.2005.02.007

3027. Prasad BB, Lakshmi D. 2005. Barbituric acid sensor based on molecularly imprinted polymer-modified hanging mercury drop electrode. Electroanalysis 17: 1260-1268. DOI: 10.1002/elan.200403226

3028. Sheridan EM, Breslin CB. 2005. Enantioselective detection of D- and Lphenylalanine using optically active polyaniline. Electroanalysis 17: 532-537. DOI: 10.1002/elan.200403192

3029. Yang L, Wei WZ, Xia JJ, Tao H, Yang PH. 2005. Capacitive biosensor for glutathione detection based on electropolymerized molecularly imprinted polymer and kinetic investigation of the recognition process. Electroanalysis 17: 969-977. DOI: 10.1002/elan.200403195

3030. Yeh WM, Ho KC. 2005. Amperometric morphine sensing using a molecularly imprinted polymer-modified electrode. Anal. Chim. Acta 542: 7682. DOI: 10.1016/j.aca.2005.01.071

3031. Zhou YX, Yu B, Levon K. 2005. Potentiometric sensor for dipicolinic acid. Biosens. Bioelectron. 20: 1851-1855. DOI: 10.1016/j.bios.2004.05.005

3032. Binet $C$, Bourrier $D$, Dilhan $M$, Estève $D$, Ferrère $S$, Garrigue JC, Granier $H$, Lattes A, Gué AM, Mauzac M, Mingotaud AF. 2006. First approach to the use of liquid crystal elastomers for chemical sensors. Talanta 69: 757-762. DOI: 10.1016/j.talanta.2005.11.027

3033. D'Agostino G, Alberti G, Biesuz R, Pesavento M. 2006. Potentiometric sensor for atrazine based on a molecular imprinted membrane. Biosens. Bioelectron. 22: 145-152. DOI: 10.1016/j.bios.2006.05.014

3034. Guzmán-Vázquez de Prada A, Reviejo AJ, Pingarrón JM. 2006. A method for the quantification of low concentration sulfamethazine residues in milk 
based on molecularly imprinted clean-up and surface preconcentration at a Nafion-modified glassy carbon electrode. J. Pharm. Biomed. Anal. 40: 281286. DOI: 10.1016/j.jpba.2005.07.022

3035. Lakshmi D, Sharma PS, Prasad BB. 2006. Development of uric acid sensor based on molecularly imprinted polymer-modified hanging mercury drop electrode. Electroanalysis 18: 918-927. DOI: 10.1002/elan.200603478

3036. Lakshmi D, Prasad BB, Sharma PS. 2006. Creatinine sensor based on a molecularly imprinted polymer-modified hanging mercury drop electrode. Talanta 70: 272-280. DOI: 10.1016/j.talanta.2006.02.038

3037. Li CY, Wang CF, Wang CH, Hu SS. 2006. Development of a parathion sensor based on molecularly imprinted nano-TiO2 self-assembled film electrode. Sens. Actuators B 117: 166-171. DOI: 10.1016/j.snb.2005.11.019

3038. Liu K, Wei WZ, Zeng JX, Liu XY, Gao YP. 2006. Application of a novel electrosynthesized polydopamine-imprinted film to the capacitive sensing of nicotine. Anal. Bioanal. Chem. 385: 724-729. DOI: 10.1007/s00216-0060489-z

3039. Luo N, Hatchett DW, Rogers KR. 2006. Impact of Polycyclic Aromatic Hydrocarbons on the Electrochemical Responses of a Ferricyanide Probe at Template-Modified Self-Assembled Monolayers on Gold Electrodes. Electroanalysis 18: 2180-2187. DOI: 10.1002/elan.200603655

3040. Prasad K, Kala R, Rao TP, Naidu GRK. 2006. Ion imprinted polymer based ion-selective electrode for the trace determination of dysprosium(III) ions. Anal. Chim. Acta 566: 69-74. DOI: 10.1016/j.aca.2006.02.064

3041. Rick J, Chou TC. 2006. Amperometric protein sensor - fabricated as a polypyrrole, poly-aminophenylboronic acid bilayer. Biosens. Bioelectron. 22: 329-335. DOI: 10.1016/j.bios.2006.04.007 
3042. Sergeyeva TA, Panasyuk-Delaney TL, Piletska OV, Piletsky SA, El'Skaya AV. 2006. Capacitive sensor for environmental monitoring based on thin films of molecularly imprinted polymers. Computational modeling for optimization of the polymers-biomimics composition. Ukr. Biokhim. Zh. 78: 121-130.

3043. Suedee R, Intakong W, Dickert FL. 2006. Molecularly imprinted polymermodified electrode for on-line conductometric monitoring of haloacetic acids in chlorinated water. Anal. Chim. Acta 569: 66-75. DOI: 10.1016/j.aca.2006.03.081

3044. Syu MJ, Chiu TC, Lai CY, Chang YS. 2006. Amperometric detection of bilirubin from a micro-sensing electrode with a synthetic bilirubin imprinted poly(MAA-co-EGDMA) film. Biosens. Bioelectron. 22: 550-557. DOI: 10.1016/j.bios.2006.07.035

3045. Tarley CRT, Segatelli MG, Kubota LT. 2006. Amperometric determination of chloroguaiacol at submicromolar levels after on-line preconcentration with molecularly imprinted polymers. Talanta 69: 259-266. DOI: 10.1016/j.talanta.2005.10.001

3046. Ulyanova YV, Blackwell AE, Minteer SD. 2006. Poly(methylene green) employed as molecularly imprinted polymer matrix for electrochemical sensing. Analyst 131: 257-261. DOI: 10.1039/b510878k

3047. Wang CH, Li CY, Wang F, Wang CF. 2006. Phosphonate electrochemical recognition by molecularly imprinted deposited film. Appl. Surf. Sci. 253: 2282-2288. DOI: 10.1016/j.apsusc.2006.04.050

3048. Wang ZH, Kang JW, Liu XY, Ma YJ. 2006. Voltammetric response of 2,4-Dmolecularly imprinted film modified glassy carbon electrodes. Indian $\mathrm{J}$. Chem. Sect. A: Inorg., Bio-inorg., Phys., Theor. Anal. Chem. 45: 1848-1851. 
3049. Wu ZY, Zhang XL, Yang YH, Shen GL, Yu RQ. 2006. A sensitive nicotine sensor based on molecularly imprinted electropolymer of $o$-aminophenol. Frontiers Chem. Chin. 1: 183-187. DOI: 10.1007/s11458-006-0004-2

3050. Zhang ZH, Nie LH, Yao SZ. 2006. Electrodeposited sol-gel-imprinted sensing film for cytidine recognition on Au-electrode surface. Talanta 69: 435-442. DOI: 10.1016/j.talanta.2005.10.008

3051. Baghel A, Boopathi M, Singh B, Pandey P, Mahato TH, Gutch PK, Sekhar K. 2007. Synthesis and characterization of metal ion imprinted nano-porous polymer for the selective recognition of copper. Biosens. Bioelectron. 22: 3326-3334. DOI: 10.1016/j.bios.2007.01.016

3052. Chen PY, Ho KC. 2007. Fabrication of molecularly imprinted uric acid biosensors based on a novel amine-imide type conducting polymer. In 2007 NSTI Nanotechnology Conference and Trade Show - NSTI Nanotech 2007, Technical Proceedings, Vol. 2, 477-480.

3053. Delaney TL, Zimin D, Rahm M, Weiss D, Wolfbeis OS, Mirsky VM. 2007. Capacitive Detection in Ultrathin Chemosensors Prepared by Molecularly Imprinted Grafting Photopolymerization. Anal. Chem. 79: 3220-3225. DOI: 10.1021/ac062143v

3054. Fuchiwaki Y, Shimizu A, Kubo I. 2007. 6-chloro-N,N-diethyl-1,3,5-triazine2,4-diamine (CAT) sensor based on biomimetic recognition utilizing a molecularly imprinted artificial receptor. Anal. Sci. 23: 49-53. DOI: 10.2116/analsci.23.49

3055. Fuchiwaki Y, Sasaki N, Kubo I. 2007. Development of an electrochemical sensing system for 6-chloro-N,N-diethyl-1,3,5-triazine-2,4-diamine (CAT) utilizing an amalgamated gold electrode and artificial sensor receptor. Electrochemistry 75: 709-714. 
3056. Gao N, Xu Z, Wang F, Dong SJ. 2007. Sensitive Biomimetic Sensor Based on Molecular Imprinting at Functionalized Indium Tin Oxide Electrodes. Electroanalysis 19: 1655-1660. DOI: 10.1002/elan.200703919

3057. Gómez-Caballero A, Unceta N, Goicolea MA, Barrio RJ. 2007. Voltammetric Determination of Metamitron with an Electrogenerated Molecularly Imprinted Polymer Microsensor. Electroanalysis 19: 356-363. DOI: 10.1002/elan.200603731

3058. Guzmán-Vázquez de Prada A, Loaiza OA, Serra B, Morales D, MartínezRuiz P, Reviejo AJ, Pingarrón JM. 2007. Molecularly imprinted polymer solid-phase extraction coupled to square wave voltammetry at carbon fibre microelectrodes for the determination of fenbendazole in beef liver. Anal. Bioanal. Chem. 388: 227-234. DOI: 10.1007/s00216-007-1233-z

3059. Hantash J, Bartlett A, Oldfield P, Dénès G, O'Rielly R, David D. 2007. Application of an in-line imprinted polymer column in a potentiometric flowinjection chemical sensor to the determination of the carbamate pesticide carbaryl in complex biological matrices. Anal. Bioanal. Chem. 387: 351-357. DOI: 10.1007/s00216-006-0898-z

3060. Heitzmann M, Bucher C, Moutet JC, Pereira E, Rivas BL, Royal G, SaintAman E. 2007. Complexation of poly(pyrrole-EDTA like) film modified electrodes: Application to metal cations electroanalysis. Electrochim. Acta 52: 3082-3087. DOI: 10.1016/j.electacta.2006.09.041

3061. Huang CY, Syu MJ, Chang YS, Chang CH, Chou TC, Liu BD. 2007. A portable potentiostat for the bilirubin-specific sensor prepared from molecular imprinting. Biosens. Bioelectron. 22: 1694-1699. DOI: 10.1016/j.bios.2006.07.036

3062. Huang HC, Huang SY, Lin Cl, Lee YD. 2007. A multi-array sensor via the integration of acrylic molecularly imprinted photoresists and 
ultramicroelectrodes on a glass chip. Anal. Chim. Acta 582: 137-146. DOI: 10.1016/j.aca.2006.09.009

3063. Ju MJ, Yang DH, Takahara N, Hayashi K, Toko K, Lee SW, Kunitake T. 2007. Landmine detection: Improved binding of 2,4-dinitrotoluene in a $\gamma$ $\mathrm{CD} /$ metal oxide matrix and its sensitive detection via a cyclic surface polarization impedance (cSPI) method. Chem. Commun. 2630-2632. DOI: $10.1039 / \mathrm{b} 703132 \mathrm{~g}$

3064. Kindschy LM, Alocilja EC. 2007. Development of a molecularly imprinted biomimetic electrode. Sensors 7: 1630-1642. DOI: 10.3390/s7081612

3065. Lakshmi D, Sharma PS, Prasad BB. 2007. Imprinted polymer-modified hanging mercury drop electrode for differential pulse cathodic stripping voltammetric analysis of creatine. Biosens. Bioelectron. 22: 3302-3308. DOI: 10.1016/j.bios.2006.12.011

3066. Li XZ, Sun YP. 2007. Evaluation of ionic imprinted polymers by electrochemical recognition of rare earth ions. Hydrometallurgy 87: 63-71. DOI: 10.1016/j.hydromet.2007.02.003

3067. Luo N, Hatchett DW, Rogers KR. 2007. Recognition of Pyrene Using Molecularly Imprinted Electrochemically Deposited Poly(2mercaptobenzimidazole) or Poly(resorcinol) on Gold Electrodes. Electroanalysis 19: 2117-2124. DOI: 10.1002/elan.200703930

3068. Metilda P, Prasad K, Kala R, Gladis JM, Rao TP, Naidu GRK. 2007. Ion imprinted polymer based sensor for monitoring toxic uranium in environmental samples. Anal. Chim. Acta 582: 147-153. DOI: 10.1016/j.aca.2006.08.052

3069. Namvar A, Warriner K. 2007. Microbial imprinted polypyrrole/poly(3methylthiophene) composite films for the detection of Bacillus endospores. Biosens. Bioelectron. 22: 2018-2024. DOI: 10.1016/j.bios.2006.08.039 
3070. Ouyang RZ, Lei JP, Ju HX, Xue YD. 2007. A Molecularly Imprinted Copolymer Designed for Enantioselective Recognition of Glutamic Acid. Adv. Funct. Mater. 17: 3223-3230. DOI: 10.1002/adfm.200700143

3071. Özcan L, Sahin Y. 2007. Determination of paracetamol based on electropolymerized-molecularly imprinted polypyrrole modified pencil graphite electrode. Sens. Actuators $B$ 127: 362-369. DOI: 10.1016/j.snb.2007.04.034

3072. Petrukhin OM, Pogorelova SP, Kharitonov AB, Shipulo EV. 2007. Molecularly selective field-effect transistors for determining nicotinamide adenine dinucleotide and its phosphates. J. Anal. Chem. 62: 894-902. DOI: 10.1134/S106193480709016X

3073. Prasad BB, Sharma PS, Lakshmi D. 2007. Molecularly imprinted polymerbased solid-phase extraction combined with molecularly imprinted polymerbased sensor for detection of uric acid. J. Chromatogr. A 1173: 18-26. DOI: 10.1016/j.chroma.2007.10.024

3074. Prasad K, Prathish KP, Gladis JM, Naidu GRK, Rao TP. 2007. Molecularly imprinted polymer (biomimetic) based potentiometric sensor for atrazine. Sens. Actuators B 123: 65-70. DOI: 10.1016/j.snb.2006.07.022

3075. Prasad K, Prathish KP, Gladis JM, Naidu GR, Rao TP. 2007. Design and Development of Imprinted Polymer Inclusion Membrane-Based Field Monitoring Device for Trace Determination of Phorate (O,O-Diethyl S-Ethyl Thiomethyl Phophorodithioate) in Natural Waters. Electroanalysis 19: 11951200. DOI: 10.1002/elan.200703842

3076. Prathish KP, Prasad K, Rao TP, Suryanarayana MVS. 2007. Molecularly imprinted polymer-based potentiometric sensor for degradation product of chemical warfare agents: Part I. Methylphosphonic acid. Talanta 71: 19761980. DOI: 10.1016/j.talanta.2006.09.002 
3077. Riskin M, Tel-Vered R, Willner I. 2007. The Imprint of Electropolymerized Polyphenol Films on Electrodes by Donor-Acceptor Interactions: Selective Electrochemical Sensing of N,N'-dimethyl-4,4'-bipyridinium (Methyl Viologen). Adv. Funct. Mater. 17: 3858-3863. DOI: 10.1002/adfm.200700303

3078. Sadeghi S, Fathi F, Abbasifar J. 2007. Potentiometric sensing of levamisole hydrochloride based on molecularly imprinted polymer. Sens. Actuators $B$ 122: 158-164. DOI: 10.1016/j.snb.2006.05.018

3079. Sekine SI, Watanabe Y, Yoshimi Y, Hattori K, Sakai K. 2007. Influence of solvents on chiral discriminative gate effect of molecularly imprinted poly(ethylene glycol dimethacrylate-co-methacrylic acid). Sens. Actuators $B$ 127: 512-517. DOI: 10.1016/j.snb.2007.05.008

3080. Sekiyama M, Katada N, Niwa M. 2007. Molecular shape-selective detection by tin oxide film sensor modified with chemical vapor deposition of molecular-sieving silica overlayer using organic template. Sens. Actuators $B$ 124: 398-406. DOI: 10.1016/j.snb.2007.01.009

3081. Sharma PS, Lakshmi D, Prasad BB. 2007. Highly Sensitive and Selective Detection of Creatinine by Combined Use of MISPE and a Complementary MIP-Sensor. Chromatographia 65: 419-427. DOI: 10.1365/s10337-0070172-3

3082. Sharma PS, Lakshmi D, Prasad BB. 2007. Molecularly imprinted solidphase extraction combined with molecularly imprinted polymer-sensor: a diagnostic tool applicable to creatine deficiency syndrome. Biomed. Chromatogr. 21: 976-986. DOI: 10.1002/bmc.842

3083. Vishnuvardhan V, Prathish KP, Naidu GRK, Prasada RT. 2007. Fabrication and topographical analysis of non-covalently imprinted polymer inclusion membranes for the selective sensing of pinacolyl methylphosphonate--A 
simulant of Soman. Electrochim. Acta 52: 6922-6928. DOI: 10.1016/j.electacta.2007.05.005

3084. Wang CH, Li CY, Wei LF, Wang CF. 2007. Electrochemical sensor for acetaminophen based on an imprinted $\mathrm{TiO} 2$ thin film prepared by liquid phase deposition. Microchim. Acta 158: 307-313. DOI: 10.1007/s00604-0060728-1

3085. Wang ZH, Kang JW, Liu XY, Ma YJ. 2007. Capacitive detection of theophylline based on electropolymerized molecularly imprinted polymer. Int. J. Polym. Anal. Charact. 12: 131-142. DOI: $10.1080 / 10236660601140821$

3086. Weng $\mathrm{CH}$, Yeh WM, Ho KC, Lee GB. 2007. A microfluidic system utilizing molecularly imprinted polymer films for amperometric detection of morphine. Sens. Actuators B 121: 576-582. DOI: 10.1016/j.snb.2006.04.111

3087. Yao LD, Tang YW, Huang ZF. 2007. Nicotinic acid voltammetric sensor based on molecularly imprinted polymer membrane-modified electrode. Anal. Lett. 40: 677-688. DOI: 10.1080/00032710601017755

3088. Zhou L, Ye GR, Yuan R, Chai YQ, Chen SM. 2007. A capacitive sensor based on molecularly imprinted polymers and poly( $p$-aminobenzene sulfonic acid) film for detection of pazufloxacin mesilate. Sci. China Chem. 50: 547553. DOI: 10.1007/s11426-007-0035-7

3089. Du D, Chen SZ, Cai J, Tao Y, Tu HY, Zhang AD. 2008. Recognition of dimethoate carried by bi-layer electrodeposition of silver nanoparticles and imprinted poly-o-phenylenediamine. Electrochim. Acta 53: 6589-6595. DOI: 10.1016/j.electacta.2008.04.027

3090. Fuchiwaki Y, Shoji R, Kubo I, Suzuki H. 2008. 6-Chloro- $N, N$-Diethyl-1,3,5Triazine-2,4-Diamine (Simazine) Electrochemical Sensing Chip Based on 
Biomimetic Recognition Utilizing a Molecularly Imprinted Polymer Layer on a Gold Chip. Anal. Lett. 41: 1398-1407. DOI: 10.1080/00032710802119319

3091. Gómez-Caballero A, Unceta N, Aranzazu Goicolea M, Barrio RJ. 2008. Evaluation of the selective detection of 4,6-dinitro-o-cresol by a molecularly imprinted polymer based microsensor electrosynthesized in a semiorganic media. Sens. Actuators B 130: 713-722. DOI: 10.1016/j.snb.2007.10.036

3092. Granot E, Tel-Vered R, Lioubashevski O, Willner I. 2008. Stereoselective and Enantioselective Electrochemical Sensing of Monosaccharides Using Imprinted Boronic Acid-Functionalized Polyphenol Films. Adv. Funct. Mater. 18: 478-484. DOI: 10.1002/adfm.200700498

3093. Hsu CW, Yang MC. 2008. Electrochemical epinephrine sensor using artificial receptor synthesized by sol-gel process. Sens. Actuators $B$ 134: 680-686. DOI: 10.1016/j.snb.2008.06.015

3094. Huang JY, Wei ZX, Chen JC. 2008. Molecular imprinted polypyrrole nanowires for chiral amino acid recognition. Sens. Actuators B 134: 573578. DOI: 10.1016/j.snb.2008.05.038

3095. Javanbakht M, Fard SE, Mohammadi A, Abdouss M, Ganjali MR, Norouzi P, Safaraliee L. 2008. Molecularly imprinted polymer based potentiometric sensor for the determination of hydroxyzine in tablets and biological fluids. Anal. Chim. Acta 612: 65-74. DOI: 10.1016/j.aca.2008.01.085

3096. Javanbakht M, Fard SE, Abdouss M, Mohammadi A, Ganjali MR, Norouzi P, Safaraliee L. 2008. A Biomimetic Potentiometric Sensor Using Molecularly Imprinted Polymer for the Cetirizine Assay in Tablets and Biological Fluids. Electroanalysis 20: 2023-2030. DOI: 10.1002/elan.200804284

3097. Kamel AH, Moreira FTC, Almeida SAA, Sales MGF. 2008. Novel Potentiometric Sensors of Molecular Imprinted Polymers for Specific Binding of Chlormequat. Electroanalysis 20: 194-202. DOI: 10.1002/elan.200704039 
3098. Kan XW, Zhao Q, Zhang Z, Wang ZL, Zhu JJ. 2008. Molecularly imprinted polymers microsphere prepared by precipitation polymerization for hydroquinone recognition. Talanta 75: 22-26. DOI: 10.1016/j.talanta.2007.08.038

3099. Kan XW, Zhao Y, Geng ZR, Wang ZL, Zhu JJ. 2008. Composites of Multiwalled Carbon Nanotubes and Molecularly Imprinted Polymers for Dopamine Recognition. J. Phys. Chem. C 112: 4849-4854. DOI: 10.1021/jp077445v

3100. Kubo I, Shoji R, Fuchiwaki Y, Suzuki H. 2008. Atrazine sensing chip based on molecularly imprinted polymer layer. Electrochemistry 76: 541-544. DOI: 10.5796/electrochemistry.76.541

3101. Lu N, Chen S, Wang HT, Quan X, Zhao HM. 2008. Synthesis of molecular imprinted polymer modified $\mathrm{TiO} 2$ nanotube array electrode and their photoelectrocatalytic activity. J. Solid State Chem. 181: 2852-2858. DOI: 10.1016/j.jssc.2008.07.004

3102. Mazzotta E, Picca RA, Malitesta C, Piletsky SA, Piletska EV. 2008. Development of a sensor prepared by entrapment of MIP particles in electrosynthesised polymer films for electrochemical detection of ephedrine. Biosens. Bioelectron. 23: 1152-1156. DOI: 10.1016/j.bios.2007.09.020

3103. Mazzotta E, Malitesta C. 2008. Preliminary Study on Electrosynthesis of a Co-Porphyrin Based Molecularly Imprinted Polymer for the Selective Detection of the Herbicide 2,4-Dichlorophenoxy Carboxylic Acid. Sensor Lett. 6: 618-622. DOI: 10.1166/sl.2008.443

3104. Özcan L, Sahin M, Sahin Y. 2008. Electrochemical preparation of a molecularly imprinted polypyrrole-modified pencil graphite electrode for determination of ascorbic acid. Sensors 8: 5792-5805. DOI: $10.3390 / \mathrm{s} 8095792$ 
3105. Özkorucuklu SP, Sahin Y, Alsancak G. 2008. Voltammetric Behaviour of Sulfamethoxazole on Electropolymerized-Molecularly Imprinted Overoxidized Polypyrrole. Sensors 8: 8463-8478. DOI: 10.3390/s8128463

3106. Patel AK, Sharma PS, Prasad BB. 2008. Development of a Creatinine Sensor Based on a Molecularly Imprinted Polymer-Modified Sol-Gel Film on Graphite Electrode. Electroanalysis 20: 2102-2112. DOI: 10.1002/elan.200804294

3107. Prasad BB, Tiwari K, Singh M, Sharma PS, Patel AK, Srivastava S. 2008. Molecularly imprinted polymer-based solid-phase microextraction fiber coupled with molecularly imprinted polymer-based sensor for ultratrace analysis of ascorbic acid. J. Chromatogr. A 1198-1199: 59-66. DOI: 10.1016/j.chroma.2008.05.059

3108. Riskin M, Tel-Vered R, Bourenko T, Granot E, Willner I. 2008. Imprinting of Molecular Recognition Sites through Electropolymerization of Functionalized Au Nanoparticles: Development of an Electrochemical TNT Sensor Based on $\pi$-Donor-Acceptor Interactions. J. Am. Chem. Soc. 130: 9726-9733. DOI: 10.1021/ja711278c

3109. Thoelen R, Vansweevelt R, Duchateau J, Horemans F, D'Haen J, Lutsen L, Vanderzande D, Ameloot M, vandeVen M, Cleij TJ, Wagner P. 2008. A MIPbased impedimetric sensor for the detection of low-MW molecules. Biosens. Bioelectron. 23: 913-918. DOI: 10.1016/j.bios.2007.08.020

3110. Vinjamuri AK, Burns SC, Dahl DB. 2008. Caffeine and Theobromine Selectivity Using Molecularly Imprinted Polypyrrole Modified Electrodes. In Physical and Analytical Electrochemistry (General) - 213th ECS Meeting, Vol. 13, Trulove P, De Long H (eds). The Electrochemical Society: 9-20. DOI: $10.1149 / 1.3002805$ 
3111. Wang YT, Zhou YX, Sokolov J, Rigas B, Levon K, Rafailovich M. 2008. A potentiometric protein sensor built with surface molecular imprinting method. Biosens. Bioelectron. 24: 162-166. DOI: 10.1016/j.bios.2008.04.010

3112. Yamazaki T, Ohta S, Sode K. 2008. Operational condition of a molecular imprinting catalyst-based fructosyl-valine sensor. Electrochemistry 76: 590593. DOI: $10.5796 /$ electrochemistry.76.590

3113. Yang DH, Ju MJ, Maeda A, Lee SW. 2008. Enhanced sensor capability of juxtaposed $\beta$-cyclodextrin rings in $\mathrm{TiO} 2$ ultrathin matrix as determined by cyclic surface-polarization impedance measurement. Sens. Mater. 20: 191200.

3114. Yokota N, Fuchiwaki Y, Kubo I. 2008. Fabrication of Bisphenol A Sensor Utilizing Electrode Modified with Molecularly Imprinted Polymer. ECS Transactions 16: 551-556. DOI: 10.1149/1.2981162

3115. Zanganeh AR, Amini MK. 2008. Polypyrrole-modified electrodes with induced recognition sites for potentiometric and voltammetric detection of copper(II) ion. Sens. Actuators B 135: 358-365. DOI: 10.1016/j.snb.2008.09.005

3116. Zhang NW, Ding MX, Liu GY, Song WW, Chai CY. 2008. Molecularly Imprinted Membrane-Based Sensor for the Detection of Chloramphenicol Succinate Residue in Milk. Chin. J. Anal. Chem. 36: 1380-1384. DOI: 10.1016/S1872-2040(08)60074-2

3117. Alizadeh T. 2009. High Selective Parathion Voltammetric Sensor Development by Using an Acrylic Based Molecularly Imprinted PolymerCarbon Paste Electrode. Electroanalysis 21: 1490-1498. DOI: 10.1002/elan.200804541

3118. Alizadeh T, Ganjali MR, Norouzi P, Zare M, Zeraatkar A. 2009. A novel high selective and sensitive para-nitrophenol voltammetric sensor, based on a 
molecularly imprinted polymer-carbon paste electrode. Talanta 79: 11971203. DOI: 10.1016/j.talanta.2009.02.051

3119. Atta NF, Abdel-Mageed AM. 2009. Smart electrochemical sensor for some neurotransmitters using imprinted sol-gel films. Talanta 80: 511-518. DOI: 10.1016/j.talanta.2009.07.014

3120. Birnbaumer GM, Lieberzeit PA, Richter L, Schirhagl R, Milnera M, Dickert FL, Bailey A, Ertl P. 2009. Detection of viruses with molecularly imprinted polymers integrated on a microfluidic biochip using contact-less dielectric microsensors. Lab Chip 9: 3549-3556. DOI: 10.1039/b914738a

3121. Buica GO, Bucher B, Moutet JM, Royal G, Saint-Aman E, Ungureanu EM. 2009. Voltammetric Sensing of Mercury and Copper Cations at Poly(EDTAlike) Film Modified Electrode. Electroanalysis 21: 77-86. DOI: 10.1002/elan.200804386

3122. Buica GO, Ungureanu EM, Bucher C, Moutet JC, Saint-Aman E. 2009. Poly(pyrrole-EDTA like) modified electrodes for mercury ions electroanalysis. J. Optoelectron. Adv. Mater. 11: 1152-1159.

3123. Cervini P, Cavalheiro ETG. 2009. Evaluation of the Analytical Potentialities of a Composite Electrode Modified with Molecularly Imprinted Polymers. Anal. Lett. 42: 1940-1957. DOI: 10.1080/00032710903082556

3124. Chen PY, Nien PC, Ho KC. 2009. Highly Selective Dopamine Sensor based on an Imprinted SAM/Mediator Gold Electrode. Procedia Chem. 1: 285-288. DOI: 10.1016/j.proche.2009.07.071

3125. Chen X, Yang ZP, Si SH. 2009. Potentiometric urea biosensor based on immobilization of urease onto molecularly imprinted $\mathrm{TiO}_{2}$ film. J. Electroanal. Chem. 635: 1-6. DOI: 10.1016/j.jelechem.2009.07.005 
3126. Choong CL, Bendall JS, Milne WI. 2009. Carbon nanotube array: A new MIP platform. Biosens. Bioelectron. 25: 652-656. DOI: 10.1016/j.bios.2008.11.025

3127. Chuang SW, Rick J, Chou TC. 2009. Electrochemical characterisation of a conductive polymer molecularly imprinted with an Amadori compound. Biosens. Bioelectron. 24: 3170-3173. DOI: 10.1016/j.bios.2009.02.033

3128. Evtugyn G, Porfireva A, Ivanov A, Konovalova O, Hianik T. 2009. Molecularly Imprinted Polymerized Methylene Green as a Platform for Electrochemical Sensing of Aptamer-Thrombin Interactions. Electroanalysis 21: 1272-1277. DOI: 10.1002/elan.200804556

3129. Fang C, Yi CL, Wang Y, Cao YH, Liu XY. 2009. Electrochemical sensor based on molecular imprinting by photo-sensitive polymers. Biosens. Bioelectron. 24: 3164-3169. DOI: 10.1016/j.bios.2009.03.030

3130. Fuchiwaki Y, Sasaki N, Kubo I. 2009. Electrochemical Sensing System Utilizing Simazine-Imprinted Polymer Receptor for the Detection of Simazine in Tap Water. J. Sens. 2009: Article No. 503464. DOI: 10.1155/2009/503464

3131. Huang CY, Tsai TC, Thomas JL, Lee MH, Liu BD, Lin HY. 2009. Urinalysis with molecularly imprinted poly(ethylene-co-vinyl alcohol) potentiostat sensors. Biosens. Bioelectron. 24: 2611-2617. DOI: 10.1016/j.bios.2009.01.016

3132. Huang CY, Lee MH, Wu ZH, Tseng HY, Huang YC, Liu BD, Lin HY. 2009. A Portable Potentiostat with Molecularly Imprinted Polymeric Electrode for Dopamine Sensing. In Testing and Diagnosis, 2009. ICTD 2009. IEEE Circuits and Systems International Conference on, 529-532. DOI: 10.1109/CAS-ICTD.2009.4960767 
3133. Kamel AH, Sayour HEM. 2009. Flow-Through Assay of Quinine Using Solid Contact Potentiometric Sensors Based on Molecularly Imprinted Polymers. Electroanalysis 21: 2701-2708. DOI: 10.1002/elan.200904699

3134. Kan XW, Geng ZR, Wang ZL, Zhu JJ. 2009. Core-Shell Molecularly Imprinted Polymer Nanospheres for the Recognition and Determination of Hydroquinone. J. Nanosci. Nanotechnol. 9: 2008-2013. DOI: 10.1166/jnn.2009.378

3135. Kang JW, Zhang HN, Wang ZH, Wu GF, Lu XQ. 2009. A Novel Amperometric Sensor for Salicylic Acid Based on Molecularly Imprinted Polymer-Modified Electrodes. Polym. -Plast. Technol. Eng. 48: 639-645. DOI: 10.1080/03602550902824499

3136. Kugimiya A, Kohara K. 2009. Biomimetic sensor for cAMP using an ionsensitive field-effect transistor. Mater. Sci. Eng.: C 29: 959-962. DOI: 10.1016/j.msec.2008.09.001

3137. Lakshmi D, Bossi A, Whitcombe MJ, Chianella I, Fowler SA, Subrahmanyam S, Piletska EV, Piletsky SA. 2009. Electrochemical Sensor for Catechol and Dopamine Based on a Catalytic Molecularly Imprinted Polymer-Conducting Polymer Hybrid Recognition Element. Anal. Chem. 81: 3576-3584. DOI: 10.1021/ac802536p

3138. Li JP, Zhao J, Wei XP. 2009. A sensitive and selective sensor for dopamine determination based on a molecularly imprinted electropolymer of 0 aminophenol. Sens. Actuators B 140: 663-669. DOI: 10.1016/j.snb.2009.04.067

3139. Liang RN, Zhang RM, Qin W. 2009. Potentiometric sensor based on molecularly imprinted polymer for determination of melamine in milk. Sens. Actuators B 141: 544-550. DOI: 10.1016/j.snb.2009.05.024 
3140. Lin CF, Tsai HH, Juang YZ, Su YK, Li WL, Wang RL, Lin HY, Guo JY, Tsai TJ, Liu BD. 2009. A CMOS-Compatible Biological Transducer for Creatinine Detection: MIP-Gate ISFET. In Testing and Diagnosis, 2009. ICTD 2009. IEEE Circuits and Systems International Conference on, IEEE: 525-528. DOI: 10.1109/CAS-ICTD.2009.4960739

3141. Liu Y, Song QJ, Wang L. 2009. Development and characterization of an amperometric sensor for triclosan detection based on electropolymerized molecularly imprinted polymer. Microchem. J. 91: 222-226. DOI: 10.1016/j.microc.2008.11.007

3142. Menaker A, Syritski V, Reut J, Öpik A, Horváth V, Gyurcsányi RE. 2009. Electrosynthesized Surface-Imprinted Conducting Polymer Microrods for Selective Protein Recognition. Adv. Mater. 21: 2271-2275. DOI: 10.1002/adma.200803597

3143. Pardieu E, Cheap H, Vedrine C, Lazerges M, Lattach Y, Garnier F, Remita S, Pernelle C. 2009. Molecularly imprinted conducting polymer based electrochemical sensor for detection of atrazine. Anal. Chim. Acta 649: 236245. DOI: 10.1016/j.aca.2009.07.029

3144. Patel AK, Sharma PS, Prasad BB. 2009. Voltammetric sensor for barbituric acid based on a sol-gel derivated molecularly imprinted polymer brush grafted to graphite electrode. Int. J. Pharm. 371: 47-55. DOI: 10.1016/j.jpharm.2008.12.016

3145. Patel AK, Sharma PS, Prasad BB. 2009. Electrochemical sensor for uric acid based on a molecularly imprinted polymer brush grafted to tetraethoxysilane derived sol-gel thin film graphite electrode. Mater. Sci. Eng.: C 29: 1545-1553. DOI: 10.1016/j.msec.2008.12.008

3146. Pesavento M, D'Agostino G, Biesuz R, Alberti G. 2009. Molecularly Imprinted Polymer-Based Sensors for Amperometric Determination of 
Nonelectroactive Substances. Electroanalysis 21: 604-611. DOI: 10.1002/elan.200804456

3147. Prasad BB, Srivastava S, Tiwari K, Sharma PS. 2009. Trace-level sensing of dopamine in real samples using molecularly imprinted polymer-sensor. Biochem. Eng. J. 44: 232-239. DOI: 10.1016/j.bej.2008.12.013

3148. Prasad BB, Srivastava S, Tiwari K, Sharma PS. 2009. Ascorbic acid sensor based on molecularly imprinted polymer-modified hanging mercury drop electrode. Mater. Sci. Eng.: C 29: 1082-1087. DOI: 10.1016/j.msec.2008.09.025

3149. Prasad BB, Srivastava S, Tiwari K, Sharma PS. 2009. A new zwitterionic imprinted polymer sensor using ethylenediamine tetraacetic acid and chloranil precursors for the trace analysis of I-histidine. Mater. Sci. Eng.: C 29: 1781-1789. DOI: 10.1016/j.msec.2009.02.001

3150. Prasad BB, Srivastava S, Tiwari K, Sharma PS. 2009. Development of Uracil and 5-Fluorouracil Sensors Based on Molecularly Imprinted PolymerModified Hanging Mercury Drop Electrode. Sens. Mater. 21: 291-306.

3151. Qu P, Lei JP, Ouyang RZ, Ju HX. 2009. Enantioseparation and Amperometric Detection of Chiral Compounds by in Situ Molecular Imprinting on the Microchannel Wall. Anal. Chem. 81: 9651-9656. DOI: 10.1021/ac902201a

3152. Qu YH, Liu Y, Zhou TS, Shi GY, Jin LT. 2009. Electrochemical Sensor Prepared from Molecularly Imprinted Polymer for Recognition of 1,3Dinitrobenzene (DNB). Chin. J. Chem. 27: 2043-2048. DOI: 10.1002/cjoc.200990343

3153. Sergeyeva TA, Slinchenko OA, Brovko OO, Piletsky SA, Elska GV. 2009. A portable sensor system for phenols detection based on molecularlyimprinted polymers with catalytic properties. Ukr. Biokhim. Zh. 81: 41-51. 
3154. Wu CT, Chen PY, Chen JG, Suryanarayanan V, Ho KC. 2009. Detection of nicotine based on molecularly imprinted TiO2-modified electrodes. Anal. Chim. Acta 633: 119-126. DOI: 10.1016/j.aca.2008.11.038

3155. Xiang HY, Li WG. 2009. Electrochemical Sensor for trans-Resveratrol Determination Based on Indium Tin Oxide Electrode Modified with Molecularly Imprinted Self-Assembled Films. Electroanalysis 21: 1207-1210. DOI: 10.1002/elan.200804488

3156. Xu XL, Zhou GL, Li HX, Liu Q, Zhang S, Kong JL. 2009. A novel molecularly imprinted sensor for selectively probing imipramine created on ITO electrodes modified by $\mathrm{Au}$ nanoparticles. Talanta 78: 26-32. DOI: 10.1016/j.talanta.2008.10.041

3157. Yang M, Chen YT, Ma J, Huai LF. 2009. Differential pulse voltammetric determination of trace rotenone using molecularly imprinted polymer microspheres. Microchim. Acta 166: 95-99. DOI: 10.1007/s00604-009-0182y

3158. Yang QY, Sun Q, Zhou TS, Shi GY, Jin LT. 2009. Determination of Parathion in Vegetables by Electrochemical Sensor Based on Molecularly Imprinted Polyethyleneimine/Silica Gel Films. J. Agric. Food Chem. 57: 6558-6563. DOI: 10.1021/jf901286e

3159. Yao LD, Tang YW, Zeng WP, Huang ZF. 2009. An Electrochemical Sensor for Phenylephrine Based on Molecular Imprinting. Anal. Sci. 25: 1089-1093. DOI: 10.2116/analsci.25.1089

3160. Yoshimi Y, Narimatsu A, Nakayama K, Sekine S, Hattori K, Sakai K. 2009. Development of an enzyme-free glucose sensor using the gate effect of a molecularly imprinted polymer. J. Artif. Organs 12: 264-270. DOI: 10.1007/s10047-009-0473-4 
3161. Aghaei A, Milani Hosseini MR, Najafi M. 2010. A novel capacitive biosensor for cholesterol assay that uses an electropolymerized molecularly imprinted polymer. Electrochim. Acta 55: 1503-1508. DOI: 10.1016/j.electacta.2009.09.033

3162. Alizadeh T, Zare M, Ganjali MR, Norouzi P, Tavana B. 2010. A new molecularly imprinted polymer (MIP)-based electrochemical sensor for monitoring 2,4,6-trinitrotoluene (TNT) in natural waters and soil samples. Biosens. Bioelectron. 25: 1166-1172. DOI: 10.1016/j.bios.2009.10.003

3163. Alizadeh T, Akhoundian M. 2010. A novel potentiometric sensor for promethazine based on a molecularly imprinted polymer (MIP): The role of MIP structure on the sensor performance. Electrochim. Acta 55: 3477-3485. DOI: 10.1016/j.electacta.2010.02.010

3164. Alizadeh T. 2010. Comparison of different methodologies for integration of molecularly imprinted polymer and electrochemical transducer in order to develop a paraoxon voltammetric sensor. Thin Solid Films 518: 6099-6106. DOI: 10.1016/j.tsf.2010.05.110

3165. Alizadeh T, Ganjali MR, Zare M, Norouzi P. 2010. Development of a voltammetric sensor based on a molecularly imprinted polymer (MIP) for caffeine measurement. Electrochim. Acta 55: 1568-1574. DOI: 10.1016/j.electacta.2009.09.086

3166. Alizadeh T, Akhoundian M. 2010. Promethazine determination in plasma samples by using carbon paste electrode modified with molecularly imprinted polymer (MIP): Coupling of extraction, preconcentration and electrochemical determination. Electrochim. Acta 55: 5867-5873. DOI: 10.1016/j.electacta.2010.05.037

3167. Balogh D, Tel-Vered R, Riskin M, Orbach R, Willner I. 2010. Electrified Au Nanoparticle Sponges with Controlled Hydrophilic/Hydrophobic Properties. ACS Nano 5: 299-306. DOI: 10.1021/nn1027592 
3168. Bongaers E, Alenus J, Horemans F, Weustenraed A, Lutsen L, Vanderzande D, Cleij TJ, Troost FJ, Brummer RJ, Wagner P. 2010. A MIPbased biomimetic sensor for the impedimetric detection of histamine in different $\mathrm{pH}$ environments. physica status solidi (a) 207: 837-843. DOI: 10.1002/pssa.200983307

3169. Cai D, Ren L, Zhao HZ, Xu CJ, Zhang L, Yu Y, Wang HZ, Lan YC, Roberts MF, Chuang JH, Naughton MJ, Ren ZF, Chiles TC. 2010. A molecularimprint nanosensor for ultrasensitive detection of proteins. Nat. Nanotechnol. 5: 597-601. DOI: 10.1038/nnano.2010.114

3170. Chai CY, Liu GY, Li F, Liu XF, Yao B, Wang L. 2010. Towards the development of a portable sensor based on a molecularly imprinted membrane for the rapid determination of salbutamol in pig urine. Anal. Chim. Acta 675: 185-190. DOI: 10.1016/j.aca.2010.07.012

3171. Chen PY, Vittal R, Nien PC, Liou GS, Ho KC. 2010. A novel molecularly imprinted polymer thin film as biosensor for uric acid. Talanta 80: 11451151. DOI: 10.1016/j.talanta.2009.08.041

3172. Chen PY, Nien PC, Hu CW, Ho KC. 2010. Detection of uric acid based on multi-walled carbon nanotubes polymerized with a layer of molecularly imprinted PMAA. Sens. Actuators B 146: 466-471. DOI: 10.1016/j.snb.2009.11.035

3173. Chen ZD, Wei JX, Wang WC, Kong Y. 2010. Separation of tryptophan enantiomers with molecularly imprinted polypyrrole electrode column. Chin. Chem. Lett. 21: 353-356. DOI: 10.1016/j.cclet.2009.11.044

3174. Choong CL, Milne WI. 2010. Dynamic modulation of detection window in conducting polymer based biosensors. Biosens. Bioelectron. 25: 2384-2388. DOI: 10.1016/j.bios.2010.03.023 
3175. Chung IC, Chang CC, Chiu HS, Jiang SF, Lee MH, Liu BD, Huang CY, Lin HY. 2010. A portable electrochemical sensor for caffeine and ()epigallocatechin gallate (EGCG) based on molecularly imprinted poly(ethylene-co-vinyl-alcohol) recognition element. In Nanoelectronics Conference (INEC), 2010 3rd International, 362-363. DOI: 10.1109/INEC.2010.5424748

3176. Dickert FL, Lieberzeit PA, Aigner S, Langsam C, Sontimuang C, Suedee R. 2010. Mass-sensitive and resistive detection of bioanalytes - Synthetic antibodies and plastic replicae. In Nanoelectronics Conference (INEC), 2010 3rd International, 352-353. DOI: 10.1109/INEC.2010.5424741

3177. Gam-Derouich S, Nguyen MN, Madani A, Maouche N, Lang P, Perruchot C, Chehimi MM. 2010. Aryl diazonium salt surface chemistry and ATRP for the preparation of molecularly imprinted polymer grafts on gold substrates. Surf. Interf. Sci. 42: 1050-1056. DOI: 10.1002/sia.3210

3178. Gómez-Caballero A, Ugarte A, Sánchez-Ortega A, Unceta N, Goicolea MA, Barrio RJ. 2010. Molecularly imprinted poly[tetra(o-aminophenyl)porphyrin] as a stable and selective coating for the development of voltammetric sensors. J. Electroanal. Chem. 638: 246-253. DOI: 10.1016/j.jelechem.2009.11.006

3179. Guo ZY, Gai PP, Duan J, Zhang HN, Wang S. 2010. Tetracycline selective electrode based on molecularly imprinted polymer particles. Chin. Chem. Lett. 21: 1235-1238. DOI: 10.1016/j.cclet.2010.04.007

3180. Horemans F, Alenus J, Bongaers E, Weustenraed A, Thoelen R, Duchateau J, Lutsen L, Vanderzande D, Wagner P, Cleij TJ. 2010. MIP-based sensor platforms for the detection of histamine in the nano- and micromolar range in aqueous media. Sens. Actuators $B$ 148: 392-398. DOI: 10.1016/j.snb.2010.05.003 
3181. Kan XW, Liu TT, Zhou H, Li C, Fang B. 2010. Molecular imprinting polymer electrosensor based on gold nanoparticles for theophylline recognition and determination. Microchim. Acta 171: 423-429. DOI: 10.1007/s00604-0100455-5

3182. Khadro B, Sanglar C, Bonhomme A, Errachid A, Jaffrezic-Renault N. 2010. Molecularly imprinted polymers (MIP) based electrochemical sensor for detection of urea and creatinine. Procedia Eng. 5: 371-374. DOI: 10.1016/j.proeng.2010.09.125

3183. Kong Y, Zhao W, Yao SP, Xu J, Wang WC, Chen ZD. 2010. Molecularly imprinted polypyrrole prepared by electrodeposition for the selective recognition of tryptophan enantiomers. J. Appl. Polym. Sci. 115: 1952-1957. DOI: 10.1002/app.31165

3184. Li JP, Jiang FY, Wei XP. 2010. Molecularly Imprinted Sensor Based on an Enzyme Amplifier for Ultratrace Oxytetracycline Determination. Anal. Chem. 82: 6074-6078. DOI: 10.1021/ac100667m

3185. Liang RN, Song DA, Zhang RM, Qin W. 2010. Potentiometric Sensing of Neutral Species Based on a Uniform-Sized Molecularly Imprinted Polymer as a Receptor. Angew. Chem. Int. Edit. 49: 2556-2559. DOI: 10.1002/anie.200906720

3186. Mazzotta E, Malitesta C. 2010. Electrochemical detection of the toxic organohalide 2,4-DB using a Co-porphyrin based electrosynthesized molecularly imprinted polymer. Sens. Actuators B 148: 186-194. DOI: 10.1016/j.snb.2010.03.089

3187. Medyantseva EP, Varlamova RM, Plotnikova OG, Budnikov GK, Popov SA, Dmitrienko SG. 2010. A procedure for determining 2,4dichlorophenoxyacetic acid using immobilized molecular imprinted polymers and an amperometric biosensor. Russ. J. Appl. Chem. 83: 287-292. DOI: $10.1134 /$ S1070427210020199 
3188. Moreira FTC, Kamel AH, Guerreiro JRL, Sales MG. 2010. Man-tailored biomimetic sensor of molecularly imprinted materials for the potentiometric measurement of oxytetracycline. Biosens. Bioelectron. 26: 566-574. DOI: 10.1016/j.bios.2010.07.036

3189. Moreira FTC, Guerreiro JRL, Azevedo VL, Kamel AH, Sales MGF. 2010. New biomimetic sensors for the determination of tetracycline in biological samples: Batch and flow mode operations. Anal. Methods 2: 2039-2045.

3190. Moreira FTC, Kamel AH, Guerreiro RL, Azevedo V, Sales MG. 2010. New potentiometric sensors based on two competitive recognition sites for determining tetracycline residues using flow-through system. Procedia Eng. 5: 1200-1203. DOI: 10.1016/j.proeng.2010.09.327

3191. Morelli I, Chiono V, Vozzi G, Ciardelli G, Silvestri D, Giusti P. 2010. Molecularly imprinted submicronspheres for applications in a novel model biosensor-film. Sens. Actuators B 150: 394-401. DOI: 10.1016/j.snb.2010.06.046

3192. Nateghi MR, Mosslemin MH, Hakimi A, Kavoosi S. 2010. Imprinted Poly(ophenylenediamine-co-aniline) Electrode for Warfarin Assay in Human Samples by Differential Pulse Voltammetry. Asian J. Chem. 22: 3516-3524.

3193. Patel AK, Sharma PS, Prasad BB. 2010. Trace-level sensing of creatine in real sample using a zwitterionic molecularly imprinted polymer brush grafted to sol-gel modified graphite electrode. Thin Solid Films 518: 2847-2853. DOI: 10.1016/j.tsf.2009.09.009

3194. Pellicer C, Gomez-Caballero A, Unceta N, Goicolea MA, Barrio RJ. 2010. Using a portable device based on a screen-printed sensor modified with a molecularly imprinted polymer for the determination of the insecticide fenitrothion in forest samples. Anal. Methods 2: 1280-1285. DOI: 10.1039/c0ay00329h 
3195. Prasad BB, Madhuri R, Tiwari MP, Sharma PS. 2010. Electrochemical sensor for folic acid based on a hyperbranched molecularly imprinted polymer-immobilized sol-gel-modified pencil graphite electrode. Sens. Actuators B 146: 321-330. DOI: 10.1016/j.snb.2010.02.025

3196. Prasad BB, Madhuri R, Tiwari MP, Sharma PS. 2010. Enantioselective recognition of $\mathrm{d}$ - and I-tryptophan by imprinted polymer-carbon composite fiber sensor. Talanta 81: 187-196. DOI: 10.1016/j.talanta.2009.11.055

3197. Prasad BB, Madhuri R, Tiwari MP, Sharma PS. 2010. Imprinted polymercarbon consolidated composite fiber sensor for substrate-selective electrochemical sensing of folic acid. Biosens. Bioelectron. 25: 2140-2148. DOI: 10.1016/j.bios.2010.02.016

3198. Prasad BB, Madhuri R, Tiwari MP, Sharma PS. 2010. Imprinting molecular recognition sites on multiwalled carbon nanotubes surface for electrochemical detection of insulin in real samples. Electrochim. Acta 55: 9146-9156. DOI: 10.1016/j.electacta.2010.09.008

3199. Prasad BB, Madhuri R, Tiwari MP, Sharma PS. 2010. Layer-by-layer assembled molecularly imprinted polymer modified silver electrode for enantioselective detection of $d$ - and I-thyroxine. Anal. Chim. Acta 681: 1626. DOI: 10.1016/j.aca.2010.09.027

3200. Queirós RB, Noronha JP, Sales MGF, Aguilar GG. 2010. Sensors for the Detection and Quantification of Bacterial Contamination in Water for Human Use. Adv. Eng. Mater. 12: B175-B178. DOI: 10.1002/adem.200980029

3201. Singh KP, Ahalawat S, Prajapati RK, Kumar S, Singh P, Sandeef Kumar D. 2010. Electrochemical sensing for the detection of 2,4-dichlorophenoxy acetic acid using molecularly imprinted polymer membrane. lonics 16: 529537. DOI: 10.1007/s11581-010-0419-0 
3202. Song W, Chen Y, Xu JA, Yang XR, Tian DB. 2010. Dopamine sensor based on molecularly imprinted electrosynthesized polymers. J. Solid State Electrochem. 14: 1909-1914. DOI: 10.1007/s10008-010-1025-9

3203. Tehrani MS, Vardini MT, Azar PA, Husain SW. 2010. Molecularly Imprinted Polymer Based PVC-Membrane-Coated Graphite Electrode for the Determination of Metoprolol. Int. J. Electrochem. Sci. 5: 88-104.

3204. Tehrani MS, Vardini MT, Azar PA, Husain SW. 2010. Molecularly imprinted polymer based PVC-membrane-coated graphite electrode for the determination of metoprolol. J. Iranian Chem. Soc. 7: 759-769.

3205. Tsai HH, Lin CF, Juang YZ, Wang IL, Lin YC, Wang RL, Lin HY. 2010. Multiple type biosensors fabricated using the CMOS BioMEMS platform. Sens. Actuators B 144: 407-412. DOI: 10.1016/j.snb.2009.01.061

3206. Wang Y, Cao YH, Fang C, Gong QQ. 2010. Electrochemical Sensor for Parabens Based on Molecular Imprinting Polymers with Dual-templates. Anal. Chim. Acta 673: 145-150. DOI: 10.1016/j.aca.2010.05.039

3207. Wang YT, Zhang ZQ, Jain V, Yi JJ, Mueller S, Sokolov J, Liu ZX, Levon K, Rigas B, Rafailovich MH. 2010. Potentiometric sensors based on surface molecular imprinting: Detection of cancer biomarkers and viruses. Sens. Actuators B 146: 381-387. DOI: 10.1016/j.snb.2010.02.032

3208. Xie CG, Gao S, Guo QB, Xu K. 2010. Electrochemical sensor for 2,4dichlorophenoxy acetic acid using molecularly imprinted polypyrrole membrane as recognition element. Microchim. Acta 169: 145-152. DOI: 10.1007/s00604-010-0303-7

3209. Xie CG, Li HF, Li SQ, Wu J, Zhang ZP. 2010. Surface Molecular SelfAssembly for Organophosphate Pesticide Imprinting in Electropolymerized Poly(p-aminothiophenol) Membranes on a Gold Nanoparticle Modified 
Glassy Carbon Electrode. Anal. Chem. 82: 241-249. DOI: $10.1021 /$ ac901860t

3210. Zhang J, Wang YQ, Lv RH, Xu L. 2010. Electrochemical tolazoline sensor based on gold nanoparticles and imprinted poly-o-aminothiophenol film. Electrochim. Acta 55: 4039-4044. DOI: 10.1016/j.electacta.2010.02.021

3211. Zhang ZH, Hu YF, Zhang HB, Luo LJ, Yao SZ. 2010. Electrochemical layerby-layer modified imprinted sensor based on multi-walled carbon nanotubes and sol-gel materials for sensitive determination of thymidine. J. Electroanal. Chem. 644: 7-12. DOI: 10.1016/j.jelechem.2010.03.015

3212. Zhang ZH, Hu YF, Zhang HB, Luo LJ, Yao SZ. 2010. Layer-by-layer assembly sensitive electrochemical sensor for selectively probing I-histidine based on molecular imprinting sol-gel at functionalized indium tin oxide electrode. Biosens. Bioelectron. 26: 696-702. DOI: 10.1016/j.bios.2010.06.062

3213. Zhang ZH, Hu YF, Zhang HB, Yao SZ. 2010. Novel layer-by-layer assembly molecularly imprinted sol-gel sensor for selective recognition of clindamycin based on Au electrode decorated by multi-wall carbon nanotube. J. Colloid Interface Sci. 344: 158-164. DOI: 10.1016/j.jcis.2009.12.022

3214. Alizadeh T, Ganjali MR, Nourozi P, Zare M, Hoseini M. 2011. A carbon paste electrode impregnated with $\mathrm{Cd} 2+$ imprinted polymer as a new and high selective electrochemical sensor for determination of ultra-trace Cd2+ in water samples. J. Electroanal. Chem. 657: 98-106. DOI: 10.1016/j.jelechem.2011.03.029

3215. Alizadeh T, Ganjali MR, Zare M. 2011. Application of an $\mathrm{Hg} 2+$ selective imprinted polymer as a new modifying agent for the preparation of a novel highly selective and sensitive electrochemical sensor for the determination of ultratrace mercury ions. Anal. Chim. Acta 689: 52-59. DOI: 10.1016/j.aca.2011.01.036 
3216. Alizadeh T, Amjadi S. 2011. Preparation of nano-sized Pb2+ imprinted polymer and its application as the chemical interface of an electrochemical sensor for toxic lead determination in different real samples. J. Haz. Mater. 190: 451-459. DOI: 10.1016/j.jhazmat.2011.03.067

3217. Almeida SAA, Arasa E, Puyol M, Martinez-Cisneros CS, Alonso-Chamarro J, Montenegro MCBS, Sales MGF. 2011. Novel LTCC-potentiometric microfluidic device for biparametric analysis of organic compounds carrying plastic antibodies as ionophores: Application to sulfamethoxazole and trimethoprim. Biosens. Bioelectron. 30: 197-203. DOI: 10.1016/j.bios.2011.09.011

3218. Almeida SAA, Moreira FTC, Heitor AM, Montenegro MCBS, Aguilar GG, Sales MGF. 2011. Sulphonamide-imprinted sol-gel materials as ionophores in potentiometric transduction. Mater. Sci. Eng.: C 31: 1784-1790. DOI: 10.1016/j.msec.2011.08.011

3219. Apodaca DC, Pernites RB, Del Mundo FR, Advincula RC. 2011. Detection of 2,4-Dinitrotoluene (DNT) as a Model System for Nitroaromatic Compounds via Molecularly Imprinted Short-Alkyl-Chain SAMs. Langmuir 27: 6768-6779. DOI: 10.1021/la105128q

3220. Apodaca DC, Pernites RB, Ponnapati R, Del Mundo FR, Advincula RC. 2011. Electropolymerized Molecularly Imprinted Polymer Film: EIS Sensing of Bisphenol A. Macromolecules 44: 6669-6682. DOI: 10.1021/ma2010525

3221. Arvand M, Alirezanejad F. 2011. Sulfamethoxazole-Imprinted Polymeric Receptor as lonophore for Potentiometric Transduction. Electroanalysis 23: 1948-1957. DOI: 10.1002/elan.201100217

3222. BelBruno JJ, Zhang G, Gibson UJ. 2011. Capacitive sensing of amino acids in molecularly imprinted nylon films. Sens. Actuators B 155: 915-918. DOI: 10.1016/j.snb.2011.01.048 
3223. Broeders J, Duchateau S, Van Grinsven B, Vanaken W, Peeters M, Cleij T, Thoelen R, Wagner P, De Ceuninck W. 2011. Miniaturised eight-channel impedance spectroscopy unit as sensor platform for biosensor applications. physica status solidi (a) 208: 1357-1363. DOI: 10.1002/pssa.201001199

3224. Chung IC, Chang CC, Chiu HS, Jiang SF, Lee MH, Chung CL, Liu BD, Huang CY, Lin HY. 2011. A Portable Electrochemical Sensor for Caffeine and (-)Epigallocatechin Gallate Based on Molecularly Imprinted Poly(ethylene-co-vinyl alcohol) Recognition Element. J. Nanosci. Nanotechnol. 11: 10633-10638. DOI: 10.1166/jnn.2011.3938

3225. Díaz-Díaz G, Blanco-López MC, Lobo-Castañón MJ, Miranda-Ordieres AJ, Tuñón-Blanco P. 2011. Preparation and Characterization of a Molecularly Imprinted Microgel for Electrochemical Sensing of 2,4,6-Trichlorophenol. Electroanalysis 23: 201-208. DOI: 10.1002/elan.201000481

3226. Gai PP, Guo ZY, Yang F, Duan J, Hao TT, Wang S. 2011. Highly-sensitive ion selective electrode based on molecularly imprinted polymer particles for determination of tetracycline in aqueous samples. Russ. J. Electrochem. 47: 940-947. DOI: 10.1134/S1023193511080040

3227. Ganjali H, Ganjali MR, Alizadeh T, Faridbod F, Norouzi P. 2011. Bio-Mimetic Cadmium Ion Imprinted Polymer Based Potentiometric Nano-Composite Sensor. Int. J. Electrochem. Sci. 6: 6085-6093.

3228. Ganjali MR, Alizadeh T, Azimi F, Larjani B, Faridbod F, Norouzi P. 2011. Bio-Mimetic Ion Imprinted Polymer Based Potentiometric Mercury Sensor Composed of Nano-Materials. Int. J. Electrochem. Sci. 6: 5200-5208.

3229. Gholivand MB, Torkashvand M. 2011. A novel high selective and sensitive metronidazole voltammetric sensor based on a molecularly imprinted polymer-carbon paste electrode. Talanta 84: 905-912. DOI: 10.1016/j.talanta.2011.02.022 
3230. Gholivand MB, Karimian N. 2011. Development of piroxicam sensor based on molecular imprinted polymer-modified carbon paste electrode. Mater. Sci. Eng.: C 31: 1844-1851. DOI: 10.1016/j.msec.2011.08.019

3231. Gholivand MB, Khodadadian M. 2011. Rationally designed molecularly imprinted polymers for selective extraction of methocarbamol from human plasma. Talanta 85: 1680-1688. DOI: 10.1016/j.talanta.2011.06.066

3232. Guan GJ, Wang SS, Zhou HB, Zhang K, Liu RY, Mei QS, Wang SH, Zhang ZP. 2011. Molecularly imprinted polypyrrole nanonecklaces for detection of herbicide through molecular recognition-amplifying current response. Anal. Chim. Acta 702: 239-246. DOI: 10.1016/j.aca.2011.06.047

3233. Guerreiro JRL, Freitas V, Sales MGF. 2011. New sensing materials of molecularly-imprinted polymers for the selective recognition of Chlortetracycline. Microchem. J. 97: 173-181. DOI: 10.1016/j.microc.2010.08.011

3234. Guerreiro JRL, Sales MGF, Moreira FTC, Rebelo TSR. 2011. Selective recognition in potentiometric transduction of amoxicillin by molecularly imprinted materials. Eur. Food Res. Technol. 232: 39-50. DOI: 10.1007/s00217-010-1360-1

3235. Hsieh CH, Lin HY, Lin CF, Tsai HH, Juang YZ, Huang CY, Liu BD, Lee MH. 2011. A microfluidic and potentiostatic sensor integrated with neopterinimprinted poly(ethylene-co-vinyl alcohol) based electrode. In Proceedings of the 2011 IEEE International Conference on Nano/Micro Engineered and Molecular Systems (NEMS), IEEE: 711-714. DOI: 10.1109/NEMS.2011.6017453

3236. Hu YF, Zhang ZH, Zhang HB, Luo LJ, Yao SZ. 2011. Electrochemical determination of I-phenylalanine at polyaniline modified carbon electrode based on $\alpha$-cyclodextrin incorporated carbon nanotube composite material 
and imprinted sol-gel film. Talanta 84: 305-313. DOI: 10.1016/j.talanta.2011.01.010

3237. Hu YF, Li JX, Zhang ZH, Zhang HB, Luo LJ, Yao SZ. 2011. Imprinted sol-gel electrochemical sensor for the determination of benzylpenicillin based on Fe3O4@SiO2/multi-walled carbon nanotubes-chitosans nanocomposite film modified carbon electrode. Anal. Chim. Acta 698: 61-68. DOI: 10.1016/j.aca.2011.04.054

3238. Huang CY. 2011. Design of a voltammetry potentiostat for biochemical sensors. Analog Intergr. Circuits Signal Process. 67: 375-381. DOI: $10.1007 / \mathrm{s} 10470-010-9569-2$

3239. Huang JD, Xing XR, Zhang XM, He XR, Lin Q, Lian WJ, Zhu H. 2011. A molecularly imprinted electrochemical sensor based on multiwalled carbon nanotube-gold nanoparticle composites and chitosan for the detection of tyramine. Food Res. Int. 44: 276-281. DOI: 10.1016/j.foodres.2010.10.020

3240. Huang JD, Zhang XM, Liu S, Lin Q, He XR, Xing XR, Lian WJ, Tang D. 2011. Development of molecularly imprinted electrochemical sensor with titanium oxide and gold nanomaterials enhanced technique for determination of 4-nonylphenol. Sens. Actuators B 152: 292-298. DOI: 10.1016/j.snb.2010.12.022

3241. Huang JD, Zhang XM, Lin Q, He XR, Xing XR, Huai HX, Lian WJ, Zhu H. 2011. Electrochemical sensor based on imprinted sol-gel and nanomaterials for sensitive determination of bisphenol A. Food Control 22: 786-791. DOI: 10.1016/j.foodcont.2010.11.017

3242. Huang JD, Zhang XM, Liu S, Lin Q, He XR, Xing XR, Lian WJ. 2011. Electrochemical sensor for bisphenol A detection based on molecularly imprinted polymers and gold nanoparticles. J. Appl. Electrochem. 41: 13231328. DOI: $10.1007 / \mathrm{s} 10800-011-0350-8$ 
3243. Huang YX, Lian HT, Sun XY, Liu B. 2011. Preparation and Electrochemical Characters of Parathion Molecule Imprinted Polymeric Sensors. Chem. Res. Chin. Univ. 27: 28-33.

3244. Jin GP, Yu B, Yang SZ, Ma HH. 2011. Extremely sensitive electrode for melamine using a kind of molecularly imprinted nano-porous film. Microchim. Acta 174: 265-271. DOI: 10.1007/s00604-011-0618-z

3245. Jing T, Xia HA, Niu JW, Zhou YS, Dai Q, Hao QL, Zhou YK, Mei SR. 2011. Determination of trace 2,4-dinitrophenol in surface water samples based on hydrophilic molecularly imprinted polymers/nickel fiber electrode. Biosens. Bioelectron. 26: 4450-4456. DOI: 10.1016/j.bios.2011.05.001

3246. Kamel AH, Moreira FTC, Silva TI, Sales MGF. 2011. A Solid Binding Matrix/Mimic Receptor-Based Sensor System for Trace Level Determination of Iron Using Potential Measurements. Int. J. Electrochem. 2011: Article ID 643683. DOI: $10.4061 / 2011 / 643683$

3247. Kamel AH, Mahmoud WH, Mostafa MS. 2011. Biomimetic ciprofloxacin sensors made of molecularly imprinted network receptors for potential measurements. Anal. Methods 3: 957-964. DOI: 10.1039/C0AY00706D

3248. Kamel AH, Moreira FTC, Sales MG. 2011. Biomimetic Sensor Potentiometric System for Doxycycline Antibiotic Using a Molecularly Imprinted Polymer as an Artificial Recognition Element. Sensor Lett. 9: 1654-1660. DOI: 10.1166/sl.2011.1707

3249. Kamel AH, Moreira FTC, Rebelo TSR, Sales MGF. 2011. MolecularlyImprinted Materials for Potentiometric Transduction: Application to the Antibiotic Enrofloxacin. Anal. Lett. 44: 2107-2123. DOI: $10.1080 / 00032719.2010 .546021$

3250. Khadro B, Betatache A, Sanglar C, Bonhommé A, Errachid A, JaffrezicRenault N. 2011. Molecularly Imprinted Polymers (MIP) Based 
Electrochemical Sensor for Detection of Urea and Creatinine. Sensor Lett. 9: 2261-2264. DOI: 10.1166/sl.2011.1795

3251. Kong Y, Ni JH, Wang WC, Chen ZD. 2011. Enantioselective recognition of amino acids based on molecularly imprinted polyaniline electrode column. Electrochim. Acta 56: 4070-4074. DOI: 10.1016/j.electacta.2011.01.120

3252. Kubo I, Yokota N, Nakane Y, Fuchiwaki Y. 2011. The Establishment of Bisphenol A Sensing System Utilizing Molecularly Imprinted Polymer Receptor and Electrochemical Determination. Int. J. Electrochem. 2011: Article ID 534936. DOI: 10.4061/2011/534936

3253. Kugimiya A, Babe F. 2011. Phosphate ion sensing using molecularly imprinted artificial polymer receptor. Polym. Bull. 67: 2017-2024. DOI: 10.1007/s00289-011-0617-6

3254. Kumar D, Madhuri R, Tiwari MP, Sinha P, Prasad BB. 2011. Molecularly imprinted polymer-modified electrochemical sensor for simultaneous determination of copper and zinc. Adv. Mat. Lett. 2: 294-297. DOI: 10.5185/amlett.indias.207

3255. Latif U, Dickert FL. 2011. Conductometric Sensors for Monitoring Degradation of Automotive Engine Oil. Sensors 11: 8611-8625. DOI: $10.3390 / \mathrm{s} 110908611$

3256. Li JP, Chen ZQ, Li YP. 2011. A strategy for constructing sensitive and renewable molecularly imprinted electrochemical sensors for melamine detection. Anal. Chim. Acta 706: 255-260. DOI: 10.1016/j.aca.2011.08.048

3257. Liang RN, Zhang RM, Song WJ, Hu XF, Qin W. 2011. Potentiometric Sensor Based on an lon-Imprinted Polymer for Determination of Copper. Sensor Lett. 9: 557-562. DOI: 10.1166/sl.2011.1512

3258. Liang Y, Gu L, Liu XQ, Yang QY, Kajiura HS, Li YM, Zhou TS, Shi GY. 2011. Composites of Polyaniline Nanofibers and Molecularly Imprinted 
Polymers for Recognition of Nitroaromatic Compounds. Chem. Eur. J. 17: 5989-5997. DOI: 10.1002/chem.201002709

3259. Liu Y, Lynch J. 2011. The development of chloride ion selective polypyrrole thin film on a layer-by-layer carbon nanotube working electrode. In Nondestructive Characterization for Composite Materials, Aerospace Engineering, Civil Infrastructure, and Homeland Security 2011, Proceeding of the SPIE, Vol. 7983, Wu HF (ed). Art. No. 798315. DOI: $10.1117 / 12.880063$

3260. Liu YT, Deng J, Xiao XL, Ding L, Yuan YL, Li H, Li XT, Yan XN, Wang LL. 2011. Electrochemical sensor based on a poly(para-aminobenzoic acid) film modified glassy carbon electrode for the determination of melamine in milk. Electrochim. Acta 56: 4595-4602. DOI: 10.1016/j.electacta.2011.02.088

3261. Lu F, Wang LY, Wu FQ, Ying XF, Gu RA, Yao JL, Zhang HL, Deng ZW. 2011. Evaluation of the binding specificity of electrosynthesized poly-orthophenylenediamine molecularly imprinted with metal chelates. Microchim. Acta 174: 47-54. DOI: 10.1007/s00604-011-0595-2

3262. Mao Y, Bao Y, Gan SY, Li FH, Niu L. 2011. Electrochemical sensor for dopamine based on a novel graphene-molecular imprinted polymers composite recognition element. Biosens. Bioelectron. 28: 291-297. DOI: 10.1016/j.bios.2011.07.034

3263. Moreira FTC, Dutra RAF, Noronha JPC, Cunha AL, Sales MG. 2011. Artificial antibodies for troponin $\mathrm{T}$ by its imprinting on the surface of multiwalled carbon nanotubes: Its use as sensory surfaces. Biosens. Bioelectron. 28: 243-250. DOI: 10.1016/j.bios.2011.07.026

3264. Moreira FTC, Freitas VAP, Sales MGF. 2011. Biomimetic norfloxacin sensors made of molecularly-imprinted materials for potentiometric transduction. Microchim. Acta 172: 15-23. DOI: 10.1007/s00604-010-0464-4 
3265. Moreira FTC, Sales MG. 2011. Biomimetic sensors of molecularly-imprinted polymers for chlorpromazine determination. Mater. Sci. Eng.: C 31: 11211128. DOI: $10.1016 /$ j.msec.2011.04.012

3266. Moreira FTC, Dutra RAF, Noronha JPC, Sales MG. 2011. Myoglobinbiomimetic electroactive materials made by surface molecular imprinting on silica beads and their use as ionophores in polymeric membranes for potentiometric transduction. Biosens. Bioelectron. 26: 4760-4766. DOI: 10.1016/j.bios.2011.05.045

3267. Najafi M, Mehdipour R. 2011. Molecularly imprinted polymer-based potentiometric sensor for 2-aminopyridine as a potential impurity in piroxicam. Drug Testing Anal. 3: 132-137. DOI: 10.1002/dta.207

3268. Najafi M, Mollazadeh M. 2011. Selective recognition of chloroacetic acids by imprinted polyaniline film. J. Appl. Polym. Sci. 121: 292-298. DOI: 10.1002/app.33550

3269. Neto JdRM, Santos WdJR, Lima PR, Tanaka SMCN, Tanaka AA, Kubota LT. 2011. A hemin-based molecularly imprinted polymer (MIP) grafted onto a glassy carbon electrode as a selective sensor for 4-aminophenol amperometric. Sens. Actuators B 152: 220-225. DOI: 10.1016/j.snb.2010.12.010

3270. Nie DX, Jiang DW, Zhang D, Liang Y, Xue Y, Zhou TS, Jin LT, Shi GY. 2011. Two-dimensional molecular imprinting approach for the electrochemical detection of trinitrotoluene. Sens. Actuators B 156: 43-49. DOI: 10.1016/j.snb.2011.03.071

3271. Oliveira HMV, Moreira FTC, Sales MG. 2011. Ciprofloxacin-imprinted polymeric receptors as ionophores for potentiometric transduction. Electrochim. Acta 56: 2017-2023. DOI: 10.1016/j.electacta.2010.11.082 
3272. Pan MF, Fang GZ, Liu B, Qian K, Wang S. 2011. Novel amperometric sensor using metolcarb-imprinted film as the recognition element on a gold electrode and its application. Anal. Chim. Acta 690: 175-181. DOI: 10.1016/j.aca.2011.02.034

3273. Patterson ML. 2011. Molecular imprinting: Does it play a role in the reduction of oxidized redox shuttle molecules? In Proceedings Batteries and Energy Technology Joint General Session - 219th ECS Meeting, ECS Transactions, Vol. 35, The Electrochemical Society: 203-212. DOI: $10.1149 / 1.3655704$

3274. Pereira E, Rivas BL, Heitzman M, Moutet JC, Bucher C, Royal G, Aman ES. 2011. Complexing Polymer Films in The Preparation of Modified Electrodes for Detection of Metal lons. Macromol. Symp. 304: 115-125. DOI: 10.1002/masy.201150616

3275. Prasad BB, Kumar D, Madhuri R, Tiwari MP. 2011. Ascorbic acid imprinted polymer-modified graphite electrode: $A$ diagnostic sensor for hypovitaminosis $C$ at ultra trace ascorbic acid level. Sens. Actuators $B$ 160: 418-427. DOI: 10.1016/j.snb.2011.08.003

3276. Prasad BB, Tiwari MP, Madhuri R, Sharma PS. 2011. Enantioselective separation and electrochemical sensing of $d$ - and I-tryptophan at ultratrace level using molecularly imprinted micro-solid phase extraction fiber coupled with complementary molecularly imprinted polymer-fiber sensor. $J$. Chromatogr B 879: 364-370. DOI: 10.1016/j.jchromb.2010.12.023

3277. Prasad BB, Kumar D, Madhuri R, Tiwari MP. 2011. Metal ion mediated imprinting for electrochemical enantioselective sensing of I-histidine at trace level. Biosens. Bioelectron. 28: 117-126. DOI: 10.1016/j.bios.2011.07.008

3278. Prasad BB, Kumar D, Madhuri R, Tiwari MP. 2011. Sol-gel derived multiwalled carbon nanotubes ceramic electrode modified with molecularly 
imprinted polymer for ultra trace sensing of dopamine in real samples. Electrochim. Acta 56: 7202-7211. DOI: 10.1016/j.electacta.2011.04.090

3279. Qu P, Zhang L, Sheng J, Lei JP, Ju HX. 2011. Convenient enantioseparation by monolithic imprinted capillary clamped in a chip with electrochemical detection. Electrophoresis 32: 1522-1529. DOI: 10.1002/elps.201000655

3280. Rebelo TSCR, Almeida SAA, Guerreiro JRL, Montenegro MCBSM, Sales MGF. 2011. Trimethoprim-selective electrodes with molecularly imprinted polymers acting as ionophores and potentiometric transduction on graphite solid-contact. Microchem. J. 98: 21-28. DOI: 10.1016/j.microc.2010.10.006

3281. Reddy KK, Gobi KV. 2011. Artificial Biomolecular Recognition Element Based Sensor for Electrochemical Impedance Detection of Creatinine. In Proceedings of the 2011 International Conference on Nanotechnology and Biosensors, International Proceedings of Chemical, Biological and Environmental Engineering, Vol. 25, IACSIT Press: Singapore; 25-29.

3282. Reddy SM, Sette G, Phan Q. 2011. Electrochemical probing of selective haemoglobin binding in hydrogel-based molecularly imprinted polymers. Electrochim. Acta 56: 9203-9208. DOI: 10.1016/j.electacta.2011.07.132

3283. Rezaei B, Majidi N, Ensafi AA, Karimi-Maleh H. 2011. Molecularly imprintedmultiwall carbon nanotube paste electrode as a biosensor for voltammetric detection of rutin. Anal. Methods 3: 2510-2516. DOI: 10.1039/C1AY05271C

3284. Rezaei B, Rahmanian O. 2011. Nanolayer treatment to realize suitable configuration for electrochemical allopurinol sensor based on molecular imprinting recognition sites on multiwall carbon nanotube surface. Sens. Actuators B 160: 99-104. DOI: 10.1016/j.snb.2011.07.019

3285. Rezaei B, Mirahmadi-Zare SZ. 2011. Nanoscale Manipulation of Prednisolone as Electroactive Configuration Using Molecularly Imprinted- 
Multiwalled Carbon Nanotube Paste Electrode. Electroanalysis 23: 27242734. DOI: 10.1002/elan.201100261

3286. Roy AK, Nisha V, Dhand C, Malhotra BD. 2011. Molecularly imprinted polyaniline film for ascorbic acid detection. J. Mol. Recognit. 24: 700-706. DOI: $10.1002 / j m r .1104$

3287. Sadeghi HB, Ebrahimi SA, Tamaddon A, Bozorgvar F, Afifinia H, Almasian N, Mollaei S. 2011. Potentiometric Sensing of Lamotrigine Based on Molecularly Imprinted Polymers. Electroanalysis 23: 2716-2723. DOI: 10.1002/elan.201100140

3288. Santos WdJR, Santhiago M, Yoshida IVP, Kubota LT. 2011. Novel electrochemical sensor for the selective recognition of chlorogenic acid. Anal. Chim. Acta 695: 44-50. DOI: 10.1016/j.aca.2011.03.018

3289. Saumya V, Prathish KP, Rao TP. 2011. In situ copper oxide modified molecularly imprinted polypyrrole film based voltammetric sensor for selective recognition of tyrosine. Talanta 85: 1056-1062. DOI: 10.1016/j.talanta.2011.05.025

3290. Saumya V, Prathish KP, Dhanya S, Rao TP. 2011. Mechanistic aspects of tyrosine sensing on an in situ copper oxide modified molecularly imprinted polypyrrole coated glassy carbon electrode. J. Electroanal. Chem. 663: 5358. DOI: 10.1016/j.jelechem.2011.08.022

3291. Shi HJ, Zhao GH, Liu MC, Zhu ZL. 2011. A novel photoelectrochemical sensor based on molecularly imprinted polymer modified $\mathrm{TiO} 2$ nanotubes and its highly selective detection of 2,4-dichlorophenoxyacetic acid. Electrochemistry Communications 13: 1404-1407. DOI: 10.1016/j.elecom.2011.08.022

3292. Sontimuang C, Suedee R, Dickert FL. 2011. Development of Interdigitated Capacitance Electrodes Based on Rubber Elongation Factor-Imprinted Polymer for 
Determination of Rubber Elongation Factor Proteins in Rubber Latex Gloves. In Proceedings of PACCON2011 (Pure and Applied Chemistry International Conference 2011), Vol. Analytical Chemistry, 53-56.

3293. Sontimuang C, Suedee R, Dickert F. 2011. Interdigitated capacitive biosensor based on molecularly imprinted polymer for rapid detection of Hev b1 latex allergen. Anal. Biochem. 410: 224-233. DOI: 10.1016/j.ab.2010.11.043

3294. Tonelli D, Ballarin B, Guadagnini L, Mignani A, Scavetta E. 2011. A novel potentiometric sensor for I-ascorbic acid based on molecularly imprinted polypyrrole. Electrochim. Acta 56: 7149-7154. DOI: 10.1016/j.electacta.2011.05.076

3295. Trammell SA, Melde BJ, Zabetakis D, Deschamps JR, Dinderman MA, Johnson BJ, Kusterbeck AW. 2011. Electrochemical detection of TNT with in-line pre-concentration using imprinted diethylbenzene-bridged periodic mesoporous organosilicas. Sens. Actuators $B$ 155: 737-744. DOI: 10.1016/j.snb.2011.01.039

3296. Vishnuvardhan V, Kalyan Y, Prathish KP, Gangadhar B, Tharakeswar Y, Rao TP, Naidu GR. 2011. Imprinted Polymer Inclusion Membrane Based Potentiometric Sensor for Determination and Quantification of Diethyl Chlorophosphate in Natural Waters. Am. J. Anal. Chem. 2: 376-382. DOI: 10.4236/ajac.2011.23046

3297. Wang HT, Zhao HM, Quan X, Chen S. 2011. Electrochemical Determination of Tetracycline Using Molecularly Imprinted Polymer Modified Carbon Nanotube-Gold Nanoparticles Electrode. Electroanalysis 23: 1863-1869. DOI: 10.1002/elan.201100049

3298. Wang TY, Shannon C. 2011. Electrochemical sensors based on molecularly imprinted polymers grafted onto gold electrodes using click chemistry. Anal. Chim. Acta 708: 37-43. DOI: 10.1016/j.aca.2011.09.030 
3299. Wang Y, Tang J, Luo XY, Hu XY, Yang C, Xu Q. 2011. Development of a sensitive and selective kojic acid sensor based on molecularly imprinted polymer modified electrode in the lab-on-valve system. Talanta 85: 25222527. DOI: 10.1016/j.talanta.2011.08.014

3300. Wang YQ, Yang YY, Xu L, Zhang J. 2011. Bisphenol A sensing based on surface molecularly imprinted, ordered mesoporous silica. Electrochim. Acta 56: 2105-2109. DOI: 10.1016/j.electacta.2010.11.077

3301. Wang ZH, Li H, Chen J, Xue ZH, Wu BW, Lu XQ. 2011. Acetylsalicylic acid electrochemical sensor based on PATP-AuNPs modified molecularly imprinted polymer film. Talanta 85: 1672-1679. DOI: 10.1016/j.talanta.2011.06.067

3302. Wang ZH, Liu XL, Yang JM, Qin YX, Lu XQ. 2011. Copper(II) determination by using carbon paste electrode modified with molecularly imprinted polymer. Electrochim. Acta 58: 750-756. DOI: 10.1016/j.electacta.2011.10.034

3303. Willner I, Willner B, Tel-Vered R. 2011. Electroanalytical Applications of Metallic Nanoparticles and Supramolecular Nanostructures. Electroanalysis 23: 13-28. DOI: 10.1002/elan.201000506

3304. Yang YQ, Yi CL, Luo J, Liu R, Liu JK, Jiang JQ, Liu XY. 2011. Glucose sensors based on electrodeposition of molecularly imprinted polymeric micelles: A novel strategy for MIP sensors. Biosens. Bioelectron. 26: 26072612. DOI: 10.1016/j.bios.2010.11.015

3305. Yuan LH, Zhang J, Zhou P, Chen JX, Wang RY, Wen TT, Li Y, Zhou XM, Jiang HJ. 2011. Electrochemical sensor based on molecularly imprinted membranes at platinum nanoparticles-modified electrode for determination of 17 $\alpha$-estradiol. Biosens. Bioelectron. 29: 29-33. DOI: 10.1016/j.bios.2011.07.058 
3306. Zhang ZH, Luo LJ, Chen HJ, Zhang ML, Yang X, Yao SZ, Li JX, Peng MJ. 2011. A Polypyrrole-Imprinted Electrochemical Sensor Based on NanoSnO2/Multiwalled Carbon Nanotubes Film Modified Carbon Electrode for the Determination of Oleanolic Acid. Electroanalysis 23: 2446-2455. DOI: 10.1002/elan.201100231

3307. Zhao C, Jin GP, Chen LL, Li Y, Yu B. 2011. Preparation of molecular imprinted film based on chitosan/nafion/nano-silver/poly quercetin for clenbuterol sensing. Food Chem. 129: 595-600. DOI: 10.1016/j.foodchem.2011.04.072

3308. Kimaro A, Kelly LA, Murray GM. 2005. Synthesis and characterization of molecularly imprinted uranyl ion exchange resins. Sep. Sci. Technol. 40: 2035-2052. DOI: 10.1081/SS-200068451

3309. Chen HC, Lin S, Lin HY, Chou TC. 2005. Sensing lysozyme rebinding on molecularly imprinted polymers by scanning electrochemical microscopy. In Proceedings of the IEEE 31St Annual Northeast Bioengineering Conference, 2005, IEEE: New York; 138-139. DOI: 10.1109/NEBC.2005.1431962

3310. Fireman-Shoresh S, Turyan I, Mandler D, Avnir D, Marx S. 2005. Chiral electrochemical recognition by very thin molecularly imprinted sol-gel films. Langmuir 21: 7842-7847. DOI: 10.1021/la050240y

3311. Dickert FL, Hayden O, Bindeus R, Mann KJ, Blaas D, Waigmann E. 2004. Bioimprinted QCM sensors for virus detection - screening of plant sap. Anal. Bioanal. Chem. 378: 1929-1934. DOI: 10.1007/s00216-004-2521-5

3312. Dickert FL, Lieberzeit PA, Achatz P, Palfinger C, Fassnauer M, Schmid E, Werther W, Horner G. 2004. QCM array for on-line-monitoring of composting procedures. Analyst 129: 432-437. DOI: 10.1039/b315356h 
3313. Dickert FL, Lieberzeit P, Gazda-Miarecka S, Halikias K, Mann KJ. 2004. Modifying polymers by self-organisation for the mass-sensitive detection of environmental and biogeneous analytes. Sens. Actuators B 100: 112-116. DOI: 10.1016/j.snb.2003.12.034

3314. Dickert FL, Lieberzeit PA, Miarecka SG, Mann KJ, Hayden O, Palfinger C. 2004. Synthetic receptors for chemical sensors - subnano- and micrometre patterning by imprinting techniques. Biosens. Bioelectron. 20: 1040-1044. DOI: 10.1016/j.bios.2004.07.011

3315. Ebarvia BS, Binag CA, Sevilla F. 2004. Biomimetic piezoelectric quartz sensor for caffeine based on a molecularly imprinted polymer. Anal. Bioanal. Chem. 378: 1331-1337. DOI: 10.1007/s00216-003-2433-9

3316. Feng LA, Liu YJ, Hu JM. 2004. Molecularly imprinted $\mathrm{TiO}_{2}$ thin film by liquid phase deposition for the determination of L-glutamic acid. Langmuir 20: 1786-1790. DOI: 10.1021/la0357108

3317. Hoang S-H, Horng D-G, Chiang C-Y, Ko C-H, Lo Y-C, Chen C-I, Chang CK. 2004. A novel measurement device for SAW chemical sensors with FT-IR spectro-microscopic analytical capability. Tamkang J. Sci. Eng. 7: 99-102.

3318. Lieberzeit PA, Glanznig G, Leidl A, Nannen H, Dickert FL. 2004. Nanostructured functional polymers for engine oil quality sensors. In Proceedings Of The IEEE Sensors 2004, Proceedings Of The IEEE, Vol. 13, Rocha D, Sarro PM, Vellekoop MJ (eds). IEEE: New York; 449-450.

3319. Lin TY, Hu CH, Chou TC. 2004. Determination of albumin concentration by MIP-QCM sensor. Biosens. Bioelectron. 20: 75-81. DOI: 10.1016/j.bios.2004.01.028

3320. Zhang ZH, Liu YJ, Long YM, Nie LH, Yao SZ. 2004. Effect of the size of molecularly imprinted polymers sensing materials on piezoelectric quartz 
crystal sensor performance. Anal. Sci. 20: 291-295. DOI: 10.2116/analsci.20.291

3321. Zhang ZH, Long YM, Liu YJ, Yao SZ. 2004. Study of molecularly imprinted post-microspheres as dipyridamole sensors. Instr. Sci. Technol. 32: 507518. DOI: $10.1081 / \mathrm{Cl}-200029768$

3322. Barasc M, Ogier J, Durant Y, Claverie J. 2005. Preparation of molecularly surface imprinted polymeric nanoparticles for the direct detection of saxitoxin in water by quartz crystal microbalance. Polym. Prepr. 46: 11461147.

3323. Ebarvia BS, Cabanilla S, Sevilla III F. 2005. Biomimetic properties and surface studies of a piezoelectric caffeine sensor based on electrosynthesized polypyrrole. Talanta 66: 145-152. DOI: 10.1016/j.talanta.2004.10.009

3324. Ebarvia BS, Sevilla III F. 2005. Piezoelectric quartz sensor for caffeine based on molecularly imprinted polymethacrylic acid. Sens. Actuators $B$ 107: 782-790. DOI: 10.1016/j.snb.2004.12.018

3325. Ersöz A, Denizli A, Özcan A, Say RI. 2005. Molecularly imprinted ligandexchange recognition assay of glucose by quartz crystal microbalance. Biosens. Bioelectron. 20: 2197-2202. DOI: 10.1016/j.bios.2004.07.030

3326. Hoang S-H, Horng D-G, Chiang C-Y, Ko C-H, Lo Y-C, Chen C-I, Chang CK. 2005. A novel measurement device for SAW chemical sensors with FT-IR spectro-microscopic analytical capability. Tamkang J. Sci. Eng. 8: 63-66.

3327. Lee SW, Yang DH, Kunitake T. 2005. Regioselective imprinting of anthracenecarboxylic acids onto $\mathrm{TiO}_{2}$ gel ultrathin films: an approach to thin film sensor. Sens. Actuators B 104: 35-42. DOI: 10.1016/j.snb.2004.04.096

3328. Piacham T, Josell A, Arwin H, Prachayasittikul V, Ye L. 2005. Molecularly imprinted polymer thin films on quartz crystal microbalance using a surface 
bound photo-radical initiator. Anal. Chim. Acta 536: 191-196. DOl: 10.1016/j.aca.2004.12.067

3329. Piacham T, Josell A, Arwin H, Prachayasittikul V, Ye L. 2005. Erratum to "Molecularly imprinted polymer thin films on quartz crystal microbalance using a surface bound photo-radical initiator": [Analytica Chimica Acta 536 (2005) 191-196]. Anal. Chim. Acta 542: 135. DOI: 10.1016/j.aca.2005.05.036

3330. Shiigi H, Kijima D, Ikenaga Y, Hori K, Fukazawa S, Nagaoka T. 2005. Molecular recognition for bile acids using a molecularly imprinted overoxidized polypyrrole film. J. Electrochem. Soc. 152: H129-H134. DOI: $10.1149 / 1.1946367$

3331. Zhang ZH, Liao HP, Li H, Nie LH, Yao SZ. 2005. Stereoselective histidine sensor based on molecularly imprinted sol-gel films. Anal. Biochem. 336: 108-116. DOI: 10.1016/j.ab.2004.09.027

3332. Zhang ZH, Li H, Liao HP, Nie LH, Yao SZ. 2005. Influence of cross-linkers' amount on the performance of the piezoelectric sensor modified with molecularly imprinted polymers. Sens. Actuators B 105: 176-182. DOI: 10.1016/j.snb.2004.05.058

3333. Zhang ZH, Li H, Liao HP, Nie LH, Yao SZ. 2005. Effect of the extraction method on the MIP-sensor. Anal. Lett. 38: 203-217. DOI: 10.1081/AL200045105

3334. Zougagh M, Ríos A, Valcárcel M. 2005. Automatic selective determination of caffeine in coffee and tea samples by using a supported liquid membranemodified piezoelectric flow sensor with molecularly imprinted polymer. Anal. Chim. Acta 539: 117-124. DOI: 10.1016/j.aca.2005.02.060

3335. Ebarvia BS, Sevilla FI. 2006. Biomimetic quartz crystal sensors for caffeine based on conducting polymers. In 2005 Asian Conference on Sensors and 
the International Conference on new Techniques in Pharmaceutical and Biomedical Research Proceedings, Ghodgaonkar DK, Ahmad M, Heng LY, Habash RW, Wui WT, Taib MN (eds). IEEE: 34-38. DOI: 10.1109/ASENSE.2005.1564500

3336. Hayden O, Podlipna D, Chen X, Krassnig S, Leidl A, Dickert FL. 2006. Nanolithography and subnanomolecular interactions for biomimetic sensors. Mater. Sci. Eng.: C 26: 924-928. DOI: 10.1016/j.msec.2005.09.014

3337. Hayden O, Lieberzeit PA, Blaas D, Dickert FL. 2006. Artificial antibodies for bioanalyte detection-sensing viruses and proteins. Adv. Funct. Mater. 16: 1269-1278. DOI: 10.1002/adfm.200500626

3338. Hayden O, Haderspöck C, Krassnig S, Chen XH, Dickert FL. 2006. Surface imprinting strategies for the detection of trypsin. Analyst 131: 1044-1050. DOI: 10.1039/b608354b

3339. Lieberzeit PA, Glanznig G, Leidl A, Voigt G, Dickert FL. 2006. Nanostructured polymers for detecting chemical changes during engine oil degradation. IEEE Sens. J. 6: 529-535. DOI: 10.1109/JSEN.2006.872341

3340. Liu F, Liu X, Ng SC, Chan HSO. 2006. Enantioselective molecular imprinting polymer coated QCM for the recognition of I-tryptophan. Sens. Actuators $B$ 113: 234-240. DOI: 10.1016/j.snb.2005.02.058

3341. Matsuguchi M, Uno T. 2006. Molecular imprinting strategy for solvent molecules and its application for QCM-based VOC vapor sensing. Sens. Actuators B 113: 94-99. DOI: 10.1016/j.snb.2005.02.028

3342. Suedee R, Intakong W, Dickert FL. 2006. The use of trichloroacetic acid imprinted polymer coated quartz crystal microbalance as a screening method for determination of haloacetic acids in drinking water. Talanta $\mathbf{7 0}$ : 194-201. DOI: 10.1016/j.talanta.2006.04.033 
3343. Sun H, Fung YS. 2006. Piezoelectric quartz crystal sensor for rapid analysis of pirimicarb residues using molecularly imprinted polymers as recognition elements. Anal. Chim. Acta 576: 67-76. DOI: 10.1016/j.aca.2006.04.058

3344. Tsuru N, Kikuchi M, Kawaguchi $H$, Shiratori S. 2006. A quartz crystal microbalance sensor coated with MIP for "Bisphenol A" and its properties. Thin Solid Films 499: 380-385. DOI: 10.1016/j.tsf.2005.07.005

3345. Wu AH, Syu MJ. 2006. Synthesis of bilirubin imprinted polymer thin film for the continuous detection of bilirubin in an MIP/QCM/FIA system. Biosens. Bioelectron. 21: 2345-2353. DOI: 10.1016/j.bios.2006.01.017

3346. Zhang ZH, Long YM, Nie LH, Yao SZ. 2006. Molecularly imprinted thin film self-assembled on piezoelectric quartz crystal surface by the sol-gel process for protein recognition. Biosens. Bioelectron. 21: 1244-1251. DOI: 10.1016/j.bios.2005.05.009

3347. Albano DR, Sevilla F. 2007. Piezoelectric quartz crystal sensor for surfactant based on molecularly imprinted polypyrrole. Sens. Actuators $B$ 121: 129134. DOI: 10.1016/j.snb.2006.09.006

3348. Ávila M, Zougagh M, Escarpa A, Ríos A. 2007. Supported liquid membranemodified piezoelectric flow sensor with molecularly imprinted polymer for the determination of vanillin in food samples. Talanta 72: 1362-1369. DOI: 10.1016/j.talanta.2007.01.045

3349. Ayela C, Vandevelde F, Lagrange D, Haupt K, Nicu L. 2007. Combining Resonant Piezoelectric Micromembranes with Molecularly Imprinted Polymers. Angew. Chem. Int. Edit. 46: 9271-9274. DOI: 10.1002/anie.200703881

3350. Bunte G, Hurttlen J, Pontius H, Hartlieb K, Krause H. 2007. Gas phase detection of explosives such as 2,4,6-trinitrotoluene by molecularly imprinted polymers. Anal. Chim. Acta 591: 49-56. DOI: 10.1016/j.aca.2007.02.014 
3351. Guney-Altay O, Pestov D, Tepper G. 2007. Organic Zeolites from a Diolefinic Monomer. J. Am. Chem. Soc. 129: 13957-13962. DOI: $10.1021 / \mathrm{ja} 0745658$

3352. Lieberzeit PA, Afzal A, Glanzing G, Dickert FL. 2007. Molecularly imprinted sol-gel nanoparticles for mass-sensitive engine oil degradation sensing. Anal. Bioanal. Chem. 389: 441-446. DOI: 10.1007/s00216-007-1274-3

3353. Lieberzeit PA, Afzal A, Podlipna D, Krassnig S, Blumenstock H, Dickert FL. 2007. Printing materials in micro- and nano-scale: Systems for process control. Sens. Actuators B 126: 153-158. DOI: 10.1016/j.snb.2006.11.019

3354. Lieberzeit PA, Afzal A, Rehman A, Dickert FL. 2007. Nanoparticles for detecting pollutants and degradation processes with mass-sensitive sensors. Sens. Actuators B 127: 132-136. DOI: 10.1016/j.snb.2007.07.020

3355. Pestov D, Guney-Altay O, Levit N, Tepper G. 2007. Improving the stability of surface acoustic wave (SAW) chemical sensor coatings using photopolymerization. Sens. Actuators $B$ 126: 557-561. DOI: 10.1016/j.snb.2007.04.010

3356. Suedee R, Intakong W, Lieberzeit PA, Wanichapichart P, Chooto P, Dickert FL. 2007. Trichloroacetic acid-imprinted polypyrrole film and its property in piezoelectric quartz crystal microbalance and electrochemical sensors to application for determination of haloacetic acids disinfection by-product in drinking water. J. Appl. Polym. Sci. 106: 3861-3871. DOI: 10.1002/app.26934

3357. Turner NW, Wright BE, Hlady V, Britt DW. 2007. Formation of protein molecular imprints within Langmuir monolayers: A quartz crystal microbalance study. J. Colloid Interface Sci. 308: 71-80. DOI: 10.1016/j.jcis.2006.12.037 
3358. Wang W, He ST, Li SZ, Liu MH, Pan Y. 2007. Enhanced sensitivity of SAW gas sensor coated molecularly imprinted polymer incorporating high frequency stability oscillator. Sens. Actuators B 125: 422-427. DOI: 10.1016/j.snb.2007.02.037

3359. Yan SL, Fang YJ, Gao ZX. 2007. Quartz crystal microbalance for the determination of daminozide using molecularly imprinted polymers as recognition element. Biosens. Bioelectron. 22: 1087-1091. DOI: 10.1016/j.bios.2006.03.007

3360. Yan SL, Fang YJ, Gao ZX. 2007. Determination of Daminozide in Apple Sample by MIP-Coated Piezoelectric Quartz Sensor. Anal. Lett. 40: 10131021. DOI: $10.1080 / 00032710701296929$

3361. Hoshino Y, Kodama T, Okahata Y, Shea KJ. 2008. Peptide Imprinted Polymer Nanoparticles: A Plastic Antibody. J. Am. Chem. Soc. 130: 1524215243. DOI: 10.1021/ja8062875

3362. Lieberzeit PA, Chen X, Seidler K, Jenik M, Dickert FL, Blumenstock H. 2008. Functional Materials for Biosensing-From Proteins to Cells and Pollen. Sensor Lett. 6: 641-645. DOI: 10.1166/sl.2008.452

3363. Lieberzeit PA, Rehman A, Yaqub S, Dickert FL. 2008. Nanostructured Particles and Layers for Sensing Contaminants in Air and Water. Nano 3: 205-208. DOI: 10.1142/S1793292008001015

3364. Mirmohseni A, Shojaei M, Farbodi M. 2008. Application of a quartz crystal nanobalance to the molecularly imprinted recognition of phenylalanine in solution. Biotechnol. Bioprocess Eng. 13: 592-597. DOI: 10.1007/s12257008-0028-1

3365. Reimhult K, Yoshimatsu K, Risveden K, Chen S, Ye L, Krozer A. 2008. Characterization of QCM sensor surfaces coated with molecularly imprinted 
nanoparticles. Biosens. Bioelectron. 23: 1908-1914. DOI: 10.1016/j.bios.2008.02.011

3366. Sun H, Mo ZH, Choy JTS, Zhu DR, Fung YS. 2008. Piezoelectric quartz crystal sensor for sensing taste-causing compounds in food. Sens. Actuators B 131: 148-158. DOI: 10.1016/j.snb.2007.12.014

3367. Syritski V, Reut J, Menaker A, Gyurcsányi RE, Öpik A. 2008. Electrosynthesized molecularly imprinted polypyrrole films for enantioselective recognition of I-aspartic acid. Electrochim. Acta 53: 27292736. DOI: 10.1016/j.electacta.2007.10.032

3368. Yang DH, Takahara N, Lee SW, Kunitake T. 2008. Fabrication of glucosesensitive TiO2 ultrathin films by molecular imprinting and selective detection of monosaccharides. Sens. Actuators $B$ 130: 379-385. DOI: 10.1016/j.snb.2007.09.035

3369. Bi XY, Yang KL. 2009. On-Line Monitoring Imidacloprid and Thiacloprid in Celery Juice Using Quartz Crystal Microbalance. Anal. Chem. 81: 527-532. DOI: $10.1021 / a c 801786 a$

3370. Diltemiz SE, Hür D, Ersöz A, Denizli A, Say R. 2009. Designing of MIP based QCM sensor having thymine recognition sites based on biomimicking DNA approach. Biosens. Bioelectron. 25: 599-603. DOI: 10.1016/j.bios.2009.01.032

3371. Ersöz A, Diltemiz SE, Özcan AA, Denizli A, Say R. 2009. 8-OHdG sensing with MIP based solid phase extraction and QCM technique. Sens. Actuators B 137: 7-11. DOI: 10.1016/j.snb.2008.12.050

3372. Iglesias RA, Tsow F, Wang R, Forzani ES, Tao NJ. 2009. Hybrid Separation and Detection Device for Analysis of Benzene, Toluene, Ethylbenzene, and Xylenes in Complex Samples. Anal. Chem. 81: 8930-8935. DOI: 10.1021/ac9015769 
3373. Jenik M, Seifner A, Lieberzeit P, Dickert FL. 2009. Pollen-imprinted polyurethanes for QCM allergen sensors. Anal. Bioanal. Chem. 394: 523528. DOI: 10.1007/s00216-009-2718-8

3374. Jenik M, Schirhagl R, Schirk C, Hayden O, Lieberzeit P, Blaas D, Paul G, Dickert FL. 2009. Sensing Picornaviruses Using Molecular Imprinting Techniques on a Quartz Crystal Microbalance. Anal. Chem. 81: 5320-5326. DOI: $10.1021 / a c 8019569$

3375. Jenik M, Seifner A, Krassnig S, Seidler K, Lieberzeit PA, Dickert FL, Jungbauer C. 2009. Sensors for bioanalytes by imprinting--Polymers mimicking both biological receptors and the corresponding bioparticles. Biosens. Bioelectron. 25: 9-14. DOI: 10.1016/j.bios.2009.01.019

3376. Kryshtal RG, Medved AV. 2009. Study of the sorption processes in a piezoelectric-molecularly imprinted polymer film structure using Rayleigh surface acoustic waves. Tech. Phys. 54: 1363-1367. DOI: $10.1134 / \mathrm{S} 1063784209090175$

3377. Lieberzeit PA, Rehman A, Iqbal N, Najafi B, Dickert FL. 2009. QCM sensor array for monitoring terpene emissions from odoriferous plants. Monatsh. Chem. 140: 947-952. DOI: 10.1007/s00706-009-0141-7

3378. Losev VV, Medved' AV, Roshchin AV, Kryshtal' RG, Zapadinskii BI, Epinat'ev ID, Kumpanenko IV. 2009. An acoustic study of the selective absorption of vapors by microporous polymer films (Original Russian Text published in Khimicheskaya Fizika, 2009, Vol. 28, No. 11, pp. 79-92.). Russ. J. Phys. Chem. B 3: 990-1003. DOI: 10.1134/S1990793109060219

3379. Perkel JM. 2009. Pesticide monitoring with a quartz crystal microbalance. Anal. Chem. 81: 859. DOI: 10.1021/ac8025306

3380. Pietrzyk A, Suriyanarayanan S, Kutner W, Chitta R, D'Souza F. 2009. Selective Histamine Piezoelectric Chemosensor Using a Recognition Film of 
the Molecularly Imprinted Polymer of Bis(bithiophene) Derivatives. Anal. Chem. 81: 2633-2643. DOI: 10.1021/ac8025652

3381. Pietrzyk A, Kutner W, Chitta R, Zandler ME, D'Souza F, Sannicolò F, Mussini PR. 2009. Melamine Acoustic Chemosensor Based on Molecularly Imprinted Polymer Film. Anal. Chem. 81: 10061-10070. DOI: 10.1021/ac9020352

3382. Romero-Guerra M, Chianella I, Piletska EV, Karim K, Turner APF, Piletsky SA. 2009. Development of a piezoelectric sensor for the detection of methamphetamine. Analyst 134: 1565-1570. DOI: 10.1039/b819351g

3383. Say RI, Gültekin A, Özcan AA, Denizli A, Ersöz A. 2009. Preparation of new molecularly imprinted quartz crystal microbalance hybride sensor system for 8-hydroxy-2'-deoxyguanosine determination. Anal. Chim. Acta 640: 82-86. DOI: 10.1016/j.aca.2009.03.022

3384. Seidler K, Lieberzeit PA, Dickert FL. 2009. Application of yeast imprinting in biotechnology and process control. Analyst 134: 361-366. DOI: $10.1039 / \mathrm{b} 809130 \mathrm{~g}$

3385. Seidler K, Polreichová M, Lieberzeit PA, Dickert FL. 2009. Biomimetic Yeast Cell Typing-Application of QCMs. Sensors 9: 8146-8157. DOI: 10.3390/s91008146

3386. Seifner A, Lieberzeit $P$, Jungbauer $C$, Dickert FL. 2009. Synthetic receptors for selectively detecting erythrocyte ABO subgroups. Anal. Chim. Acta 651 : 215-219. DOI: 10.1016/j.aca.2009.08.021

3387. Tsow F, Forzani E, Rai A, Wang R, Tsui R, Mastroianni S, Knobbe C, Gandolfi AJ, Tao NJ. 2009. A Wearable and Wireless Sensor System for Real-Time Monitoring of Toxic Environmental Volatile Organic Compounds. IEEE Sens. J. 9: 1734-1740. DOI: 10.1109/JSEN.2009.2030747 
3388. Wyszynski B, Nakamoto T. 2009. Linking biological and artificial olfaction: biomimetic quartz crystal microbalance odor sensors. IEEJ Trans. Elec. Electron. Eng. 4: 334-338. DOI: 10.1002/tee.20414

3389. Yang ZP, Zhang CJ. 2009. Designing of MIP-based QCM sensor for the determination of $\mathrm{Cu}(\mathrm{II})$ ions in solution. Sens. Actuators B 142: 210-215. DOI: 10.1016/j.snb.2009.08.029

3390. Yao W, Gao ZX, Cheng YY. 2009. Quartz crystal microbalance for the detection of carbaryl using molecularly imprinted polymers as recognition element. J. Sep. Sci. 32: 3334-3339. DOI: 10.1002/jssc.200900181

3391. Apodaca DC, Pernites RB, Ponnapati RR, Del Mundo FR, Advincula RC. 2010. Electropolymerized Molecularly Imprinted Polymer Films of a BisTerthiophene Dendron: Folic Acid Quartz Crystal Microbalance Sensing. ACS Appl. Mater. Interf. 3: 191-203. DOI: 10.1021/am100805y

3392. Arenas LF, Ebarvia BS, Sevilla FB. 2010. Enantioselective piezoelectric quartz crystal sensor for d-methamphetamine based on a molecularly imprinted polymer. Anal. Bioanal. Chem. 397: 3155-3158. DOI: 10.1007/s00216-010-3865-7

3393. Iqbal N, Mustafa G, Rehman A, Biedermann A, Najafi B, Lieberzeit PA, Dickert FL. 2010. QCM-Arrays for Sensing Terpenes in Fresh and Dried Herbs via Bio-Mimetic MIP Layers. Sensors 10: 6361-6376. DOI: 10.3390/s100706361

3394. Lieberzeit PA, Findeisen A, Mähner J, Samardzic R, Pitkänen J, Anttalainen O, Dickert FL. 2010. Artificial receptor layers for detecting chemical and biological threats. Procedia Eng. 5: 381-384. DOI: 10.1016/j.proeng.2010.09.127

3395. Mujahid A, Afzal A, Glanzing G, Leidl A, Lieberzeit PA, Dickert FL. 2010. Imprinted sol-gel materials for monitoring degradation products in 
automotive oils by shear transverse wave. Anal. Chim. Acta 675: 53-57. DOI: 10.1016/j.aca.2010.07.005

3396. Mujahid A, Stathopulos H, Lieberzeit PA, Dickert FL. 2010. Solvent Vapour Detection with Cholesteric Liquid Crystals-Optical and Mass-Sensitive Evaluation of the Sensor Mechanism. Sensors 10: 4887-4897. DOI: $10.3390 /$ s 100504887

3397. Pietrzyk A, Suriyanarayanan S, Kutner W, Maligaspe E, Zandler ME, D'Souza F. 2010. Molecularly imprinted poly[bis(2,2'-bithienyl)methane] film with built-in molecular recognition sites for a piezoelectric microgravimetry chemosensor for selective determination of dopamine. Bioelectrochemistry 80: 62-72. DOI: 10.1016/j.bioelechem.2010.03.004

3398. Pietrzyk A, Suriyanarayanan S, Kutner W, Chitta R, Zandler ME, D'Souza F. 2010. Molecularly imprinted polymer (MIP) based piezoelectric microgravimetry chemosensor for selective determination of adenine. Biosens. Bioelectron. 25: 2522-2529. DOI: 10.1016/j.bios.2010.04.015

3399. Schirhagl R, Seifner A, Husain FT, Cichna-Markl M, Lieberzeit PA, Dickert FL. 2010. Antibodies and Their Replicae in Microfluidic Sensor SystemsLabelfree Quality Assessment in Food Chemistry and Medicine. Sensor Lett. 8: 399-404. DOI: 10.1166/sl.2010.1285

3400. Schirhagl R, Lieberzeit PA, Blaas D, Dickert FL. 2010. Chemosensors for Viruses Based on Artificial Immunoglobulin Copies. Adv. Mater. 22: 20782081. DOI: 10.1002/adma.200903517

3401. Schirhagl R, Podlipna D, Lieberzeit PA, Dickert FL. 2010. Comparing biomimetic and biological receptors for insulin sensing. Chem. Commun. 3128-3130. DOI: 10.1039/c000936a

3402. Sener G, Ozgur E, Yilmaz E, Uzun L, Say RI, Denizli A. 2010. Quartz crystal microbalance based nanosensor for lysozyme detection with lysozyme 
imprinted nanoparticles. Biosens. Bioelectron. 26: 815-821. DOI: 10.1016/j.bios.2010.06.003

3403. Tai DF, Jhang MH, Chen GY, Wang SC, Lu KH, Lee YD, Liu HT. 2010. Epitope-Cavities Generated by Molecularly Imprinted Films Measure the Coincident Response to Anthrax Protective Antigen and Its Segments. Anal. Chem. 82: 2290-2293. DOI: 10.1021/ac9024158

3404. von Hauff E, Fuchs K, Hellmann DC, Parisi J, Weiler R, Burkhardt C, Kraushaar U, Guenther E. 2010. Biocompatible molecularly imprinted polymers for the voltage regulated uptake and release of I-glutamate in neutral pH solutions. Biosens. Bioelectron. 26: 596-601. DOI: 10.1016/j.bios.2010.07.022

3405. Zhou WH, Tang SF, Yao QH, Chen FR, Yang HH, Wang XR. 2010. A quartz crystal microbalance sensor based on mussel-inspired molecularly imprinted polymer. Biosens. Bioelectron. 26: 585-589. DOI: 10.1016/j.bios.2010.07.024

3406. Zyablov AN, Kalach AV, Zhibrova Y, Selemenev VF, D'yakonova OV. 2010. Determination of glycine in aqueous solutions using a molecularly imprinted polymer-modified piezosensor (published in Zhurnal Analiticheskoi Khimii, 2010, Vol. 65, No. 1, pp. 93-95.). J. Anal. Chem. 65: 91-93. DOI: $10.1134 / \mathrm{S} 106193481001017 \mathrm{X}$

3407. Cela-Pérez MC, López-Vilariño JM, González-Rodríguez MV. 2011. Impact of functional cross-linker on recognition properties of a Bisphenol-A imprinted polymer film for coating a Quartz Crystal Microbalance. In Sensors, 2011 IEEE, IEEE: 1740-1743. DOI: 10.1109/ICSENS.2011.6126966

3408. Hwang MJ, Shim WG, Yang CY, Moon H. 2011. Preparation of Molecularly Imprinted Polymers for the Detection of Aromatic Hydrocarbons. J. Nanosci. Nanotechnol. 11: 7206-7209. DOI: 10.1166/jnn.2011.4812 
3409. Latif U, Mujahid A, Afzal A, Sikorski R, Lieberzeit PA, Dickert FL. 2011. Dual and tetraelectrode QCMs using imprinted polymers as receptors for ions and neutral analytes. Anal. Bioanal. Chem. 400: 2507-2515. DOI: 10.1007/s00216-011-4927-1

3410. Latif U, Rohrer A, Lieberzeit PA, Dickert FL. 2011. QCM gas phase detection with ceramic materials-VOCs and oil vapors. Anal. Bioanal. Chem. 400: 2457-2462. DOI: 10.1007/s00216-011-4684-1

3411. Lattach Y, Fourati N, Zerrouki C, Fougnion JM, Garnier F, Remita S. 2011. Surface Acoustic Wave Sensor Combining Gravimetric and Electrochemical Transductions: Application for Atrazine Detection. Sensor Lett. 9: 22492252. DOI: 10.1166/sI.2011.1783

3412. Lee MH, Thomas JL, Tseng HY, Lin WC, Liu BD, Lin HY. 2011. Sensing of Digestive Proteins in Saliva with a Molecularly Imprinted Poly(ethylene-covinyl alcohol) Thin Film Coated Quartz Crystal Microbalance Sensor. ACS Appl. Mater. Interf. 3: 3064-3071. DOI: 10.1021/am2005724

3413. Lee SW, Takahara N, Korposh S, Yang DH, Kunitake T. 2011. A Novel Mass-Sensitive Sensor Based on $\beta$-Cyclodextrin-Anchored Bisphenol AImprinted TiO(2) Ultrathin Layers. Sens. Mater. 23: 229-236.

3414. Lieberzeit PA, Bajwa S, Mustafa G, Wanchareansak T, Dickert FL. 2011. From metal ions to biospecies: template-assisted synthesis as a strategy to generate artificial receptor materials. Adv. Mat. Lett. 2: 319-321. DOI: 10.5185/amlett.2011.1024am2011

3415. Madhuri R, Tiwari MP, Kumar D, Mukharji A, Prasad BB. 2011. Biomimetic piezoelectric quartz sensor for folic acid based on a molecular imprinting technology. Adv. Mat. Lett. 2: 264-267. DOI: 10.5185/amlett.indias.194

3416. Ni Y, Zheng ST, Zhao C, Hu LL. 2011. Study of explosives detected system based on molecularly imprinted piezoelectric sensor. In Proceedings of the 
4th International Conference on Biomedical Engineering and Informatics (BMEI), 2011, Vol. 2, IEEE: 828-831. DOI: 10.1109/BMEI.2011.6098375

3417. Ni Y, Zheng ST, Zhao C, Hu LL. 2011. Study of explosives detected system based on molecularly imprinted piezoelectric sensor. In Proceedings of the 4th International Conference on Biomedical Engineering and Informatics (BMEI), 2011, Vol. 3, IEEE: 1170-1173. DOI: 10.1109/BMEI.2011.6098518

3418. Polreichova M, Latif U, Dickert FL. 2011. Functionalized Polymers as Receptors for Detection of Cells. Aust. J. Chem. 64: 1254-1258. DOI: $10.1071 / \mathrm{CH} 11181$

3419. Schirhagl R, Latif U, Dickert FL. 2011. Atrazine detection based on antibody replicas. J. Mater. Chem. 21: 14594-14598. DOI: 10.1039/C1JM11576F

3420. Sontimuang C, Suedee R, Canyuk B, Phadoongsombut N, Dickert FL. 2011. Development of a Rubber Elongation Factor Surface-Quartz Crystal Microbalance Sensor for Determination of Natural Rubber Latex Allergens in Glove Samples. In Proceedings of PACCON2011 (Pure and Applied Chemistry International Conference 2011), Vol. Analytical Chemistry, 26-29.

3421. Sontimuang C, Suedee R, Canyuk B, Phadoongsombut N, Dickert FL. 2011. Development of a rubber elongation factor, surface-imprinted polymerquartz crystal microbalance sensor, for quantitative determination of $\mathrm{Hev}$ b1 rubber latex allergens present in natural rubber latex products. Anal. Chim. Acta 687: 184-192. DOI: 10.1016/j.aca.2010.12.021

3422. Troïani D, Dion JR, Burns DH. 2011. Ultrasonic quantification using smart hydrogel sensors. Talanta 83: 1371-1375. DOI: 10.1016/j.talanta.2010.11.009

3423. Wangchareansak T, Sangma C, Choowongkomon K, Dickert F, Lieberzeit P. 2011. Surface molecular imprints of WGA lectin as artificial receptors for 
mass-sensitive binding studies. Anal. Bioanal. Chem. 400: 2499-2506. DOI: $10.1007 / \mathrm{s} 00216-011-4952-0$

3424. Yang ZP, Yan JL, Zhang CJ, Luo SQ. 2011. Enhanced removal of bilirubin on molecularly imprinted titania film. Colloids Surf., B 87: 187-191. DOI: 10.1016/j.colsurfb.2011.05.022

3425. Yang ZP, Zhang CJ. 2011. Molecularly imprinted hydroxyapatite thin film for bilirubin recognition. Biosens. Bioelectron. 29: 167-171. DOI: 10.1016/j.bios.2011.08.012

3426. Yaqub S, Latif U, Dickert FL. 2011. Plastic antibodies as chemical sensor material for atrazine detection. Sens. Actuators B 160: 227-233. DOI: 10.1016/j.snb.2011.07.039

3427. Zhang CJ, Luo SQ. 2011. Piezoelectric atrazine sensor based on a molecularly imprinted film of titanium dioxide. Microchim. Acta 175: 63-68. DOI: 10.1007/s00604-011-0644-x

3428. Zhou DZ, Guo TY, Yang Y, Zhang ZP. 2011. Surface imprinted macroporous film for high performance protein recognition in combination with quartz crystal microbalance. Sens. Actuators B 153: 96-102. DOI: 10.1016/j.snb.2010.10.012

3429. Kikuchi M, Tsuru N, Shiratori S. 2006. Recognition of terpenes using molecular imprinted polymer coated quartz crystal microbalance in air phase. Sci. Technol. Adv. Mater. 7: 156-161. DOI: 10.1016/j.stam.2005.12.004

3430. Lieberzeit PA, Rehman A, Najafi B, Dickert FL. 2008. Real-life application of a QCM-based e-nose: quantitative characterization of different plantdegradation processes. Anal. Bioanal. Chem. 391: 2897-2903. DOI: 10.1007/s00216-008-2222-6 
3431. Lettau K, Katterle M, Warsinke A, Scheller FW. 2008. Sequential conversion by catalytically active MIP and immobilized tyrosinase in a thermistor. Biosens. Bioelectron. 23: 1216-1219. DOI: 10.1016/j.bios.2007.10.021

3432. Rajkumar R, Katterle M, Warsinke A, Mohwald H, Scheller FW. 2008. Thermometric MIP sensor for fructosyl valine. Biosens. Bioelectron. 23: 1195-1199. DOI: 10.1016/j.bios.2007.09.015

3433. Rajkumar R, Warsinke A, Möhwald H, Scheller FW, Katterle M. 2008. Analysis of recognition of fructose by imprinted polymers. Talanta 76: 11191123. DOI: 10.1016/j.talanta.2008.05.022

3434. Athikomrattanakul U, Gajovic-Eichelmann N, Scheller FW. 2011. Thermometric Sensing of Nitrofurantoin by Noncovalently Imprinted Polymers Containing Two Complementary Functional Monomers. Anal. Chem. 83: 7704-7711. DOI: 10.1021/ac201099h

3435. Chen CT, Chen YC. 2004. Molecularly imprinted $\mathrm{TiO}_{2}$-matrix-assisted laser desorption/ionization mass spectrometry for selectively detecting $\alpha$ cyclodextrin. Anal. Chem. 76: 1453-1457. DOI: 10.1021/ac034986h

3436. Jafari MT, Rezaei B, Zaker B. 2009. Ion Mobility Spectrometry as a Detector for Molecular Imprinted Polymer Separation and Metronidazole Determination in Pharmaceutical and Human Serum Samples. Anal. Chem. 81: 3585-3591. DOI: 10.1021/ac802557t

3437. Yoshikawa M, Asano Y, Guiver MD. 2001. Selective Recognition and Permeation of Bisphenol A with Molecularly Imprinted Polyamide Membranes. Membrane 26: 185-188. DOI: 10.5360/membrane.26.185

3438. Brüggemann O, Visnjevski A, Burch R, Patel P. 2004. Selective extraction of antioxidants with molecularly imprinted polymers. Anal. Chim. Acta 504: 8188. DOI: 10.1016/S0003-2670(03)01099-7 
3439. Ciardelli G, Cioni B, Netri R, Porciani F, Silvestri D, Cristallini C, Barbani N, Giusti P. 2004. Engineering membranes for molecular recognition. In Molecularly Imprinted Polymers-2003, MRS Symposium Proceedings, Vol. 787, Kofinas P, Sellergren B, Roberts MJ (eds). Materials Research Society: Warrendale; 91-96. DOI: 10.1557/PROC-787-G6.4

3440. Cristallini C, Ciardelli G, Barbani N, Giusti P. 2004. Acrylonitrile-acrylic acid copolymer membrane imprinted with uric acid for clinical uses. Macromol. Biosci. 4: 31-38. DOI: 10.1002/mabi.200300026

3441. El-Toufaili FA, Visnjevski A, Brüggemann O. 2004. Screening combinatorial libraries of molecularly imprinted polymer films casted on membranes in single-use membrane modules. J. Chromatogr. B 804: 135-139. DOI: 10.1016/j.jchromb.2004.02.016

3442. Hattori K, Hiwatari M, liyama C, Yoshimi Y, Kohori F, Sakai K, Piletsky SA. 2004. Gate effect of theophylline-imprinted polymers grafted to the cellulose by living radical polymerization. J. Membr. Sci. 233: 169-173. DOI: 10.1016/j.memsci.2003.12.013

3443. Liao HP, Zhang ZH, Nie LH, Yao SZ. 2004. Electrosynthesis of imprinted polyacrylamide membranes for the stereospecific L-histidine sensor and its characterization by AC impedance spectroscopy and piezoelectric quartz crystal technique. J. Biochem. Biophys. Meth. 59: 75-87. DOI: 10.1016/j.jbbm.2003.12.001

3444. Malaisamy R, Ulbricht M. 2004. Evaluation of molecularly imprinted polymer blend filtration membranes under solid phase extraction conditions. Sep. Purif. Technol. 39: 211-219. DOI: 10.1016/j.seppur.2003.12.005

3445. Minoura N, Idei K, Rachkov A, Choi YW, Ogiso M, Matsuda K. 2004. Preparation of azobenzene-containing polymer membranes that function in photoregulated molecular recognition. Macromolecules 37: 9571-9576. DOI: 10.1021/ma040010a 
3446. Park JK, Kim SJ. 2004. Separation of phenylalanine by ultrafiltration using D-Phe imprinted polyacrylonitrile-poly(acrylic acid)-poly(acryl amide) terpolymer membrane. Kor. J. Chem. Eng. 21: 994-998. DOI: 10.1007/BF02705583

3447. Silvestri D, Cristallini C, Ciardelli G, Giusti P, Barbani N. 2004. Molecularly imprinted bioartificial membranes for the selective recognition of biological molecules. J. Biomater. Sci. Polym. Ed. 15: 255-278. DOI: $10.1163 / 156856204322977175$

3448. Suedee R, Srichana T, Chuchome T, Kongmark U. 2004. Use of molecularly imprinted polymers from a mixture of tetracycline and its degradation products to produce affinity membranes for the removal of tetracycline from water. J. Chromatogr B 811: 191-200. DOI: 10.1016/j.jchromb.2004.08.044

3449. Wang HY, Xia SL, Sun H, Liu YK, Cao SK, Kobayashi T. 2004. Molecularly imprinted copolymer membranes functionalized by phase inversion imprinting for uracil recognition and permselective binding. J. Chromatogr. $B$ 804: 127-134. DOI: 10.1016/j.jchromb.2004.01.036

3450. Xia SL, Wang HY, Kobayashi T. 2004. Phase inversion molecularly imprinting of uracil targeted membranes made of polyacrylonitrile copolymers having methacrylic acid and acrylic acid segments for recognition and permselective binding. In Molecularly Imprinted Materials2003, MRS Symposium Proceedings, Vol. 787, Kofinas P, Sellergren B, Roberts MJ (eds). Materials Research Society: Warrendale; 103-108. DOI: 10.1557/PROC-787-G7.5

3451. Yang $H H$, Zhang $S Q$, Yang $W$, Chen XL, Zhuang ZX, Xu JG, Wang XR. 2004. Molecularly imprinted sol-gel nanotubes membrane for biochemical separations. J. Am. Chem. Soc. 126: 4054-4055. DOI: 10.1021/ja0389570

3452. Aoki T, Kaneko T. 2005. New macromolecular architectures for permselective membranes - Gas permselective membranes from 
dendrimers and enantioselectively permeable membranes from one-handed helical polymers. Polym. J. 37: 717-735. DOI: 10.1295/polymj.37.717

3453. Araki K, Maruyama T, Kamiya N, Goto M. 2005. Metal ion-selective membrane prepared by surface molecular imprinting. J. Chromatogr B 818: 141-145. DOI: 10.1016/j.jchromb.2004.12.030

3454. Kielczynski R, Bryjak M. 2005. Molecularly imprinted membranes for cinchona alkaloids separation. Sep. Purif. Technol. 41: 231-235. DOI: 10.1016/j.seppur.2004.03.021

3455. Silvestri D, Cristallini C, Ciardelli G, Giusti P, Barbani N. 2005. Molecularly imprinted bioartificial membranes for the selective recognition of biological molecules. Part 2: Release of components and thermal analysis. J. Biomater. Sci. Polym. Ed. 16: 397-410. DOI: 10.1163/1568562053654130

3456. Takeda K, Abe M, Kobayashi T. 2005. Molecular-imprinted Nylon membranes for the permselective binding of phenylalanine as opticalresolution membrane adsorbents. J. Appl. Polym. Sci. 97: 620-626. DOI: 10.1002/app.21753

3457. Trotta F, Baggiani C, Luda MP, Drioli E, Massari T. 2005. A molecular imprinted membrane for molecular discrimination of tetracycline hydrochloride. J. Membr. Sci. 254: 13-19. DOI: 10.1016/j.memsci.2004.11.013

3458. Yoshikawa M, Koso K, Yonetani K, Kitamura S, Kimura S. 2005. Optical resolution of racemic amino acid derivatives with molecularly imprinted membranes bearing oligopeptide tweezers. J. Polym. Sci. A,Polym. Chem. 43: 385-396. DOI: 10.1002/pola.20504

3459. Zhao BH, Li CX, Lu Y, Wang XD, Liu ZL, Zhang J. 2005. Formation of ordered macroporous membranes from random copolymers by the breath figure method. Polymer 46: 9508-9513. DOI: 10.1016/j.polymer.2005.07.035 
3460. Chen CB, Chen YJ, Zhou J, Wu CH. 2006. A 9-vinyladenine-based molecularly imprinted polymeric membrane for the efficient recognition of plant hormone 1H-indole-3-acetic acid. Anal. Chim. Acta 569: 58-65. DOI: 10.1016/j.aca.2006.03.062

3461. Conesa A, Palet C. 2006. Molecularly imprinted membranes (MIM) for the enantioseparation of selenoaminoacid compounds. Desalination 200: 110111. DOI: 10.1016/j.desal.2006.03.261

3462. Jiang ZY, Yu YX, Wu H. 2006. Preparation of CS/GPTMS hybrid molecularly imprinted membrane for efficient chiral resolution of phenylalanine isomers. J. Membr. Sci. 280: 876-882. DOI: 10.1016/j.memsci.2006.03.006

3463. Liu XY, Li CY, Wang CF, Li T, Hu SS. 2006. The preparation of molecularly imprinted poly(o-phenylenediamine) membranes for the specific $O, O$ dimethyl- $\alpha$-hydroxylphenyl phosphonate sensor and its characterization by AC impedance and cyclic voltammetry. J. Appl. Polym. Sci. 101: 2222-2227. DOI: 10.1002/app.23514

3464. Salam A, Ulbricht M. 2006. Enantio-selective MIP "nano-monolith" composite membranes. Desalination 199: 532-534. DOI: 10.1016/j.desal.2006.03.121

3465. Silvestri D, Coluccio ML, Barbani N, Ciardelli G, Cristallini C, Pegoraro C, Giusti P. 2006. Poly(ethylene-co-vinyl alcohol) membranes with specific adsorption properties for potential clinical application. Desalination 199: 138140. DOI: 10.1016/j.desal.2006.03.030

3466. Silvestri D, Barbani N, Cristallini C, Giusti P, Ciardelli G. 2006. Molecularly imprinted membranes for an improved recognition of biomolecules in aqueous medium. J. Membr. Sci. 282: 284-295. DOI: 10.1016/j.memsci.2006.05.031 
3467. Takeda K, Kobayashi T. 2006. Hybrid molecularly imprinted membranes for targeted bisphenol derivatives. J. Membr. Sci. 275: 61-69. DOI: 10.1016/j.memsci.2005.09.004

3468. Yoshikawa M, Murakoshi K, Kogita T, Hanaoka K, Guiver MD, Robertson GP. 2006. Chiral separation membranes from modified polysulfone having myrtenal-derived terpenoid side groups. Eur. Polym. J. 42: 2532-2539.

3469. Zhu XL, Su QD, Cai JB, Yang J, Gao Y. 2006. Molecularly imprinted polymer membranes for substance-selective solid-phase extraction from aqueous solutions. J. Appl. Polym. Sci. 101: 4468-4473. DOI: 10.1002/app.24183

3470. Bing NC, Xu ZL, Wang XJ, Yang ZG, Yang H. 2007. Recognition properties of poly(vinylidene fluoride) hollow-fiber membranes modified by levofloxacinimprinted polymers. J. Appl. Polym. Sci. 106: 71-76. DOI: 10.1002/app.26428

3471. Bossi A, Andreoli M, Bonini F, Piletsky S. 2007. "Gate effect" in templated polyacrylamide membranes influences the electrotransport of proteins and finds applications in proteome analysis. Anal. Bioanal. Chem. 389: 447-454. DOI: 10.1007/s00216-007-1258-3

3472. Chen H, Zeng G, Wang Z, Zhang X. 2007. To Construct "lon Traps" for Enhancing the Permselectivity and Permeability of Polyelectrolyte Multilayer Films. Macromolecules 40: 653-660. DOI: 10.1021/ma061406k

3473. Qu XJ, Chen CB, Zhou J, Wu CH. 2007. Transport Selectivity of a Diethylene Glycol Dimethacrylate-Based Thymine-imprinted Polymeric Membrane over a Cellulose Support for Nucleic Acid Bases. Chin. J. Chem. 25: 213-218. DOI: 10.1002/cjoc.200790043

3474. Salam A, Ulbricht M. 2007. Effect of Surface Modification on the Synthesis of Pore-Filling Polymeric Monoliths in Microfiltration Membranes Made from 
Poly(propylene) and Poly(ethylene terephthalate). Macromol. Mater. Eng. 292: 310-318. DOI: 10.1002/mame.200600411

3475. Sergeyeva TA, Brovko OO, Piletska EV, Piletsky SA, Goncharova LA, Karabanova LV, Sergeyeva LM, El'Skaya AV. 2007. Porous molecularly imprinted polymer membranes and polymeric particles. Anal. Chim. Acta 582: 311-319. DOI: 10.1016/j.aca.2006.09.011

3476. Silvestri D, Barbani N, Coluccio ML, Pegoraro C, Giusti P, Cristallini C, Ciardelli G. 2007. Poly(ethylene-co-vinyl alcohol) Membranes with Specific Adsorption Properties for Potential Clinical Application. Sep. Sci. Technol. 42: 2829-2847. DOI: 10.1080/01496390701558292

3477. Silvestri D, Cristallini C, Borrelli C, Barbani N, Giusti P, Ciardelli G. 2007. Composite membranes modified with recognition-able nanobeads as potential adsorbers for purification of biological fluids. J. Appl. Biomater. Biomech. 5: 166-175.

3478. Son $\mathrm{SH}$, Jegal J. 2007. Chiral separation of $\mathrm{D}, \mathrm{L}$-serine racemate using a molecularly imprinted polymer composite membrane. J. Appl. Polym. Sci. 104: 1866-1872. DOI: 10.1002/app.25845

3479. Takeda K, Uemura K, Kobayashi T. 2007. Hybrid molecular imprinted membranes having selectivity and separation behavior to targeted indole derivatives. Anal. Chim. Acta 591: 40-48. DOI: 10.1016/j.aca.2007.02.017

3480. Xia SL, Sun H, Wang HY, Takeda K, Kobayashi T. 2007. Ion Complex Membranes of Acrylonitrile Copolymers Having Methacrylic Acid and Amphiphilic Quaternized Ammonium Groups for Uracil Molecular Imprinting. J. Mater. Online 3: DOI: 10.2240/azojomo0239

3481. Yoshikawa M, Nakai K, Matsumoto H, Tanioka A, Guiver MD, Robertson GP. 2007. Molecularly Imprinted Nanofiber Membranes from Carboxylated 
Polysulfone by Electrospray Deposition. Macromol. Rapid Commun. 28: 2100-2105. DOI: 10.1002/marc.200700359

3482. Zhang QQ, Kusunoki T, Xu Q, Wang HY, Kobayashi T. 2007. Porous imprinted polymer membranes prepared by phase separation in compressed liquid CO2. Anal. Bioanal. Chem. 388: 665-673. DOI: 10.1007/s00216-007$1252-9$

3483. Cai ZF, Dai HJ, Si SH, Ren FL. 2008. Molecular imprinting and adsorption of metallothionein on nanocrystalline titania membranes. Appl. Surf. Sci. 254: 4457-4461. DOI: 10.1016/j.apsusc.2008.01.015

3484. Faizal CK, Hoshina Y, Kobayashi T. 2008. Scaffold membranes for selective adsorption of $\alpha$-tocopherol by phase inversion covalently imprinting technique. J. Membr. Sci. 322: 503-511. DOI: 10.1016/j.memsci.2008.05.046

3485. Faizal CKM, Kobayashi T. 2008. Tocopherol-targeted membrane adsorbents prepared by hybrid molecular imprinting. Polym. Eng. Sci. 48: 1085-1093. DOI: 10.1002/pen.21053

3486. Itou Y, Nakano M, Yoshikawa M. 2008. Optical resolution of racemic amino acid derivatives with molecularly imprinted membranes from tetrapetide consisting of glycinyl residues. J. Membr. Sci. 325: 371-375. DOI: 10.1016/j.memsci.2008.07.051

3487. Kobayashi T, Leong SS, Zhang QQ. 2008. Using polystyrene-co-maleic acid for molecularly imprinted membranes prepared in supercritical carbon dioxide. J. Appl. Polym. Sci. 108: 757-768. DOI: 10.1002/app.27734

3488. Lee MH, Tsai TC, Thomas JL, Lin HY. 2008. Recognition of creatinine by poly(ethylene-co-vinylalcohol) molecular imprinting membrane. Desalination 234: 126-133. DOI: 10.1016/j.desal.2007.09.078 
3489. Lin $Y$, Tang SQ, Mao X, Bao L. 2008. Protein recognition via molecularly imprinted agarose gel membrane. J. Biomed. Mater. Res. Part A 85A: 573581. DOI: 10.1002/jbm.a.31361

3490. Pegoraro C, Silvestri D, Ciardelli G, Cristallini C, Barbani N. 2008. Molecularly imprinted poly(ethylene-co-vinyl alcohol) membranes for the specific recognition of phospholipids. Biosens. Bioelectron. 24: 748-755. DOI: 10.1016/j.bios.2008.06.050

3491. Qu XJ, Meng QX, Ai SY, Zhou J, Zhu LS. 2008. Recognition of 6benzyladenine using a molecularly imprinted membrane on a cellulose acetate support. J. Anal. Chem. 63: 999-1004. DOI: $10.1134 / \mathrm{S} 1061934808100134$

3492. Sergeyeva TA, Piletska OV, Piletsky SA, Sergeeva LM, Brovko OO, El'ska GV. 2008. Data on the structure and recognition properties of the templateselective binding sites in semi-IPN-based molecularly imprinted polymer membranes. Mater. Sci. Eng.: C 28: 1472-1479. DOI: 10.1016/j.msec.2008.04.006

3493. Tasselli F, Donato L, Drioli E. 2008. Evaluation of molecularly imprinted membranes based on different acrylic copolymers. J. Membr. Sci. 320: 167172. DOI: 10.1016/j.memsci.2008.03.071

3494. Ul-Haq N, Khan T, Park JK. 2008. Enantioseparation with D-Phe- and LPhe-imprinted PAN-based membranes by ultrafiltration. J. Chem. Technol. Biotechnol. 83: 524-533. DOI: 10.1002/jctb.1827

3495. Ul-Haq N, Park JK. 2008. Optical resolution of phenylalanine using D-Pheimprinted poly(acrylic acid-co-acrylonitrile) membrane-Racemate solution concentration effect. Polym. Compos. 29: 1006-1013. DOI: 10.1002/pc.20479 
3496. Wang P, Hu WM, Su WK. 2008. Molecularly imprinted poly (methacrylamide-co-methacrylic acid) composite membranes for recognition of curcumin. Anal. Chim. Acta 615: 54-62. DOI: 10.1016/j.aca.2008.03.040

3497. Wang XJ, Xu ZL, Feng JL, Bing NC, Yang ZG. 2008. Molecularly imprinted membranes for the recognition of lovastatin acid in aqueous medium by a template analogue imprinting strategy. J. Membr. Sci. 313: 97-105. DOI: 10.1016/j.memsci.2007.12.067

3498. Wang XJ, Xu ZL, Bing NC, Yang ZG. 2008. Preparation and characterization of metal-complex imprinted PVDF hollow fiber membranes. J. Appl. Polym. Sci. 109: 64-73. DOI: 10.1002/app.26805

3499. Zhai YH, Liu YW, Chang XJ, Ruan XF, Liu JL. 2008. Metal ion-small molecule complex imprinted polymer membranes: Preparation and separation characteristics. Reac. Func. Polym. 68: 284-291. DOI: 10.1016/j.reactfunctpolym.2007.08.013

3500. Zhang CJ, Zhong S, Yang ZP. 2008. Cellulose acetate-based molecularly imprinted polymeric membrane for separation of vanillin and o-vanillin. Braz. J. Chem. Eng. 25: 365-373. DOI: 10.1590/S0104-66322008000200014

3501. Zhao CS, Yu BY, Qian BS, Wei Q, Yang KG, Zhang AM. 2008. BPA transfer rate increase using molecular imprinted polyethersulfone hollow fiber membrane. J. Membr. Sci. 310: 38-43. DOI: 10.1016/j.memsci.2007.10.042

3502. He DM, Susanto H, Ulbricht M. 2009. Photo-irradiation for preparation, modification and stimulation of polymeric membranes. Prog. Polym. Sci. 34: 62-98. DOI: 10.1016/j.progpolymsci.2008.08.004

3503. Zhang $Y Q$, Xiang L, Zhang $Y H$, Gao XQ. 2009. Study on preparation of composite membrane with molecular recognizing property and its selective permeance mechanism. Sep. Purif. Technol. 65: 130-136. DOI: 10.1016/j.seppur.2008.10.049 
3504. Faizal CK, Kikuchi Y, Kobayashi T. 2009. Molecular imprinting targeted for $\alpha$-tocopherol by calix[4]resorcarenes derivative in membrane scaffold prepared by phase inversion. J. Membr. Sci. 334: 110-116. DOI: 10.1016/j.memsci.2009.02.019

3505. Liu W, Wang B. 2009. Preparation and application of NorfloxacinMIP/polysulfone blending molecular imprinted polymer membrane. J. Appl. Polym. Sci. 113: 1125-1132. DOI: 10.1002/app.30052

3506. Hillberg AL, Brain KR, Allender CJ. 2009. Design and evaluation of thin and flexible theophylline imprinted polymer membrane materials. J. Mol. Recognit. 22: 223-231. DOI: 10.1002/jmr.935

3507. Nemulenzi O, Mhaka B, Cukrowska E, Ramström O, Tutu H, Chimuka L. 2009. Potential of combining of liquid membranes and molecularly imprinted polymers in extraction of $17 \beta$-estradiol from aqueous samples. J. Sep. Sci. 32: 1941-1948. DOI: 10.1002/jssc.200800659

3508. Wu H, Zhao YY, Nie MC, Jiang ZY. 2009. Molecularly imprinted organicinorganic hybrid membranes for selective separation of phenylalanine isomers and its analogue. Sep. Purif. Technol. 68: 97-104. DOI: 10.1016/j.seppur.2009.04.014

3509. Zhang YQ, Gao XQ, Wang YL, Zhang YH, Lu GQ. 2009. Study on the build of channels in accurate separation membrane and its selective mechanism. J. Membr. Sci. 339: 100-108. DOI: 10.1016/j.memsci.2009.04.035

3510. Ceolin G, Navarro-Villoslada F, Moreno-Bondi MC, Horvai G, Horvath V. 2009. Accelerated Development Procedure for Molecularly Imprinted Polymers Using Membrane Filterplates. J. Comb. Chem. 11: 645-652. DOI: $10.1021 /$ cc900022u 
3511. Shawky HA. 2009. Synthesis of ion-imprinting chitosan/PVA crosslinked membrane for selective removal of $\mathrm{Ag}(\mathrm{I})$. J. Appl. Polym. Sci. 114: 26082615. DOI: 10.1002/app.30816

3512. Yoshimi Y, Yoshiizumi R, Arai R, Nakano I, Sekine S. 2009. ChiralDiscriminative Gate Effect in Self-Supporting Phenylalanine-Imprinted Poly(Methacrylic Acid-co-2-Vinylpyridine-co-Triethyleneglycol Dimethacrylate) Membrane. J. Chem. Eng. Jpn. 42: 600-606. DOI: 10.1252/jcej.08we251

3513. Wang $P$, Chen SS, Zhu XY, Xie J. 2009. Daidzein-imprinted membranes using co-functional monomers. J. Chromatogr. A 1216: 7639-7644. DOI: 10.1016/j.chroma.2009.08.093

3514. Dima SO, Sarbu A, Dobre T, Bradu C, Antohe N, Radu AL, Nicolescu TV, Lungu A. 2009. Molecularly Imprinted Membranes for Selective Separations. Materiale Plastice 46: 372-378.

3515. Fan PM, Wang B. 2009. Preparation of molecularly imprinted polymer membrane with blending trimethoprim-MIP and polysulfone and its transport properties. Kor. J. Chem. Eng. 26: 1813-1820. DOI: 10.1007/s11814-0090256-x

3516. Lee MH, Thomas JL, Tasi SB, Liu BD, Lin HY. 2009. Formation and Recognition Characteristics of Albumin-imprinted Poly(Ethylene-co-VinylAlcohol) Membranes. J. Nanosci. Nanotechnol. 9: 3469-3477. DOI: 10.1166/jnn.2009.NS18

3517. Bing NC, Tian Z, Zhu LP, Jin HY, Wang LL, Wang LJ. 2010. Controlled grafting of S-naproxen imprinted layer on PVDF microporous membrane by ATRP. In Manufacturing Processes and Systems, Advanced Materials Research, Vol. 148-149, Liu XH, Jiang ZY, Han JT (eds). Scientific.net: 1026-1030. DOI: 10.4028/www.scientific.net/AMR.148-149.1026 
3518. Chen RR, Qin L, Jia M, He XW, Li WY. 2010. Novel surface-modified molecularly imprinted membrane prepared with iniferter for permselective separation of lysozyme. J. Membr. Sci. 363: 212-220. DOI: 10.1016/j.memsci.2010.07.026

3519. Donato L, Tasselli F, Drioli E. 2010. Molecularly Imprinted Membranes with Affinity Properties for Folic Acid. Sep. Sci. Technol. 45: 2373-2379. DOI: 10.1080/01496395.2010.510089

3520. Faizal CKM, Kikuchi Y. 2010. Molecular imprinting membrane having calix[4]resorcarenes moieties for selective separation of $\alpha$-tocopherol. In Chemical, Biological and Environmental Engineering (ICBEE), 2010 2nd International Conference on, IEEE: 274-276. DOI: 10.1109/ICBEE.2010.5648796

3521. Kochkodan V, Hilal N, Melnik V, Kochkodan O, Vasilenko O. 2010. Selective recognition of organic pollutants in aqueous solutions with composite imprinted membranes. Adv. Colloid Interface Sci. 159: 180-188. DOI: 10.1016/j.cis.2010.06.006

3522. Li JP, Jiang FY. 2010. A Chlortoluron Sensor based on Molecularly Imprinted Sensitive Membranes. Chem. Lett. 39: 478-479. DOI: $10.1246 / \mathrm{cl} .2010 .478$

3523. Liu XF, Zhou J, Chen CB. 2010. Transport selectivity of tribenuron-methyl imprinted polymer nanowire membrane prepared using $\mathrm{N}, \mathrm{O}$-bismethacryloyl ethanolamine as a functional crosslinking monomer. J. Appl. Polym. Sci. 118: 678-684. DOI: 10.1002/app.32456

3524. Singh KP, Dobhal R, Prajapati RK, Kumar S, Sanjesh, Ansarid MA. 2010. Preparation of isoproturon and 2,4-dichlorophenoxy acetic acid imprinted membranes: Ion transport study. Desal. Water Treat. 24: 176-189. DOI: 10.5004/dwt.2010.1497 
3525. Sueyoshi Y, Fukushima C, Yoshikawa M. 2010. Molecularly imprinted nanofiber membranes from cellulose acetate aimed for chiral separation. $J$. Membr. Sci. 357: 90-97. DOI: 10.1016/j.memsci.2010.04.005

3526. Ul-Haq N, Park JK. 2010. Chiral resolution of phenylalanine by d-Phe imprinted membrane considering rejection property. Bioprocess Biosystems Eng. 33: 79-86. DOI: 10.1007/s00449-009-0352-7

3527. Wang JY, Liu F, Xu ZL, Li K. 2010. Theophylline molecular imprint composite membranes prepared from poly(vinylidene fluoride) (PVDF) substrate. Chem. Eng. Sci. 65: 3322-3330. DOI: 10.1016/j.ces.2010.02.024

3528. Wu SG, Tan WG, Xu HH. 2010. Protein molecularly imprinted polyacrylamide membrane: for hemoglobin sensing. Analyst 135: 25232527. DOI: $10.1039 / C 0 A N 00191 \mathrm{~K}$

3529. Yoshimi Y, Arai R, Nakayama S. 2010. Influence of the solvent on nature of gate effect in molecularly imprinted membrane. Anal. Chim. Acta 682: 110116. DOI: 10.1016/j.aca.2010.09.050

3530. Zhang MS, Huang JR, Yu P, Chen X. 2010. Preparation and characteristics of protein molecularly imprinted membranes on the surface of multiwalled carbon nanotubes. Talanta 81: 162-166. DOI: 10.1016/j.talanta.2009.11.052

3531. Zhang YQ, Gao XQ, Xiang L, Zhang YH, Diniz da Costa JC. 2010. Study on composite membranes with high selective permeance properties. J. Membr. Sci. 346: 318-326. DOI: 10.1016/j.memsci.2009.09.053

3532. Barahona F, Turiel E, Martín-Esteban A. 2011. Supported liquid membraneprotected molecularly imprinted fibre for solid-phase microextraction of thiabendazole. Anal. Chim. Acta 694: 83-89. DOI: 10.1016/j.aca.2011.03.052 
3533. Bing NC, Tian Z, Jin HY, Wang LJ, Zhu LP, Xu ZL. 2011. Separation of Naproxen Enantiomers Using Hollow Fiber Molecularly Imprinted Membrane Chromatography. Chem. Lett. 40: 266-267. DOI: 10.1246/cl.2011.266

3534. Bryjak M, Duraj I. 2011. Molecularly Imprinted Membranes for Removal of Bisphenol A. Solv. Extract. Ion Exch. 29: 432-439. DOI: 10.1080/07366299.2011.573446

3535. Büttiker R, Ebert J, Hinderling C, Adlhart C. 2011. Membranes for Specific Adsorption: Immobilizing Molecularly Imprinted Polymer Microspheres using Electrospun Nanofibers. Chimia 65: 182-186. DOI: 10.2533/chimia.2011.182

3536. De Luca G, Donato L, García Del Blanco S, Tasselli F, Drioli E. 2011. On the Cause of Controlling Affinity to Small Molecules of Imprinted Polymeric Membranes Prepared by Noncovalent Approach: A Computational and Experimental Investigation. J. Phys. Chem. B 115: 9345-9351. DOI: $10.1021 /$ jp2006638

3537. Donato L, Greco MC, Drioli E. 2011. Preparation of molecularly imprinted membranes and evaluation of their performance in the selective recognition of dimethoate. Desal. Water Treat. 30: 171-177. DOI: 10.5004/dwt.2011.1960

3538. Donato L, Chiappetta G, Drioli E. 2011. Surface Functionalization of PVDF Membrane with a Naringin-Imprinted Polymer Layer Using PhotoPolymerization Method. Sep. Sci. Technol. 46: 1555-1562. DOI: $10.1080 / 01496395.2011 .575429$

3539. Faizal CKM, Kobayashi T. 2011. Molecularly Imprinted Membrane Applied for Selective Separation. J. Appl. Sci. 11: 2411-2415. DOI: 10.3923/jas.2011

3540. Hatanaka M, Nishioka Y, Yoshikawa M. 2011. Polyurea With L-Lysinyl Residues as Components: Application to Membrane Separation of 
Enantiomers. Macromol. Chem. Phys. 212: 1351-1359. DOI: 10.1002/macp.201100054

3541. Hattori K, Sasaki A, Yoshimi Y, Kohori F, Sakai K. 2011. Chiral-selectivity on gate effect of phenylalanine-imprinted polymers grafted onto cellulosic membrane. Chem Sens 20: 384-385.

3542. Kochkodan V, Hilal N, Melnik V, Kochkodan O, Remizovska A. 2011. The express monitoring of organic pollutants in water with composite imprinted membranes. J. Membr. Sci. 377: 151-158. DOI: 10.1016/j.memsci.2011.04.033

3543. Li XF, Zhong SA, Chen L, Whittaker A. 2011. Computer simulation and preparation of molecularly imprinted polymer membranes with chlorogenic acid as template. Polym. Int. 60: 592-598. DOI: 10.1002/pi.2985

3544. Ma XL, Chen RY, Zheng X, Youn H, Chen Z. 2011. Preparation of molecularly imprinted CS membrane for recognizing naringin in aqueous media. Polym. Bull. 66: 853-863. DOI: 10.1007/s00289-011-0453-8

3545. Mi ZY, Ma Z, Li XL, Xiong WD, Zhang YQ. 2011. Recognition of Papain by Konjac Glucomannan-Based Molecularly Imprinted Membrane. In Advanced Research on Material Engineering, Chemistry, Bioinformatics, Advanced Materials Research, Vol. 282-283, Zhang H, Jin D (eds). Trans Tech Publications: 687-690. DOI: 10.4028/www.scientific.net/AMR.282-283.687

3546. Renkecz T, Ceolin G, Horváth V. 2011. Selective solid phase extraction of propranolol on multiwell membrane filter plates modified with molecularly imprinted polymer. Analyst 136: 2175-2182. DOI: 10.1039/C0AN00906G

3547. Vatanpour V, Madaeni SS, Zinadini S, Rajabi HR. 2011. Development of ion imprinted technique for designing nickel ion selective membrane. J. Membr. Sci. 373: 36-42. DOI: 10.1016/j.memsci.2011.02.030 
3548. Xu SP, Liu Y, Sun LQ, Luo AQ. 2011. Preparation and Recognition Properties of Levodropopizine Molecularly Imprinted Composite Membranes. In New Materials, Applications and Processes, Vol. 399-401, Zeng JM, Kim YH, Chen YF (eds). Scientific.net: 355-358. DOI: 10.4028/www.scientific.net/AMR.399-401.355

3549. Zhang YQ, Shan X, Gao XQ. 2011. Development of a molecularly imprinted membrane for selective separation of flavonoids. Sep. Purif. Technol. 76: 337-344. DOI: 10.1016/j.seppur.2010.10.024

3550. Herold M, Lehmann M, Brunner H, Tovar GEM. 2006. Research note: Smart material composite membranes based on molecularly imprinted nanoparticles used for selective filtration. Filtration 6: 250-253.

3551. Guan P, Hu XL, Zhao YM. 2009. Influences of preparative conditions on properties of erythromycin- molecularly imprinted membrane. In Advanced Polymer Processing, Advanced Materials Research, Ma LX, Wang CS, Yang WM (eds). Scientific.Net: 119-124. DOI: 10.4028/www.scientific.net/AMR.87-88.119

3552. Yu JY, Hu XL, Jiao CC, Zhao YM, Yang WW. 2009. Synthesis and properties of molecularly imprinted composite membranes of roxithromycin. In Advanced Polymer Processing, Advanced Materials Research, Ma LX, Wang CS, Yang WM (eds). Scientific.Net: 80-85. DOI: 10.4028/www.scientific.net/AMR.87-88.80

3553. Suedee R, Srichana T, Sangpagai C, Tunthana C, Vanichapichat P. 2004. Development of trichloroacetic acid sensor based on molecularly imprinted polymer membrane for the screening of complex mixture of haloacetic acids in drinking water. Anal. Chim. Acta 504: 89-100. DOI: 10.1016/S00032670(03)00951-6

3554. Kobayashi T, Faizal CKM, Son LT. 2009. Hybrid molecularly imprinted membranes for targeted tocopherol: Uses of cross-linked copolymer 
particles prepared by surfactant-free dispersion technique. J. Sep. Sci. 32: 3327-3333. DOI: 10.1002/jssc.200900307

3555. Baggiani C, Giraudi G, Giovannoli C, Tozzi C, Anfossi L. 2004. Adsorption isotherms of a molecular imprinted polymer prepared in the presence of a polymerisable template - Indirect evidence of the formation of template clusters in the binding site. Anal. Chim. Acta 504: 43-52. DOI: 10.1016/S0003-2670(03)00671-8

3556. Bass JD, Anderson SL, Katz A. 2004. The synthesis and catalytic application of a new class of imprinted silica. In Molecularly Imprinted Materials-2003, MRS Symposium Proceedings, Vol. 787, Kofinas P, Sellergren B, Roberts MJ (eds). Materials Research Society: Warrendale; 1 6. DOI: 10.1557/PROC-787-G1.3

3557. Busi E, Basosi R, Ponticelli F, Olivucci M. 2004. An innovative approach to the design of plastic antibodies: molecular imprinting via a non-polar transition state analogue. J. Mol. Catal. A 217: 31-36. DOI: 10.1016/j.molcata.2004.03.044

3558. Gxoyiya BSB, Hagemann JR, Kaye PT. 2004. Designer ligands. Part 12. Synthesis and evaluation of novel palladium (II)-selective ligand systems. J. Chem. Res. -S 252-256. DOI: 10.3184/0308234041209248

3559. Hedin-Dahlström J, Shoravi S, Wikman S, Nicholls IA. 2004. Stereoselective reduction of menthone by molecularly imprinted polymers. Tetrahedron: Asymmetry 15: 2431-2436. DOI: 10.1016/j.tetasy.2004.06.002

3560. Huang JT, Zheng SH, Zhang JQ. 2004. Molecularly imprinting of polymeric nucleophilic catalysts containing 4-alkylaminopyridine functions. Polymer 45: 4349-4354. DOI: 10.1016/j.polymer.2004.03.050

3561. Lettau K, Warsinke A, Laschewski A, Mosbach K, Yilmaz E, Scheller FW. 2004. An esterolytic imprinted polymer prepared via a silica-supported 
transition state analogue. Chem. Mater. 16: 2745-2749. DOl: $10.1021 / \mathrm{cm} 0352394$

3562. Liu JQ, Wulff G. 2004. Molecularly imprinted polymers with strong carboxypeptidase A- like activity: Combination of an amidinium function with a zinc-ion binding site in transition-state imprinted cavities. Angew. Chem. Int. Edit. 43: 1287-1290. DOI: 10.1002/anie.200352770

3563. Liu JQ, Wulff G. 2004. Functional mimicry of the active site of carboxypeptidase A by a molecular imprinting strategy: Cooperativity of an amidinium and a copper ion in a transition-state imprinted cavity giving rise to high catalytic activity. J. Am. Chem. Soc. 126: 7452-7453. DOI: 10.1021/ja048372I

3564. Maddock SC, Pasetto P, Resmini M. 2004. Novel imprinted soluble microgels with hydrolytic catalytic activity. Chem. Commun. 536-537. DOI: 10.1039/b312631e

3565. Meng ZH, Yamazaki T, Sode K. 2004. A molecularly imprinted catalyst designed by a computational approach in catalysing a transesterification process. Biosens. Bioelectron. 20: 1068-1075. DOI: 10.1016/j.bios.2004.02.032

3566. Motherwell WB, Bingham MJ, Pothier J, Six Y. 2004. A study of some molecularly imprinted polymers as protic catalysts for the isomerisation of $\alpha$ pinene oxide to trans-carveol. Tetrahedron 60: 3231-3241. DOI: 10.1016/j.tet.2004.02.016

3567. Svenson J, Zheng N, Nicholls IA. 2004. A molecularly imprinted polymerbased synthetic transaminase. J. Am. Chem. Soc. 126: 8554-8560. DOI: 10.1021/ja039622।

3568. Tada M, Sasaki T, Iwasawa Y. 2004. Design of a novel molecular-imprinted Rh-amine complex on $\mathrm{SiO}_{2}$ and its shape-selective catalysis for $\alpha$ - 
methylstyrene hydrogenation. J. Phys. Chem. B 108: 2918-2930. DOI: 10.1021/jp036421v

3569. Visnjevski A, Yilmaz E, Brüggemann O. 2004. Catalyzing a cycloaddition with molecularly imprinted polymers obtained via immobilized templates. Appl. Catal., A 260: 169-174. DOI: 10.1016/j.apcata.2003.10.015

3570. Anderson CD, Shea KJ, Rychnovsky SD. 2005. Strategies for the generation of molecularly imprinted polymeric nitroxide catalysts. Org. Lett. 7: 4879-4882. DOI: 10.1021/ol051749n

3571. Burri E, Ohm M, Daguenet C, Severin K. 2005. Site-isolated porphyrin catalysts in imprinted polymers. Chem. Eur. J. 11: 5055-5061. DOI: 10.1002/chem.200500375

3572. Defreese JL, Katz A. 2005. Synthesis of a confined class of chiral organic catalysts via bulk imprinting of silica. Chem. Mater. 17: 6503-6506. DOI: $10.1021 / \mathrm{cm} 050730 \mathrm{~b}$

3573. Gilliland JW, Yokoyama K, Yip WT. 2005. Solvent effect on mobility and photostability of organic dyes embedded inside silica sol-gel thin films. Chem. Mater. 17: 6702-6712. DOI: 10.1021/cm050658h

3574. Kalim R, Schomäcker R, Yüce $S$, Brüggemann O. 2005. Catalysis of a $\beta$ elimination applying membranes with incorporated molecularly imprinted polymer particles. Polym. Bull. 55: 287-297. DOI: 10.1007/s00289-0050438-6

3575. Luo $\mathrm{Y}$, Lin J. 2005. Synthesis and characterization of $\mathrm{Co}(\mathrm{II})$ salen functionalized MCM-41-type hybrid mesoporous silicas and their applications in catalysis for styrene oxidation with $\mathrm{H} 2 \mathrm{O} 2$. Microporous Mesoporous Mater. 86: 23-30. DOI: 10.1016/j.micromeso.2005.07.016 
3576. Meng ZH, Sode K. 2005. The molecular reaction vessels for a transesterification process created by molecular imprinting technique. $J$. Mol. Recognit. 18: 262-266. DOI: 10.1002/jmr.732

3577. Meng ZH, Yamazaki T, Sode K. 2005. Construction of artificial cyclic amide amidohydrolases using molecular imprinting technique. Biotechnol. Lett. 27: 471-475. DOI: 10.1007/s10529-005-2223-x

3578. Pasetto P, Maddock SC, Resmini M. 2005. Synthesis and characterisation of molecularly imprinted catalytic microgels for carbonate hydrolysis. Anal. Chim. Acta 542: 66-75. DOI: 10.1016/j.aca.2005.01.069

3579. Piletsky SA, Nicholls IA, Rozhko MI, Sergeyeva TA, Piletska EV, El'Skaya AV, Karube I. 2005. Molecularly imprinted polymers - Tyrosinase mimics. Ukr. Biokhim. Zh. 77: 63-67.

3580. Rathbone DL, Ali A, Antonaki P, Cheek S. 2005. Towards a polymeric binding mimic for cytochrome CYP2D6. Biosens. Bioelectron. 20: 23532363. DOI: 10.1016/j.bios.2005.01.009

3581. Say RI, Erdem M, Ersöz A, Turk H, Denizli A. 2005. Biomimetic catalysis of an organophosphate by molecularly surface imprinted polymers. Appl. Catal., A 286: 221-225. DOI: 10.1016/j.apcata.2005.03.015

3582. Schiller A, Scopelliti R, Benmelouka M, Severin K. 2005. Highly cross-linked polymers containing $\mathrm{N}, \mathrm{N}$ ',N"-chelate ligands for the $\mathrm{Cu}(\mathrm{II})$-mediated hydrolysis of phosphoesters. Inorg. Chem. 44: 6482-6492. DOI: $10.1021 /$ ic0504588

3583. Silvestri D, Borrelli C, Giusti P, Cristallini C, Ciardelli G. 2005. Polymeric devices containing imprinted nanospheres: a novel approach to improve recognition in water for clinical uses. Anal. Chim. Acta 542: 3-13. DOI: 10.1016/j.aca.2004.12.005 
3584. Tada M, Iwasawa Y. 2005. Chemical design and in situ characterization of active surfaces for selective catalysis. In The Materials Design and Chemistry of Environmentally Acceptable Catalysts, Annual Review of Materials Research, Vol. 35, Clarke DR, Rühle M (eds). Annual Reviews: Palo Alto; 397-426. DOI: 10.1146/annurev.matsci.35.102003.142526

3585. Visnjevski A, Schomäcker R, Yilmaz E, Brüggemann O. 2005. Catalysis of a Diels-Alder cycloaddition with differently fabricated molecularly imprinted polymers. Catal. Commun. 6: 601-606. DOI: 10.1016/j.catcom.2005.06.001

3586. Visnjevski A, Schomacker R, Yilmaz E, Brüggemann O. 2006. Erratum to "Catalysis of a Diels-Alder cycloaddition with differently fabricated molecularly imprinted polymers" [Catal. Commun. 6 (9) (2005) 601-606]. Catal. Commun. 7: 114. DOI: 10.1016/j.catcom.2005.08.004

3587. Bass JD, Solovyov A, Pascall AJ, Katz A. 2006. Acid-base bifunctional and dielectric outer-sphere effects in heterogeneous catalysis: A comparative investigation of model primary amine catalysts. J. Am. Chem. Soc. 128: 3737-3747. DOI: 10.1021/ja057395c

3588. Cheng ZY, Li YZ. 2006. The role of molecular recognition in regulating the catalytic activity of peroxidase-like polymers imprinted by a reductant substrate. J. Mol. Catal. A 256: 9-15. DOI: 10.1016/j.molcata.2006.04.042

3589. Jakubiak A, Kolarz BN, Jezierska J. 2006. Catalytic activity of Copper(II) enzyme-like catalysts, prepared by molecular imprinting technique in oxidation of phenols. Macromol. Symp. 235: 127-135. DOI: 10.1002/masy.200650316

3590. Lettau K, Warsinke A, Katterle M, Danielsson B, Scheller FW. 2006. A Bifunctional Molecularly Imprinted Polymer (MIP): Analysis of Binding and Catalysis by a Thermistor. Angew. Chem. Int. Edit. 45: 6986-6990. DOI: 10.1002/anie.200601796 
3591. Matsui J, Ochi Y, Tamaki K. 2006. Photodimerization of anthryl moieties in a poly(methacrylic acid) derivative as reversible cross-linking step in molecular imprinting. Chem. Lett. 35: 80-81. DOI: 10.1246/cl.2006.80

3592. Schiller A, Scopelliti R, Severin K. 2006. Enhanced hydrolytic activity of $\mathrm{CU}(\mathrm{II})$ and $\mathrm{Zn}$ (II) complexes in highly cross-linked polymers. Dalton Trans. 3858-3867. DOI: 10.1039/b605676h

3593. Tada M, Iwasawa Y. 2006. Advanced chemical design with supported metal complexes for selective catalysis. Chem. Commun. 2833-2844. DOI: $10.1039 / \mathrm{b} 601507 \mathrm{~g}$

3594. Volkmann A, Brüggemann O. 2006. Catalysis of an ester hydrolysis applying molecularly imprinted polymer shells based on an immobilised chiral template. Reac. Func. Polym. 66: 1725-1733. DOI: 10.1016/j.reactfunctpolym.2006.07.007

3595. Yane T, Shinmori H, Takeuchi T. 2006. Atrazine transforming polymer prepared by molecular imprinting with post-imprinting process. Org. Biomol. Chem. 4: 4469-4473. DOI: 10.1039/b612407k

3596. Yoshikawa M, Kawamura K, Ejima A, Aoki T, Sakurai S, Hayashi K, Watanabe K. 2006. Green polymers from Geobacillus thermodenitrificans DSM465 - Candidates for molecularly imprinted materials. Macromol. Biosci. 6: 210-215. DOI: 10.1002/mabi.200500187

3597. Zhang HQ, Piacham T, Drew M, Patek M, Mosbach K, Ye L. 2006. Molecularly imprinted nanoreactors for regioselective Huisgen 1,3-dipolar cycloaddition reaction. J. Am. Chem. Soc. 128: 4178-4179. DOI: 10.1021/ja057781u

3598. Chen ZY, Hua ZD, Wang J, Guan Y, Zhao MP, Li YZ. 2007. Molecularly imprinted soluble nanogels as a peroxidase-like catalyst in the oxidation 
reaction of homovanillic acid under aqueous conditions. Appl. Catal., A 328: 252-258. DOI: 10.1016/j.apcata.2007.05.040

3599. Johnson-White B, Zeinali M, Malanoski AP, Dinderman MA. 2007. Sunlightcatalyzed conversion of cyclic organics with novel mesoporous organosilicas. Catal. Commun. 8: 1052-1056. DOI: 10.1016/j.catcom.2006.10.002

3600. Li Q, Su HJ, Li J, Tan TW. 2007. Studies of adsorption for heavy metal ions and degradation of methyl orange based on the surface of ion-imprinted adsorbent. Process Biochem. 42: 379-383. DOI: 10.1016/j.procbio.2006.09.020

3601. Santos W, Lima PR, Tarley CRT, Kubota LT. 2007. A catalytically active molecularly imprinted polymer that mimics peroxidase based on hemin: application to the determination of p-aminophenol. Anal. Bioanal. Chem. 389: 1919-1929. DOI: 10.1007/s00216-007-1601-8

3602. Shen XT, Zhu LH, Tang HQ. 2007. Synthesis of molecular imprinted polymer coated photocatalysts with high selectivity. Chem. Commun. 11631165. DOI: 10.1039/b615303h

3603. Wang J, Chen ZY, Zhao MP, Li YZ. 2007. Catalytical oxidation of styrene by molecularly imprinted polymer with phenylacetic acid as template and hemin as co-monomer. Chin. Chem. Lett. 18: 981-984. DOI: 10.1016/j.cclet.2007.06.009

3604. Zhang DN, Li SJ, Li WK, Chen YF. 2007. Biomimic recognition and catalysis by an imprinted catalysts: a rational design of molecular self-assembly toward predetermined high specificity. Catal. Lett. 115: 169-175. DOI: 10.1007/s10562-007-9084-y 
3605. Carboni D, Flavin K, Servant A, Gouverneur V, Resmini M. 2008. The First Example of Molecularly Imprinted Nanogels with Aldolase Type I Activity. Chem. Eur. J. 14: 7059-7065. DOI: 10.1002/chem.200800675

3606. Christoforidis KC, Louloudi M, Rutherford AW, Deligiannakis Y. 2008. Semiquinone in Molecularly Imprinted Hybrid Amino Acid-SiO2 Biomimetic Materials. An Experimental and Theoretical Study. J. Phys. Chem. C 112: 12841-12852. DOI: 10.1021/jp800430n

3607. Huang X, Liu Y, Liang K, Tang Y, Liu JQ. 2008. Construction of the Active Site of Glutathione Peroxidase on Polymer-Based Nanoparticles. Biomacromolecules 9: 1467-1473. DOI: 10.1021/bm701386b

3608. Li SJ, Tong KJ, Zhang DN, Huang X. 2008. Rationally Designing Active Molecularly Imprinted Polymer Toward a Highly Specific Catalyst by Using Metal as an Assembled Pivot. J. Inorg. Organomet. Polym. Mater. 18: 264271. DOI: $10.1007 / \mathrm{s} 10904-007-9172-x$

3609. Liu JQ, Wulff G. 2008. Functional Mimicry of Carboxypeptidase A by a Combination of Transition State Stabilization and a Defined Orientation of Catalytic Moieties in Molecularly Imprinted Polymers. J. Am. Chem. Soc. 130: 8044-8054. DOI: $10.1021 / \mathrm{ja} 8012648$

3610. Tai DF, Lin YF. 2008. Molecularly imprinted cavities template the macrocyclization of tetrapeptides. Chem. Commun. 5598-5600. DOI: 10.1039/b813439a

3611. Terry TJ, Stack TDP. 2008. Covalent Heterogenization of a Discrete Mn(II) Bis-Phen Complex by a Metal-Template/Metal-Exchange Method: An Epoxidation Catalyst with Enhanced Reactivity. J. Am. Chem. Soc. 130: 4945-4953. DOI: 10.1021/ja0742030

3612. Tong KJ, Xiao S, Li SJ, Wang J. 2008. Molecular Recognition and Catalysis by Molecularly Imprinted Polymer Catalysts: Thermodynamic and Kinetic 
Surveys on the Specific Behaviors. J. Inorg. Organomet. Polym. Mater. 18: 426-433. DOI: 10.1007/s10904-008-9217-9

3613. Tsvelikhovsky D, Pessing D, Avnir D, Blum J. 2008. Forcing a cis-Product by Matrix Imprinting: Heck Reaction Catalyzed by Palladium Acetate Entrapped within cis-Imprinted Sol-Gel Derived Silicates. Adv. Synth. Catal. 350: 2856-2858. DOI: 10.1002/adsc.200800590

3614. Weyland M, Ferrère S, Lattes A, Mingotaud AF, Mauzac M. 2008. Artificial enzymes based on imprinted liquid-crystalline materials. Liq. Cryst. 35: 219231. DOI: $10.1080 / 02678290701751566$

3615. Huang X, Yin YZ, Liu Y, Bai XL, Zhang ZM, Xu JY, Shen JC, Liu JQ. 2009. Incorporation of glutathione peroxidase active site into polymer based on imprinting strategy. Biosens. Bioelectron. 25: 657-660. DOI: 10.1016/j.bios.2009.01.033

3616. Huo HY, Su HJ, Jiang W, Tan TW. 2009. Effect of trace Ag+ adsorption on degradation of organic dye wastes. Biochem. Eng. J. 43: 2-7. DOI: 10.1016/j.bej.2008.07.012

3617. Ikegami S, Hamamoto H. 2009. Novel Recycling System for Organic Synthesis via Designer Polymer-Gel Catalysts. Chem. Rev. 109: 583-593. DOI: $10.1021 / \mathrm{cr} 800481 \mathrm{x}$

3618. Kirsch N, Hedin-Dahlström J, Henschel H, Whitcombe MJ, Wikman S, Nicholls IA. 2009. Molecularly imprinted polymer catalysis of a Diels-Alder reaction. J. Mol. Catal. B 58: 110-117. DOI: 10.1016/j.molcatb.2008.12.002

3619. Lee J, Bernard S, Liu XC. 2009. Nanostructured biomimetic catalysts for asymmetric hydrogenation of enamides using molecular imprinting technology. Reac. Func. Polym. 69: 650-654. DOI: 10.1016/j.reactfunctpolym.2009.04.006 
3620. Li SJ, Gong SQ. 2009. A Substrate-Selective Nanoreactor Made of Molecularly Imprinted Polymer Containing Catalytic Silver Nanoparticles. Adv. Funct. Mater. 19: 2601-2606. DOI: 10.1002/adfm.200900428

3621. Li SJ, Gong SQ. 2009. A Positively Temperature-Responsive, SubstrateSelective Ag Nanoreactor. J. Phys. Chem. B 113: 16501-16507. DOI: 10.1021/jp907527x

3622. Lin YF, Lee SM, Chen CT, Lu KH, Tai DF. 2009. On-surface Cyclization of Tetrapeptides using Molecularly Imprinted Polymers as Non-covalent Auxiliaries. J. Chin. Chem. Soc. 56: 127-134.

3623. Liu XH, Yue H, Li SJ, Li WK. 2009. A Highly Substrate-Selective Metal Nanoreactor Using a Template-Imprinted Memory. J. Inorg. Organomet. Polym. Mater. 19: 335-341. DOI: 10.1007/s10904-009-9265-9

3624. Pachón LD, Yosef I, Markus TZ, Naaman R, Avnir D, Rothenberg G. 2009. Chiral imprinting of palladium with cinchona alkaloids. Nat. Chem. 1: 160164. DOI: $10.1038 /$ nchem. 180

3625. Santos WdJR, Lima PR, Tarley CRT, Höehr NF, Kubota LT. 2009. Synthesis and application of a peroxidase-like molecularly imprinted polymer based on hemin for selective determination of serotonin in blood serum. Anal. Chim. Acta 631: 170-176. DOI: 10.1016/j.aca.2008.10.034

3626. Shen XT, Zhu LH, Huang CX, Tang HQ, Yu ZW, Deng F. 2009. Inorganic molecular imprinted titanium dioxide photocatalyst: synthesis, characterization and its application for efficient and selective degradation of phthalate esters. J. Mater. Chem. 19: 4843-4851. DOI: 10.1039/b900196d

3627. Shen XT, Zhu LH, Liu GX, Tang HQ, Liu SS, Li WY. 2009. Photocatalytic removal of pentachlorophenol by means of an enzyme-like molecular imprinted photocatalyst and inhibition of the generation of highly toxic intermediates. New J. Chem. 33: 2278-2285. DOI: 10.1039/b9nj00255c 
3628. Shen XT, Zhu LH, Yu HW, Tang HQ, Liu SS, Li WY. 2009. Selective photocatalysis on molecular imprinted $\mathrm{TiO}_{2}$ thin films prepared via an improved liquid phase deposition method. New J. Chem. 33: 1673-1679. DOI: 10.1039/b901087d

3629. Wu XY, Shimizu KD. 2009. Development of molecularly imprinted polymers as tailored templates for the solid-state [2+2] photodimerization. Biosens. Bioelectron. 25: 640-646. DOI: 10.1016/j.bios.2009.01.038

3630. Yu CY, Chen S, Quan X, Ou XX, Zhang YB. 2009. Separation of Phthalocyanine-like Substances from Humic Acids Using a Molecular Imprinting Method and Their Photochemical Activity under Simulated Sunlight Irradiation. J. Agric. Food Chem. 57: 6927-6931. DOI: $10.1021 / \mathrm{jf} 9016459$

3631. Zayas HA, Bowyer MC, Gordon CP, Holdsworth Cl, McCluskey A. 2009. Synthesis of biaryl-styrene monomers by microwave-assisted Suzuki coupling. Tetrahedron Lett. 50: 5894-5895. DOI: 10.1016/j.tetlet.2009.07.117

3632. Chen ZY, Xu L, Liang YA, Zhao MP. 2010. pH-Sensitive Water-Soluble Nanospheric Imprinted Hydrogels Prepared as Horseradish Peroxidase Mimetic Enzymes. Adv. Mater. 22: 1488-1492. DOI: 10.1002/adma.200903122

3633. Erdem M, Say RI, Ersöz A, Denizli A, Türk H. 2010. Biomimicking, metalchelating and surface-imprinted polymers for the degradation of pesticides. Reac. Func. Polym. 70: 238-243. DOI: 10.1016/j.reactfunctpolym.2009.12.005

3634. Erdem M, Say RI, Ersöz A, Denizli A, Türk H. 2010. Imprinted polymer/organo-smectite nanocomposites for paraoxon hydrolysis. Appl. Clay Sci. 47: 223-228. DOI: 10.1016/j.clay.2009.10.011 
3635. Han DM, Li YM, Jia WP. 2010. Preparation and characterization of molecularly imprinted $\mathrm{SiO}_{2}-\mathrm{TiO}_{2}$ and photo-catalysis for 2, 4-dichlorophenol. Adv. Mat. Lett. 1: 188-192. DOI: 10.5185/amlett.2010.7137

3636. Han DM, Dai GL, Jia WP, Liang HD. 2010. Preparation and photocatalytic activity of Cu2+-doped 2, 4-dichlorophenol molecularly imprinted SiO2-TiO2 nanocomposite. Micro \& Nano Letters 5: 76-80. DOI: 10.1049/mnl.2009.0114

3637. Karakhanov EA, Maximov AL. 2010. Molecular imprinting technique for the design of cyclodextrin based materials and their application in catalysis. Curr. Org. Chem. 14: 1284-1295. DOI: 10.2174/138527210791616902

3638. Liu YT, Liu RH, Liu CB, Luo SL, Yang LX, Sui F, Teng YR, Yang RB, Cai QY. 2010. Enhanced photocatalysis on TiO2 nanotube arrays modified with molecularly imprinted TiO2 thin film. J. Haz. Mater. 182: 912-918. DOI: 10.1016/j.jhazmat.2010.07.007

3639. Sergeyeva TA, Slinchenko OA, Gorbach LA, Matyushov VF, Brovko OO, Piletsky SA, Sergeeva LM, Elska GV. 2010. Catalytic molecularly imprinted polymer membranes: Development of the biomimetic sensor for phenols detection. Anal. Chim. Acta 659: 274-279. DOI: 10.1016/j.aca.2009.11.065

3640. Sharabi D, Paz Y. 2010. Preferential photodegradation of contaminants by molecular imprinting on titanium dioxide. Applied Catalysis B: Environmental 95: 169-178. DOI: 10.1016/j.apcatb.2009.12.024

3641. Shiraishi Y, Suzuki T, Hirai T. 2010. Selective photooxidation of chlorophenols with molecularly imprinted polymers containing a photosensitizer. New J. Chem. 34: 714-717. DOI: 10.1039/b9nj00732f

3642. Song H, Ozkan US. 2010. The role of impregnation medium on the activity of ceria-supported cobalt catalysts for ethanol steam reforming. J. Mol. Catal. A 318: 21-29. DOI: 10.1016/j.molcata.2009.11.003 
3643. Tang Y, Zhou LP, Li JX, Luo QA, Huang X, Wu P, Wang YG, Xu JY, Shen JC, Liu JQ. 2010. Giant Nanotubes Loaded with Artificial Peroxidase Centers: Self-Assembly of Supramolecular Amphiphiles as a Tool To Functionalize Nanotubes. Angew. Chem. Int. Edit. 49: 3920-3924. DOI: 10.1002/anie.200907036

3644. Wu ZF, Dong MX, Lu M, Li ZQ. 2010. Encapsulation of $\alpha$-galactosidase from Aspergillus oryzae based on "fish-in-net" approach with molecular imprinting technique. J. Mol. Catal. $B$ 63: 75-80. DOI: 10.1016/j.molcatb.2009.12.012

3645. Yu CY, Zhang YB, Quan X, Chen S, Han JB, Ou XX, Zhao JC. 2010. Photochemical Effect of Humic Acid Components Separated Using Molecular Imprinting Method Applying Porphyrin-like Substances as Templates in Aqueous Solution. Environ. Sci. Technol. 44: 5812-5817. DOI: 10.1021/es100806d

3646. Abbate V, Bassindale AR, Brandstadt KF, Taylor PG. 2011. Biomimetic catalysis at silicon centre using molecularly imprinted polymers. J. Catal. 284: 68-76. DOI: 10.1016/j.jcat.2011.08.019

3647. Díaz-Díaz G, Diñeiro Y, Menéndez MI, Blanco-López MC, Lobo-Castañón MJ, Miranda-Ordieres AJ, Tuñón-Blanco P. 2011. Molecularly imprinted catalytic polymers with biomimetic chloroperoxidase activity. Polymer 52: 2468-2473. DOI: 10.1016/j.polymer.2011.04.004

3648. Hassanzadeh-Khayyat M, Lai EPC, Kollu K, Ormeci B. 2011. Degradation of Diclofenac in Molecularly Imprinted Polymer Submicron Particles by UV Light Irradiation and $\mathrm{HCl}$ Acid Treatment. Journal of Water Resource and Protection 3: 643-654. DOI: 10.4236/jwarp.2011.39074

3649. Henschel $H$, Kirsch $N$, Hedin-Dahlström J, Whitcombe MJ, Wikman S, Nicholls IA. 2011. Effect of the cross-linker on the general performance and temperature dependent behaviour of a molecularly imprinted polymer 
catalyst of a Diels-Alder reaction. J. Mol. Catal. B 72: 199-205. DOI: 10.1016/j.molcatb.2011.06.006

3650. Huo PW, Lu ZY, Wang HQ, Pan JM, Li HM, Wu XY, Huang WH, Yan YS. 2011. Enhanced photodegradation of antibiotics solution under visible light with $\mathrm{Fe} 2+/ \mathrm{Fe} 3+$ immobilized on $\mathrm{TiO} /$ fly-ash cenospheres by using ions imprinting technology. Chem. Eng. J. 172: 615-622. DOI: 10.1016/j.cej.2011.06.003

3651. Keçili R, Özcan AA, Ersöz A, Hür D, Denizli A, Say R. 2011. Superparamagnetic nanotraps containing MIP based mimic lipase for biotransformations uses. J. Nanopart. Res. 13: 2073-2079. DOI: 10.1007/s11051-010-9962-x

3652. Li SJ, Ge Y, Tiwari A, Wang SQ, Turner APF, Piletsky SA. 2011. 'On/off'switchable catalysis by a smart enzyme-like imprinted polymer. J. Catal. 278: 173-180. DOI: 10.1016/j.jcat.2010.11.011

3653. Li SJ, Ge Y, Turner APF. 2011. A Catalytic and Positively Thermosensitive Molecularly Imprinted Polymer. Adv. Funct. Mater. 21: 1194-1200. DOI: 10.1002/adfm.201001906

3654. Okutucu B, Çelem EB, Önal S. 2010. Immobilization of $\alpha$-galactosidase on galactose-containing polymeric beads. Enzyme Microb. Technol. 46: 200205. DOI: 10.1016/j.enzmictec.2009.12.005

3655. Guo Y, Yang Y, Guo TY. 2011. Preparation of a PTE simulacrum based on surface molecular imprinting. Chin. Chem. Lett. 22: 493-496. DOI: 10.1016/j.cclet.2010.10.050

3656. Sartori LR, Santos WdJR, Kubota LT, Segatelli MG, Tarley CRT. 2011. Flow-based method for epinephrine determination using a solid reactor based on molecularly imprinted poly(FePP-MAA-EGDMA). Mater. Sci. Eng.: C 31: 114-119. DOI: 10.1016/j.msec.2010.08.012 
3657. Servant A, Haupt K, Resmini M. 2011. Tuning Molecular Recognition in Water-Soluble Nanogels with Enzyme-Like Activity for the Kemp Elimination. Chem. Eur. J. 17: 11052-11059. DOI: 10.1002/chem.201002747

3658. Striegler S, Barnett JD, Dunaway NA. 2011. Glycoside Hydrolysis with Sugar-Templated Microgel Catalysts. ACS Catalysis 2: 50-55. DOI: $10.1021 /$ cs200512w

3659. Tominaga $\mathrm{Y}$, Kubo T, Hosoya K. 2011. Surface modification of TiO2 for selective photodegradation of toxic compounds. Catal. Commun. 12: 785789. DOI: 10.1016/j.catcom.2011.01.021

3660. Wang HF, Yang H, Zhang LM. 2011. Temperature-sensitive molecularly imprinted microgels with esterase activity. Sci. China Chem. 54: 515-520. DOI: $10.1007 / \mathrm{s} 11426-010-4200-z$

3661. Weng ZH, Muratsugu S, Ishiguro N, Ohkoshi S, Tada M. 2011. Preparation of surface molecularly imprinted Ru-complex catalysts for asymmetric transfer hydrogenation in water media. Dalton Trans. 40: 2338-2347.

3662. Xie YT, Li HB, Wang L, Liu Q, Shi Y, Zheng HY, Zhang M, Wu YT, Lu B. 2011. Molecularly imprinted polymer microspheres enhanced biodegradation of bisphenol A by acclimated activated sludge. Water Res. 45: 1189-1198. DOI: 10.1016/j.watres.2010.11.014

3663. Sagawa T, Togo K, Miyahara C, Ihara H, Ohkubo K. 2004. Rateenhancement of hydrolysis of long-chain amino acid ester by cross-linked polymers imprinted with a transition-state analogue: evaluation of imprinting effect in kinetic analysis. Anal. Chim. Acta 504: 37-41. DOI: 10.1016/S00032670(03)00945-0

3664. Aït-Haddou H, Leeder SM, Gagné MR. 2004. Amino-acid containing metallomonomers copolymerized into porous organic polymers: applicability 
to allylic alkylation catalysis. Inorg. Chim. Acta 357: 3854-3864. DOl: 10.1016/j.ica.2004.04.017

3665. Cheng ZY, Zhang LW, Li YZ. 2004. Synthesis of an enzyme-like imprinted polymer with the substrate as the template, and its catalytic properties under aqueous conditions. Chem. Eur. J. 10: 3555-3561. DOI: 10.1002/chem.200305370

3666. Takeuchi T, Ugata S, Masuda S, Matsui J, Yane T, Takase M. 2004. Atrazine transformation using synthetic enzymes prepared by molecular imprinting. Org. Biomol. Chem. 2: 2563-2566. DOI: 10.1039/b407002j

3667. Takeuchi T, Ugata S, Masuda S, Matsui J, Yane T, Takase M. 2004. Atrazine transformation using synthetic enzymes prepared by molecular imprinting (vol 2, pg 2563, 2004). Org. Biomol. Chem. 2: 2884.

3668. Karakhanov EA, Maksimov AL, Zhuchkova AY, Filippova TY, Karapetyan LM. 2005. Molecular imprinting as a method for the creation of cyclodextrinbased supramolecular catalysts used in the Wacker oxidation of unsaturated compounds. Pet. Chem. 45: 79-86.

3669. Santos WJR, Lima PR, Tarley CRT, Kubota LT. 2009. Synthesis, Characterization and Kinetics of Catalytically Active Molecularly Imprinted Polymers for the Selective Recognition of 4-Aminophenol. J. Braz. Chem. Soc. 20: 820-825. DOI: 10.1590/S0103-50532009000500003

3670. Burri E, Severin K. 2006. Molecular imprinting with ruthenium porphyrin catalysts. Chimia 60: 182-184. DOI: 0.2533/000942906777674868

3671. Hedin-Dahlström J, Rosengren-Holmberg JP, Legrand S, Wikman S, Nicholls IA. 2006. A class II aldolase mimic. J. Org. Chem. 71: 4845-4853. DOI: $10.1021 /$ jo060608b 
3672. Wu XY, Shimizu KD. 2006. Development of molecularly imprinted polymers as catalysts in selective solid-state [2+2] photodimerization. Polym. Prepr. 47: 328.

3673. Kulkarni MG. 1996. Pendent Chain Linked Delivery Systems: From Concept to Practice. In Proceedings of the Fifteenth Southern Biomedical Engineering Conference, IEEE: 241-244. DOI: 10.1109/SBEC.1996.493160

3674. Alvarez-Lorenzo C, Concheiro A. 2004. Molecularly imprinted polymers for drug delivery. J. Chromatogr. $B$ 804: 231-245. DOI: 10.1016/j.jchromb.2003.12.032

3675. Cai WS, Gupta RB. 2004. Molecularly-imprinted polymers selective for tetracycline binding. Sep. Purif. Technol. 35: 215-221. DOI: 10.1016/S13835866(03)00143-6

3676. Ciardelli G, Cioni B, Cristallini C, Barbani N, Silvestri D, Giusti P. 2004. Acrylic polymeric nanospheres for the release and recognition of molecules of clinical interest. Biosens. Bioelectron. 20: 1083-1090. DOI: 10.1016/j.bios.2004.06.028

3677. Hiratani H, Alvarez-Lorenzo C. 2004. The nature of backbone monomers determines the performance of imprinted soft contact lenses as timolol drug delivery systems. Biomaterials 25: 1105-1113. DOI: 10.1016/S01429612(03)00622-7

3678. Hiratani H, Fujiwara A, Tamiya Y, Mizutani Y, Alvarez-Lorenzo C. 2005. Ocular release of timolol from molecularly imprinted soft contact lenses. Biomaterials 26: 1293-1298. DOI: 10.1016/j.biomaterials.2004.04.030

3679. Hiratani H, Mizutani Y, Alvarez-Lorenzo C. 2005. Controlling drug release from imprinted hydrogels by modifying the characteristics of the imprinted cavities. Macromol. Biosci. 5: 728-733. DOI: 10.1002/mabi.200500065 
3680. Kotrotsiou O, Kotti K, Dini E, Kammona O, Kiparissides C. 2005. Nanostructured materials for selective recognition and targeted drug delivery. J. Phys. Conf. Ser. 10: 281-284. DOI: 10.1088/1742-6596/10/1/069

3681. Piletska EV, Turner NW, Turner APF, Piletsky SA. 2005. Controlled release of the herbicide simazine from computationally designed molecularly imprinted polymers. J. Controlled Release 108: 132-139. DOI: 10.1016/j.jconrel.2005.07.016

3682. Alvarez-Lorenzo C, Yanez F, Barreiro-Iglesias R, Concheiro A. 2006. Imprinted soft contact lenses as norfloxacin delivery systems. J. Controlled Release 113: 236-244. DOI: 10.1016/j.jconrel.2006.05.003

3683. Bodhibukkana C, Srichana T, Kaewnopparat S, Tangthong N, Bouking P, Martin GP, Suedee R. 2006. Composite membrane of bacterially-derived cellulose and molecularly imprinted polymer for use as a transdermal enantioselective controlled-release system of racemic propranolol. $\mathrm{J}$. Controlled Release 113: 43-56. DOI: 10.1016/j.jconrel.2006.03.007

3684. Bögershausen A, Pas SJ, Hill AJ, Koller H. 2006. Drug release from selfassembled inorganic-organic hybrid gels and gated porosity detected by positron annihilation lifetime spectroscopy. Chem. Mater. 18: 664-672. DOI: $10.1021 / \mathrm{cm} 0518590$

3685. Duarte ARC, Casimiro T, Aguiar-Ricardo A, Simplicio AL, Duarte CMM. 2006. Supercritical fluid polymerisation and impregnation of molecularly imprinted polymers for drug delivery. J. Supercrit. Fluids 39: 102-106. DOI: 10.1016/j.supflu.2006.01.013

3686. Kaffashi B, Zandieh A, Khadiv-Parsi P. 2006. Drug release study of systems containing the tragacanth and collagen composite: Release characterization and viscoelastic measurements. Macromol. Symp. 239: 120-129. DOI: 10.1002/masy.200690088 
3687. Ki CD, Chang JY. 2006. Preparation of a molecularly imprinted polymeric nanocapsule with potential use in delivery applications. Macromolecules 39: 3415-3419. DOI: 10.1021/ma052480q

3688. Mitchell-Koch JT, Padden KM, Borovik AS. 2006. Modification of immobilized metal complexes toward the design and synthesis of functional materials for nitric oxide delivery. J. Polym. Sci. A,Polym. Chem. 44: 22822292. DOI: 10.1002/pola.21314

3689. Ali M, Horikawa S, Venkatesh S, Saha J, Hong JW, Byrne ME. 2007. Zeroorder therapeutic release from imprinted hydrogel contact lenses within in vitro physiological ocular tear flow. J. Controlled Release 124: 154-162. DOI: 10.1016/j.jconrel.2007.09.006

3690. Puoci F, lemma F, Cirillo G, Picci N, Matricardi P, Alhaique F. 2007. Molecularly imprinted polymers for 5 -fluorouracil release in biological fluids. Molecules 12: 805-814. DOI: 10.3390/12040805

3691. Ulubayram K, Tunc Y, Baykara E. 2007. Molecularly imprinted acrylic-based microspheres for colonic delivery of 5-aminosalicylic acid. J. Optoelectron. Adv. Mater. 9: 3479-3483.

3692. Ulubayram K, Tunc Y, Baykara E. 2007. Molecularly imprinted acrylic-based microspheres for colonic delivery of 5-aminosalicylic acid. Optoelectron. Adv. Mater. Rapid Commun. 1: 312-316.

3693. Venkatesh S, Sizemore SP, Byrne ME. 2007. Biomimetic hydrogels for enhanced loading and extended release of ocular therapeutics. Biomaterials 28: 717-724. DOI: 10.1016/j.biomaterials.2006.09.007

3694. Byrne ME, Hilt JZ, Peppas NA. 2008. Recognitive biomimetic networks with moiety imprinting for intelligent drug delivery. J. Biomed. Mater. Res. Part A 84A: 137-147. DOI: 10.1002/jbm.a.31443 
3695. Gong CB, Wong KL, Lam MHW. 2008. Photoresponsive Molecularly Imprinted Hydrogels for the Photoregulated Release and Uptake of Pharmaceuticals in the Aqueous Media. Chem. Mater. 20: 1353-1358. DOI: $10.1021 / \mathrm{cm} 7019526$

3696. Jantarat C, Tangthong N, Songkro S, Martin GP, Suedee R. 2008. SPropranolol imprinted polymer nanoparticle-on-microsphere composite porous cellulose membrane for the enantioselectively controlled delivery of racemic propranolol. Int. J. Pharm. 349: 212-225. DOI: 10.1016/j.ijpharm.2007.07.030

3697. Puoci F, Cirillo G, Curcio M, lemma F, Parisi Ol, Castiglione M, Picci N. 2008. Molecularly Imprinted Polymers for $\alpha$-Tocopherol Delivery. Drug Delivery 15: 253-258. DOI: 10.1080/10717540802006724

3698. Singh B, Chauhan N. 2008. Molecular Imprinted Polymers for use as Drug Delivery Devices: Preliminary Evaluation. J. Macromol. Sci. A 45: 776-784. DOI: 10.1080/10601320802223242

3699. Singh B, Chauhan N. 2008. Preliminary evaluation of molecular imprinting of 5-fluorouracil within hydrogels for use as drug delivery systems. Acta Biomater. 4: 1244-1254. DOI: 10.1016/j.actbio.2008.03.017

3700. Suedee R, Bodhibukkana C, Tangthong N, Amnuaikit C, Kaewnopparat S, Srichana T. 2008. Development of a reservoir-type transdermal enantioselective-controlled delivery system for racemic propranolol using a molecularly imprinted polymer composite membrane. J. Controlled Release 129: 170-178. DOI: 10.1016/j.jconrel.2008.05.001

3701. Sumi VS, Kala R, Praveen RS, Prasada Rao T. 2008. Imprinted polymers as drug delivery vehicles for metal-based anti-inflammatory drug. Int. J. Pharm. 349: 30-37. DOI: 10.1016/j.jpharm.2007.07.017 
3702. Venkatesh S, Saha J, Pass S, Byrne ME. 2008. Transport and structural analysis of molecular imprinted hydrogels for controlled drug delivery. Eur. J. Pharm. Biopharm. 69: 852-860. DOI: 10.1016/j.ejpb.2008.01.036

3703. Ali M, Byrne ME. 2009. Controlled Release of High Molecular Weight Hyaluronic Acid from Molecularly Imprinted Hydrogel Contact Lenses. Pharm. Res. 26: 714-726. DOI: 10.1007/s11095-008-9818-6

3704. Ali M, Vaughan AD, Zhang J, Venkatesh S, Byrne ME. 2009. Templated hydrogels for combination devices: therapeutic contact lenses. In Proceedings of the Annual International Conference of the IEEE Engineering in Medicine and Biology Society. IEEE Engineering in Medicine and Biology Society.Conference, Vol. 2009, IEEE: 242-245. DOI: 10.1109/IEMBS.2009.5332862

3705. Cirillo G, lemma F, Puoci F, Parisi OI, Curcio M, Spizzirri UG, Picci N. 2009. Imprinted hydrophilic nanospheres as drug delivery systems for 5fluorouracil sustained release. J. Drug Target. 17: 72-77. DOI: $10.1080 / 10611860802455813$

3706. Kan XW, Geng ZR, Zhao Y, Wang ZL, Zhu JJ. 2009. Magnetic molecularly imprinted polymer for aspirin recognition and controlled release. Nanotechnology 20: Article No. 165601. DOI: 10.1088/0957$4484 / 20 / 16 / 165601$

3707. Kryscio DR, Peppas NA. 2009. Mimicking biological delivery through feedback-controlled drug release systems based on molecular imprinting. AlChE J 55: 1311-1324. DOI: 10.1002/aic.11779

3708. Lauten EH, Peppas NA. 2009. Intelligent drug release using molecular imprinting methods. Recognitive systems for angiotensin II. J. Drug. Deliv. Sci. Technol. 19: 391-399. 
3709. Vendamme R, Eevers W, Kaneto M, Minamizaki Y. 2009. Influence of Polymer Morphology on the Capacity of Molecularly Imprinted Resins to Release or to Retain their Template. Polym. J. 41: 1055-1066. DOI: 10.1295/polymj.PJ2009098

3710. Azodi-Deilami S, Abdouss M, Rezvaneh Seyedi S. 2010. Synthesis and characterization of molecularly imprinted polymer for controlled release of tramadol. Cent. Eur. J. Chem. 8: 687-695. DOI: 10.2478/s11532-010-0035-x

3711. Azodi-Deilami S, Abdouss M, Rezvaneh Seyedi S. 2011. Erratum to: "Synthesis and characterization of molecularly imprinted polymer for controlled release of tramadol". Cent. Eur. J. Chem. 9: 499. DOI: 10.2478/s11532-011-0025-7

3712. Braga MEM, Yañez F, Alvarez-Lorenzo C, Concheiro A, Duarte CMM, Gil $\mathrm{MH}$, de Sousa HC. 2010. Improved drug loading/release capacities of commercial contact lenses obtained by supercritical fluid assisted molecular imprinting methods. J. Controlled Release 148: e102-e104. DOI: 10.1016/j.jconrel.2010.07.077

3713. Cirillo G, Curcio M, Parisi OI, Puoci F, lemma F, Spizzirri UG, Picci N. 2010. Gastro-intestinal sustained release of phytic acid by molecularly imprinted microparticles. Pharmaceut. Dev. Technol. 15: 526-531. DOI: 10.3109/10837450903397602

3714. Cirillo G, Parisi OI, Curcio M, Puoci F, lemma F, Spizzirri UG, Picci N. 2010. Molecularly imprinted polymers as drug delivery systems for the sustained release of glycyrrhizic acid. J. Pharm. Pharmacol. 62: 577-582. DOI: 10.1211/jpp.62.05.0003

3715. del Sole R, Lazzoi MR, Vasapollo G. 2010. Synthesis of nicotinamide-based molecularly imprinted microspheres and in vitro controlled release studies. Drug Delivery 17: 130-137. DOI: 10.3109/10717541003587418 
3716. Li SJ, Tiwari A, Ge Y, Fei D. 2010. A pH-responsive, low crosslinked, molecularly imprinted insulin delivery system. Adv. Mat. Lett. 1: 4-10. DOI: 10.5185/amlett.2010.4110

3717. Suedee R, Jantarat $C$, Lindner W, Viernstein $H$, Songkro S, Srichana T. 2010. Development of a pH-responsive drug delivery system for enantioselective-controlled delivery of racemic drugs. J. Controlled Release 142: 122-131. DOI: 10.1016/j.jconrel.2009.10.011

3718. Vaughan AD, Zhang JB, Byrne ME. 2010. Enhancing therapeutic loading and delaying transport via molecular imprinting and living/controlled polymerization. AlChE J 56: 268-279. DOI: 10.1002/aic.11949

3719. Wang CY, Javadi A, Ghaffari M, Gong SQ. 2010. A pH-sensitive molecularly imprinted nanospheres/hydrogel composite as a coating for implantable biosensors. Biomaterials 31: 4944-4951. DOI: 10.1016/j.biomaterials.2010.02.073

3720. Abdouss M, Asadi E, Azodi-Deilami S, Beik-mohammadi N, Aslanzadeh SA. 2011. Development and characterization of molecularly imprinted polymers for controlled release of citalopram. J. Mater. Sci.: Mater. Med. 22: 22732281. DOI: $10.1007 / \mathrm{s} 10856-011-4395-3$

3721. Anirudhan TS, Sandeep S. 2011. Synthesis and characterization of molecularly imprinted polymer of $\mathrm{N}$-maleoylchitosan-grafted-2-acrylamido-2methylpropanesulfonic acid and its controlled delivery and recognition of bovine serum albumin. Polym. Chem. 2: 2052-2061. DOI: 10.1039/C1PY00059D

3722. Azodi-Deilami S, Abdouss M, Javanbakht M. 2011. The Syntheses and Characterization of Molecularly Imprinted Polymers for the Controlled Release of Bromhexine. Appl. Biochem. Biotechnol. 164: 133-147. DOI: 10.1007/s12010-010-9121-y 
3723. Chu HJ, Wei HL, Zhu J, Nong CP. 2011. Synthesis and Performance of Molecular Imprinted Polymers as Drug Release Controller. In Mechanical and Electronics Engineering III, Vol. 130-134, Zhao H (ed). Trans Tech Publications: Switzerland; 446-449.

DOI: 10.4028/www.scientific.net/AMM.130-134.446

3724. da Silva MS, Viveiros R, Morgado PI, Aguiar-Ricardo A, Correia IJ, Casimiro T. 2011. Development of 2-(dimethylamino)ethyl methacrylate-based molecular recognition devices for controlled drug delivery using supercritical fluid technology. Int. J. Pharm. 416: 61-68. DOI: 10.1016/j.jpharm.2011.06.004

3725. da Silva MS, Nobrega FL, Aguiar-Ricardo A, Cabrita EJ, Casimiro T. 2011. Development of molecularly imprinted co-polymeric devices for controlled delivery of flufenamic acid using supercritical fluid technology. J. Supercrit. Fluids 58: 150-157. DOI: 10.1016/j.supflu.2011.05.010

3726. Esfandyari-Manesh M, Javanbakht M, Atyabi F, Mohammadi A, Mohammadi S, Akbari-adergani B, Dinarvand R. 2011. Dipyridamole recognition and controlled release by uniformly sized molecularly imprinted nanospheres. Mater. Sci. Eng.: C 31: 1692-1699. DOI: 10.1016/j.msec.2011.07.019

3727. Javanbakht M, Mohammadi S, Esfandyari-Manesh M, Abdouss M. 2011. Molecularly imprinted polymer microspheres with nanopore cavities prepared by precipitation polymerization as new carriers for the sustained release of dipyridamole. J. Appl. Polym. Sci. 119: 1586-1593. DOI: 10.1002/app.32798

3728. Mahkam M, Poorgholy N. 2011. Imprinted polymers as drug delivery vehicles for anti-inflammatory drugs. Nature and Science 9: 163-168.

3729. Pavlyuchenko VN, Ivanchev SS, Primachenko ON, Khaikin SYa, Danilichev VF, Proshina VS, Trunov VA, Lebedev VT, Kul'velis Y. 2011. Polymer 
hydrogels with the memory effect for immobilization of drugs. Polym. Sci. Ser. A 53: 323-335. DOI: 10.1134/S0965545X11040080

3730. Ribeiro A, Veiga F, Santos D, Torres-Labandeira JJ, Concheiro A, AlvarezLorenzo C. 2011. Bioinspired Imprinted PHEMA-Hydrogels for Ocular Delivery of Carbonic Anhydrase Inhibitor Drugs. Biomacromolecules 12: 701-709. DOI: 10.1021/bm101562v

3731. Ribeiro A, Veiga F, Santos D, Torres-Labandeira JJ, Concheiro A, AlvarezLorenzo C. 2011. Receptor-based biomimetic NVP/DMA contact lenses for loading/eluting carbonic anhydrase inhibitors. J. Membr. Sci. 383: 60-69. DOI: 10.1016/j.memsci.2011.08.030

3732. Singh B, Chauhan N, Sharma V. 2011. Design of Molecular Imprinted Hydrogels for Controlled Release of Cisplatin: Evaluation of Network Density of Hydrogels. Ind. Eng. Chem. Res. 50: 13742-13751. DOI: 10.1021/ie200758b

3733. Tang Q, Gong CB, Lam M, Fu XK. 2011. Photoregulated uptake and release of drug by an organic-inorganic hybrid sol-gel material. J. Sol-Gel Sci. Technol. 59: 495-504. DOI: 10.1007/s10971-011-2518-4

3734. White CJ, McBride MK, Pate KM, Tieppo A, Byrne ME. 2011. Extended release of high molecular weight hydroxypropyl methylcellulose from molecularly imprinted, extended wear silicone hydrogel contact lenses. Biomaterials 32: 5698-5705. DOI: 10.1016/j.biomaterials.2011.04.044

3735. Yañez F, Martikainen L, Braga MEM, Alvarez-Lorenzo C, Concheiro A, Duarte CMM, Gil MH, de Sousa HC. 2011. Supercritical fluid-assisted preparation of imprinted contact lenses for drug delivery. Acta Biomater. 7: 1019-1030. DOI: 10.1016/j.actbio.2010.10.003

3736. Yañez F, Chauhan A, Concheiro A, Alvarez-Lorenzo C. 2011. Timololimprinted soft contact lenses: Influence of the template: Functional monomer 
ratio and the hydrogel thickness. J. Appl. Polym. Sci. 122: 1333-1340. DOI: 10.1002/app.34022

3737. Yin JF, Cui Y, Yang GL, Wang HL. 2010. Molecularly imprinted nanotubes for enantioselective drug delivery and controlled release. Chem. Commun. 46: 7688-7690. DOI: 10.1039/C0CC01782E

3738. Bolisay LDV, March JF, Bentley WE, Kofinas P. 2004. Separation of baculoviruses using molecularly imprinted polymer hydrogels. In Molecularly Imprinted Materials-2003, MRS Symposium Proceedings, Vol. 787, Kofinas P, Sellergren B, Roberts MJ (eds). Materials Research Society: Warrendale; 29-33. DOI: 10.1557/PROC-787-G3.1

3739. Cunliffe D, Pennadam S, Alexander C. 2004. Synthetic and biological polymers - merging the interface. Eur. Polym. J. 40: 5-25. DOI: 10.1016/j.eurpolymj.2003.10.020

3740. Egan TJ, Rodgers AL, Siele T. 2004. Nucleation of calcium oxalate crystals on an imprinted polymer surface from pure aqueous solution and urine. $\mathrm{J}$. Biol. Inorg. Chem. 9: 195-202. DOI: 10.1007/s00775-003-0517-9

3741. Huval CC, Chen X, Holmes-Farley SR, Mandeville WH, Polomoscanik SC, Sacchiero RJ, Dhal PK. 2004. Molecularly imprinted bile acid sequestrants: Synthesis and biological studies. In Molecularly Imprinted Materials-2003, MRS Symposium Proceedings, Vol. 787, Kofinas P, Sellergren B, Roberts MJ (eds). Materials Research Society: Warrendale; 85-90. DOI: 10.1557/PROC-787-G6.3

3742. Piletska E, Piletsky S, Karim K, Terpetschnig E, Turner A. 2004. Biotinspecific synthetic receptors prepared using molecular imprinting. Anal. Chim. Acta 504: 179-183. DOI: 10.1016/S0003-2670(03)00813-4 
3743. Slinchenko O, Rachkov A, Miyachi H, Ogiso M, Minoura N. 2004. Imprinted polymer layer for recognizing double-stranded DNA. Biosens. Bioelectron. 20: 1091-1097. DOI: 10.1016/j.bios.2004.06.027

3744. Wang H, Huang L, Sun Y. 2004. Preparation and characterization of theophylline crystal-imprinted polymers. Acta Polym. Sin. 288-291.

3745. Foresti ML, Alimenti GA, Ferreira ML. 2005. Interfacial activation and bioimprinting of Candida rugosa lipase immobilized on polypropylene: effect on the enzymatic activity in solvent-free ethyl oleate synthesis. Enzyme Microb. Technol. 36: 338-349. DOI: 10.1016/j.enzmictec.2004.09.012

3746. Sreenivasan K. 2005. Surface-imprinted polyurethane having affinity sites for ampicillin. Macromol. Biosci. 5: 187-191. DOI: 10.1002/mabi.200400127

3747. Venkatesh S, Sizemore SP, Byrne ME. 2005. Biomimetic recognitive polymer networks for ocular delivery of anti-histamines. Vol. 897, Materials Research Society: 81-86.

3748. Bolisay LD, Culver JN, Kofinas P. 2006. Molecularly imprinted polymers for tobacco mosaic virus recognition. Biomaterials 27: 4165-4168. DOI: 10.1016/j.biomaterials.2006.03.018

3749. Ciardelli G, Montevecchi FM, Giusti P, Silvestri D, Morelli I, Cristallini C, Vozzi G. 2006. Molecular imprinted nanostructures in biomedical applications. In Proceedings of the 8th Biennial ASME Conference on Engineering Systems Design and Analysis, Vol. 2006, American Society of Mechanical Engineers: New York; 561-567.

3750. Hayden O, Mann KJ, Krassnig S, Dickert FL. 2006. Biomimetic ABO BloodGroup Typing. Angew. Chem. Int. Edit. 45: 2626-2629. DOI: 10.1002/anie.200502857

3751. Stephenson CJ, Carroll WR, Yehl MA, Shimizu KD. 2006. Virus imprinting and reuptake in polymer films. Polym. Prepr. 47: 463. 
3752. Vitale U, Rechichi A, D'Alonzo M, Cristallini G, Barbani N, Ciardelli G, Giusti P. 2006. Selective peptide recognition with molecularly imprinted polymers in designing new biomedical devices. In Proceedings of the 8th Biennial ASME Conference on Engineering Systems Design and Analysis, Vol. 2, American Society of Mechanical Engineers: New York; 545-553.

3753. Yavuz H, Andaç M, Uzun L, Say R, Denizli A. 2006. Molecular recognition based iron removal from human plasma with imprinted membranes. International Journal of Artificial Organs 29: 900-911.

3754. Bolisay LD, Culver JN, Kofinas P. 2007. Optimization of Virus Imprinting Methods To Improve Selectivity and Reduce Nonspecific Binding. Biomacromolecules 8: 3893-3899.

3755. Esmaeili MA, Yazdanparast R. 2007. Molecularly imprinted poly $\alpha$ cyclodextrin polymer: Application in protein refolding. Biochim. Biophys. Acta,Gen. Subjects 1770: 943-950. DOI: 10.1016/j.bbagen.2007.02.007

3756. Haruki M, Konnai Y, Shimada A, Takeuchi H. 2007. Molecularly Imprinted Polymer-Assisted Refolding of Lysozyme. Biotechnol. Prog. 23: 1254-1257. DOI: $10.1021 / \mathrm{bp} 070130 \mathrm{c}$

3757. Rechichi A, Cristallini C, Vitale U, Ciardelli G, Barbani N, Vozzi G, Giusti P. 2007. New biomedical devices with selective peptide recognition properties. Part 1: Characterization and cytotoxicity of molecularly imprinted polymers. J. Cell. Mol. Med. 11: 1367-1376. DOI: 10.1111/j.1582-4934.2007.00102.x

3758. Breton F, Delépée R, Jégourel D, Deville-Bonne D, Agrofoglio LA. 2008. Selective adenosine-5'-monophosphate uptake by water-compatible molecularly imprinted polymer. Anal. Chim. Acta 616: 222-229. DOI: 10.1016/j.aca.2008.04.025

3759. Ghasemzadeh N, Nyberg F, Hjertén S. 2008. Highly selective artificial gel antibodies for detection and quantification of biomarkers in clinical samples. 
II. Albumin in body fluids of patients with neurological disorders. J. Sep. Sci. 31: 3954-3958. DOI: 10.1002/jssc.200800386

3760. Ghasemzadeh N, Nyberg F, Hjertén S. 2008. Highly selective artificial gel antibodies for detection and quantification of biomarkers in clinical samples. I. Spectrophotometric approach to design the calibration curve for the quantification. J. Sep. Sci. 31: 3945-3953. DOI: 10.1002/jssc.200800385

3761. Hjertén M, Rezeli M, Kilár F, Hjertén S. 2008. Renewable enzyme reactors based on beds of artificial gel antibodies. J. Biochem. Biophys. Meth. 70: 1188-1191. DOI: 10.1016/j.jprot.2008.01.006

3762. Yang Y, Long YY, Cao Q, Li KA, Liu F. 2008. Molecularly imprinted polymer using $\alpha$-cyclodextrin as functional monomer for the efficient recognition of bilirubin. Anal. Chim. Acta 606: 92-97. DOI: 10.1016/j.aca.2007.10.044

3763. Barbani N, Cristallini C, Rosellini E, Morini F, Roccasalvo IM, Giusti P. 2009. Intelligent molecularly imprinted scaffolds for tissue growth technology. J. Appl. Biomater. Biomech. 7: 53.

3764. Cutivet A, Schembri C, Kovensky J, Haupt K. 2009. Molecularly Imprinted Microgels as Enzyme Inhibitors. J. Am. Chem. Soc. 131: 14699-14702. DOI: $10.1021 / \mathrm{ja} 901600 \mathrm{e}$

3765. Fukazawa K, Ishihara K. 2009. Fabrication of a cell-adhesive protein imprinting surface with an artificial cell membrane structure for cell capturing. Biosens. Bioelectron. 25: 609-614. DOI: 10.1016/j.bios.2009.02.034

3766. Uzun L, Say R, Ünal S, Denizli A. 2009. Hepatitis B surface antibody purification with hepatitis B surface antibody imprinted poly(hydroxyethyl methacrylate-N-methacryloyl-I-tyrosine methyl ester) particles. $J$. Chromatogr B 877: 181-188. DOI: 10.1016/j.jchromb.2008.12.004 
3767. Xue XH, Pan J, Xie HM, Wang JH, Zhang S. 2009. Specific recognition of staphylococcus aureus by staphylococcus aureus protein A-imprinted polymers. Reac. Func. Polym. 69: 159-164. DOI: 10.1016/j.reactfunctpolym.2008.12.013

3768. Dai CL, Liu CS, Wei J, Hong H, Zhao QH. 2010. Molecular imprinted macroporous chitosan coated mesoporous silica xerogels for hemorrhage control. Biomaterials 31: 7620-7630. DOI: 10.1016/j.biomaterials.2010.06.049

3769. Derazshamshir A, Baydemir G, Andac M, Say RI, Galaev IY, Denizli A. 2010. Molecularly Imprinted PHEMA-Based Cryogel for Depletion of Hemoglobin from Human Blood. Macromol. Chem. Phys. 211: 657-668. DOI: 10.1002/macp.200900425

3770. Rosellini E, Barbani N, Giusti P, Ciardelli G, Cristallini C. 2010. Molecularly imprinted nanoparticles with recognition properties towards a laminin $\mathrm{H}-\mathrm{Tyr}$ Ile-Gly-Ser-Arg-OH sequence for tissue engineering applications. Biomed. Mater. 5: Art. No. 065007. DOI: 10.1088/1748-6041/5/6/065007

3771. Rosellini E, Barbani N, Giusti P, Ciardelli G, Cristallini C. 2010. Novel bioactive scaffolds with fibronectin recognition nanosites based on molecular imprinting technology. J. Appl. Polym. Sci. 118: 3236-3244. DOI: 10.1002/app.32622

3772. Silvestri D, Cristallini C, Domenichini M, Gagliardi M, Giusti P. 2010. Non Conventional Surface Functionalization of Porous Poly-[epsilon]Caprolactone Scaffolds Using Bioactive Molecularly Imprinted Nanospheres. Biomed. Eng. Appl. Basis Commun. 22: 337-349. DOI: $10.4015 / \mathrm{S} 1016237210002109$

3773. Vozzi G, Morelli I, Vozzi F, Andreoni C, Salsedo E, Morachioli A, Giusti P, Ciardelli G. 2010. SOFT-MI: A novel microfabrication technique integrating 
soft-lithography and molecular imprinting for tissue engineering applications. Biotechnol. Bioeng. 106: 804-817. DOI: 10.1002/bit.22740

3774. Khurshid SS, Schmidt CE, Peppas NA. 2011. Optimization of molecularly imprinted polymers of serotonin for biomaterial applications. J. Biomater. Sci. Polym. Ed. 22: 343-362. DOI: 10.1163/092050610X486955

3775. Piletska EV, Stavroulakis G, Larcombe LD, Whitcombe MJ, Sharma A, Primrose S, Robinson GK, Piletsky SA. 2011. Passive Control of Quorum Sensing: Prevention of Pseudomonas aeruginosa Biofilm Formation by Imprinted Polymers. Biomacromolecules 12: 1067-1071. DOI: $10.1021 / \mathrm{bm} 101410 \mathrm{q}$

3776. Zhang XF, Ding CL, Liu H, Liu LH, Zhao CQ. 2011. Protective effects of ionimprinted chitooligosaccharides as uranium-specific chelating agents against the cytotoxicity of depleted uranium in human kidney cells. Toxicology 286: 75-84. DOI: 10.1016/j.tox.2011.05.011

3777. Piletsky SA, Piletska EV, Sergeyeva TA, Nicholls IA, Weston D, Turner APF. 2006. Synthesis of Biologically Active Molecules by Imprinting Polymerisation. Biopolym. Cell 22: 63-68.

3778. Hoshino Y, Koide H, Urakami T, Kanazawa H, Kodama T, Oku N, Shea KJ. 2010. Recognition, Neutralization, and Clearance of Target Peptides in the Bloodstream of Living Mice by Molecularly Imprinted Polymer Nanoparticles: A Plastic Antibody. J. Am. Chem. Soc. 132: 6644-6645. DOI: $10.1021 / \mathrm{ja} 102148 \mathrm{f}$

3779. Lauterbur PC. 2008. The Spontaneous Development of Biology from Chemistry. Astrobiology 8: 3-8. DOI: 10.1089/ast.2008.1109 